\title{
Continuous Variable Cluster State Computation
}

\author{
Larsen, Mikkel Vilsbøll
}

Publication date:

2021

Document Version

Publisher's PDF, also known as Version of record

Link back to DTU Orbit

Citation (APA):

Larsen, M. V. (2021). Continuous Variable Cluster State Computation. Department of Physics, Technical University of Denmark.

\section{General rights}

Copyright and moral rights for the publications made accessible in the public portal are retained by the authors and/or other copyright owners and it is a condition of accessing publications that users recognise and abide by the legal requirements associated with these rights.

- Users may download and print one copy of any publication from the public portal for the purpose of private study or research.

- You may not further distribute the material or use it for any profit-making activity or commercial gain

- You may freely distribute the URL identifying the publication in the public portal

If you believe that this document breaches copyright please contact us providing details, and we will remove access to the work immediately and investigate your claim 


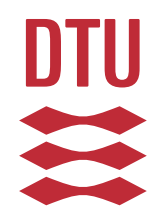

\section{Continuous Variable Cluster State Computation}

$\mathrm{PhD}$ thesis

Mikkel Vilsbøll Larsen

Supervised by Senior researcher Jonas Schou Neergaard-Nielsen Professor Ulrik Lund Andersen

DTU Physics Technical University of Denmark 
A thesis submitted in partial fulllment of the requirements for the degree of Doctor of Philosophy

Title: Continuous variable cluster state computation

Author: Mikkel Vilsbøll Larsen

Supervisors: Senior researcher Jonas Schou Neergaard-Nielsen Professor Ulrik Lund Andersen

Period: $\quad$ October 1, 2017 - January 31, 2021

Department: Technical University of Denmark, DTU Physics

Section for Quantum Physics and Information Technology (QPIT) 


\section{Abstract}

In measurement-based quantum computation (MBQC), or cluster state computation, gates are implemented on a multi-mode entangled cluster state by projective measurements. In the optical continuous variable $(\mathrm{CV})$ regime, such cluster state can be deterministically generated while a class of measurements is efficiently implemented by homodyne detection. This immediately allows for the deterministic implementation of Gaussian gates in a scalable optical computation platform.

In this thesis, work towards the realization of CV MBQC is presented. In MBQC, a cluster state of at least two dimensions is required, and in this thesis, the generation of such two-dimensional (2D) cluster state is proposed and experimentally demonstrated. Assuming the availability of Gottesman-Kitaev-Preskill (GKP) encoded input qubits, a universal computation scheme for the $2 \mathrm{D}$ cluster state is proposed, and noise analysis of the computation scheme is carried out and compared to other computation schemes on $2 \mathrm{D}$ cluster states. Following the proposed computation scheme, a universal Gaussian gate set is implemented on the generated cluster state by projective measurements, and to demonstrate the programmability, gates are combined into a small quantum circuit. Gate noise, caused by finite squeezing, is characterized, and the requirements for faulttolerant computation are discussed. Finally, a new computation scheme is proposed where gates are implemented on a three-dimensional cluster state allowing topological error correction. Taking finite squeezing in both the cluster state generation and approximate GKP-states into account, fault-tolerant computation is shown to be possible by simulation when the squeezing level is above a certain squeezing threshold.

To aid the experimental implementations, the focus throughout this thesis is on temporal encoding where resources are reused in time minimizing the required spatial resources, i.e. time multiplexing. To this end, the thesis starts with a demonstration of two-mode squeezed state generation in two spatial modes from a single time-multiplexed squeezed light source using optical switching and delay. In this demonstration, multiple experimental techniques are developed, including efficient free-space to fiber coupling, in-fiber phase control, and fiber-based homodyne detection, each of which plays important roles in the experimental demonstration of the following cluster state generation and gate implementation. 


\section{Resumé (danish)}

I målebaseret kvanteberegning (MBQC), eller klyngetilstandsberegning, implementeres gates på en multimode sammenvinklet klyngetilstand via projektive målinger. I det optiske kontinuertvariable $(\mathrm{CV})$ regime kan sådan klyngetilstand genereres deterministisk, mens en klasse af målinger effektivt implementeres med homodyne detektorer. Dette giver straks mulighed for deterministisk implementering af Gaussiske gates i en skalerbar optisk beregningsplatform.

I denne afhandling præsenteres arbejde mod realisering af CV MBQC. I MBQC kræves en klyngetilstand på mindst to dimensioner, og i denne afhandling foreslås og demonstreres genereringen af en sådan todimensionel (2D) klyngetilstand eksperimentelt. Under forudsætning af tilgængelige Gottesman-Kitaev-Preskill (GKP)-kodede input qubits foreslås en universelt beregningsprotokol for 2D-klyngetilstanden, og støjanalyse af beregningsprotokollen udføres og sammenlignes med andre beregningsprotokoller på 2D-klyngetilstande. Med den foreslåede beregningsprotokol implementeres et universelt Gaussisk gate-sæt på den genererede klyngetilstand ved projektive målinger, og for at demonstrere programmerbarheden, kombineres gates til et lille kvantekredsløb. Gatestøj forårsaget af begrænset klemning er karakteriseret, og kravene til fejltolerant beregning diskuteres. Endelig foreslås en ny beregningsprotokollen, hvor gates implementeres i en tredimensionel klyngetilstand, der muligg ør topologisk fejlkorrektion. Ved medregning af begrænset klemning i både klyngetilstandsgenerering og approksimative GKP-tilstande, vises fejltolerant beregning ved simulering at være mulig, når klemmeniveauet er over en bestemt klemmetærskel.

For at simplificere de eksperimentelle implementeringer, er der i hele denne afhandling fokuseret på tidsmæssig kodning, hvor ressourcer genbruges i tide og minimerer de nødvendige rumlige ressourcer, dvs. tidslig multiplexing. Til dette formål starter afhandlingen med en demonstration af to-mode klemte tilstand i to rumlige modes fra en enkelt tidslig multiplexet klemt lyskilde, genereret vha. en optisk switch og optisk forsinkelse. I denne demonstration udvikles flere eksperimentelle teknikker, herunder effektiv kobling fra fritgående lys til optisk fiber, fasekontrol i fiber og detektering med fiberbaseret homodyne detektorer, som hver især spiller vigtige roller i den eksperimentelle demonstration af følgende klyngetilstandsgenerering og gateimplementering. 


\section{Acknowledgments}

The work of this thesis is carried out in the QPIT (Quantum Physics and Information Technology) group at DTU Physics, supported by the Danish National Research Foundation, and was supervised by group leader professor Ulrik L. Andersen and senior researcher Jonas S. Neergaard-Nielsen. I joined the group for my master project, and in 2017, Ulrik gave me the opportunity to continue my work as a PhD student for which I am greatly thankful. QPIT is a diverse group of many research topics within quantum mechanics, driven by a large and diverse group of people representing every corner of the world. It has been inspiring and great fun working in the QPIT group for the last 4 years. Here you can always find people of opposite opinions to discuss everything from quantum physics to topics I will not mention here, and you can count on some social activity to balance the lab-life.

Besides thanks to everyone in the QPIT group, great thanks goes to Ulrik who has been open for me to follow research directions I find most interesting and supervising me on the way, and to Jonas for day-to-day supervision where I always can count on help and feedback whenever needed. Ulrik and Jonas have made it easy for me to stay motivated continuously throughout my 4 years in QPIT making my research both fun and rewarding. I also want to thank the QPIT administrator, Tine. Tine usually knows the paths to solutions of problems not related to physics, and I appreciate her help with paperwork and other DTU related things that at times are harder than quantum mechanics.

Special thanks go to office mates, lab colleagues, and friends in QPIT. While Casper and Xueshi have been greatly involved in my experimental work, they are also good friends outside the lab, and I have enjoyed being able to spend both work and private time with you. Jens is a great friend as well who, without failure, contributes with joy every day. Another outstanding friend in QPIT is Shuro. I highly appreciate the time spent with you including discussion, countless dinners, and beers, both within and outside Denmark. Thank you all. I also thank Subhashish and Abhi for joining me in lab in the final time of my $\mathrm{PhD}$. I welcome your interest in my experimental work, and I hope you will enjoy it as I did.

Of people outside the QPIT group, I want to thank Peter van Loock for hosting me on my external stay in his group at the Johannes Gutenberg University of Mainz. I want to thank Rafael N. Alexander for useful discussions and explanations, as well as Christopher Chamberland and Kyungjoo Noh for collaboration.

Finally, I want to thank friends and family. In particular, I sent my deepest love and appreciation to Yini who supports and, to some extent, understands my dedication to my studies, while she at the same time brings me back to an enjoyable real life. Thank you.

Mikkel Vilsbøll Larsen 


\section{Contents}

$\begin{array}{ll}\text { Abstract } & \text { iii }\end{array}$

Resumé (danish) $\quad$ v

$\begin{array}{lc}\text { Acknowledgments } & \text { vii }\end{array}$

1 Introduction $\quad 1$

1.1 Thesis structure . . . . . . . . . . . . . . . . . . . . . . 4

2 Fiber coupled EPR-state generation using a single temporally multiplexed squeezed light source $\quad \mathbf{5}$

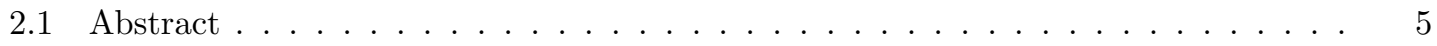

2.2 Introduction . . . . . . . . . . . . . . . . . . . 6

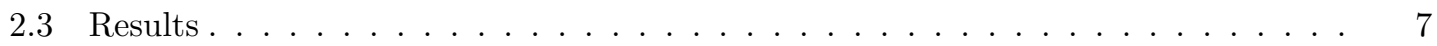

2.3.1 Experimental setup . . . . . . . . . . . . . . . . . . . . 7

2.3 .2 Experimental results . . . . . . . . . . . . . . . . . 8

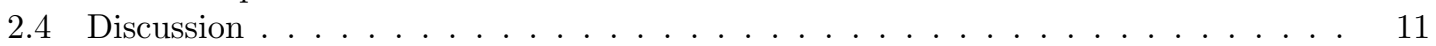

2.5 Methods . . . . . . . . . . . . . . . . . . . . . . . . 12

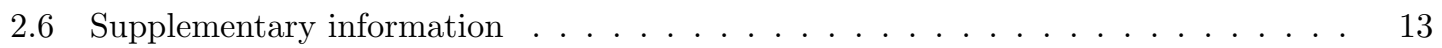

2.6.1 Quadrature relations . . . . . . . . . . . . . . 13

2.6.2 Experimental methods . . . . . . . . . . . . . . . . . . . . . . . . . . . . . . . . . . 16

2.6.3 Temporal data processing . . . . . . . . . . . . . . . . . . . . . . . . . . . . . . . . 16

2.6.4 Sources of noise and theoretical prediction . . . . . . . . . . . . . . 18

2.6.5 Squeezing spectrum with seed beam noise . . . . . . . . . . . . . 20

2.6 .6 Bound on covariances . . . . . . . . . . . . . . . . . 23

3 Deterministic generation of a two-dimensional cluster state $\quad 27$

3.1 Abstract ............................ . . 27

3.2 Main text . . . . . . . . . . . . . . . . . . . . . . . . 28

3.3 Methods . . . . . . . . . . . . . . . . . . . . . . . . 32

3.4 Suppl. Inf.: Theory on cluster state . . . . . . . . . . . . . . . . . 33

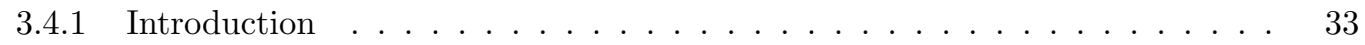

3.4.1.1 Approximate cluster states . . . . . . . . . . . . . . . 34

3.4.1.2 $\mathcal{H}$-graph states . . . . . . . . . . . . . . . . . . . . . . . . . . . . . . 35

3.4.2 Cluster state generation . . . . . . . . . . . . . . . . . . . . . . . . . . . . . . . .

3.4.2.1 EPR-state generation . . . . . . . . . . . . . 36

3.4.2.2 1D cluster states . . . . . . . . . . . . . . . . . . . . . . . . . . . . . . . . . 36

$3.4 .2 .32 \mathrm{D}$ cluster states . . . . . . . . . . . . . . . . . . . . . . . . . . . . . 37

3.4 .3 Nullifiers . . . . . . . . . . . . . . . . . . . . . . . . . . . . . . . . . . . . . . . . . . .

3.4.4 Cluster state computation . . . . . . . . . . . . . . . . . . . . . . 42

3.5 Suppl. Inf.: Inseparability criterion . . . . . . . . . . . . . . . . . . . . . . . . . . . . . . . . . . . .

3.6 Suppl. Inf.: Experimental setup . . . . . . . . . . . . . . . . . . . . . 47 
3.6.1 Efficiency and phase stability . . . . . . . . . . . . . . . . 47

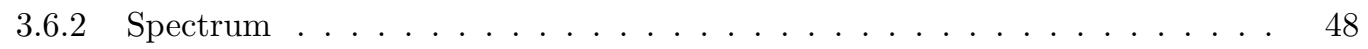

3.6 .3 Temporal mode function . . . . . . . . . . . . . . . . . . 50

3.7 Suppl. Inf.: Results . . . . . . . . . . . . . . . . . . . 51

4 Architecture and noise analysis of continuous-variable quantum gates using twodimensional cluster states $\quad 59$

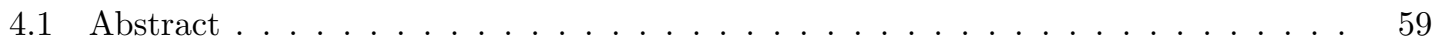

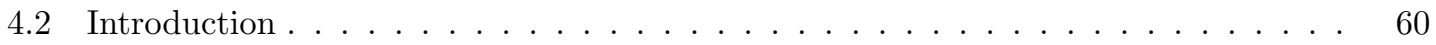

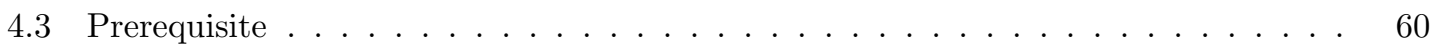

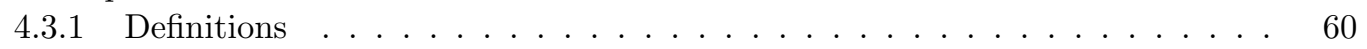

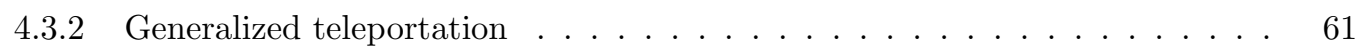

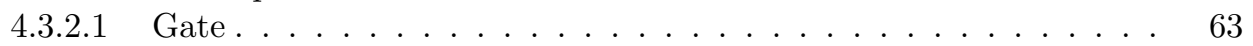

4.3.2.2 Gate noise . . . . . . . . . . . . . . . . . . . . . . . . . 63

4.3.2.3 Displacement by-product . . . . . . . . . . . . . . . 64

4.3.2.4 Wigner function representation . . . . . . . . . . . . 64

4.3 .3 Error correction . . . . . . . . . . . . . . . . . . 65

4.4 Double bilayer square lattice . . . . . . . . . . . . . . . . . . . . 66

4.4.1 Efficient computation scheme . . . . . . . . . . . . . . 67

4.4 .2 Gate noise analysis . . . . . . . . . . . . . . . . . . . . . 69

4.4.3 Variable control mode basis . . . . . . . . . . . . . . . . . 73

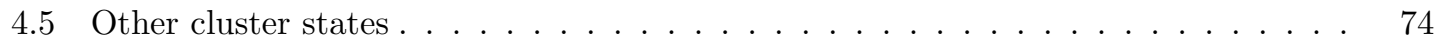

4.5.1 Bilayer square lattice . . . . . . . . . . . . . . . . . . . . . . . . . . . . . . . . . . . . . . . . 74

4.5.2 Modified bilayer square lattice . . . . . . . . . . . . . . . . . . 77

4.5.3 Quad-rail lattice ..................... . . . 80

4.6 Discussion . . . . . . . . . . . . . . . . . . . . 83

4.6 .1 Cluster state comparison _.................. 83

4.6.2 Towards universality and fault-tolerance . . . . . . . . . . . . 85

4.7 Conclusion . . . . . . . . . . . . . . . . . . . 87

4.8 Appendix: Calculation of quadrature tansformations . . . . . . . . . . . . . . 88

4.9 Appendix: Wigner function transformations . . . . . . . . . . . . . . . . . 90

4.10 Appendix: Cluster state comparison cheat sheet . . . . . . . . . . . . . . 91

5 Deterministic multi-mode gates on a scalable photonic quantum computing plat$\begin{array}{ll}\text { form } & 93\end{array}$

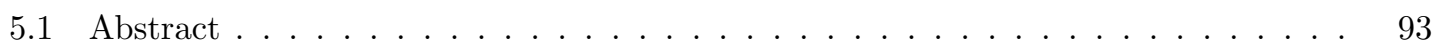

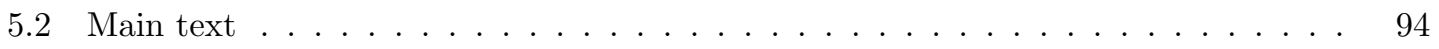

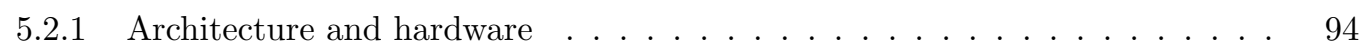

5.2 .2 Computation scheme . . . . . . . . . . . . . . . . . 95

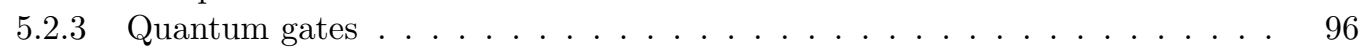

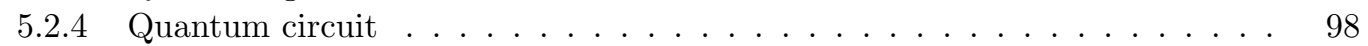

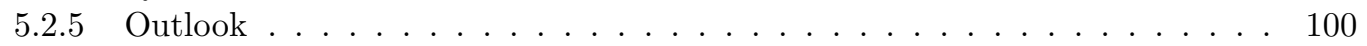

5.3 Suppl. Inf.: Experimental setup . . . . . . . . . . . . . . . . . . . 100

5.4 Suppl. Inf.: Computation scheme . . . . . . . . . . . . . . . . . . 102

5.4 Single mode gates . . . . . . . . . . . . . . . . . . . . . . . . . . . . . . . . . 105

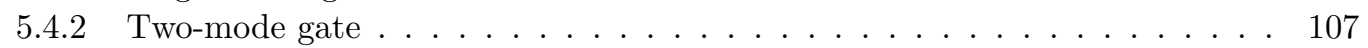

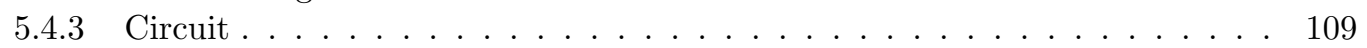

5.5 Suppl. Inf.: Gate tomography . . . . . . . . . . . . . . . . . . . . . . 113

5.5.1 Single-mode gates . . . . . . . . . . . . . . . . . . . . . . . . . . . . . . . . . . . . . . . . . . . .

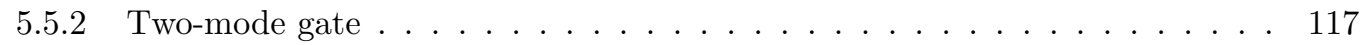

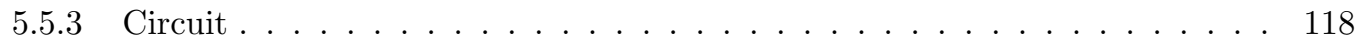

5.6 Suppl. Inf.: Gate noise . . . . . . . . . . . . . . . . . . . . 119

5.6.1 Gate noise in optimal settings . . . . . . . . . . . . . . . . 120 
6 A fault-tolerant continuous-variable measurement-based quantum computation $\begin{array}{lr}\text { architecture } & 123\end{array}$

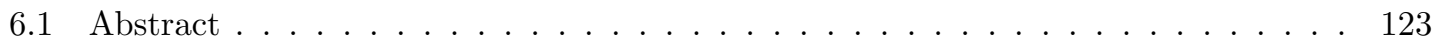

6.2 Introduction . . . . . . . . . . . . . . . . . . . . . . . . . . 124

6.3 Computation scheme . . . . . . . . . . . . . . . . . . 125

6.4 GKP quadrature correction . . . . . . . . . . . . . . . . . . . 128

6.5 Surface code . . . . . . . . . . . . . . . . . . . . . . 130

6.5.1 Implementation of the rotated surface code . . . . . . . . . . . . . . 131

6.5.2 Simulation results . . . . . . . . . . . . . . . . . 134

6.6 Discussion and conclusion . . . . . . . . . . . . . . . . . 135

6.7 Appendix: Gates by projective measurements . . . . . . . . . . . . . . . 137

6.8 Appendix: Simulation . . . . . . . . . . . . . . . . . . . . . 139

7 Conclusion $\quad 143$

7.1 Outlook ............................. 144

$\begin{array}{lr}\text { A Optical table } & 147\end{array}$

B Phase locking in 2D cluster state generation $r$

$\begin{array}{ll}\text { C Waveform optimization for gate implentation } & 153\end{array}$

Bibliography $r$ 


\section{Chapter 1}

\section{Introduction}

Since proposed in 2001 by Raussendorf and Briegel [1], measurement-based quantum computation (MBQC) has been a viable strategy towards scalable quantum computation. In MBQC, gates are implemented by local projective measurements on a multi-mode entangled cluster state, circumventing the coherent dynamics required in traditional gate-based quantum computation. The principle is sketched in Fig. 1.1a,b. In Fig. 1.1b a cluster state is prepared with nodes corresponding to qubits prepared in $|+\rangle \propto|0\rangle+|1\rangle$ states and edges corresponding to entangling controlled-Z gates, $\hat{C}_{Z}=|0\rangle\langle 0|\otimes \hat{I}+| 1\rangle\langle 1| \otimes \hat{Z}$. Here, $\hat{I}$ and $\hat{Z}$ are the identity and Pauli-Z operators, respectively. Multi-qubit input states are then connected to the cluster state, possibly by controlled-Z gates or other entangling gates. By projective measurements, the input states are teleported through the entangled cluster state, and depending on the measurement basis settings, a desired quantum algorithm (Fig. 1.1a) is implemented on the input states when teleported. As a result, all the coherent dynamics of implementing an algorithm is packed away in the preparation of the multi-mode entangled cluster state. Given the preparation of a universal cluster state, this approach has the advantage that the cluster state is independent on the algorithm. We can then focus on preparing a high-quality cluster state while implementing different algorithms by local measurements becomes easy. Here, a universal cluster state refers to a cluster state of some structure and topology that allows for implementing arbitrary algorithms by local measurements. To this, a cluster state of at least two dimensions is required: One dimension for encoding the input information, and one dimension for encoding the desired algorithm. Finally, the output states may be measured as well to reveal the computation outcome.

In 2006, Menicucci et al. proposed MBQC in the continuous variable (CV) regime [2]. In the $\mathrm{CV}$ regime, qubits are replaced by bosonic qumodes, or just modes, each being a harmonic oscillator. Each node of the cluster state in Fig. 1.1b is then prepared in an eigenstate of the momentum quadrature operator, $\hat{p}$, while edges correspond to the $\mathrm{CV}$ version of the controlled- $\mathrm{Z}$ gate, $\hat{C}_{Z}=e^{i \hat{x} \otimes \hat{x}}$ with $\hat{x}$ being the position quadrature. Similar to before, input states are teleported through the cluster state by projective measurements with an applied algorithm dictated by the measurement basis settings. In practice, this approach has advantages on optical platforms where approximate CV cluster states can be deterministically generated, and a group of measurements, specifically homodyne measurements measuring in the quadrature bases, is efficiently implemented. Here, an approximate cluster state refers to finite squeezing in the cluster state preparation: The momentum quadrature eigenstates are non-physical since they require infinite squeezing and thus infinite energy. In approximate cluster states, the ideal quadrature eigenstates are replaced by momentum squeezed states, and the approximate cluster state goes towards an ideal cluster state for increasing squeezing levels. The price of using an approximate cluster state for computation is computation noise caused by the finite squeezing, which eventually leads to computation errors. Similar to other platforms, including gate-based computation, fortunately, such errors can be corrected using quantum error correction [3].

Prior to this thesis, since proposed in Ref. [2], theory on CV MBQC and CV cluster states has been developed and matured [3-6], and a toolbox of graphical calculus of Gaussian states 

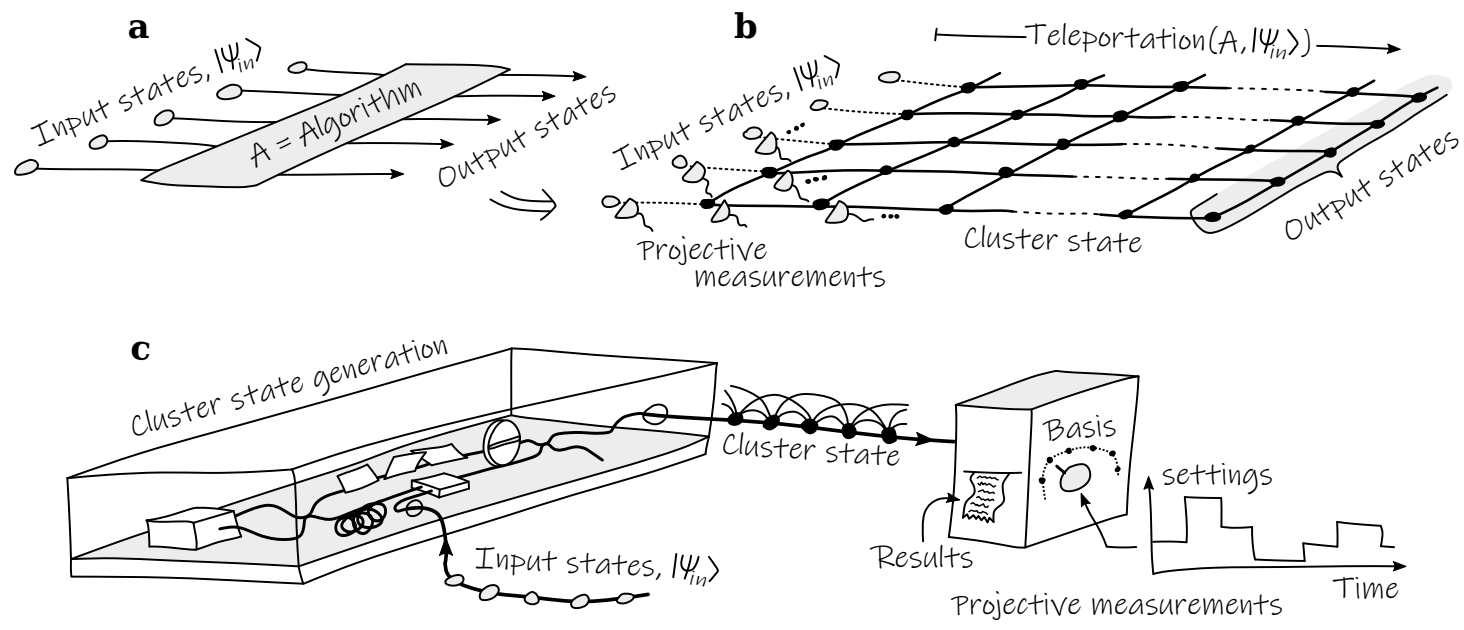

Figure 1.1: (a) A quantum algorithm, $A$, with multi-mode input states, $\left|\psi_{\text {in }}\right\rangle$, sketched in a gatebased fashion. (b) The principle of MBQC, where input states are connected to a cluster state, here in two dimensions, and teleported through the cluster state by projective measurements while the desired algorithm is implemented depending on the measurement bases. (c) Schematics of temporal encoded MBQC comprising a cluster state generator and a measurement device. Each node of the generated cluster state, here temporally encoded in a single spatial mode, occupy a temporal mode in time with entangling edges between temporal modes at different times. The measurement device is reused in time to measure each temporal mode with different basis settings dictating the implemented algorithm, and the measurement results are recorded to analyze the computation outcome.

has been presented in Ref. [7], which is used extensively in this thesis. Several MBQC schemes for cluster state generation and computation have been proposed [8-12], each of which utilizes temporal or frequency multiplexing to keep the required spatial resources low, and error correction in the CV regime has been proposed [13-15] and applied in CV MBQC [3]. On the experimental side, small cluster states were first generated [16-18], followed by the generation of large onedimensional cluster states based on time and frequency multiplexing [19-21], while quantum gates, implemented by projective measurements, have been demonstrated on small cluster states [22-29].

In this thesis, comprising Refs. [30-34], I present both experimental and theoretical work further towards the realization of CV MBQC. On the experimental side, this includes the demonstration of a two-dimensional (2D) CV cluster state, implementation of a universal Gaussian gate set by projective measurements, combining gates in a programmable fashion, and demonstration of optical switching in a quantum setting. On the theoretical side, besides the theory behind 2D cluster state generation and the derivation of a computation scheme for gate implementation, noise analysis of different computation schemes on 2D cluster stated is carried out and compared, and a new faulttolerant computation scheme is proposed allowing topological error correction.

Throughout this thesis, the focus is on temporal encoded computation schemes where, by temporal multiplexing, the number of required spatial resources is kept low [9]. This is mainly to ease the proof-of-principle experiments of this work. A schematic of the principles in temporal encoded MBQC is presented in Fig. 1.1c. The implementation consists of two parts: A cluster state generator, generating a temporal encoded cluster state in few spatial modes, and a measurement device that is reused in time to perform projective measurements on the spatial modes in which the cluster state is encoded in time. For temporal encoded cluster states, the cluster state generator often comprises optical delays, interfering beam-splitters, and an optical switch for coupling input states into the cluster state. Depending on the configuration, cluster state in one, two, or more dimensions can be generated with each dimension "folded" in time, such that cluster state edges exist in time between different temporal modes. In Fig. 1.1c, for simplicity, the generated cluster 
state is sketched to occupy a single spatial mode, while for most schemes proposed, the temporal encoded cluster state is generated in two or four spatial modes. Scalability of the cluster state size, and thereby the computation size, is immediately obtained in the cluster state generation by scaling optical delay lengths, while the number of spatial modes required remains fixed. As an example, in this thesis, a 2D cluster state is generated with a cylindrical topology [31]. Here the cylinder length and circumference represent the two dimensions, and while the length in principle is unlimited, the circumference is increased by adjusting optical delays in the generation. Finally, each temporal mode is measured using a measurement device with a number of inputs that equals the number of spatial modes in which the cluster state is temporally encoded. By adjusting the measurement basis for each temporal mode, a desired computation algorithm is implemented depending on the basis settings, and the measurement result for each measurement is read out to analyze the computation outcome classically. Note that no changes are required to the measurement device when scaling the temporal encoded cluster state.

While temporal encoded MBQC has advantages with a fixed number of spatial resources when scaling up the computation, temporal encoding, as well as frequency encoding, also possesses some limitations that eventually limit the possible computation size. In temporal encoding, the maximum delay length that can be used for scaling up is naturally limited by propagation losses leading to computation noise. Above a certain delay length depending on the quantum error correction to be applied, fault-tolerant computation is no longer possible. To keep scaling up, the temporal mode duration is shortened, such that more temporal modes fit into the longest possible delay. This, on the other hand, requires increasing bandwidths of squeezing sources and experimental control, which as well is limited. For frequency-encoded computation, it is the same limitation of the squeezing bandwidth, together with spectral resolution, that limits scalability. Nevertheless, temporal and frequency encoding allows for near-term realizations and applications using relatively simple experimental setups, and scaling is straightforward until optical losses become detrimental and the setup is operated with a maximum possible bandwidth. As of today, we are far from this limit. Assuming realizations with recent experimental demonstrations of large detection and squeezing bandwidths $[35,36]$ in the tera-hertz regime, and assuming comparable experimental control (though this may be challenging), temporal encoded computation schemes can potentially perform computation on information encoded in millions of modes.

During the work presented in this thesis, the field of CV quantum computation has advanced rapidly aiding CV MBQC. In the following, I list few examples that are relevant to this thesis (note, this is far from a complete list of all the exciting work carried out within CV quantum information during the work of this thesis). On the experimental side, in parallel with 2D CV cluster state generation in this work, a similar 2D cluster state was demonstrated using temporal encoding in Ref. [37], while single-mode Gaussian gates were implemented by projective measurements on a temporal encoded one-dimensional cluster state in Ref. [25]. As mentioned above, large bandwidth detection and squeezed light sources have been demonstrated for temporal encoded computation schemes [35, 36]. On the theoretical side, excess anti-squeezing in mixed squeezed states was shown in Ref. [38] to not affect the squeezing threshold for fault-tolerant MBQC. To correct for finite squeezing, Gottesman-Kitaev-Preskill (GKP) encoding [14] is widely accepted as a favorable qubit encoding introducing the required redundancy for error correction. For such encoded GKP-qubits, qubit magic states are shown in Ref. [39] to be distillable with only Gaussian gates, rendering non-Gaussian gates unnecessary for universal quantum computation [40], and in Ref. [41] this approach is even shown to be preferable over implementing the non-Gaussian cubic phase gate on GKP-qubits. In Ref. [42] a GKP quadrature correction scheme suitable for optical platforms was proposed, dispensing the need for on-line active coupling to ancilla GKP-qubits. While using GKP quadrature correction to correct CV noise with the cost of inducing qubit errors, in Ref. [43] GPK quadrature correction was proposed combined with topological qubit error correction to achieve fault-tolerant computation with realistic squeezing thresholds. Such implementation requires threedimensional cluster states, and besides the proposal in the work of this thesis, such schemes were proposed with frequency encoding in Ref. [44], temporal encoding in Ref. [45], and spatial encoding in Ref. [46]. 


\section{$1.1 \quad$ Thesis structure}

This thesis is a compilation of Refs. [30-34], and each of the chapters 2-6 can be read independently. As such, some of the same introduction and motivation is repeated in each chapter, while notation may vary from chapter to chapter. The thesis is organized as described in the following:

Chapter 2 presents the paper "Fiber coupled EPR-state generation using a single temporally multiplexed squeezed light source" of Ref. [30], in which two-mode entangled states in two spatial modes are prepared from a single temporal multiplexed squeezed light source.

Chapter 3 presents the paper "Deterministic generation of a two-dimensional cluster state" of Ref. [31] where a simple temporal encoded 2D cluster state generation scheme is proposed and demonstrated.

Chapter 4 presents the paper "Architecture and noise analysis of continuous-variable quantum gates using two-dimensional cluster states" of Ref. [32] where a computation scheme on the cluster state in chapter 3 is presented. Furthermore, a noise analysis of the computation scheme is carried out together with three other relevant computation schemes on $2 \mathrm{D}$ cluster states, and their performances are finally compared.

Chapter 5 presents the paper "Deterministic multi-mode gates on a scalable photonic quantum computing platform" of Ref. [33] where, following the proposed computation scheme in chapter 4, Gaussian gates and a small quantum circuit is implemented on the cluster state of chapter 3 .

Chapter 6 presents the paper "A fault-tolerant continuous-variable measurement-based quantum computation architecture" of Ref. [34], where a new scheme for generation and computation on a three-dimensional cluster state is proposed. The proposed scheme supports GKP quadrature correction and topological qubit error correction, and fault-tolerant computation is shown to be possible.

Chapter 7 summarizes and concludes the results, and an outlook of future work toward the realization of CV MBQC is presented.

Besides these chapters, appendix A, B, and $\mathrm{C}$ of this thesis includes additional technical information complementing the supplementary information in chapter 3 and 5, describing the experimental setup for cluster state generation and gate implementation. The information in these appendices is not published elsewhere. 


\section{Chapter 2}

\section{Fiber coupled EPR-state generation using a single temporally multiplexed squeezed light source}

In this chapter, the paper "Fiber coupled EPR-state generation using a single temporally multiplexed squeezed light source" of Ref. [30] is presented. This paper is authored by Mikkel V. Larsen, Xueshi Guo, Casper R. Breum, Jonas S. Neergaard-Nielsen, and Ulrik L. Andersen, and published in npj Quantum Information 5, 46 (2019).

In this work, we prepare two-mode squeezed states in two spatial modes by interfering two single-mode squeezed states from a single temporal multiplexed squeezing source. This is done by utilizing an optical switch for guiding different temporal modes of the squeezing source into different spatial modes, and further using an optical delay for aligning temporal modes in time. As such, this is an experimental work, demonstrating the combination of temporal and spatial multiplexing, as well as demonstrating optical switching and delay in a quantum setting with quantum entanglement being a figure of merit.

On the practical side, experimental techniques are developed and demonstrated, including efficient coupling of free-space light into optical fiber, efficient in-fiber phase control, and fiber-based homodyne detection. These techniques have shown ot be useful in the following experimental work and plays an important role in the experimental realizations of chapter 3 and $5[31,33]$. Besides this, compensation of systematic electronic noise is demonstrated, while a useful model is developed taking seed-noise in the squeezing source into account in the quadrature squeezing spectra, and allows for more accurate estimation of squeezing levels, efficiencies, and phase fluctuations.

From npj Quantum Information 5, 46 (2019).

\subsection{Abstract}

A prerequisite for universal quantum computation and other large-scale quantum information processors is the careful preparation of quantum states in massive numbers or of massive dimension. For continuous variable approaches to quantum information processing (QIP), squeezed states are the natural quantum resources, but most demonstrations have been based on a limited number of squeezed states due to the experimental complexity in up-scaling. The number of physical resources can however be significantly reduced by employing the technique of temporal multiplexing. Here, we demonstrate an application to continuous variable QIP of temporal multiplexing in fiber: Using just a single source of squeezed states in combination with active optical switching and a $200 \mathrm{~m}$ 


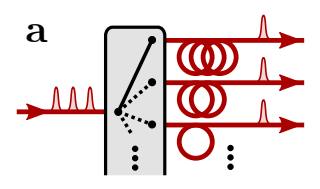

d

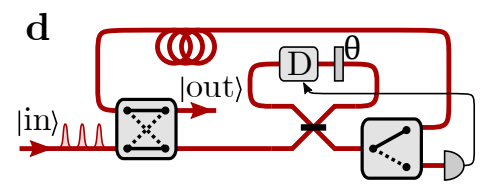

b

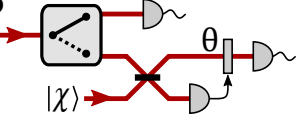

Switches

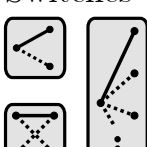

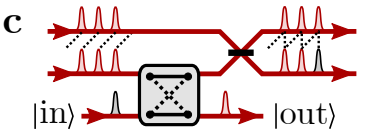

D Homodyne detector $\Lambda$ Temporal mode (O) Delay * Beam splitter (BS) BS transformation

Figure 2.1: Quantum information processing architectures using optical switching and optical delay. (a) Switching and delay lines applied to a single squeezed state resource in order to generate multiple time-synchronized squeezed state. (b) Switching between homodyne detection and the more demanding cubic-phase gate-teleportation measurement with $|\chi\rangle$ being an ancillary cubicphase-state [12]. (c) Example of switching temporal modes into or out of a cluster state [58]. (d) Loop-based architecture for fully temporally encoded MBQC utilizing switching and delay [60].

fiber delay line, we generate fiber-coupled Einstein-Podolsky-Rosen entangled quantum states. Our demonstration is a critical enabler for the construction of an in-fiber, all-purpose quantum information processor based on a single or few squeezed state quantum resources.

\subsection{Introduction}

The realization of quantum computation (QC) with demonstrated quantum supremacy requires a scalable platform of quantum resources [47, 48]: Usually hundreds of logical qubits, or thousands of physical qubits, are needed to reach this longstanding goal [49]. In one-way measurement based quantum computation (MBQC) $[1,50]$, universal computation is performed with only single-qubit projective measurements of an entangled cluster state [51]. Thereby, scalability is relaxed to the generation of a cluster state of suitable size [5]. Cluster states of multiple modes of light are readily accessible in continuous variable optical platforms, but most demonstrations have been limited by the amount of spatial resources [52-56]. However, by time and frequency multiplexing with squeezed states of light, large cluster states can be deterministically generated as demonstrated with 60 frequency modes in $[18,20]$ and $10^{6}$ temporal modes in $[19,21]$. This allows for excellent scalability, thereby rendering the need for spatially distributed resources unnecessary.

MBQC based on temporally encoded cluster states $[9,12]$ from a single squeezed state resource [57] requires optical switching and passive optical storage (such as an optical delay line) in different configurations as illustrated in Fig. 2.1. Multiple time-synchronized squeezed states can be generated in the network illustrated in Fig.2.1a, allowing 2D cluster state generation from a single squeezing source $[9,12]$. Moreover, in MBQC, sequential measurements are performed on the cluster in which each measurement strategy is adaptively changed based on previous measurement outcomes. In some cases, switching between completely different measurement schemes, e.g. homodyne detection and a non-Gaussian measurement, is required [12] (Fig. 2.1b). As an alternative to switching between Gaussian and non-Gaussian measurement schemes, one might fix the measurement setting to Gaussian homodyne detection and switch ancillary non-Gaussian states into selected modes of the cluster state [58] (Fig. 2.1c). Finally, it is possible to realize MBQC by applying optical switching in loop-based architectures [59,60] as illustrated in Fig. 2.1d. No matter which of the strategies is chosen, switching and delay lines are key functionalities in managing temporal modes in optical MBQC.

In this article, we demonstrate optical fiber switching combined with an optical fiber delay in a continuous variable (CV) quantum setting in the telecom band. This enables us to generate an Einstein-Podolsky-Rosen (EPR) state [61] between two fiber modes by time multiplexing of a single source of squeezed states of light. Our demonstration of optical switching and optical delay in a CV, fiber-integrated and low-loss setting is a critical step towards the realization of a scalable 


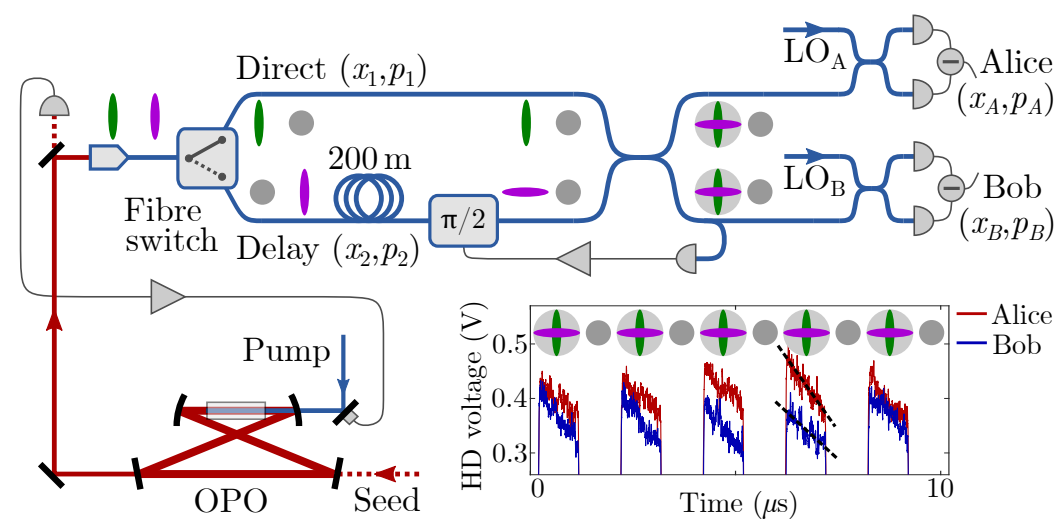

Figure 2.2: Schematics of the experiment. Bright amplitude squeezed states of light are generated using type-0 parametric down conversion in an optical resonator (OPO) at the wavelength of $1550 \mathrm{~nm}$, seeded with a coherent beam for phase locks. The squeezed states of light are coupled into a single mode fiber network (marked by blue lines) in which the generation of two-mode squeezing takes place: Using a fiber switch, two consecutive temporal modes (marked by green and purple) are guided in different directions. Subsequently, the two modes are synchronized by a fiber delay of $200 \mathrm{~m}$ in one of the modes. Finally, the two spatial modes interfere in a 50:50 fiber coupler, thereby forming a two-mode squeezed state in the output as the phase difference of the two input modes are locked to $\pi / 2$ (using active feedback to a fiber-stretching device described in the methods section 2.5). The quadratures of the two-mode squeezed state are measured with two fiber-based homodyne detection stations, Alice and Bob. A typical measurement output in time-domain is shown in the inset together with illustrations of the corresponding states in phase space, alternating between two-mode squeezed states and vacuum states.

platform for CV quantum information processing and ultimately universal quantum computation.

\subsection{Results}

The quadrature entangled EPR-state is an important resource in numerous quantum information and sensing protocols ranging from CV teleportation [62] and cryptography [63] to CV computing [2]. The most wide-spread realization of quadrature entanglement is based on cavity-enhanced spontaneous parametric down-conversion in an optical parametric oscillator (OPO). Correlations can be established between different polarization or frequency modes from a single non-degenerate OPO [64-71], or by combining the squeezed state outputs of two degenerate OPOs onto a balanced beam splitter [62, 72-74]. Here we use the latter approach of combining two squeezed states on a beam splitter, but instead of using two OPOs, we exploit time multiplexing of a single source.

\subsubsection{Experimental setup}

The experimental setup is sketched in Fig. 2.2. We inject a single $\sim 7 \mathrm{~dB}$ squeezed beam into a fiber switch that alternately guides the squeezed beam into two different fibers at a frequency of $500 \mathrm{kHz}$; thereby delaying one mode by $1 \mu \mathrm{s}$ with respect to the other. To compensate for the delay and thus synchronize the two modes in time, the mode ahead propagates through a $200 \mathrm{~m}$ fiber spool. The two modes interfere with a relative phase shift of $\pi / 2$ in a balanced fiber coupler, thereby forming a two-mode squeezed state.

For state characterization, we sample on an oscilloscope the quadratures of the fiber coupler outputs measured by two homodyne detection stations, Alice and Bob. Typical time traces of such measurements are shown in the inset of Fig. 2.2. A single data set consists of 16000 time traces triggered by the switching signal. Each time trace is affected by a frequency dependent response 
of the detector giving rise to the negative slope seen in the inset of Fig. 2.2, and a noisy oscillatory response of the fiber switch. Besides this, there is a variation in the slope of each time trace due to spurious interferences - both effects occur from the coherent amplitude of the initial bright squeezed state together with limited detection, switching and feedback bandwidths. However, since these effects are systematic, repeatable and synchronized with the switching process, they can be tracked and compensated in the data processing - see methods section 2.5.

We have striven to reduce the loss of all components to maintain as much of the non-classicality as possible. We used an anti-reflection coated graded-index lens to couple the squeezed light into the fiber with an efficiency of $97 \%$ (by matching counter-propagating light in the OPO cavity), we spliced together all fiber components to minimize fiber-to-fiber coupling losses and by using the wavelength of $1550 \mathrm{~nm}$, fiber propagation loss was negligible: Even through the $200 \mathrm{~m}$ fiber delay (standard SMF-28e+ fiber), the propagation loss is $\leq 1 \%$. The largest loss contribution is caused by the fiber switch (Nanona by Boston Applied Technologies Inc.), where light is coupled into a bulk electro-optic material and back into fiber leading to $17 \%$ loss. Including OPO escape efficiency, detection efficiency and various tapping for phase locks, the total transmission from the squeezed state source to the detected signal becomes $\eta \approx 68 \%$ (for more details see methods section $2.5)$.

\subsubsection{Experimental results}

To perform partial tomography of the generated two-mode squeezed states, we measure the quadratures $\hat{q}_{A}(\theta) \pm \hat{q}_{B}(-\theta)$ and $\hat{q}_{A}(\theta) \pm \hat{q}_{B}(\theta-\pi / 2)$ as a function of the local oscillator phase $\theta$. Here $\hat{q}_{i}(\theta)=x_{i} \cos \theta+\hat{p}_{i} \sin \theta$ where $\hat{x}_{i}$ is the amplitude and $\hat{p}_{i}$ the phase quadrature at Alice $(i=A)$ and Bob $(i=B)$. The resulting noise variances at the 3 and $10 \mathrm{MHz}$ side band frequencies are shown in Fig. 2.3 together with theoretical predictions. We observe a maximum shot noise suppression of $3.8 \mathrm{~dB}$. The very small discrepancy of the measurements at $3 \mathrm{MHz}$ results from technical noise of the seed beam as well as additional noise added in the delay line - both noise effects are discussed and analyzed below and in the supplementary information section 2.6.4.

The variances of $\hat{q}_{A}(\theta) \pm \hat{q}_{B}(-\theta)$,

$$
\begin{aligned}
& \left\langle\Delta\left(\hat{q}_{A}(\theta)+\hat{q}_{B}(-\theta)\right)^{2}\right\rangle \\
& \quad=2\left(\left\langle\Delta \hat{x}_{1}^{2}\right\rangle \cos ^{2} \theta+\left\langle\Delta \hat{x}_{2}^{2}\right\rangle \sin ^{2} \theta\right), \\
& \left\langle\Delta\left(\hat{q}_{A}(\theta)-\hat{q}_{B}(-\theta)\right)^{2}\right\rangle \\
& \quad=2\left(\left\langle\Delta \hat{p}_{2}^{2}\right\rangle \cos ^{2} \theta+\left\langle\Delta \hat{p}_{1}^{2}\right\rangle \sin ^{2} \theta\right),
\end{aligned}
$$

associated with the maximally squeezed and anti-squeezed quadratures, respectively, are seen to be constant with $\theta$, indicating symmetric two-mode squeezing. This is expected as the individual single mode squeezed states in the direct $\left(\hat{x}_{1}, \hat{p}_{1}\right)$ and delay $\left(\hat{x}_{2}, \hat{p}_{2}\right)$ line originate from the same squeezing source, that is $\left\langle\Delta \hat{x}_{1}^{2}\right\rangle=\left\langle\Delta \hat{x}_{2}^{2}\right\rangle$ and $\left\langle\Delta \hat{p}_{1}^{2}\right\rangle=\left\langle\Delta \hat{p}_{2}^{2}\right\rangle$. From the data sets at $\theta=0^{\circ}$ and $90^{\circ}$, entanglement can be verified by the inseparability criterion [75] which reads

$$
\left\langle\Delta\left(\hat{x}_{A}+\hat{x}_{B}\right)^{2}\right\rangle+\left\langle\Delta\left(\hat{p}_{A}-\hat{p}_{B}\right)^{2}\right\rangle=1.72 V_{0}<4 V_{0},
$$

at $3 \mathrm{MHz}$, and $2.42 V_{0}<4 V_{0}$ at $10 \mathrm{MHz}$. Here $V_{0}$ is the variance of the vacuum state.

When measuring the variances of

$$
\begin{aligned}
\hat{q}_{A}(\theta) \pm \hat{q}_{B}(\theta-\pi / 2)=\hat{x}_{A} \cos \theta \pm \hat{x}_{B} \sin \theta \\
+\hat{p}_{A} \sin \theta \mp \hat{p}_{B} \cos \theta
\end{aligned}
$$

as a function of $\theta$, we trace out one specific projection that in particular realizes the squeezed and anti-squeezed quadratures. Maximum squeezing and anti-squeezing are measured at $\theta=45^{\circ}$ where correlations are strongest, corresponding to the measurements of $\hat{q}_{A}(\theta) \pm \hat{q}_{B}(-\theta)$. At $\theta=0^{\circ}$ and $90^{\circ}$ we expect no correlations and measure the variances $\left(\left\langle\Delta \hat{x}_{1}^{2}\right\rangle+\left\langle\Delta \hat{p}_{1}^{2}\right\rangle+\left\langle\Delta \hat{x}_{2}^{2}\right\rangle+\left\langle\Delta \hat{p}_{2}^{2}\right\rangle\right) / 2$ corresponding to the added noise of thermal states at Alice and Bob when tracing out one mode. 


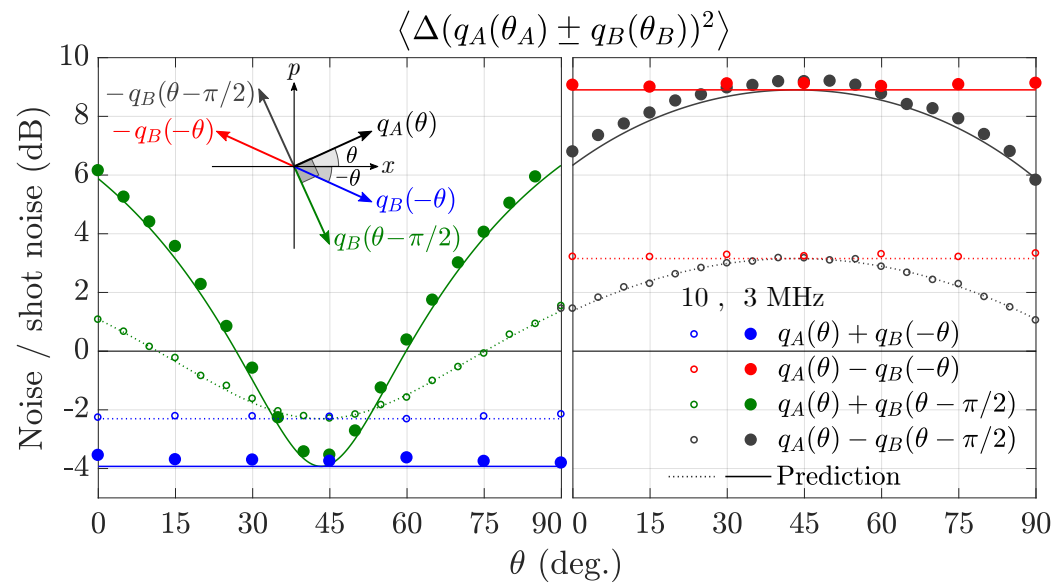

Figure 2.3: Partial tomography of the generated two-mode squeezed state. We plot the noise variance (normalized to the shot noise variances) of the quadratures $\hat{q}_{A}\left(\theta_{A}\right) \pm \hat{q}_{B}\left(\theta_{B}\right)$ with $\theta_{A}$ restricted to $\theta_{A}=\theta$ and $\theta_{B}$ restricted to $\theta_{B}=-\theta$ (blue and red), and $\theta_{B}=\theta-\pi / 2$ (green and black). Each point corresponds to one dataset of 16000 processed time traces as in Fig. 2.5. To extract the 3 and $10 \mathrm{MHz}$ frequency modes, each time trace is digitally mixed with a 3 or $10 \mathrm{MHz}$ sine curve and integrated to one value. The noise is then the variance of these 16000 values, added/subtracted for Alice and Bob. With the time trace length of about $900 \mathrm{~ns}$, the frequency mode bandwidth is around $1 \mathrm{MHz}$. The solid and dashed line shows theoretical noise predicted from measured efficiency, OPO bandwidth, pump power and fitted phase fluctuations in Fig. 2.4. The predictions include $1.7^{\circ}$ phase offset. The inset illustrates the measured quadratures in a phase-space diagram.

From the partial tomography, we reconstruct the covariance matrix of the two-mode squeezed state at the $3 \mathrm{MHz}$ side band frequency [76]:

$$
\gamma=V_{0}\left(\begin{array}{cccc}
4.36 & - & -3.84 & 0.36 \\
- & 4.43 & 0.45 & 3.92 \\
-3.84 & 0.45 & 4.17 & - \\
0.36 & 3.92 & - & 4.26
\end{array}\right)
$$

Here, the entries with '-' were not measured as it would require a more elaborate measurement scheme, but they should in principle be zero due to the symmetry of the states. However, due to uncertainties in the phase control and non-perfect phase-space alignments, the values will in practice be slightly different from zero. This is also clear from the off-diagonal correlation terms $\left\langle\hat{x}_{A} \hat{p}_{B}\right\rangle$ and $\left\langle\hat{x}_{B} \hat{p}_{A}\right\rangle$ which in practice are non-zero as seen in the measured co-variance matrix but in theory should be zero for a perfectly aligned system (see supplementary information section 2.6.6). Finally, from the covariance matrix we determine the conditional variances between Alice and Bob's measurements from which we test the EPR-criterion [77]:

$$
\begin{aligned}
& \Delta_{\text {inf. }}^{2} \hat{x}_{A \mid B} \cdot \Delta_{\text {inf. }}^{2} \hat{p}_{A \mid B}=0.69 V_{0}^{2}<V_{0}^{2}, \\
& \Delta_{\text {inf. }}^{2} \hat{x}_{B \mid A} \cdot \Delta_{\text {inf. }}^{2} \hat{p}_{B \mid A}=0.64 V_{0}^{2}<V_{0}^{2},
\end{aligned}
$$

where $\Delta_{\text {inf. }}^{2} \hat{q}_{i \mid j}=\min _{g}\left\langle\Delta\left(\hat{q}_{i}-g \hat{q}_{j}\right)^{2}\right\rangle=\left\langle\Delta \hat{q}_{i}^{2}\right\rangle-\left\langle\hat{q}_{i} \hat{q}_{j}\right\rangle^{2} /\left\langle\Delta \hat{q}_{j}^{2}\right\rangle$ is the conditional uncertainty in predicting $\hat{q}_{i}$ when measuring $\hat{q}_{j}$. Since both conditional variance products are below $V_{0}^{2}$, the generated states are EPR entangled in both directions.

As seen from Eq. (2.1) for $\theta=0^{\circ}$ and $90^{\circ}$, the measured two-mode squeezing is equivalent to the squeezing of the single mode states in the direct and delayed paths, respectively. The spectra of such measurements are shown in Fig. 2.4. The squeezing spectra are Lorentzian and resemble that of the OPO cavity. Furthermore, the anti-squeezing is seen to be symmetric, while the squeezing 


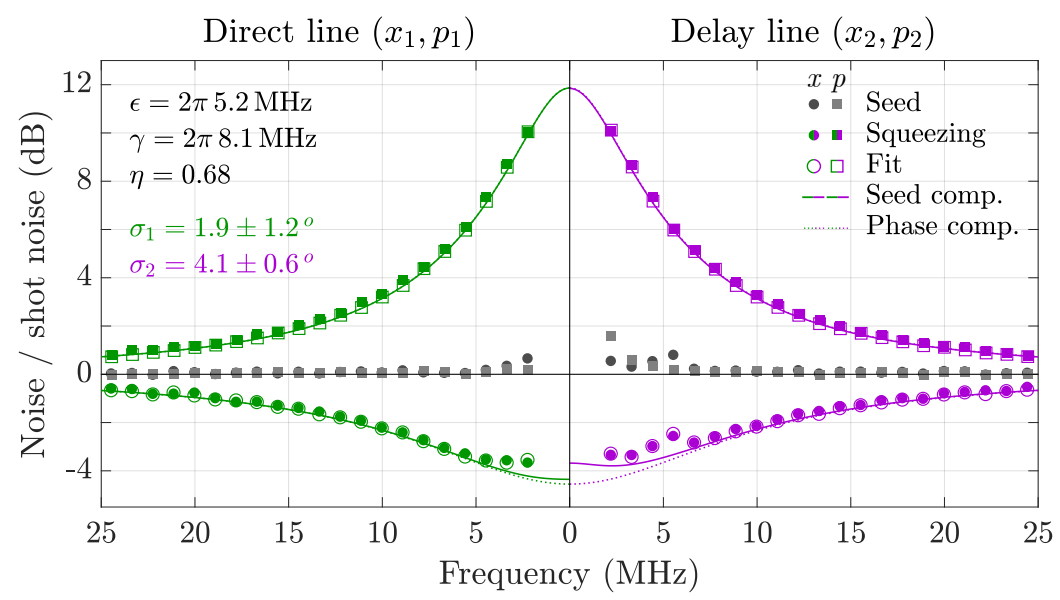

Figure 2.4: Spectrum of squeezing. Noise spectrum of $\hat{x}_{1}=\left(\hat{x}_{A}+\hat{x}_{B}\right) / \sqrt{2}, \hat{p}_{1}=\left(\hat{p}_{A}+\hat{p}_{B}\right) / \sqrt{2}$, $\hat{x}_{2}=\left(\hat{p}_{A}-\hat{p}_{B}\right) / \sqrt{2}$ and $\hat{p}_{2}=\left(\hat{x}_{A}-\hat{x}_{B}\right) / \sqrt{2}$ relative to shot noise. Solid points correspond to the average of Fourier transformed time traces in the measured datasets $\hat{q}_{A}(\theta) \pm \hat{q}_{B}(-\theta)$ for $\theta=0^{\circ}$ and $90^{\circ}$. Here, coloured points are with pumped OPO, while gray points are the seed noise when blocking the pump. Hollow points are the result of fitting a squeezing spectrum with phase fluctuations $\sigma_{1}$ and $\sigma_{2}$ in the direct and delay line respectively. From the fit, the solid lines indicates the expected squeezing when compensating for seed noise, while the dashed lines indicates the expected squeezing in case of no phase fluctuation, and thus the best squeezing achievable with the given efficiency.

has degraded slightly in the delay line due to additional phase noise. To characterize this, we measure the seed spectrum by blocking the pump to the squeezing cavity. The low frequency noise that can be observed in the direct line results from technical noise of the seed beam. Even more low frequency noise is apparent in the squeezed state of the delay line. We believe it originates from phase noise generated by the $200 \mathrm{~m}$ fiber and amplitude noise from the fiber switch which is most prominent at $5-6 \mathrm{MHz}$.

To infer the phase fluctuations, $\sigma_{i}$, associated with the direct $(i=1)$ and delay $(i=2)$ line, the squeezing spectra including a normal distributed phase with $\sigma_{i}$ standard deviation, approximated to $\left\langle\Delta \hat{x}_{i}^{2}\right\rangle \cos ^{2}\left(\theta+\sigma_{i}\right)+\left\langle\Delta \hat{p}_{i}^{2}\right\rangle \sin ^{2}\left(\theta+\sigma_{i}\right)$ for $\theta=0$ and $\pi / 2$ [78], is fitted with $\sigma_{i}$ as the only fitting parameter. Here, following [79] with additional seed noise coupled into the OPO and $V_{0}=1 / 2$,

$$
\left\langle\Delta \hat{q}_{i}^{2}\right\rangle=\frac{1}{2} \mp \frac{2 \varepsilon \gamma \eta}{(\gamma \pm \varepsilon)^{2}+\omega^{2}}+\frac{K_{q}}{(\gamma \pm \varepsilon)^{2}+\omega^{2}}
$$

where $q=x, p, \varepsilon$ is the pump rate, $\gamma$ is the total OPO decay rate, $\eta$ is the overall efficiency and $\omega$ is the angular frequency, while $K_{q}=4 \gamma \gamma_{s} \eta_{i}\left(\left\langle\Delta \hat{q}_{s}^{2}\right\rangle-1 / 2\right)$ with $\gamma_{s}$ being the decay rate due to the seed beam coupling mirror and $\left\langle\Delta \hat{q}_{s}^{2}\right\rangle$ is the seed beam quadrature noise before injection into the OPO (for detailed derivation see supplementary information section 2.6.5). We find a decay rate of $\gamma / 2 \pi=8.1 \mathrm{MHz}$ by measuring the OPO intracavity losses $(0.55 \%)$, the cavity length $(320 \mathrm{~mm})$ and the transmissivity of the coupling mirror $(10 \%)$, and we estimate the pump rate to $\varepsilon / 2 \pi=5.2 \mathrm{MHz}$ for a pump power of $350 \mathrm{~mW}$ and a measured OPO threshold power of $833 \mathrm{~mW}$. $K_{q}$ is estimated as $K_{q}=\left(\gamma^{2}+\omega^{2}\right)\left(\left\langle\Delta \hat{q}_{0}^{2}\right\rangle-1 / 2\right)$ where $\left\langle\Delta \hat{q}_{0}^{2}\right\rangle$ is the quadrature noise measured with no pump ( $\varepsilon=0$, gray points in direct line of Fig. 2.4). Finally, to include excess noise of the delay line, the seed noise difference of the direct and delay line is added to the fit in the delay line. The fit is shown as hollow points in Fig. 2.4, and is seen to fit very well with the measured data. The resulting phase fluctuations obtained from the fit are $\sigma_{1}=1.9 \pm 1.2^{\circ}$ and $\sigma_{2}=4.1 \pm 0.6^{\circ}$ with uncertainties estimated as the $95 \%$ confidence interval. These values are included in the theoretical model used for Fig. 2.3.

From the theoretical model with fitted phase fluctuations, the solid lines in Fig. 2.4 indicate 
the expected squeezing spectra if the seed beam were shot noise limited and no additional noise existed in the delay line. In that case, we can expect more than $4 \mathrm{~dB}$ two-mode squeezing. The phase fluctuation in the delay line, $\sigma_{2}=4.1 \pm 0.6^{\circ}$, is more than double that in the direct line, $\sigma_{1}=1.9 \pm 1.2^{\circ}$. This is mainly due to limited phase control bandwidth of the fiber delay and low signal-to-noise ratio of the feedback signal. Finally, the dotted line in Fig. 2.4 shows the squeezing spectrum we would expect if we had perfect phase control, and thus the optimum squeezing we may measure with the given efficiency.

\subsection{Discussion}

The fast switching frequency of $500 \mathrm{kHz}$ demonstrated here is suitable for encoding temporal modes of megahertz bandwidth and is thus applicable in the optical schemes in Fig. 2.1. Similarly, the low loss of the $200 \mathrm{~m}$ fiber allows for an efficient delay of almost $1 \mu \mathrm{s}$, compatible with the temporal modes defined by the switching. However, the $17 \%$ loss of the particular switch used here, as well as the phase fluctuations of $4^{\circ}$ standard deviation in the fiber delay, leads to decoherence and results in some limitations when used in quantum settings: For cluster state generation from a temporal multiplexed source, as in Fig. 2.1a, or when switching modes in and out of a cluster state, as in Fig. 2.1c, the switching loss and phase fluctuation leads to limited entanglement even when large amount of initial squeezing is available. Yet, it does not accumulate through the cluster state as the loss and phase fluctuation on each mode is local, and so it does not limit the cluster state size. It will be more detrimental in loop based architectures, as in Fig. 2.1d, where a temporal mode passes through the same switch and delay line multiple times, and so the switch efficiency and delay phase fluctuations limit the number of passes possible and thereby the computation depth.

High efficient fast switching is demonstrated in free-space [80], while one can imagine more compact fiber coupled switching based on Mach-Zehnder interferometry. However, in either case care must be taken not to compromise the high switching frequency, as this leads to longer delay lines necessary and thereby larger phase fluctuations. In work towards temporal encoded optical quantum information processing, faster switching is preferable as it minimizes the required delay lengths and increases the computational speed. Thus the ideal switch, besides being efficient, is as fast as the detection or squeezing source bandwidth.

In conclusion, using a single squeezing source with optical switching and delay, we have successfully generated in-fiber EPR-states with nearly $4 \mathrm{~dB}$ of two-mode squeezing, characterized by fiber-coupled homodyne detection. Our setup has great scalability potentials: Adding an additional delay line, it is possible to extend the setup to generate one-dimensional cluster states [19, 21], and by adding a multi-port switch and more delay lines, two-dimensional cluster states $[9,12]$ can be generated from a single squeezing source. Moreover, by inserting the switch inside a loop, as in Fig. 2.1d, combined with dynamical control, various entangled states can be generated and in principle universal quantum computation can be realized. Since all switches and delay lines are fiber components, the setup remains very small and flexible despite the increasing complexity in generating more complex states. Moreover, since fiber propagation losses are extremely low at the operating wavelength of $1550 \mathrm{~nm}$, decoherence is not a big issue despite the increasing number of fiber delays. The largest decoherence source in the current setup is the optical switch which introduces a loss of $17 \%$. However, with future developments of the optical switch, we expect that the in-fiber temporal multiplexing technique demonstrated here will play a significant role in reducing the resources in future large-scale photonic circuits for continuous variable quantum information processing, including quantum computing [5], quantum teleportation [81], distributed sensing [82] and multi-partite quantum key distribution. 


\subsection{Methods}

\section{Squeezing source}

The experimental setup is outlined in Fig. 2.2. As squeezing source, we use an optical parametric oscillator (OPO) based on a periodically poled potassium titanyl phosphate (PPKTP) crystal in a bowtie shaped cavity, locked by a counter propagating coherent beam. A pump beam at a wavelength of $775 \mathrm{~nm}$ is used to drive the parametric process and thus produce squeezed light at $1550 \mathrm{~nm}$ via type- 0 phase matching. The OPO has a bandwidth of $\gamma / \pi=16 \mathrm{MHz}$. Stable phase locking at different stages of the experiment is facilitated by an excitation of the squeezed state, realized by injecting a bright seed beam into the OPO. To lock the phase of the input pump beam to the deamplification point of the parametric process, thereby producing amplitude squeezed states, we tap off and detect $1 \%$ of the excited squeezed beam for feedback to a piezo-mounted mirror in the pump beam. This, as well as all other feedback controls in the experiment, is realized by the open-source software package PyRPL [83] running on Red Pitaya boards that integrate an FPGA system-on-chip with fast ADCs and DACs.

\section{In-fiber phase control}

For locking the $\pi / 2$ relative phase difference when interfering the two beams of bright squeezed states in a balanced fiber coupler for EPR-state generation, $1 \%$ is tapped off one of the fiber coupler output arms, and fed back to a homemade fiber stretcher in the delay line based on [84]. Here, using a piezoelectric actuator, a phase shift is induced by stretching the fiber. For more details, see the supplementary information section 2.6.2. The optical transmission efficiency is near unity, as it simply depends on the fiber which has negligible loss at $1550 \mathrm{~nm}$ wavelength. This allows high-efficient in-fiber phase control, and the same design is used for phase control of the local oscillators in the homodyne detection.

\section{Fiber-coupled homodyne detection}

To detect quadratures of the in-fiber generated EPR-state, we developed a fiber-coupled homodyne detector (HD) where signal and local oscillator (LO) is interfered in a balanced fiber coupler before detection. For schematics and details, see supplementary information section 2.6.2. This has the benefit of being mobile, and the visibility between signal and LO is easily optimized to near unity due to the single mode nature of the fiber used.

The fiber coupler is not exactly symmetric, but has a coupling ratio of approximately 48:52. To compensate for this, the HD is balanced by attenuation in the fiber coupler output arm of stronger LO by inducing bending losses. With an asymmetry of $4 \%$ in the fiber coupler, after balancing this leads to $4 \%$ loss.

Finally, to couple and focus light from the fiber onto the HD photo diodes of $100 \mu \mathrm{m}$ diameter (Laser Components Nordic AB), anti-reflective coated graded-index (GRIN) lens are used in front of the diode, leading to a free-space waist diameter of $13 \mu \mathrm{m}$ at $5 \mathrm{~mm}$ from the GRIN lens facet. The quantum efficiency is measured to be $97 \%$, and so together with $4 \%$ loss from balancing and $99 \%$ visibility, the total HD efficiency achieved is $91 \%$.

\section{Overall efficiency}

With the OPO escape efficiency of $95 \%$, and $1 \%$ tapping for gain lock, the efficiency in free-space before fiber coupling is $94 \%$. In fiber, including $97 \%$ fiber coupling efficiency, $17 \%$ loss in the fiber switch and $1 \%$ tapping for phase control, the efficiency is $80 \%$. Finally, with $91 \%$ detection efficiency, the overall efficiency becomes

$$
\eta=0.94 \cdot 0.80 \cdot 0.91=68 \% .
$$




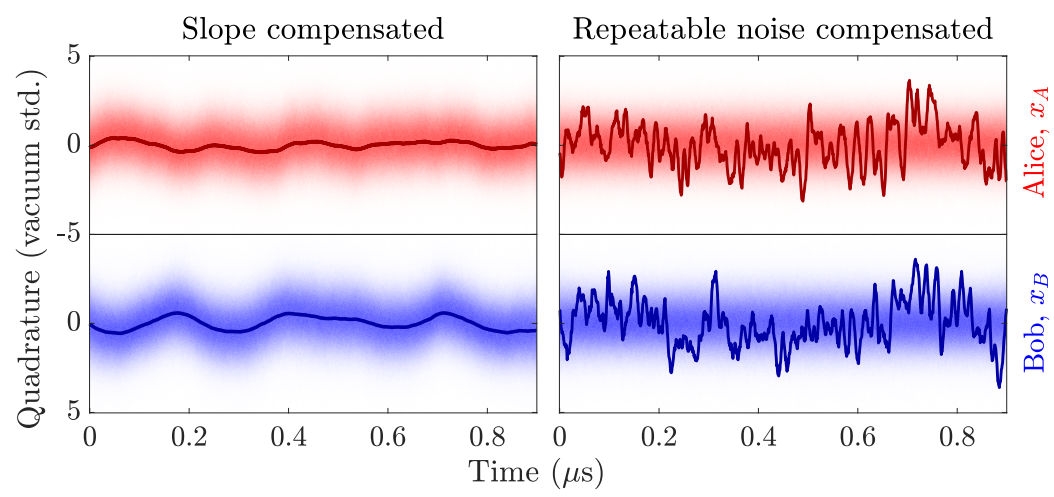

Figure 2.5: Temporal filtering by data processing. (left) Temporal histogram of a data set with 16000 time traces associated with amplitude quadratures of a two-mode squeezed state at Alice (red) and Bob (blue) compensated for slope variations and decaying detector response. The solid lines show the dataset average time trace indicating the remaining repeating oscillations from the switching process. (right) Temporal histogram of the dataset in (left) compensated for any systematic and repeatable noise responses from the switching process. Here, the solid lines indicate a single pair of synchronized time traces (at Alice and Bob) in which quadrature anti-correlations are visible (note the inverted axis on Bob).

\section{Temporal data processing}

To recover two-mode squeezing from the acquired time traces affected by a frequency dependent detector response (leading to a negative slope), spurious interference (leading to slope variations) and an oscillating response from the switch, we use the statistic of 16000 time traces in a dataset synchronized with the switching process. To compensate for the negative and varying slope of each time trace, linear regression lines (as the dashed lines in the inset of Fig. 2.2) are subtracted from each individual trace of the dataset. The result is shown in Fig. 2.5(left). Here, the repeatable oscillating noise is visible, and compensated for by subtracting the average time trace of the dataset from every single time trace. The final processed dataset is seen in Fig. 2.5(right) with a constant temporal histogram and a single time trace at Alice and Bob showing anti-correlations as in [85]. For detailed discussion on the data processing, see supplementary information section 2.6.3.

\subsection{Supplementary information}

This section includes the supplementary information of Ref. [30].

\subsubsection{Quadrature relations}

To generate two-mode squeezed states from a single squeezer, two amplitude squeezed states from the same squeezing source, but in different temporal modes, are guided into two different spatial modes, synchronized in time by a delay in one spatial mode, and finally interfered on a symmetric beam splitter with a $\pi / 2$ phase difference. The phase-space in the two spatial modes after the temporal modes of squeezing are synchronized, after $\pi / 2$ rotation, and after interfering the two modes, are illustrated in Fig. 2.6a. In the following quadrature relations of the generated twomode squeezed states are derived, assuming the input states being squeezed vacuum, perfect phase control, and the beam splitter (or fiber coupler) being symmetric.

After synchronization, the two spatial modes - the direct $\left(x_{1}, \hat{p}_{1}\right)$ and delay $\left(x_{2}, \hat{p}_{2}\right)$ line - are both amplitude squeezed states, i.e.

$$
\left\langle\Delta \hat{x}_{1}^{2}\right\rangle,\left\langle\Delta \hat{x}_{2}^{2}\right\rangle<V_{0} \quad, \quad\left\langle\Delta \hat{p}_{1}^{2}\right\rangle,\left\langle\Delta \hat{p}_{2}^{2}\right\rangle>V_{0},
$$




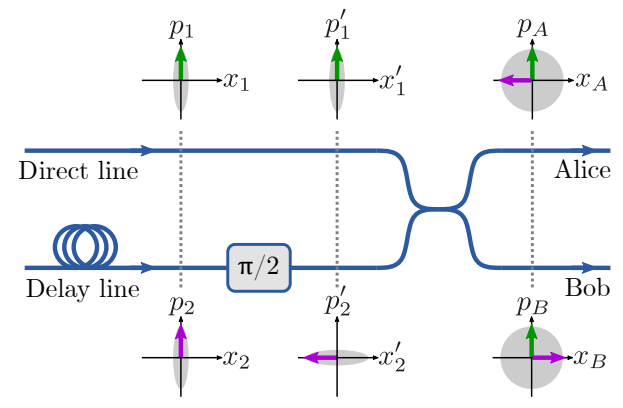

(a)

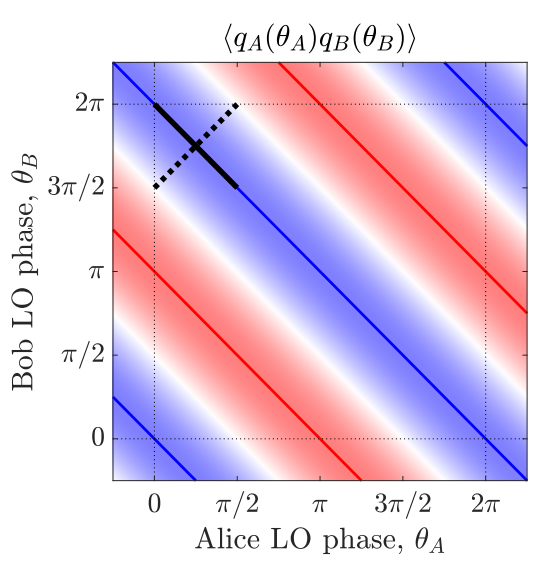

(b)

Figure 2.6: (a) Quadrature transformations of the two-mode squeezed state generation, where simultaneous amplitude squeezed states in the direct and delay line are interfered at a symmetric beam splitter (fiber coupler) with a $\pi / 2$ phase difference in the delay line. (b) Quadrature covariance in Eq. (2.11) with red and blue indicating areas of correlation and anti-correlation respectively, and solid coloured lines indicates maximum correlations. The two measured sets of quadratures for partial tomography are marked by the black solid and dotted line for set $1\left(\theta_{B}=-\theta_{A}\right)$ and set $2\left(\theta_{B}=\theta_{B}-\pi / 2\right)$ respectively.

where $V_{0}$ is the vacuum variance. After a $\pi / 2$ phase-space rotation in the delay line, the new quadratures read

$$
\begin{array}{ll}
\hat{x}_{1}^{\prime}=\hat{x}_{1} & , \quad \hat{p}_{1}^{\prime}=\hat{p}_{1}, \\
\hat{x}_{2}^{\prime}=-\hat{p}_{2} \quad & , \quad \hat{p}_{2}^{\prime}=\hat{x}_{2} .
\end{array}
$$

Finally, after a beam splitter transformation of the symmetric fiber coupler, we get

$$
\begin{array}{ll}
\hat{x}_{A}=\frac{1}{\sqrt{2}}\left(\hat{x}_{1}^{\prime}+\hat{x}_{2}^{\prime}\right)=\frac{1}{\sqrt{2}}\left(\hat{x}_{1}-\hat{p}_{2}\right) \quad, \quad \hat{p}_{A}=\frac{1}{\sqrt{2}}\left(\hat{p}_{1}^{\prime}+\hat{p}_{2}^{\prime}\right)=\frac{1}{\sqrt{2}}\left(\hat{p}_{1}+\hat{x}_{2}\right), \\
\hat{x}_{B}=\frac{1}{\sqrt{2}}\left(\hat{x}_{1}^{\prime}-\hat{x}_{2}^{\prime}\right)=\frac{1}{\sqrt{2}}\left(\hat{x}_{1}+\hat{p}_{2}\right) \quad, \quad \hat{p}_{B}=\frac{1}{\sqrt{2}}\left(\hat{p}_{1}^{\prime}-\hat{p}_{2}^{\prime}\right)=\frac{1}{\sqrt{2}}\left(\hat{p}_{1}-\hat{x}_{2}\right) .
\end{array}
$$

Thus, when measuring the noise of $\hat{x}_{A}+\hat{x}_{B}=\sqrt{2} \hat{x}_{1}$ and $\hat{p}_{A}-\hat{p}_{B}=\sqrt{2} \hat{x}_{2}$, the squeezing of $\hat{x}_{1}$ and $\hat{x}_{2}$ are visible, while when measuring the noise of $\hat{x}_{A}-\hat{x}_{B}=-\sqrt{2} \hat{p}_{2}$ and $\hat{p}_{A}+\hat{p}_{B}=\sqrt{2} \hat{p}_{1}$, the anti-squeezing of $\hat{p}_{1}$ and $\hat{p}_{2}$ are visible. Or in other words: $\hat{x}_{A}$ and $\hat{x}_{B}$ are correlated while $\hat{p}_{A}$ and $\hat{p}_{B}$ are anti-correlated, both below the standard quantum limit demonstrating quadrature entanglement.

The two modes of the two-mode squeezed state at the fiber coupler output are sent to two homodyne detection stations, Alice and Bob, where an arbitrary quadrature, $\hat{q}_{i}\left(\theta_{i}\right)$, is measured depending on the local oscillator phase $\theta_{i}$ at Alice $(i=A)$ and Bob $(i=B)$,

$$
\begin{gathered}
\hat{q}_{A}\left(\theta_{A}\right)=\hat{x}_{A} \cos \theta_{A}+\hat{p}_{A} \sin \theta_{A}=\frac{1}{\sqrt{2}}\left(\hat{x}_{1}-\hat{p}_{2}\right) \cos \theta_{A}+\frac{1}{\sqrt{2}}\left(\hat{p}_{1}+\hat{x}_{2}\right) \sin \theta_{A} \\
\hat{q}_{B}\left(\theta_{B}\right)=\hat{x}_{B} \cos \theta_{B}+\hat{p}_{B} \sin \theta_{B}=\frac{1}{\sqrt{2}}\left(\hat{x}_{1}+\hat{p}_{2}\right) \cos \theta_{B}+\frac{1}{\sqrt{2}}\left(\hat{p}_{1}-\hat{x}_{2}\right) \sin \theta_{B} .
\end{gathered}
$$

The quadrature entanglement can then be illustrated as correlations, or covariance, between $\hat{q}_{A}\left(\theta_{A}\right)$ 
and $\hat{q}_{B}\left(\theta_{B}\right)$ :

$$
\begin{aligned}
\operatorname{cov}\left\{\hat{q}_{A}\left(\theta_{A}\right), \hat{q}_{B}\left(\theta_{B}\right)\right\}= & \left\langle\hat{q}_{A}\left(\theta_{A}\right) \hat{q}_{B}\left(\theta_{B}\right)\right\rangle-\left\langle\hat{q}_{A}\left(\theta_{A}\right)\right\rangle\left\langle\hat{q}_{B}\left(\theta_{B}\right)\right\rangle=\left\langle\hat{q}_{A}\left(\theta_{A}\right) \hat{q}_{B}\left(\theta_{B}\right)\right\rangle \\
= & \frac{1}{2}\left\langle\left[\left(\hat{x}_{1}-\hat{p}_{2}\right) \cos \theta_{A}+\left(\hat{p}_{1}+\hat{x}_{2}\right) \sin \theta_{A}\right] \times\right. \\
& \left.\quad\left[\left(\hat{x}_{1}+\hat{p}_{2}\right) \cos \theta_{B}+\left(\hat{p}_{1}-\hat{x}_{2}\right) \sin \theta_{B}\right]\right\rangle \\
= & \frac{1}{2}\left(\left\langle\hat{x}_{1}^{2}\right\rangle-\left\langle\hat{p}_{2}^{2}\right\rangle\right) \cos \theta_{A} \cos \theta_{B}+\frac{1}{2}\left(\left\langle\hat{p}_{1}^{2}\right\rangle-\left\langle\hat{x}_{2}^{2}\right\rangle\right) \sin \theta_{A} \sin \theta_{B} \\
= & \frac{1}{2}\left(\left\langle\Delta \hat{x}_{1}^{2}\right\rangle-\left\langle\Delta \hat{p}_{2}^{2}\right\rangle\right) \cos \theta_{A} \cos \theta_{B}-\frac{1}{2}\left(\left\langle\Delta \hat{x}_{2}^{2}\right\rangle-\left\langle\Delta \hat{p}_{1}^{2}\right\rangle\right) \sin \theta_{A} \sin \theta_{B},
\end{aligned}
$$

where it is used that $\left\langle\hat{q}_{A}\left(\theta_{A}\right)\right\rangle=\left\langle\hat{q}_{B}\left(\theta_{B}\right)\right\rangle=0$ for two-mode squeezed states, $\left\langle\hat{q}_{1} \hat{q}_{2}\right\rangle=0$ for $\hat{q}_{1}=\left(\hat{x}_{1}, \hat{p}_{1}\right)$ and $\hat{q}_{2}=\left(\hat{x}_{2}, \hat{p}_{2}\right)$ since the amplitude squeezed states before interfering are separable squeezed vacuum states, $\left\langle\hat{x}_{i} \hat{p}_{i}\right\rangle=0$ for $i=(1,2)$ as amplitude and phase quadrature are independent for squeezing exactly along $\hat{x}$ or $\hat{p}$, and $\left\langle\Delta \hat{q}_{i}^{2}\right\rangle=\left\langle\hat{q}_{i}^{2}\right\rangle-\left\langle\hat{q}_{i}\right\rangle^{2}=\left\langle\hat{q}_{i}^{2}\right\rangle$ for $q=(x, p)$ and $i=(1,2)$ as $\left\langle\hat{q}_{i}\right\rangle=0$ for squeezed vacuum. Thus, since $\left.\left\langle\Delta \hat{p}_{1}^{2}\right\rangle,\left\langle\Delta \hat{p}_{1}^{2}\right\rangle\right\rangle\left\langle\Delta \hat{x}_{1}^{2}\right\rangle,\left\langle\Delta \hat{x}_{1}^{2}\right\rangle$, we expect strong anti-correlation when both $\theta_{A}$ and $\theta_{B}$ equals $\pi n$ with $n$ being an integer number, while strong correlation when both $\theta_{A}$ and $\theta_{B}$ equals $\pi(n+1 / 2)$. This is easier seen in the ideal case where the direct and delay line are identical so that $\left\langle\Delta \hat{x}_{1}^{2}\right\rangle=\left\langle\Delta \hat{x}_{2}^{2}\right\rangle \equiv V_{x}$ and $\left\langle\Delta \hat{p}_{1}^{2}\right\rangle=\left\langle\Delta \hat{p}_{2}^{2}\right\rangle \equiv V_{p}$, in which case the above covariance can be simplified using $\cos (\alpha+\beta)=\cos \alpha \cos \beta-\sin \alpha \sin \beta$ :

$$
\operatorname{cov}\left\{\hat{q}_{A}\left(\theta_{A}\right), \hat{q}_{B}\left(\theta_{B}\right)\right\}=\left\langle\hat{q}_{A}\left(\theta_{A}\right) \hat{q}_{B}\left(\theta_{B}\right)\right\rangle=\frac{1}{2}\left(V_{x}-V_{p}\right) \cos \left(\theta_{A}+\theta_{B}\right) .
$$

The quadrature covariance for the ideal case in (2.11) is shown in Fig. 2.6b. Here, two-mode squeezing and anti-squeezing can be measured in areas of strong correlations, and is constant along constant $\theta_{A}+\theta_{B}$. As a result, for partial tomography of the generated two-mode squeezed state, we measure two sets of quadratures $\left(\hat{q}_{A}\left(\theta_{A}\right), \hat{q}_{B}\left(\theta_{B}\right)\right)$ : Set 1 along constant correlation (maximum squeezing and anti-squeezing) with $\theta_{A}=-\theta_{B}=\theta$; set 2 perpendicular to constant squeezing (across maximum squeezing and anti-squeezing) with $\theta_{A}=\theta_{B}+\pi / 2=\theta$. Both set are measured with $\theta$ in the range from 0 to $\pi / 2$, and is marked in Fig. 2.6b. By adding and subtracting $\hat{q}_{A}$ and $\hat{q}_{B}$ of these two measured sets, we observe two-mode squeezing, anti-squeezing and all in between.

When adding/subtracting quadratures from Alice and Bob measured in set $1\left(\theta_{B}=-\theta_{A}\right)$, from Eq. (2.9-2.10) the added and subtracted quadrature noise becomes

$$
\begin{aligned}
& \left\langle\Delta\left(\hat{q}_{A}(\theta)+\hat{q}_{B}(-\theta)\right)^{2}\right\rangle=2\left(\left\langle\Delta \hat{x}_{1}^{2}\right\rangle \cos ^{2} \theta+\left\langle\Delta \hat{x}_{2}^{2}\right\rangle \sin ^{2} \theta\right), \\
& \left\langle\Delta\left(\hat{q}_{A}(\theta)-\hat{q}_{B}(-\theta)\right)^{2}\right\rangle=2\left(\left\langle\Delta \hat{p}_{2}^{2}\right\rangle \cos ^{2} \theta+\left\langle\Delta \hat{p}_{1}^{2}\right\rangle \sin ^{2} \theta\right),
\end{aligned}
$$

and thus we expect to measure two-mode squeezing and anti-squeezing for all $\theta$, and constant squeezing levels in the case of symmetric two-mode squeezing where $\left\langle\Delta \hat{x}_{1}^{2}\right\rangle=\left\langle\Delta \hat{x}_{2}^{2}\right\rangle$ and $\left\langle\Delta \hat{p}_{1}^{2}\right\rangle=$ $\left\langle\Delta \hat{p}_{2}^{2}\right\rangle$.

Finally, when adding/subtracting quadratures from Alice and Bob measured in set $2\left(\theta_{B}=\right.$ $\left.\theta_{A}-\pi / 2\right)$ we obtain

$$
\begin{aligned}
\hat{q}_{A}(\theta) \pm \hat{q}_{B}(\theta-\pi / 2) & =\hat{x}_{A} \cos \theta+\hat{p}_{A} \sin \theta \pm \hat{x}_{B} \cos (\theta-\pi / 2) \pm \hat{p}_{B} \sin (\theta-\pi / 2) \\
& =\hat{x}_{A} \cos \theta+\hat{p}_{A} \sin \theta \pm \hat{x}_{B} \sin \theta \mp \hat{p}_{B} \cos \theta .
\end{aligned}
$$

For $\theta=\pi / 4$ we extract again two-mode squeezing and anti-squeezing:

$$
\begin{aligned}
& \left.\left\langle\Delta \hat{q}_{A}(\pi / 4)+\hat{q}_{B}(\pi / 4-\pi / 2)\right)^{2}\right\rangle=\frac{1}{2}\left\langle\Delta\left(\hat{x}_{A}+\hat{p}_{A}+\hat{x}_{B}-\hat{p}_{B}\right)^{2}\right\rangle=2\left(\left\langle\Delta \hat{x}_{1}^{2}\right\rangle+\left\langle\Delta \hat{x}_{2}^{2}\right\rangle\right), \\
& \left.\left\langle\Delta \hat{q}_{A}(\pi / 4)-\hat{q}_{B}(\pi / 4-\pi / 2)\right)^{2}\right\rangle=\frac{1}{2}\left\langle\Delta\left(\hat{x}_{A}+\hat{p}_{A}-\hat{x}_{B}+\hat{p}_{B}\right)^{2}\right\rangle=2\left(\left\langle\Delta \hat{p}_{1}^{2}\right\rangle+\left\langle\Delta \hat{p}_{2}^{2}\right\rangle\right),
\end{aligned}
$$




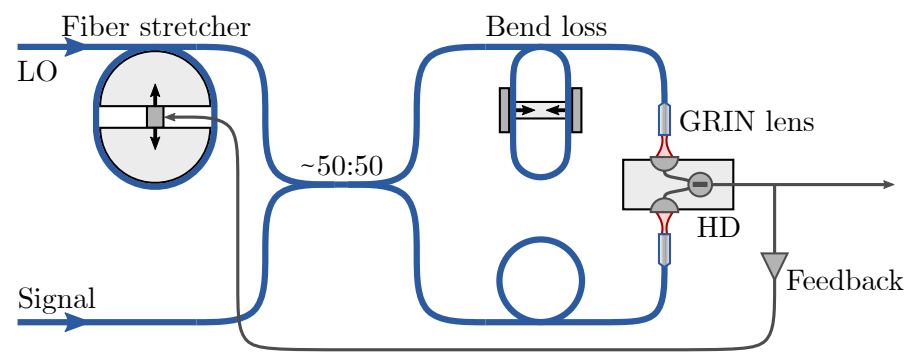

(a)

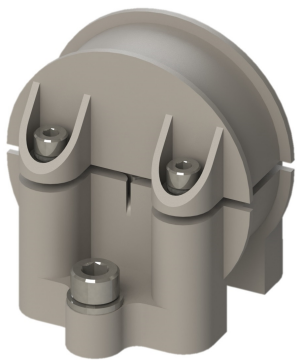

(b)

Figure 2.7: (a) Schematics of the fiber coupled homodyne detection (HD) setup, where blue lines indicates single mode fiber. (b) Illustration of the homemade fiber stretcher consisting of a top and bottom part around a piezoelectric actuator. Pre-load can be applied by tightening the screw with O-rings attaching the top part to the bottom part.

using (2.8). Moving away from $\theta=\pi / 4$ we measure less and less two-mode squeezing and antisqueezing, until $\theta=0$ or $\pi / 2$ where, using (2.8),

$$
\begin{aligned}
\left\langle\Delta\left(\hat{q}_{A}(0) \pm \hat{q}_{B}(0-\pi / 2)\right)^{2}\right\rangle & \left.=\left\langle\Delta\left(\hat{x}_{A} \mp \hat{p}_{B}\right)^{2}\right\rangle\right)=\frac{1}{2}\left(\left\langle\Delta \hat{x}_{1}^{2}\right\rangle+\left\langle\Delta \hat{p}_{2}^{2}\right\rangle+\left\langle\Delta \hat{p}_{1}^{2}\right\rangle+\left\langle\Delta \hat{x}_{2}^{2}\right\rangle\right) \\
\left\langle\Delta\left(\hat{q}_{A}(\pi / 2) \pm \hat{q}_{B}(\pi / 2-\pi / 2)\right)^{2}\right\rangle & \left.=\left\langle\Delta\left(\hat{p}_{A} \pm \hat{x}_{B}\right)^{2}\right\rangle\right)=\frac{1}{2}\left(\left\langle\Delta \hat{p}_{1}^{2}\right\rangle+\left\langle\Delta \hat{x}_{2}^{2}\right\rangle+\left\langle\Delta \hat{x}_{1}^{2}\right\rangle+\left\langle\Delta \hat{p}_{2}^{2}\right\rangle\right)
\end{aligned}
$$

and we simply measure added uncorrelated noise at Alice and Bob corresponding to thermal states when tracing out one mode of the two-mode squeezed state.

\subsubsection{Experimental methods}

The experimental setup is outlined in section 2.3.1. In Fig. 2.7a schematics of the fiber-coupled homodyne detection (HD) is seen. Here, the local oscillator (LO) phase is controlled by a fiber stretcher illustrated in Fig. 2.7b, and mixed with the signal in a 50:50 fiber coupler. The HD is balanced by inducing bend loss in one fiber coupler output arm: The fiber is coiled up in between two plates, which are squeezed towards each other with a micrometer screw. Finally, light is coupled from fiber onto the photo diodes using anti-reflective coated graded-index (GRIN) lenses.

The fiber stretcher based on [84] is illustrated in Fig. 2.7b. With a piezoelectric actuator the fiber stretcher top and bottom part are pushed away from each other, stretching a single mode fiber coiled around it. The bandwidth is set by the mechanical resonance frequency of the top part and optimized to $2.5 \mathrm{kHz}$ by applying pre-load. This can be improved by a lighter design of the top-part, but it is sufficient for our purpose.

\subsubsection{Temporal data processing}

As two-mode squeezing is generated by temporal multiplexing a single squeezing source, we need to filter temporal modes for analysis. Due to the switching and synchronization of temporal modes, two-mode squeezing at the 50:50 fiber coupler output is only present half of the time in a switching period (whereas vacuum is present the other half time, which is the cost of using a single squeezing source).

The HD signal from Alice and Bob is shown in Fig. 2.8a (left) as function of time, where the two-mode squeezing is seen with an offset due to the coherent seed beam transmitted through the setup for phase locks. Here we can select time traces of $900 \mathrm{~ns}$ well within the temporal region of two-mode squeezing with 1 ps length. Doing so, one measurement set consist of such 16000 time traces, with the temporal histogram shown in Fig. 2.8a (center). The quadratures measured in Fig. 2.8 are $\hat{x}_{A}$ and $\hat{x}_{B}$, and thus by adding and subtracting the measured quadrature traces, we 
(a) Raw data
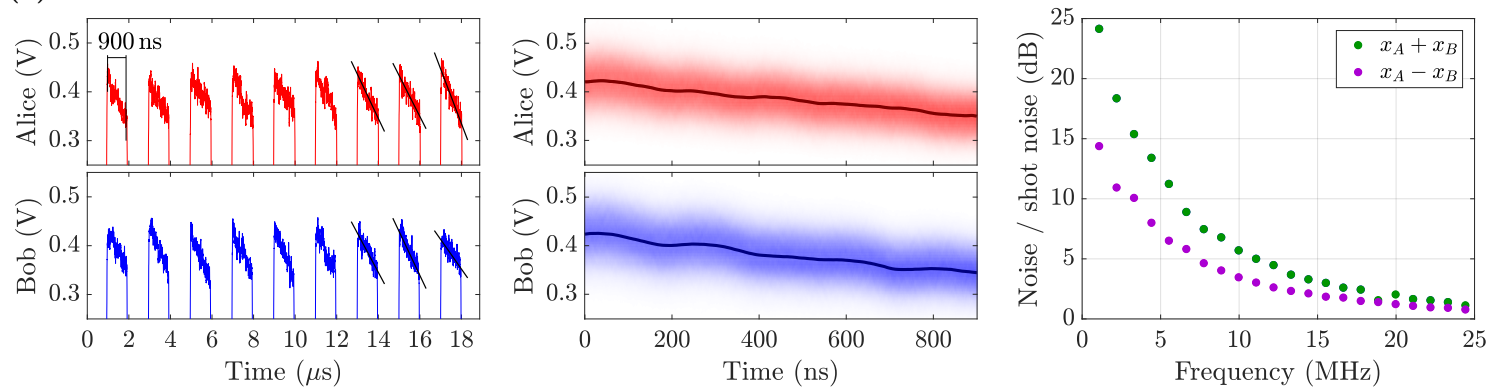

(b) Slope compensated
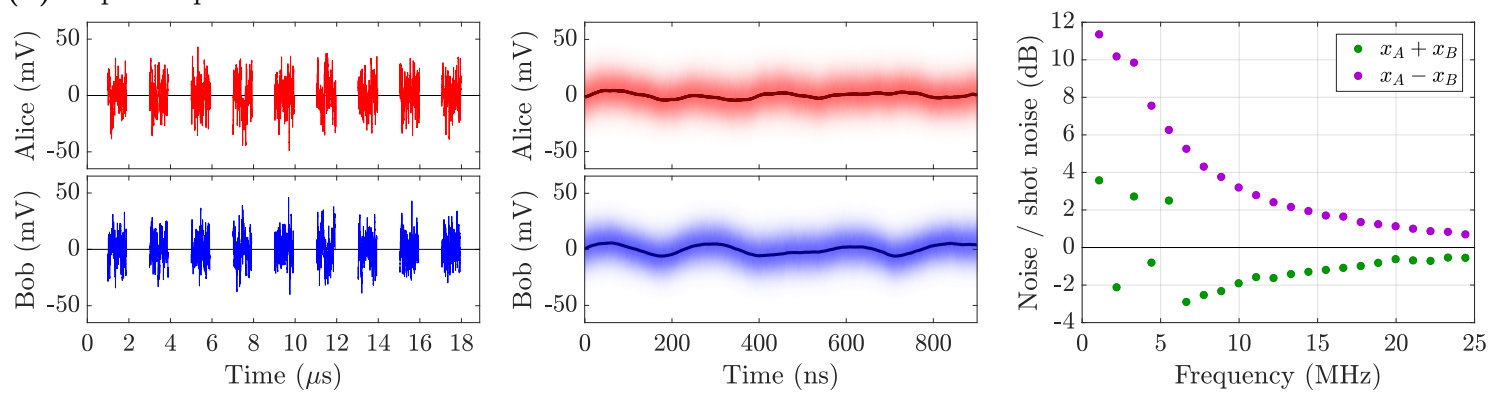

(c) Repeating noise compensated
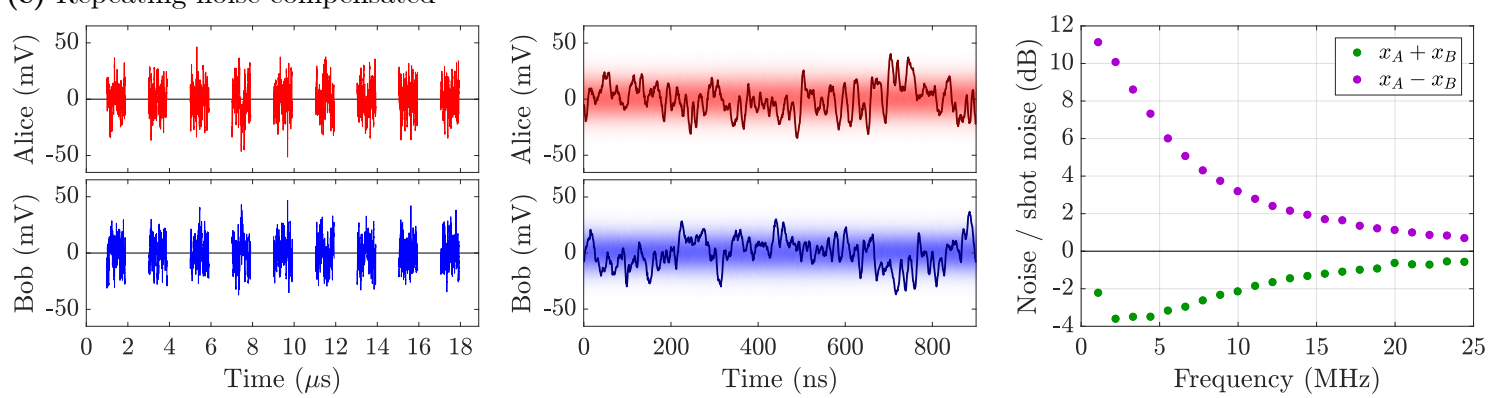

Figure 2.8: Example of the temporal post processing procedure of measured data, here of $\hat{x}_{A}$ and $\hat{x}_{B}$. (a) Raw data with HD signals from Alice and Bob shown in (left), temporal histogram of 16000 time traces of $900 \mathrm{~ns}$ length as indicated in (left) shown in (center), and spectrum of added and subtracted signals in (right). (b) Data in (a) compensated for non-zero slope by subtracting linear regression lines as shown in (a,left). (c) Data in (b) compensated for repeating noise by subtracting the average of the 16000 slope compensated time traces shown as solid lines in (b,center). Here, the solid lines in (center) is a single time trace from Alice and Bob indicating quadrature correlations, while the final two-mode squeezing spectrum after processing is shown in (right). 
expect squeezing and anti-squeezing according to Eq. (2.12-2.13). However, as seen in Fig. 2.8a (right) where the Fourier transform of $\hat{x}_{A} \pm \hat{x}_{B}$ is shown, this is not the case. This is due to the slope seen on each time trace in Fig. 2.8a (left) and (center), which is caused by a decaying detector response when the coherent seed beam is turned on and off in the detector.

To compensate for the non-zero slope on each time trace, we cannot simply high pass filter the HD signals, as the slope includes frequency components in the MHz-regime, and we would filter out the two-mode squeezing. Instead, we subtract the slope from each individual time trace. However, due to spurious interference of the coherent seed beam and small phase fluctuations, the slope of each time trace varies, and we cannot subtract the same slope from each time trace. Instead, assuming the decaying detector response is linear on this time scale, a linear regression line is fitted to each time trace, as shown on the last three pulses in Fig. 2.8a (left), and subtracted.

The result of compensating for non-zero slope on each time trace is shown in Fig. 2.8b, where the HD signals in (left) are effectively high pass filtered without affecting the squeezing spectrum, and two-mode squeezing below added shot noise is visible in (right). Yet, peaks are seen in the spectrum due to oscillations visible in the temporal histogram in Fig. 2.8b (center). These are caused by the switching process and maybe oscillating detection response. However, since they are repeating with the switching process, they can be compensated for by subtracting an average of the 16000 time traces, indicated by the solid lines in Fig. 2.8b (center).

Finally, the result of compensating for the repeating noise is shown in Fig. 2.8c, where in (center) the temporal histogram is seen to be nicely constant, and a two-mode squeezing spectrum in (right) resembling the OPO spectrum without additional noise peaks. Here the degrading of squeezing in the first frequency component shown is due to the coherent seed beam not being shot noise limited, and limiting bandwidth of $\sim 1 \mathrm{MHz}$ due to the $900 \mathrm{~ns}$ short time traces. The solid line shown in Fig. 2.8c (center) is a single time trace at Alice and Bob showing quadrature correlations. To observe stronger correlations, only frequency components within the OPO bandwidth should be observed, which is the case in Fig. 2.3.

\subsubsection{Sources of noise and theoretical prediction}

To theoretically predict the measured two-mode squeezing, we include several sources of noise. Besides the squeezed vacuum noise itself, the seed beam transmitted through the optical parametric oscillator (OPO) contains noise, while additional noise is added after the OPO. Besides this, due to phase fluctuations, when measuring one quadrature, noise of the other quadrature is observed as well. Finally, electronic noise is also considered. When adding and subtracting the measured $\hat{x}$ - or $\hat{p}$-quadrature of the two-mode squeezed state at Alice and Bob, the single mode squeezed states in the direct or delay line is extracted according to (2.12-2.13) for $\theta=0$ and $\pi / 2$. So, for simplicity, in the following discussion we consider the case of measuring single mode squeezing in the direct and delay line. The different sources of quadrature noise are summarized in Fig. 2.9 as a function of the pump power.

Electronic noise is present in both measured quadratures and shot noise. It is independent on pump power, and we deal with it by subtracting the electronic noise variance from both measured quadrature variance and shot noise variance. However, the electronic noise is less than $-20 \mathrm{~dB}$ relative to shot noise, and can in principle be neglected.

Noise unrelated to the squeezing process is characterized by measuring the seed spectrum by blocking the pump to the OPO, and the result is seen in Fig. 2.4 (grey points). From the direct line, low frequency noise resulting from technical noise of the seed beam is observed. Even more low frequency noise is apparent in the squeezed state of the delay line, and we believe it originates from phase noise generated by the $200 \mathrm{~m}$ fiber, and amplitude noise from the fiber switch which is most prominent at 5-6 $\mathrm{MHz}$.

The measured seed beam noise undergoes squeezing in the OPO, and thus depends on the pump power. The squeezed and anti-squeezed quadrature noise spectrum from the OPO including the noisy seed beam is derived below in section 2.6 .5 to be

$$
\left\langle\Delta \hat{q}^{2}\right\rangle=\frac{1}{2} \mp \frac{2 \varepsilon \gamma \eta}{(\gamma \pm \varepsilon)^{2}+\omega^{2}}+\frac{K_{q}}{(\gamma \pm \varepsilon)^{2}+\omega^{2}} \quad, \quad q=x, p,
$$




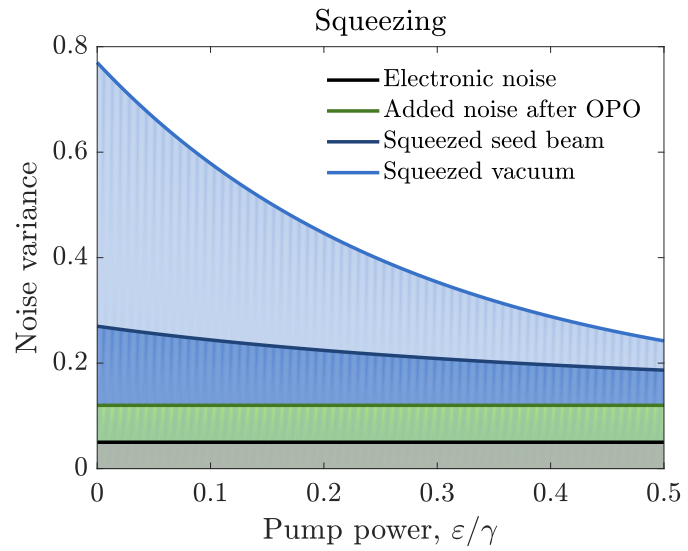

(a)

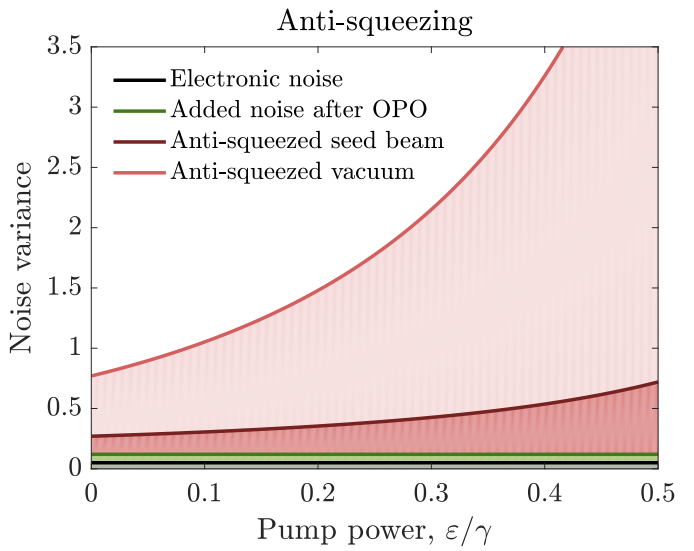

(b)

Figure 2.9: Illustration of quadrature noise when measuring single mode squeezing in the direct or delay line, including vacuum squeezing, squeezing of seed beam noise, added noise after the OPO and electronic noise. (a) Illustrates how the different noise sources behave in the squeezed quadrature when increasing the pump power, and in (b) the anti-squeezed quadrature is illustrated. The sources or noise shown here are not to scale.

where $\varepsilon$ is the pump rate, $\gamma$ is the total OPO decay rate, $\eta$ is the overall efficiency and $\omega$ is the angular frequency. The first two terms corresponds to vacuum squeezing when subtracted and antisqueezing when added (with $1 / 2$ being the vacuum variance), while the third term corresponds to squeezing/anti-squeezing of the seed beam noise $\left\langle\Delta \hat{q}_{s}^{2}\right\rangle$ with $K_{q}=4 \eta \gamma \gamma_{s}\left(\left\langle\Delta \hat{q}_{s}^{2}\right\rangle-1 / 2\right)$, where $\gamma_{s}$ is the coupling rate of the mirror through which the seed beam is leaked into the OPO. From the measured seed beam spectrum when blocking the pump $(\varepsilon=0), K_{q}$ can be estimated as

$$
K_{q}=\left(\gamma^{2}+\omega^{2}\right)\left(\left\langle\Delta \hat{q}_{0}^{2}\right\rangle-1 / 2\right),
$$

where $\left\langle\Delta \hat{q}_{0}^{2}\right\rangle$ then corresponds to grey points in the direct line of Fig. 2.4. Notice how the squeezed vacuum in (2.14) (the first two terms) goes towards 0 for $\varepsilon \rightarrow \gamma$ and $\eta=1$ at $\omega=0$, while the seed beam noise goes towards $\left(\left\langle\Delta \hat{q}_{0}^{2}\right\rangle-1 / 2\right) / 4$, and thus the seed beam noise can only be eliminated when $\left\langle\Delta \hat{q}_{0}^{2}\right\rangle=1 / 2$ is shot noise limited. The OPO decay rate is estimated to $\gamma=2 \pi 8.1 \mathrm{MHz}$ by measuring the OPO intracavity losses $(0.55 \%)$, the cavity length $(320 \mathrm{~mm})$ and the transmittivity of the coupling mirror $(10 \%)$, while we estimate the pump rate to $\varepsilon=2 \pi 5.2 \mathrm{MHz}$ for a pump power of $350 \mathrm{~mW}$ and an estimated OPO threshold power of $833 \mathrm{~mW}$. The overall efficiency is $\eta=68 \%$ as discussed in section 2.6.2.

To predict the squeezing, the direct line Eq. (2.14) is sufficient, since negligible noise is added after the OPO. However, as apparent in Fig. 2.4 and discussed above, both amplitude and phase noise is added in the delay line, and is independent on the pump power. To encounter this, the difference in measured noise spectrum in the direct and delay line when blocking the pump (grey points in Fig. 2.4) is added to Eq. (2.14) for the squeezing spectrum in the delay line.

Finally, phase fluctuations are included by applying a normal distribution $P(\sigma, \theta)$ of width $\sigma$ to the measured quadrature as

$$
\begin{aligned}
\left\langle\Delta \hat{x}_{\sigma}^{2}(\theta)\right\rangle & =\int P(\sigma, \theta)\left(\left\langle\Delta \hat{x}^{2}\right\rangle \cos ^{2} \theta+\left\langle\Delta \hat{p}^{2}\right\rangle \sin ^{2} \theta\right) \mathrm{d} \theta \\
& \approx\left\langle\Delta \hat{x}^{2}\right\rangle \cos ^{2}(\theta+\sigma)+\left\langle\Delta \hat{p}^{2}\right\rangle \sin ^{2}(\theta+\sigma)
\end{aligned}
$$

and similar for $\left\langle\Delta \hat{p}^{2}\right\rangle$ with $\cos (\theta+\sigma)$ and $\sin (\theta+\sigma)$ interchanged, and with the the approximation valid for small $\sigma$ [78]. Since we expect different phase fluctuations in the direct line and the $200 \mathrm{~m}$ long delay line, different normal distributions of width $\sigma_{1}$ and $\sigma_{2}$ are used respectively. By using $\sigma_{1}$ and $\sigma_{2}$ as fitting parameters, the theoretical predicted squeezing in Eq. (2.14), with additional 


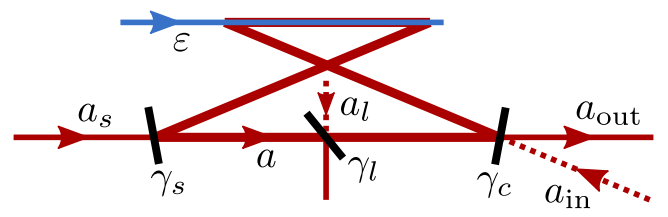

Figure 2.10: Schematic model of the OPO as squeezing source. Here, $\gamma_{c}$ is the decay rate due to the cavity coupling mirror of $10 \%$ transmission, while $\gamma_{s}$ is the decay rate due to the high reflective mirror through which the seed is leaked into the cavity. The internal cavity loss is modelled by a beams splitter transformation leading to the decay rate $\gamma_{l}$. $\hat{a}$ is the annihilation operator of the cavity mode, $\hat{a}_{i}$ for $i=$ in, out, $s, l$ are annihilation operators of modes mixing with the cavity mode, and $\varepsilon$ is the effective pump intensity which is treated classically. Dotted line corresponds to modes with vacuum.

added noise in the delay line, is fitted to the experimental data, and shown as hollow points in Fig. 2.4. From the fitting we get $\sigma_{1}=1.9 \pm 1.2^{\circ}$ and $\sigma_{2}=4.1 \pm 0.6^{\circ}$ (uncertainty estimated as $95 \%$ confidence interval), and the theoretical prediction is seen to agree excellent with measured data.

\subsubsection{Squeezing spectrum with seed beam noise}

In order to derive the squeezing spectrum of the OPO in Eq. (2.14) with a seed beam leaked into the cavity, we follow the approach in [79] where the spectrum is derived from the quantum Langevin equations of a cavity. However, in [79] the leakage of a seed beam into the cavity is not considered, and so the model is here extended for this purpose - a schematic of the model is sketched in Fig. 2.10.

Treating the pump power classically with the effective pump intensity $\varepsilon$, and frequency of double the cavity resonant mode frequency $\omega_{0}$, the system Hamiltonian of the OPO cavity mode is

$$
\hat{H}_{\mathrm{sys}}=\hbar \omega_{0} \hat{a}^{\dagger} \hat{a}+\frac{1}{2}\left(\varepsilon e^{-i 2 \omega_{0} t} \hat{a}^{\dagger 2}-\varepsilon^{*} e^{i 2 \omega_{0} t} \hat{a}^{2}\right),
$$

where $\hat{a}$ is the annihilation operator of the cavity mode. Here the second term is the Hamiltonian of the parametric down conversion process of the pump in a second order non-linear crystal, and the pump is assumed non-depleted (i.e. far below the OPO threshold). Using $\left[\hat{a}, \hat{H}_{\text {sys }}\right]=$ $\hbar \omega_{0} \hat{a}+i \hbar \varepsilon e^{-i 2 \omega_{0} t} \hat{a}^{\dagger}$, the Langevin equations becomes

$$
\begin{aligned}
\frac{d \hat{a}}{d t} & =-\frac{i}{\hbar}\left[\hat{a}, \hat{H}_{\mathrm{sys}}\right]-\gamma \hat{a}+\hat{\Gamma} \\
& =-i \omega_{0} \hat{a}+\varepsilon e^{-i 2 \omega_{0} t} \hat{a}^{\dagger}-\gamma \hat{a}+\hat{\Gamma},
\end{aligned}
$$

where $\gamma$ is the cavity damping rate, and $\hat{\Gamma}$ is the noise operator. Cavity damping is caused by out coupling of the cavity mode, internal cavity loss, and leakage through the high reflective mirror through which the seed is coupled, each leading to corresponding decay rates $\gamma_{c}, \gamma_{l}$ and $\gamma_{s}$ respectively so that $\gamma=\gamma_{c}+\gamma_{l}+\gamma_{s}$. Where light is coupled out, vacuum in the mode $\hat{a}_{\text {in }}$ is coupled in. Similar, the cavity internal loss can be modelled by a beam splitter transformation coupling vacuum in mode $\hat{a}_{l}$ into the cavity mode. Finally, with the seed beam in mode $\hat{a}_{s}$ leaked through a high reflector, the noise operator is $\hat{\Gamma}=\sqrt{2 \gamma_{c}} \hat{a}_{\text {in }}+\sqrt{2 \gamma_{l}} \hat{a}_{l}+\sqrt{2 \gamma_{s}} \hat{a}_{s}$. With the mirror between $\hat{a}_{s}$ and $\hat{a}$ being high reflective, we have $\gamma_{s} \ll \gamma_{c}, \gamma_{l}$ and so $\gamma \approx \gamma_{c}+\gamma_{l}$. However, we cannot choose $\gamma_{s}=0$ so that $\hat{\Gamma}=\sqrt{2 \gamma_{c}} \hat{a}_{\text {in }}+\sqrt{2 \gamma_{l}} \hat{a}_{l}$, as then no seed beam leaks into the cavity mode and the model simplifies to that in [79].

Moving to a rotating frame with frequency $\omega_{0}$,

$$
\hat{a} \rightarrow \hat{a} e^{-i \omega_{0} t} \quad, \quad \frac{d \hat{a}}{d t} \rightarrow \frac{d}{d t}\left\{\hat{a} e^{-i \omega_{0} t}\right\}=\frac{d \hat{a}}{d t} e^{-i \omega_{0} t}-i \omega_{0} \hat{a} e^{-i \omega_{0} t},
$$


Eq. (2.15) simplifies to

$$
\frac{d \hat{\boldsymbol{a}}}{d t}=(\mathbf{A}-\gamma \mathbf{1}) \boldsymbol{a}+\hat{\boldsymbol{\Gamma}},
$$

where

$$
\hat{\boldsymbol{a}}=\left(\begin{array}{c}
\hat{a} \\
\hat{a}^{\dagger}
\end{array}\right) \quad, \quad \mathbf{A}=\left(\begin{array}{cc}
0 & \varepsilon \\
\varepsilon^{*} & 0
\end{array}\right) \quad, \quad \hat{\boldsymbol{\Gamma}}=\left(\begin{array}{c}
\hat{\Gamma} \\
\hat{\Gamma}^{\dagger}
\end{array}\right) .
$$

Eq. (2.16) is easiest solved in frequency domain, and by the Fourier transform,

$$
\begin{aligned}
\hat{a}(t) & =\frac{1}{\sqrt{2 \pi}} \int_{-\infty}^{\infty} \hat{\tilde{a}}(\omega) e^{-i \omega t} d \omega, \\
\hat{a}^{\dagger}(t) & =\frac{1}{\sqrt{2 \pi}} \int_{-\infty}^{\infty} \hat{\tilde{a}}^{\dagger}(\omega) e^{i \omega t} d \omega=\frac{1}{\sqrt{2 \pi}} \int_{-\infty}^{\infty} \hat{\tilde{a}}^{\dagger}(-\omega) e^{-i \omega t} d \omega,
\end{aligned}
$$

Eq. (2.16) becomes

$$
\begin{gathered}
-i \omega \hat{\tilde{\boldsymbol{a}}}=(\mathbf{A}-\gamma \mathbf{1}) \hat{\tilde{\boldsymbol{a}}}+\hat{\tilde{\boldsymbol{\Gamma}}}, \\
\hat{\tilde{\boldsymbol{a}}}=\left(\begin{array}{c}
\hat{\tilde{a}}(\omega) \\
\hat{\tilde{a}}^{\dagger}(-\omega)
\end{array}\right) \quad, \quad \hat{\tilde{\boldsymbol{\Gamma}}}=\left(\begin{array}{c}
\hat{\tilde{\Gamma}}(\omega) \\
\hat{\tilde{\Gamma}}^{\dagger}(-\omega)
\end{array}\right) .
\end{gathered}
$$

Solving for $\hat{\tilde{\boldsymbol{a}}}$ in $(2.17)$,

$$
\hat{\tilde{\boldsymbol{a}}}=-(\mathbf{A}+(i \omega-\gamma) \mathbf{1})^{-1} \hat{\tilde{\boldsymbol{\Gamma}}},
$$

leads to the solution

$$
\begin{aligned}
\hat{\tilde{a}}(\omega) & =\frac{(i \omega-\gamma) \hat{\tilde{\Gamma}}(\omega)-\varepsilon \hat{\tilde{\Gamma}}(-\omega)}{|\varepsilon|^{2}-(i \omega-\gamma)^{2}}, \\
\hat{\tilde{a}}^{\dagger}(-\omega) & =\frac{(i \omega-\gamma) \hat{\tilde{\Gamma}}(-\omega)-\varepsilon \hat{\tilde{\Gamma}}^{\dagger}(\omega)}{|\varepsilon|^{2}-(i \omega-\gamma)^{2}},
\end{aligned}
$$

of the annihilation and creation operator of the cavity frequency modes.

With the solution of the Langevin equations in (2.18), and writing the effective pump intensity as $\varepsilon=|\varepsilon| e^{i \phi}$, the cavity mode quadrature in frequency domain, $\tilde{q}_{a}(\theta)$, becomes

$$
\begin{aligned}
& \hat{\tilde{q}}_{a}(\theta)=\frac{1}{\sqrt{2}}\left(e^{-i \theta} \hat{\tilde{a}}(\omega)+e^{i \theta} \hat{\tilde{a}}^{\dagger}(-\omega)\right) \\
& =\frac{1}{\sqrt{2}} \frac{e^{-i \theta}\left[(i \omega-\gamma) \hat{\tilde{\Gamma}}(\omega)-|\varepsilon| e^{i \phi} \hat{\tilde{\Gamma}}^{\dagger}(-\omega)\right]+e^{i \theta}\left[(i \omega-\gamma) \hat{\tilde{\Gamma}}^{\dagger}(-\omega)-|\varepsilon| e^{-i \phi} \hat{\tilde{\Gamma}}(\omega)\right]}{|\varepsilon|^{2}-(i \omega-\gamma)^{2}} \\
& =\frac{(i \omega-\gamma) \frac{1}{\sqrt{2}}\left[e^{-i \theta} \hat{\tilde{\Gamma}}(\omega)+e^{i \theta} \hat{\tilde{\Gamma}}(-\omega)\right]-|\varepsilon| \frac{1}{\sqrt{2}}\left[e^{-i(\phi-\theta)} \hat{\tilde{\Gamma}}(\omega)+e^{i(\phi-\theta)} \hat{\tilde{\Gamma}}^{\dagger}(-\omega)\right]}{|\varepsilon|^{2}-(i \omega-\gamma)^{2}} \\
& =\frac{(i \omega-\gamma) \hat{\tilde{\tilde{q}}}_{\Gamma}(\theta)-|\varepsilon| \hat{\tilde{q}}_{\Gamma}(\phi-\theta)}{|\varepsilon|^{2}-(i \omega-\gamma)^{2}} .
\end{aligned}
$$

Here,

$$
\begin{aligned}
\hat{\tilde{q}}_{\Gamma}(\theta)= & \frac{1}{\sqrt{2}}\left(e^{-i \theta} \hat{\tilde{\Gamma}}(\omega)+e^{i \theta} \hat{\tilde{\Gamma}}^{\dagger}(-\omega)\right) \\
= & \frac{1}{\sqrt{2}}\left(e^{-i \theta}\left[\sqrt{2 \gamma_{c}} \hat{\tilde{a}}_{\text {in }}(\omega)+\sqrt{2 \gamma_{l}} \hat{\tilde{a}}_{l}(\omega)+\sqrt{2 \gamma_{s}} \hat{\tilde{a}}_{s}(\omega)\right]\right. \\
& \left.\quad+e^{i \theta}\left[\sqrt{2 \gamma_{c}} \hat{\tilde{a}}_{\text {in }}^{\dagger}(-\omega)+\sqrt{2 \gamma_{l}} \hat{\tilde{a}}_{l}^{\dagger}(-\omega)+\sqrt{2 \gamma_{s}} \hat{\tilde{a}}_{s}^{\dagger}(-\omega)\right]\right) \\
= & \sqrt{2 \gamma_{c}} \hat{\tilde{q}}_{\text {in }}(\theta)+\sqrt{2 \gamma_{l}} \hat{\tilde{q}} l(\theta)+\sqrt{2 \gamma_{s}} \hat{\tilde{q}}_{s}(\theta)
\end{aligned}
$$




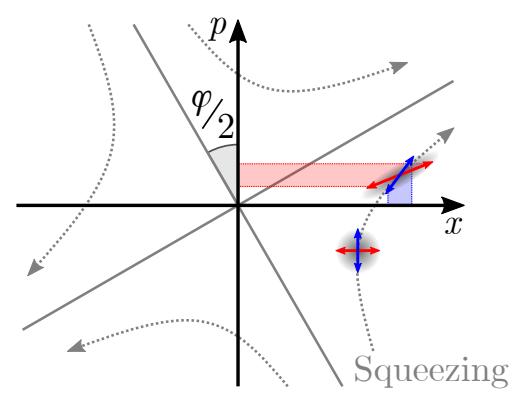

Figure 2.11: Illustration of the phase space transformation under squeezing with arbitrary $\phi$ (grey). Red illustrates how noise initially in the $\hat{x}$-quadrature is mixed into the $\hat{p}$-quadrature when squeezing, and similar (by blue) how noise in the $\hat{p}$-quadrature is mixed into the $\hat{x}$-quadrature.

is a weighted sum of quadratures in the modes of the noise operator $\Gamma$. Writing the noise operator quadrature in terms of amplitude and phase quadrature, $\hat{\tilde{q}}_{\Gamma}(\theta)=\hat{\tilde{x}}_{\Gamma} \cos \theta+\hat{\tilde{p}}_{\Gamma} \sin \theta$, the amplitude and phase quadrature of the cavity mode becomes

$$
\begin{gathered}
\hat{\tilde{x}}_{a}=\hat{\tilde{q}}_{a}(0)=\frac{(i \omega-\gamma) \hat{\tilde{q}}_{\Gamma}(0)-|\varepsilon| \hat{\tilde{q}}_{\Gamma}(\phi)}{|\varepsilon|^{2}-(i \omega-\gamma)^{2}}=\frac{(i \omega-\gamma-|\varepsilon| \cos \phi) \hat{\tilde{x}}_{\Gamma}-|\varepsilon| \sin \phi \hat{\tilde{p}}_{\Gamma}}{|\varepsilon|^{2}-(i \omega-\gamma)^{2}} \\
\hat{\tilde{p}}_{a}=\hat{\tilde{q}}_{a}(\pi / 2)=\frac{(i \omega-\gamma) \hat{\tilde{q}}_{\Gamma}(\pi / 2)-|\varepsilon| \hat{\tilde{q}}_{\Gamma}(\phi-\pi / 2)}{|\varepsilon|^{2}-(i \omega-\gamma)^{2}}=\frac{(i \omega-\gamma+|\varepsilon| \cos \phi) \hat{\tilde{p}}_{\Gamma}-|\varepsilon| \sin \phi \hat{\tilde{x}}_{\Gamma}}{|\varepsilon|^{2}-(i \omega-\gamma)^{2}},
\end{gathered}
$$

where it is used that $\hat{\tilde{q}}_{\Gamma}(\phi-\pi / 2)=\hat{\tilde{x}}_{\Gamma} \sin \phi-\hat{\tilde{p}}_{\Gamma} \cos \phi$. In conclusion, when $\phi=0$ or $\pi$ the cavity mode amplitude quadrature does not depend on the external noise phase quadrature, and similarly, the cavity phase quadrature does not depend on the external noise amplitude quadrature. This is expected, as for $\phi=0$ and $\pi$ we have squeezing exactly along the $\hat{p}$ - and $\hat{x}$-quadrature respectively. However, when that is not the case $(\phi \neq 0, \pi)$, the quadratures mix as the phase space is stretched in some direction not along $\hat{x}$ or $\hat{p}$. This is illustrated in Figure 2.11.

In the experimental setup, we have squeezing in the $\hat{p}$-quadrature, $\phi=\pi$, and (2.19) and (2.20) simplify to

$$
\begin{aligned}
& \hat{\tilde{x}}_{a}=\frac{i \omega-\gamma+|\varepsilon|}{|\varepsilon|^{2}-(i \omega-\gamma)^{2}} \hat{\tilde{x}}_{\Gamma}=\frac{\hat{\tilde{x}}_{\Gamma}}{\gamma+\varepsilon-i \omega}, \\
& \hat{\tilde{p}}_{a}=\frac{i \omega-\gamma-|\varepsilon|}{|\varepsilon|^{2}-(i \omega-\gamma)^{2}} \hat{\tilde{p}}_{\Gamma}=\frac{\hat{\tilde{x}}_{\Gamma}}{\gamma-\varepsilon-i \omega} .
\end{aligned}
$$

The output field mode, $a_{\text {out }}$, is related to the cavity mode by $a_{\text {out }}=\sqrt{2 \gamma_{c}} a-a_{\text {in }}$ [79], and the quadrature of the electric field coupled out of the cavity through the coupling mirror becomes

$$
\begin{aligned}
& \hat{\tilde{x}}_{\text {out }}=\sqrt{2 \gamma_{c}} \frac{\hat{\tilde{x}}_{\Gamma}}{\gamma+\varepsilon-i \omega}-\hat{\tilde{x}}_{\text {in }}=\frac{\gamma_{c}-\gamma_{l}-\gamma_{s}-\varepsilon+i \omega}{\gamma+\varepsilon-i \omega} \hat{\tilde{x}}_{\text {in }}+\frac{2 \sqrt{\gamma_{c} \gamma_{l}}}{\gamma+\varepsilon-i \omega} \hat{\tilde{x}}_{l}+\frac{2 \sqrt{\gamma_{c} \gamma_{s}}}{\gamma+\varepsilon-i \omega} \hat{\tilde{x}}_{s}, \\
& \hat{\tilde{p}}_{\text {out }}=\sqrt{2 \gamma_{c}} \frac{\hat{\tilde{p}}_{\Gamma}}{\gamma+\varepsilon-i \omega}-\hat{\tilde{p}}_{\text {in }}=\frac{\gamma_{c}-\gamma_{l}-\gamma_{s}+\varepsilon+i \omega}{\gamma-\varepsilon-i \omega} \hat{\tilde{p}}_{\text {in }}+\frac{2 \sqrt{\gamma_{c} \gamma_{l}}}{\gamma-\varepsilon-i \omega} \hat{\tilde{p}}_{l}+\frac{2 \sqrt{\gamma_{c} \gamma_{s}}}{\gamma-\varepsilon-i \omega} \hat{\tilde{p}}_{s} .
\end{aligned}
$$

Finally, when the cavity output field is detected by homodyne detection, where $\hat{x}_{\text {out }}$ and $\hat{p}_{\text {out }}$ are measured in the rotating frame, it is the photo current we measure. Here, the photo current is a direct measure of $\hat{x}_{\text {out }}$ and $\hat{p}_{\text {out }}$, and the photo current power spectrum is proportional to the spectral density $S_{q}(\omega)$ of the quadrature $q(\theta)$ defined by $S_{q}(\omega) \delta\left(\omega+\omega^{\prime}\right)=\left\langle\hat{\tilde{q}}(\omega) \hat{\tilde{q}}\left(\omega^{\prime}\right)\right\rangle[86]$. Using that $\left\langle\hat{q}_{i} \hat{q}_{j}\right\rangle=\left\langle\hat{q}_{i}\right\rangle\left\langle\hat{q}_{j}\right\rangle$ for independent modes $i$ and $j$, and that the only input mode to the cavity which is not simply vacuum is the seed, i.e. $\left\langle\hat{q}_{\text {in }}\right\rangle=\left\langle\hat{q}_{l}\right\rangle=0$ and $\left\langle\Delta \hat{q}_{\text {in }}^{2}\right\rangle=\left\langle\Delta \hat{q}_{l}^{2}\right\rangle=1 / 2$ for $q=x, p$, the spectral density of $\hat{x}_{\text {out }}$ and $\hat{p}_{\text {out }}$ becomes, after some cumbersome simplification,

$$
S_{x}(\omega)=\left\langle\hat{\tilde{x}}_{\text {out }}(\omega) \hat{\tilde{x}}_{\text {out }}(-\omega)\right\rangle=\frac{1}{2}-\frac{2 \gamma_{c} \varepsilon}{(\gamma+\varepsilon)^{2}+\omega^{2}}+\frac{4 \gamma_{c} \gamma_{s}}{(\gamma+\varepsilon)^{2}+\omega^{2}}\left(\left\langle\Delta \hat{x}_{s}^{2}\right\rangle-\frac{1}{2}\right)
$$




$$
S_{p}(\omega)=\left\langle\hat{\tilde{p}}_{\text {out }}(\omega) \hat{\tilde{p}}_{\text {out }}(-\omega)\right\rangle=\frac{1}{2}+\frac{2 \gamma_{c} \varepsilon}{(\gamma-\varepsilon)^{2}+\omega^{2}}+\frac{4 \gamma_{c} \gamma_{s}}{(\gamma-\varepsilon)^{2}+\omega^{2}}\left(\left\langle\Delta \hat{p}_{s}^{2}\right\rangle-\frac{1}{2}\right) .
$$

In conclusion, for the seed beam simply being vacuum or a pure coherent state, i.e. $\left\langle\Delta \hat{x}_{s}^{2}\right\rangle=$ $\left\langle\Delta \hat{p}_{s}^{2}\right\rangle=1 / 2,(2.21)$ and $(2.22)$ reduces to the well known squeezing spectra, where the first term is the vacuum noise, to which the second term add or subtract to form an anti-squeezed or squeezed spectrum respectively. However, for seed beam noise larger than the vacuum noise, the third term in (2.21) and (2.22) becomes positive and adds to the squeezing spectra. To encounter any loss in experimental setup, the decay rate $\gamma_{c}$ in the numerators of the two last terms in (2.21) and (2.22) can be replaced by $\eta \gamma$, where $\eta$ is the total efficiency of the experimental setup, ranging from the OPO escape efficiency, $\gamma_{c} / \gamma$, to the homodyne detection efficiency.

In the experimental setup, even with the mirror through which the seed beam is coupled into the cavity being a high reflector $\left(\gamma_{s}\right.$ being small), the seed beam noise is large enough for the third term in (2.21) and (2.22) to be visible. To take this into account when predicting the squeezing spectra, we need to estimate $\gamma_{s}$ and $\left\langle\Delta \hat{q}_{s}^{2}\right\rangle$ for $q=x, p$. To do this the spectral density of $\hat{x}$ and $\hat{p}$ are measured without pump power in the cavity $(\varepsilon=0), S_{q}^{0}(\omega)$, leading to

$$
\begin{gathered}
S_{q}^{0}(\omega)=\frac{1}{2}+4 \frac{4 \eta \gamma \gamma_{s}}{\gamma^{2}+\omega^{2}}\left(\left\langle\Delta \hat{q}_{s}^{2}\right\rangle-\frac{1}{2}\right)=\frac{1}{2}+\frac{K_{q}}{\gamma^{2}+\omega} \quad, \quad q=x, p \\
\Uparrow \\
K_{q}=4 \eta \gamma \gamma_{s}\left(\left\langle\Delta \hat{q}_{s}^{2}\right\rangle-\frac{1}{2}\right)=\left(\gamma^{2}+\omega^{2}\right)\left(S_{q}^{0}(\omega)-\frac{1}{2}\right),
\end{gathered}
$$

where the total efficiency, $\eta$, of the setup is included. Here, since $\gamma_{s} \ll \gamma_{c}, \gamma_{l}$,

$$
\gamma=\gamma_{c}+\gamma_{l}+\gamma_{s} \approx \gamma_{c}+\gamma_{l}=\frac{1-\sqrt{1-T_{c}}}{\tau}+\frac{1-\sqrt{1-\mathcal{L}}}{\tau}=2 \pi 8.1 \mathrm{MHz}
$$

with $T_{c}=10 \%$ coupling mirror transmission, $\mathcal{L}=0.55 \%$ intracavity losses and a OPO round trip time of $\tau=320 \mathrm{~mm} /\left(3 \times 10^{8} \mathrm{~m} / \mathrm{s}\right)$. Thus by measuring $S_{q}^{0}(\omega)$ and $\gamma$ we get $K_{q}$ from (2.23), we predict the squeezed and anti-squeezed spectra as

$$
S_{q}(\omega)=\frac{1}{2} \mp \frac{2 \varepsilon \gamma \eta}{(\gamma \pm \varepsilon)^{2}+\omega^{2}}+\frac{K_{q}}{(\gamma \pm \varepsilon)^{2}+\omega^{2}} \quad, \quad q=x, p .
$$

\subsubsection{Bound on covariances}

From measurements we got the covariance matrix for the operators $\boldsymbol{\xi}=\left(\hat{x}_{A}, \hat{p}_{A}, \hat{x}_{B}, \hat{p}_{B}\right)^{T}$ to be

$$
\gamma=V_{0}\left(\begin{array}{cccc}
4.36 & a & -3.84 & 0.36 \\
a & 4.43 & 0.45 & 3.92 \\
-3.84 & 0.45 & 4.17 & b \\
0.36 & 3.92 & b & 4.26
\end{array}\right)
$$

where $V_{0}=1 / 2$ is the vacuum variance.

In the experiment, we aim at setting the phase angles such that $a=b=0$, but since $a$ and $b$ are not directly measured they could in principle deviate from zero. In the following we first estimate the allowed range of values for $a$ and $b$ using the uncertainty relation and given the actual measured entries of the covariance matrix. Second, we investigate how a phase off-set of the individually squeezed beams will lead to non-zero values of $a$ and $b$.

To determine the bounds of $a$ and $b$ for $\gamma$ to be physical, we use the covariance matrix uncertainty relation $[87]$

$$
\gamma+\frac{i}{2} \boldsymbol{\Omega} \geq 0
$$



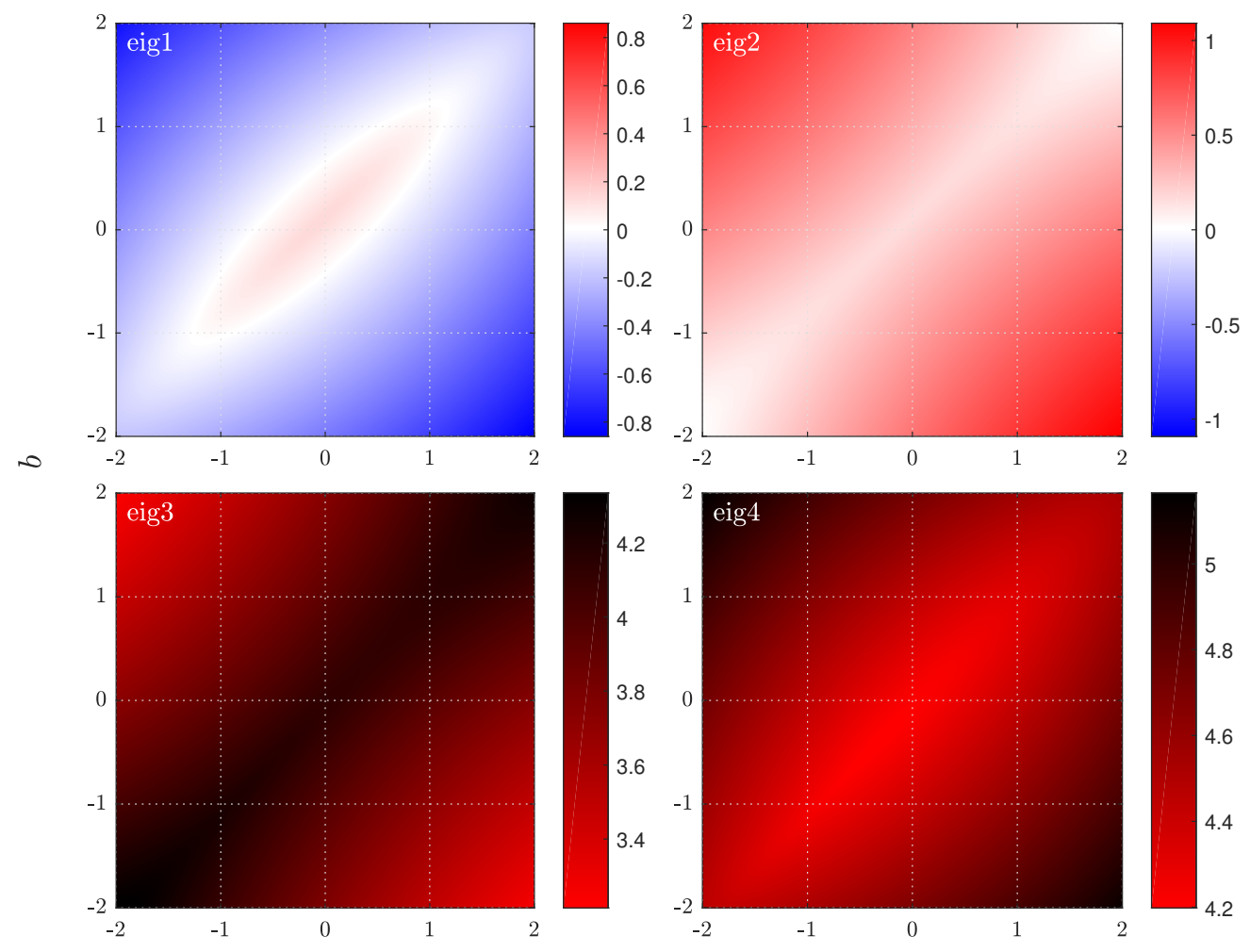

Figure 2.12: Numerical calculations of the eigenvalues of $\gamma+\frac{i}{2} \Omega$ for the covariance matrix shown in (2.24).

where the elements of $\boldsymbol{\Omega}$ are given by the commutator relations $\left[\xi_{i}, \xi_{j}\right]=i \Omega_{i j}$, hence

$$
\boldsymbol{\Omega}=\left(\begin{array}{cccc}
0 & 1 & 0 & 0 \\
-1 & 0 & 0 & 0 \\
0 & 0 & 0 & 1 \\
0 & 0 & -1 & 0
\end{array}\right)
$$

From (2.25), all eigenvalues of $\gamma+\frac{i}{2} \boldsymbol{\Omega}$ must be non-negative. In Fig. 2.12 the 4 eigenvalues of $\gamma+\frac{i}{2} \Omega$ for $a$ and $b$ in the range of -2 to 2 is shown. In this interval only the first eigenvalue is seen to limit $a$ and $b$ in (2.25). The bounds of $a$ and $b$ are seen not to be independent, but they both share an absolute lower bound of zero (corresponding to the ideal case of $\hat{x}$ and $\hat{p}$ being completely uncorrelated). The lower and upper bound of $a$ (depending on $b$ ) and $b$ (depending on $a$ ) is

$$
\begin{aligned}
& a \in[-1.24,1.17] \\
& b \in[-1.10,1.21] .
\end{aligned}
$$

It is thus clear that by using a bona fide criterion for the covariance matrix, the range of possible xp-covarainces is quite large. In the following, we instead estimate the potential values for $a$ and $b$ by propagating a phase off-set of the input squeezed state through the system. We consider the setup in Fig. 2.13. Two squeezed states undergo a phase rotation, $\sigma$, and loss, $1-\eta$, and interfere on a balanced beam splitter $90^{\circ}$ out of phase to produce a two-mode squeezed state. If the phase off-set is zero, the xp-covariances will be exactly zero. However, for a phase rotation, 


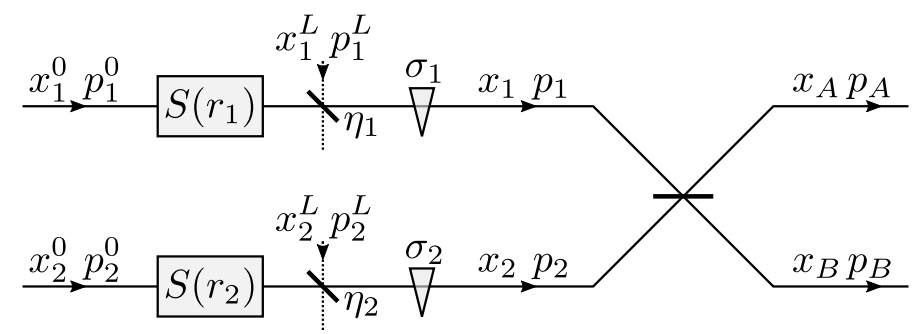

Figure 2.13: Two modes of vacuum, $\hat{x}_{1}^{0}, \hat{p}_{1}^{0}$ and $\hat{x}_{2}^{0}, \hat{p}_{2}^{0}$, are squeezed by $S\left(r_{1}\right)$ and $S\left(r_{2}\right)$ before they are subjected to loss $\left(1-\eta_{1}\right.$ and $\left.1-\eta_{2}\right)$ and some phase change $\left(\sigma_{1}\right.$ and $\left.\sigma_{2}\right)$. Finally they are interfered on a beam splitter to form two-mode squeezing. Here, mode 1 and 2 corresponds to the direct and delay path in the experimental setup. Notice, in the experimental setup the two modes 1 and 2 share the same temporal multiplexed squeezing source, such that the squeezing parameters $r_{1}=r_{2}$.

the quadratures are transformed as

$$
\begin{aligned}
& \hat{x}_{1}=\left(\sqrt{\eta_{1}} e^{-r_{1}} \hat{x}_{1}^{0}-\sqrt{1-\eta_{1}} \hat{x}_{1}^{L}\right) \cos \sigma_{1}-\left(\sqrt{\eta_{1}} e^{r_{1}} \hat{p}_{1}^{0}+\sqrt{1-\eta_{1}} \hat{p}_{1}^{L}\right) \sin \sigma_{1} \\
& \hat{p}_{1}=\left(\sqrt{\eta_{1}} e^{-r_{1}} \hat{x}_{1}^{0}-\sqrt{1-\eta_{1}} \hat{x}_{1}^{L}\right) \sin \sigma_{1}+\left(\sqrt{\eta_{1}} e^{r_{1}} \hat{p}_{1}^{0}+\sqrt{1-\eta_{1}} \hat{p}_{1}^{L}\right) \cos \sigma_{1} \\
& \hat{x}_{2}=\left(\sqrt{\eta_{2}} e^{r_{2}} \hat{x}_{2}^{0}-\sqrt{1-\eta_{2}} \hat{x}_{2}^{L}\right) \cos \sigma_{2}-\left(\sqrt{\eta_{2}} e^{-r_{2}} \hat{p}_{2}^{0}+\sqrt{1-\eta_{2}} \hat{p}_{2}^{L}\right) \sin \sigma_{2} \\
& \hat{p}_{2}=\left(\sqrt{\eta_{2}} e^{r_{2}} \hat{x}_{2}^{0}-\sqrt{1-\eta_{2}} \hat{x}_{2}^{L}\right) \sin \sigma_{2}+\left(\sqrt{\eta_{2}} e^{-r_{2}} \hat{p}_{2}^{0}+\sqrt{1-\eta_{2}} \hat{p}_{2}^{L}\right) \cos \sigma_{2}
\end{aligned}
$$

and with $\hat{x}_{A}=\frac{1}{\sqrt{2}}\left(\hat{x}_{1}+\hat{x}_{2}\right), \hat{x}_{B}=\frac{1}{\sqrt{2}}\left(\hat{x}_{1}-\hat{x}_{2}\right), \hat{p}_{A}=\frac{1}{\sqrt{2}}\left(\hat{p}_{1}+\hat{p}_{2}\right), \hat{p}_{B}=\frac{1}{\sqrt{2}}\left(\hat{p}_{1}-\hat{p}_{2}\right)$, we find the correlation

$$
\begin{aligned}
C_{\hat{x}_{A} \hat{p}_{B}} & =\frac{1}{2}\left(\left\langle\hat{x}_{1} \hat{p}_{1}\right\rangle-\left\langle\hat{x}_{2} \hat{p}_{2}\right\rangle\right) \\
& =\frac{1}{2} \eta_{1}\left(e^{-2 r_{1}}-e^{2 r_{1}}\right) V_{0} \cos \sigma_{1} \sin \sigma_{1}+\frac{1}{2} \eta_{2}\left(e^{-2 r_{2}}-e^{2 r_{2}}\right) V_{0} \cos \sigma_{2} \sin \sigma_{2}
\end{aligned}
$$

It is thus clear that a phase off-set of the individually squeezed modes results in non-zero xpcovariances. Interestingly, the covariances are identical to the intra-mode covariances:

$$
C_{\hat{x}_{A} \hat{p}_{A}}=C_{\hat{x}_{B} \hat{p}_{B}}=C_{\hat{x}_{A} \hat{p}_{B}}=C_{\hat{x}_{B} \hat{p}_{A}}
$$

which means that the expected off-set values of $a$ and $b$ should be similar to the measured values for $C_{\hat{x}_{A} \hat{p}_{A}}$ and $C_{\hat{x}_{B} \hat{p}_{B}}$ under the above mentioned assumptions. We have now seen how a phase off-set will give rise to non-zero covariances. It is however important to note that symmetric phase diffusion noise centered around the perfectly aligned phase will not produce non-zero values of the covariances. 


\section{Chapter 3}

\section{Deterministic generation of a two-dimensional cluster state}

In this chapter, the paper "Deterministic generation of a two-dimensional cluster state" of Ref. [31] is presented. This papers is authored by Mikkel V. Larsen, Xueshi Guo, Casper R. Breum, Jonas S. Neergaard-Nielsen, and Ulrik L. Andersen, and published in Science 366, 369 (2019) back-to-back with a similar work by Asavanant et al. in Ref. [37].

In this work, we propose and demonstrate generation of a continuous-variable cluster state in two dimensions. With the motivation being scalable measurement-based quantum computation without requiring similar scaling of spatial resources, the cluster state is generated using temporal multiplexing of a minimum of spatial resources. As such, this work is partly experimental, and partly theoretical. The experimental setup is mainly based on optical fiber components operated at the telecom wavelength, and we utilize experimental techniques presented in chapter 2 [30].

While two-dimensional cluster state generation is realized in this work, computation on this cluster state is only discussed briefly in this chapter. In chapter 4 [32] an efficient computation scheme on the cluster state generated here is proposed, while in chapter 5 [33] gates, implemented by projective measurements of the cluster state, is demonstrated.

Appendix A and B (page 147 and 149) complements the method section 3.3 of this chapter, describing the optical table and phase lock configurations for cluster state generation. Experimental data and analysis code are freely available at figshare.com [88].

From Science 366, 369 (2019). Reprinted with permission from AAAS.

\subsection{Abstract}

Measurement-based quantum computation offers exponential computational speed-up via simple measurements on a large entangled cluster state. We propose and demonstrate a scalable scheme for the generation of photonic cluster states suitable for universal measurement-based quantum computation. We exploit temporal multiplexing of squeezed light modes, delay loops, and beam-splitter transformations to deterministically generate a cylindrical cluster state with a two-dimensional (2D) topological structure as required for universal quantum information processing. The generated state consists of more than 30000 entangled modes arranged in a cylindrical lattice with 24 modes on the circumference, defining the input register, and a length of 1250 modes, defining the computation depth. Our demonstrated source of $2 \mathrm{D}$ cluster states can be combined with quantum error correction to enable fault-tolerant quantum computation. 


\subsection{Main text}

Quantum computing represents a new paradigm for information processing that harnesses the inherent non-classical features of quantum physics to find solutions to problems that are computationally intractable on classical processors [89]. In measurement-based, or cluster state, quantum computing (MBQC), the processing is performed via simple single-site measurements on a large entangled cluster state [1]. This constitutes a simplification over the standard gate-based model of quantum computing, as it replaces complex coherent unitary dynamics with simple projective measurements. However, one of the outstanding challenges in realizing cluster state computation is the reliable, deterministic and scalable generation of non-classical entangled states suitable for universal information processing.

Several candidate platforms for scalable cluster state generation have been proposed and some experimentally realized, including solid state superconducting qubits [90], trapped ion qubits [29, 91] and photonic qubits or qumodes, in which qubits can be encoded, generated by parametric down-conversion [19, 20, 28, 92] or by quantum dots [93]. However, none of these implementations have demonstrated true scalability combined with computational universality. The largest cluster state generated to date is a temporally multiplexed photonic state comprising entangled modes in a long chain which however does not allow for universal computation due to its one-dimensional (1D) topological structure $[19,21]$. To achieve universality, the dimension of the cluster state must be at least two. Several proposals for generating two-dimensional (2D) cluster states in different systems have been proposed $[9,11,94,95]$ but due to technical challenges, scalable and computationally universal cluster states have yet to be produced in any physical system.

We propose and demonstrate a highly scalable scheme for the generation of cluster states for universal quantum computation based on quantum continuous variables (CV) where information is encoded in the position or momentum quadratures of photonic harmonic oscillators [76]. We use a temporally multiplexed source of optical Einstein-Podolsky-Rosen (EPR) states [61] to generate a long string of entangled modes that is curled up and fused to form a $2 \mathrm{D}$ cylindrical array of entangled modes. Specifically, we generate a massive cluster state of more than 30000 entangled modes comprising an input register of $2 \times 12=24$ modes on which the input state may be encoded, and a length of 1250 modes for encoding operations by projective measurements, only limited by the phase stability of our setup. In addition to being universal and deterministically generated, the source is operated under ambient conditions in optical fibers at the low-loss telecom wavelength of $1550 \mathrm{~nm}$. These favorable operational conditions and specifications significantly facilitate further upscaling of the entangled state as well as its use in applications and fundamental studies.

The canonical approach to CV cluster state generation is to apply two-mode controlled-Z gates onto pairs of individually prepared eigenstates of the momentum (or phase quadrature) operators $\hat{p}_{i}, \hat{p}_{j}$ in adjacent modes $i, j$. The gate is described by the unitary operation $\hat{C}_{Z}=e^{i g \hat{x}_{i} \hat{x}_{j}}$ where $\hat{x}_{i}, \hat{x}_{j}$ are the position (amplitude quadrature) operators of mode $i$ and $j$, while $g$ is the interaction strength. Applying this gate to two modes leads to entanglement in the form of quantum correlations of the two modes' quadratures. The operations and resulting state can be represented by a graph in which the nodes represent the momentum eigenstates while the edges (links) between the nodes represent the application of a controlled- $Z$ operation where the interaction strength is given by the edge weight. In a practical implementation, the unphysical momentum eigenstates are replaced by highly squeezed states while the controlled-Z operations can be imitated by phase shifts and beam splitter transformations. To enable scalability, it has been suggested to use multiplexing of spatial modes [17], frequency modes [8, 96], or temporal modes [9, 12]. For example, Menicucci suggested using temporal multiplexing to form a 2D cluster state combining four squeezed state generators, five beam splitters, and two delay lines [9].

We propose a simpler approach to $2 \mathrm{D}$ cluster state generation lowering the experimental requirements (Fig. 3.1). The state is produced in four steps: i) Pairs of squeezed vacuum states are generated at $1550 \mathrm{~nm}$ wavelength from two bow-tie shaped optical parametric oscillators (OPOs) by parametric down conversion [57]. The states are defined in consecutive temporal modes of duration $\tau$ of the continuously generated OPO output. ii) The squeezed vacuum pairs in spatial modes $A$ and $B$ are interfered on a balanced beam splitter (denoted $\mathrm{BS}_{1}$ ). This produces a train 


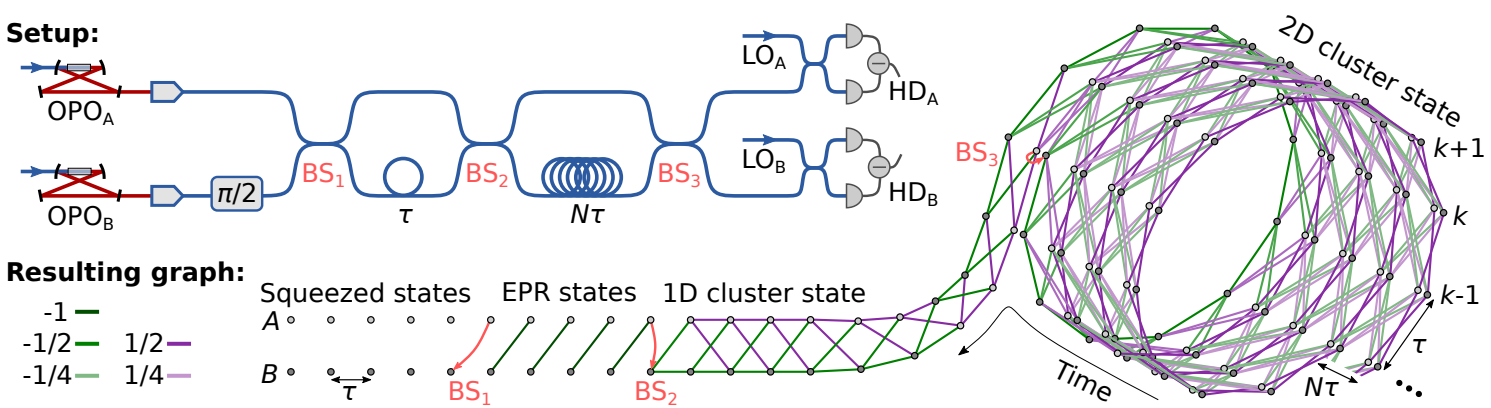

Figure 3.1: Scheme of 2D cluster state generation. Squeezing is produced by two OPOs $\left(\mathrm{OPO}_{\mathrm{A}}\right.$ and $\left.\mathrm{OPO}_{\mathrm{B}}\right)$, and coupled into fiber with $97 \%$ coupling efficiency. There, temporal modes are interfered with fiber coupled beam splitters to generate a $2 \mathrm{D}$ cluster state. The corresponding graph is shown: Temporal modes of squeezing with mode index $k$ in two spatial modes $A$ and $B$ (bright and dark nodes) are interfered to generate EPR-states at $\mathrm{BS}_{1}$. The EPR pairs are entangled to form a $1 \mathrm{D}$ cluster state using a $\tau$ delay in mode $B$ and $\mathrm{BS}_{2}$, and the $1 \mathrm{D}$ cluster state is curled up to a $2 \mathrm{D}$ cluster state by another delay of $N \tau$ and $\mathrm{BS}_{3}$. Using homodyne detectors $\left(\mathrm{HD}_{\mathrm{A}}\right.$ and $\left.\mathrm{HD}_{\mathrm{B}}\right)$, the temporal mode quadratures are measured from which the nullifiers are calculated. In the experimental implementation, the short delay is a $50.5 \mathrm{~m}$ fiber leading to temporal modes of 247 ns duration, while the long delay is a $606 \mathrm{~m}$ fiber such that $N=12$ as the illustrated graph. The temporal modes are defined by an asymmetric shaped temporal mode function within the 247 ns duration which filters out low frequency noise and leads to less than $10^{-3}$ mode overlap [21]. For more information, see the methods section 3.3.

of pairwise EPR-entangled temporal modes exhibiting quantum correlation between the position and momentum quadratures. Each EPR pair can be represented by a simple graph of a single edge connecting two nodes. iii) A $1 \mathrm{D}$ cluster state is formed by delaying one arm of the interferometer by $\tau$ with respect to the other arm and interfering the resulting time-synchronized modes on another balanced beam splitter (denoted $\mathrm{BS}_{2}$ ). The interference entangles EPR pairs along an indefinitely long chain creating a $1 \mathrm{D}$ graph. iv) In the final step, the $2 \mathrm{D}$ cluster state is produced by introducing another delay to one interferometer arm of duration $N \tau$ and interfering the resulting time-synchronized modes on a final beam splitter (denoted $\mathrm{BS}_{3}$ ). This effectively curls up the graph and fuses the modes into an indefinitely long cylinder with $N$ nodes on the circumference as illustrated in Fig. 3.1 for $N=12$, leading to $2 \times N=24$ input modes distributed on the two spatial modes $A$ and $B$. For detailed description of experimental implementations see the methods section 3.3 .

All states and operations involved are Gaussian, meaning they can be described by Gaussian distributions of the quadrature variables in phase space. In the formalism of graphical calculus for Gaussian states [7], the generated graphs are so-called $\mathcal{H}$-graphs as they can be generated from vacuum by a single Hamiltonian, and have an edge weight of $g=i \sinh (2 r) G$ where $r$ is the squeezing parameter of the two squeezing operations and $G=-1$ for the EPR-states, $\pm 1 / 2$ for the $1 \mathrm{D}$ graph and $\pm 1 / 4,1 / 2$ for the $2 \mathrm{D}$ graph. Due to the particular structure of the $\mathcal{H}$-graph generated here (it is self-inverse and bipartite - see the supplementary information section 3.4.1 for details), it can be transformed into a cluster state by $\pi / 2$ rotations in phase space leading to real edges of weight $g=\tanh (2 r) G \rightarrow G$ for $r \rightarrow \infty$. Finally, as the $\pi / 2$ phase space rotations can be absorbed into the measurement basis, or simply by appropriate re-definitions of quadratures on the rotated modes, the generated $\mathcal{H}$-graph state and its corresponding cluster state are completely equivalent. See the supplementary information section 3.4.2 for details on the cluster state generation scheme.

The produced cylindrical 2D cluster state can be shown to be a universal resource for quantum computing: In Fig. 3.2, the generated cylindrical cluster state is unfolded and projected into a square lattice by projective measurements in the position basis and $\pi / 2$ phase-space rotations of different modes. Such a square lattice is a well-known universal resource for quantum computing [5], and thus the initial cylindrical cluster state is itself universal. For computation it is not necessary 


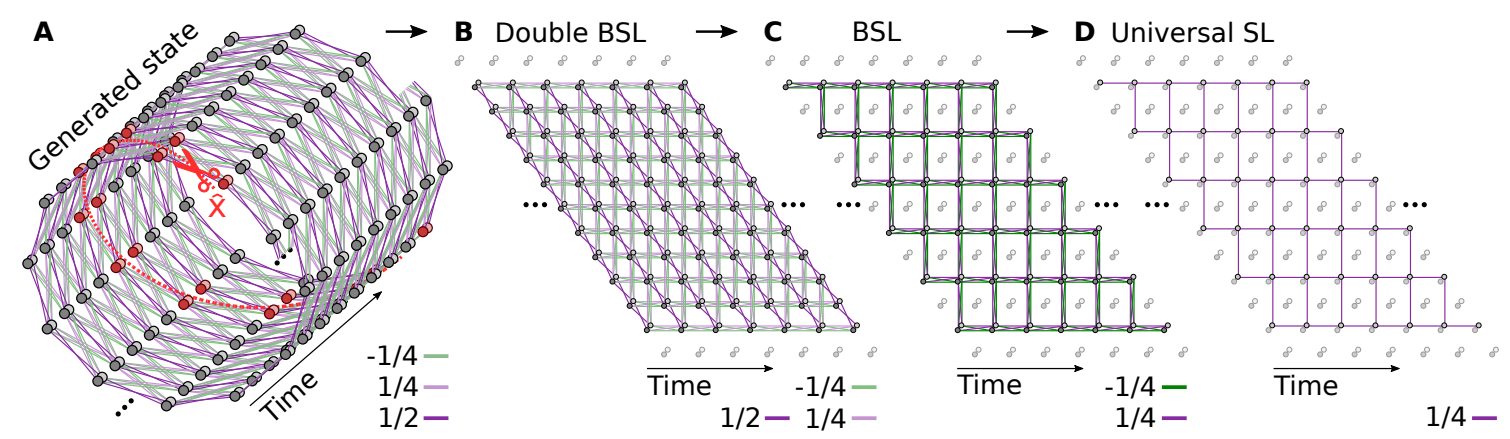

Figure 3.2: Universality of generated 2D cluster state. (A) Graph of the generated 2D cluster state. Measuring the nodes marked by red in the position basis removes all edges connected to the measured nodes, and the cylindrical graph unfolds to a plane. (B) Resulting plane 2D cluster state after the projective measurements in (A), consisting of two bilayer square lattices (double BSL) connected by edges of weight $1 / 2$. (C) Single BSL after projective measurement of half the modes in (B) in the position basis. (D) Square lattice (SL) after projective position measurements of all modes in spatial mode $B$ (dark nodes), and applying the Fourier gate $(\pi / 2$ phase delay) on half the modes in spatial mode $A$ (bright nodes). This SL is a traditional universal resource state for MBQC.

to project the generated cluster state into a square lattice - rather, one would in general optimize the detector settings required for the gate to be implemented. For instance, with proper settings the cluster state can be projected into 1D dual-rail wires along the cylinder, an efficient resource for one-mode computation $[12,19]$ and with possible two-mode interactions between them-for details see supplementary information section 3.4.4. Doing so requires fast control of the measurement bases in between temporal modes, while in this work the cluster state is measured in fixed bases for state verification.

Multi-partite cluster state inseparability can be witnessed through the measurement of the uncertainties of the state nullifiers - linear combinations of position and momentum operators for which the cluster states are eigenstates with eigenvalue 0. E.g. for the ideal two-mode EPR state, the well-known nullifiers are $\hat{n}_{\mathrm{EPR}}^{x}=\hat{x}_{A}-\hat{x}_{B}$ and $\hat{n}_{\mathrm{EPR}}^{p}=\hat{p}_{A}+\hat{p}_{B}$ since $\hat{n}_{\mathrm{EPR}}^{x}|\mathrm{EPR}\rangle=0$ and $\hat{n}_{\mathrm{EPR}}^{p}|\mathrm{EPR}\rangle=0$. For our $2 \mathrm{D}$ cluster state, $|2 \mathrm{D}\rangle$, the nullifiers consist of 8 modes and are given by

$$
\begin{aligned}
& \hat{n}_{k}^{x}=\hat{x}_{k}^{A}+\hat{x}_{k}^{B}-\hat{x}_{k+1}^{A}-\hat{x}_{k+1}^{B} \\
&-\hat{x}_{k+N}^{A}+\hat{x}_{k+N}^{B}-\hat{x}_{k+N+1}^{A}+\hat{x}_{k+N+1}^{B}, \\
& \hat{n}_{k}^{p}=\hat{p}_{k}^{A}+\hat{p}_{k}^{B}+\hat{p}_{k+1}^{A}+\hat{p}_{k+1}^{B} \\
& \quad-\hat{p}_{k+N}^{A}+\hat{p}_{k+N}^{B}+\hat{p}_{k+N+1}^{A}-\hat{p}_{k+N+1}^{B},
\end{aligned}
$$

as $\hat{n}_{k}^{x}|2 \mathrm{D}\rangle=0$ and $\hat{n}_{k}^{p}|2 \mathrm{D}\rangle=0$ (derived in the supplementary information section 3.4.3), where the subscript indicates the temporal mode index with $N$ being the number of temporal modes in the cluster state circumference.

The practically realizable cluster state is never an exact eigenstate of the nullifiers since such a state is unphysical. The measurement outcomes of the nullifiers are therefore not exactly zero in every measurement but possess some uncertainties around zero. A condition for complete inseparability of the $2 \mathrm{D}$ cluster state (derived in the supplementary information section 3.5) leads to a bound on the variances of all nullifiers of $3 \mathrm{~dB}$ squeezing below the shot noise level. Therefore, to witness full inseparability, we must observe more than $3 \mathrm{~dB}$ squeezing for all nullifiers. In Fig. 3.3, the measured nullifier variances are shown for a dataset of 1500 nullifiers and they are all observed to be well below the $-3 \mathrm{~dB}$ bound; we measure an averaged variance of $-4.7 \mathrm{~dB}$ and $-4.3 \mathrm{~dB}$ for $\hat{n}_{k}^{x}$ and $\hat{n}_{k}^{p}$, respectively. In the inset of Fig. 3.3, we present the measurement of a longer cluster state of 15000 temporal modes corresponding to a measurement time of $4 \mathrm{~ms}$. Although phase instabilities are clearly seen to affect the performance in terms of variations of the 


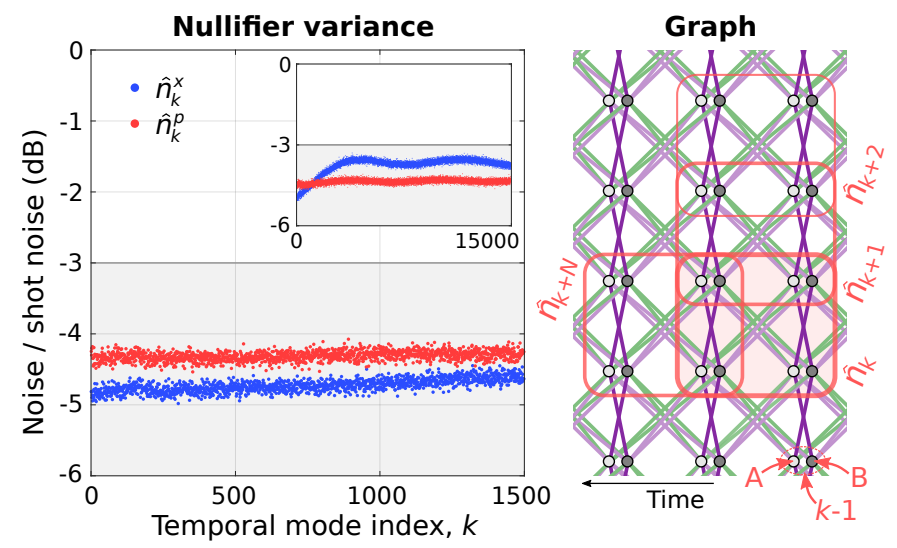

Figure 3.3: Experimental result. On the right graph, the nullifiers in Eq. (3.1) and (3.2) are shown on the $2 \mathrm{D}$ cluster state lattice with the measured variance of 1500 consecutive nullifiers shown in the left plot. Here, the variance is calculated from 10000 measurements of each nullifier. All nullifier variances are seen to be well below the $-3 \mathrm{~dB}$ inseparability bound derived in the supplementary information section 3.5, and thus the generated cluster state is completely inseparable. In the insert, the nullifier variance of a larger data set with $2 \times 15000=30000$ modes are shown. Again, with all modes below the $-3 \mathrm{~dB}$ inseparability bound, we conclude the successful generation of a 30000 mode $2 \mathrm{D}$ cluster state. The rapid increase of the variance in $\hat{n}_{k}^{x}$ and its periodic variation is caused by phase fluctuation of the squeezing sources as described in the supplementary information section 3.7 .

nullifier variances, all variances stay below the $-3 \mathrm{~dB}$ bound. The $2 \mathrm{D}$ cluster of $2 \times 15000=30000$ modes is thus fully inseparable. Note that not all 30000 modes of the cluster state need to exist simultaneously when performing projective measurements for computation. In fact, only a single temporal mode of the cluster state needs to exist while the remaining modes of the state are under construction. Hence, the cluster state can be immediately consumed for computation while being generated, with no additional state storage necessary — see supplementary information section 3.4.4 for a possible measurement scheme for computation on the cluster state.

With the deterministic generation of a universal 2D cluster state, we have for the first time (in parallel with Asavanant et al. [37]) in any system constructed a platform for universal MBQC. Its scalability was demonstrated by entangling 30000 optical modes in a 2D lattice that includes 24 input modes and allows for a computation depth of 1250 modes. Since only a few modes exist simultaneously, we are not limited by the coherence time of the light source, and thus the number of operations depends only on the phase stability of the system. The computational depth can therefore be unlimited by implementing continuous feedback control of the system for phase stabilization as demonstrated for the 1D photonic cluster state in [21]. The results presented here and in [37] are similar: Both 2D cluster states are generated deterministically in the CV regime with comparable size and amount of squeezing in the nullifier variance. However, with only two squeezing sources, three interference points, and operation in fiber, the experimental setup demonstrated here is simpler, while in [37] larger bandwidth OPOs are demonstrated resulting in shorter delay lines. In both systems, the number of input modes can be readily increased by using OPOs with larger bandwidths, possibly combined with a longer time delay of the second interferometer. E.g. using OPOs with a $1 \mathrm{GHz}$ bandwidth (65 times wider) and a twice as long interferometer delay, a state with $\sim 1500$ input modes can be generated. Large bandwidth OPOs have been demonstrated, but phase stability and losses in the delay lines are more challenging. While phase fluctuation is only a matter of experimental control on which we expect to improve with continuous phase stabilization, delay losses are unavoidable and increasing the OPO bandwidth may be a better solution than increasing the delay lengths.

CV cluster states are described by Gaussian statistics, but it is known that an element (state, 
operation, or measurement) of non-Gaussian quadrature statistics is required for universal quantum computing [97]. Such an element could be a photon number resolving detector (PNRD) or an ancillary cubic-phase state $[12,14]$. Despite recent experimental efforts in developing highefficiency PNRD [98] and deterministically generating optical states with non-Gaussian statistic [99], the formation of the required non-Gaussianity of the cluster state still constitutes an important challenge to be tackled in the future. Another currently limiting factor towards quantum computation is the existence of finite squeezing in the cluster leading to excess quantum noise and thus computational errors. However, these errors can be circumvented using Gottesman-KitaevPreskill (GKP) state encoding [14] concatenated with traditional qubit error correction schemes leading to fault-tolerant computation with a $15-17 \mathrm{~dB}$ squeezing threshold [38]. Another recently discovered advantage of the GKP encoding is that in addition to fault-tolerance, it also allows for universality without adding extra non-Gaussian states or operations [39]. While GKP states have recently been produced in the microwave regime [100] and in trapped-ion mechanical oscillators [101], their production in the optical regime remains a task for future work. For further discussion on quantum computation using the generated cluster state, see supplementary information section 3.4.4. Although a path towards fault-tolerant universal quantum computing using CV cluster states has been established, it is highly likely that the first demonstrations of CV quantum computation will be non-universal algorithmic sub-routines such as boson sampling and instantaneous quantum computing [102]. With the large, but noisy cluster state demonstrated here, interesting future work will be to implement basic Gaussian circuits and investigate e.g. the attainable circuit depth. Furthermore, the technique of folding a 1D cluster state into a 2D structure could be extended, using an additional interferometer, to form 3D cluster states which might be suitable for topologically protected MBQC.

\subsection{Methods}

The experimental setup is shown in detail in Fig. 3.4. Amplitude squeezed light at $1550 \mathrm{~nm}$ wavelength is generated by type- 0 parametric down conversion in two bow-tie shaped optical parametric oscillators $\left(\mathrm{OPO}_{\mathrm{A}}\right.$ and $\left.\mathrm{OPO}_{\mathrm{B}}\right)$ with periodically poled potassium titanyl phosphate (PPKTP) crystals, pumped by light at $775 \mathrm{~nm}$ wavelength generated from a second harmonic generator (SHG). For cavity and phase locking throughout the setup, we use a sample-hold locking scheme where the two OPOs are periodically seeded with a coherent probe chopped by two acoustooptic modulators (AOM): During the sample-time the probe is left on and active feedback is used for cavities and phase locks. After $10 \mathrm{~ms}$ of sample-time with active feedback, the probe is turned off for $5 \mathrm{~ms}$ (denoted hold-time) where all feedback loops are kept constant and quadrature data of the generated $2 \mathrm{D}$ cluster state is acquired from the two homodyne detectors $\left(\mathrm{HD}_{\mathrm{A}}\right.$ and $\left.\mathrm{HD}_{\mathrm{B}}\right)$. The cavities are locked by the Pound-Drever-Hall locking technique using a counter propagating lock beam with $28 \mathrm{MHz}$ phase modulation by an electro-optic modulator (not shown in Fig. 3.4). For the generation of amplitude squeezing, the classical parametric gains in $\mathrm{OPO}_{\mathrm{A}}$ and $\mathrm{OPO}_{\mathrm{B}}$ are locked to de-amplification using an AC-locking scheme: Phase modulated probe beams (at frequencies $f_{A}=90 \mathrm{kHz}$ and $f_{B}=55 \mathrm{kHz}$ ) are injected into the OPOs, a fraction (1\%) is measured and subsequently fed back to piezoelectric mounted mirrors.

The beams of squeezed light are coupled into single mode fibers (SMF) using gradient-index (GRIN) lenses with $97 \%$ coupling efficiency. Here, the two beams of squeezed light are interfered in a 50:50 fiber coupler $\left(\mathrm{BS}_{1}\right)$, where $1 \%$ of one output arm is tapped, detected, and fed back to a phase controlling fiber-stretcher for locking the relative phase between the two input beams. For more information on this fiber-stretcher, see previous experimental work described in section 2.6.2 [30]. Using a manual polarization controller, the visibility is optimized to near unity. By locking the relative phase difference to $\pi / 2$ using a DC-locking scheme, EPR-states are generated.

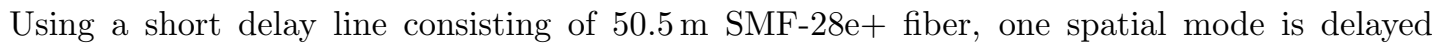
by $\tau=247 \mathrm{~ns}$. This delay defines the temporal mode width. Again, the two spatial modes are interfered on a 50:50 fiber coupler $\left(\mathrm{BS}_{2}\right)$ with phase control by tapping and detecting $1 \%$ of the output and feeding back to a fiber-stretcher, while visibility is optimized with a manual polarization 


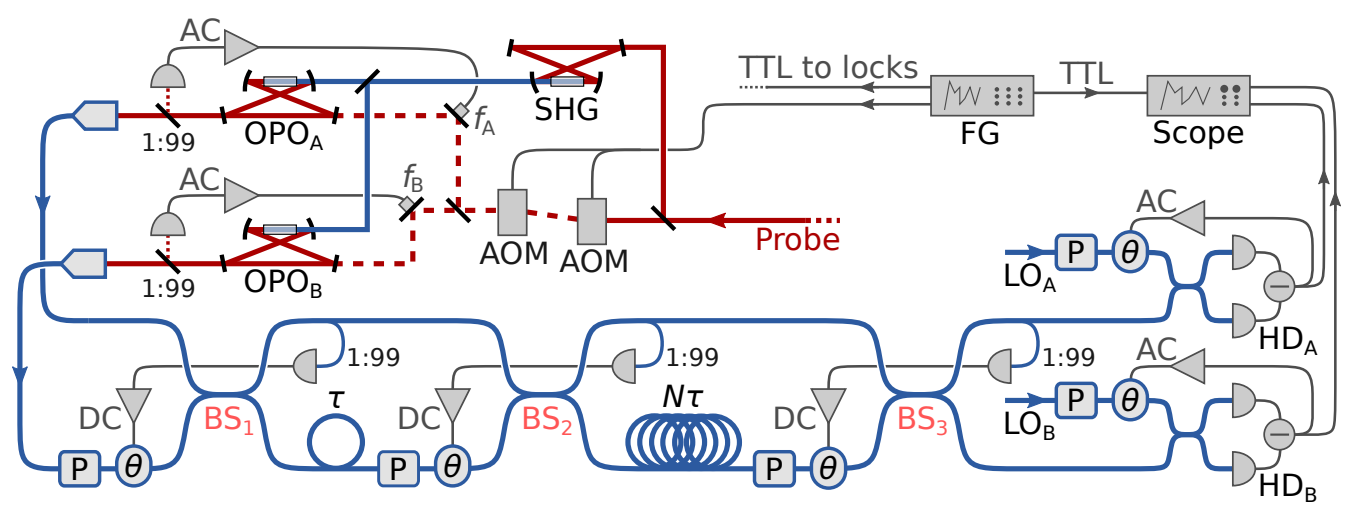

Figure 3.4: Detailed schematic of the experimental setup for 2D cluster state generation. Here the free space squeezing sources are marked by red (besides second harmonic generated light at $775 \mathrm{~nm}$ wavelength which is marked by blue), while optical fibers in which the cluster state is generated are marked by blue. Electronics for experimental control are marked by black. A function generator (FG) generates a logic signal (TTL) for switching on and off the probe and activating/deactivating feedback for cavity and phase locks. Data is acquired on an oscilloscope (Scope) when the probe is turned off and feedback is kept constant. The fiber components marked by $\mathrm{P}$ and $\theta$ represents manual polarization controllers and phase control by fiber-stretchers respectively.

controller. Locking the phase with a DC-locking scheme leads to a 1D cluster state with temporal modes defined by the short $\tau$-delay.

Finally, using a long delay of $606 \mathrm{~m}$, one spatial mode is delayed by $N=12$ temporal modes. Interfering the two spatial mode in the 50:50 fiber coupler $\left(\mathrm{BS}_{3}\right)$ corresponds to "coiling up" the $1 \mathrm{D}$ cluster state generated in $\mathrm{BS}_{2}$, leading to a $2 \mathrm{D}$ cluster state as illustrated in the main text Fig. 3.1 and described in the supplementary information section 3.4.2.3. Here, too, the relative phase is locked by tapping and detecting $1 \%$ of the output and feeding back to a fiber-stretcher, while polarization is controlled with a manual polarization controller.

For characterizing the generated 2D cluster state, amplitude $(\hat{x})$ and phase $(\hat{p})$ quadratures of the two spatial modes are continuously measured by two fiber-based homodyne detectors (HD). For more information on these fiber-based HDs, see previous experimental work described in section in section 2.6.2 [30]. The local oscillator phases for the two HDs are locked using an AC-locking scheme, where for measuring in the $\hat{x}$ - and $\hat{p}$-basis, demodulation by $f_{A}$ and $f_{B}$ are used, respectively.

For more details and characterization of the experimental implementation given here, see supplementary information section 3.6. Furthermore, the corresponding optical table is shown in appendix A (page 147), while in appendix B (page 149) the configurations of phase locks are described in more detail.

\subsection{Suppl. Inf.: Theory on cluster state}

In this section, cluster states are first introduced in section 3.4.1 before the generated $2 \mathrm{D}$ cluster state is derived in section 3.4.2 and its nullifiers in section 3.4.3. We use the convention of $\hbar=1$.

\subsubsection{Introduction}

Cluster states are a resource for measurement based quantum computation (MBQC) and are well described in [5] for the case of continuous variables (CV). For CV a cluster state is a set of modes, all initially in the momentum eigenstate $|0\rangle_{p}$, entangled by a number of controlled-Z operations of weight $g, \hat{C}_{Z}=\exp [i g \hat{x} \otimes \hat{x}]$ where $\hat{x}$ is the position quadrature. In the following we follow the 


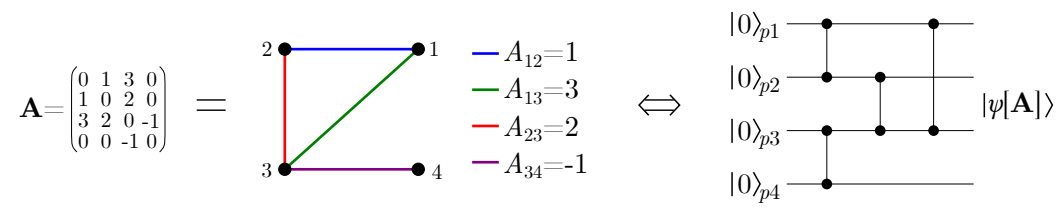

Figure 3.5: Adjacency matrix with its corresponding graph and equivalent circuit model.

conventions of graphical calculus for Gaussian pure states outlined in [7], and more details on the theory summarized here can be found in $[5,7,9]$.

A cluster state $\left|\psi_{\mathbf{A}}\right\rangle$ of $m$ modes can be defined by a symmetric real valued $m \times m$ adjacency matrix $\mathbf{A}$ as

$$
\left|\psi_{\mathbf{A}}\right\rangle=\hat{C}_{Z}[\mathbf{A}]|0\rangle_{p}^{\otimes m}=\prod_{j=1}^{m} \prod_{k=j}^{m} e^{i A_{j k} \hat{x}_{j} \hat{x}_{k}}|0\rangle_{p}^{\otimes m}=\exp \left[\frac{i}{2} \hat{\mathbf{x}}^{\mathrm{T}} \mathbf{A} \hat{\mathbf{x}}\right]|0\rangle_{p}^{\otimes m}
$$

where $\hat{\mathbf{x}}=\left(\hat{x}_{1}, \hat{x}_{2}, \cdots, \hat{x}_{m}\right)^{\mathrm{T}}$ is vector of position operators. For ideal cluster states, $\mathbf{A}$ is zero in the diagonal, while the off-diagonal term $A_{j k}$ describes a link (an edge) between mode $j$ and $k$

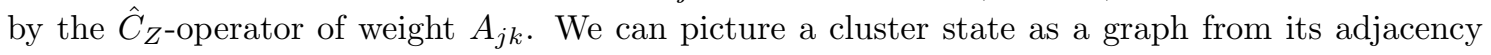
matrix as in Fig. 3.5.

The cluster state in Eq. (3.3) is most easily described in the stabilizer formalism in which $\hat{p}_{j}-\sum_{k} A_{j k} \hat{x}_{k}$ is a nullifier:

$$
(\hat{\mathbf{p}}-\mathbf{A} \hat{\mathbf{x}})\left|\psi_{\mathbf{A}}\right\rangle=\mathbf{0},
$$

where $\hat{\mathbf{p}}=\left(\hat{p}_{1}, \hat{p}_{2}, \cdots, \hat{p}_{m}\right)^{\mathrm{T}}$ is a vector of momentum operators. In conclusion, when measuring the nullifier $\hat{p}_{j}-\sum_{k} A_{j k} \hat{x}_{k}$ we expect vanishing variance. A gives a complete description of the state $\left|\psi_{\mathbf{A}}\right\rangle$.

\subsubsection{Approximate cluster states}

Eq. (3.4) is only valid for true momentum eigenstates as in Eq. (3.3), which require infinite squeezing and are not physical. Finite squeezing leads to non-zero variance when measuring the nullifier, and the variance increases with decreasing squeezing. Finite squeezing can be accounted for in the adjacency matrix by allowing it to be complex. We denote this complex adjacency matrix

$$
\mathbf{Z}=\mathbf{V}+i \mathbf{U}
$$

where $\mathbf{V}$ and $\mathbf{U}$ are real valued and symmetric. Again, $\mathbf{V}$ is zero in its diagonal and corresponds to $\mathbf{A}$ in the ideal case, while most often $\mathbf{U}$ is non-zero in the diagonal and corresponds to the deviation from the ideal case. We can still illustrate the corresponding graph state as in Fig. 3.5, but with complex weight and with self-loops on each node corresponding to the imaginary non-zero diagonal terms of $\mathbf{Z}$.

The physical graph state described by $\mathbf{Z}$ is said to be an approximate cluster state with adjacency matrix $\mathbf{A}$ if

$$
\lim _{r \rightarrow \infty} \mathbf{Z}(r)=\mathbf{A}
$$

where $r$ is the squeezing parameter of the initial states. As an example, applying $\hat{C}_{Z}[\mathbf{A}]$ to a number of finitely squeezed momentum states leads to

$$
\mathbf{Z}=\mathbf{A}+i e^{-2 r} \mathbf{I} \rightarrow \mathbf{A} \text { for } r \rightarrow \infty .
$$

Here $\mathbf{V}=\mathbf{A}$ and $\mathbf{U}=e^{-2 r} \mathbf{I}$. 


\subsubsection{2 $\quad \mathcal{H}$-graph states}

The controlled-Z operation, $\hat{C}_{Z}$, for entanglement generation is not easily implemented experimentally. Instead, quadrature entanglement (two-mode squeezing) is generated directly by nondegenerate down conversion or by interference of squeezed states, and the resulting graph state can be expressed by the adjacency matrix

$$
\mathbf{Z}=i e^{-2 r \mathbf{G}}
$$

where $\mathbf{G}$ is a real symmetric matrix. The state is called an $\mathcal{H}$-graph state, since it can be generated by the Hamiltonian

$$
\hat{\mathcal{H}}(\mathbf{G})=\hbar \kappa\left(\hat{\mathbf{x}}^{\mathrm{T}} \mathbf{G} \hat{\mathbf{p}}+\hat{\mathbf{p}}^{\mathrm{T}} \mathbf{G} \hat{\mathbf{x}}\right),
$$

with $\kappa$ being the squeezing parameter per unit time, $r=2 \kappa t$. It is not easy to illustrate this graph state with its exponential map, but in the case of $\mathbf{G}$ being self-inverse $\left(\mathbf{G}^{2}=\mathbf{I}\right)$, Eq. (3.5) simplifies to

$$
\mathbf{Z}=i \cosh (2 r) \mathbf{I}-i \sinh (2 r) \mathbf{G},
$$

and it can be pictured as in Fig. 3.5 with complex weights. However, it is not an approximate cluster state as $\mathbf{Z}$ does not go to some real valued matrix with zero in the diagonal for $r \rightarrow \infty$. But in the case of $\mathbf{G}$ also being bipartite (meaning the nodes can be separated into two sets with no connecting edges in between modes of the same set), it can be transformed into an approximate cluster state by applying the Fourier gate ( $\pi / 2$ rotation in phase-space) on some of its modes. Finally, since this Fourier gate can be absorbed into the measurement basis when measuring each mode of the graph state, we consider generation of a self-inverse bipartite $\mathcal{H}$-graph state as cluster state generation.

\subsubsection{Cluster state generation}

In the approach to cluster state generation, we start with modes of quadrature squeezed light to which we apply beam-splitters and Fourier gates. Traditionally, the starting point is the complex adjacency matrix for $m$ modes squeezed in the phase (or momentum) quadrature,

$$
\mathbf{Z}=i e^{-2 r} \mathbf{I},
$$

with $r$ being the squeezing parameter. In the experimental implementation we start with states squeezed in the amplitude (or position) quadrature, but this makes no difference to the theoretical derivation of the cluster state, and is merely a question on quadrature definition or $\pi / 2$ phase-space rotation. The quadrature transformation under beam-splitter transformations and/or phase-space rotations in the Heisenberg picture can be expressed by a $2 m \times 2 m$ symplectic matrix $\mathbf{S}$ as

$$
\left(\begin{array}{c}
\hat{\mathbf{x}}^{\prime} \\
\hat{\mathbf{p}}^{\prime}
\end{array}\right)=\mathbf{S}\left(\begin{array}{c}
\hat{\mathbf{x}} \\
\hat{\mathbf{p}}
\end{array}\right) \quad, \quad \mathbf{S}=\left(\begin{array}{ll}
\mathbf{A} & \mathbf{B} \\
\mathbf{C} & \mathbf{D}
\end{array}\right)
$$

where $\mathbf{A}, \mathbf{B}, \mathbf{C}$ and $\mathbf{D}$ are real $m \times m$ matrices. The corresponding transformation of the adjacency matrix $\mathbf{Z}$ is shown in [7] to be

$$
\mathbf{Z}^{\prime}=(\mathbf{C}+\mathbf{D Z})(\mathbf{A}+\mathbf{B Z})^{-1},
$$

with the resulting graph described by $\mathbf{Z}^{\prime}$.

The scheme of $2 \mathrm{D}$ cluster state generation in the main text Fig. 3.1 is summarized in Fig. 3.6. First a $1 \mathrm{D} \mathcal{H}$-graph state is generated as in [19], by applying a $\pi / 2$ phase-space rotation in the spatial mode $B$, beam-splitter transformation, delay of one spatial mode and another beam-splitter transformation. The phase-space rotation and beam-splitter is described by symplectic operations, while the delay is included by keeping track of the temporal mode index of simultaneously existing temporal modes in the two spatial modes $A$ and $B$. In the following sections, each step is described in detail. 


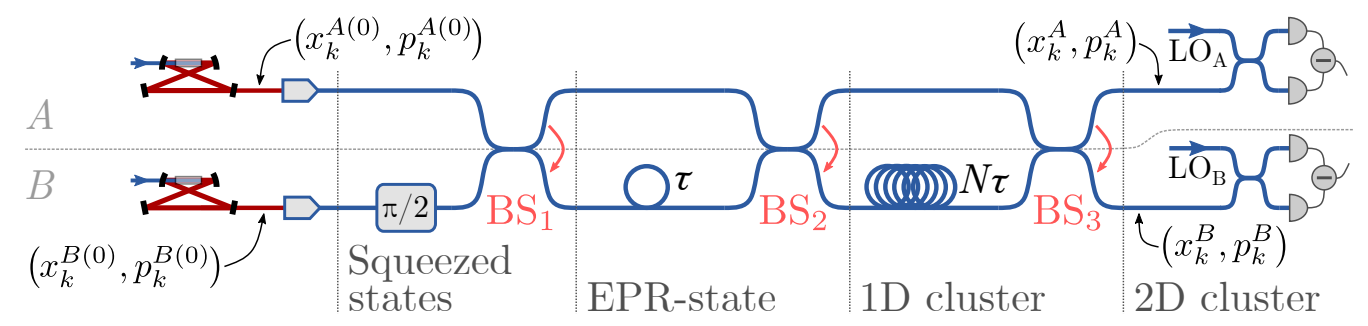

Figure 3.6: Sketch of setup for 2D cluster state generation. Following [19], first a 1D cluster state ( $\mathcal{H}$-graph state) is generated with temporal modes separated by the time $\tau$ using beam-splitters $\mathrm{BS}_{1}$ and $\mathrm{BS}_{2}$ together with the optical delay $\tau$. This $1 \mathrm{D}$ cluster state is then coiled up in a cylinder with the $N \tau$ delay, such that temporal modes at times $k \tau$ in the spatial mode $A$ overlap in time with the temporal modes of initial times $(k-N) \tau$ in the spatial $B$, where $k$ is an integer. From the side of the cylinder, we can see it as parallel 1D cluster states, which are then connected by the last beam-splitter $\mathrm{BS}_{3}$ to form a $2 \mathrm{D}$ cylindrical cluster state. The arrows on the beam-splitters points from the first to the second mode of the beam-splitter transformation $\mathbf{S}_{\mathrm{BS}}^{A B}$ in Eq. (3.10).

\subsubsection{EPR-state generation}

As the first step in Fig. 3.6, consider two modes $A$ and $B$ squeezed in the phase quadratures. To generate an EPR-state, mode $B$ is rotated by $\pi / 2$ in phase-space, and we apply the beam-splitter transformation $\mathrm{BS}_{1}$ between $A$ and $B$. The symplectic matrix is

$$
\mathbf{S}=\mathbf{S}_{\mathrm{BS}}^{A B} \mathbf{S}_{\pi / 2}^{B} \quad, \quad \mathbf{S}_{\pi / 2}^{B}=\left(\begin{array}{cccc}
1 & 0 & 0 & 0 \\
0 & 0 & 0 & -1 \\
0 & 0 & 1 & 0 \\
0 & 1 & 0 & 0
\end{array}\right) \quad, \quad \mathbf{S}_{\mathrm{BS}}^{A B}=\frac{1}{\sqrt{2}}\left(\begin{array}{cccc}
1 & -1 & 0 & 0 \\
1 & 1 & 0 & 0 \\
0 & 0 & 1 & -1 \\
0 & 0 & 1 & 1
\end{array}\right)
$$

Identifying $\mathbf{A}, \mathbf{B}, \mathbf{C}$ and $\mathbf{D}$ in (3.9) from (3.10) and inserting (3.8) we get

$$
\mathbf{Z}_{\mathrm{EPR}}=\left(\begin{array}{cc}
i \cosh (2 r) & -i \sinh (2 r) \\
-i \sinh (2 r) & i \cosh (2 r)
\end{array}\right)
$$

which is an $\mathcal{H}$-graph with the exact form of (3.7) where

$$
\mathbf{G}=\left(\begin{array}{ll}
0 & 1 \\
1 & 0
\end{array}\right)
$$

Note that the same EPR-state can then be generated by the Hamiltonian in (3.6), corresponding to non-degenerate parametric down conversion as expected. $\mathbf{G}$ is self-inverse and bipartite, and if we were to rotate mode $B$ (applying $\mathbf{S}_{\pi / 2}^{B}$ ) we would get

$$
\mathbf{Z}_{\mathrm{EPR}}^{\prime}=\left(\begin{array}{cc}
i \operatorname{sech}(2 r) & \tanh (2 r) \\
\tanh (2 r) & i \operatorname{sech}(2 r)
\end{array}\right) \rightarrow\left(\begin{array}{ll}
0 & 1 \\
1 & 0
\end{array}\right) \equiv \mathbf{A} \text { for } r \rightarrow \infty
$$

and so the $\mathcal{H}$-graph for the EPR-state has a corresponding approximate cluster state. From Eq. (3.4), the nullifiers of this cluster state are $\hat{p}_{A}-\hat{x}_{B}$ and $\hat{p}_{B}-\hat{x}_{A}$, which transform into $\hat{p}_{A}+\hat{p}_{B}$ and $\hat{x}_{B}-\hat{x}_{A}$ after rotating mode $B$ by $\pi / 2$. These relations are expected for an EPR-state.

\subsubsection{1D cluster states}

To generate 1D cluster states as in [19], we continue with pairs of EPR-states as described by the adjacency matrix in Eq. (3.11). Instead of the matrix notation, we will use the more convenient 
graph notation:

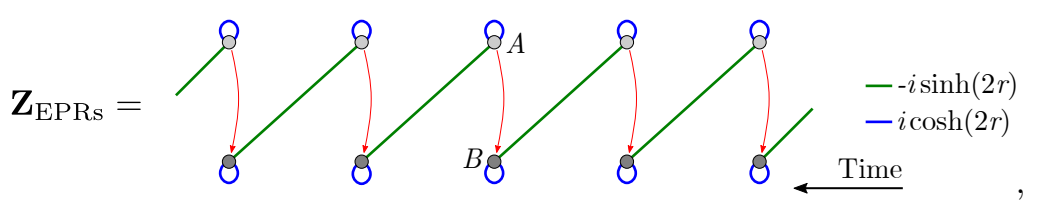

with the beam-splitter transformation marked by red arrows corresponding to $\mathrm{BS}_{2}$ in Fig. 3.6. Here, the bright and dark grey nodes symbolize temporal modes of the two different spatial modes of $A$ and $B$ respectively, and has no other meaning than distinguishing spatial modes. Note also that $\mathbf{Z}_{\mathrm{EPRs}}$ is the graph just after the delay, $\tau$, in Fig. 3.6. After the beam-splitter transformation connecting the pairs of EPR-states, we attain the 1D $\mathcal{H}$-graph state

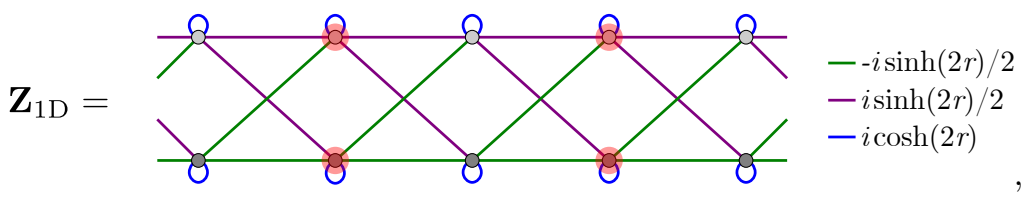

which is self-inverse and bipartite, and so it can be transformed into an approximative cluster state by applying the Fourier gate on all modes in one of the bipartitions: Rotating every second pairs of spatial modes marked with red in $\mathbf{Z}_{1 \mathrm{D}}$ leads to

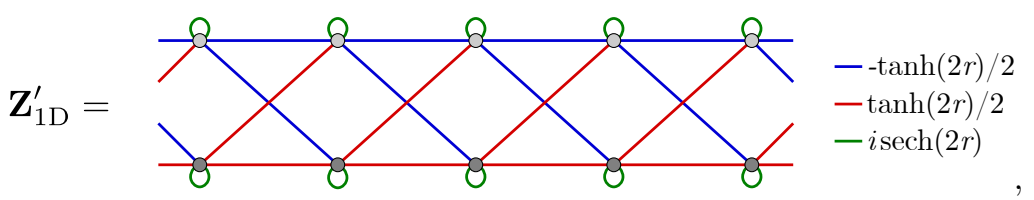

with only real edges and vanishing self-loops when $r \rightarrow \infty$ as $\tanh (2 r) / 2 \rightarrow 1 / 2$ and $i \operatorname{sech}(2 r) \rightarrow 0$. By determining the nullifiers in the limit $r \rightarrow \infty$, and rotating every second pair of modes back again (as for the EPR-state in section 3.4.2.1) we can determine the nullifiers of $\mathbf{Z}_{1 \mathrm{D}}$, which each will include 5 modes according to Eq. (3.4) (all modes connected to a single mode). These nullifiers can be simplified, as all linear combinations of nullifiers are also nullifiers, and the nullifiers including the least modes are

$$
\hat{x}_{A k}+\hat{x}_{B k}-\hat{x}_{A k+1}+\hat{x}_{B k+1} \quad, \quad \hat{p}_{A k}+\hat{p}_{B k}+\hat{p}_{A k+1}-\hat{p}_{B k+1},
$$

where the index $k$ and $k+1$ denote different temporal mode numbers. Since the nullifiers are linear combination of $\hat{x}$ or $\hat{p}$, they are easily measured in order to verify the entanglement of the cluster state.

\subsubsection{2D cluster states}

After the $N \tau$ delay in Fig. 3.6, the $1 \mathrm{D}$ cluster, $\mathbf{Z}_{1 \mathrm{D}}$, is coiled up into a cylinder as illustrated in Fig. 3.7. To begin with, we consider only a section of the cylinder:

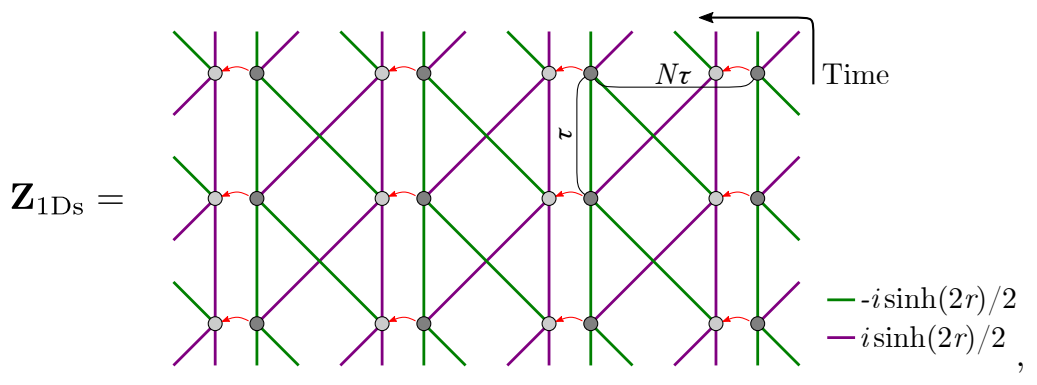




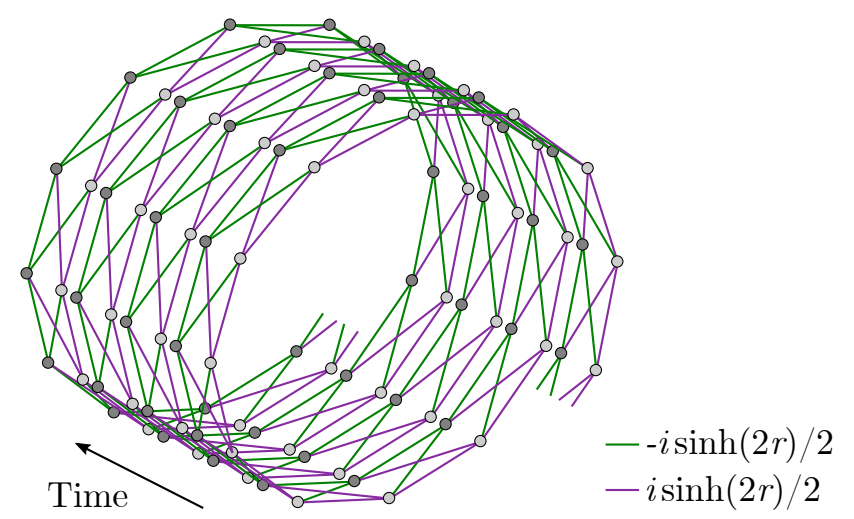

Figure 3.7: Complex adjacency matrix, $\mathbf{Z}_{1 \mathrm{Ds}}$, of the coiled up 1D $\mathcal{H}$-graph state just after the $N \tau$ delay in Fig. 3.6 with $N=12$ as in the experimental implementation. For simplicity, self-loops of $i \cosh (2 r)$ are omitted, but they are still present in the diagonal of $\mathbf{Z}_{1 \text { Ds }}$.

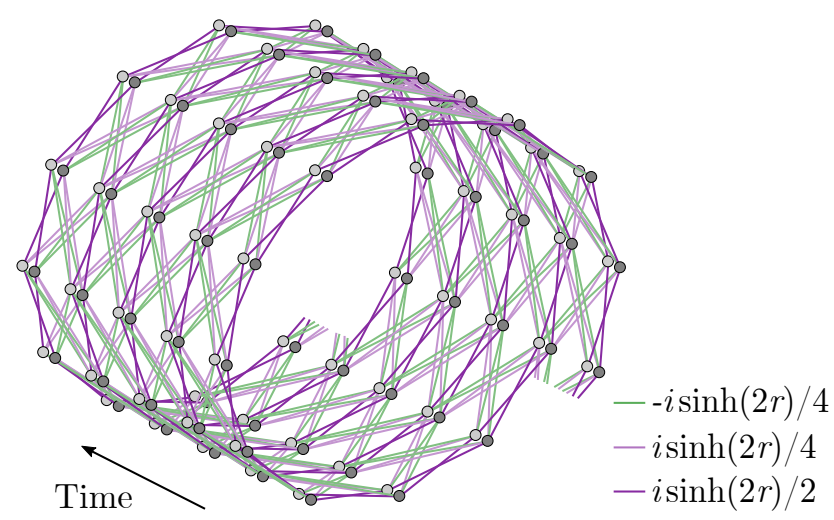

Figure 3.8: 2D $\mathcal{H}$-graph, $\mathbf{Z}_{2 \mathrm{D}}$, generated in Fig. 3.6 with $N=12$. Self-loops of $i \cosh (2 r)$ are omitted for simplicity, but they are present in the diagonal of $\mathbf{Z}_{2 \mathrm{D}}$. 
where each parallel $1 \mathrm{D}$ cluster state is separated by $N \tau$ in time corresponding to one circumference of the cylinder. Note that the self-loops of $i \cosh (2 r)$ have been omitted, and will be omitted in the following, but they are still present in the diagonal of $\mathbf{Z}_{1 \text { Ds }}$. Here, two closer spaced spatial modes $A$ and $B$ overlap in time, and the red arrows represent the last beam-splitter transformation $\mathrm{BS}_{3}$ in Fig. 3.6, leading to the $2 \mathrm{D} \mathcal{H}$-graph state

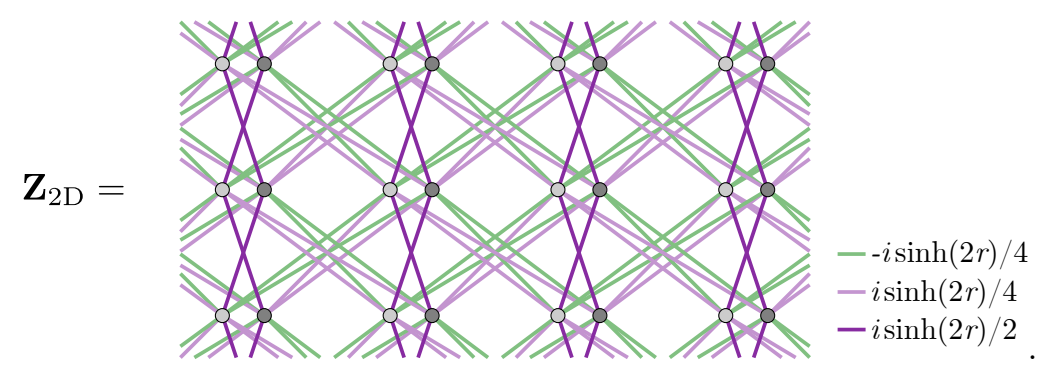

$\mathbf{Z}_{2 \mathrm{D}}$ is self-inverse, and if we consider $\mathbf{Z}_{2 \mathrm{D}}$ as a infinite plane instead of a cylinder it is also bipartite, and by $\pi / 2$ phase-space rotations on all modes in one bipartion, namely every second horizontal row shown in $\mathbf{Z}_{2 \mathrm{D}}$ above (corresponding to every second pair of modes arriving simultaneously at the homodyne detectors in Fig. 3.6), we get the approximate cluster state

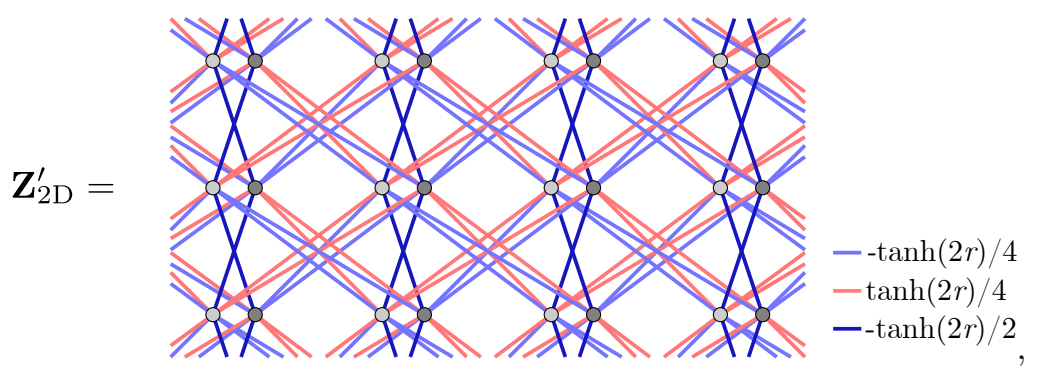

where again we have omitted self-loops of $i \operatorname{sech}(2 r) \rightarrow 0$ for $r \rightarrow \infty$.

Finally, considering the $\mathbf{Z}_{2 \mathrm{D}}$ as a cylinder, the resulting $\mathcal{H}$-graph state is shown in Fig. 3.8 with $N$ temporal modes in the cylinder circumference. Only in the case of even $N, \mathbf{Z}_{2 \mathrm{D}}$ is a bipartite graph, and can be transformed as described above into the approximate cluster state $\mathbf{Z}_{2 \mathrm{D}}^{\prime}$ by $\pi / 2$ phase-space rotation on half of its modes. As previously mentioned, such $\pi / 2$ phase-space rotation of modes in the generated state can be absorbed into the measurement basis in the homodyne detection, and therefore the generated self-inverse bipartite $\mathcal{H}$-graph state is considered equivalent to its corresponding cluster state. In the experimental implementation we have chosen $N=12$ as in Fig. 3.8.

\subsubsection{Nullifiers}

The nullifiers of the generated 2D cluster state can be determined from its graph $\mathbf{Z}_{2 \mathrm{D}}^{\prime}$ in the same way as for the $1 \mathrm{D}$ cluster state in section 3.4.2.2. However, to give a clear picture of the quadrature transformation, here we will calculate the quadrature relations throughout the setup, from which we can finally derive the resulting nullifiers.

Consider the circuit in Fig. 3.9 corresponding to the experimental setup in Fig. 3.6, but with temporal modes and the effect of optical delays clearly illustrated. Here, different stages of the setup are numbered from 0 to 7 , where at stage 0 all modes are initially in a vacuum state, while at stage 1 each mode are squeezed in the amplitude quadratures:

$$
\hat{x}_{k}^{A(1)}=e^{-r_{A}} \hat{x}_{k}^{A(0)} \quad, \quad \hat{p}_{k}^{A(1)}=e^{r_{A}} \hat{p}_{k}^{A(0)} \quad, \quad \hat{x}_{k}^{B(1)}=e^{-r_{B}} \hat{x}_{k}^{B(0)} \quad, \quad \hat{p}_{k}^{B(1)}=e^{r_{B}} \hat{p}_{k}^{B(0)},
$$

where $r_{A}$ and $r_{B}$ are the squeezing coefficients in spatial modes $A$ and $B$ and the stage is indicated 


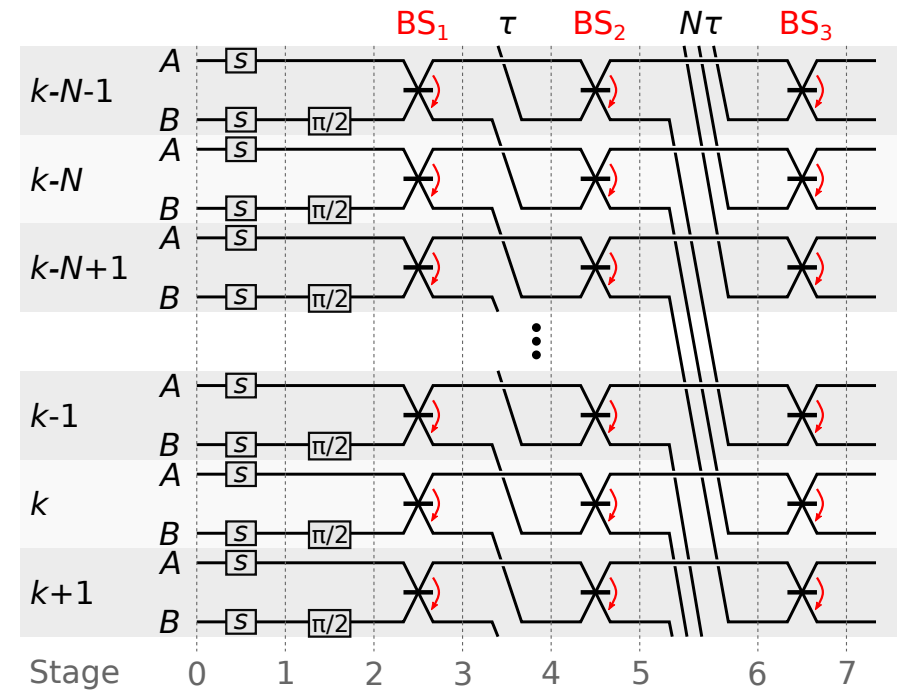

Figure 3.9: Corresponding circuit diagram of the experimental setup in Fig. 3.6 for 2D cluster state generation.

in the superscript. At stage 2 , the spatial mode $B$ is rotated by $\pi / 2$ in phase space such that

$$
\begin{array}{ccc}
\hat{x}_{k}^{A(2)}=\hat{x}_{k}^{A(1)}=e^{-r_{A}} \hat{x}_{k}^{A(0)} \quad, \quad \hat{p}_{k}^{A(2)}=\hat{p}_{k}^{A(1)}=e^{r_{A}} \hat{p}_{k}^{A(0)}, \\
\hat{x}_{k}^{B(2)}=-\hat{p}_{k}^{B(1)}=-e^{r_{B}} \hat{p}_{k}^{B(0)} \quad, \quad \hat{p}_{k}^{B(2)}=\hat{x}_{k}^{B(1)}=e^{-r_{B}} \hat{x}_{k}^{B(0)} .
\end{array}
$$

From stage 2 to 3 , a beam-splitter interaction is applied onto the spatial modes $A$ to $B$,

$$
\begin{aligned}
& \hat{x}_{k}^{A(3)}=\frac{1}{\sqrt{2}}\left(\hat{x}_{k}^{A(2)}-\hat{x}_{k}^{B(2)}\right)=\frac{1}{\sqrt{2}}\left(e^{-r_{A}} \hat{x}_{k}^{A(0)}+e^{r_{B}} \hat{p}_{k}^{B(0)}\right), \\
& \hat{p}_{k}^{A(3)}=\frac{1}{\sqrt{2}}\left(\hat{p}_{k}^{A(2)}-\hat{p}_{k}^{B(2)}\right)=\frac{1}{\sqrt{2}}\left(e^{r_{A}} \hat{p}_{k}^{A(0)}-e^{-r_{B}} \hat{x}_{k}^{B(0)}\right), \\
& \hat{x}_{k}^{B(3)}=\frac{1}{\sqrt{2}}\left(\hat{x}_{k}^{A(2)}+\hat{x}_{k}^{B(2)}\right)=\frac{1}{\sqrt{2}}\left(e^{-r_{A}} \hat{x}_{k}^{A(0)}-e^{r_{B}} \hat{p}_{k}^{B(0)}\right), \\
& \hat{p}_{k}^{A(3)}=\frac{1}{\sqrt{2}}\left(\hat{p}_{k}^{A(2)}+\hat{p}_{k}^{B(2)}\right)=\frac{1}{\sqrt{2}}\left(e^{r_{A}} \hat{p}_{k}^{A(0)}+e^{-r_{B}} \hat{x}_{k}^{B(0)}\right) .
\end{aligned}
$$

From stage 3 to 4 , the spatial mode $B$ is delayed by one temporal mode index,

$$
\begin{aligned}
& \hat{x}_{k}^{A(4)}=\hat{x}_{k}^{A(3)}=\frac{1}{\sqrt{2}}\left(e^{-r_{A}} \hat{x}_{k}^{A(0)}+e^{r_{B}} \hat{p}_{k}^{B(0)}\right), \\
& \hat{p}_{k}^{A(4)}=\hat{p}_{k}^{A(3)}=\frac{1}{\sqrt{2}}\left(e^{r_{A}} \hat{p}_{k}^{A(0)}-e^{-r_{B}} \hat{x}_{k}^{B(0)}\right), \\
& \hat{x}_{k}^{B(4)}=\hat{x}_{k-1}^{B(3)}=\frac{1}{\sqrt{2}}\left(e^{-r_{A}} \hat{x}_{k-1}^{A(0)}-e^{r_{B}} \hat{p}_{k-1}^{B(0)}\right), \\
& \hat{p}_{k}^{B(4)}=\hat{p}_{k-1}^{B(3)}=\frac{1}{\sqrt{2}}\left(e^{r_{A}} \hat{p}_{k-1}^{A(0)}+e^{-r_{B}} \hat{x}_{k-1}^{B(0)}\right) .
\end{aligned}
$$


From stage 4 to 5 , a beam-splitter interaction is applied on the spatial modes $A$ to $B$,

$$
\begin{aligned}
& \hat{x}_{k}^{A(5)}=\frac{1}{\sqrt{2}}\left(\hat{x}_{k}^{A(4)}-\hat{x}_{k}^{B(4)}\right)=\frac{1}{2}\left(e^{-r_{A}}\left[\hat{x}_{k}^{A(0)}-\hat{x}_{k-1}^{A(0)}\right]+e^{r_{B}}\left[\hat{p}_{k}^{B(0)}+\hat{p}_{k-1}^{B(0)}\right]\right), \\
& \hat{p}_{k}^{A(5)}=\frac{1}{\sqrt{2}}\left(\hat{p}_{k}^{A(4)}-\hat{p}_{k}^{B(4)}\right)=\frac{1}{2}\left(e^{-r_{B}}\left[-\hat{x}_{k}^{B(0)}-\hat{x}_{k-1}^{B(0)}\right]+e^{r_{A}}\left[\hat{p}_{k}^{A(0)}-\hat{p}_{k-1}^{A(0)}\right]\right), \\
& \hat{x}_{k}^{B(5)}=\frac{1}{\sqrt{2}}\left(\hat{x}_{k}^{A(4)}+\hat{x}_{k}^{B(4)}\right)=\frac{1}{2}\left(e^{-r_{A}}\left[\hat{x}_{k}^{A(0)}+\hat{x}_{k-1}^{A(0)}\right]+e^{r_{B}}\left[\hat{p}_{k}^{B(0)}-\hat{p}_{k-1}^{B(0)}\right]\right), \\
& \hat{p}_{k}^{B(5)}=\frac{1}{\sqrt{2}}\left(\hat{p}_{k}^{A(4)}+\hat{p}_{k}^{B(4)}\right)=\frac{1}{2}\left(e^{-r_{B}}\left[-\hat{x}_{k}^{B(0)}+\hat{x}_{k-1}^{B(0)}\right]+e^{r_{A}}\left[\hat{p}_{k}^{A(0)}+\hat{p}_{k-1}^{A(0)}\right]\right) .
\end{aligned}
$$

From stage 5 to 6 , the spatial mode $B$ is delayed by $N$ temporal modes indices,

$$
\begin{aligned}
& \hat{x}_{k}^{A(6)}=\hat{x}_{k}^{A(5)}=\frac{1}{2}\left(e^{-r_{A}}\left[\hat{x}_{k}^{A(0)}-\hat{x}_{k-1}^{A(0)}\right]+e^{r_{B}}\left[\hat{p}_{k}^{B(0)}+\hat{p}_{k-1}^{B(0)}\right]\right), \\
& \hat{p}_{k}^{A(6)}=\hat{p}_{k}^{A(5)}=\frac{1}{2}\left(e^{-r_{B}}\left[-\hat{x}_{k}^{B(0)}-\hat{x}_{k-1}^{B(0)}\right]+e^{r_{A}}\left[\hat{p}_{k}^{A(0)}-\hat{p}_{k-1}^{A(0)}\right]\right), \\
& \hat{x}_{k}^{B(6)}=\hat{x}_{k-N}^{B(5)}=\frac{1}{2}\left(e^{-r_{A}}\left[\hat{x}_{k-N}^{A(0)}+\hat{x}_{k-N-1}^{A(0)}\right]+e^{r_{B}}\left[\hat{p}_{k-N}^{B(0)}-\hat{p}_{k-N-1}^{B(0)}\right]\right), \\
& \hat{p}_{k}^{B(6)}=\hat{p}_{k-N}^{B(5)}=\frac{1}{2}\left(e^{-r_{B}}\left[-\hat{x}_{k-N}^{B(0)}+\hat{x}_{k-N-1}^{B(0)}\right]+e^{r_{A}}\left[\hat{p}_{k-N}^{A(0)}+\hat{p}_{k-N-1}^{A(0)}\right]\right) .
\end{aligned}
$$

Finally, from stage 6 to 7 , a beam-splitter interaction is executed from spatial mode $A$ to $B$,

$$
\begin{aligned}
\hat{x}_{k}^{A} & =\frac{1}{\sqrt{2}}\left(\hat{x}_{k}^{A(6)}-\hat{x}_{k}^{B(6)}\right) \\
& =\frac{1}{2 \sqrt{2}}\left(e^{-r_{A}}\left[\hat{x}_{k}^{A(0)}-\hat{x}_{k-1}^{A(0)}-\hat{x}_{k-N}^{A(0)}-\hat{x}_{k-N-1}^{A(0)}\right]+e^{r_{B}}\left[\hat{p}_{k}^{B(0)}+\hat{p}_{k-1}^{B(0)}-\hat{p}_{k-N}^{B(0)}+\hat{p}_{k-N-1}^{B(0)}\right]\right), \\
\hat{p}_{k}^{A} & =\frac{1}{\sqrt{2}}\left(\hat{p}_{k}^{A(6)}-\hat{p}_{k}^{B(6)}\right) \\
& =\frac{1}{2 \sqrt{2}}\left(e^{-r_{B}}\left[-\hat{x}_{k}^{B(0)}-\hat{x}_{k-1}^{B(0)}+\hat{x}_{k-N}^{B(0)}-\hat{x}_{k-N-1}^{B(0)}\right]+e^{r_{A}}\left[\hat{p}_{k}^{A(0)}-\hat{p}_{k-1}^{A(0)}-\hat{p}_{k-N}^{A(0)}-\hat{p}_{k-N-1}^{A(0)}\right]\right), \\
\hat{x}_{k}^{B} & =\frac{1}{\sqrt{2}}\left(\hat{x}_{k}^{A(6)}+\hat{x}_{k}^{B(6)}\right) \\
& =\frac{1}{2 \sqrt{2}}\left(e^{-r_{A}}\left[\hat{x}_{k}^{A(0)}-\hat{x}_{k-1}^{A(0)}+\hat{x}_{k-N}^{A(0)}+\hat{x}_{k-N-1}^{A(0)}\right]+e^{r_{B}}\left[\hat{p}_{k}^{B(0)}+\hat{p}_{k-1}^{B(0)}+\hat{p}_{k-N}^{B(0)}-\hat{p}_{k-N-1}^{B(0)}\right]\right), \\
\hat{p}_{k}^{B} & =\frac{1}{\sqrt{2}}\left(\hat{p}_{k}^{A(6)}+\hat{p}_{k}^{B(6)}\right) \\
& =\frac{1}{2 \sqrt{2}}\left(e^{-r_{B}}\left[-\hat{x}_{k}^{B(0)}-\hat{x}_{k-1}^{B(0)}-\hat{x}_{k-N}^{B(0)}+\hat{x}_{k-N-1}^{B(0)}\right]+e^{r_{A}}\left[\hat{p}_{k}^{A(0)}-\hat{p}_{k-1}^{A(0)}+\hat{p}_{k-N}^{A(0)}+\hat{p}_{k-N-1}^{A(0)}\right]\right),
\end{aligned}
$$

where the superscript (7) has been omitted on this final stage. Solving for the initially squeezed amplitude quadratures $e^{-r_{A}} \hat{x}_{k}^{A(0)}$ and $e^{-r_{B}} \hat{x}_{k}^{B(0)}$, a set of nullifiers are found to be

$$
\begin{aligned}
& \hat{n}_{k}^{x}=\hat{x}_{k}^{A}+\hat{x}_{k}^{B}-\hat{x}_{k+1}^{A}-\hat{x}_{k+1}^{B}-\hat{x}_{k+N}^{A}+\hat{x}_{k+N}^{B}-\hat{x}_{k+N+1}^{A}+\hat{x}_{k+N+1}^{B}=2 \sqrt{2} e^{-r_{A}} \hat{x}_{k}^{A(0)}, \\
& \hat{n}_{k}^{p}=\hat{p}_{k}^{A}+\hat{p}_{k}^{B}+\hat{p}_{k+1}^{A}+\hat{p}_{k+1}^{B}-\hat{p}_{k+N}^{A}+\hat{p}_{k+N}^{B}+\hat{p}_{k+N+1}^{A}-\hat{p}_{k+N+1}^{B}=-2 \sqrt{2} e^{-r_{B}} \hat{x}_{k}^{B(0)},
\end{aligned}
$$

with the variance

$$
\left\langle\Delta \hat{n}_{k}^{x 2}\right\rangle=4 e^{-2 r_{A}} \quad, \quad\left\langle\Delta \hat{n}_{k}^{p 2}\right\rangle=4 e^{-2 r_{B}}
$$

going towards zero when $r \rightarrow \infty$ in the spatial modes $A$ and $B$. With $\hbar=1,\left\langle\Delta \hat{x}_{k}^{A(0)}\right\rangle=\left\langle\Delta \hat{x}_{k}^{B(0)}\right\rangle=$ $1 / 2$. 


\subsubsection{Cluster state computation}

In the main text Fig. 3.2, the generated double bilayer square lattice cluster state (2xBSL), $\mathbf{Z}_{2 \mathrm{D}}^{\prime}$, is projected into a regular square lattice using mode deletion by measuring in the $\hat{x}$-basis. This serves as a proof of the generated cluster state being a universal cluster state: As the square lattice extracted from the $2 \mathrm{xBSL}$ is a universal resource [5], the 2xBSL is itself a universal resource, also without projective measurements into a square lattice. In fact, one would in general optimize the projective measurements required for the specific quantum circuit to be implemented. In this section we will give an example of such optimized setting, and discuss the experimental requirements for universal quantum computation.

Modes are wasted when projecting the cluster state into a square lattice, but more important is the resulting $1 / 4$ edge weights of the square lattice (in the simplified picture of infinite squeezing levels). For each computation step, edge weights less than unity leads to squeezing of the state in computation as a known byproduct of the computation. In the ideal case of the cluster state prepared from momentum eigenstates, this is not a problem as the amount of byproduct squeezing is known, and can be compensated for in the following computation steps. Yet, the resource states for physical cluster state generation are finitely squeezed approximated momentum eigenstates leading to noise in the measurements of each computation step and thereby errors. These errors can be corrected by error correction (which we will come back to), thereby enabling fault-tolerant computation, as long as the squeezing of the initial resource states surpasses a given threshold. However, with the squeezing of the state in computation as byproduct in each computation step due to edge weights less than unity, less noise is required in the measurements of the computation, thereby increasing the squeezing threshold of the initial resource states for fault-tolerance. As a result, when projecting the $2 x B S L$ into a regular square lattice with $1 / 4$ edge weights, not only modes are wasted, but squeezing is wasted as well.

To avoid squeezing waste when deleting modes by $\hat{x}$-measurements, entanglement from the modes can be rearranged in the cluster state by phase delay before measurements, or in other words, by measuring in different bases, $\hat{q}(\theta)=\hat{x} \cos \theta+\hat{p} \sin \theta$. That way, the $2 \mathrm{xBSL}$ can be projected into a simpler lattice without wasting squeezing. One example of this is shown in [11] for the bilayer square lattice cluster state (BSL), and a similar approach can be used here for the 2xBSL: Measuring the two spatial modes $A$ and $B$ for every second temporal mode $k+2 n$ in basis $\hat{q}\left((-1)^{n} \pi / 4\right)$ leads to dual-rail wires of edge weights $\pm 1 / 2$ as illustrated in Fig. 3.10. Such dual-rail wires are well known efficient resources for single mode computation, where each wire corresponds to a one-mode computer [19] with input states encoded in the macronodes consisting of spatial modes $A$ and $B$ within the same temporal mode as indicated in Fig. 3.10. Another benefit of projecting into these dual-rail wires is that the wires are along the length of the cylindrical structure of $2 x B S L$, and so it is unnecessary to "cut up" the cylinder while the one-mode computation length can be as long as the cylinder itself. For the generated 2xBSL with $N=12$ temporal modes in the circumference, 6 parallel dual-rail wires can be constructed.

With two spatial modes $A$ and $B$ in each temporal macronode of the dual-rail wire, the macronodes include a symmetric $(+)$ and an anti-symmetric mode $(-)$ via

$$
\hat{a}_{k}^{ \pm}=\frac{1}{\sqrt{2}}\left(\hat{a}_{k}^{A} \pm \hat{a}_{k}^{B}\right)
$$

where $\hat{a}_{i}$ is the annihilation operator of mode $i$, and $\hat{a}_{k}^{ \pm}$corresponds to the two spatial modes before the beam splitter $\mathrm{BS}_{3}$. Thus, as in [11], to encode information in a macronode, a switch can be placed before $\mathrm{BS}_{3}$ as illustrated in Fig. 3.11, switching the input state $|\psi\rangle_{\text {in }}$ into the anti-symmetric macronode with the given direction of $\mathrm{BS}_{3}$ used in Fig. 3.6 (alternatively, we can switch into the symmetric macronode by placing the switch in spatial mode $A$ before $\mathrm{BS}_{3}$ ). With this encoding, the multiple edges of $\pm 1 / 2$ weight between each macronode of the dual-rail wire correspond to an edge of weight 1 between the encoded logic mode of each macronode, allowing efficient computation without squeezing waste.

Concurrently with the projection of the state into dual-rail wires, one-mode computation is performed in each wire by measuring the macronodes in an adaptive basis depending on the 


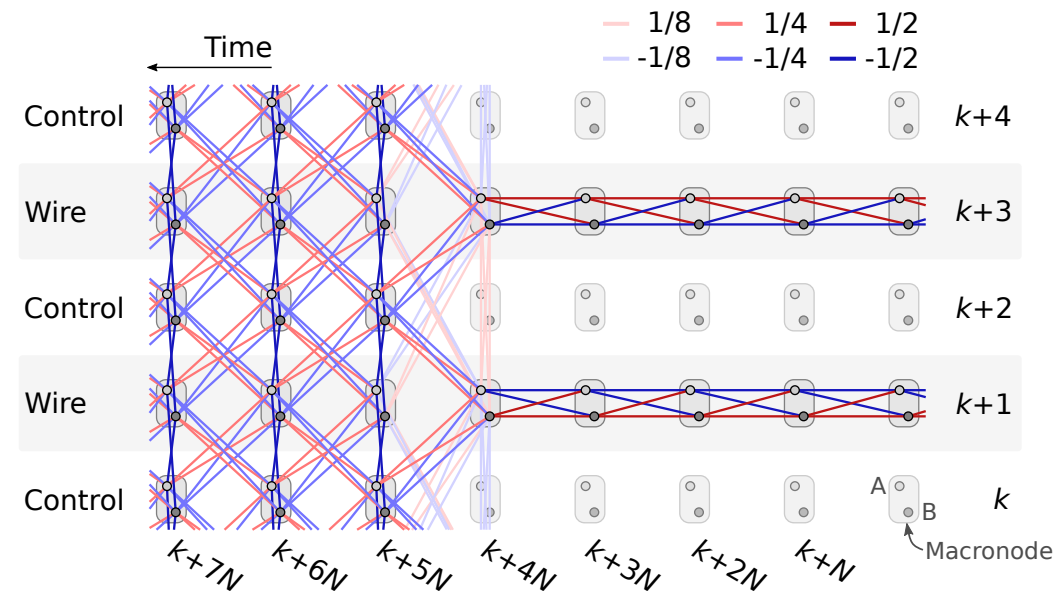

Figure 3.10: Depending on the measurement basis of control modes, the double bilayer square lattice can efficiently be projected into dual-rail wires with possible entanglement in between neighbouring wires depending on the basis in which the modes in the control line is measured. It is convenient to define macronodes of the two spatial modes $A$ and $B$ in the same temporal mode. In the situation depicted here, the spatial modes $A$ and $B$ of the first 5 macronodes in the control lines have been measured in bases $(\hat{x} \pm \hat{p}) / \sqrt{2}$ leading to separated dual-rail wires.

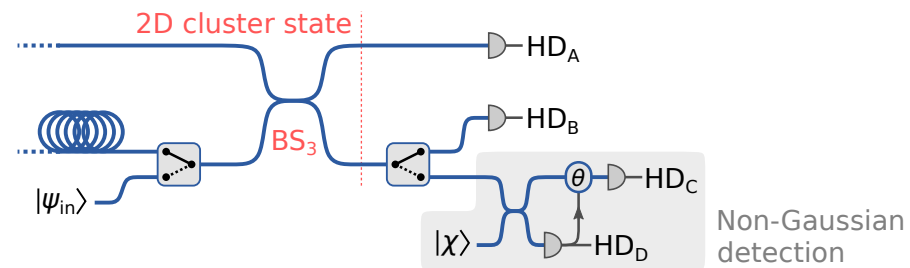

Figure 3.11: With a switch before beam splitter $\mathrm{BS}_{3}$, input states can be encoded into the symmetric or anti-symmetric macronode given by Eq. (3.16), while Gaussian computation is performed with homodyne detectors $\mathrm{HD}_{A}$ and $\mathrm{HD}_{B}$. With a switch after $\mathrm{BS}_{3}$ we can change to non-Gaussian measurements when required, necessary for universal quantum computation. One proposal for non-Gaussian detection is illustrated in the grey shaded area with $|\chi\rangle$ being the cubic-phase state [12].

gate to be implemented and previous measurement outcomes. However, with the cluster state being a Gaussian state (fully described by the first and second moments of the quadratures), only Gaussian computation is possible using homodyne detectors which project Gaussian states into Gaussian states. For universal computation, some non-Gaussian element is needed. As proposed in [12], a universal gate set can be realized using the measurement scheme illustrated in the shaded area of Fig. 3.11. A switch in spatial mode $B$ will enable the choice between this measurement and the homodyne detection as needed for the computation. With the ancillary input $|\chi\rangle$ being the highly non-Gaussian cubic-phase state, $|\chi\rangle=\int e^{i \chi s^{3}}|s\rangle_{x} \mathrm{~d} s$, this measurement implements the cubic-phase gate which, together with Gaussian gates, completes the universal one-mode gate set. Like the infinitely squeezed momentum eigenstate, the cubic-phase state is unphysical due to its infinite energy, while approximate cubic-phase states are demanding to prepare, and have not yet been generated in optical settings. Other possibilities for non-Gaussian operations exist, such as non-Gaussian projection by photon counting [14], and non-Gaussian operations for a universal gate set is today an active research topic.

For a universal multi-mode gate set, we need interaction between the dual-rail wires in Fig. 3.10. By measuring macronodes between two wires in a different basis than $\hat{q}\left((-1)^{n} \pi / 4\right)$, entanglement between the wires can be prepared depending on the measurement basis used. This is an appealing 
setting, where by measuring the so-called control macronodes, we can control the connectivity between neighbouring wires. However, the basis required in a macronode measurement for a given two-mode interaction is not trivial, and the detailed implementation of the $\hat{C}_{Z}$-gate, which together with the universal single mode gate set constitutes a universal multi-mode gate set, is left for future work.

Finally, for fault-tolerant computation error correction is necessary: With finite squeezing of the approximate momentum eigenstates from which the cluster state is generated and without error correction, only a limited number of computational steps are possible before the encoded state is lost in noise from the finite squeezing. While error correction in continuous variables is challenging, a possible way around this is to encode the information in a discrete subspace of the continuous variables concatenated with a conventional discrete error correction code. One popular example is the Gottesman-Kitaev-Preskill (GKP) encoding and correction, where a qubit is encoded as periodic peaks in the quadrature wave functions [14]. The squeezing level required for fault-tolerant quantum computation with the GKP-encoding depends on the error rate threshold of the concatenated error correction code to be used. For a rather conservative error correction scheme with $10^{-6}$ error rate threshold, a squeezing level of $20.5 \mathrm{~dB}$ is required [3]. With more modern error correction codes, this threshold can be brought down to $15-17 \mathrm{~dB}$ of squeezing [38], while the required squeezing using topological error correction is shown to be even lower [43].

An advantage of GKP-encoding of qubits is the recent result by B. Baragiola et al. [39] showing that the magic state can be distilled in the encoded subspace of the qubit allowing universal computation in the qubit subspace, rendering the need for non-Gaussian measurements discussed above unnecessary. However, with GKP-encoded states being highly non-Gaussian, they are as the cubic-phase state difficult to prepare, and their generation is as well an active research topic with recent demonstration in the microwave regime [100] and in trapped-ion mechanical oscillators [101].

\subsection{Suppl. Inf.: Inseparability criterion}

In this section, we derive an upper bound on nullifier variance for complete inseparability of modes in the generated cluster state based on the van Loock-Furusawa criterion [103]. In the van LoockFurusawa criterion, a number of modes are divided into two or more sets from which an inequality with combined quadrature variance is derived. A violation of this inequality means that the sets are inseparable.

For simplicity, we will consider only two sets of modes, $\mathcal{S}_{1}$ and $\mathcal{S}_{2}$, and define

$$
\hat{X}=\sum_{j \in \mathcal{S}_{1} \cup \mathcal{S}_{2}} h_{j} \hat{x}_{j} \quad, \quad \hat{P}=\sum_{j \in \mathcal{S}_{1} \cup \mathcal{S}_{2}} g_{j} \hat{p}_{j},
$$

for arbitrary coefficients $h_{j}$ and $g_{j}$. The van Loock-Furusawa criterion for separability then reads

$$
\left\langle\Delta \hat{X}^{2}\right\rangle+\left\langle\Delta \hat{P}^{2}\right\rangle \geq\left|\sum_{j \in \mathcal{S}_{1}} h_{j} g_{j}\right|+\left|\sum_{j \in \mathcal{S}_{2}} h_{j} g_{j}\right|,
$$

with $\hbar=1$. The goal is to find suitable $h_{j}$ and $g_{j}$ such that Eq. (3.18) is violated, thus proving inseparability of the two sets. Doing so for all possible bipartitions of modes then proves complete inseparability.

Since the generated cluster state is periodic, it is only necessary to consider the modes of a single unit cell of the cluster state lattice, and show complete inseparability of the modes within this unit cell. A good example of this approach is shown in the supplementary material of [21] for a 1D cluster states. The 8 modes of the nullifiers $\hat{n}_{k}^{x}$ and $\hat{n}_{k}^{p}$ in Eq. (3.13) and (3.14) make up a unit cell of the generated 2D cluster state, and is illustrated in Fig. 3.12 with the modes numbered from 1 to 8 . Hence, complete inseparability of the 2D cluster state can be proven by demonstrating a violation of the separability inequality in Eq. (3.18) for each of the $2^{8-1}-1=127$ possible bipartitions of these 8 modes. Below, we give three examples with different bipartitions. 


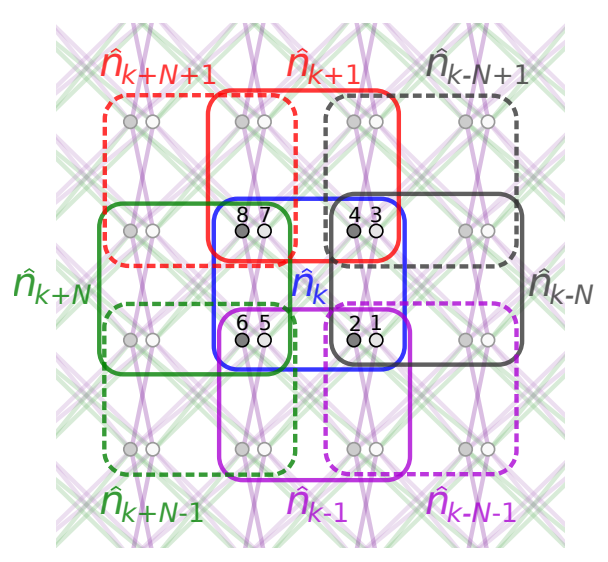

Figure 3.12: Graph of the generated 2D cluster state with the nullifier $\hat{n}_{k}\left(\hat{n}_{k}^{x}\right.$ or $\left.\hat{n}_{k}^{p}\right)$ and its neighbouring nullifiers indicated. In the van Loock-Furusawa inseparability criterion we consider a unit cell of 8 modes in common with $\hat{n}_{k}$, numbered as $(A, k) \rightarrow 1,(B, k) \rightarrow 2,(A, k+1) \rightarrow 3$, $(B, k+1) \rightarrow 4,(A, k+N) \rightarrow 5,(B, k+N) \rightarrow 6,(A, k+N+1) \rightarrow 7$ and $(B, k+N+1) \rightarrow 8$.

The mode numbering in Fig. 3.12 is used to shorten the notation:

Example 1: Consider the two sets of modes $\mathcal{S}_{1}=\{1,2,5,6\}$ and $\mathcal{S}_{2}=\{3,4,7,8\}$. Choosing

$$
\hat{X}=\hat{n}_{k}^{x}=\hat{x}_{1}+\hat{x}_{2}-\hat{x}_{3}-\hat{x}_{4}-\hat{x}_{5}+\hat{x}_{6}-\hat{x}_{7}+\hat{x}_{8}
$$

such that $\left(h_{1}, h_{2}, h_{3}, h_{4}, h_{5}, h_{6}, h_{7}, h_{8}\right)=(1,1,-1,-1,-1,1,-1,1)$, and

$$
\hat{P}=\hat{n}_{k}^{p}=\hat{p}_{1}+\hat{p}_{2}+\hat{p}_{3}+\hat{p}_{4}-\hat{p}_{5}+\hat{p}_{6}+\hat{p}_{7}-\hat{p}_{8}
$$

such that $\left(g_{1}, g_{2}, g_{3}, g_{4}, g_{5}, g_{6}, g_{7}, g_{8}\right)=(1,1,1,1,-1,1,1-1)$, then Eq. (3.18) becomes

$$
\begin{aligned}
\left\langle\Delta \hat{X}^{2}\right\rangle+\left\langle\Delta \hat{P}^{2}\right\rangle=\left\langle\Delta n_{k}^{x 2}\right\rangle+\left\langle\Delta n_{k}^{p 2}\right\rangle \geq & \left|\sum_{j \in S_{1}} h_{j} g_{j}\right|+\left|\sum_{j \in S_{2}} h_{j} g_{j}\right| \\
= & |1 \cdot 1+1 \cdot 1+(-1) \cdot(-1)+1 \cdot 1| \\
& \quad+|(-1) \cdot 1+(-1) \cdot 1+(-1) \cdot 1+1 \cdot(-1)| \\
= & .
\end{aligned}
$$

We may measure different variances of $\hat{n}_{k}^{x}$ and $\hat{n}_{k}^{p}$, but if we measure both below 4 , the above inequality will for sure be violated and the two mode sets $\mathcal{S}_{1}$ and $\mathcal{S}_{2}$ are inseparable. From Eq. (3.15) this requires $4 e^{-2 r_{i}}<4$ for $i=A, B$, and thus measuring the variance of $\hat{n}_{k}^{x}$ and $\hat{n}_{k}^{p}$ with more than $0 \mathrm{~dB}$ squeezing below shot noise.

Example 2: Consider now the two mode sets $\mathcal{S}_{1}=\{1,2,3,4\}$ and $\mathcal{S}_{2}=\{5,6,7,8\}$. Choosing $\hat{X}$ and $\hat{P}$ as in example 1 leads to

$$
\begin{aligned}
\left\langle\Delta \hat{X}^{2}\right\rangle+\left\langle\Delta \hat{P}^{2}\right\rangle=\left\langle\Delta \hat{n}_{k}^{x 2}\right\rangle+\left\langle\Delta \hat{n}_{k}^{p 2}\right\rangle \geq \mid & \sum_{j \in S_{1}} h_{j} g_{j}|+| \sum_{j \in S_{2}} h_{j} g_{j} \mid \\
= & |1 \cdot 1+1 \cdot 1+(-1) \cdot 1+(-1) \cdot 1| \\
& +|(-1) \cdot(-1)+1 \cdot 1+(-1) \cdot 1+1 \cdot(-1)| \\
= & 0,
\end{aligned}
$$

which is impossible to violate. If we instead choose

$$
\hat{P}=\hat{n}_{k+1}^{p}=\hat{p}_{3}+\hat{p}_{4}-\hat{p}_{7}+\hat{p}_{8}+\hat{p}_{k+2}^{A}+\hat{p}_{k+2}^{B}+\hat{p}_{k+N+2}^{A}+\hat{p}_{k+N+2}^{B},
$$


such that $\left(g_{1}, g_{2}, g_{3}, g_{4}, g_{5}, g_{6}, g_{7}, g_{8}\right)=(0,0,1,1,0,0,-1,1)$, Eq. (3.18) becomes

$$
\begin{aligned}
\left\langle\Delta \hat{X}^{2}\right\rangle+\left\langle\Delta \hat{P}^{2}\right\rangle=\left\langle\Delta \hat{n}_{k}^{x 2}\right\rangle+\left\langle\Delta \hat{n}_{k+1}^{p 2}\right\rangle \geq \mid & \left|\sum_{j \in S_{1}} h_{j} g_{j}\right|+\left|\sum_{j \in S_{2}} h_{j} g_{j}\right| \\
= & |1 \cdot 0+1 \cdot 0+(-1) \cdot 1+(-1) \cdot 1| \\
& +|(-1) \cdot 0+1 \cdot 0+(-1) \cdot(-1)+1 \cdot 1| \\
= & 4,
\end{aligned}
$$

which is violated if the variance of the two nullifiers $\hat{n}_{k}^{x}$ and $\hat{n}_{k}^{p}$ are less than 2 , requiring $3 \mathrm{~dB}$ of squeezing. The additional modes included in $\hat{P},(A, k+2),(B, k+2),(A, k+N+2)$ and $(B, k+N+2)$, are not included in the above inequality since they are not common modes with any in $\hat{X}=\hat{n}_{k}^{x}$, and thus will not contribute to the right hand side of Eq. (3.18). However, when including 4 extra modes, we should consider all new possible bipartitions: Given the two sets $\mathcal{S}_{1}$ and $\mathcal{S}_{2}$, we can add the additional 4 modes into these two sets in any arbitrary way without any change to Eq. (3.18). As a result, by violating Eq. (3.18) we prove inseparability of all bipartitions where each of the 4 extra modes are added to $\mathcal{S}_{1}$ or $\mathcal{S}_{2}$ in all possible ways.

Example 3: Consider the two mode sets $\mathcal{S}_{1}=\{3,5\}$ and $\mathcal{S}_{2}=\{1,2,4,6,7,8\}$. For this bipartition, there exists no single nullifier for $\hat{X}$ and $\hat{P}$ of the form in Eq. (3.13) and (3.14) which forms an inequality we can hope to violate experimentally. However, since linear combinations of nullifiers are also nullifiers, more exotic choices for $\hat{X}$ and $\hat{P}$ exist which leads to an inequality we can violate experimentally:

$$
\begin{aligned}
& \hat{X}=-\hat{n}_{k}^{x}+\hat{n}_{k+N}^{x}=-\hat{x}_{1}-\hat{x}_{2}+\hat{x}_{3}+\hat{x}_{4}+2 \hat{x}_{5}-2 \hat{x}_{8}-\hat{x}_{k+2 N}^{A}+\hat{x}_{k+2 N}^{B}-\hat{x}_{k+2 N+1}^{A}+\hat{x}_{k+2 N+1}^{B} \\
& \hat{P}=\hat{n}_{k-1}^{p}+\hat{n}_{k+N}^{p}+\hat{n}_{k+N+1}^{p}+\hat{n}_{k+N-1}^{p} \\
& =\hat{p}_{1}+\hat{p}_{2}+3 \hat{p}_{5}+\hat{p}_{6}+2 \hat{p}_{7}+2 \hat{p}_{8}+\hat{p}_{k-1}^{A}+\hat{p}_{k-1}^{B}+2 \hat{p}_{k+N-1}^{B} \\
& +\hat{p}_{k+N+2}^{A}+\hat{p}_{k+N+2}^{B}-\hat{p}_{k+2 N-1}^{A}+\hat{p}_{k+2 N-1}^{B}+\hat{p}_{k+2 N+2}^{A}-\hat{p}_{k+2 N+2}^{B}
\end{aligned}
$$

leading to

$$
\begin{aligned}
\left(h_{1}, h_{2}, h_{3}, h_{4}, h_{5}, h_{6}, h_{7}, h_{8}\right) & =(-1,-1,1,1,2,0,0,-2), \\
\left(g_{1}, g_{2}, g_{3}, g_{4}, g_{5}, g_{6}, g_{7}, g_{8}\right) & =(1,1,0,0,3,1,2,2),
\end{aligned}
$$

and so (3.18) becomes

$$
\begin{aligned}
\left\langle\Delta \hat{X}^{2}\right\rangle+\left\langle\Delta \hat{P}^{2}\right\rangle & =\left\langle\Delta \hat{n}_{k}^{x 2}\right\rangle+\left\langle\Delta \hat{n}_{k+N}^{x 2}\right\rangle+\left\langle\Delta \hat{n}_{k-1}^{p 2}\right\rangle+\left\langle\Delta \hat{n}_{k+N}^{p 2}\right\rangle+\left\langle\Delta \hat{n}_{k+N+1}^{p 2}\right\rangle+\left\langle\Delta \hat{n}_{k+N-1}^{p 2}\right\rangle \\
& \geq\left|\sum_{j \in S_{1}} h_{j} g_{j}\right|+\left|\sum_{j \in S_{2}} h_{j} g_{j}\right| \\
& =|1 \cdot 0+2 \cdot 3|+|(-1) \cdot 1+(-1) \cdot 1+1 \cdot 0+0 \cdot 1+0 \cdot 2+(-2) \cdot 2|=12,
\end{aligned}
$$

which is violated if the variance of each of the 6 nullifiers in the inequality is less than 2 , corresponding to $3 \mathrm{~dB}$ of squeezing. Here, the cross terms between different nullifiers in $\left\langle\Delta \hat{X}^{2}\right\rangle$ and $\left\langle\Delta \hat{P}^{2}\right\rangle$ are zero, as the nullifiers in Eq. (3.13) and (3.14) correspond to different temporal modes at the squeezing sources before $\mathrm{BS}_{1}$, and thus there are no correlations between different nullifiers. Notice how the nullifiers in $\hat{X}$ and $\hat{P}$ are chosen such that the 4 and 9 extra modes included in $\hat{X}$ and $\hat{P}$, respectively, are not the same. Thus, by the same argument as in example 2 , violating the above inequality proves inseparability of all bipartitions where each of the $4+9$ extra modes are added to $\mathcal{S}_{1}$ or $\mathcal{S}_{2}$ in all possible ways.

Using the same approach as in the above three examples, nullifiers for $\hat{X}$ and $\hat{P}$ are found for all 127 possible bipartitions of the 8 modes in the studied unit cell, resulting in a sufficient condition for the squeezing degree among all nullifiers of $3 \mathrm{~dB}$ below shot noise. As a result, with the generated 


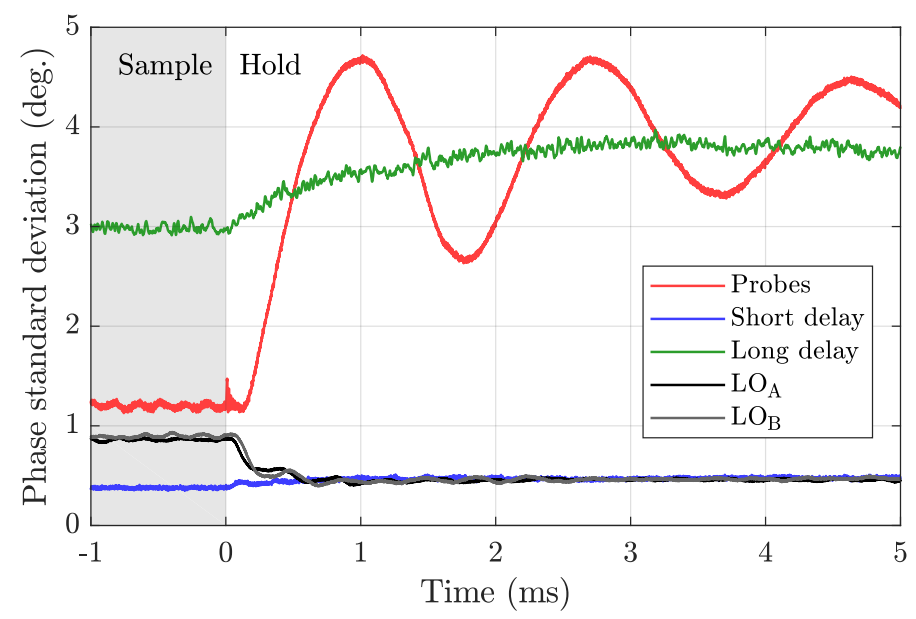

Figure 3.13: Standard deviation of the phase fluctuations in different parts of the setup measured by sending a coherent probe through the particular part of the setup while feedback is kept constant (hold-time). The phase fluctuations of the short and long delay lengths are measured by coupling a probe into the setup before $\mathrm{BS}_{1}$ and $\mathrm{BS}_{2}$ while measuring the interference after $\mathrm{BS}_{2}$ and $\mathrm{BS}_{3}$ respectively. The relative phase fluctuation of the two probes from $\mathrm{OPO}_{\mathrm{A}}$ and $\mathrm{OPO}_{\mathrm{B}}$ is measured by the interference after $\mathrm{BS}_{1}$. The local oscillator $(\mathrm{LO})$ phase fluctuations are measured by coupling a probe into the setup before $\mathrm{BS}_{3}$ and measuring the probe quadrature.

2D cluster state being periodic with this unit cell, measuring every temporal nullifier $\left(\hat{n}_{k}^{x}\right.$ and $\hat{n}_{k}^{p}$ for every $k$ ) with a variance less than $3 \mathrm{~dB}$ below shot noise leads to complete inseparability of the cluster state. The resulting $\hat{X}$ and $\hat{P}$ for every bipartition are listed in table 3.1, page 54, and as pointed out in example 2 and 3 , each choice of $\hat{X}$ and $\hat{P}$ are made such that they do not share any modes outside the studied unit cell.

\subsection{Suppl. Inf.: Experimental setup}

In this section, more details and characterization is given on the experimental implementation described in the methods section 3.3 .

\subsubsection{Efficiency and phase stability}

In the following, all loss contributions are summarized, and a combined efficiency of the setup is estimated: The $\mathrm{OPO}_{\mathrm{A}}$ and $\mathrm{OPO}_{\mathrm{B}}$ escape efficiencies are measured to be 0.98 and 0.95 , respectively, while $1 \%$ is tapped off in both squeezing sources for gain locks. The two spatial modes, $A$ and $B$, are coupled from free-space into fiber with a 0.97 coupling efficiency, where $3 \times 1 \%$ is tapped off for phase locking the three interference points at $\mathrm{BS}_{1}, \mathrm{BS}_{2}$ and $\mathrm{BS}_{3}$, each with an estimated visibility of 0.99 . To minimize the propagation losses, all fibers are spliced together, while short and long delay lines of SMF-28e+ fiber with $0.2 \mathrm{~dB} / \mathrm{km}$ attenuation each leads to $0.2 \%$ and $2.7 \%$ propagation loss, respectively. Finally, the fiber based homodyne detectors each have a detection efficiency of 0.91. For more information on the OPO, fiber coupling and homodyne detection efficiencies, see previous experimental work in chapter 2 [30]. In total, the estimated efficiencies add up to 0.81 and 0.78 in spatial mode $A$ and $B$, respectively.

Besides loss, the generated 2D cluster state is affected by phase fluctuations. In Fig. 3.13, the standard deviation of the phase is shown. The phases were measured while probing different parts of the setup with a coherent beam while turning off the feedback for cavity or phase locks. As expected, we see around 6 times more phase fluctuation of the long delay line compared to the short delay line. Another, and maybe more surprising, contribution to the phase fluctuation is 


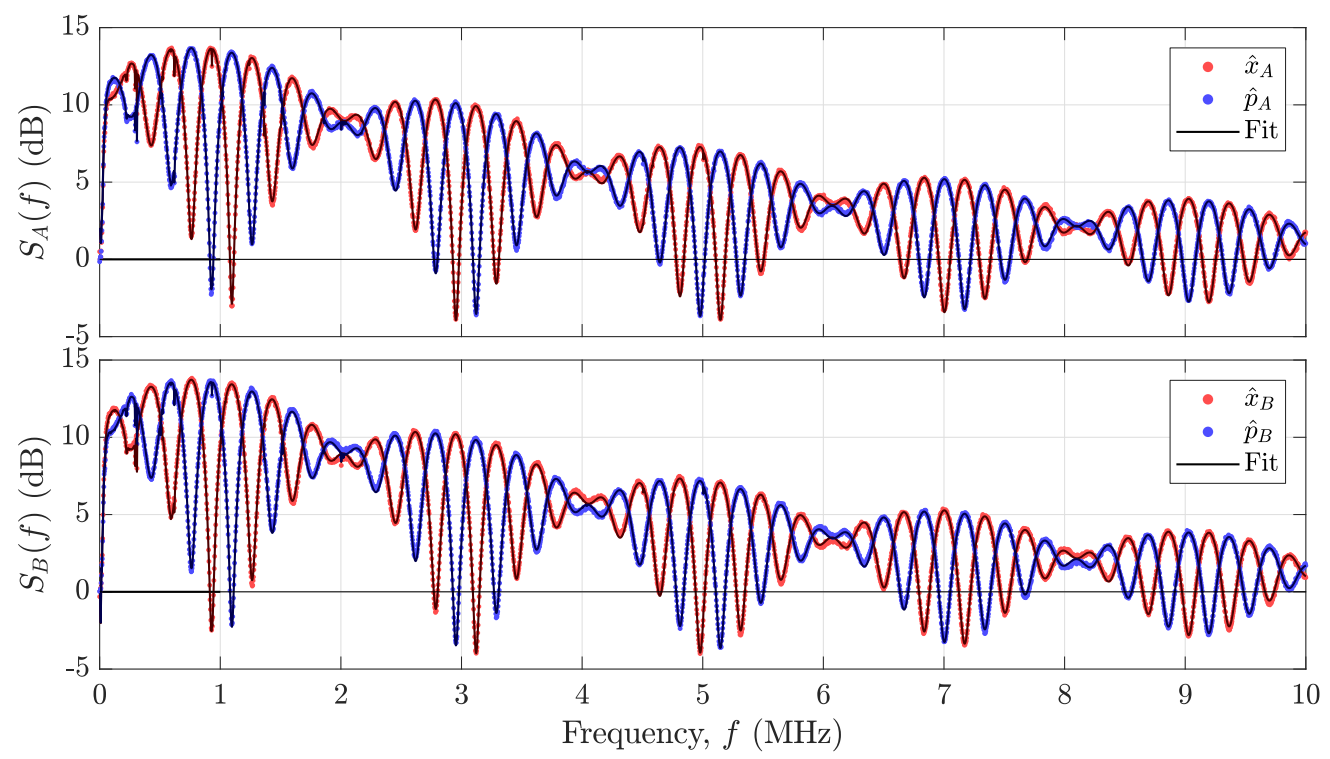

Figure 3.14: Power spectrum of the temporal encoded cluster state measured at the homodyne detectors in the spatial mode $A$ and $B$. The solid lines shows the result of fitting the power spectra in Eq. (3.21) with the squeezing spectra in Eq. (3.22) including phase fluctuations by Eq. (3.23) and measured electronic noise. The resulting fitting parameters are listed in Eq. (3.24).

from the probe phase which is seen to fluctuate fast as soon as the feedback is kept constant (holdtime). This is explained by the strong phase dependence in the OPO cavities around resonance. Furthermore, the probe phase standard deviation is seen to fluctuate, indicating systematic phase fluctuations which we believe are due to mechanical resonance and limited feedback bandwidth leading to a large impulse response when the feedback is suddenly kept constant when changing from sample- to hold-time. However, from this phase measurement, it is not clear whether the large phase fluctuation is from the probes of both OPO cavities, or if mainly one OPO cavity is more unstable. Finally, the standard deviation of the local oscillator (LO) phases appears to decrease during hold-time. This is simply caused by the fact that the probe quadrature fluctations (in addition to the LO noise) are measured during the sample-time while during the hold-time, only the LO noise is measured.

\subsubsection{Spectrum}

The generated 2D cluster state is temporally encoded in 2 spatial modes, $A$ and $B$. As a result, modes of the cluster state are measured by acquiring time traces from the two homodyne detectors in $A$ and $B$, on which a temporal mode function is applied for each mode as will be described in section 3.6.3. However, by analyzing the acquired time traces in frequency domain, we can obtain useful information about the setup and the two squeezing sources. In Fig. 3.14 the power spectra of the acquired time traces are shown, calculated by fast Fourier transform of $320 \mu$ s long time traces corresponding to 1300 consecutive temporal modes. To understand these power spectra, we derive them theoretically in the following.

According to the Wiener-Khinchin theorem, the quadrature power spectrum is expressed by the Fourier transform of the quadrature autocorrelation function,

$$
S_{j}^{q}(\omega)=\int_{-\infty}^{\infty}\left\langle\hat{q}_{j}(t) \hat{q}_{j}(0)\right\rangle e^{i \omega t} \mathrm{~d} t \quad, \quad j=A, B, q=x, p,
$$

where $\omega$ is the angular frequency. The time dependent amplitude and phase quadratures, $\hat{x}(t)$ and $\hat{p}(t)$, are derived in the exact same way as the temporal mode quadratures in Eq. (3.12), and 
the result is the same but with time dependency instead of temporal mode index, i.e. $\hat{q}_{j, k-m} \rightarrow$ $\hat{q}_{j}(t-m \tau)$ with $m=0,1, N, N+1$, as neighbouring temporal modes are spaced in time by $\tau$. Considering first the $\hat{x}$-quadrature in mode $A$, the autocorrelation function, using the quadratures expressed in Eq. (3.12), becomes

$$
\begin{aligned}
&\left\langle\hat{x}_{A}(t) \hat{x}_{A}(0)\right\rangle=\frac{1}{8}\left(4\left\langle\hat{x}_{A}^{(1)}(t) \hat{x}_{A}^{(1)}(0)\right\rangle\right.-\left\langle\hat{x}_{A}^{(1)}(t+N \tau+\tau) \hat{x}_{A}^{(1)}(0)\right\rangle+\left\langle\hat{x}_{A}^{(1)}(t+N \tau-\tau) \hat{x}_{A}^{(1)}(0)\right\rangle \\
&+\left.\left\langle\hat{x}_{A}^{(1)}(t-N \tau+\tau) \hat{x}_{A}^{(1)}(0)\right\rangle-\left\langle\hat{x}_{A}^{(1)}(t-N \tau-\tau) \hat{x}_{A}^{(1)}(0)\right\rangle\right) \\
&+\frac{1}{8}\left(4\left\langle\hat{p}_{B}^{(1)}(t) \hat{p}_{B}^{(1)}(0)\right\rangle+\left\langle\hat{p}_{B}^{(1)}(t+N \tau+\tau) \hat{p}_{B}^{(1)}(0)\right\rangle-\left\langle\hat{p}_{B}^{(1)}(t+N \tau-\tau) \hat{p}_{B}^{(1)}(0)\right\rangle\right. \\
&\left.-\left\langle\hat{p}_{B}^{(1)}(t-N \tau+\tau) \hat{p}_{B}^{(1)}(0)\right\rangle+\left\langle\hat{p}_{B}^{(1)}(t-N \tau-\tau) \hat{p}_{B}^{(1)}(0)\right\rangle\right)
\end{aligned}
$$

where the property of the autocorrelation $\left\langle\hat{q}_{j}(t) \hat{q}_{j}(y)\right\rangle=\left\langle\hat{q}_{j}(t-y) \hat{q}_{j}(0)\right\rangle$ is used. Substituting $\left\langle\hat{x}_{A}(t) \hat{x}_{A}(0)\right\rangle$ into Eq. (3.20), and using that

$$
\int_{-\infty}^{\infty}\left\langle\hat{q}_{j}(t+y) \hat{q}_{j}(0)\right\rangle e^{i \omega t} \mathrm{~d} t=\int_{-\infty}^{\infty}\left\langle\hat{q}_{j}(t) \hat{q}_{j}(0)\right\rangle e^{i \omega(t-y)} \mathrm{d} t=e^{-i \omega y} S_{j}^{q}(\omega),
$$

the power spectrum measured in mode $A$ in the $\hat{x}$-quadrature becomes

$$
\begin{aligned}
S_{A}^{x}(\omega)= & \frac{1}{8}\left(4-e^{-i \omega(N \tau+\tau)}+e^{-i \omega(N \tau-\tau)}+e^{-i \omega(-N \tau+\tau)}-e^{-i \omega(-N \tau-\tau)}\right) S_{A}^{x(1)}(\omega) \\
& +\frac{1}{8}\left(4+e^{-i \omega(N \tau+\tau)}-e^{-i \omega(N \tau-\tau)}-e^{-i \omega(-N \tau+\tau)}+e^{-i \omega(-N \tau-\tau)}\right) S_{B}^{p(1)}(\omega) \\
= & \frac{1}{4}(2-\cos (\omega N \tau+\omega \tau)+\cos (\omega N \tau-\omega \tau)) S_{A}^{x(1)}(\omega) \\
& +\frac{1}{4}(2+\cos (\omega N \tau+\omega \tau)-\cos (\omega N \tau-\omega \tau)) S_{B}^{p(1)}(\omega),
\end{aligned}
$$

where $S_{A}^{x(1)}$ and $S_{B}^{p(1)}$ are power spectra at stage 1 in Fig. 3.9, and corresponds to the squeezing and the anti-squeezing spectrum of the amplitude squeezing sources in mode $A$ and $B$ respectively. Following the same approach for the $\hat{p}$-quadrature and for the quadratures in mode $B$, and using that $2 \mp \cos (\omega N \tau+\omega \tau) \pm \cos (\omega N \tau-\omega \tau)=2 \pm 2 \sin (\omega N \tau) \sin (\omega \tau)$, the power spectra displayed in Fig. 3.14 are expressed as

$$
\begin{aligned}
& S_{A}^{x}(\omega)=\frac{1}{2}(1+\sin (\omega N \tau) \sin (\omega \tau)) S_{A}^{x(1)}(\omega)+\frac{1}{2}(1-\sin (\omega N \tau) \sin (\omega \tau)) S_{B}^{p(1)}(\omega), \\
& S_{A}^{p}(\omega)=\frac{1}{2}(1-\sin (\omega N \tau) \sin (\omega \tau)) S_{B}^{x(1)}(\omega)+\frac{1}{2}(1+\sin (\omega N \tau) \sin (\omega \tau)) S_{A}^{p(1)}(\omega), \\
& S_{B}^{x}(\omega)=\frac{1}{2}(1-\sin (\omega N \tau) \sin (\omega \tau)) S_{A}^{x(1)}(\omega)+\frac{1}{2}(1+\sin (\omega N \tau) \sin (\omega \tau)) S_{B}^{p(1)}(\omega), \\
& S_{B}^{p}(\omega)=\frac{1}{2}(1+\sin (\omega N \tau) \sin (\omega \tau)) S_{B}^{x(1)}(\omega)+\frac{1}{2}(1-\sin (\omega N \tau) \sin (\omega \tau)) S_{A}^{p(1)}(\omega) .
\end{aligned}
$$

Finally, the squeezing spectra $S_{j}^{q(1)}$ from the OPO squeezing sources, squeezed in the amplitude quadrature, are derived in [79] to be

$$
S_{j}^{x(1)}(\omega)=\frac{1}{2}-\frac{2 \varepsilon_{j} \gamma_{j} \eta_{j}}{\left(\gamma_{j}+\varepsilon_{j}\right)^{2}+\omega^{2}} \quad, \quad S_{j}^{p(1)}(\omega)=\frac{1}{2}+\frac{2 \varepsilon_{j} \gamma_{j} \eta_{j}}{\left(\gamma_{j}-\varepsilon_{j}\right)^{2}+\omega^{2}} \quad, j=A, B,
$$

where $\varepsilon_{j}, \gamma_{j}$ and $\eta_{j}$ is the pump rate, total OPO decay rate and squeezing source efficiency, respectively, in mode $j=A, B$.

To include phase fluctuations in the spectra, we should ideally include phase fluctuation in the quadrature transformation at every stage in Fig. 3.9. However, for simplicity, we include all phase fluctuations either before or after the beam-splitter array from stage 2 to 7 . Since the sensitive OPO 
cavities are one of the dominating sources of phase fluctuations, here we include phase fluctuations in the squeezing source, i.e. at stage 1. Assuming the statistics of the phase fluctuations to follow a normal distribution of phase, $\theta$, with the width $\sigma, P(\theta, \sigma)$, the phase fluctuations are included in the squeezing spectrum as

$$
\begin{aligned}
S_{j}^{x(1)}(\omega, \sigma) & =\int P\left(\sigma_{j}, \theta\right)\left(S_{j}^{x(1)}(\omega) \cos ^{2} \theta+S_{j}^{p(1)} \sin ^{2} \theta\right) \mathrm{d} \theta \\
& \approx S_{j}^{x(1)}(\omega) \cos ^{2} \sigma_{j}+S_{j}^{p(1)} \sin ^{2} \sigma_{j} \quad, j=A, B,
\end{aligned}
$$

where the approximation holds for small $\sigma$, and the same for $S_{j}^{p(1)}(\omega, \sigma)$ with cos and sin interchanged.

In Fig. 3.14, we present the fitted power spectra of Eq. (3.21) accounting for phase fluctuations (as in Eq. (3.23)) and electronic noise by including a frequency dependent electronic efficiency determined from a measured electronic power spectrum. The fitting parameters are $\varepsilon_{j}, \gamma_{j}, \eta_{j}$ and $\sigma_{j}(j=A, B)$ and we use $N=12$ and $247 \mathrm{~ns}$. The result of the fitting routine is

$$
\begin{aligned}
\varepsilon_{A} & =2 \pi \times 5.38 \pm 0.02 \mathrm{MHz} & & \varepsilon_{B}=2 \pi \times 5.57 \pm 0.02 \mathrm{MHz} \\
\gamma_{A} & =2 \pi \times 7.59 \pm 0.02 \mathrm{MHz} & & \gamma_{B}=2 \pi \times 7.80 \pm 0.02 \mathrm{MHz} \\
\eta_{A} & =0.789 \pm 0.004 & & \eta_{B}=0.764 \pm 0.004 \\
\sigma_{A} & =5.17 \pm 0.12^{\circ} & \sigma_{B} & =5.90 \pm 0.10^{\circ}
\end{aligned}
$$

where uncertainties are estimated as the $95 \%$ confidence interval. The fit is seen to agree very well with the measured data, and supports $N=12$ with $\tau=247 \mathrm{~ns}$. The fitted $\eta_{A}$ and $\eta_{B}$ differ by 0.025 , which is expected due to $3 \%$ lower escape efficiency of the $\mathrm{OPO}_{\mathrm{B}}$ compared to $\mathrm{OPO}_{\mathrm{A}}$. The fitted OPO decay rates are as expected for the OPO design, while $\mathrm{OPO}_{\mathrm{B}}$ is pumped slightly harder to compensate for the lower escape efficiency. Both OPOs are pumped to around half the threshold $\left(\varepsilon^{2} / \gamma^{2}=0.50\right.$ for $\mathrm{OPO}_{\mathrm{A}}$ and 0.51 for $\left.\mathrm{OPO}_{\mathrm{B}}\right)$. The fitted phase fluctuations, $\sigma_{A}$ and $\sigma_{B}$, are seen to be comparable with the measured phase fluctuations in Fig. 3.13. However, with the model used for the phase fluctuations, $\sigma_{A}$ and $\sigma_{B}$ do not represent the phase fluctuation of the squeezing sources only, but a combination of phase fluctuations throughout the setup, and thus we cannot conclude the squeezing sources to have similar phase fluctuation from this fit. Finally, $\eta_{A}$ and $\eta_{B}$ are not only the efficiency of the squeezing sources, but includes efficiency throughout the setup, and can be compared with the estimated efficiencies in section 3.6.1 of 0.81 and 0.78 in spatial mode $A$ and $B$ respectively. The fitted efficiency is slightly lower than the estimated efficiency, which may be explained by experimental imperfections (e.g. lossy fiber splicing and polarization drift) which are not included in the estimation.

\subsubsection{Temporal mode function}

A temporal mode $k$ is defined by its temporal mode function $f_{k}(t)$. In the experimental setup a quadrature, $\hat{q}(t)$, is continuously measured by homodyne detection, and by integrating the acquired quadrature time trace weighted by the temporal mode function, we obtain the measured quadrature of the corresponding temporal mode,

$$
\hat{q}_{k}=\int f_{k}(t) \hat{q}(t) \mathrm{d} t
$$

Defined by the short delay length, the temporal mode function is restricted to a temporal window of $\tau=247 \mathrm{~ns}$ to avoid temporal overlap with neighbouring modes. However, within this window, the shape of the mode function may be optimized to exploit the squeezing spectrum of limited bandwidth and to avoid low frequencies where technical noise dominate. In this work, inspired by [21], we use an uneven temporal mode function given by

$$
f_{k}(t)= \begin{cases}\mathcal{N}(t-k \tau) e^{-\kappa^{2}(t-k \tau)^{2} / 2} & ,|t-k \tau|<\frac{\tau}{2} \\ 0 & , \text { otherwise }\end{cases}
$$




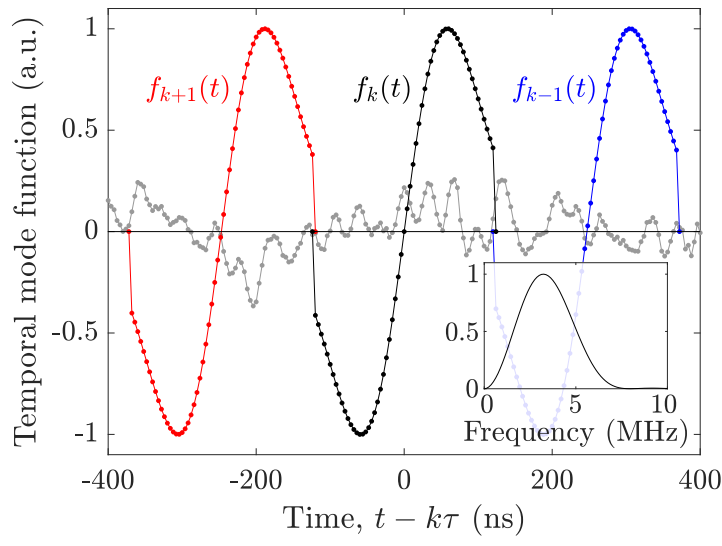

(a)

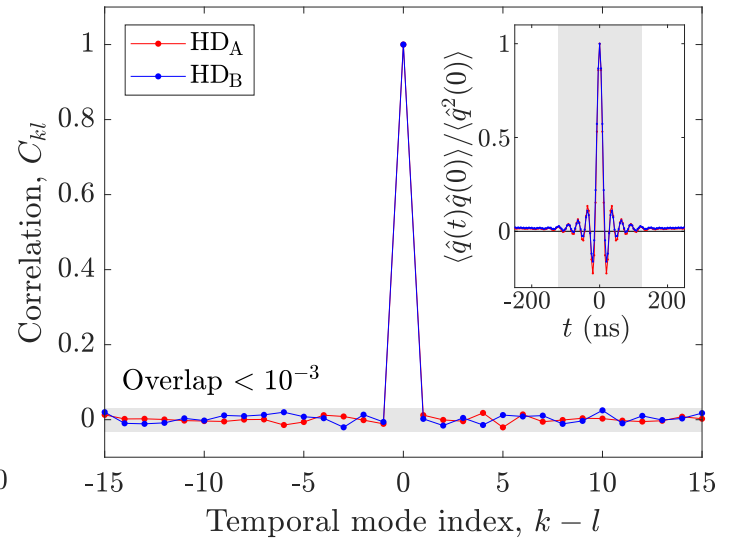

(b)

Figure 3.15: (a) Temporal mode function of three neighbouring modes with the form in Eq. (3.25), together with an acquired quadrature time trace (grey). The insert shows the corresponding spectrum of a temporal mode function. (b) Correlations of neighbouring temporal modes with the mode function in (a). Here, the grey area indicates overlap of less than $10^{-3}$. The insert shows the normalized autocorrelation function with the shaded area indicating the time window of a temporal mode.

where $\mathcal{N}$ is a normalization factor of unit $s^{-1}$, and $\kappa=2 \pi \times 2.7 \mathrm{MHz}$ is optimized to reduce the nullifier variance. Three neighbouring temporal mode functions are shown in Fig. 3.15(a) together with an acquired time trace. The mode function is a product of a Gaussian function and a linear term $t-k \tau$ : The Gaussian function width defines the mode function bandwidth $\kappa$ which should be within the squeezing source bandwidth, $\gamma_{A}, \gamma_{B}$, while the linear term filters out noisy low frequencies. The mode function spectrum is shown in the insert of Fig. 3.15(a).

Even though different temporal mode functions do not overlap in time, neighbouring temporal modes may show some overlap due to electronic filtering in the homodyne detectors and electronic noise which can be correlated across multiple temporal modes. To quantify the mode overlap, we measure correlations between different temporal modes of shot noise and the overlap is defined as this correlation squared,

$$
\text { [Overlap }]=C_{k l}^{2}=\left(\frac{\left\langle\hat{q}_{k} \hat{q}_{l}\right\rangle}{\left\langle\hat{q}_{k}^{2}\right\rangle}\right)^{2} .
$$

In Fig. 3.15(b), correlations between neighbouring modes from a set of 10000 quadrature measurements are shown, indicating mode overlap of less than $10^{-3}$. This low overlap is achieved with the uneven mode function where any offset of the acquired data is cancelled, together with little electronic filtering leading to zero autocorrelation outside the temporal mode function window as shown in the insert of Fig. 3.15(b).

\subsection{Suppl. Inf.: Results}

Two sets of data are acquired: A small set comprising 1500 temporal modes acquired over $371 \mu \mathrm{s}$, and a large set of 15000 modes with an acquisition time of $3.71 \mathrm{~ms}$. Each set includes 10000 time traces measured both in the $\hat{x}$ - and $\hat{p}$-basis for building up quadrature statistics to calculate the variances. The sets are acquired with a sampling rate of $250 \mathrm{MHz}$ in order to have a large resolution and thus large flexibility in optimizing the delay times.

Using the temporal mode functions described in Eq. (3.25), the 10000 quadrature measurements for each temporal mode are extracted from the 10000 time traces and normalized to shot noise. Finally, the nullifiers $\hat{n}_{k}^{x}$ and $\hat{n}_{k}^{p}$ are calculated from the measured quadratures by Eq. (3.13-3.14) 


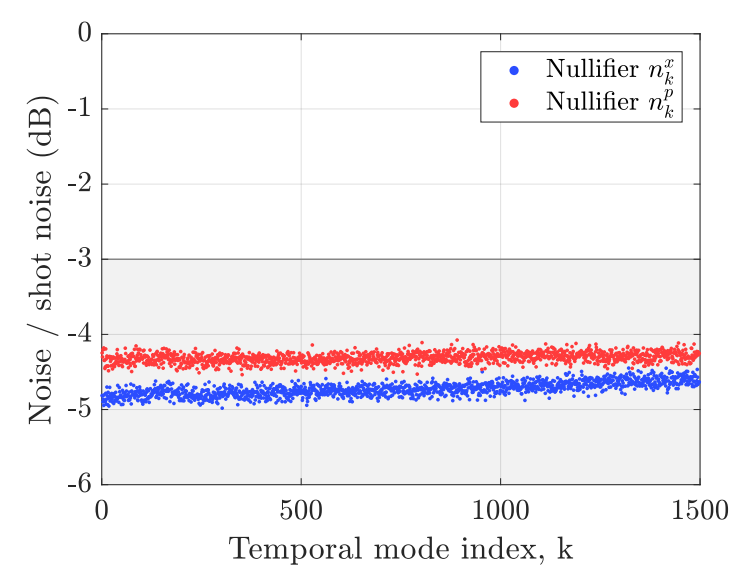

(a)

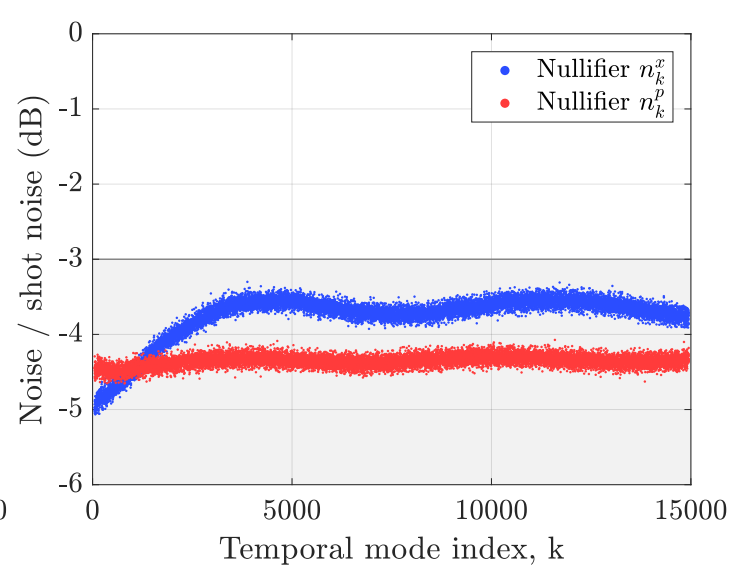

(b)

Figure 3.16: Nullifier variance from (a) a short data set of $371 \mu$ s long time traces, and from (b) a long data setup of $3.71 \mathrm{~ms}$ long time traces. In both figures, the $-3 \mathrm{~dB}$ separability bound, derived in section 3.5 from the van Loock-Furusawa criterion, is marked. With all nullifier variances below this bound, the generated 2D cluster state is completely inseparable.

and the nullifier variance is determined. In Fig. 3.16(a) and (b), the resulting nullifier variances are shown for the short and long data set, respectively.

From the short data set, the average variance of $\hat{n}_{k}^{x}$ and $\hat{n}_{k}^{p}$ is $-4.7 \mathrm{~dB}$ and $-4.3 \mathrm{~dB}$ below shot noise, respectively, while the maximum nullifier squeezing measured (an average of 10 neighbouring nullifiers) is $-4.8 \mathrm{~dB}$ and $-4.4 \mathrm{~dB}$ respectively. All measured nullifiers show a variance below the $-3 \mathrm{~dB}$ separability bound derived in section 3.5, and we conclude that the generated $2 \mathrm{D}$ cluster state is completely inseparable. For completeness, we plot in Fig. 3.17, page 57, the combined variances $\left\langle\Delta \hat{X}^{2}\right\rangle+\left\langle\Delta \hat{P}^{2}\right\rangle$ from the van Loock-Furusawa criterion, Eq. (3.18), for the specific choice of nullifier combinations $\hat{X}$ and $\hat{P}$ used in each of the 127 bipartitions. For all cases, the variances are below the right-hand side of Eq. (3.18), as indicated by the gray areas, hence explicitly demonstrating the inseparability.

In an attempt to reach a point where the generated cluster state does not violate the $-3 \mathrm{~dB}$ separability bound due to phase drift when the feedback of cavity and phase locks are kept constant during hold-time, the large data set was acquired. As expected, the nullifier variance increase with time, but even after 15000 temporal modes $(3.71 \mathrm{~ms})$ the phase is stable enough to stay below the separability bound, and we conclude that also the generated $2 \times 15000=30000$ mode $(2$ spatial modes) 2D cluster state is completely inseparable, while we expect that even larger cluster states may be generated before reaching the separability bound. In the large data set, the average nullifier squeezing of $\hat{n}_{k}^{x}$ and $\hat{n}_{k}^{p}$ are $-3.8 \mathrm{~dB}$ and $-4.4 \mathrm{~dB}$ below shot noise, respectively, while up to (an average of 10 neighbouring nullifiers) $-5.0 \mathrm{~dB}$ and $-4.5 \mathrm{~dB}$ of squeezing are measured, respectively.

The periodic variation observed in the nullifier variance in Fig. 3.16(b) is explained by the systematic phase drift from the OPO cavities as discussed in section 3.6.1. We observe a rapid increase of the variance associated with $\hat{n}_{k}^{x}$ which may be explained by phase fluctuations of one of the squeezing sources: From Eq. (3.12) it can be seen that when measuring in the $\hat{x}$-basis, we measure squeezing from the squeezing source in the spatial mode $A$ and anti-squeezing from the the spatial mode $B$ (whereas when measuring in the $\hat{p}$-basis, squeezing and anti-squeezing from the the spatial modes $B$ and $A$ are measured, respectively). When calculating the nullifiers, the antisqueezing cancels, and we are left with squeezing from one of the two squeezing sources. However, when phases from the squeezing sources drifts, anti-squeezing is mixed into the otherwise squeezed quadrature, and since $\hat{n}_{k}^{x}$ only includes squeezing from spatial mode $A$ (and $\hat{n}_{k}^{p}$ from spatial mode $B$ ), we suspect the large relative probe phase fluctuation seen in Fig. 3.13 to be mainly caused by phase drift from the OPO cavity in mode $A$, leading to a rapid increase of $\left\langle\Delta \hat{n}_{k}^{x 2}\right\rangle$ but not $\left\langle\Delta \hat{n}_{k}^{p 2}\right\rangle$. 
Hence, we expect $\mathrm{OPO}_{\mathrm{A}}$ to be the dominant source of phase fluctuations that contaminates the measured nullifiers, and not the $606 \mathrm{~m}$ long fiber delay since we would expect this to affect both $\hat{n}_{k}^{x}$ and $\hat{n}_{k}^{p}$. Thus, the setup stability may be improved simply by keeping the feedback to cavity locks active at all times. Unfortunately, this was not possible with the current version of the experimental setup, as the cavity lock beams were chopped together with the probe beams in the sample-hold locking scheme described in the methods section 3.3. 
Table 3.1: In the table below, modes of the studied unit cell in the van Loock-Furusawa criterion discussed in the supplementary information section 3.5 are numbered as indicated in Fig. 3.12. Every bipartition is systematically given an ID between 1 and 127: Consider the 8 bit long binary form of this ID with the least significant bit to the left (e.g. ID $=3=\left[\begin{array}{lllll}1 & 1000 & 0 & 0 & 0\end{array}\right]_{\text {binary }}$ ). We then let $\mathcal{S}_{1}$ include modes with mode number equal to the bit number of bits equal 1 in this binary form of the ID (e.g. ID $=3 \Rightarrow \mathcal{S}_{1}=\{1,2\}$ and thus $\mathcal{S}_{2}=\{3,4,5,6,7,8\}$ ). The table includes Var., the combined variance $\left\langle\Delta \hat{X}^{2}\right\rangle+\left\langle\Delta \hat{P}^{2}\right\rangle ; f$, the right hand side of Eq. (3.18); and Sq., the required variance squeezing of each nullifier below shot noise to violate Eq. (3.18) with the listed choice of $\hat{X}$ and $\hat{P}$. For clarity, the resulting combined variance $\left\langle\Delta \hat{X}^{2}\right\rangle+\left\langle\Delta \hat{P}^{2}\right\rangle$ from acquired data is plotted and compared with $f$ for each bipartition in Fig. 3.17, page 57. Note that the squeezing levels listed here are not necessarily the lowest squeezings required to show inseparability for the given bipartition, and for each bipartition a better choice of $\hat{X}$ and $\hat{P}$ may exist which lowers the necessary squeezing. (Table ends on page 56)

\begin{tabular}{|c|c|c|c|c|c|c|}
\hline ID & $\mathcal{S}_{1}$ & $\hat{X}$ & $\hat{P}$ & Var. & $f$ & Sq. \\
\hline 1 & 1 & $-\hat{n}_{k}^{x}+\hat{n}_{k-1}^{x}$ & $-\hat{n}_{k}^{p}+\hat{n}_{k-N}^{p}$ & $16 e^{-2 r}$ & 8 & $3 \mathrm{~dB}$ \\
\hline 2 & 2 & $-\hat{n}_{k}^{x}+\hat{n}_{k-1}^{x-1}$ & $\hat{n}_{k}^{p}+\hat{n}_{k-N}^{p}$ & $16 e^{-2 r}$ & 8 & $3 \mathrm{~dB}$ \\
\hline 3 & 1,2 & $\hat{n}_{k}^{x}{ }^{k-1}$ & $\hat{n}_{k}^{p}{ }^{k-N}$ & $8 e^{-2 r}$ & 4 & $3 \mathrm{~dB}$ \\
\hline 4 & 3 & $-\hat{n}_{k}^{x}+\hat{n}_{k+1}^{x}$ & $\hat{n}_{k}^{p}+\hat{n}_{k-N}^{p}$ & $16 e^{-2 r}$ & 8 & $3 \mathrm{~dB}$ \\
\hline 5 & 1,3 & $\hat{n}_{k}^{x}{ }^{k+1}$ & ${ }^{k} \hat{n}_{k-N}^{p}{ }^{k-N}$ & $8 e^{-2 r}$ & 4 & $3 \mathrm{~dB}$ \\
\hline 6 & 2,3 & $-\hat{n}_{k+1}^{x}+\hat{n}_{k-1}^{x}$ & $\begin{array}{l}\hat{n}_{k}^{p}+\hat{n}_{k-N}^{p} \\
\text { pon }\end{array}$ & $16 e^{-2 r}$ & 8 & $3 \mathrm{~dB}$ \\
\hline 7 & $1,2,3$ & $\hat{n}_{k}^{x+1}$ & $\hat{n}_{k-1}^{p}$ & $8 e^{-2 r}$ & 4 & $3 \mathrm{~dB}$ \\
\hline 8 & 4 & $-\hat{n}_{k}^{x}+\hat{n}_{k+1}^{x}$ & $\begin{array}{l}-\hat{n}_{k}^{p}+\hat{n}_{k-N}^{p}\end{array}$ & $16 e^{-2 r}$ & 8 & $3 \mathrm{~dB}$ \\
\hline 9 & 1,4 & $-\hat{n}_{k+1}^{x}+\hat{n}_{k-1}^{x+1}$ & $-\hat{n}_{k}^{p}+\hat{n}_{k-N}^{p-N}$ & $16 e^{-2 r}$ & 8 & $3 \mathrm{~dB}$ \\
\hline 10 & 2,4 & $\hat{n}_{k}^{x+1}$ & $\hat{n}_{k-N}^{p}$ & $8 e^{-2 r}$ & 4 & $3 \mathrm{~dB}$ \\
\hline 11 & $1,2,4$ & $\hat{n}_{k}^{x}$ & $\begin{array}{l}\hat{n}_{k-1}^{p-N} \\
\hat{n}_{k-1}^{p}\end{array}$ & $8 e^{-2 r}$ & 4 & $3 \mathrm{~dB}$ \\
\hline 12 & 3,4 & $\hat{n}_{k}^{x}$ & $\hat{n}_{k}^{p-1}$ & $8 e^{-2 r}$ & 4 & $3 \mathrm{~dB}$ \\
\hline 13 & $1,3,4$ & $\hat{n}_{k}^{x}$ & $\hat{n}_{k+1}^{p^{k}}$ & $8 e^{-2 r}$ & 4 & $3 \mathrm{~dB}$ \\
\hline 14 & $2,3,4$ & $\hat{n}_{k}^{x}$ & $\hat{n}_{k+1}^{p+1}$ & $8 e^{-2 r}$ & 4 & $3 \mathrm{~dB}$ \\
\hline 15 & $1,2,3,4$ & $\hat{n}_{k}^{x}$ & $\hat{n}_{k+1}^{p+1}$ & $8 e^{-2 r}$ & 4 & $3 \mathrm{~dB}$ \\
\hline 16 & 5 & $\hat{n}_{k}^{x}+\hat{n}_{k-1}^{x}$ & $\begin{array}{l}-\hat{n}_{k}^{p}+\hat{n}_{k+N}^{p} \\
\text { knt }\end{array}$ & $16 e^{-2 r}$ & 8 & $3 \mathrm{~dB}$ \\
\hline 17 & 1,5 & $\hat{n}_{k}^{x-1}$ & $\hat{n}_{k}^{p+N}$ & $8 e^{-2 r}$ & 4 & $3 \mathrm{~dB}$ \\
\hline 18 & 2,5 & $\hat{n}_{k}^{\kappa}$ & $\hat{n}_{k}^{p}$ & $8 e^{-2 r}$ & 4 & $3 \mathrm{~dB}$ \\
\hline 19 & $1,2,5$ & $\hat{n}_{k}^{x}$ & $\hat{n}_{k}^{p}$ & $8 e^{-2 r}$ & 6 & $1.2 \mathrm{~dB}$ \\
\hline 20 & 3,5 & $-\hat{n}_{k}^{x}+\hat{n}_{k+N}^{x}$ & $\hat{n}_{k-1}^{p}+\hat{n}_{k+N}^{p}+\hat{n}_{k+N+1}^{p}+\hat{n}_{k+N-1}^{p}$ & $24 e^{-2 r}$ & 12 & $3 \mathrm{~dB}$ \\
\hline 21 & $1,3,5$ & $\hat{n}_{k}^{x}$ & $\begin{array}{cccc}\kappa-1 & \kappa+N & \hat{n}_{k-N}^{p+N+1} & \kappa+N-1 \\
& & \end{array}$ & $8 e^{-2 r}$ & 4 & $3 \mathrm{~dB}$ \\
\hline 22 & $2,3,5$ & $-\hat{n}_{k}^{x}+\hat{n}_{k+N}^{x}$ & $-\hat{n}_{k}^{p}+\hat{n}_{k+N}^{p}+\hat{n}_{k+N+1}^{p}+\hat{n}_{k+N-1}^{p}$ & $24 e^{-2 r}$ & 12 & $3 \mathrm{~dB}$ \\
\hline 23 & $1,2,3,5$ & $\hat{n}_{k}^{x}$ & $\begin{array}{llll}\kappa & \kappa+N & \hat{n}_{k}^{p+N+1} & \kappa+N-1 \\
& & \end{array}$ & $8 e^{-2 r}$ & 4 & $3 \mathrm{~dB}$ \\
\hline 24 & 4,5 & $-\hat{n}_{k}^{x}+\hat{n}_{k+N}^{x}$ & $\hat{n}_{k-1}^{p}+\hat{n}_{k+N}^{p}+\hat{n}_{k+N+1}^{p}+\hat{n}_{k+N-1}^{p}$ & $24 e^{-2 r}$ & 12 & $3 \mathrm{~dB}$ \\
\hline 25 & $1,4,5$ & $-\hat{n}_{k}^{x}+\hat{n}_{k+N}^{x+N}$ & $-\hat{n}_{k}^{p}+\hat{n}_{k+N}^{p+N}+\hat{n}_{k+N+1}^{p+N+1}+\hat{n}_{k+N-1}^{p+N-1}$ & $24 e^{-2 r}$ & 12 & $3 \mathrm{~dB}$ \\
\hline 26 & $2,4,5$ & $\hat{n}_{k}^{x}$ & $\begin{array}{ll}\kappa & \kappa+N \\
\hat{n}_{k-N}^{p+N+1} & \kappa+N-1\end{array}$ & $8 e^{-2 r}$ & 4 & $3 \mathrm{~dB}$ \\
\hline 27 & $1,2,4,5$ & $\hat{n}_{k}^{x}$ & $\hat{n}_{b}^{p^{p}}$ & $8 e^{-2 r}$ & 4 & $3 \mathrm{~dB}$ \\
\hline 28 & $3,4,5$ & $\hat{n}_{k}^{x}$ & $\hat{n}_{k+1}^{p^{k}}$ & $8 e^{-2 r}$ & 4 & $3 \mathrm{~dB}$ \\
\hline 29 & $1,3,4,5$ & $\hat{n}_{k}^{x}$ & $\hat{n}_{k+1}^{p+1}$ & $8 e^{-2 r}$ & 4 & $3 \mathrm{~dB}$ \\
\hline 30 & $2,3,4,5$ & $\hat{n}_{k}^{x}$ & $\hat{n}_{k+1}^{p+1}$ & $8 e^{-2 r}$ & 4 & $3 \mathrm{~dB}$ \\
\hline 31 & $1,2,3,4,5$ & $\hat{n}_{k}^{x}$ & $\hat{n}_{k+1}^{p+1}$ & $8 e^{-2 r}$ & 4 & $3 \mathrm{~dB}$ \\
\hline 32 & 6 & $\hat{n}_{k}^{x}+\hat{n}_{k-1}^{x}$ & $\hat{n}_{k}^{p}+\hat{n}_{k+N}^{p}$ & $16 e^{-2 r}$ & 8 & $3 \mathrm{~dB}$ \\
\hline 33 & 1,6 & $\hat{n}_{k}^{x-1}$ & $\hat{n}_{k}^{p+N}$ & $8 e^{-2 r}$ & 4 & $3 \mathrm{~dB}$ \\
\hline 34 & 2,6 & $\hat{n}_{k}^{x}$ & $\hat{n}_{k}^{p}$ & $8 e^{-2 r}$ & 4 & $3 \mathrm{~dB}$ \\
\hline 35 & $1,2,6$ & $\hat{n}_{k}^{x}$ & $\hat{n}_{k}^{p}$ & $8 e^{-2 r}$ & 6 & $1.2 \mathrm{~dB}$ \\
\hline 36 & 3,6 & $\hat{n}_{k}^{x}+\hat{n}_{k+N}^{x}$ & $-\hat{n}_{k-1}^{p}+\hat{n}_{k+N}^{p}+\hat{n}_{k+N+1}^{p}+\hat{n}_{k+N-1}^{p}$ & $24 e^{-2 r}$ & 12 & $3 \mathrm{~dB}$ \\
\hline 37 & $1,3,6$ & $\hat{n}_{k}^{x}$ & $\begin{array}{ccc}k-1 & k+N \\
\hat{n}_{k-N}^{p} k+N+1 & k+N-1\end{array}$ & $8 e^{-2 r}$ & 4 & $3 \mathrm{~dB}$ \\
\hline 38 & $2,3,6$ & $\hat{n}_{k}^{x}+\hat{n}_{k+N}^{x}$ & $\hat{n}_{k}^{p}+\hat{n}_{k+N}^{p}+\hat{n}_{k+N+1}^{k-N}+\hat{n}_{k+N-1}^{p}$ & $24 e^{-2 r}$ & 12 & $3 \mathrm{~dB}$ \\
\hline 39 & $1,2,3,6$ & $\hat{n}_{k}^{x+N}$ & & $8 e^{-2 r}$ & 4 & $3 \mathrm{~dB}$ \\
\hline 40 & 4,6 & $\hat{n}_{k}^{x}+\hat{n}_{k+N}^{x}$ & $-\hat{n}_{k-1}^{p}+\hat{n}_{k+N}^{p}+\hat{n}_{k+N+1}^{p}+\hat{n}_{k+N-1}^{p}$ & $24 e^{-2 r}$ & 12 & $3 \mathrm{~dB}$ \\
\hline 41 & $1,4,6$ & $\hat{n}_{k}^{x}+\hat{n}_{k+N}^{x+N}$ & $\hat{n}_{k}^{p}+\hat{n}_{k+N}^{p}+\hat{n}_{k+N+1}^{p}+\hat{n}_{k+N-1}^{p}$ & $24 e^{-2 r}$ & 12 & $3 \mathrm{~dB}$ \\
\hline 42 & $2,4,6$ & $\hat{n}_{k}^{x+N}$ & 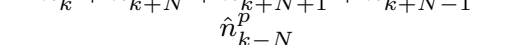 & $8 e^{-2 r}$ & 4 & $3 \mathrm{~dB}$ \\
\hline 43 & $1,2,4,6$ & $\hat{n}_{k}^{x}$ & $\hat{n}_{k}^{p}$ & $8 e^{-2 r}$ & 4 & $3 \mathrm{~dB}$ \\
\hline 44 & $3,4,6$ & $\hat{n}_{k}^{x}$ & $\hat{n}_{k+1}^{p^{k}}$ & $8 e^{-2 r}$ & 4 & $3 \mathrm{~dB}$ \\
\hline 45 & $1,3,4,6$ & $\hat{n}_{k}^{x}$ & $\hat{n}_{k+1}^{p+1}$ & $8 e^{-2 r}$ & 4 & $3 \mathrm{~dB}$ \\
\hline 46 & $2,3,4,6$ & $\hat{n}_{k}^{x}$ & $\hat{n}_{k+1}^{p+1}$ & $8 e^{-2 r}$ & 4 & $3 \mathrm{~dB}$ \\
\hline 47 & $1,2,3,4,6$ & $\hat{n}_{k}^{x}$ & $\hat{n}_{k+1}^{p+1}$ & $8 e^{-2 r}$ & 4 & $3 \mathrm{~dB}$ \\
\hline
\end{tabular}




\begin{tabular}{|c|c|c|c|c|c|}
\hline 5,6 & $\hat{n}_{k}^{x}$ & $\hat{n}_{k}^{p}$ & $8 e^{-2 r}$ & 4 & $3 \mathrm{~dB}$ \\
\hline $1,5,6$ & $\hat{n}_{k}^{x}$ & $\hat{n}_{k}^{p}$ & $8 e^{-2 r}$ & 6 & $1.2 \mathrm{~dB}$ \\
\hline $2,5,6$ & $\hat{n}_{k}^{x}$ & $\hat{n}_{k}^{p}$ & $8 e^{-2 r}$ & 6 & $1.2 \mathrm{~dB}$ \\
\hline $1,2,5,6$ & $\hat{n}_{k}^{x}$ & $\hat{n}_{k}^{p}$ & $8 e^{-2 r}$ & 8 & $0 \mathrm{~dB}$ \\
\hline $3,5,6$ & $\hat{n}_{k}^{x}$ & $\hat{n}_{k-1}^{p^{\kappa}}$ & $8 e^{-2 r}$ & 4 & $3 \mathrm{~dB}$ \\
\hline $1,3,5,6$ & $\hat{n}_{k}^{x}$ & $\hat{n}_{k}^{p}$ & $8 e^{-2 r}$ & 4 & $3 \mathrm{~dB}$ \\
\hline $2,3,5,6$ & $\hat{n}_{k}^{\kappa}$ & $\hat{n}_{k}^{\mathcal{F}}$ & $8 e^{-2 r}$ & 4 & $3 \mathrm{~dB}$ \\
\hline $1,2,3,5,6$ & $\hat{n}_{k}^{x}$ & $\hat{n}_{k}^{\tilde{p}}$ & $8 e^{-2 r}$ & 6 & $1.2 \mathrm{~dB}$ \\
\hline $4,5,6$ & $\hat{n}_{k}^{x}$ & $\hat{n}_{k-1}^{p^{k}}$ & $8 e^{-2 r}$ & 4 & $3 \mathrm{~dB}$ \\
\hline $1,4,5,6$ & $\hat{n}_{k}^{x}$ & $\hat{n}_{k}^{p}$ & $8 e^{-2 r}$ & 4 & $3 \mathrm{~dB}$ \\
\hline $2,4,5,6$ & $\hat{n}_{k}^{x}$ & $\hat{n}_{k}^{p}$ & $8 e^{-2 r}$ & 4 & $3 \mathrm{~dB}$ \\
\hline $1,2,4,5,6$ & $\hat{n}_{k}^{x}$ & $\hat{n}_{k}^{p}$ & $8 e^{-2 r}$ & 6 & $1.2 \mathrm{~dB}$ \\
\hline $3,4,5,6$ & $\hat{n}_{k}^{x}$ & $\hat{n}_{k+1}^{p^{k}}$ & $8 e^{-2 r}$ & 4 & $3 \mathrm{~dB}$ \\
\hline $1,3,4,5,6$ & $\hat{n}_{k}^{x}$ & $\hat{n}_{k+1}^{p+1}$ & $8 e^{-2 r}$ & 4 & $3 \mathrm{~dB}$ \\
\hline $2,3,4,5,6$ & $\hat{n}_{k}^{x}$ & $\hat{n}_{k+}^{k^{p+}}$ & $8 e^{-2 r}$ & 4 & $3 \mathrm{~dB}$ \\
\hline $1,2,3,4,5,6$ & $\hat{n}_{k}^{x}$ & & $8 e^{-2 r}$ & 4 & $3 \mathrm{~dB}$ \\
\hline 7 & $\hat{n}_{k}^{x}+\hat{n}_{k+1}^{x}$ & $\hat{n}_{k}^{p}+\hat{n}_{k+N}^{p}$ & $16 e^{-2 r}$ & 8 & $3 \mathrm{~dB}$ \\
\hline 1,7 & $\hat{n}_{k}^{x}+\hat{n}_{k+N}^{x}$ & $-\hat{n}_{k+1}^{p}+\hat{n}_{k+N}^{p}+\hat{n}_{k+N+1}^{p}+\hat{n}_{k+N-1}^{p}$ & $24 e^{-2 r}$ & 12 & $3 \mathrm{~dB}$ \\
\hline 2,7 & $\hat{n}_{k}^{x}+\hat{n}_{k+N}^{x+N}$ & $-\hat{n}_{k+1}^{\hat{p}^{p+1}}+\hat{n}_{k+N}^{p+1}+\hat{n}_{k+N+1}^{p+1}+\hat{n}_{k+N-1}^{p+1}$ & $24 e^{-2 r}$ & 12 & $3 \mathrm{~dB}$ \\
\hline $1,2,7$ & $\hat{n}_{k}^{x}$ & $\hat{n}_{k-1}^{p}$ & $8 e^{-2 r}$ & 4 & $3 \mathrm{~dB}$ \\
\hline 3,7 & $\hat{n}_{k}^{x}$ & $\hat{n}_{k}^{\bar{p}}$ & $8 e^{-2 r}$ & 4 & $3 \mathrm{~dB}$ \\
\hline $1,3,7$ & $\hat{n}_{k}^{x}$ & $\hat{n}_{k}^{p}{ }^{n}$ & $8 e^{-2 r}$ & 4 & $3 \mathrm{~dB}$ \\
\hline $2,3,7$ & $\hat{n}_{k}^{x}+\hat{n}_{k+N}^{x}$ & $\hat{n}_{k}^{p}+\hat{n}_{k+N}^{p}+\hat{n}_{k+N+1}^{p}+\hat{n}_{k+N-1}^{p}$ & $24 e^{-2 r}$ & 12 & $3 \mathrm{~dB}$ \\
\hline $1,2,3,7$ & $\hat{n}_{k}^{x+1 v}$ & $\hat{n}_{k-1}^{p}$ & $8 e^{-2 r}$ & 4 & $3 \mathrm{~dB}$ \\
\hline 4,7 & $\hat{n}_{k}^{x}$ & & $8 e^{-2 r}$ & 4 & $3 \mathrm{~dB}$ \\
\hline $1,4,7$ & $\hat{n}_{k}^{x}+\hat{n}_{k+N}^{x}$ & $\hat{n}_{k}^{p}+\hat{n}_{k+N}^{p}+\hat{n}_{k+N+1}^{p^{k}}+\hat{n}_{k+N-1}^{p}$ & $24 e^{-2 r}$ & 12 & $3 \mathrm{~dB}$ \\
\hline $2,4,7$ & $\hat{n}_{k}^{x}$ & $\hat{n}_{k-N}^{p}$ & $8 e^{-2 r}$ & 4 & $3 \mathrm{~dB}$ \\
\hline $1,2,4,7$ & $\hat{n}_{k}^{x}$ & $\hat{n}_{k-1}^{p-N}$ & $8 e^{-2 r}$ & 4 & $3 \mathrm{~dB}$ \\
\hline $3,4,7$ & $\hat{n}_{k}^{x}$ & $\hat{n}_{k}^{\kappa}$ & $8 e^{-2 r}$ & 6 & $1.2 \mathrm{~dB}$ \\
\hline $1,3,4,7$ & $\hat{n}_{k}^{x}$ & $\hat{n}_{k}^{p}$ & $8 e^{-2 r}$ & 4 & $3 \mathrm{~dB}$ \\
\hline $2,3,4,7$ & $\hat{n}_{k}^{x}$ & $\hat{n}_{k}^{\hat{P}}$ & $8 e^{-2 r}$ & 4 & $3 \mathrm{~dB}$ \\
\hline $1,2,3,4,7$ & $\hat{n}_{k}^{x}$ & $\hat{n}_{k-1}^{p^{k}}$ & $8 e^{-2 r}$ & 4 & $3 \mathrm{~dB}$ \\
\hline 5,7 & $\hat{n}_{k}^{x}$ & $\hat{n}_{k+N}^{p-1}$ & $8 e^{-2 r}$ & 4 & $3 \mathrm{~dB}$ \\
\hline $1,5,7$ & $\hat{n}_{k}^{x}$ & $\hat{n}_{k+}^{p+}$ & $8 e^{-2 r}$ & 4 & $3 \mathrm{~dB}$ \\
\hline $2,5,7$ & $\hat{n}_{k}^{x}$ & $\hat{n}_{k+}^{p+}$ & $8 e^{-2 r}$ & 4 & $3 \mathrm{~dB}$ \\
\hline $1,2,5,7$ & $\hat{n}_{k}^{x}$ & & $8 e^{-2 r}$ & 4 & $3 \mathrm{~dB}$ \\
\hline $3,5,7$ & $\hat{n}_{k}^{x}$ & $\hat{n}_{k+N}^{p}$ & $8 e^{-2 r}$ & 4 & $3 \mathrm{~dB}$ \\
\hline $1,3,5,7$ & $\hat{n}_{k}^{x}$ & $\hat{n}_{k+N}^{p}$ & $8 e^{-2 r}$ & 4 & $3 \mathrm{~dB}$ \\
\hline $2,3,5,7$ & $\hat{n}_{k}^{x}$ & $\hat{n}_{k+N}^{p}$ & $8 e^{-2 r}$ & 4 & $3 \mathrm{~dB}$ \\
\hline $1,2,3,5,7$ & $\hat{n}_{k}^{x}$ & $\hat{n}_{k-}^{p-}$ & $8 e^{-2 r}$ & 4 & $3 \mathrm{~dB}$ \\
\hline $4,5,7$ & $\hat{n}_{k}^{x}$ & $\hat{n}_{k+}^{p-}$ & $8 e^{-2 r}$ & 4 & $3 \mathrm{~dB}$ \\
\hline $1,4,5,7$ & $\hat{n}_{k}^{x}$ & $\hat{n}_{k+}^{p+}$ & $8 e^{-2 r}$ & 4 & $3 \mathrm{~dB}$ \\
\hline $2,4,5,7$ & $\hat{n}_{k}^{x}$ & $\hat{n}_{k+}^{p+}$ & $8 e^{-2 r}$ & 4 & $3 \mathrm{~dB}$ \\
\hline $1,2,4,5,7$ & $\hat{n}_{k}^{x}$ & & $8 e^{-2 r}$ & 4 & $3 \mathrm{~dB}$ \\
\hline $3,4,5,7$ & $\hat{n}_{k}^{x}$ & & $8 e^{-2 r}$ & 4 & $3 \mathrm{~dB}$ \\
\hline $1,3,4,5,7$ & $\hat{n}_{k}^{x}$ & $\hat{n}_{k+}^{p k}$ & $8 e^{-2 r}$ & 4 & $3 \mathrm{~dB}$ \\
\hline $2,3,4,5,7$ & $\hat{n}_{k}^{x}$ & $\hat{n}_{k+}^{p+}$ & $8 e^{-2 r}$ & 4 & $3 \mathrm{~dB}$ \\
\hline $1,2,3,4,5,7$ & $\hat{n}_{k}^{x}$ & $\hat{n}_{k+N}^{p+N}$ & $8 e^{-2 r}$ & 4 & $3 \mathrm{~dB}$ \\
\hline 6,7 & $-\hat{n}_{k+1}^{x}+\hat{n}_{k-1}^{x}$ & $\hat{n}_{k}^{p}+\hat{n}_{k+N}^{p}$ & $16 e^{-2 r}$ & 8 & $3 \mathrm{~dB}$ \\
\hline $1,6,7$ & $-\hat{n}_{k}^{x+1}+\hat{n}_{k-N}^{x}$ & $\hat{n}_{k}^{p}-\hat{n}_{k-N}^{p}+\hat{n}_{k-N+1}^{p}+\hat{n}_{k-N-1}^{p}$ & $24 e^{-2 r}$ & 12 & $3 \mathrm{~dB}$ \\
\hline $2,6,7$ & $\hat{n}_{k}^{x}+\hat{n}_{k-N}^{x}$ & $-\hat{n}_{k}^{p}-\hat{n}_{k-N}^{p}+\hat{n}_{k-N+1}^{p-N+1}+\hat{n}_{k-N-1}^{p-N-1}$ & $24 e^{-2 r}$ & 12 & $3 \mathrm{~dB}$ \\
\hline $1,2,6,7$ & $\hat{n}_{k}^{x-N}$ & & $8 e^{-2 r}$ & 4 & $3 \mathrm{~dB}$ \\
\hline $3,6,7$ & $\hat{n}_{k}^{x}+\hat{n}_{k-N}^{x}$ & $-\hat{n}_{k}^{p}-\hat{n}_{k-N}^{p}+\hat{n}_{k-N+1}^{k^{k}}+\hat{n}_{k-N-1}^{p}$ & $24 e^{-2 r}$ & 12 & $3 \mathrm{~dB}$ \\
\hline $1,3,6,7$ & $\hat{n}_{k}^{x}$ & $\begin{array}{cl}\kappa & \kappa-N \\
\hat{n}_{k-N}^{p-N+1} & \kappa-N-1\end{array}$ & $8 e^{-2 r}$ & 4 & $3 \mathrm{~dB}$ \\
\hline $2,3,6,7$ & $-\hat{n}_{k+1}^{x}+\hat{n}_{k-1}^{x}$ & $-\hat{n}_{k+N}^{p}+\hat{n}_{k-N}^{p}$ & $16 e^{-2 r}$ & 8 & $3 \mathrm{~dB}$ \\
\hline $1,2,3,6,7$ & $-\hat{n}_{k}^{x+1}+\hat{n}_{k-N}^{x}$ & $\hat{n}_{k}^{p}-\hat{n}_{k-N}^{p}+\hat{n}_{k-N+1}^{p}+\hat{n}_{k-N-1}^{p}$ & $24 e^{-2 r}$ & 12 & $3 \mathrm{~dB}$ \\
\hline $4,6,7$ & $-\hat{n}_{k}^{x}+\hat{n}_{k-N}^{x-N}$ & $\hat{n}_{k}^{p}-\hat{n}_{k-N}^{p}+\hat{n}_{k-N+1}^{p}+\hat{n}_{k-N-1}^{p}$ & $24 e^{-2 r}$ & 12 & $3 \mathrm{~dB}$ \\
\hline $1,4,6,7$ & $-\hat{n}_{k+1}^{x}+\hat{n}_{k-1}^{x}$ & $\kappa \quad \hat{n}_{k+N}^{p}+\hat{n}_{k-N}^{p+1}$ & $16 e^{-2 r}$ & 8 & $3 \mathrm{~dB}$ \\
\hline $2,4,6,7$ & $\hat{n}_{k}^{x+1}$ & $\kappa+N p \quad k-N$ & $8 e^{-2 r}$ & 4 & $3 \mathrm{~dB}$ \\
\hline $1,2,4,6,7$ & $\hat{n}_{k}^{x}+\hat{n}_{k-N}^{x}$ & $-\hat{n}_{k}^{p}-\hat{n}_{k-N}^{p}+\hat{n}_{k-N+1}^{p}+\hat{n}_{k-N-1}^{p}$ & $24 e^{-2 r}$ & 12 & $3 \mathrm{~dB}$ \\
\hline $3,4,6,7$ & $\begin{array}{c}\hat{n}_{k}^{x} \\
\hat{n}^{x}+\hat{n}^{x}\end{array}$ & $\begin{array}{c}\hat{n}_{k}^{p} \\
-\hat{n}_{l}^{p}-\hat{n}_{l}^{p}+\hat{n}_{l}^{p}\end{array}+\hat{n}_{l}^{p}$ & $\begin{array}{c}8 e^{-2 r} \\
24 e^{-2 r}\end{array}$ & $\begin{array}{c}4 \\
12\end{array}$ & $\begin{array}{l}3 \mathrm{~dB} \\
3 \mathrm{~dB}\end{array}$ \\
\hline $2,3,4,6,7$ & & $\begin{array}{c}-\hat{n}_{k}^{p}-\hat{n}_{k-N}^{p}+\hat{n}_{k-N+1}^{p}+\hat{n}_{k-N-1}^{p} \\
\hat{n}_{k}^{p}-\hat{n}_{l}^{p}+\hat{n}_{b}^{p}\end{array}$ & $24 e^{-2 r}$ & 12 & $3 \mathrm{~dB}$ \\
\hline
\end{tabular}




\begin{tabular}{|c|c|c|c|c|c|c|}
\hline 111 & $1,2,3,4,6,7$ & $-\hat{n}_{k+1}^{x}+\hat{n}_{k-1}^{x}$ & $-\hat{n}_{k}^{p}+\hat{n}_{k+N}^{p}$ & $16 e^{-2 r}$ & 8 & $3 \mathrm{~dB}$ \\
\hline 112 & $5,6,7$ & $\hat{n}_{k}^{x}$ & $\hat{n}_{k-1}^{p}$ & $8 e^{-2 r}$ & 4 & $3 \mathrm{~dB}$ \\
\hline 113 & $1,5,6,7$ & $\hat{n}_{k}^{x}$ & $\hat{n}_{k}^{p}$ & $8 e^{-2 r}$ & 4 & $3 \mathrm{~dB}$ \\
\hline 114 & $2,5,6,7$ & $\hat{n}_{k}^{x}$ & $\hat{n}_{k}^{p}$ & $8 e^{-2 r}$ & 4 & $3 \mathrm{~dB}$ \\
\hline 115 & $1,2,5,6,7$ & $\hat{n}_{k}^{x}$ & $\hat{n}_{k}^{\hat{p}}$ & $8 e^{-2 r}$ & 6 & $1.2 \mathrm{~dB}$ \\
\hline 116 & $3,5,6,7$ & $\hat{n}_{k}^{x}$ & $\hat{n}_{k-1}^{p^{n}}$ & $8 e^{-2 r}$ & 4 & $3 \mathrm{~dB}$ \\
\hline 117 & $1,3,5,6,7$ & $\hat{n}_{k}^{x}$ & $\hat{n}_{k-N}^{p}$ & $8 e^{-2 r}$ & 4 & $3 \mathrm{~dB}$ \\
\hline 118 & $2,3,5,6,7$ & $-\hat{n}_{k}^{x}+\hat{n}_{k+N}^{x}$ & $-\hat{n}_{k}^{p}+\hat{n}_{k+N}^{p}+\hat{n}_{k+N+1}^{p}+\hat{n}_{k+N-1}^{p}$ & $24 e^{-2 r}$ & 12 & $3 \mathrm{~dB}$ \\
\hline 119 & $1,2,3,5,6,7$ & $\hat{n}_{k}^{x}$ & $\hat{n}_{k}^{p}$ & $8 e^{-2 r}$ & 4 & $3 \mathrm{~dB}$ \\
\hline 120 & $4,5,6,7$ & $\hat{n}_{k}^{x}$ & $\hat{n}_{k-1}^{p^{k}}$ & $8 e^{-2 r}$ & 4 & $3 \mathrm{~dB}$ \\
\hline 121 & $1,4,5,6,7$ & $-\hat{n}_{k}^{x}+\hat{n}_{k+N}^{x}$ & $-\hat{n}_{k}^{p}+\hat{n}_{k+N}^{p}+\hat{n}_{k+N+1}^{p}+\hat{n}_{k+N-1}^{p}$ & $24 e^{-2 r}$ & 12 & $3 \mathrm{~dB}$ \\
\hline 122 & $2,4,5,6,7$ & $\hat{n}_{k}^{x}$ & $\hat{n}_{k-N}^{p}$ & $8 e^{-2 r}$ & 4 & $3 \mathrm{~dB}$ \\
\hline 123 & $1,2,4,5,6,7$ & $\hat{n}_{k}^{x}$ & $\hat{n}_{k}^{p}$ & $8 e^{-2 r}$ & 4 & $3 \mathrm{~dB}$ \\
\hline 124 & $3,4,5,6,7$ & $\hat{n}_{k}^{x}$ & $\hat{n}_{k-1}^{p^{\kappa}}$ & $8 e^{-2 r}$ & 4 & $3 \mathrm{~dB}$ \\
\hline 125 & $1,3,4,5,6,7$ & $-\hat{n}_{k}^{x}+\hat{n}_{k+N}^{x}$ & $\hat{n}_{k+1}^{p}+\hat{n}_{k+N}^{p}+\hat{n}_{k+N+1}^{p}+\hat{n}_{k+N-1}^{p}$ & $24 e^{-2 r}$ & 12 & $3 \mathrm{~dB}$ \\
\hline 126 & $2,3,4,5,6,7$ & $-\hat{n}_{k}^{x}+\hat{n}_{k+N}^{x}$ & $\hat{n}_{k+1}^{p}+\hat{n}_{k+N}^{p}+\hat{n}_{k+N+1}^{p}+\hat{n}_{k+N-1}^{p}$ & $24 e^{-2 r}$ & 12 & $3 \mathrm{~dB}$ \\
\hline 127 & $1,2,3,4,5,6,7$ & $\hat{n}_{k}^{x}+\hat{n}_{k+1}^{x}$ & $-\hat{n}_{k}^{p}+\hat{n}_{k+N}^{p}$ & $16 e^{-2 r}$ & 8 & $3 \mathrm{~dB}$ \\
\hline
\end{tabular}




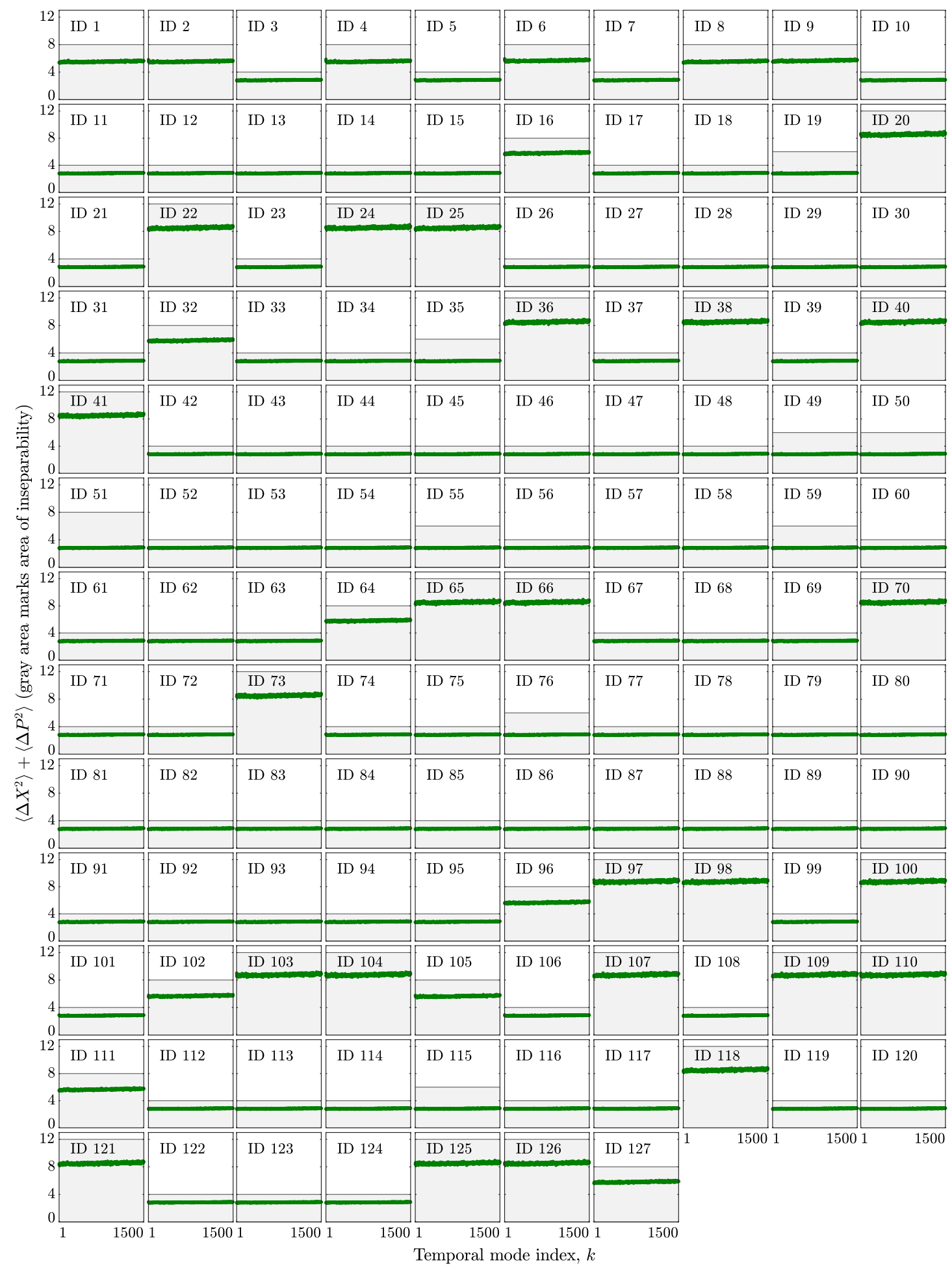

Figure 3.17: Plot of the van Loock-Furusawa separability criterion's left-hand-side, $\left\langle\Delta \hat{X}^{2}\right\rangle+\left\langle\Delta \hat{P}^{2}\right\rangle$, for each bipartition and chosen nullifier combination listed in table 3.1 using acquired data. Here, the gray area marks values less than the van Loock-Furusawa criterion right-hand-side, $f$, listed in table 3.1, and corresponds to the given bipartition being inseparable. 


\section{Chapter 4}

\section{Architecture and noise analysis of continuous-variable quantum gates using two-dimensional cluster states}

In this chapter, the paper "Architecture and noise analysis of continuous-variable quantum gates using two-dimensional cluster states" of Ref. [32] is presented. This paper is authored by Mikkel V. Larsen, Jonas S. Neergaard-Nielsen, and Ulrik L. Andersen, and published in Physical Review A 102, 042608 (2020).

In this theoretical work, we propose and analyze a quantum computation scheme based on projective measurements of the cluster state generated in chapter 3 [31]. The analysis is extended to include similar computation schemes on two-dimensional cluster states, including the demonstrated cluster state by Asavanant et al. in Ref. [37], and we finally compare their performances.

With the computation scheme proposed in this work, in chapter 5 [33] the implementation of quantum gates is demonstrated by projective measurements on the cluster state generated in chapter 3 [31]. Besides proposing an efficient computation scheme, in this work, we demonstrate a search method to determine the required basis settings for implementing desired gates by projective measurements. This method has shown useful to search for basis settings to implement gates on various cluster states, and the method is applied in both chapter 5 and $6[33,34]$.

From Physical Review A 102, 042608 (2020). (c) American Physical Society.

\subsection{Abstract}

Due to its unique scalability potential, continuous variable quantum optics is a promising platform for large scale quantum computing. In particular, very large cluster states with a two-dimensional topology that are suitable for universal quantum computing and quantum simulation can be readily generated in a deterministic manner, and routes towards fault-tolerance via bosonic quantum error-correction are known. In this article we propose a complete measurement-based quantum computing architecture for the implementation of a universal set of gates on the recently generated two-dimensional cluster states [31, 37]. We analyze the performance of the various quantum gates that are executed in these cluster states as well as in other two-dimensional cluster states (the bilayer-square lattice and quad-rail lattice cluster states $[9,11]$ ) by estimating and minimizing the associated stochastic noise addition as well as the resulting gate error probability. We compare the four different states and find that, although they all allow for universal computation, the 
quad-rail lattice cluster state performs better than the other three states which all exhibit similar performance.

\subsection{Introduction}

Measurement-based quantum computation (QC) [1] on continuous variable (CV) cluster states [2, $5]$, also known as cluster state computation, shows great potential for scalable quantum information processing. This is due to the simplicity of generating a deterministic and scalable cluster state resource, and the efficiency by which Gaussian gates can be implemented with high-efficiency homodyne detection as already experimentally demonstrated on few-mode cluster states [22-24, 104, 105]. The generation of large one-dimensional (1D) cluster states was realized several years ago [19-21], but for QC at least two dimensions are required - one for encoding and another for computation. There are multiple feasible proposals for the generation of two-dimensional (2D) cluster states $[8,9,11,12,106]$, and recently two different 2D cluster states were experimentally realized [31, 37] (with Ref. [31] being the content of chapter 3). A natural question is then, how do the different $2 \mathrm{D}$ cluster states compare with regards to their suitability for QC?

Computation schemes for some of the popular 2D cluster states already exist [11, 12, 58, 107]. Here, we summarize these schemes and propose new computation schemes for the recently experimentally realized states. Since physical CV cluster states always include noise due to finite squeezing, we furthermore perform a noise analysis of the discussed computation schemes. While similar noise analyses have been done for the 1D dual-rail wire cluster state [10] and the regular 2D square lattice cluster state [3], such analysis on experimentally feasible 2D cluster states has not yet, to our knowledge, been carried out. Here, we aim to find the best noise performance for QC with the discussed schemes on each cluster state.

As such, this work is partly a review of existing computation schemes, an introduction to new computation schemes of experimentally realized cluster states, a detailed noise analysis of QC on the different 2D CV cluster states, and a comparison of these. The paper begins with an introduction to the notation and a review of basic concepts in section 4.3. In section 4.4, we introduce a new computation scheme for the $2 \mathrm{D}$ cluster state experimentally realized by us in [31], as described in chapter 3, and perform a noise analysis of this scheme. In section 4.5, we describe corresponding computation schemes on three other popular 2D cluster states, namely the quad-rail lattice [107], the bilayer square lattice [11], and the recently generated cluster state by Asavanant et al. [37]. For each of them, we repeat the same noise analysis as presented in section 4.4. In section 4.6, we compare the topology and noise performance of the different cluster states and discuss the requirements for universal QC. Finally, we conclude on the results in section 4.7. Depending on the reader's motivation and prior knowledge of cluster state computation, the reader may skip sections and jump to that of interest - we have carefully cross-referenced the sections of this work.

\subsection{Prerequisite}

In this section we review the basic concepts of continuous variable cluster state quantum computation that we will be using in this work. In case the reader is familiar with these concepts, the section can be skipped.

\subsubsection{Definitions}

Throughout the paper we assume that $\hbar=1$ and $[\hat{x}, \hat{p}]=i$ such that the light field amplitude, $\hat{x}$, (or position) and phase, $\hat{p}$, (or momentum) quadratures can be written as $\hat{x}=\left(\hat{a}+\hat{a}^{\dagger}\right) / \sqrt{2}$ and $\hat{p}=-i\left(\hat{a}-\hat{a}^{\dagger}\right) / \sqrt{2}$, respectively, where $\hat{a}$ is the annihilation operator. With these definitions, the variance of the vacuum is $1 / 2$. We will make use of six different unitary operators: The identity operator $\hat{I}$, the phase rotation operator $\hat{R}(\theta)=e^{-i \theta\left(\hat{x}^{2}+\hat{p}^{2}\right) / 2}$ (where $\theta$ is the rotation angle) with 
the Fourier operator $\hat{F}=\hat{R}(\pi / 2)$ as a special case, the squeezing operator $\hat{S}(s)=e^{i \ln (s)(\hat{x} \hat{p}+\hat{p} \hat{x}) / 2}$ (where $r=-\ln (s)$ is the standard squeezing parameter), the shear operator $\hat{P}(p)=e^{i p \hat{x}^{2} / 2}$ (where $p$ is a shearing parameter), the controlled-Z operator $\hat{C}_{Z}(g)=e^{i g \hat{x} \otimes \hat{x}}$ (where $g$ is the coupling coefficient), and the balanced beam splitter operator $\hat{B}=e^{-i \pi(\hat{x} \otimes \hat{p}-\hat{p} \otimes \hat{x}) / 4}$. Each of these operators are Gaussian and can be described by symplectic matrices representing the evolution of the quadrature operators, arranged in a vector $\left(\hat{x}_{1}, \cdots, \hat{x}_{n}, \hat{p}_{1}, \cdots, \hat{p}_{n}\right)^{T}$ for $n$ modes, in the Heisenberg picture:

$$
\begin{gathered}
\mathbf{I}=\left(\begin{array}{ll}
1 & 0 \\
0 & 1
\end{array}\right), \quad \mathbf{R}=\left(\begin{array}{cc}
\cos \theta & \sin \theta \\
-\sin \theta & \cos \theta
\end{array}\right), \quad \mathbf{S}=\left(\begin{array}{cc}
\frac{1}{s} & 0 \\
0 & s
\end{array}\right), \quad \mathbf{P}=\left(\begin{array}{ll}
1 & 0 \\
p & 1
\end{array}\right), \\
\mathbf{C}_{\mathbf{Z}}=\left(\begin{array}{llll}
1 & 0 & 0 & 0 \\
0 & 1 & 0 & 0 \\
0 & g & 1 & 0 \\
g & 0 & 0 & 1
\end{array}\right),
\end{gathered}
$$

and

$$
\mathbf{B}=\frac{1}{\sqrt{2}}\left(\begin{array}{cccc}
1 & -1 & 0 & 0 \\
1 & 1 & 0 & 0 \\
0 & 0 & 1 & -1 \\
0 & 0 & 1 & 1
\end{array}\right)
$$

for $\hat{I}, \hat{R}(\theta), \hat{S}(s), \hat{P}(p), \hat{C}_{Z}(g)$ and $\hat{B}$, respectively.

To allow for quantum error correction, in this paper we consider the encoding of qubits in bosonic modes of computation, $\left|\psi_{\text {in }}\right\rangle$. Numerous different qubit encodings have been proposed such as encoding in cat states $[13,108]$ and binomial states $[15,109]$ but here we will consider the efficient Gottesman-Kitaev-Preskill (GKP) encoding [14]. For these codes, a qubit is encoded on a square lattice in phase-space in a way that allows for the suppression of relevant errors (such as loss) to a certain extend. To combat residual qubit errors, the GKP code must be concatenated with another qubit error correction code such as the 7 -qubit Steane code [110] or Knill's $C_{4} / C_{6}$ code [111]. GKP-encoding is the only known bosonic code for which a universal Gaussian gate set allows logic Clifford computation and error correction of encoded qubits. A logic single-mode Clifford gate set is realized by the Gaussian gate set $\{\hat{I}, \hat{F}, \hat{P}(1)\}$ together with displacements, while two-mode gates are enabled by the $\hat{C}_{Z}(1)$-gate. A non-Clifford gate completes the universal encoded qubit gate set. While the non-Clifford gate requires challenging non-Gaussian transformations, practical proposals do exists which are further discussed in section 4.6.2. Here in section 4.3, and in section 4.4 and 4.5 , we will focus on the implementations of the Gaussian gates $\hat{I}, \hat{F}, \hat{P}(1)$ and $\hat{C}_{Z}(1)$.

Gaussian gates are implemented on a cluster state by quadrature measurement of each mode in different bases rotated by $\theta$ with respect to the $\hat{x}$-quadrature, i.e. measuring $\hat{x}(\theta)=\hat{x} \cos \theta+\hat{p} \sin \theta$. In this paper we use the Heisenberg picture, in which we simulate the evolution of the quadrature operators and where the noise contributions simply appear as additive Gaussian noise terms. In the following we consider the generalized teleportation circuit as an example, which as well plays an important role in the quantum computation schemes presented in section 4.4 and 4.5.

\subsubsection{Generalized teleportation}

An arbitrary Gaussian transformation on a single bosonic mode can be realized by means of the generalized teleportation circuit, as diagrammatically depicted in Fig. 4.1a. Here, the term generalized teleportation is in terms of the generalized measurement bases, where different gates are implemented on a teleported state depending on the basis setting. Generalized teleportation consists of an input state, an entangled multi-mode ancillary state, and a measurement device. In conventional single-mode teleportation, the ancillary entangled state is a two-mode squeezed state [62], while traditionally for MBQC we consider a cluster state. The two-mode squeezed state and two-mode cluster state are equivalent (up to a phase rotation), but since well-developed theoretical tools exist for cluster states in the language of graphical calculus [7], here we focus on 
a)

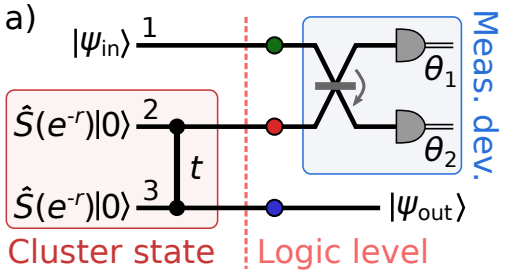

b)

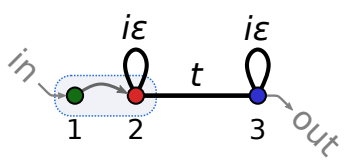

Figure 4.1: (a) Generalized teleportation circuit implementing single mode Gaussian operation on the input state $\left|\psi_{\text {in }}\right\rangle$ by joint measurement of the input state with one mode of an ancillary approximate cluster state using a two-mode measurement device (meas. dev.). The measurement device consists of a beam splitter, with the arrow pointing from the first to the second mode in Eq. (4.1), followed by homodyne detection measuring in bases $\hat{x}_{i}\left(\theta_{i}\right)=\hat{x}_{i} \cos \theta_{i}+\hat{p}_{i} \sin \theta_{i}$. (b) Short graphical notation of the circuit in (a) used in this paper, with the nodes representing the modes in (a). Here, $\varepsilon=e^{-2 r}$. The node colors have no physical meaning and are only used to identify the modes in (a). The graphical notation can be thought of as a "snap-shot" of the logic level in (a) where the computation takes place.

cluster states. In practice the ancillary entangled state is an approximate cluster state composed of finitely momentum-squeezed states that are entangled by a controlled- $Z$ gate of weight $t$ [5]. In Fig. 4.1a, the momentum variance is $\varepsilon / 2$ where $\varepsilon=e^{-2 r}$ with $r$ being the squeezing parameter. To implement a gate, a joint measurement is performed on the input state and one mode of the cluster state using the measurement device marked on Fig. 4.1a consisting of a beam splitter and two homodyne detectors. The resulting transformation of the quadratures in the Heisenberg picture is

$$
\left(\begin{array}{l}
\hat{x}_{3}^{\prime} \\
\hat{p}_{3}^{\prime}
\end{array}\right)=\mathbf{G}\left(\begin{array}{l}
\hat{x}_{1} \\
\hat{p}_{1}
\end{array}\right)+\mathbf{N}\left(\begin{array}{l}
\hat{p}_{2} \\
\hat{p}_{3}
\end{array}\right)+\mathbf{D}\left(\begin{array}{l}
m_{1} \\
m_{2}
\end{array}\right) \text {. }
$$

Here, the first term represents the implemented Gaussian gate with $\mathbf{G}$ corresponding to the gate symplectic matrix. The second term, with $\mathbf{N}$ being a matrix, represents noise added to the quadratures due to finite squeezing in the cluster state; it vanishes in the infinite squeezing limit as $\left\langle\hat{p}_{2,3}\right\rangle=0$ and $\operatorname{Var}\left(\hat{p}_{2,3}\right)=e^{-2 r} / 2 \rightarrow 0$ for $r \rightarrow \infty$. This term represents the gate noise. The last term, with $\mathbf{D}$ being a matrix, is the computational by-product in the form of a displacement, where $m_{1}$ and $m_{2}$ are the measurement outcomes of mode 1 and 2 , respectively. $\mathbf{G}, \mathbf{N}$, and $\mathbf{D}$ are each described in detail in the following subsections.

When considering multi-mode computing schemes with large cluster states, the circuit model in Fig. 4.1a becomes tedious. Instead it is customary to use a graph notation as illustrated in Fig. 4.1b, where the cluster state is represented by its corresponding graph with imaginary self-loops indicating the finite squeezing of the cluster state modes [7], the beam splitter of the measurement device is represented by an arrow, and the input state is represented by a free node. For the schemes presented in this work, we assume that all cluster state modes are equally squeezed. Hence, we will omit the identical $i \varepsilon$ self-loops on the cluster state nodes - they are always there, and only vanish in the non-physical infinite squeezing limit. Finally, we will define the logic level as being the level in the circuit diagram where the computation takes place, i.e. after the cluster state generation where the input state appears, and before the measurement device for computation. The logic level is marked on Fig. 4.1a, and the graph notation in Fig. 4.1b is a "snap-shot" of this logic level with the arrow indicating the subsequent beam splitter operation of the measurement device. An alternative formulation is to use macronodes as in [10, 11], where, instead of joint measurements of localized modes in the logic level, one considers single-mode measurements of distributed modes. In Fig. 4.1a this macronode formulation corresponds to locating the logic level right after the beam-splitter transformation, and keeping in mind, that in the logic level the mode under computation is distributed between mode 1 and 2 as $\left(\hat{a}_{1}+\hat{a}_{2}\right) / \sqrt{2}$.

In the following we describe each term of Eq. (4.2) in more detail: 


\subsubsection{Gate}

The implemented gate in Eq. (4.2) depends on the measurement bases of the two quadrature measurements (defined as $\theta_{1}$ and $\theta_{2}$ ) as

$$
\mathbf{G}=\frac{1}{\sin \theta_{-}}\left(\begin{array}{cc}
\frac{1}{t} \cos \theta_{+}+\frac{1}{t} \cos \theta_{-} & \frac{1}{t} \sin \theta_{+} \\
-t \sin \theta_{+} & t \cos \theta_{+}-t \cos \theta_{-}
\end{array}\right)
$$

where $\theta_{ \pm}=\theta_{1} \pm \theta_{2}$, and corresponds to the operation [6]

$$
\hat{S}(t) \hat{R}\left(\frac{\theta_{+}}{2}\right) \hat{S}\left(\tan \frac{\theta_{-}}{2}\right) \hat{R}\left(\frac{\theta_{+}}{2}\right) .
$$

By implementing such operation twice - corresponding to two consecutive runs of the teleportation circuit in Fig 4.1a - it is possible to induce an arbitrary single mode Gaussian transformation [10], $\mathbf{U}(\hat{x}, \hat{p})^{T}+\mathbf{c}$, where $\mathbf{U}=\mathbf{G}_{2} \mathbf{G}_{1}$ while the displacement $\mathbf{c}$ is ubiquitous and simply implemented by shifting the measurement result and updating the bases of the subsequent measurements $[2,5]$. However, in the following we consider only the subset of single-mode Gaussian transformation that is required for GKP state computation, namely the set $\{\hat{I}, \hat{F}, \hat{P}(1)\}$.

The identity operator, $\hat{I}$, can be executed in a single computation step of the teleportation circuit in Fig. 4.1a by choosing

$$
\left(\theta_{+}, \theta_{-}\right)_{I}=(0,2 \arctan 1 / t),
$$

as easily seen from Eq. (4.3). For the Fourier gate, $\hat{F}$, and the $\hat{P}(1)$-gate two computation steps are necessary: If we let the output state $|\psi\rangle_{\text {out }}$ of the first computation step in Fig. 4.1a, with bases $\left(\theta_{+1}, \theta_{-1}\right)$, be the input state on a second identical circuit, but with bases $\left(\theta_{+2}, \theta_{-2}\right)$, the gate

$$
\hat{S}(t) \hat{R}\left(\frac{\theta_{+2}}{2}\right) \hat{S}\left(\tan \frac{\theta_{-2}}{2}\right) \hat{R}\left(\frac{\theta_{+2}}{2}\right) \times \hat{S}(t) \hat{R}\left(\frac{\theta_{+1}}{2}\right) \hat{S}\left(\tan \frac{\theta_{-1}}{2}\right) \hat{R}\left(\frac{\theta_{+1}}{2}\right)
$$

is implemented. Choosing

$$
\left(\theta_{+1}, \theta_{-1}, \theta_{+2}, \theta_{-2}\right)_{F}=\left(\frac{\pi}{2}, \frac{\pi}{2}, 0,2 \arctan \frac{1}{t^{2}}\right)
$$

implements the $\hat{F}$-gate, while

$$
\left(\theta_{+1}, \theta_{-1}, \theta_{+2}, \theta_{-2}\right)_{P}=\left(\arctan 2,-\arctan 2, \frac{\pi}{2}, \frac{\pi}{2}\right)
$$

implements the $\hat{P}(1)$-gate. To implement the two-mode $\hat{C}_{Z}$-gate a scheme with at least two input modes is required. This will be discussed in section 4.4 and 4.5 , where we find that also two teleportation steps are necessary.

\subsubsection{Gate noise}

The second term in Eq. (4.2) represents the gate noise and is governed by the matrix

$$
\mathbf{N}=\left(\begin{array}{cc}
-\frac{1}{t} & 0 \\
0 & 1
\end{array}\right),
$$

which is independent on the measurement bases. However, for gates realized in two steps, as the $\hat{F}$ - and $\hat{P}(1)$-gate described above, the gate noise of the first step enters the gate of the second step, leading to the final gate noise that depends on the bases of the second computation step. For two concatenated circuits of the type in Fig. 4.1a, with the cluster state of the second circuit being denoted mode 4 and 5 , the combined gate noise becomes

$$
\mathbf{G}_{2} \mathbf{N}_{1}\left(\begin{array}{c}
\hat{p}_{2} \\
\hat{p}_{3}
\end{array}\right)+\mathbf{N}_{2}\left(\begin{array}{c}
\hat{p}_{4} \\
\hat{p}_{5}
\end{array}\right) \equiv \mathbf{N}\left(\begin{array}{c}
\hat{p}_{2} \\
\hat{p}_{3} \\
\hat{p}_{4} \\
\hat{p}_{5}
\end{array}\right)
$$


where $\mathbf{N}_{1}\left(\hat{p}_{2}, \hat{p}_{3}\right)^{T}$ is the gate noise of the first step, and $\mathbf{G}_{2}$ and $\mathbf{N}_{2}\left(\hat{p}_{4}, \hat{p}_{5}\right)^{T}$ is the gate symplectic matrix and gate noise of the second step. Here, the combined gate noise matrix $\mathbf{N}$ is a $2 \times 4$ matrix. Note, that in the following sections III and IV, $\mathbf{N}$ is in general the combined gate noise matrix of an implemented gate in one or more teleportation steps, with the number of columns equal to the number of quadratures in the output mode(s), and the number of rows equal to the number of ancillary cluster state modes involved in the implemented gate.

Assuming that all cluster state modes in Eq. (4.4) are equally squeezed, $\operatorname{Var}\left(\hat{p}_{l}\right)=\varepsilon / 2$ for $l=2,3,4,5$, the gate noise variance amounts to $\sum_{j=1}^{4} N_{i j}^{2} \varepsilon / 2$ for $i=1,2$. From this expression, it is clear that the gate noise can be reduced by both increasing the degree of squeezing of the cluster state modes (reducing $\varepsilon / 2$ ) and by minimizing the sums $\sum_{j=1}^{4} N_{i j}^{2}, i=1$, 2 . We will refer to these two sums as the quadrature noise factors, one for each quadrature. In the schemes for gate implementation presented in section 4.4 and 4.5 , the focus is on optimizing the quadrature noise factors for the gate set $\left\{\hat{I}, \hat{F}, \hat{P}(1), \hat{C}_{Z}(1)\right\}$ in order to minimize the probability of inducing errors on the GKP-encoded qubit as discussed in section 4.3.3.

\subsubsection{Displacement by-product}

The displacement matrix in Eq. (4.2) reads

$$
\mathbf{D}=\frac{\sqrt{2}}{\sin \theta_{-}}\left(\begin{array}{cc}
-\frac{1}{t} \cos \theta_{2} & -\frac{1}{t} \cos \theta_{1} \\
t \sin \theta_{2} & t \sin \theta_{1}
\end{array}\right),
$$

and so, since we know the measurement bases, $\left(\theta_{1}, \theta_{2}\right)$, together with the measurement outcome, $\left(m_{1}, m_{2}\right)$, the amount of displacement of a single computation step is known. By keeping track of the displacements in each computation step, the displacement can be accounted for in the following steps by updating the measurement bases and results (i.e. feed-forward) [5]. When all gates are Gaussian, the displacement by-product can be compensated for in the measurement result of the final output state $\left|\psi_{\text {out }}\right\rangle$, known as Gaussian parallelism [2].

In this work we will ignore the displacement by-product as it has no effect on the gate noise performance. However, for the actual experimental implementations of the schemes discussed in this work, the compensation for the displacement is important.

\subsubsection{Wigner function representation}

To understand the effect of the quadrature transformation and gate noise in Eq. (4.2), it is useful to analyse the generalized teleportation in the Wigner function representation. This does not add anything new, but considering the gate noise from a different perspective helps to understand it. Here, the Wigner function of the input state to the teleportation circuit in Fig. 4.1, $\left|\psi_{\text {in }}\right\rangle_{1} \hat{S}\left(e^{-r}\right)|0\rangle_{2} \hat{S}\left(e^{-r}\right)|0\rangle_{3}$, is

$$
W_{\text {in }}\left(x_{1}, p_{1}\right) G_{1 / \varepsilon}\left(x_{2}\right) G_{\varepsilon}\left(p_{2}\right) G_{1 / \varepsilon}\left(x_{3}\right) G_{\varepsilon}\left(p_{3}\right),
$$

where $G_{\delta}$ is a normalized Gaussian function of variance $\delta / 2$, and $W_{\text {in }}$ is the Wigner function corresponding to $\left|\psi_{\text {in }}\right\rangle_{1}$, but not necessarily of a pure state. After the quadrature transformation in the generalized teleportation circuit and measurement of $\hat{x}_{1}\left(\theta_{1}\right)$ and $\hat{x}_{2}\left(\theta_{2}\right)$, the output Wigner function corresponding to $\left|\psi_{\text {out }}\right\rangle$ on mode 3 in Fig. 4.1 becomes

$$
\begin{aligned}
W_{\text {out }}\left(x_{3}, p_{3}\right)=\mathcal{N} G_{1 / \varepsilon}\left(x_{3}\right) \int & \mathrm{d} \eta_{2} G_{\varepsilon}\left(\eta_{2}\right) G_{t^{2} / \varepsilon}\left(p_{3}-\eta_{2}\right) \times \\
& \int \mathrm{d} \eta_{1} G_{\varepsilon / t^{2}}\left(\eta_{1}\right) W_{\text {in }}\left(\mathbf{G}^{-1}\left(\begin{array}{c}
x_{3}-d_{x}-\eta_{1} \\
p_{3}-d_{p}-\eta_{2}
\end{array}\right)\right),
\end{aligned}
$$

where $\mathcal{N}$ is a normalization factor depending on the basis setting $\left(\theta_{1}, \theta_{2}\right)$ and measurement outcome $\left(m_{1}, m_{2}\right)$, and $\left(d_{x}, d_{p}\right)^{T}=\mathbf{D}\left(m_{1}, m_{2}\right)^{T}$ subtracted from the $\left(x_{3}, p_{3}\right)^{T}$ argument in $W_{\text {in }}$ corresponds to the displacement by-product. Here, each index in the vector argument of $W_{\text {in }}$ should be understood as the two arguments in $W_{\text {in }}(\cdot, \cdot)$. It is clear from the expression that the input state 
undergoes an operation of symplectic matrix $\mathbf{G}$ by the transformation of its Wigner function arguments by $\mathbf{G}^{-1}$. This corresponds to the implemented gate. The gate noise $\mathbf{N}\left(\hat{p}_{2}, \hat{p}_{3}\right)^{T}$ with variance $\left(\varepsilon / t^{2}, \varepsilon\right)^{T} / 2$ is seen to become a Gaussian convolution of the Wigner function with corresponding variance after applying the gate $\mathbf{G}$. Finally, the Wigner function is subjected to a Gaussian envelope in both quadratures: One of variance $t^{2} / 2 \varepsilon$ in the $\hat{p}$-quadrature after convolution in $\hat{x}$-quadrature with $G_{\varepsilon / t^{2}}$, followed by one of variance $1 / 2 \varepsilon$ in $\hat{x}$-quadrature after convolution in $\hat{p}$-quadrature with $G_{\varepsilon}$. These envelopes in each quadratures are the result of convolutions in orthogonal quadratures, as the quadratures are related by the Fourrier transform: Convoluting a Wigner function in $\hat{x}$-quadrature with $G_{\delta}$ leads to an envelope of the Wigner function in the $\hat{p}$-quadrature of $G_{1 / \delta}(p)$, and vice versa.

Two limits of Eq. (4.5) are interesting: In the ideal infinite squeezing limit, the convolution functions $G_{\varepsilon / t^{2}}$ and $G_{\varepsilon}$ becomes delta functions, while the Gaussian envelopes becomes infinitely broad, and so $W_{\text {out }}\left(x_{3}+d_{x}, p_{3}+d_{p}\right)^{T}=W_{\text {in }}\left(\mathbf{G}^{-1}\left(x_{3}, p_{3}\right)^{T}\right)$. In the limit $t=0$, where we expect no information to transfer from $W_{\text {in }}$ to $W_{\text {out }}$, the envelope on $W_{\text {in }}\left(\mathbf{G}^{-1}\left(x_{3}, p_{3}\right)^{T}\right)$ becomes a delta function in $\hat{p}$-quadrature, which is then convoluted by $G_{\varepsilon}$, while the $\hat{x}$-quadrature is convoluted by an infinitely broad Gaussian followed by an envelope of $G_{1 / \varepsilon}$. As a result, for $t=0, W_{\text {out }}\left(x_{3}, p_{3}\right)=$ $G_{1 / \varepsilon}\left(x_{3}\right) G_{\varepsilon}\left(p_{3}\right)$ corresponding to the initial input squeezed state in the cluster state as expected.

\subsubsection{Error correction}

It is now clear that cluster state quantum computation will inevitably suffer from gate noise that will accumulate throughout the computation. To avoid noise accumulation, quadrature error correction is required in between every implemented gate. For this purpose, symmetric GKP states are particularly useful, not only as the qubit but also as ancillaries for error correction. GKP states have been thoroughly reviewed in several places $[112,113]$, while here it is reviewed in terms of MBQC focusing on the added gate noise caused by finite squeezing in the cluster states. The GKP state Wigner function, $W_{\text {in }}(\mathbf{x})$ (with $\mathbf{x}=\left(x_{1}, \cdots, x_{n}, p_{1}, \cdots, p_{n}\right)^{T}$ for a $n$-mode state), consists of delta functions arranged on a square lattice in phase space of each mode with a lattice constant of $\sqrt{\pi}$, and its qubit eigenstates of the Pauli-Z and -X operators are $\left|j_{L}\right\rangle_{X, Z}=\sum_{i \in \mathbb{Z}}|(2 i+j) \sqrt{\pi}\rangle_{x, p}$ in the quadrature eigenstate basis, $|s\rangle_{x}$ and $|s\rangle_{p}[14]$.

As a result of the execution of a $n$-mode gate $\mathbf{G}$ in one or more computation steps, the gate noise $\mathbf{N}\left(\hat{p}_{c 1}, \hat{p}_{c 2}, \cdots\right)^{T}$ (where $p_{c i}$ are ancillary cluster state modes) leads to a broadening of the GKP delta functions into Gaussian functions of variances in $\boldsymbol{\sigma}^{\mathbf{2}}=\operatorname{Var}\left\{\mathbf{N}\left(\hat{p}_{c 1}, \hat{p}_{c 2}, \cdots\right)^{T}\right\}$ in the $2 n$ quadratures ( $\boldsymbol{\sigma}^{2}$ is a $2 n$ vector). Furthermore, as the ideal GKP-encoding with vanishing variance (represented by delta functions) is non-physical, we instead consider the physical approximate GKP-states in $W_{\text {in }}(\mathbf{x})$ with delta functions replaced by symmetric Gaussian functions of identical variance, $\delta$, in $\hat{x}$ - and $\hat{p}$-quadrature. The quadrature variance of the Gaussian spikes in the approximate GKP-state after the implementation of a noisy gate is then

$$
\delta^{\prime}=\operatorname{Var}\left\{\mathbf{G} \hat{\mathbf{x}}_{\delta}\right\}+\sigma^{2}
$$

where $\hat{\mathbf{x}}$ is decomposed into a sum, $\hat{\mathbf{x}}_{\mathbf{0}}+\hat{\mathbf{x}}_{\boldsymbol{\delta}}$, where $\hat{\mathbf{x}}_{\mathbf{0}}$ and $\hat{\mathbf{x}}_{\boldsymbol{\delta}}$ are the centers and variance of the GKP spikes, respectively. Note that for ideal GKP states $\hat{\mathbf{x}}=\hat{\mathbf{x}}_{\mathbf{0}}$. As examples, $\operatorname{Var}\left\{\mathbf{G x}_{\boldsymbol{\delta}}\right\}=(\delta, \delta)^{T}$ for the $\hat{I}$ - and $\hat{F}$-gate, $\operatorname{Var}\left\{\mathbf{G x}_{\boldsymbol{\delta}}\right\}=(\delta, 2 \delta)^{T}$ for the $\hat{P}(1)$-gate, and $\operatorname{Var}\left\{\mathbf{G x}_{\boldsymbol{\delta}}\right\}=(\delta, \delta, 2 \delta, 2 \delta)^{T}$ for the two-mode $\hat{C}_{Z}(1)$-gate.

To avoid gate noise accumulating on the GKP-encoded qubit state, after every implemented $\hat{I}$-, $\hat{F}_{-}, \hat{P}(1)$ - and $\hat{C}_{Z}(1)$-gate, we measure the quadratures $\hat{x} \bmod \sqrt{\pi}$ and $\hat{p} \bmod \sqrt{\pi}$ using ancillary GKP-states, and perform quadrature error correction by displacing back the state depending on 
the measurement outcome:

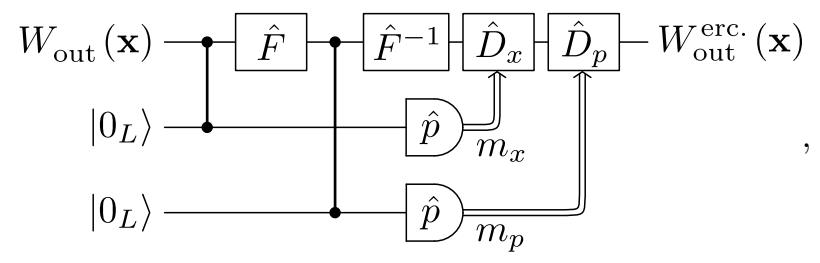

where $\left|0_{L}\right\rangle$ are approximate GKP-states with spike variances in both quadratures of $\delta$. Note that while this circuit illustrates the correction algorithm for a single mode of $W_{\text {out }}$, similar circuits are required for each other mode. After the two measurements with outcome $m_{x}$ and $m_{p}$, the encoded qubit is projected into a "fresh" GKP-state, but displaced in $\hat{x}$ - and $\hat{p}$-quadrature depending on the values of $m_{x}$ and $m_{p}$ : If $m_{x(p)} \bmod \sqrt{\pi}$ is smaller than $\sqrt{\pi} / 2$, the encoded state is displaced back by $m_{x(p)} \bmod \sqrt{\pi}$ in $\hat{x}(\hat{p})$, while if it is larger than $\sqrt{\pi} / 2$, the encoded states is displaced forward by $\sqrt{\pi}-\left(m_{x(p)} \bmod \sqrt{\pi}\right)$, and so we obtain an error corrected version of $W_{\text {out }}(\mathbf{x}) \rightarrow W_{\text {out }}^{\text {erc. }}(\mathbf{x})$, which is then the input to the next gate. The possible values of $m_{x}$ and $m_{p}$ are Gaussian distributed with variance $\delta_{i}^{\prime}+\delta$, where $\delta_{i}^{\prime}$ in $\boldsymbol{\delta}^{\prime}$ of Eq. (4.6) is the corresponding spike quadrature variance of the encoded state after gate implementation, $W_{\text {out }}$, and $\delta$ is the spike variance of the $\left|0_{L}\right\rangle$ ancillary states. As a result, for large $\delta_{i}^{\prime}$ and/or $\delta$, there is a risk of measuring a GKP spike closer to its neighbouring spikes of the orthogonal qubit state, i.e. outside the bin range $\left[x_{0}-\sqrt{\pi} / 2 ; x_{0}+\sqrt{\pi} / 2\right]$ where $x_{0}$ is the spike center, and thereby inducing a qubit error when "correcting" the state by displacing it in the wrong direction. The combined probability of displacing a $n$-mode encoded state with $2 n$ quadrature corrections in the wrong direction is shown to be [3]

$$
P_{\text {err. }}\left(\boldsymbol{\delta}^{\prime}, \delta\right)=1-\prod_{i=1}^{2 n} \operatorname{erf}\left(\frac{\sqrt{\pi}}{2 \sqrt{2\left(\delta_{i}^{\prime}+\delta\right)}}\right)
$$

where each factor in the product term is the probability of a successful quadrature correction. It is important to mention that $P_{\text {err. }}$ is not a true qubit error probability, as it does not account for the probability of measuring a spike at its neighbours neighbour bin range, $\left[x_{0} \pm 3 \sqrt{\pi} / 2 ; x_{0} \pm 5 \sqrt{\pi} / 2\right]$, which leads to a $2 \sqrt{\pi}$ displacement of the GKP state when corrected, and thereby not a qubit error although it is an error. This leads for example to $P_{\text {err. }} \rightarrow 1$ for large $\delta_{i}^{\prime}+\delta$ while the actual error probability should be $1 / 2$. Furthermore, Eq. (4.8) does not account for the overall envelope on the spikes of the GKP-state, and for the fact that the error probability is qubit-dependent: Displacing the $\hat{p}$-quadrature by $\sqrt{\pi}$ leads to an error on $\left|+_{L}\right\rangle$, but no error on $\left|0_{L}\right\rangle$. Therefore, for a true estimation of the qubit error probability, we need to take these effects into account. However, despite these issues, in this work (as in [3]) we will use $P_{\text {err. }}$ as a figure of merit as it constitutes a good approximation to the actual error probability for reasonably large squeezing levels in which $\delta_{i}^{\prime}+\delta$ is small enough for $2 \sqrt{\pi}$ (or larger) displacements to be neglected during quadrature corrections.

Since the two-mode $\hat{C}_{Z}(1)$-gate requires four quadrature corrections, while the $\hat{I}$-, $\hat{F}$ - and $\hat{P}(1)$ gate only requires two, the error probability after the $\hat{C}_{Z}(1)$-gate is in general larger. In the schemes presented in section 4.4 and 4.5 , when possible, we search for a basis setting for the $\hat{C}_{Z}(1)$-gate that minimizes $P_{\text {err. }}$.

\subsection{Double bilayer square lattice}

Having discussed the general concept of CV quantum computation and the associated error analysis, we are now equipped with the relevant tools to rigorously analyze the performance of cluster state computation based on different types of cluster states. In this section, we will consider the double bilayer square lattice (DBSL) cluster state while in the following section 4.5 we will consider three other known clusters states. 

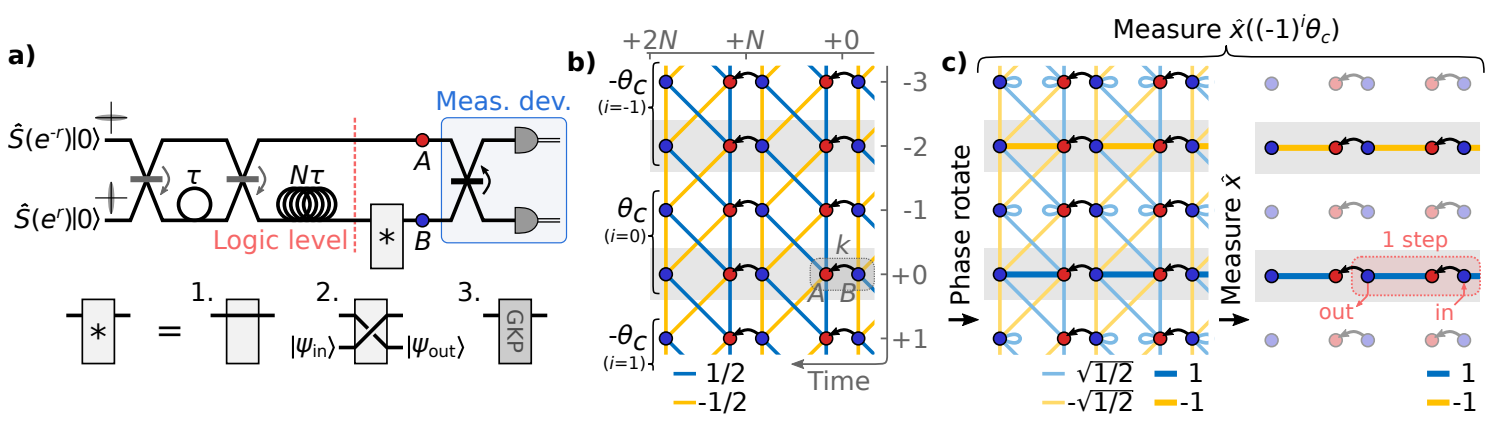

Figure 4.2: (a) Temporal encoded DBSL cluster state generation and computation setup. While the DBSL cluster state is generated after the third beam splitter, for computation we consider the cluster state in the marked logic level, and the third beam splitter as part of a joint measurement device for computation. The cluster state temporal mode duration $\tau$ is defined by the short delay line. The device marked by asterisk is an identity gate when implementing gates (1.), an optical switch when switching in a state for computation (2.), or the circuit in Eq. (4.7) for correcting gate noise after each implemented gate (3.). (b) Cluster state in the logic level: A dual-rail wire coiled up by the $N \tau$ long delay, leading to a cylinder with $N$ temporal modes in the circumference - the temporal mode indices are written in grey. Computation is performed in wires including the modes in the marked grey areas, while modes in between the grey areas are control modes. Measuring the control modes in an alternating basis of $(-1)^{i} \theta_{c}$ induces edges between the wire modes as shown in (c), allowing single mode computation in each $N / 2$ wire with one computation step marked in red. Measuring a control mode in a different basis creates coupling between the neighbouring two wires as shown in Fig. 4.3 allowing multi-mode gates. The edge weights shown here is in the limit of infinite squeezing and $\theta_{c}=\pi / 4$. For finite squeezing the edge weights are multiplied by $\tanh (2 r)$ while self-loops of $i \operatorname{sech}(2 r)$ are present on each cluster state mode. For easy comparison, the experimental setup, logic cluster state and its projection into wires are shown in the appendix section 4.10 together with the schemes considered in section 4.5.

The cylindrical 2D cluster state produced in chapter 3 [31], can be straightforwardly projected into a universal DBSL cluster which will be analysed in the following. The cylindrical DBSL cluster state with a 2D topology of Ref. [31] (corresponding to a cylindrical $\mathcal{H}$-graph state) was generated by "coiling up" a 1D cluster state (a dual-rail wire [19]) of temporal mode duration $\tau$ using a $N \tau$ long delay line, and interfering it with itself - the generation setup is summarized in Fig. 4.2a. As this $\mathcal{H}$-graph state is self-inverse and bipartite for even $N$, it is transformed into a DBSL cluster state through $\pi / 4$ phase rotations of all modes. Since this transformation simply corresponds to a redefinition of the quadratures, the DBSL $\mathcal{H}$-graph state and the corresponding cluster state are equivalent $[7,31]$. In the following we will therefore only consider the DBSL cluster state.

It is also important to note that in chapter 3 [31], it was shown that the DBSL cluster state can be projected into a regular square lattice cluster state which is known to be a universal resource for quantum computing. However, due to the resulting low edge weights of this square lattice, this approach is rather inefficient and leads to unnecessary large gate noise. In the following, we present a more efficient computation scheme of the DBSL and quantify it by a gate noise analysis.

\subsubsection{Efficient computation scheme}

Similar to the generalized teleportation scheme in section 4.3, we define a multi-mode measurement device that includes the third beam splitter as marked in Fig. 4.2a. The resulting logic level is located just before the measurement device, where the generated 1D cluster state is coiled up, but not yet interfered with itself. A section of the cylindrical coiled up 1D cluster state at the logic level is shown in Fig. 4.2b. Here the horizontal direction follows the cluster state cylinder axis while the vertical direction corresponds to the circumference of the cylinder whose size is limited by the long delay line to $N$ temporal modes. 
In the following we assume that the cylindrical cluster state has an even number of temporal modes in the circumference $(N$ is an even number which is necessary for the generated $\mathcal{H}$-graph state to be bipartite), each with a temporal mode index $k$. Every second temporal mode $(k+2 i$ for $i \in \mathbb{Z}$ ) form wires for computation along the cylinder (shaded area in Fig. 4.2b), while the remaining temporal modes $(k+2 i-1$ for $i \in \mathbb{Z})$ are control modes that are used to control couplings between wires. In this way we have $N / 2$ wires, and thereby $N / 2$ modes for computation. We will further assume that the number of wires is even, i.e. $N / 2$ being an even number. As an example, the experimental realization of the DBSL in chapter 3 had $N=12$ leading to 6 wires. Using an optical switch in the lower spatial mode at point $B$ in the logic level in Fig. 4.2a, an input state can be switched into the circuit. It corresponds to adding input states to the blue nodes in Fig. 4.2b. Optical switches have previously been demonstrated in quantum settings [30, 114] with Ref. [30] being the content of chapter 2 .

By inducing certain phase rotations, $(-1)^{i} \theta_{c}$, of the control modes it is possible to create new edges along the wires as illustrated in Fig. 4.2c [7]. If these phase rotations are followed by measurement of the control modes in the $\hat{x}$-basis, the modes and their edges are "deleted", and we are consequently left with $N / 2$ parallel wires suitable for single mode computation of $N / 2$ modes. It is also worth noting that this combination of phase rotation and $\hat{x}$-measurement corresponds to measuring the quadrature $\hat{x}\left((-1)^{i} \theta_{c}\right)$ on each control mode individually:

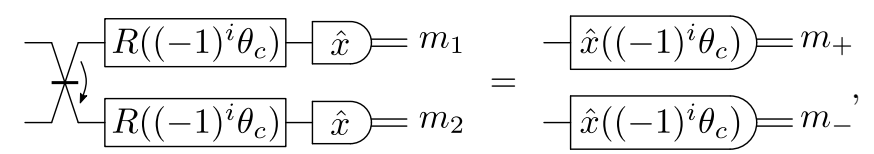

where the beam-splitter of the left-hand-side is the beam-splitter of the measurement device, and $m_{ \pm}=\left(m_{1} \pm m_{2}\right) / \sqrt{2}$. As an example, we may consider the case of infinite squeezing as pictured in Fig. 4.2c. Here the edge weights of the wires tend towards the optimal values of \pm 1 (where the sign alternates between neighbouring wires) by choosing $\theta_{c}=\pi / 4$. For finite, thus practical, squeezing levels, the induced wire edge weight is lower, while $\theta_{c}$ may be optimised for minimizing the gate noise. For simplicity, in the computation scheme presented here, we keep $\theta_{c}=\pi / 4$ for all squeezing levels while in section 4.4 .3 we discuss the effect of varying $\theta_{c}$.

The projected wires in Fig. 4.2c are now suitable for single mode Gaussian computation: One computation step (one horizontal time step from temporal mode $k$ to $k+N$ ) corresponds to the generalized teleportation circuit in section 4.3.2 with an input from the previous computation step, or switched into the cluster using an optical switch as previously mentioned. Similar to the generalized teleportation, the resulting operation of one single-mode computation step on a wire is

$$
\hat{S}\left((-1)^{i} 4 t^{2}\right) \hat{R}\left(\frac{\theta_{+}}{2}\right) \hat{S}\left(\tan \frac{\theta_{-}}{2}\right) \hat{R}\left(\frac{\theta_{+}}{2}\right)
$$

where $\theta_{ \pm}=\theta_{B k} \pm \theta_{A k}$, and $t$ is the absolute edge weight in the logic dual-rail wire cluster state in Fig. $4.2 \mathrm{~b}$ which equals $1 / 2$ in the infinite squeezing limit, while the case of finite squeezing is discussed in section 4.4.2. For derivation of (4.10) see appendix section 4.8. The negative edge weight on every second wire (uneven $i$ ) leads to a $\pi$ phase rotation in each computation step which then cancels out in every second step, or can be compensated for in the required basis setting for the desired gate. As for the generalized teleportation, any Gaussian single-mode gate can be implemented in two steps.

Now let us discuss how a two-mode gate can be implemented by coupling two neighboring wires. In Fig. 4.3a all control modes except one has been measured in the basis $(-1)^{i} \theta_{c}=(-1)^{i} \pi / 4$ in order to separate wires as described above - the remaining two central control modes in Fig. 4.3a have only been phase shifted by $\pi / 4$, but not measured. Phase rotating these remaining control modes further before measurements (i.e. measuring them in another bases than the neighbouring control modes), leads to coupling between the two neighbouring wires which is seen as direct edges in Fig. 4.3b. In this way, controlling the measurement bases of a temporal control mode, together with the measurement bases of its neighbouring wires, a desired two-mode gates can be 
a)

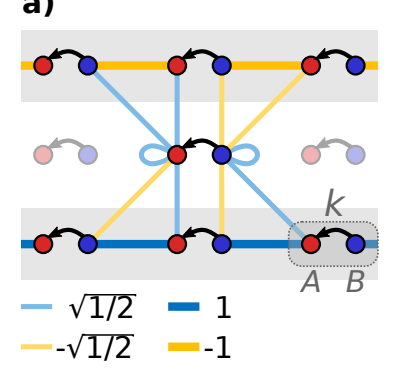

b)

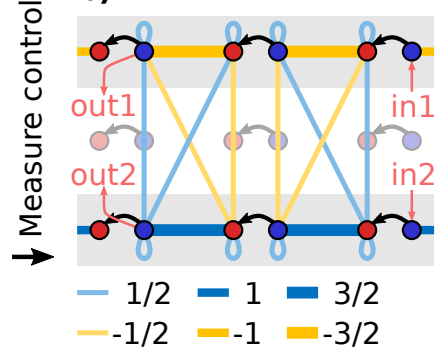

Figure 4.3: (a) Logic cluster state after measuring all temporal control modes in Fig. 4.2c except control modes in temporal mode $k+N-1$. (b) Cluster state after further phase rotation and measurement of the remaining two control modes in (a), leading to direct edges between the neighbouring two wires. The edge weights shown here is for the case of infinite squeezing, $\theta_{c}=\pi / 4$, and the central control modes further phase rotated by $\arctan (1 / 2)$, i.e. $g=1 \mathrm{in} \mathrm{Eq.} \mathrm{(4.11).}$

implemented. As an example, in the infinite squeezing limit of Fig. 4.3, the base setting

$$
\boldsymbol{\theta} \equiv\left(\begin{array}{c}
\theta_{A k-2} \\
\theta_{B k-2} \\
\theta_{A k} \\
\theta_{B k} \\
\theta_{A k+N-2} \\
\theta_{B k+N-2} \\
\theta_{A k+N-1} \\
\theta_{B k+N-1} \\
\theta_{A k+N} \\
\theta_{B k+N}
\end{array}\right)=\left(\begin{array}{c}
(-1)^{i} 3 \pi / 8 \\
-(-1)^{i} \pi / 8 \\
(-1)^{i} 3 \pi / 8 \\
-(-1)^{i} \pi / 8 \\
(-1)^{i} \pi / 4-\arctan (g / 2) \\
-(-1)^{i} \pi / 4 \\
(-1)^{i} \pi / 4+\arctan (g / 2) \\
(-1)^{i} \pi / 4+\arctan (g / 2) \\
(-1)^{i} \pi / 4-\arctan (g / 2) \\
-(-1)^{i} \pi / 4
\end{array}\right)
$$

leads to an implementation of the gate $(\hat{R}(\pi / 4) \otimes \hat{R}(\pi / 4)) \hat{C}_{Z}(g)$ between the blue input modes in temporal mode $k-2$ and $k$, where $\hat{R}(\pi / 4) \otimes \hat{R}(\pi / 4)$ can be compensated for with the following single mode gates.

In summary, we have now shown that a universal Gaussian gate set can be efficiently implemented on a DBSL cluster state: Single mode gates can be realized along parallel wires in the cluster while the two-mode controlled-Z gate can be realized between neighboring wires.

\subsubsection{Gate noise analysis}

As mentioned previously, if the squeezed states used to construct the cluster state are infinitely squeezed, the gates will be realized perfectly without noise addition, thus without adding any processing errors. However, in a realistic setting, the degree of squeezing is finite which inevitably will result in processing noise. In the following we will be analysing the impact it has when using the DBSL for computation.

Assuming that the two squeezed inputs states of the circuit in Fig. 4.2a have squeezed variances of $e^{-2 r}$ in the $\hat{x}$ - or $\hat{p}$-quadrature, the edge weights and self-loops of the coiled up 1D cluster state at the logic level becomes $\pm t= \pm \tanh (2 r) / 2$ and $i \varepsilon=i \operatorname{sech}(2 r)$ respectively [7,31]. Note that the existence of self-loops is a result of the finite input squeezing while $\sqrt{\varepsilon}$ can be considered as the effective momentum squeezing in the cluster state modes.

The finite squeezing leads to two effects: Gate noise appearing in each computation step and distortion of the implemented gate. As seen in Eq. (4.10), for single mode gates the distortion is caused by an additional squeezing transformation, $\hat{S}\left((-1)^{i} \tanh ^{2}(2 r)\right)$, on the output of each computation step. However, as for generalized teleportation, the unwanted squeezing transformation can be compensated for simply by tuning the basis settings. The gate noise (introduced in section 4.3.2 and corresponding to the second term of Eq. (4.2)) of one single-mode computation step from 
temporal mode $k$ to $k+N$ (derived in the appendix section 4.8) is represented by the following quadratures

$$
\mathbf{N}\left(\hat{p}_{A k-1}, \hat{p}_{A k}, \hat{p}_{A k+1}, \hat{p}_{B k+N-1}, \hat{p}_{B k+N}, \hat{p}_{B k+N+1}\right)^{T}
$$

with

$$
\mathbf{N}=\left(\begin{array}{cccccc}
\frac{1}{4 t} & \frac{-1}{4 t^{2}} & \frac{-1}{4 t} & \frac{1}{4 t} & 0 & \frac{1}{4 t} \\
t & 0 & t & t & 1 & -t
\end{array}\right)
$$

leading to quadrature noise factors (introduced in section 4.3.2) of

$$
\begin{aligned}
& N_{x}=\sum_{j} N_{1 j}^{2}=\frac{1}{\tanh ^{4}(2 r)}+\frac{1}{\tanh ^{2}(2 r)} \\
& N_{p}=\sum_{j} N_{2 j}^{2}=\tanh ^{2}(2 r)+1
\end{aligned}
$$

in $\hat{x}$ and $\hat{p}$ respectively.

To avoid accumulating gate noise during computation, we consider the usage of GKP-encoded qubit states, in which the gate noise is translated into qubit errors by quadrature corrections after each implemented gate using auxiliary GKP-states as described in section 4.3.3. To prevent erroneous computation, the qubits may then be error corrected by including a qubit error correction scheme in the computation. Within the GKP-encoded qubit subspace, a logic complete Clifford gate set is realized by the Gaussian gate set $\left\{\hat{I}, \hat{F}, \hat{P}(1), \hat{C}_{Z}(1)\right\}$ on the bosonic modes. We therefore only consider the implementation of this gate set in the noisy cluster state. An additional nonClifford gate in the GKP-qubit subspace completes the gate set for universal qubit computation, and is further discussed in section 4.6.2.

Similar to the generalized teleportation circuit in section 4.3 .2 (Eq. (4.3)), but by substituting the edge weights, $t$, with $(-1)^{i} \tanh ^{2}(2 r)$, the single-mode $\hat{I}$-gate is implemented from temporal mode $k$ to $k+N$ with the basis setting

$$
\left(\begin{array}{l}
\theta_{+} \\
\theta_{-}
\end{array}\right)_{I}=\left(\begin{array}{c}
0 \\
(-1)^{i} 2 \arctan \left(\tanh ^{-2}(2 r)\right)
\end{array}\right),
$$

where $\theta_{ \pm}=\theta_{B k} \pm \theta_{A k}$, and with gate noise variance of $N_{x} \varepsilon / 2$ and $N_{p} \varepsilon / 2$ in $\hat{x}$ - and $\hat{p}$-quadrature respectively. The $\hat{F}$ - and $\hat{P}(1)$-gate are implemented in two computation steps from mode $k$ to $k+2 N$ : Choosing

$$
\left(\begin{array}{l}
\theta_{+1} \\
\theta_{-1} \\
\theta_{+2} \\
\theta_{-2}
\end{array}\right)_{F}=\left(\begin{array}{c}
\pi / 2 \\
\pi / 2 \\
0 \\
2 \arctan \left(\tanh ^{-4}(2 r)\right)
\end{array}\right)
$$

implements $\hat{F}$ with equal gate noise variance of $\left(N_{x}+N_{p}\right) \varepsilon / 2$ in $\hat{x}$ and $\hat{p}$, while

$$
\left(\begin{array}{c}
\theta_{+1} \\
\theta_{-1} \\
\theta_{+2} \\
\theta_{-2}
\end{array}\right)_{P}=\left(\begin{array}{c}
\arctan 2 \\
-\arctan 2 \\
\pi / 2 \\
\pi / 2
\end{array}\right)
$$

implements $\hat{P}(1)$ with gate noise variance of $2 N_{x} \varepsilon / 2$ and $2 N_{p} \varepsilon / 2$ in $\hat{x}$ and $\hat{p}$ respectively. Here

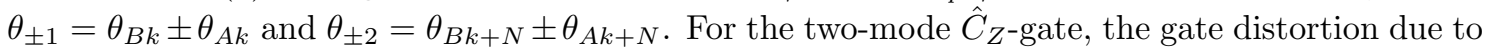
finite squeezing, and how it is compensated for, is less trivial. In the following, we search for basis settings that compensate for finite squeezing and optimizes the gate noise in order to minimize the error probability of the encoded qubit after quadrature corrections.

The GKP quadrature corrections can be realized by implementing the circuit in Eq. (4.7) at mode $B$ in the logic level in Fig. 4.2 where the processed state is encoded. This may be challenging, as it requires tunable $\hat{C}_{Z}(g)$-coupling strengths with $g=1$ when performing error correction, and 
otherwise $g=0$. An alternative is to occupy the free wires with ancillary $\left|0_{L}\right\rangle$ GKP-states, and then implement the required $\hat{C}_{Z}(1)$-gates through measurements. However, with this approach, the error-correcting gate is subjected to the same kind of gate noise as we are trying to correct for in the encoded state. For simplicity, we assume successful implementation of the quadrature correction circuit in Eq. (4.7) at the logic level using a supply of ancillary GKP-states with quadrature symmetric spike variance equal the variance of the resource squeezing, $\delta=e^{-2 r} / 2$.

As discussed in section 4.4.1 and illustrated in Fig. 4.3, the $\hat{C}_{Z}(1)$-gate between two wires is implemented in two computation steps, and while staying within the encoded qubit subspace, we are allowed to implement any $\hat{C}_{Z}(1)$-gate with a bi-product of gates in $\{\hat{F}, \hat{P}(1)\}$ in order to minimize the resulting GKP-encoded qubit errors. The $\hat{P}(1)$-gate transforms quadratures as $(\hat{x}, \hat{p}) \rightarrow(\hat{x}, \hat{x}+\hat{p})$, which, before adding gate noise, already leads to an increase of the spike variances in the GKP-encoded state as $(\delta, \delta) \rightarrow(\delta, 2 \delta)$, where the first and second index corresponds to variance in the $\hat{x}$ - and $\hat{p}$-quadrature respectively. Thus adding $\hat{P}(1)$-gates to $\hat{C}_{Z}(1)$ will hardly improve the error probability. On the other hand, the $\hat{F}$-gate transforms the quadratures as $(\hat{x}, \hat{p}) \rightarrow(\hat{p},-\hat{x})$, and the GKP-spike variance in each quadrature (before adding gate noise) is unchanged. Hence, we may improve the error probability if we can improve the resulting gate noise by adding $\hat{F}$-gates to the $\hat{C}_{Z}(1)$-gate. We have investigated the gates $\left(\hat{F}^{n} \otimes \hat{F}^{m}\right) \hat{C}_{Z}(1)$ for all $n, m \in\{-1,0,1,2\}$, and find that gates with $n, m= \pm 1$ are optimal. We choose $n=1$ and $m=(-1)^{i}$, where the index $i$ denotes the control modes between the two coupled wires. The improvement on the $\hat{C}_{Z}(1)$ gate noise by adding $\hat{F} \otimes \hat{F}^{ \pm 1}$ may be explained intuitively: The $\hat{F}$-gates rotate the states in computation during the two computation steps implementing $\left(\hat{F} \otimes \hat{F}^{ \pm 1}\right) \hat{C}_{Z}(1)$, and leads to the gate noise being better distributed on the quadratures, similar to the symmetrical distributed gate noise when implementing the single-mode $\hat{F}$-gate as described above. The biproduct of $\hat{F} \otimes \hat{F}^{ \pm 1}$ can then be compensated for by applying the associated single mode gates after GKP error correction. In the following, we first consider the case for even $i$, and to shorten the notation we write $\hat{F} \hat{F} \hat{C}_{Z}$ where the tensor product and $\hat{C}_{Z}$-weight have been ignored.

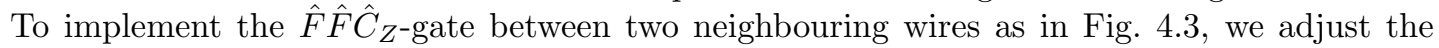
basis setting $\boldsymbol{\theta}$ in Eq. (4.11). Using a global search algorithm, we search for $\boldsymbol{\theta}$ minimizing the objective function

$$
f(\boldsymbol{\theta})=\|\mathbf{G}-\mathbf{T}\|_{1}+w \log P_{\text {err. }}\left(\boldsymbol{\delta}^{\prime}, \delta\right),
$$

where $\mathbf{G}$ and $\mathbf{T}$ are the symplectic matrices of the implemented gate, governed by $\boldsymbol{\theta}$, and the target gate, $\hat{F} \hat{F} \hat{C}_{Z}$ (see appendix section 4.8 for the procedure of calculating $\mathbf{G}$ ), $\|\mathbf{A}\|_{1}=\sum_{i, j}\left|A_{i j}\right|$ is the entrywise matrix 1-norm, and $P_{\text {err. }}$ is the error probability in Eq. (4.8). Here, $\delta=e^{-2 r} / 2$ for the ancillary GKP states, and $\boldsymbol{\delta}^{\prime}=(2 \delta, 2 \delta, \delta, \delta)^{T}+\boldsymbol{\sigma}^{2}$ for the $\hat{F} \hat{F} \hat{C}_{Z}$-gate with gate noise variance $\boldsymbol{\sigma}^{\mathbf{2}}=\left(N_{x 1}, N_{x 2}, N_{p 1}, N_{p 2}\right)^{T} \operatorname{sech}(2 r) / 2$ where $N_{i}$ are basis-dependent quadrature noise factors. The first term of $f(\boldsymbol{\theta})$ in Eq. (4.13) is minimized for $\mathbf{G}=\mathbf{T}$, and thus helps us find the basis setting implementing the target gate $\mathbf{T}$. Since multiple solutions, $\boldsymbol{\theta}$, leading to $\mathbf{G}=\mathbf{T}$ may exist, we search for a solution that also minimizes the error probability, $P_{\text {err. }}$, which is the purpose of the second term in Eq. (4.13). To well resolve $P_{\text {err. }}$ close to 0 we use the logarithm of $P_{\text {err. }}$, while the weight $w$ is varied in the range $10^{-8}$ to 1 for different resource squeezing in order for the global search algorithm not to favour one term in (4.13) while ignoring the other term. Finally, the objective function is considered successfully minimized only when the resulting gate is close to the target gate. To check this, we use the condition

$$
\|\mathbf{G}-\mathbf{T}\|_{1}<10^{-5}
$$

with all results not satisfying this condition being discarded. Depending on the global search algorithm used, we are not guaranteed to find the best basis settings minimizing the error probability. However, repeating the algorithm many times with different $w$ and starting points increases the confidence of the resulting basis settings being optimal.

The resulting bases minimizing the objective function $f(\boldsymbol{\theta})$ for the $\hat{F} \hat{F} \hat{C}_{Z}$ target gate (with even $i$ for the central control mode) is presented in Fig. 4.4a for different resource squeezing levels as input in Fig. 4.2a. In the following we will refer to the mode numbering labelled in Fig. 4.4a. According to Eq. (4.10), with $\left(\theta_{1,3}, \theta_{2,4}\right)=(-\pi / 4, \pi / 4)$ in the first computation step, ignoring 

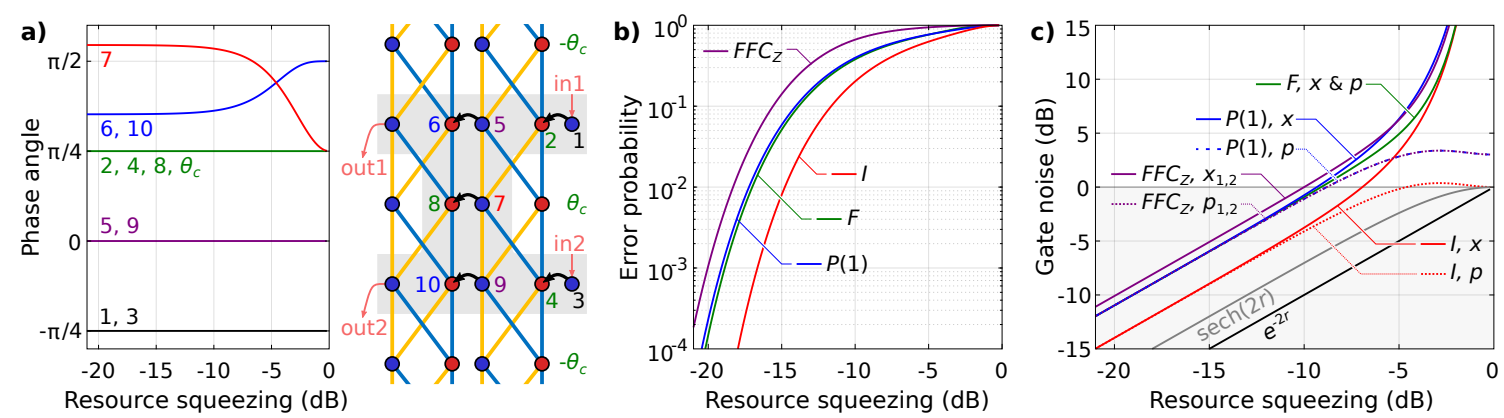

Figure 4.4: (a) Basis settings as function of resource squeezing found by mimizing $f(\boldsymbol{\theta})$ in Eq. (4.13) for implementing the $\hat{C}_{Z}(1)$-gate as $(\hat{F} \otimes \hat{F}) \hat{C}_{Z}(1)$ (in short $\hat{F} \hat{F} \hat{C}_{Z}$ ) for even $i$ on the central control modes. The mode numbers are labelled on the graph to the right, where control modes outside the shaded area are measuring in basis $(-1)^{i} \theta_{c}=(-1)^{i} \pi / 4$. For uneven $i,\left(\hat{F} \otimes \hat{F}^{-1}\right) \hat{C}_{Z}(1)$ is implemented with the same gate noise by changing the sign of the bases in mode $3,4,6,7$, 8, and 10. (b) Resulting error probabilities of Eq. (4.8) for the $\hat{I}-\hat{F}$ - and $\hat{P}(1)$-gate and the $\left(\hat{F} \otimes \hat{F}^{ \pm 1}\right) \hat{C}_{Z}(1)$-gate implemented with the basis settings in (a). (c) Gate noise responsible for the error probability in (b) together with appriximate ancillary GKP states of $e^{-2 r} / 2$ spike variance. Here the resource variance squeezing, $e^{-2 r}$, and effective variance squeezing in the cluster state modes, $\operatorname{sech}(2 r)$, is shown as well (black and grey respectively). The resource squeezing and gate noise in $\mathrm{dB}$-scale is relative to vacuum variance of $1 / 2$. Note that the $\hat{p}$-quadrature gate noise

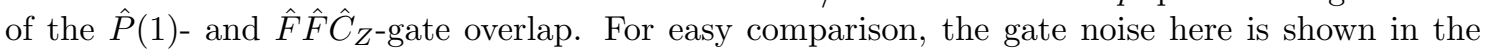
appendix section 4.10 together with gate noise of the schemes considered in section 4.5.

the coupling between wires, the input mode is simply teleported to the second computation step with a bi-product (beside displacement) of $\hat{S}\left(\tanh ^{2}(2 r)\right)$. Here, control mode 8 is measured in the same $\theta_{c}=\pi / 4$ basis used for separating wires, while control mode 7 is measured in a different basis in order to couple the two wires. With the combined basis setting of mode 5, 6, 7, 9 and 10 the bi-product squeezing of the first step is compensated, and the $\hat{F} \hat{F} \hat{C}_{Z}$-gate is implemented. Finally, for uneven $i$ on the control modes between the two coupled wires, the $\left(\hat{F} \otimes \hat{F}^{-1}\right) \hat{C}_{Z}(1)$-gate is implemented by changing the sign on mode $3,4,6,7,8$, and 10 . The resulting gate noise and error probability is the same as for $\hat{F} \hat{F} \hat{C}_{Z}$ with even $i$.

After quadrature correction in the GKP-scheme the resulting error probability of the above described basis settings for the $\hat{I}, \hat{F}$ and $\hat{P}(1)$ single-mode gates and the two-mode $\hat{F} \hat{F} \hat{C}_{Z}$-gate is shown in Fig. 4.4b. As expected, the error probability is seen to go towards 0 for increasing resource squeezing, and towards 1 for vanishing squeezing. Furthermore, the $\hat{F} \hat{F} \hat{C}_{Z}$-gate is seen to have the highest error probability due to four successful quadrature corrections necessary to avoid qubit error, while the $\hat{I}$-gate leads to the lowest error probability as it is implemented in a single computation step. In section 4.6.1 these error probabilities are compared with error probabilities when using other relevant cluster states and computing schemes.

To gain a better understanding of the error probabilities, we consider the responsible gate noise. The gate noise variance, for the basis settings in Fig. 4.4a and described above, is plotted in Fig. 4.4c. In the large squeezing limit, the effective variance squeezing in the cluster state modes of $\operatorname{sech}(2 r)$ is a factor of $2(3 \mathrm{~dB})$ larger than the resource variance squeezing of $e^{-2 r}$, which is the cost of preparing the cluster state with off-line squeezing [5]. The $\hat{I}$-gate, implemented in a single computation step, has a gate noise in the range two times higher than the effective squeezing due to $N_{x}, N_{p} \rightarrow 2$ for $r \rightarrow \infty$. The $\hat{F}$ - and $\hat{P}(1)$-gate have further gate noise of around a factor two, since they are implemented in two computation steps. Finally, the $\hat{F} \hat{F} \hat{C}_{Z}$-gate, also implemented in two computation steps, has similar gate noise, but slightly higher due to the noise of an additional control mode included in the gate to couple two neighbouring wires. The gate noise is in general asymmetric in the quadratures (besides for the $\hat{F}$-gate with equal noise factor in the two quadratures), also for the $\hat{F} \hat{F} \hat{C}_{Z}$-gate with optimized basis settings: Since $P_{\text {err. }}$ in 

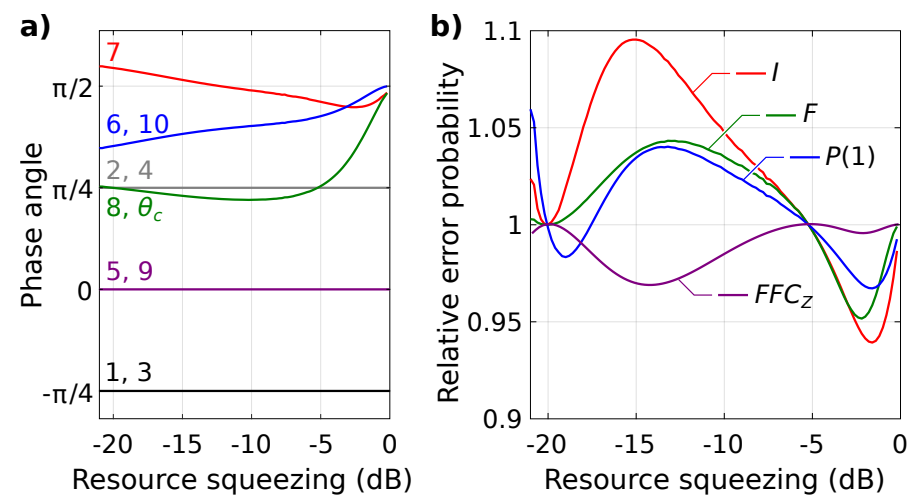

Figure 4.5: (a) Basis setting implementing $(\hat{F} \otimes \hat{F}) \hat{C}_{Z}(1)$ (shortened $\hat{F} \hat{F} \hat{C}_{Z}$ ) for even $i$ with variable control basis $\theta_{c}$ minimizing the error probability of Eq. (4.8), while $\left(\hat{F} \otimes \hat{F}^{-1}\right) \hat{C}_{Z}(1)$ is implemented for uneven $i$ by changing the sign on mode $3,4,6,7,8$ and 10 . Here the mode numbering used is that of Fig. 4.4a. (b) Resulting error probabilities using variable $\theta_{c}$ in (a) optimized for $\hat{F} \hat{F} \hat{C}_{Z}$, relative to the corresponding error probabilities in Fig. 4.4b for fixed $\theta_{c}=\pi / 4$.

Eq. (4.8) rely on the product of quadrature correction success, and because the encoded GKP spike noise is also asymmetric after the $\hat{F} \hat{F} \hat{C}_{Z^{-}}$and $\hat{P}(1)$-gate, the error probability is not necessary minimum with quadrature symmetric gate noise, and at low squeezing we see the majority of the gate noise in one quadrature. Finally, in the vanishing squeezing limit the gate noise diverges. To understand this, consider the Wigner function transformation of the generalized teleportation in Eq. (4.5): With the diverging gate noise variance, the Wigner function is convoluted with infinitely broad Gaussian functions in $\hat{x}$ and subjected to corresponding delta function envelopes in $\hat{p}$, erasing all information of the encoded state. Together with convolutions in the $\hat{p}$-quadrature and corresponding envelopes in the $\hat{x}$-quadrature, the Wigner function is ensured to go towards vacuum for $0 \mathrm{~dB}$ resource squeezing. This is further described in the appendix section 4.9 with the Wigner function transformation of single-mode gates on the DBSL.

\subsubsection{Variable control mode basis}

For simplicity, so far we fixed control mode basis to $\theta_{c}=\pi / 4$, which only leads to unity wire edge weight in the infinite squeezing limit. Allowing variable $\theta_{c}$ implements

$$
\hat{S}\left((-1)^{i} 4 t^{2} \tan \theta_{c}\right) \hat{R}\left(\frac{\theta_{+}}{2}\right) \hat{S}\left(\tan \frac{\theta_{-}}{2}\right) \hat{R}\left(\frac{\theta_{+}}{2}\right)
$$

single-mode gates in each computation step with

$$
\mathbf{N}=\left(\begin{array}{cccccc}
\frac{1}{4 t} & \frac{-1}{4 t^{2} \tan \theta_{c}} & \frac{-1}{4 t} & \frac{1}{4 t} & 0 & \frac{1}{4 t} \\
t \tan \theta_{c} & 0 & t \tan \theta_{c} & t \tan \theta_{c} & 1 & -t \tan \theta_{c}
\end{array}\right)
$$

for the gate noise leading to

$$
\begin{aligned}
& N_{x}=\sum_{j} N_{1 j}^{2}=\frac{1}{\tanh ^{4}(2 r) \tan ^{2} \theta_{c}}+\frac{1}{\tanh ^{2}(2 r)} \\
& N_{p}=\sum_{j} N_{2 j}^{2}=\tanh ^{2}(2 r) \tan ^{2} \theta_{c}+1
\end{aligned}
$$

noise factors. As a result, by varying $\theta_{c}$ we are able to distribute the gate noise between the quadratures in order to minimize the GKP-encoded qubit errors.

To prevent unwanted couplings between wires, $\theta_{c}$ needs to be the same for all gates. With the two-mode $\hat{C}_{Z}(1)$-gate being the gate of largest error probability, we may optimize $\theta_{c}$ to minimize 
the error probability of the $\left(\hat{F} \otimes \hat{F}^{ \pm 1}\right) \hat{C}_{Z}(1)$-gate. In Fig. 4.5 optimized basis settings, and corresponding error probabilities relative to the error probabilities for fixed $\theta_{c}=\pi / 4$, is shown as function of resource squeezing when including $\theta_{c}$ in the objective function in Eq. (4.13). The error probability for the $\left(\hat{F} \otimes \hat{F}^{ \pm 1}\right) \hat{C}_{Z}(1)$-gate, for which $\theta_{c}$ is optimized, is seen at best to decrease to 0.97 of the error probability with fixed $\theta_{c}$, and thus the gain of variable $\theta_{c}$ is little. Furthermore, since $\theta_{c}$ is only optimized for the $\left(\hat{F} \otimes \hat{F}^{ \pm 1}\right) \hat{C}_{Z}(1)$-gate, for some ranges of resource squeezing, the error probabilities for the $\hat{I}$-, $\hat{F}$ - and $\hat{P}(1)$-gate is seen to becomes worse. In conclusion, there may be a small advantage of optimizing $\theta_{c}$, but this depends on the amount of resource squeezing available, and what gates dominate the quantum algorithm to be implemented.

\subsection{Other cluster states}

Besides the DBSL, there are three other interesting cluster states with corresponding self-inverse and bipartite $\mathcal{H}$-graph states and thus realizable with off-line squeezing and linear optics. It counts the quad-rail lattice (QRL) [9] with the efficient computation scheme in Ref. [107]; the bilayer square lattice (BSL) [11, 12], also with an efficient computation scheme; and the recently demonstrated cluster state by Asavanant et al. [37]. In the following, we refer to this last cluster state as the modified bilayer square lattice (MBSL) since computation on this state is similar to computation on the BSL with few modifications. Below, we summarize the computation schemes for each cluster state focusing on the $\left\{\hat{I}, \hat{F}, \hat{P}(1), \hat{C}_{Z}(1)\right\}$ gate set which, together with $\sqrt{\pi}$ displacements in $\hat{x}$ - and $\hat{p}$-quadratures, constitute a universal Clifford gate set in the GKP-encoded qubit subspace. Here, we apply the same search for basis settings that optimize the gate noise in order to minimize qubits errors - as figure of merit, we use the the error probability of Eq. (4.8). For easy comparison, the figures summarizing the different considered schemes and the resulting gate noise are also put together in the appendix section 4.10. The resulting error probabilities are then compared with the error probabilities for the DBSL in the section 4.6.1, while universality through the implementation of a non-Clifford gate in the various schemes is discussed in section 4.6.2.

\subsubsection{Bilayer square lattice}

The 2-dimensional BSL can be generated in the time-frequency domain using a single optical parametric oscillator [11] or solely in time domain using four squeezing sources [12] as summarized in Fig. 4.6a. We emphasize that the time-only encoding of the BSL in [12] is not necessarily more favourable than the frequency-time encoding in [11] — one may even argue that the frequency-time encoding has a better scaling performance. Here, we simply present the time-only encoded version of the setup, since it is comparable to that of the QRL and the MBSL, but it is important to note that the analysis presented in this work holds also for the time-frequency encoded BSL.

The setup in Fig. 4.6a produces a self-inverse and bipartite $\mathcal{H}$-graph state, which under phase rotations is transformed into a cluster state. An efficient universal computation scheme is well described by Alexander et al. $[11,12]$ in the language of macronodes in which each macronode corresponds to the logic level marked in Fig. 4.6a. The computation takes place at this level and the logic cluster state consists of square cluster states as presented in Fig. $4.6 \mathrm{~b}$ with $\pm t=$ $\pm \tanh (2 r) / \sqrt{2}$ edge weight and $i \varepsilon=i \operatorname{sech}(2 r)$ self-loops. The measuring system comprises two joint measurements for each temporal mode $k$ : A joint measurement of the control modes $B$ and $C$ in basis $(-1)^{k} \theta_{c}$ to project the cluster state into wires as shown in Fig. 4.6c, and a joint measurement of the wire modes $A$ and $D$ to implement gates on these wires. As for the DBSL, we find that $\theta_{c}=\pi / 4$ is near optimal. Measuring wire modes $A$ and $B$ of temporal mode $k$ in bases $\theta_{A k}$ and $\theta_{D k}$ implements the single mode gate

$$
\hat{S}\left((-1)^{k+1} 2 t^{2}\right) \hat{R}\left(\frac{\theta_{+}}{2}\right) \hat{S}\left(\tan \frac{\theta_{-}}{2}\right) \hat{R}\left(\frac{\theta_{+}}{2}\right)
$$

from temporal mode $k$ to $k+N$ (1 computation step) where $\theta_{ \pm}=\theta_{D k} \pm \theta_{A k}$. The resulting gate 
a)

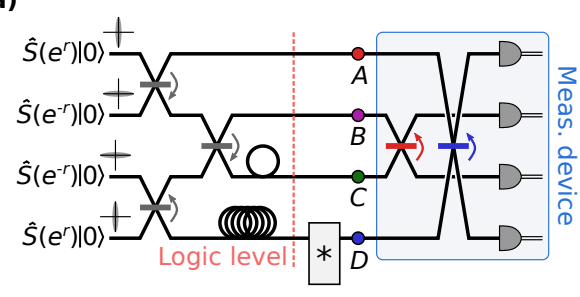

b)

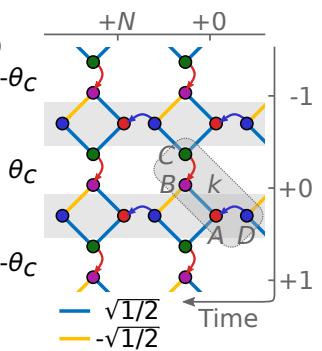

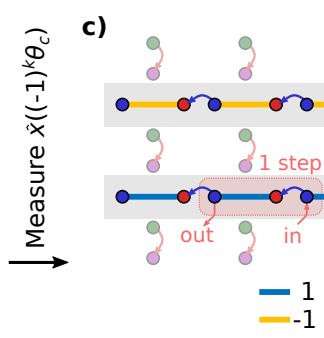
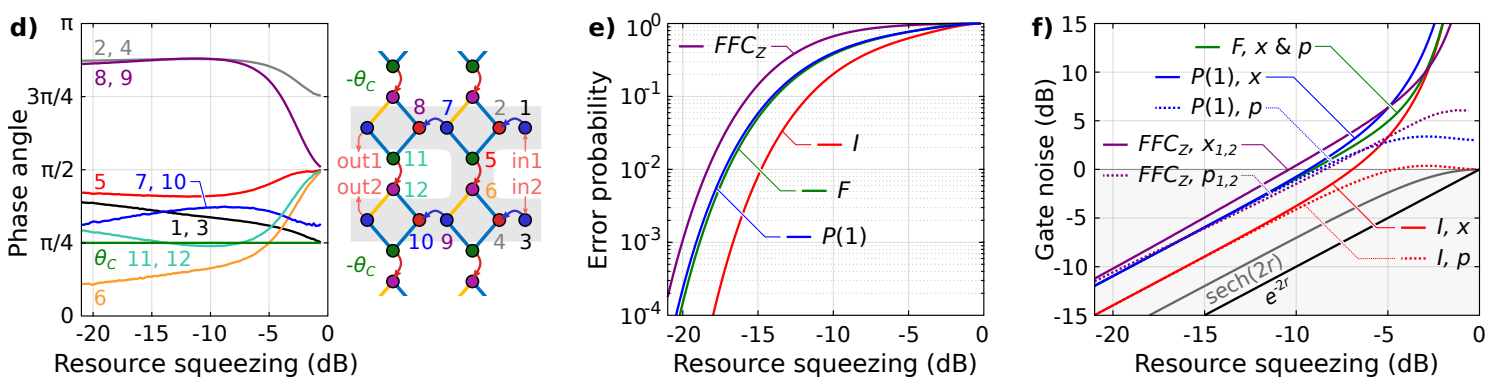

Figure 4.6: Bilayer square lattice (BSL): (a) Experimental setup for generating the $\mathcal{H}$-graph state corresponding to the BSL cluster state [11, 12]. Here the device marked by asterisk is described in Fig. 4.2 for the DBSL, and represents a switch for switching in and out states or a GKP quadrature correction circuit. The logic level in which the computation takes place is marked, and the corresponding logic cluster state is shown in (b) with arrows representing the beam splitters of the measurement device, while in (c) the logic cluster state is projected into wires for computation. The edge weights shown here are in the limit of infinite squeezing and $\theta_{c}=\pi / 4$. (d) Basis setting implementing the $\left(\hat{F} \otimes \hat{F}^{-1}\right) \hat{C}_{Z}(1)$-gate for even temporal modes $k$ with a minimum error probability. For uneven $k,(\hat{F} \otimes \hat{F}) \hat{C}_{Z}(1)$ is implemented by changing the sign of the bases. The error probability in Eq. (4.8) of the single mode $\hat{I}$-, $\hat{F}$-, $\hat{P}(1)$-gate with $\theta_{c}=\pi / 4$, and the $\left(\hat{F} \otimes \hat{F}^{ \pm 1}\right) \hat{C}_{Z}(1)$-gate, are presented in (e) with the corresponding gate noise shown in (f)-here, $\hat{F} \hat{F} \hat{C}_{Z}$ is short for $\left(\hat{F} \otimes \hat{F}^{ \pm 1}\right) \hat{C}_{Z}(1)$. The experimental setup, logic cluster state and its projection into wires, and the resulting gate noise, is shown together with the other considered schemes in the appendix section 4.10 for easy comparison. 
noise for one computation step is $\mathbf{N}\left(\hat{p}_{A k}, \hat{p}_{B k}, \hat{p}_{C k+1}, \hat{p}_{D k+N}\right)^{T}$ where

$$
\mathbf{N}=\left(\begin{array}{cccc}
\frac{1}{2 t^{2}} & \frac{1}{2 t} & -\frac{1}{2 t} & 0 \\
0 & -t & -t & 1
\end{array}\right)
$$

Thus, the variance of the gate noise added to the output quadratures in each computation step is $N_{x} \varepsilon / 2$ and $N_{p} \varepsilon / 2$ for the $\hat{x}$ - and $\hat{p}$-quadrature respectively, where

$$
\begin{aligned}
& N_{x}=\frac{1}{\tanh ^{4}(2 r)}+\frac{1}{\tanh ^{2}(2 r)} \\
& N_{p}=\tanh ^{2}(2 r)+1
\end{aligned}
$$

are quadrature noise factors, $\sum_{j} N_{i j}^{2}$, introduced in section 4.3.2.2, and we note that they are identical to the noise factors of the DBSL. The $\hat{I}$-gate is implemented in a single computation step by choosing

$$
\left(\begin{array}{l}
\theta_{+} \\
\theta_{-}
\end{array}\right)_{I}=\left(\begin{array}{c}
0 \\
(-1)^{k+1} 2 \arctan \left(\tanh ^{-2}(2 r)\right)
\end{array}\right) .
$$

The $\hat{F}$ - and $\hat{P}(1)$-gate are implemented in two computation steps from temporal mode $k$ to $k+2 N$. Choosing basis settings

$$
\left(\begin{array}{c}
\theta_{+1} \\
\theta_{-1} \\
\theta_{+2} \\
\theta_{-2}
\end{array}\right)_{F}=\left(\begin{array}{c}
\pi / 2 \\
\pi / 2 \\
0 \\
2 \arctan \left(\tanh ^{-4}(2 r)\right)
\end{array}\right)
$$

$\hat{F}$ is implemented with equal gate noise variance of $\left(N_{x}+N_{p}\right) \varepsilon / 2$ in $\hat{x}$ - and $\hat{p}$-quadrature, while $\hat{P}(1)$ is realized with

$$
\left(\begin{array}{l}
\theta_{+1} \\
\theta_{-1} \\
\theta_{+2} \\
\theta_{-2}
\end{array}\right)_{P}=\left(\begin{array}{c}
\arctan 2 \\
-\arctan 2 \\
\pi / 2 \\
\pi / 2
\end{array}\right)
$$

resulting in gate noise variances of $2 N_{x} \varepsilon / 2$ and $2 N_{p} \varepsilon / 2$ in $\hat{x}$ - and $\hat{p}$-quadrature, respectively. Here $\theta_{ \pm 1}=\theta_{D k} \pm \theta_{A k}$ and $\theta_{ \pm 2}=\theta_{D k+N} \pm \theta_{A k+N}$. Notice the similarity with the DBSL: The basis settings and gate noises are identical, and the BSL and DBSL are expected to perform single mode gates equally well.

Measuring control modes $B$ and $C$ of one temporal mode in different bases leads to coupling between the two neighbouring wires and thus allow for the implementation of two-mode gates. In Ref. [11], the basis setting for implementing the $\hat{C}_{Z}(g)$-gate is given for the case of infinite squeezing. Here, we extend this analysis by searching the basis setting that minimizes the error probability of two encoded qubits after the $\hat{C}_{Z}(1)$-gate for the more relevant case of finite squeezing. To do so, we use the same technique as for the DBSL by minimizing the objective function in Eq. (4.13). Note that to compensate for finite squeezing distortion $\left(\right.$ as $\hat{S}\left( \pm \tanh ^{2}(2 r)\right)$ in Eq. (4.14) for single mode gates), two computation steps are required to implement $\hat{C}_{Z}(1)$. For all $\left(\hat{F}^{n} \otimes \hat{F}^{m}\right) \hat{C}_{Z}(1)$ gates with $n, m=0,1,2,3$ we find the lowest error probability for $n, m= \pm 1$ and we choose $(n, m)=\left(1,(-1)^{k+1}\right)$ where $k$ is the temporal mode index of the control modes coupling the two wires. The resulting basis settings implementing the $\left(\hat{F} \otimes \hat{F}^{-1}\right) \hat{C}_{Z}(1)$-gate are shown in Fig. 4.6d for even $k$, while for uneven $k$ the $(\hat{F} \otimes \hat{F}) \hat{C}_{Z}(1)$-gate is implemented with equal error probability by changing the sign of all bases in Fig. 4.6d. In case we allow for a variable $\theta_{c}$ in the objective function of Eq. (4.13), we find no improvement of the error probability, and we conclude there will be no gain of a variable $\theta_{c}$ when implementing the $\left(\hat{F} \otimes \hat{F}^{ \pm 1}\right) \hat{C}_{Z}(1)$-gate.

Note how the basis settings in Fig. 4.6d, different from the DBSL in Fig. 4.4a and the MBSL later in Fig. 4.7d, seem to depend on the resource squeezing in the full shown squeezing range. The reason for this is that there exist multiple solutions for basis settings that implement a desired $\hat{C}_{Z}(1)$-gate with a minimum error probability. The same is the case for the DBSL and MBSL, 
however, in Fig 4.4a and 4.7d a more consistent solution set of basis settings as function of resource squeezing is shown, while here for the BSL a slightly inconsistent solution set is shown. This effect often occurs when unnecessarily large degrees of freedom in the basis settings are used when minimizing the objective function in Eq. (4.13). However, this does not mean that the basis settings in Fig. 4.6d are not optimal, but is rather an example of the existence of multiple basis setting solutions and proper use of Eq. (4.13) to derive a suitable solution for a given experimental implementation.

The resulting error probabilities of Eq. (4.8) when correcting the quadratures after the $\hat{I}-, \hat{F}$-, $\hat{P}(1)$ - and $\left(\hat{F} \otimes \hat{F}^{ \pm 1}\right) \hat{C}_{Z}(1)$-gate as described above are presented in Fig. 4.6e. As expected, the two-mode $C_{Z}(1)$-gate is seen to have the highest error probability since four successful quadrature corrections are necessary to avoid inducing an error on the encoded qubits. Finally, the gate noise variances are presented in Fig. 4.6f, and here we clearly see similar behavior as for the DBSL: For infinite squeezing, the $\hat{I}$-gate in one computation step has a gate noise variance of twice the effective variance squeezing, $\operatorname{sech}(2 r)$ (as $N_{x}, N_{p} \rightarrow 2$ when $r \rightarrow \infty$ ), while the $\hat{F}$ - and $\hat{P}(1)$-gates implemented in two computation steps have gate noise variances of four times that. In the other extreme of vanishing squeezing, the gate noise diverges in the $\hat{x}$-quadrature, thereby erasing all information of the encoded state as previously explained for the DBSL. This can also be seen from the corresponding Wigner function transformation in the appendix section 4.9.

\subsubsection{Modified bilayer square lattice}

The experimental setup of the MBSL cluster state, recently generated by Asavanant et al. [37] and summarized in Fig. 4.7a, is very similar to the setup of the all-time encoded BSL in Fig. 4.6a, and we can therefore adopt the computation scheme for the BSL with only a few changes. The corresponding cluster state at the logic level is shown in Fig. 4.7b in which we see that the square clusters of the BSL have been replaced with "butterfly" clusters. As for the BSL, the edge weight and self-loops are $\pm t= \pm \tanh (2 r) / \sqrt{2}$ and $i \varepsilon=i \operatorname{sech}(2 r)$ respectively. The spatial modes $C$ and $D$ of each temporal mode $k$ constitutes wire modes, while $A$ and $B$ are control modes. In contrast to the square clusters in the BSL, the butterfly clusters already contain direct edges in the wires before potential phase rotation of the control modes. Thus we can directly "delete" the control modes by measuring them in the $\hat{x}$-basis, i.e. $\theta_{c}=0$, and implement the operations

$$
\hat{S}(t) \hat{R}\left(\frac{\theta_{+}}{2}\right) \hat{S}\left(\tan \frac{\theta_{-}}{2}\right) \hat{R}\left(\frac{\theta_{+}}{2}\right) \quad\left(\theta_{c}=0\right)
$$

in one computation step from temporal mode $k$ to $k+N$ with $\theta_{ \pm}=\theta_{C k} \pm \theta_{D k}$. The resulting gate noise is $\mathbf{N}\left(\hat{p}_{D k}, \hat{p}_{A k}, \hat{p}_{B k+1}, \hat{p}_{C k+N}\right)^{T}$ with

$$
\mathbf{N}=\left(\begin{array}{cccc}
-\frac{1}{t} & 0 & 0 & 0 \\
0 & 0 & 0 & 1
\end{array}\right) \quad\left(\theta_{c}=0\right)
$$

such that the gate noise variance is $N_{x} \varepsilon / 2$ and $N_{p} \varepsilon / 2$ in $\hat{x}$ - and $\hat{p}$-quadrature respectively with quadrature noise factors of

$$
\begin{array}{ll}
N_{x} & =2 / \tanh ^{2}(2 r) \quad\left(\theta_{c}=0\right) . \\
N_{p} & =1
\end{array}
$$

Alternatively, we can measure the control modes in the $\hat{p}$-basis, i.e. $\theta_{c}=\pi / 2$, rearranging the edge weights of the butterfly cluster states to increase the edge weight between wire modes as shown in Fig. 4.7c. In this case, the operation

$$
\hat{S}(2 t) \hat{R}\left(\frac{\theta_{+}}{2}\right) \hat{S}\left(\tan \frac{\theta_{-}}{2}\right) \hat{R}\left(\frac{\theta_{+}}{2}\right) \quad\left(\theta_{c}=\pi / 2\right)
$$

is implemented with the gate noise,

$$
\mathbf{N}=\left(\begin{array}{cccc}
-\frac{1}{2 t} & -\frac{1}{2 t} & 0 & 0 \\
0 & 0 & -1 & 1
\end{array}\right) \quad\left(\theta_{c}=\pi / 2\right)
$$


a)

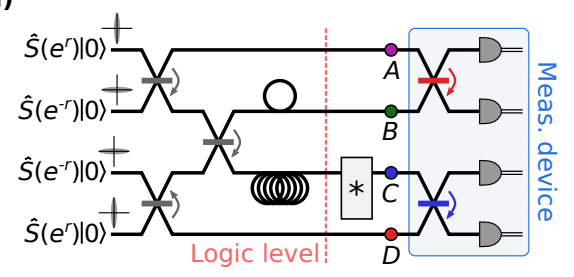

b)

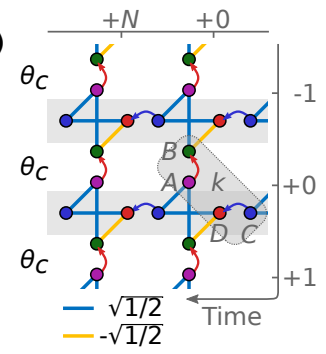

c)

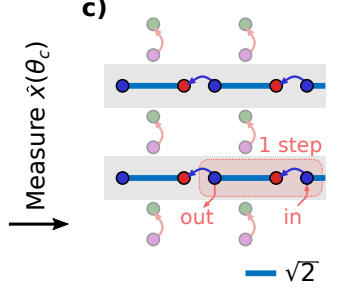

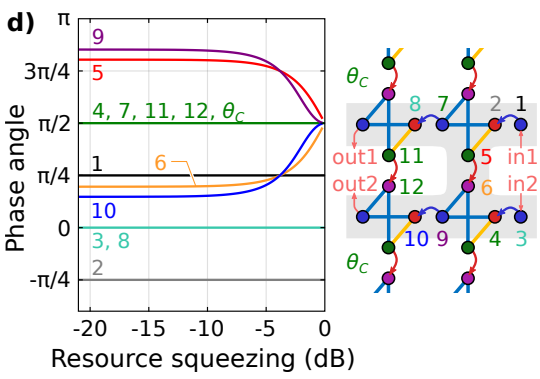
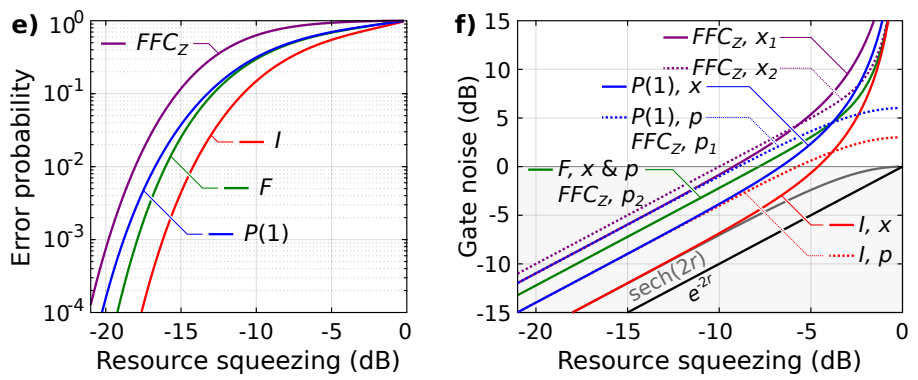

Figure 4.7: Modified bilayer square lattice (MBSL): (a) Experimental setup for generating the $\mathcal{H}$-graph state corresponding to the MBSL cluster state by Asavanant et al. [37]. Here the device marked by asterisk is described in Fig. 4.2 for the DBSL, and represents a switch for switching in and out states or a GKP quadrature corrections circuit. The logic level in which the computation takes place is marked, and the corresponding logic cluster state is shown in (b) with arrows representing the beam splitters of the measurement device, while in (c) the logic cluster state is projected into wires for computation. The edge weights shown here are in the limit of infinite squeezing and $\theta_{c}=\pi / 2$. (d) shows a basis setting implementing the $(\hat{F} \otimes \hat{F}) \hat{C}_{Z}(1)$-gate with a minimum error probability. The error probability in Eq. (4.8) of the single-mode $\hat{I}$-, $\hat{F}$-, and $\hat{P}(1)$-gate with $\theta_{c}=\pi / 2$, and the $\left(\hat{F} \otimes \hat{F}^{ \pm 1}\right) \hat{C}_{Z}(1)$-gate, is presented in (e) with the corresponding gate noise shown in (f)-here $\hat{F} \hat{F} \hat{C}_{Z}$ is short for $(\hat{F} \otimes \hat{F}) \hat{C}_{Z}(1)$. Note that the gate noise variance in the $\hat{p}$-quadratures of each mode for the $(\hat{F} \otimes \hat{F}) \hat{C}_{Z}(1)$-gate each equals that of the $\hat{P}(1)$ and $\hat{F}$ gate. The experimental setup, logic cluster state and its projection into wires, and the resulting gate noise, is shown together with the other considered schemes in the appendix section 4.10 for easy comparison. 
such that

$$
\begin{aligned}
& N_{x}=1 / \tanh ^{2}(2 r) \quad\left(\theta_{c}=\pi / 2\right) . \\
& N_{p}=2
\end{aligned}
$$

Other values of $\theta_{c}$ are also possible, but in this case the implemented gate as well as gate noise is less trivial. However, in the later analysis of the $\hat{C}_{Z}(1)$-gate we do find that $\theta_{c}=\pi / 2$ is indeed optimal. Notice that - unlike the BSL with square cluster states - all control modes are measured in the same basis without an alternating sign for different temporal modes. This is because the wire modes are directly connected with equal edge weights for all temporal modes, or connected with three edges through two control modes, while for the square cluster states, wire modes have two connections, each through a single control mode, but with different sign on the edge weights depending on whether the control mode is in the next or previous temporal mode.

For $\theta_{c}=\pi / 2$, the basis setting

$$
\left(\begin{array}{l}
\theta_{+} \\
\theta_{-}
\end{array}\right)_{I}=\left(\begin{array}{c}
0 \\
2 \arctan \left(\tanh ^{-1}(2 r) / \sqrt{2}\right)
\end{array}\right)
$$

with $\theta_{ \pm}=\theta_{C k} \pm \theta_{D k}$ implements the $\hat{I}$-gate in one computation step from temporal mode $k$ to $k+N$ and gate noise variance $N_{x} \varepsilon / 2$ and $N_{p} \varepsilon / 2$ in $\hat{x}$ - and $\hat{p}$-quadrature respectively. The basis setting

$$
\left(\begin{array}{c}
\theta_{+1} \\
\theta_{-1} \\
\theta_{+2} \\
\theta_{-2}
\end{array}\right)_{F}=\left(\begin{array}{c}
\pi / 2 \\
\pi / 2 \\
0 \\
2 \arctan \left(\tanh ^{-2}(2 r) / 2\right)
\end{array}\right)
$$

implements the $\hat{F}$-gate in two computation steps with equal $\left(N_{x}+N_{p}\right) \varepsilon / 2$ gate noise variance in $\hat{x}$ - and $\hat{p}$-quadrature, while

$$
\left(\begin{array}{l}
\theta_{+1} \\
\theta_{-1} \\
\theta_{+2} \\
\theta_{-2}
\end{array}\right)_{P}=\left(\begin{array}{c}
\arctan 2 \\
-\arctan 2 \\
\pi / 2 \\
\pi / 2
\end{array}\right)
$$

implements the $\hat{P}(1)$-gate in two computation steps with $2 N_{x} \varepsilon / 2$ and $2 N_{p} \varepsilon / 2$ gate noise variance in $\hat{x}$ - and $\hat{p}$-quadrature respectively. Here, $\theta_{ \pm 1}=\theta_{C k} \pm \theta_{D k}$ and $\theta_{ \pm 2}=\theta_{C k+N} \pm \theta_{D k+N}$ when implementing $\hat{F}$ and $\hat{P}(1)$ from temporal mode $k$ to $k+2 N$.

To couple pairs of wires for the implementation of a two-mode gate, one measures the control modes $A$ and $B$ of one temporal mode $k$ in different bases by which a coupling between the wires in temporal modes $k-1$ and $k$ is induced. The $\hat{C}_{Z}(1)$-gate is again implemented in two computation steps, and similar as for the BSL and the DBSL, we search the basis setting that minimizes the objective function in Eq. (4.13) and thus the error probability in Eq. (4.8) of that particular gate. Again, we need to investigate all $\left(\hat{F}^{n} \otimes \hat{F}^{m}\right) \hat{C}_{Z}(1)$-gates for $n, m=0,1,2,3$ and find $n=m=1$ to be optimal. The resulting basis setting is shown in Fig. $4.7 \mathrm{~d}$ where $\theta_{c}=\pi / 2$ is found to be optimal. Note that, unlike the DBSL and BSL, this basis setting is independent on the temporal mode index $k$, as the control basis does not have an alternating sign governed by $k$.

The resulting error probability of the single mode $\hat{I}$-, $\hat{F}$ - and $\hat{P}(1)$-gate, and the two-mode $(\hat{F} \otimes \hat{F}) \hat{C}_{Z}(1)$-gate, with the basis settings described above and in Fig. 4.7d, is shown in Fig. 4.7e. The single mode gates are all seen to have a lower error probability than in computations with the DBSL and BSL cluster states. This is explained by the lower quadrature noise factors, $N_{x}$ and $N_{p}$, due to the structure of the butterfly cluster states with initial edges between wire modes before projecting the logic cluster state into wires. As expected, due to the four quadrature corrections, the error probability of the $(\hat{F} \otimes \hat{F}) \hat{C}_{Z}(1)$-gate is largest. Gate noise variances are shown in Fig. 4.7f. For single mode gates, in general we see lower gate noise variance than for the DBSL and BSL, and in the large squeezing limit where $N_{x} \rightarrow 1$ for $r \rightarrow \infty$ and $\theta_{c}=\pi / 2$, while $N_{p}=2$, we see the gate noise variances in $\hat{x}$-quadratures of the $\hat{I}$-gate to equal the effective squeezing variance of $\operatorname{sech}(2 r)$. For vanishing squeezing, the gate noise variance diverges in the $\hat{x}$-quadrature, erasing 

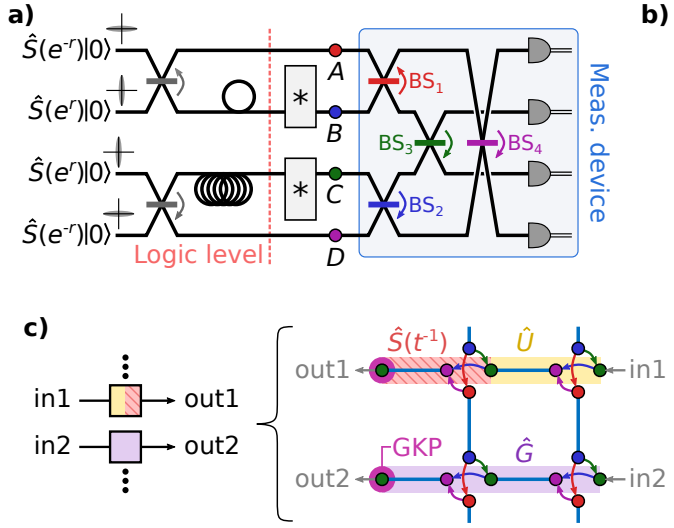

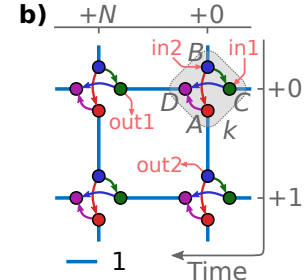

d)

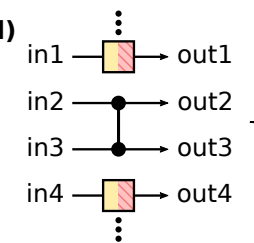

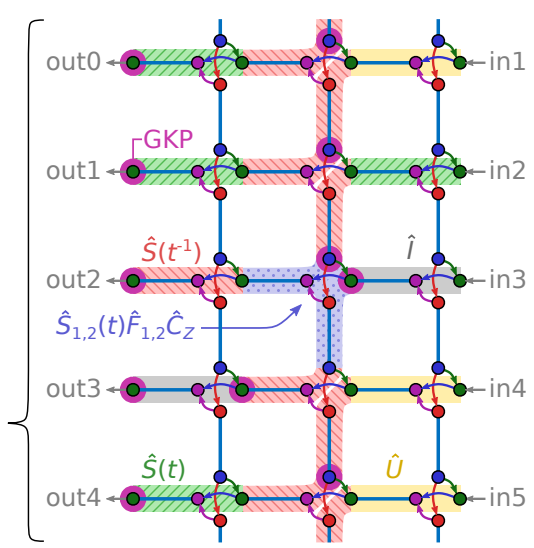

Figure 4.8: Quad-rail lattice (QRL): (a) Experimental setup for generating the $\mathcal{H}$-graph state corresponding the the QRL cluster state. Here, the devices marked by asterisk are described in Fig. 4.2 and represent optical switches switching in and out states or GKP quadrature correction circuits as in Eq. (4.7). The logic level in which the computation takes place is marked, with the corresponding logic cluster state shown in (b) with arrows representing the beam-splitter network of the measurement device. The edge weight shown is in the infinite squeezing limit, while for finite squeezing it is $t=\tanh (2 r)$, while $i \varepsilon=i \operatorname{sech}(2 r)$ self-loops are present on all nodes. Temporal mode indices are written in grey, while a single computation step is marked with input modes 'in1' and 'in2' and output modes 'out1' and 'out2'. (c) Example of single mode computation along the cluster state cylinder by restricting the bases with $\theta_{A k}=\theta_{D k}$ and $\theta_{B k}=\theta_{C k}$, thereby implementing $\hat{U}$ in Eq. (4.17) in each computation step. With two computation steps, the $\hat{S}(t)$ distortion in Eq. (4.17) due to finite squeezing can be compensated in the second step by implementing $\hat{S}\left(t^{-1}\right)$. Or, more generally, any single-mode Gaussian gate can be implemented as $\hat{G}=\hat{U}_{1} \hat{U}_{2}$. Here, input modes in spatial modes $B$ (blue) are ignored. After implemention of gates, the output modes marked with a pink circle are quadrature corrected. (d) Example of implementing the $(\hat{F} \otimes \hat{F}) \hat{C}_{Z}(1)$-gate between input modes 'in2' and 'in3'. Since all gates are implemented on pairs of modes, first one computation step is required to guide the in2 and in3 modes to the $(\hat{F} \otimes \hat{F}) C Z(1)$-gate, while gates of the form $\hat{S}\left(t^{-1}\right) \hat{U}$ can be implemented on other computation modes. After the $(\hat{F} \otimes \hat{F}) C Z(1)$ gate, all computation modes are aligned to the same vertical position in the lattice using $\hat{I}$-gates (notice that $\hat{S}\left(t^{ \pm 1}\right) \hat{S}\left(t^{\mp 1}\right)=\hat{I}$ ). To prevent accumulating gate noise, GKP quadrature correction are performed after every implemented gate on modes marked with pink circle. The experimental setup and logic cluster state is shown together with the other considered schemes in the appendix section 4.10 for easy comparison.

all information of the encoded state as is also the case for computing with BSL and DBSL (also eluded by the Wigner function transformation in the appendix section 4.9). Notice that, unlike the DBSL and BSL, the gate noise of the $(\hat{F} \otimes \hat{F}) \hat{C}_{Z}(1)$-gate is not symmetric in the quadratures of the two modes.

\subsubsection{Quad-rail lattice}

In Ref. [8], it was proposed to generate a cluster state with a quad-rail lattice (QRL) structure in the frequency domain from a single optical parametric oscillator while in Ref. [9] it was suggested to construct a time domain version of the QRL clusters state. With temporal encoding, the generated state has a cylindrical topology reminiscent of the DBSL, BSL and MBSL, allowing for computation along the cylinder with information encoded on the circumference of the cylinder. The scheme for generating the temporally encoded QRL state is summarized in Fig. 4.8a. Since the QRL is self-inverse and bipartite, this QRL $\mathcal{H}$-graph state has a corresponding QRL cluster state (under phase rotations) which we consider in the following. 
An efficient computation scheme on the QRL cluster state is presented in [107] in the language of macronodes. It corresponds to the logic level marked on Fig. 4.8a which is followed by a measurement device consisting of a beam-splitter network of four beam splitters $\left(\mathrm{BS}_{1-4}\right)$ and four homodyne detections. The cluster state at the logic level is shown in Fig. 4.8b. With the logic level at a beam-splitter depth of only one, the edge weight of $t=\tanh (2 r)$ is larger than in the DBSL, BSL and MBSL, while the self-loops are equal, $i \varepsilon=i \operatorname{sech}(2 r)$. The logic cluster state consists of two-mode entangled states as in the generalized teleportation circuit in section 4.3.2, and no projection of the cluster state into wires before computation is necessary. This, together with the larger edge weight, reduces the gate noise and thus makes computation on the QRL more efficient. On the other hand, the increased complexity of the measurement device (a joint measurements of four modes) makes the computation scheme presented here more tricky and may seem less intuitive.

One computation step is marked in Fig. 4.8b. It implements a two-mode operation from input modes $C k$ (in1) and $B k$ (in2) to the output modes $C k+N$ (out1) and $B k+1$ (out2). In the following, we will refer to the mode in computation from in1(2) to out1(2) as computation mode $1(2)$. It is possible to decouple the two computation modes 1 and 2 by restricting the basis settings to $\theta_{A k}=\theta_{D k}$ and $\theta_{B k}=\theta_{C k}$. In the same way as in Eq. (4.9), this effectively cancel the the beam splitter $\mathrm{BS}_{3}$ and $\mathrm{BS}_{4}$, since equal phase shifts commute with the beam splitter. Then, single-mode gates can be implemented using $\mathrm{BS}_{1}$ and $\mathrm{BS}_{2}$ in the same way as for the generalized teleportation in section 4.3.2, but the same gate will be applied to both computation modes 1 and 2 due to the basis restriction. That is, $\hat{U} \otimes \hat{U}$ will be implemented, where

$$
\hat{U}=\hat{S}(\tanh (2 r)) \hat{R}\left(\frac{\theta_{+}}{2}\right) \hat{S}\left(\tan \frac{\theta_{-}}{2}\right) \hat{R}\left(\frac{\theta_{+}}{2}\right)
$$

with $\theta_{ \pm}=\theta_{C k} \pm \theta_{D k}$. Similarly, using the basis permutation rules in [107], restricting to $\theta_{D k}=\theta_{B k}$ and $\theta_{A k}=\theta_{C k}$ implements $\hat{U} \otimes \hat{U}$ on modes from in1 and in2 to out2 and out1, respectively. As a result, when implementing $\hat{U} \otimes \hat{U}$, the modes in computation may travel straight across each other on the cluster state lattice or they may do a $90^{\circ}$ change in their computation direction on the lattice depending on the basis restriction. For implementation of single mode gates, we will mainly focus on the former case in which one mode (computation mode 1) travels in the direction of the cluster state cylinder, while the other mode (computation mode 2), travelling around the cylinder, is ignored as illustrated in Fig. 4.8c. Regardless of the basis restriction, the gate noise of one computation step is $\mathbf{N}\left(\hat{p}_{A k}, \hat{p}_{D k}, \hat{p}_{B k+1}, \hat{p}_{C k+N}\right)^{T}$ with

$$
\mathbf{N}=\left(\begin{array}{cccc}
-\frac{1}{\tanh (2 r)} & 0 & 0 & 0 \\
0 & -\frac{1}{\tanh (2 r)} & 0 & 0 \\
0 & 0 & 1 & 0 \\
0 & 0 & 0 & 1
\end{array}\right)
$$

leading to equal quadrature noise factors in the two computation modes of

$$
\begin{aligned}
& N_{x}=\frac{1}{\tanh ^{2}(2 r)} \\
& N_{p}=1
\end{aligned}
$$

in $\hat{x}$ - and $\hat{p}$-quadratures, respectively.

As for the generalized teleportation circuit, the single-mode $\hat{I}$-gate is implemented in a single computation step with basis setting

$$
\left(\begin{array}{c}
\theta_{+} \\
\theta_{-}
\end{array}\right)_{I}=\left(\begin{array}{c}
0 \\
2 \arctan \left(\tanh ^{-1}(2 r)\right)
\end{array}\right)
$$

with gate noise variance $N_{x} \varepsilon / 2$ and $N_{p} \varepsilon / 2$ in $\hat{x}$ - and $\hat{p}$-quadratures. The $\hat{F}$ - and $\hat{P}(1)$-gates are 
implemented in two computation steps: With basis setting

$$
\left(\begin{array}{l}
\theta_{+1} \\
\theta_{-1} \\
\theta_{+2} \\
\theta_{-2}
\end{array}\right)_{F}=\left(\begin{array}{c}
\pi / 2 \\
\pi / 2 \\
0 \\
2 \arctan \left(\tanh ^{-2}(2 r)\right)
\end{array}\right)
$$

$\hat{F}$ is implemented with equal gate noise variance in $\hat{x}$ and $\hat{p}$ of $\left(N_{x}+N_{p}\right) \varepsilon / 2$, while

$$
\left(\begin{array}{l}
\theta_{+1} \\
\theta_{-1} \\
\theta_{+2} \\
\theta_{-2}
\end{array}\right)_{P}=\left(\begin{array}{c}
\arctan 2 \\
-\arctan 2 \\
\pi / 2 \\
\pi / 2
\end{array}\right)
$$

implements $\hat{P}(1)$ with gate noise variances of $2 N_{x} \varepsilon / 2$ and $2 N_{p} \varepsilon / 2$ in $\hat{x}$ and $\hat{p}$, respectively. Here, for the mode in computation travelling straight along the cylinder, $\theta_{ \pm 1}=\theta_{C k} \pm \theta_{D k}$ and $\theta_{ \pm 2}=$ $\theta_{C k+N} \pm \theta_{D k+N}$ while $\left(\theta_{B k}, \theta_{A k}\right)=\left(\theta_{C k}, \theta_{D k}\right)$ and $\left(\theta_{B k+N}, \theta_{A k+N}\right)=\left(\theta_{C k+N}, \theta_{D k+N}\right)$.

To implement the $\hat{C}_{Z}(1)$-gate, we have investigated $\left(\hat{F}^{n} \otimes \hat{F}^{m}\right) \hat{C}_{Z}(1)$ for $n, m=0,1,2,3$ and find that $n=m=1$ leads to the lowest error probability in Eq. (4.8) of the GKP-encoded qubits. With the basis setting

$$
\left(\begin{array}{l}
\theta_{A k} \\
\theta_{B k} \\
\theta_{C k} \\
\theta_{D k}
\end{array}\right)_{C_{Z}}=\left(\begin{array}{c}
\pi / 2-\arctan (1 / 2) \\
0 \\
\pi / 2+\arctan (1 / 2) \\
0
\end{array}\right)
$$

$(\hat{S}(t) \otimes \hat{S}(t))(\hat{F} \otimes \hat{F}) \hat{C}_{Z}(1)$ is implemented in a single computation step, where the two modes in computation goes from in1 and in2 to out2 and out1 respectively (i.e. they do not cross, but each mode is redirected $\left.90^{\circ}\right)$. Here, $(\hat{S}(t) \otimes \hat{S}(t))$ is the distortion due to finite squeezing, and is compensated for in each computation mode in a second computation step with basis setting

$$
\left(\begin{array}{l}
\theta_{+} \\
\theta_{-}
\end{array}\right)_{S\left(t^{-1}\right)}=\left(\begin{array}{c}
0 \\
2 \arctan \left(\tanh ^{-2}(2 r)\right)
\end{array}\right) .
$$

As a result, $(\hat{F} \otimes \hat{F}) \hat{C}_{Z}(1)$ is implemented in two computation steps with equal gate noise variance in all four quadratures of $\left(N_{x}+N_{p}\right) \varepsilon / 2$ as for the $\hat{F}$-gate. As gates on the QRL are in general performed on pairs of modes in computation and requires two computation steps (with the exception of the $\hat{I}$-gate), implementing $(\hat{F} \otimes \hat{F}) \hat{C}_{Z}(1)$ among other computation modes may be tricky. However, an example of a possible implementation is shown in Fig. 4.8d.

The gate noise variance for each of the implemented gates in $\left\{\hat{I}, \hat{F}, \hat{P}(1),(\hat{F} \otimes \hat{F}) \hat{C}_{Z}(1)\right\}$ is shown in Fig. $4.9 \mathrm{a}$ as a function of the initial squeezing of the $\hat{p}$-quadrature variance in the resource state, $e^{-2 r}$. Notice that in the high squeezing limit, the gate noise of the $\hat{I}$-gate is equal to the effective variance squeezing of the cluster state modes, $\operatorname{sech}(2 r)$, which is better than seen for the other computation schemes presented in this work, and is due to the large edge weight in the logic cluster state with no projection of the cluster state necessary before computation. The $\hat{F}$ - and $\hat{P}(1)$-gate, implemented in two computation steps, naturally has double gate noise compared to the $\hat{I}$-gate, and so does the $(\hat{F} \otimes \hat{F}) \hat{C}_{Z}(1)$-gate, unlike the $\hat{C}_{Z}(1)$-gates implemented on the DBSL, BSL and MBSL. This improvement for the $(\hat{F} \otimes \hat{F}) \hat{C}_{Z}(1)$-gate happens because no extra control modes are included when coupling two computation modes. In the limit of vanishing resource squeezing, the gate noise variance of each computation diverges in the $\hat{x}$-quadrature, erasing all information of the encoded state as for the generalized teleportation circuit in Eq. (4.5).

To prevent gate noise accumulating on the GKP-encoded qubits, quadrature corrections as described in section 4.3.3 should be performed on modes in computation after each implemented gate. Here, with two computation modes in each computation step, two quadrature correction devices are necessary: One in each spatial mode $B$ and $C$ as marked in Fig. 4.8a. After quadrature correcting modes as shown on the examples in Fig. 4.8c and d, the error probabilities of Eq. (4.8) 

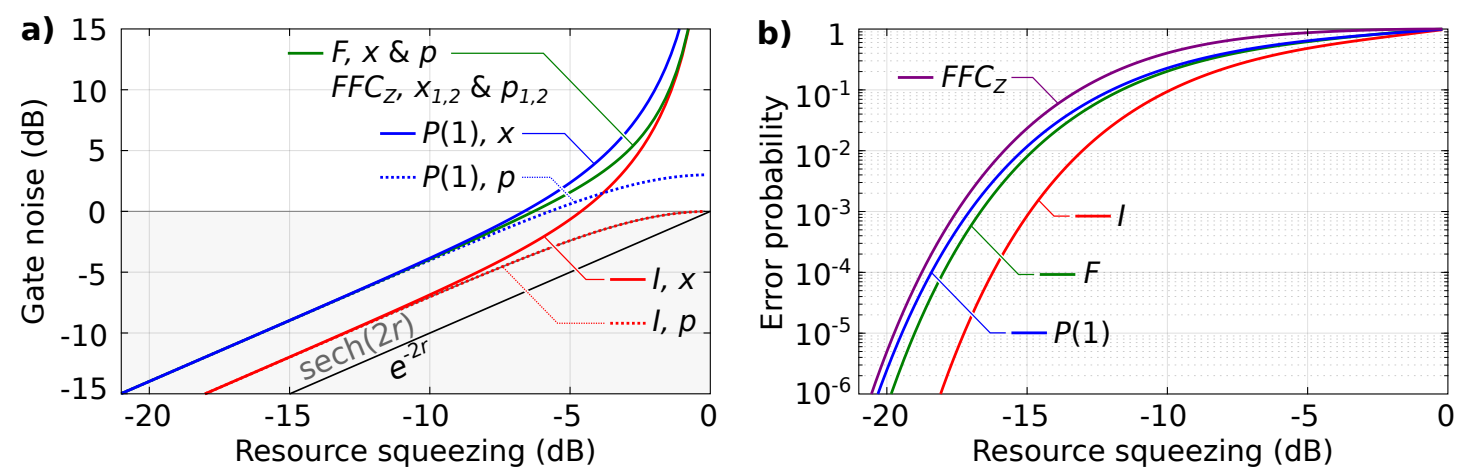

Figure 4.9: (a) Gate noise variance of the $\hat{I}-, \hat{F}$-, $\hat{P}(1)$ - and $(\hat{F} \otimes \hat{F}) \hat{C}_{Z}(1)$-gate (in short $\hat{F} \hat{F} \hat{C}_{Z}$ ) on the QRL cluster state as functions of input resource squeezing in Fig. 4.8a. Here, $e^{-2 r}$ and $\operatorname{sech}(2 r)$ marks the resource and effective squeezing variance. Note that the gate noise variance in each of the four quadratures when implementing $\hat{F} \hat{F} \hat{C}_{Z}$ is equal to the gate noise variance when implementing the $\hat{F}$-gate. The gate noise here is shown together with the other considered schemes in the appendix section 4.10 for easy comparison. (b) Resulting error probabilities of Eq. (4.8) after quadrature corrections.

are shown in Fig. $4.9 \mathrm{~b}$ for each of the four gates $\hat{I}, \hat{F}, \hat{P}(1)$ and $(\hat{F} \otimes \hat{F}) \hat{C}_{Z}(1)$. As expected, with four successful quadrature corrections required to avoid qubit error, the $(\hat{F} \otimes \hat{F}) \hat{C}_{Z}(1)$-gate has the highest error probability. In the following section 4.6.1, it is compared with the DBSL, BSL and MBSL.

\subsection{Discussion}

Below, in section 4.6.1 we compare the cluster states and computation schemes presented and discussed in section 4.4 and 4.5, while the figures summarizing the different computation schemes and resulting gate noise is shown side-by-side in the appendix section 4.10. In section 4.6.2 we then comment on computation universality with these cluster states.

\subsubsection{Cluster state comparison}

For all the four cluster states considered in section 4.4 and 4.5, the implemented two-mode $\hat{C}_{Z}(1)$ gates lead to the highest error probability of the GKP-encoded qubits among the gates of the set $\left\{\hat{I}, \hat{F}, \hat{P}(1), \hat{C}_{Z}(1)\right\}$. An indicative measure of the performance of a particular cluster state for quantum computing is thus the error probability associated with the implementation of the $\hat{C}_{Z}(1)$-gate. In Fig. 4.10a, these are plotted for the DBSL, BSL, MBSL and the QRL. Here, the error probability of $\hat{C}_{Z}(1)$ implemented on a canonically generated square lattice (SL) cluster state in [3] is plotted for comparison.

As discussed in section 4.3.3, the error probability in Eq. (4.8) is fuelled by the gate noise, the noise of the GKP qubits as well as the noise introduced in quadrature error correction. Gate noise is governed by the amount of squeezing of cluster state while the noise of the qubits and correction is produced by the finite squeezing of the peaks in the GKP state. Here, as described in section 4.4.2, we have assumed the peak variances of both quadratures in the GKP-states to equal the squeezing resource variance of $e^{-2 r} / 2$. To see how much the finite squeezing in the GKP-encoding and correction contributes to the error probability, the $\hat{C}_{Z}(1)$ error probability in the case of zero gate noise (corresponding to setting $\boldsymbol{\sigma}^{\mathbf{2}}=\mathbf{0}$ in Eq. (4.6)) is also plotted in Fig. 4.10a. No matter what computation scheme is considered with the GKP-encoding used here, we will not be able to perform better than the case of zero gate noise, as the noise contributions from the GKP-encoding and quadrature correction are unavoidable. 

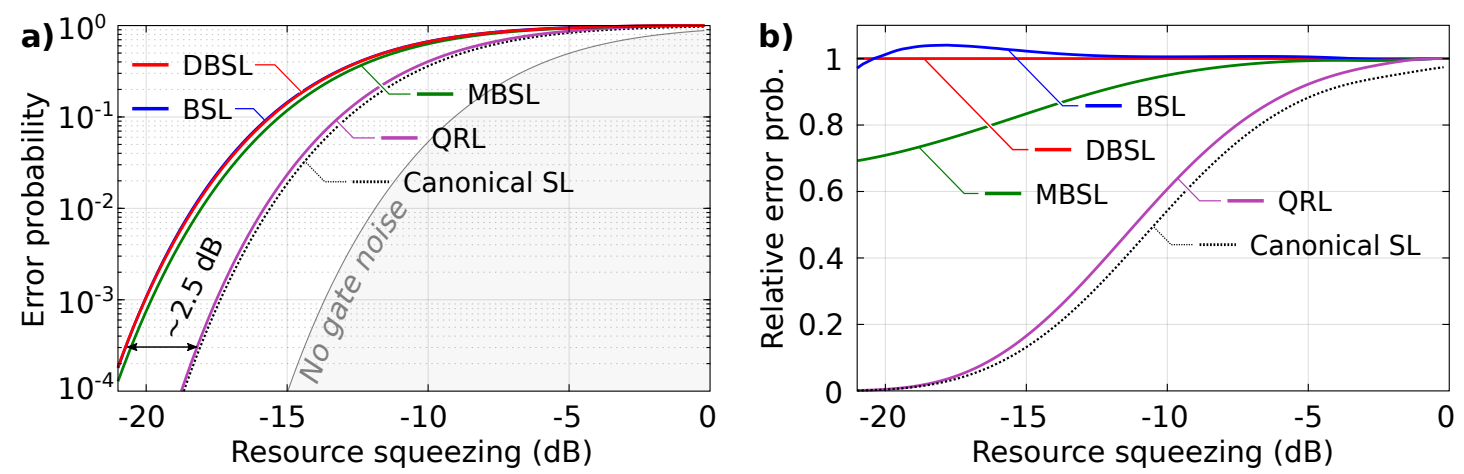

Figure 4.10: (a) Error probabilities for $\hat{C}_{Z}(1)$-gates implemented on the DBSL in section 4.4, the BSL, MBSL and QRL in section 4.5, and on the canonically generated square lattice (SL) cluster state in [3]. Depending on the cluster state, the implemented $\hat{C}_{Z}(1)$-gates have a Fourier gate biproduct on each mode. Note that the error probabilities for the DBSL and BSL are overlapping. The grey area marks the error probability in case of zero gate noise, where qubit errors are caused only by the available squeezing in the GKP-encoding. (b) Error probabilities in (a) relative to that of the DBSL.

The DBSL, BSL and MBSL are seen to have similar performance, while the QRL is superior and almost match the performance of the canonically generated SL cluster state. Approximately $2.5 \mathrm{~dB}$ additional squeezing is necessary in the squeezing resources for the DBSL and BSL to match the performance of the QRL. This performance advantage of the QRL is due to the larger cluster state edge weight in the logic level, and that the cluster state needs no projection by measurement of control modes, which adds additional noise to the state in computation. It is worth considering whether similar computation schemes can be developed for the DBSL, BSL and MBSL, possibly by placing the logic levels closer to the squeezing sources in the setups after the first beam-splitters (leading to temporally delocalized macronodes in the macronode language).

To quantify further the performance difference of the DBSL, BSL and MBSL, the $\hat{C}_{Z}(1)$ error probabilities are plotted in Fig. $4.10 \mathrm{~b}$ relative to the $\hat{C}_{Z}(1)$ error probability of the DBSL. Here, the BSL and DBSL are seen to have very similar performance in the investigated range of resource squeezing. The MBSL performs better with an error probability down to $70 \%$ of the error probability in the DBSL at $21 \mathrm{~dB}$ resource squeezing, while the relative error probability is approximately $83 \%$ using the currently achievable squeezing of $15 \mathrm{~dB}$ [115]. However, in practice one also has to account for experimental imperfections and setup complexity when deciding which setup to use: The generation scheme of the DBSL is technically simpler than that of MBSL as it requires only two squeezing sources and three interference points contra four squeezing sources and five interference points.

At first sight, with only two squeezing sources, the DBSL seems to require less resources than the BSL and MBSL. However, in the DBSL cluster state, only every second temporal mode holds control modes, while for the BSL and MBSL every single temporal mode includes both control and wire modes. As a result, the DBSL only contains half as many modes for computation in the same number of temporal modes. Doubling the long delay, $N \tau$, in the generation setup in Fig. 4.2a doubles the cylindrical cluster state circumference and compensates for only having wire modes in every second temporal modes. However, by doubling the circumference, the time needed to implement gates doubles as well. As a result, there is a cost of using only two squeezing sources in the DBSL, which unfolds as fewer computation modes or longer computation time, but not as additional computation noise.

Finally, we compare the architecture of the computation schemes on the considered cluster states. The DBSL, BSL and MBSL all use the same principles of measuring control modes to control coupling between wires with modes in computation. Turning on and off coupling between wires makes it intuitive to implement multi-mode gates decomposed into single- and two-mode 
gates, while on the QRL one has to take care of the surrounding modes when implementing twomode gates as for the $\hat{C}_{Z}(1)$-gate in Fig. 4.8d. However, the control-mode based architectures only allow coupling between neighbouring wires, whereas the QRL is more "flexible" as introduced in [107]. As an example, consider an arbitrary swap-gate, $\hat{X}_{i j}$, swapping the modes in computation on wires $i$ and $j$. On the DBSL, a swap-gate (with an unimportant Fourier gate applied to the two output modes) can be performed between two neighbouring wires in two computation steps from temporal mode $(k-2, k)$ to $(k+2 N, k+2 N-2)$ with the basis setting

$$
\left(\begin{array}{c}
\theta_{A k-2} \\
\theta_{B k-2} \\
\theta_{A k} \\
\theta_{B k} \\
\theta_{A k+N-2} \\
\theta_{B k+N-2} \\
\theta_{A k+N-1} \\
\theta_{B k+N-1} \\
\theta_{A k+N} \\
\theta_{B k+N}
\end{array}\right)_{X}=\left(\begin{array}{c}
\pi / 4 \\
-\pi / 4 \\
\pi / 4 \\
-\pi / 4 \\
\pi / 2 \\
0 \\
\pi / 2 \\
0 \\
\pi / 2 \\
0
\end{array}\right)
$$

independent on the amount of resource squeezing, and with a gate noise variance of $N_{x} \varepsilon / 2$ and $N_{p} \varepsilon / 2$ in $\hat{x}$ - and $\hat{p}$-quadratures, respectively, where $N_{x}=\tanh ^{-4}(2 r)+3 \tanh ^{-2}(2 r)$ and $N_{p}=$ $\tanh ^{2}(2 r)+3$. Thus, to swap two modes on wires separated by $n$ wires in-between, $n+1$ swapgates are required on each mode leading to $2(n+1)$ required computation steps. On the QRL, on the other hand, using vertically travelling modes in Fig. 4.8, two modes can be swapped in only a single horizontal computation step independent on the initial distance between the modes on the cluster state lattice. This is illustrated in Fig. 4.11 for $\hat{X}_{14}$ with a mode distance of 2 . As a result, depending on the interconnectivity required in the quantum algorithm to be implemented, computation times can be shorter on the QRL than on the DBSL, BSL and MBSL.

Lastly, we want to comment on the performance of the QRL compared to the canonically generated SL cluster state. It is clear that the QRL performs almost as well as the SL, while the SL is much more challenging to generate since it requires on-line squeezing to perform canonical $\hat{C}_{Z}(g)$ operations and the total squeezing cost is in general larger [5, 116]. However, for a fair comparison, it should be mentioned that the $\hat{C}_{Z}(1)$ implemented in [3] on the SL was not optimised. It was implemented with four computation steps, where each of the $\hat{x}$ - and $\hat{p}$-quadrature corrections in the GKP-scheme were performed in two different computation steps, both leading to more noise on the GKP-encoded qubits. The $\hat{C}_{Z}(1)$ error probability on the SL may be improved by optimizing the required basis settings to implement the $\hat{C}_{Z}(1)$-gate in fewer computation steps, and performing GKP quadrature corrections of both the $\hat{x}$ - and $\hat{p}$-quadrature on the last cluster state mode as for the computation schemes considered in this work.

\subsubsection{Towards universality and fault-tolerance}

The four different computation schemes in section 4.4 and 4.5 involve only Gaussian measurements (in the form of homodyne detection) on Gaussian cluster states. In this pure Gaussian realm, one is only able to perform universal Gaussian computation [5], which with Gaussian input-modes may be simulated classically $[97,117]$. To achieve universal quantum computation, non-Gaussian operations or resources are required [118]. There exist different proposals on how to achieve a universal gate set, which we summarize and discuss in the following. Non-Gaussianity of states and operations has been obtained in numerous systems [119], including some recent results on optical non-Gaussian state preparation [99] and non-Gaussian transformations on cluster states [120].

In many $\mathrm{CV}$ quantum computing architecture proposals, the Gaussian gate set is complemented with the non-Gaussian cubic phase gate, $\hat{K}(\chi)=e^{i \chi \hat{x}^{3} / 3}[5,14]$ to achieve universal quantum computation on the bosonic modes [118]. Such a non-Gaussian gate can for example be implemented 

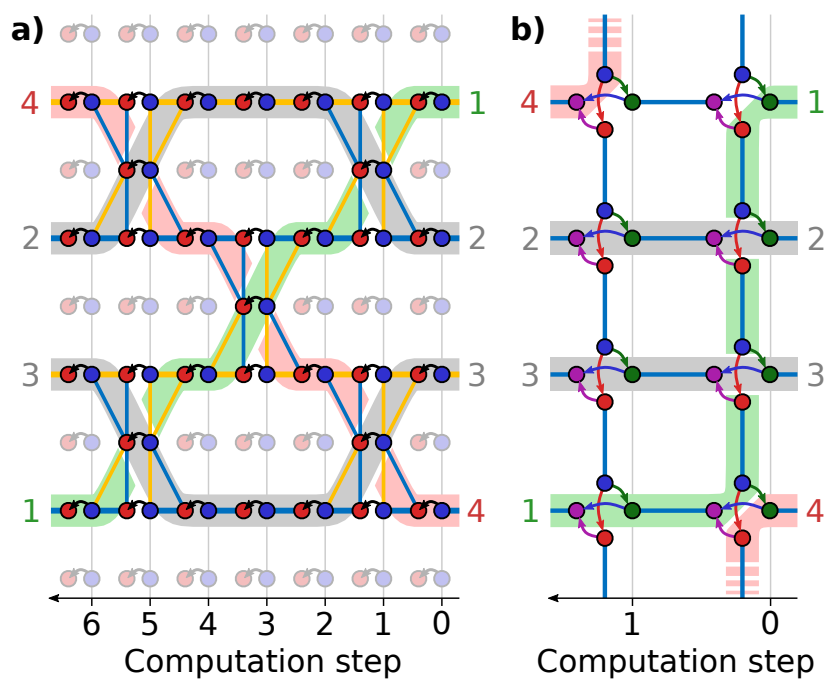

Figure 4.11: Implementation of a swap-gate between computation mode 1 and $4, \hat{X}_{14}$, separated by computation mode 2 and 3, on (a) the DBSL and on (b) the QRL. Since only coupling between neighbouring wires is possible on the DBSL in (a), a circuit depth of 3 is required, corresponding to 6 horizontal computation steps necessary along the cylindrical cluster state. The same goes for the BSL and the MBSL. On the QRL in (b), the same swap-gate can be implemented in a single horizontal computation step using crossing identity gates, $\hat{I}$. Here, computation mode 1 crosses computation mode 2 and 3, while computation mode 4 is lead all the way around the cluster state cylinder to appear in the next horizontal computation step, crossing other computation modes on its way.

by redirecting specific modes of the cluster to a photon counter, thereby realizing a measurement induced non-Gaussian gate transformation [5]. Moreover, in [12] it is shown how $\hat{K}(\chi)$ may be implemented on the BSL using an ancillary cubic phase state, $|\chi\rangle=\int \mathrm{d} s e^{i \chi s^{3} / 3}|s\rangle_{x}$, as a nonGaussian resource switched into the logic level of the computation scheme as an input state. Such cubic phase state may be prepared using photon counting [14]. Given the similarities between computation on the BSL, the DBSL and the MBSL, it is straight forward to adopt this method of implementing $\hat{K}(\chi)$ by inputting $|\chi\rangle$ in these computation schemes. A similar approach may also be viable on the QRL.

Using GKP-states with symmetric quadrature noise, one can expect bad performance of the cubic phase gate due to the applied phase by $\hat{K}(\chi)$ on the finitely squeezed GKP peaks being a cubic function of $\hat{x}[14,41]$. A more efficient approach to quantum universality is to consider a gate set that is only universal in the encoded logic space rather than in the full, infinite-dimensional Hilbert space. This requires an ample supply of qubit magic states such as the Hadamard eigenstates, $\left|H_{L}\right\rangle=\cos \pi / 8\left|0_{L}\right\rangle+\sin \pi / 8\left|1_{L}\right\rangle$. By injecting these states into the computation wires as input states using an optical switch, the non-Clifford $\pi / 8$-gate can be executed with only Gaussian transformations of the bosonic modes $[14,40]$. Such magic GKP states may be prepared similarly to the GKP-encoded input states, or directly distilled using GKP $\left|0_{L}\right\rangle$-states [39]. In conclusion, the inherent non-Gaussianity of the GKP-states is sufficient to achieve universal quantum computation in the GKP-encoded qubit subspace using solely Gaussian transformations. Moreover, adding magic state distillation to the scheme may not increase the experimental requirements significantly since the squeezing needed for the distillation is expected to be lower than the squeezing already required to reach fault-tolerant Clifford computation [3, 39].

Finally, for fault-tolerant computation, the qubit error correction scheme - concatenated with the GKP error correction scheme - should be considered when estimating the required squeezing. With the quadrature corrections of states in computation after each implemented gate, gate noise and finite squeezing in the approximate GKP-encoded qubit states are translated to qubit errors. 
For fault-tolerant computation, these qubit errors are corrected with an appropriate qubit error correction scheme, where a logic qubit is encoded in multiple GKP-qubits. Here, it is not appropriate just to choose a qubit error correction scheme with a large qubit error threshold, as considerations on how to practically implement the scheme are also of critical importance. For this reason, here we will not estimate a squeezing threshold for fault-tolerance. As an example, the 7-qubit Steane code with $\mathrm{a} \sim 10^{-3}$ error threshold requires two-mode gates between arbitrary modes in computation [110], while the discussed computation schemes in section 4.4 and 4.5 only implement two-mode gates between neighbouring computation modes. Thus, to implement the 7-qubit Steane code a number of swap-gates are required for each syndrome measurement, each leading to an increase in the combined qubit error probability before qubit error correction. The QRL may have an advantage when considering the implementation of a qubit error correction scheme owing to its flexibility as previously discussed and illustrated for a swap-gate in Fig. 4.11. Future work includes considerations on the practical implementation of qubit error correction on a suitable and realizable cluster states - such proposal is outlined in chapter 6 .

\subsection{Conclusion}

In summary, we have reviewed the principles of CV measurement-based QC based on generalized teleportation, we have proposed an efficient computation scheme for the DBSL cluster state that was experimentally generated in chapter 3 [31], and we have carefully analysed and compared quantum computation based on that state with the BSL, QRL, and MBSL cluster states.

Through a careful study of the added gate noise for the different cluster states, we find that the DBSL, the BSL and the MBSL exhibit similar performance. We also find that the QRL is superior in terms of performance and flexibility, allowing implementation of quantum circuits in a minimum number of time steps. Finally, we have reviewed proposals for implementation of a universal gate set, either on the bosonic modes or just in the GKP-encoded qubit subspace, and conclude that universal qubit computation is possible in all four considered cluster states, given the availability of GKP-states.

To optimise the performance of the various computation schemes, we introduced a tool to find the basis setting implementing a desired gate with minimum GKP-encoded qubit errors. We believe that this technique for finding the optimal basis settings will be important for future developments and optimizations of new types of gates and algorithms. It should however be noted that the technique of optimizing the basis setting might not be the only strategy for minimizing the error probability: We have only considered GKP-qubit encodings on a square grid in phase space which is appropriate for symmetric noise addition among conjugate quadratures. However, since the considered computation schemes in general add noise asymmetrically in the quadratures, it may be beneficial to encode the qubits in a rectangular lattice. Since different gates have different gate noise asymmetry in $\hat{x}$ - and $\hat{p}$-quadrature, the optimal lattice ratio depends on which gates dominate the circuit to be implemented: As an example, the Fourier gate, $\hat{F}$, in general adds symmetric gate noise in which case a square lattice is optimal, while the gate noise asymmetry of the identity gate, $\hat{I}$, depends on the resource squeezing. One can argue, that with the $\hat{C}_{Z}$-gate being the noisiest gate, the GKP lattice ratio should be optimized to minimize qubit error for this gate. In this case, for the DBSL, BSL and MBSL, the optimal ratio again depends on the resource squeezing, while for the superior QRL with symmetric gate noise, the square lattice seems optimal. However, one further complication is that when performing the $\hat{C}_{Z}$-gate or the $\hat{P}$-gate, not only gate noise is added, but also noise from the state in computation is added due to the addition of quadratures in these gates. Thus, the optimal lattice ratio depends as well on the noise performance of the states in computation, and to determine a general optimal lattice ratio for a given application is outside the scope of this work. Finally, one has to keep in mind that changing the lattice ratio also alters the logic operators in the GKP-encoding. As an example, with a rectangular lattice the logic Hadamard gate becomes a combination of the Fourier and squeezing gates.

Throughout this article, we have assumed all cluster states to be pure, while in practice, the cluster state will have some degree of mixedness in the form of excess noise in the anti-squeezed 
a)

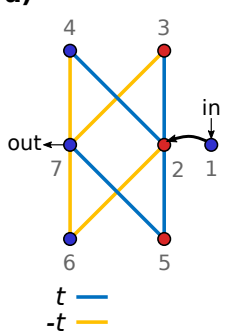

b)

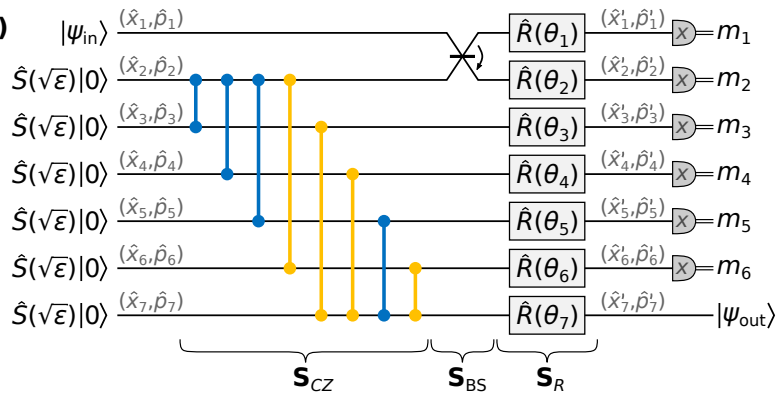

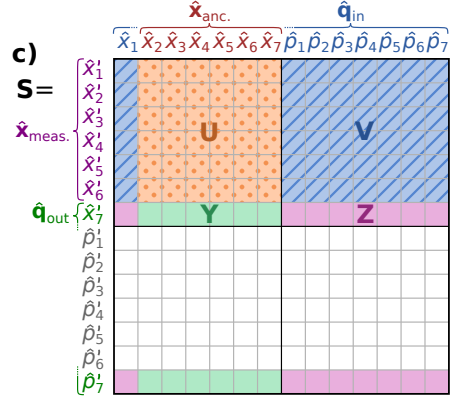

Figure 4.12: (a) Graph notation of a single-mode computation step on the DBSL. For an input in temporal mode $k$, the mode numbering translates as $(1,2,3,4,5,6,7)=(B k, A k, A k-1, B k+N-$ $1, A k+1, B k+N+1, B k+N)$ where $A$ and $B$ are spatial modes in Fig. 4.2a. (b) Corresponding circuit where the blue and yellow two-mode gates represent $\hat{C}_{Z}(g)$-gates of weights $t$ and $-t$, respectively. (c) Outline of the symplectic matrix $\mathbf{S}=\mathbf{S}_{R} \mathbf{S}_{B S} \mathbf{S}_{C Z}$ representing the quadrature transformation in (b).

quadratures. However, it has been shown in Ref. [38] that excess noise in the anti-squeezed quadrature does not affect the performance of the computation, and thus our purity assumption in this article is well justified. It is however worth mentioning that in practice it is still favorable to produce highly pure squeezed states as large excess noise will decrease the amount of squeezing due to inevitable phase instabilities of the experimental setup.

In this chapter, we have not studied the actual implementation of qubit error correction. Thus, as an outlook, it would be interesting to study how a qubit error correction algorithm is most efficiently implemented such that the squeezing threshold for fault-tolerant quantum computation is minimized. An interesting solution could be topological QC, for which the resulting squeezing threshold is within the already experimental demonstrated range [43, 121]. In topological QC, the qubits are encoded in a two-dimensional plane while the actual computation takes place in a third dimension, thus rendering the need for the construction of 3D cluster states. Proposals do exist for the generation of $3 \mathrm{D}$ cluster states [44, 45], and the next interesting step is thus to analyse the performance of these states using the techniques developed in this article.

\subsection{Appendix: Calculation of quadrature tansformations}

In this appendix section, we present an example of the quadrature transformation of the singlemode computation step on the DBSL that leads to the expressions Eq. (4.10) and (4.12) in section 4.4. The modes involved are shown on the graph in Fig. 4.12a with the corresponding circuit in Fig. 4.12b. We will use the mode numbering labelled in Fig. 4.12a.

The approximate cluster state (ancillary mode 2-7) consists of vacuum states squeezed by $\sqrt{\varepsilon}$ and connected by $\hat{C}_{Z}(g)$-operations of weights that are described by the adjacency matrix

$$
\mathbf{A}=\left(\begin{array}{ccccccc}
0 & 0 & 0 & 0 & 0 & 0 & 0 \\
0 & 0 & t & t & t & -t & 0 \\
0 & t & 0 & 0 & 0 & 0 & -t \\
0 & t & 0 & 0 & 0 & 0 & -t \\
0 & t & 0 & 0 & 0 & 0 & t \\
0 & t & 0 & 0 & 0 & 0 & -t \\
0 & 0 & -t & -t & t & -t & 0
\end{array}\right)
$$

Thus, in the Heisenberg picture, we consider the generation of the cluster state as a quadrature 
transformation described by the symplectic matrix

$$
\mathbf{S}_{C Z}=\left(\begin{array}{cc}
\mathbf{I} & \mathbf{0} \\
\mathbf{A} & \mathbf{I}
\end{array}\right)
$$

on the input and initially squeezed ancillary modes, where $\mathbf{I}$ and $\mathbf{0}$ are the $7 \times 7$ identity and zero matrix, respectively (note that the quadratures of the input mode 1 are left unchanged by $\mathbf{S}_{C Z}$ ). The input mode 1 is then connected to the cluster state by a beam-splitter (the beam-splitter of the measurement device in Fig. 4.2a), leading to the quadrature transformation

$$
\mathbf{S}_{B S}=\left(\begin{array}{ll}
\mathbf{B} & \mathbf{0} \\
\mathbf{0} & \mathbf{B}
\end{array}\right)
$$

where

$$
\mathbf{B}=\left(\begin{array}{ccccccc}
1 / \sqrt{2} & -1 / \sqrt{2} & 0 & 0 & 0 & 0 & 0 \\
1 / \sqrt{2} & 1 / \sqrt{2} & 0 & 0 & 0 & 0 & 0 \\
0 & 0 & 1 & 0 & 0 & 0 & 0 \\
0 & 0 & 0 & 1 & 0 & 0 & 0 \\
0 & 0 & 0 & 0 & 1 & 0 & 0 \\
0 & 0 & 0 & 0 & 0 & 1 & 0 \\
0 & 0 & 0 & 0 & 0 & 0 & 1
\end{array}\right)
$$

Here we have ignored beam-splitter operations on the control modes $3-6$, as these operations can be directly compensated for by adding/subtracting the measurement outcomes of the homodyne detectors as shown in Eq. (4.9). Finally, each mode $j$, except the output mode 7, is measured in basis $\hat{x}\left(\theta_{j}\right)$. This is represented first by a phase rotation, $\hat{R}\left(\theta_{j}\right)$, followed by a homodyne measurement of $\hat{x}_{j}$. Thus, before the $\hat{x}$-measurements, the quadratures are transformed as

$$
\mathbf{S}_{R}=\left(\begin{array}{cc}
\mathbf{c} & \mathbf{s} \\
-\mathbf{s} & \mathbf{c}
\end{array}\right),
$$

where $\mathbf{c}$ and $\mathbf{s}$ are matrices with $\left(\cos \theta_{1}, \cdots, \cos \theta_{7}\right)$ and $\left(\sin \theta_{1}, \cdots, \sin \theta_{7}\right)$ in the diagonal, respectively, and zero elsewhere. For implementing single-mode gates, the control mode measurement bases are set to $\left(\theta_{3}, \theta_{4}, \theta_{5}, \theta_{6}\right)=(-1)^{i} \theta_{c}(1,1,-1,-1)$, where $\theta_{c}=\pi / 4$ for simplicity, and the output mode cannot be phase rotated, $\theta_{7}=0$. Here, $i$ is the wire number as shown in Fig. $4.2 \mathrm{~b}$. The total quadrature transformation of the input and squeezed cluster state modes just before measurements is then

$$
\hat{\mathbf{q}}^{\prime}=\mathbf{S}_{R} \mathbf{S}_{B S} \mathbf{S}_{C Z} \hat{\mathbf{q}}=\mathbf{S} \hat{\mathbf{q}}
$$

where $\hat{\mathbf{q}}=\left(\hat{x}_{1}, \cdots, \hat{x}_{7}, \hat{p}_{1}, \cdots, \hat{p}_{7}\right)^{T}$ and $\hat{\mathbf{q}}^{\prime}=\left(\hat{x}_{1}^{\prime}, \cdots, \hat{x}_{7}^{\prime}, \hat{p}_{1}^{\prime}, \cdots, \hat{p}_{7}^{\prime}\right)^{T}$ are vectors of quadrature operators before and after the transformation as marked in Fig. 4.12b. It should be noted that for the cluster state prepared as a $\mathcal{H}$-graph with off-line squeezing only, the effective amount of squeezing of the cluster state modes is $\varepsilon=\operatorname{sech}(2 r)$, where $r$ is the squeezing parameter of the initially prepared off-line squeezed state with variance $e^{-2 r}[5]$.

Next, we solve for the anti-squeezed $\hat{x}$-quadratures of the cluster state, $\hat{\mathbf{x}}_{\text {anc. }}=\left(\hat{x}_{2}, \cdots, \hat{x}_{7}\right)^{T}$, as a function of the measured $\hat{x}$-quadratures, $\hat{\mathbf{x}}_{\text {meas. }}=\left(\hat{x}_{1}^{\prime}, \cdots, \hat{x}_{6}^{\prime}\right)^{T}$ :

$$
\begin{gathered}
\hat{\mathbf{x}}_{\text {meas. }}=\mathbf{U} \hat{\mathbf{x}}_{\text {anc. }}+\mathbf{V} \hat{\mathbf{q}}_{\text {in }} \\
\Uparrow \\
\hat{\mathbf{x}}_{\text {anc. }}=\mathbf{U}^{-1} \hat{\mathbf{x}}_{\text {meas. }}-\mathbf{U}^{-1} \mathbf{V} \hat{\mathbf{q}}_{\text {in }},
\end{gathered}
$$

where $\hat{\mathbf{q}}_{\text {in }}=\left(\hat{x}_{1}, \hat{p}_{1}, \cdots, \hat{p}_{7}\right)^{T}$ while $\mathbf{U}$ and $\mathbf{V}$ are the parts of $\mathbf{S}$ that transform $\hat{\mathbf{x}}_{\text {anc. }}$ and $\hat{\mathbf{q}}_{\text {in }}$ to $\hat{\mathbf{x}}_{\text {meas. }}$ as shown in Fig. 4.12c. Finally, we substitute the $\hat{x}$-quadratures of the cluster state with the quadratures of output mode $7, \hat{\mathbf{q}}_{\text {out }}=\left(\hat{x}_{7}^{\prime}, \hat{p}_{7}^{\prime}\right)$ :

$$
\begin{aligned}
\hat{\mathbf{q}}_{\text {out }} & =\mathbf{Y} \hat{\mathbf{x}}_{\text {anc. }}+\mathbf{Z} \hat{\mathbf{q}}_{\text {in }} \\
& =\mathbf{Y}\left(\mathbf{U}^{-1} \hat{\mathbf{x}}_{\text {meas. }}-\mathbf{U}^{-1} \mathbf{V} \hat{\mathbf{q}}_{\text {in }}\right)+\mathbf{Z} \hat{\mathbf{q}}_{\text {in }} \\
& =\left(\mathbf{Z}-\mathbf{Y} \mathbf{U}^{-1} \mathbf{V}\right) \hat{\mathbf{q}}_{\text {in }}+\mathbf{Y} \mathbf{U}^{-1} \hat{\mathbf{x}}_{\text {meas. }} .
\end{aligned}
$$


With $\hat{\mathbf{x}}_{\text {meas. }} \rightarrow\left(m_{1}, \cdots, m_{6}\right)$ when measuring, $\mathbf{Y U}^{-1} \hat{\mathbf{x}}_{\text {meas. }}$. corresponds to the by-product displacement, while $\mathbf{M} \equiv \mathbf{Z}-\mathbf{Y} \mathbf{U}^{-1} \mathbf{V}$ of size $2 \times 8$ corresponds to the combined gate symplectic matrix $\mathbf{G}$ and gate noise matrix $\mathbf{N}$ in Eq. (4.2) as $\mathbf{M}=(\mathbf{G} \mathbf{N})$. Extracting $\mathbf{G}$ as the first two columns of $\mathbf{M}$ transforming $\left(\hat{x}_{1}, \hat{p}_{1}\right)$ to $\left(\hat{x}_{7}^{\prime}, \hat{p}_{7}^{\prime}\right)$, we get

$$
\mathbf{G}=\frac{1}{\sin \theta_{-}}\left(\begin{array}{cc}
\frac{1}{t^{\prime}} \cos \theta_{+}+\frac{1}{t^{\prime}} \cos \theta_{-} & \frac{1}{t^{\prime}} \sin \theta_{+} \\
-t^{\prime} \sin \theta_{+} & t^{\prime} \cos \theta_{+}-t^{\prime} \cos \theta_{-}
\end{array}\right)
$$

which is the symplectic matrix corresponding to the operation in Eq. (4.10) where $t^{\prime}=(-1)^{i} 4 t^{2}$ and $\theta_{ \pm}=\theta_{1} \pm \theta_{2} . \mathbf{N}$ is associated with the remaining 6 columns of $\mathbf{M}$;

$$
\mathbf{N}=\left(\begin{array}{cccccc}
-\frac{1}{4 t^{2}} & \frac{1}{4 t} & \frac{1}{4 t} & -\frac{1}{4 t} & \frac{1}{4 t} & 0 \\
0 & t & t & t & -t & 1
\end{array}\right)
$$

which leads to the quadrature noise factors coined in Eq. (4.12) when $t=\tanh (2 r) / 2$.

The procedure shown here for calculating the gate symplectic matrix, $\mathbf{G}$, and gate noise matrix, $\mathbf{N}$, is not limited to the single-mode one computation step on the DBSL, but represents a general procedure that can be used to analyse the noise of all gates in this work (irrespective of the cluster state) as done in section 4.4 and 4.5: If $\mathbf{S}_{C Z}$ represents the construction of any cluster state and $\mathbf{S}_{R} \mathbf{S}_{B S}$ represents any Gaussian measurement, we can determine the resulting linear quadrature transformation corresponding to an arbitrary Gaussian operation on a single- or multimode input state. For each case, we need to keep track of the following quadratures: $\hat{\mathbf{x}}_{\text {anc. }}$ including anti-squeezed $\hat{x}$-quadratures of the cluster state; $\hat{\mathbf{q}}_{\text {in }}$ including the input mode quadratures and squeezed $\hat{p}$-quadratures of the cluster state leading to gate noise; $\hat{\mathbf{x}}_{\text {meas. }}$ including the transformed $\hat{x}$-quadratures to be measured; and $\hat{\mathbf{q}}_{\text {out }}$ including the output mode quadratures of non-measured modes.

\subsection{Appendix: Wigner function transformations}

In this appendix section we discuss the single-mode computation step in the DBSL, BSL and MBSL in the Wigner function representation. Here, for simplicity, the basis setting for implementing the $\hat{I}$-gate is chosen, while to shorten the notation, we have post-selected on measurement outcomes equal zero. As described in the section 4.3.2, non-zero measurement outcomes lead to an unimportant displacement in phase-space. For the QRL, the two-mode cluster state corresponds to the one considered for the generalized teleportation in section 4.3.2, and so the Wigner function transformation is similar to that presented in Eq. (4.5).

For a single-mode $\hat{I}$-gate performed on the DBSL in one computation step, described in sections 4.4 , the transformation of the Wigner function can be calculated in the same way as we did for the generalized teleportation in section 4.3.2, resulting in

$$
\begin{aligned}
& W_{\text {out }}(x, p)=\mathcal{N} G_{1 / \varepsilon}(x) \int \mathrm{d} \eta_{4} G_{\varepsilon}\left(\eta_{4}\right) G_{4 t^{2} / \varepsilon}\left(p-\eta_{4}\right) \int \mathrm{d} \eta_{3} G_{\varepsilon /\left(4 t^{2}\right)}\left(\eta_{3}\right) G_{1 /\left(4 t^{2} \varepsilon\right)}\left(x-\eta_{3}\right) \times \\
& \int \mathrm{d} \eta_{2} G_{4 t^{2} \varepsilon}\left(\eta_{2}\right) G_{16 t^{4} / \varepsilon}\left(p-\eta_{2}-\eta_{4}\right) \int \mathrm{d} \eta_{1} G_{\varepsilon /\left(16 t^{4}\right)}\left(\eta_{1}\right) W_{\text {in }}\left(\begin{array}{c}
x-\eta_{1}-\eta_{3} \\
p-\eta_{2}-\eta_{4}
\end{array}\right),
\end{aligned}
$$

where $\mathcal{N}$ is a normalization factor and $G_{\delta}$ is a normalized Gaussian function of $\delta / 2$ variance. The transformation includes two convolutions in each quadrature and corresponding envelopes in the conjugate quadrature due to the Fourier relation between quadratures. Comparing with Eq. (4.18), and referring to the mode numbering in Fig. 4.12a, in the $\hat{x}$-quadrature the first convolution with $G_{\varepsilon /\left(16 t^{4}\right)}$ corresponds to noise from the finitely squeezed mode 2 , while the third convolution with $G_{\varepsilon /\left(4 t^{2}\right)}$ corresponds to noise of control modes $3,4,5$ and 6 . In the $\hat{p}$-quadrature, the second convolution with $G_{4 t^{2} \varepsilon}$ corresponds to noise from the control modes 3, 4, 5 and 6 , while the last convolution with $G_{\varepsilon}$ corresponds to the finite squeezing noise of the output mode 7 . In the limit of infinite squeezing, $r \rightarrow \infty$, (assuming $t \neq 0$ ) the convolution functions become 
delta functions since $\varepsilon=\operatorname{sech}(2 r) \rightarrow 0$, while their corresponding envelopes in the orthogonal quadratures become infinitely broad, and so $W_{\text {out }}(x, p) \rightarrow W_{\text {in }}(x, p)$. In the limit of $t=0$ where we expect no information to pass from the input mode 1 to the output mode 7 , the first three convolutions lead to the Wigner function of an infinitely squeezed state in $\hat{p}$, erasing all information of the input state, while the last convolution with $G_{\varepsilon}$ in $\hat{p}$-quadrature ensures that the output Wigner function equals the initial squeezed Wigner function of mode $7, W_{\text {out }}(x, p)=G_{1 / \varepsilon}(x) G_{\varepsilon}(p)$, which equals vacuum for no squeezing as $\varepsilon=\operatorname{sech}(2 r)=1$ when $r=0$.

On the BSL, the single-mode $\hat{I}$-gate performed in one computation step transforms the Wigner function as

$$
\begin{array}{r}
W_{\text {out }}(x, p)=\mathcal{N} G_{1 / \varepsilon}(x) \int \mathrm{d} \eta_{4} G_{\varepsilon}\left(\eta_{4}\right) G_{2 t^{2} / \varepsilon}\left(p-\eta_{4}\right) \int \mathrm{d} \eta_{3} G_{\varepsilon /\left(2 t^{2}\right)}\left(\eta_{3}\right) G_{1 /\left(2 t^{2} \varepsilon\right)}\left(x-\eta_{3}\right) \times \\
\int \mathrm{d} \eta_{2} G_{2 t^{2} \varepsilon}\left(\eta_{2}\right) G_{4 t^{2} / \varepsilon}\left(p-\eta_{2}-\eta_{4}\right) \int \mathrm{d} \eta_{1} G_{\varepsilon /\left(4 t^{4}\right)}\left(\eta_{1}\right) W_{\text {in }}\left(\begin{array}{c}
x-\eta_{1}-\eta_{3} \\
p-\eta_{2}-\eta_{4}
\end{array}\right) .
\end{array}
$$

Similar to the DBSL, comparing with $\mathbf{N}$ in Eq. (4.15), the convolutions with $G_{\varepsilon /\left(4 t^{4}\right)}$ and $G_{\varepsilon /\left(2 t^{2}\right)}$ in the $\hat{x}$-quadrature correspond respectively to noise added from the first wire mode $A k$ and the two control modes $B k+1$ and $C k+N$ in a square cluster of the BSL in Fig. 4.6a,b. In the $\hat{p}$-quadrature, the convolutions with $G_{2 t^{2} \varepsilon}$ corresponds to noise from the control modes $B k+1$ and $C k+N$, while the convolution with $G_{\varepsilon}$ corresponds to noise from the output mode $D k+N$. Again, in the limit of infinite squeezing, $W_{\text {out }}(x, p) \rightarrow W_{\text {in }}(x, p)$, while for $t=0$ the output Wigner function becomes $W_{\text {out }}(x, p)=G_{1 / \varepsilon}(x) G_{\varepsilon}(p)$ as expected.

For the MBSL, the Wigner function transformation of the $\hat{I}$-gate in one computation step with the control basis $\theta_{c}=\pi / 2$ is

$$
\begin{gathered}
W_{\text {out }}(x, p)=\mathcal{N} G_{1 / \varepsilon}(x) G_{2 \varepsilon+4 t^{2} / \varepsilon}(p) \int \mathrm{d} \eta_{3} G_{\varepsilon /\left(4 t^{2}\right)}\left(\eta_{3}\right) G_{1 / \varepsilon}\left(x-2 \eta_{3}\right) \int \mathrm{d} \eta_{2} G_{2 \varepsilon}\left(\eta_{2}\right) \times \\
G_{4 t^{2} / \varepsilon}\left(p-\eta_{2}\right) \int \mathrm{d} \eta_{1} G_{\varepsilon /\left(4 t^{2}\right)}\left(\eta_{1}\right) W_{\text {in }}\left(\begin{array}{c}
x-\eta_{1}-\eta_{3} \\
p-\eta_{2}
\end{array}\right) .
\end{gathered}
$$

Due to the direct edges along the computation wires of the butterfly cluster states in Fig. 4.7, the Wigner function transformation becomes less intuitive. Here, the envelope $G_{2 \varepsilon+4 t^{2} / \varepsilon}(p)$ corresponds to an envelope of $G_{4 t^{2} / \varepsilon}(p)$ convoluted with $G_{2 \varepsilon}$. Comparing with Eq. (4.16), the first and third convolution in the $\hat{x}$-quadrature, both with $G_{\varepsilon /\left(4 t^{2}\right)}$, correspond to noise added from wire mode $D k$ and control mode $A k$. The second convolution with $G_{2 \varepsilon}$ in the $\hat{p}$-quadrature corresponds to noise from both control mode $B k+1$ and the output mode $C k+N$. In the infinite squeezing limit, $W_{\text {out }}(x, p) \rightarrow W_{\text {in }}(x, p)$. For $t=0$ we get $W_{\text {out }}(x, p)=G_{1 / \varepsilon}(x) G_{2 \varepsilon}(p) G_{2 \varepsilon}(p)=G_{1 / \varepsilon}(x) G_{\varepsilon}(p)$.

If, instead of the identity gate $\hat{I}$, an arbitrary single-mode Gaussian gate of one computation step is implemented with symplectic matrix $\mathbf{G}$, the resulting Wigner function transformation corresponds to that presented above, but with the arguments of $W_{\text {in }}$ transformed by $\mathbf{G}^{-1}$ as shown in Eq. (4.5) for the generalized teleportation. For single-mode gates implemented in two computation steps, the output Wigner function of the first step becomes the input Wigner function of the second step, leading to addition of the gate noise variance since a convolution of two Gaussian functions is a Gaussian function with the combined variance, i.e. additive Gaussian gate noise. For multi-mode gates, more modes are involved leading to more convolutions in the expression of the output Wigner function, and the Wigner function representation becomes tedious. However, the principle is the same as for single-mode gates: The gate noise leads to convolutions with Gaussian functions of variance equal the gate noise variance.

\subsection{Appendix: Cluster state comparison cheat sheet}

In section 4.4 and 4.5 the computation schemes on the different cluster states are analysed separately in order to facilitate easy lookup of a specific cluster state. The different schemes are discussed and compared in section 4.6, while in this appendix section the schemes are arranged side by side in Fig. 4.13 for easy comparison. 


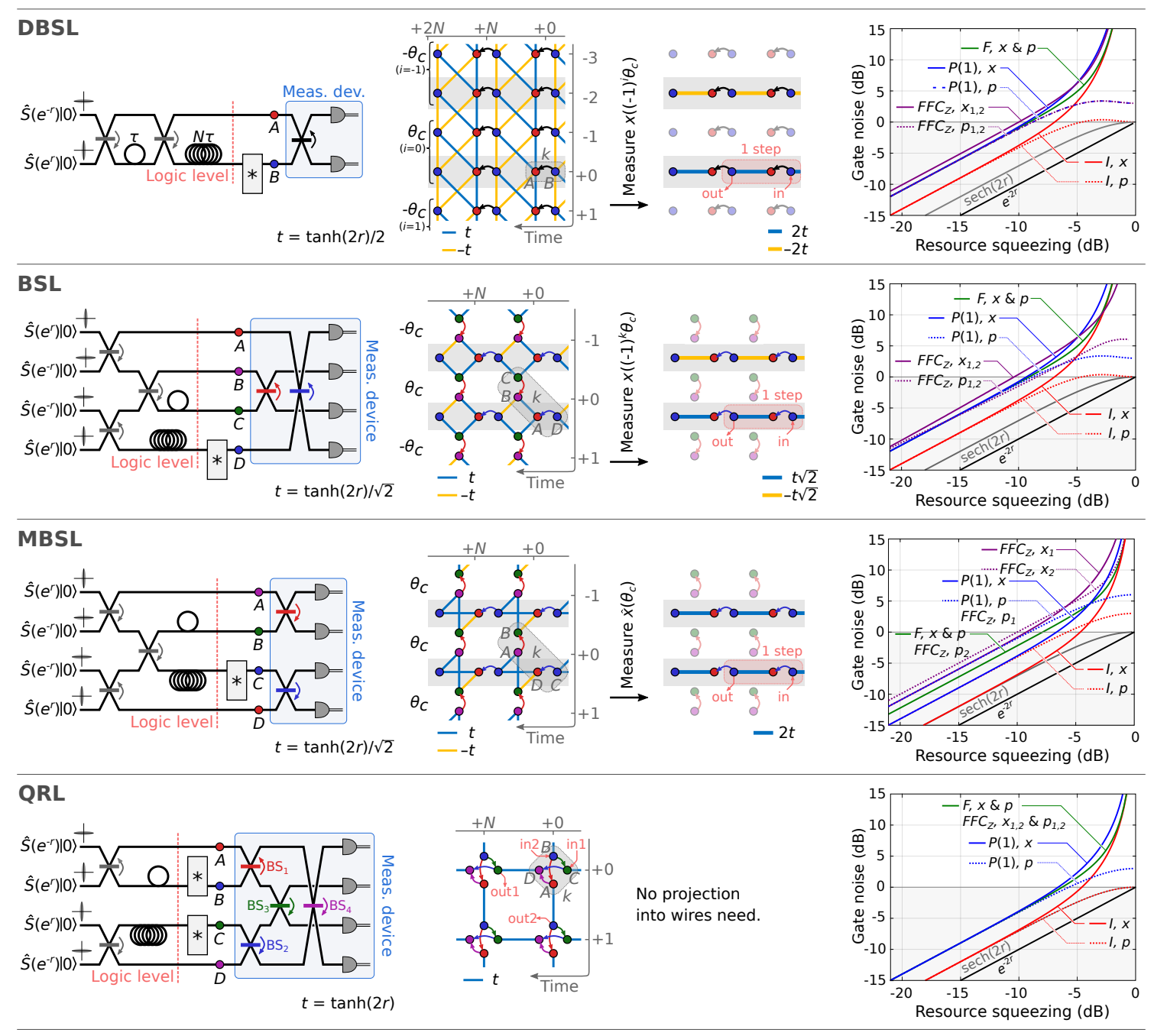

Figure 4.13: Setup, cluster state in the logic level, and resulting gate noise of each the DBSL, BSL, MBSL, and QRL cluster states studied in the sections 4.4 and 4.5. For the DBSL, BSL, and MBSL, the projection into wires is shown as well, while this is not required for computation on the QRL. 


\section{Chapter 5}

\section{Deterministic multi-mode gates on a scalable photonic quantum computing platform}

In this chapter, the paper "Deterministic multi-mode gates on a scalable photonic quantum computing platform" of Ref. [33] is presented. This paper is authored by Mikkel V. Larsen, Xueshi Guo, Casper R. Breum, Jonas S. Neergaard-Nielsen, and Ulrik L. Andersen, and is submitted for publication.

In this work, following the computation scheme presented in chapter 4 [32] with few variations, we implement quantum gates by projective measurements on the cluster state generated in chapter 3 [31]. We then characterize the implemented gates by multi-mode gate tomography using entangle inputs. This work is partly experimental and partly theoretical.

Both single- and two-mode gates are demonstrated, and together they constitute a universal multi-mode Gaussian gate set that allows for universal quantum computation if combined with Gottesman-Kitaev-Preskill encoded qubits [14]. However, with the inherent noise from finite squeezing, the implemented gates are noisy, and we study the gate performance as gate noise comprising additive Gaussian noise in the quadratures of the computation modes. In this work we characterize gate noise by gate tomography, and we discuss how to reduce and correct the gate noise, while in chapter 6 [34] a complete implementation of quantum error correction for fault-tolerant computation is proposed.

Appendix A and C (page 147 and 153) complements the supplementary information section 5.3 of this chapter, describing the experimental optical table and optimization of basis control.

From www.arXiv.org as pre-print arXiv:2010.14422 (2020).

\subsection{Abstract}

Quantum computing can be realized with numerous different hardware platforms and computational protocols. A highly promising approach to foster scalability is to apply a photonic platform combined with a measurement-induced quantum information processing protocol where gate operations are realized through optical measurements on a multipartite entangled quantum state - a so-called cluster state $[1,2]$. Heretofore, a few quantum gates on non-universal or non-scalable cluster states have been realized [22-29], but a full set of gates for universal scalable quantum computing has not been realized. We propose and demonstrate the deterministic implementation of a multi-mode set of measurement-induced quantum gates in a large two-dimensional (2D) optical cluster state using phase-controlled continuous variable quadrature measurements $[2,5]$. Each gate is simply programmed into the phases of the high-efficiency quadrature measurements which 
execute the transformations by teleportation through the cluster state. Using these programmable gates, we demonstrate a small quantum circuit consisting of 10 single-mode gates and 2 two-mode gates on a three-mode input state. The demonstrated quantum computing platform operates at the telecom wavelength and is therefore easily network connectable. Moreover, fault-tolerant and universal quantum computing can be realized by increasing the amount of entanglement and combining it with error-correctable Gottesman-Kitaev-Preskill qubits [14, 39-41].

\subsection{Main text}

Recent remarkable advances in developing fully programmable quantum computing platforms have led to a plethora of groundbreaking results in quantum information science including the demonstration of fault-tolerant operations on an error-corrected logical ion-trap qubit [122] and the demonstration of quantum sampling at a super-classical rate in a 53-qubit superconducting quantum computer [123]. Albeit marked progress, the currently realized qubit-based platforms for quantum computing are still strongly limited in size while the proposed methods for up-scaling are stymied by significant technical challenges.

An alternative is the continuous variable (CV) photonic platform which has recently gained interest due to its proven scalability potential for measurement-based quantum computation (MBQC) as exemplified by the generation of $2 \mathrm{D}$ cluster states with thousands of modes [31, 37] (where Ref. [31] is the content of chapter 3) and the sequential operation of one hundred single-mode gates [25]. In CV quantum computing [2, 14, 108, 118], information is encoded and processed in bosonic harmonic oscillators - e.g. the optical field - that are described by states in infinitedimensional Hilbert spaces [76, 119]. Although the idea of using CVs for quantum computing dates back more than 20 years [118], it is only within the last few years that feasible models for fault-tolerant large-scale CV MBQC were conceived [3, 39, 40, 43, 121]. Our demonstration represents a critical step towards these CV computing models. It constitutes the first realization of a fully deterministic and programmable multi-mode computation platform for MBQC.

\subsubsection{Architecture and hardware}

In CV MBQC, quantum information processing is realized by teleporting the constituent gates through a computationally universal cluster state, comprising quadrature entangled modes in a 2D grid [2]. For Gaussian gates, these teleportation protocols are effectuated by quadrature measurements of the cluster state modes, where the determined gate or sequence of gates is fully programmed into the phases of the quadrature measurements, i.e. the measurement bases. The reconfigurable and programmable nature of the cluster state quantum computer is illustrated in Fig. 5.1a by the different layers of operation from software to hardware. First, the quantum algorithm is specified and subsequently resolved into a certain sequence of single- and two-mode gates, such as the rotation, shear, squeezing and the controlled- $\mathrm{Z}$ gate. This sequence of gates is then converted into a sequence of phases that finally controls the consecutive quadrature measurements to effect the quantum algorithm on the cluster state.

At the hardware level, the processor comprises a 2D cluster state of time-encoded entangled modes generated by injecting squeezed light (produced by optical parametric oscillators (OPOs) at the wavelength of $1550 \mathrm{~nm}$ ) to a setup of two consecutive highly asymmetric fiber Mach-Zehnder interferometers as shown in Fig. 5.1b. The first interferometer with a delay of $\tau$ produces a timeencoded one-dimensional (1D) cluster state $[9,19]$ with two spatial modes, $A$ and $B$, while the second interferometer of delay $N \tau$ coils up the 1D cluster state to form a 2D cluster state [31] represented by a cylindrical graph as illustrated in Fig. 5.1c. The computational logic level of the cluster state is located just prior to the termination of the second interferometer. Each node of the graph corresponds to spatial modes $A$ and $B$ in different temporal modes, $k$, with duration $\tau$, and the number of temporal modes on the cylinder circumference is $N$. We have chosen fiberinterferometer delays of $50 \mathrm{~m}$ and $600 \mathrm{~m}$ leading to a temporal mode duration of $\tau \approx 250 \mathrm{~ns}$ and $N=12$. 


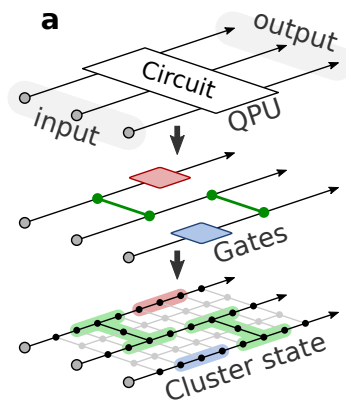

b

Cluster state generation

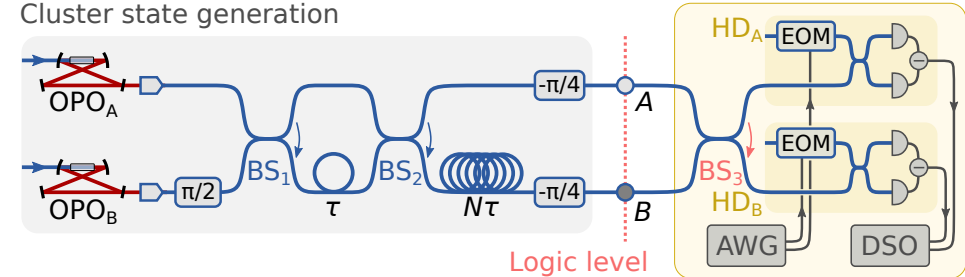

d

-Single-mode gates:

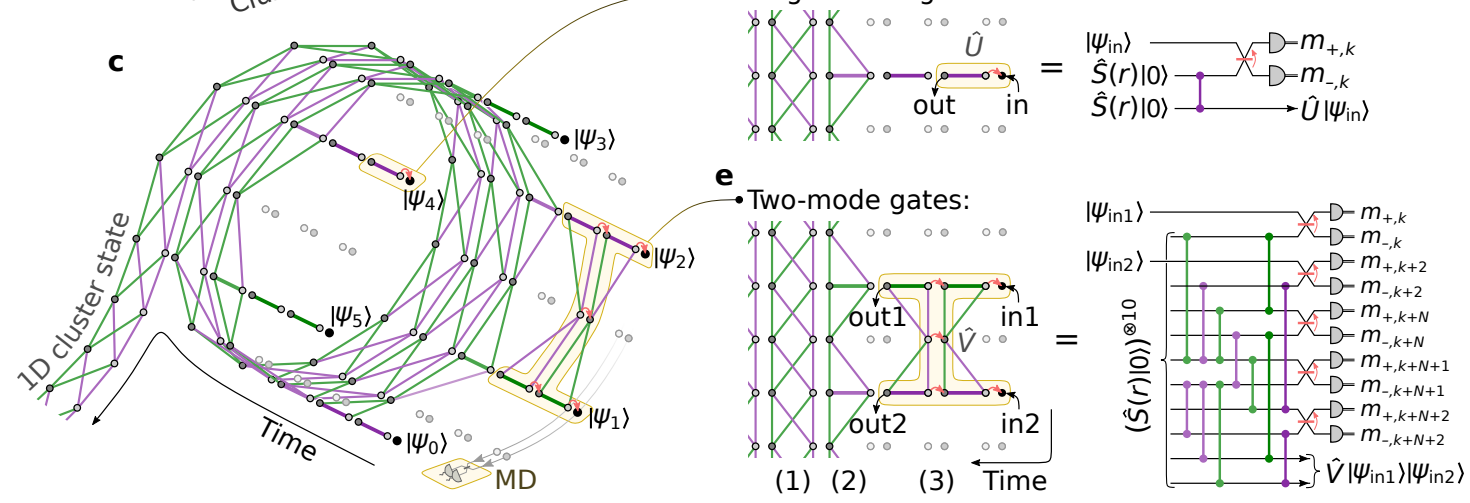

Figure 5.1: Experimental setup and computation scheme. (a) Decomposition of a quantum circuit in a quantum processing unit (QPU) into gates implemented on a cluster state by projective measurements of the input and cluster state modes. (b) Experimental setup generating a coiled-up $1 \mathrm{D}$ cluster state in the logic level where quantum information is encoded. The $-\pi / 4$ phase-shifts transforms the generated $\mathcal{H}$-graph state into a cluster state [32]. Computation takes place in the logic level using a two-mode measurement device (MD) consisting of a beam-splitter $\left(\mathrm{BS}_{3}\right)$ and two homodyne detectors $\left(\mathrm{HD}_{\mathrm{A}}\right.$ and $\left.\mathrm{HD}_{\mathrm{B}}\right)$ with basis settings controlled using electro-optical modulators (EOM) driven by an arbitrary wavefrom generator (AWG). Measurement outcomes are acquired using a digital storage oscilloscope (DSO). The experimental setup is further described in the supplementary information section 5.3. (c) Coiled-up 1D cluster state in the logic level on which input states, $\left|\psi_{0, \cdots, 5}\right\rangle$, can be encoded on the circumference. By measuring control modes, the cluster state is projected into wires on which single- and two-mode gates can be implemented by gate teleportation. Bright and dark nodes indicate spatial modes $A$ and $B$ respectively, while the red arrows indicate $\mathrm{BS}_{3}$-operation of the MD. $(\mathrm{d}, \mathrm{e})$ Cut-outs of the cluster state showing implementations of single- and two-mode gate operations, $\hat{U}$ and $\hat{V}$, defined by the MD basis settings. Here the coiled-up cluster state at (1) is seen to be projected into wires at (2) prior to the implementation of gates at (3). The corresponding circuits are shown as well and are further described in supplementary information section 5.4 together with the computation scheme.

\subsubsection{Computation scheme}

Input quantum states for computation may be encoded on the circumference of the cluster state, and for the implementation of the desired quantum gates, they are teleported along the cylinder by projective measurement of each mode. The actually implemented gate depends on the measurement bases of the teleportation protocols. Note that the measurements are performed chronologicallyswirling around the cylinder - and that the order of the measurements is inconsequential. In the following, we summarize the computation scheme with details described in the supplementary information section 5.4 .

At the logic level, a joint measurement is performed on spatial modes $A$ and $B$ of every temporal mode $k$. The two-mode measurement device consists of a beam-splitter $\left(\mathrm{BS}_{3}\right)$ followed by two homodyne detectors each measuring in a basis determined by a phase $\theta$-i.e. measuring the 
quadrature $\hat{x}(\theta)=\hat{x} \cos \theta+\hat{p} \sin \theta$ where $\hat{x}$ and $\hat{p}$ are the electric field amplitude and phase (or position and momentum) quadratures, respectively. Temporal modes of odd $k$ are used as control modes: Measuring these in basis $\theta_{c}=(-1)^{(k-1) / 2} \pi / 4$ of $A$ and $B$, the cluster state is projected into $N / 2$ wires along the cylinder as illustrated in Fig. 5.1c [7]. These wires, which at the logic level consist of segments of two-mode entangled states, can be used for single-mode computation by gate teleportation [10]. Performing a joint measurement between mode $B, k$ representing an input state and one mode, $A, k$, of a wire segment, the input state is teleported through a gate, $\hat{U}$, to mode $B, k+N$ of the same wire where the operation $\hat{U}$ depends on the measurement basis setting, $\left(\theta_{A, k}, \theta_{B, k}\right)_{U}$ (see Fig. 5.1d). To implement two-mode gates, modes of neighboring wires must be coupled which can be done by changing the measurement basis, $\theta_{c}$, of some of the control modes $[11,32]$. Depending on the basis setting on the wire modes and the coupling control mode, $\left(\left\{\theta_{A, i}, \theta_{B, i}\right\}_{i=k, k+2, k+N, k+N+1, k+N+2}\right)_{V}$, a two-mode gate operation $\hat{V}$ is implemented as illustrated in Fig. 5.1e.

In the Heisenberg picture, an implemented Gaussian $n$-mode gate operation transforms the quadratures as

$$
\hat{\boldsymbol{q}}^{\prime}=\mathbf{G} \hat{\boldsymbol{q}}+\mathbf{N} \hat{\boldsymbol{p}}_{\boldsymbol{i}}+\mathbf{D} \boldsymbol{m},
$$

where $\hat{\boldsymbol{q}}=\left(\hat{x}_{1}, \cdots, \hat{x}_{n}, \hat{p}_{1}, \cdots, \hat{p}_{n}\right)^{T}$ and $\hat{\boldsymbol{q}}^{\prime}=\left(\hat{x}_{1}^{\prime}, \cdots, \hat{x}_{n}^{\prime}, \hat{p}_{1}^{\prime}, \cdots, \hat{p}_{n}^{\prime}\right)^{T}$ are $2 n$ vectors of quadratures of the gate input and output modes, respectively. For single- and two-mode gates, $n=1$ and 2. In Eq. (5.1), the first term represents the Gaussian gate with $\mathbf{G}$ being the corresponding symplectic matrix that depends on the measurement basis setting. The last term, $\mathbf{D} \boldsymbol{m}$, represents a teleportation by-product of displacements with $\boldsymbol{m}$ being a vector of measurement outcomes, transformed by the basis setting dependent matrix D. Finally, the middle term, $\mathbf{N} \hat{\boldsymbol{p}}_{\boldsymbol{i}}$, represents noise occurring in the gate with $\hat{\boldsymbol{p}}_{\boldsymbol{i}}$ being a vector of initial momentum squeezed quadratures of the cluster state modes, transformed by the gate noise matrix $\mathbf{N}$. G, $\mathbf{N}$ and $\mathbf{D}$ are given in the supplementary information section 5.4 for different basis settings.

An ideal gate transformation is performed when the gate noise and displacement terms are zero. Since the measurement outcomes, $\boldsymbol{m}$, are known, the displacement by-product can be compensated for by feedforward, which is done here by adding $-\mathbf{D} \boldsymbol{m}$ to the measurement outcomes of output state quadratures, $\hat{\boldsymbol{q}}^{\prime}$. The gate noise, however, is only negligible for cluster states generated from infinitely squeezed vacuum states, i.e. $\operatorname{Var}\left\{\hat{\boldsymbol{p}}_{\boldsymbol{i}}\right\}=\mathbf{0}$. Such states are however nonphysical and in practice, non-zero additive Gaussian noise will inevitably occur and must eventually be accounted for by quantum error-correction. It is interesting to note that while the gate noise critically depends on the amount of multi-mode squeezing in the cluster, the transformation matrix $\mathbf{G}$ can be perfectly realized independent on this squeezing. This feature is similar to conventional CV teleportation $[124]$.

\subsubsection{Quantum gates}

For single-mode gates, the implemented operation corresponding to $\mathbf{G}$ in Eq. (5.1) is

$$
(-1)^{w} \hat{R}\left(\theta_{+} / 2\right) \hat{S}\left(\tan \theta_{-} / 2\right) \hat{R}\left(\theta_{+} / 2\right),
$$

where $w=(k \bmod N) / 2 \in\{0, \cdots, 5\}$ is the wire number, $\hat{R}(\theta)=e^{i \theta\left(\hat{x}^{2}+\hat{p}^{2}\right) / 2}$ and $\hat{S}(s)=$ $e^{i \ln (s)(\hat{x} \hat{p}+\hat{p} \hat{x})}$ are rotation and squeezing operations, and $\theta_{ \pm}$depends on the basis settings as $\theta_{ \pm}=$ $\pm \theta_{A, k}+\theta_{B, k}$. With the basis settings described in the supplementary information section 5.4.1 we implement the rotation gate, $\hat{R}(\theta)$, a modified shear gate, $\hat{F}^{j} \hat{P}(\sigma)=\hat{F}^{j} e^{i \sigma \hat{x}^{2} / 2}$, and the squeezing gate, $\hat{S}\left(e^{r}\right)$. Note, this modified version of the shear gate, with $\hat{F}^{j}=\hat{R}(j \pi / 2)$ where $j=(-1)^{w}$, makes it possible to implement shear in a single computation step of Eq. (5.2), while $\hat{F}^{j}$ can be compensated for in a second computation step if necessary. $\left\{\hat{R}(\theta), \hat{S}\left(e^{r}\right)\right\}$ constitutes a universal single-mode Gaussian gate-set [6], while $\left\{\hat{R}(\pi / 2), \hat{F}^{j} \hat{P}(1)\right\}$ constitutes a universal single-mode Clifford gate-set on GKP-encoded qubits [14]. Note that phase-space displacements are ubiquitous in MBQC, and they are simply accounted for in the measurement results.

We characterize the implemented gates with gate tomography by letting the input mode be entangled to a reference mode and measuring the quadrature correlations between the reference and 


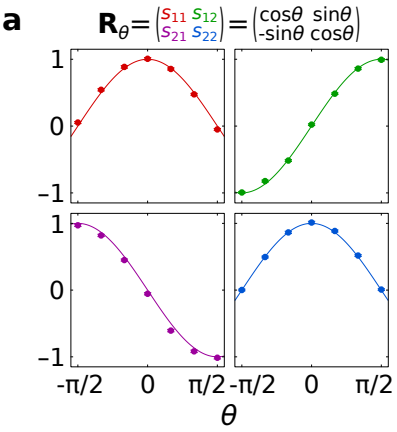

C

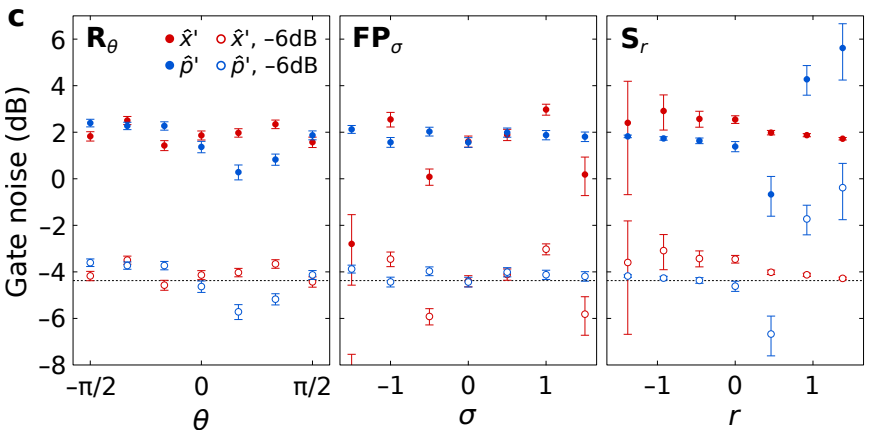

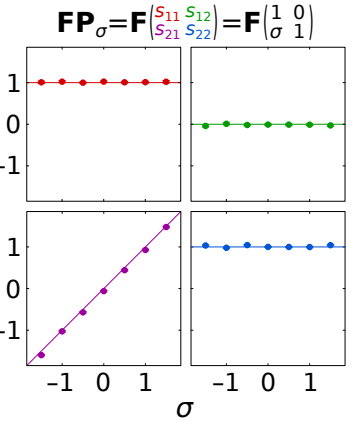

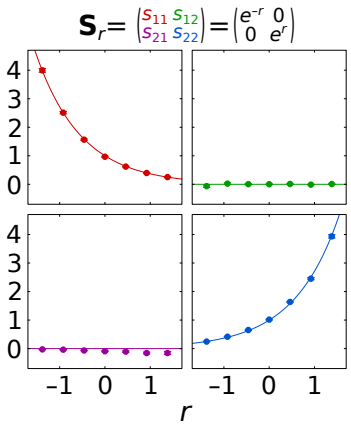

b
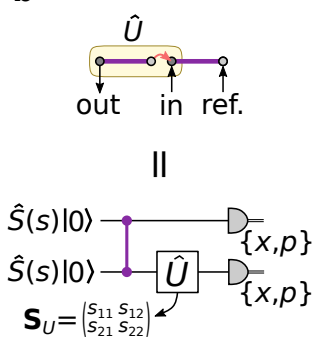

Figure 5.2: Single-mode gates. (a) Symplectic matrices corresponding to $\mathbf{G}$ in Eq. (5.1) for the implemented rotation, shear (modified by $\hat{F}$ ), and squeezing gates, measured by gate tomography as described in supplementary information section 5.5 and summarized in (b). (c) Measured gate noise variance in each output quadrature for each implemented gate (solid points). Also, gate noise compensated by $-6 \mathrm{~dB}$ to account for the effect of the gate noise matrix $\mathbf{N}$ in Eq. (5.1) is shown (hollow points), in which case it should agree with the initial momentum squeezing variance, which is independently measured to be $-4.4 \mathrm{~dB}$ (dashed line). Each point in (a) and (c) is extracted from 10000 measurements, while the error bars are estimated as standard deviation when binning the 10000 measurements into smaller datasets. The same is the case for datapoints in Fig. 5.3 and 5.4. (d) Single-mode gate noise as a function of pump power, where zero pump power corresponds to no squeezing in which case the cluster state is replaced by vacuum. Here the measured gate noise (solid points) is for $\hat{R}(\theta)$, averaged over $\theta, \hat{x}$ and $\hat{p}$, and error bars are estimated as standard deviation hereof. Black-solid line represents the estimated gate noise using the setup parameters, while the dashed line corresponds to the case with no correlations. Green and purple lines show the expected gate noise for more optimal parameters with $\geq 90 \%$ optical efficiencies and for $7.7 \mathrm{MHz}$ squeezing bandwidth as in the experimental setup (green), together with a broader squeezing bandwidth of $100 \mathrm{MHz}$ (purple) - see supplementary information section 5.6 for a derivation and further discussion.

the gate-teleported output modes [25]. The resulting symplectic matrices are shown in Fig. 5.2a with the corresponding circuit summarized in Fig. 5.2b-for details, see supplementary information section 5.5.1. The symplectic matrix elements are seen to agree well with the theoretical values. The gate noise is shown in Fig. 5.2c. For our computation scheme, we expect a gate noise of $\operatorname{Var}\left\{\mathbf{N} \hat{\boldsymbol{p}}_{\boldsymbol{i}}\right\}=4 e^{-2 r} V_{0}$ (supplementary information section 5.4.1) which is four times larger than the initially squeezed state variance of $e^{-2 r} V_{0}$ (where $V_{0}$ is the vacuum variance). Therefore, by compensating the measured gate noise by the four vacuum units, $1 / 4 \approx-6 \mathrm{~dB}$, we expect to regain the initially measured squeezing variance of $4.4 \mathrm{~dB}$. The compensated noise level is illustrated in Fig. 5.2c and is seen to agree well with the expected value except for the squeezing gate where the squeezing level, $e^{r}=\tan \theta_{-} / 2$, becomes highly sensitive to phase fluctuations in $\theta_{-}=-\theta_{A, k}+\theta_{B, k}$ for large $|r|$.

To further demonstrate the impact of the cluster state entanglement, the measured gate noise is 

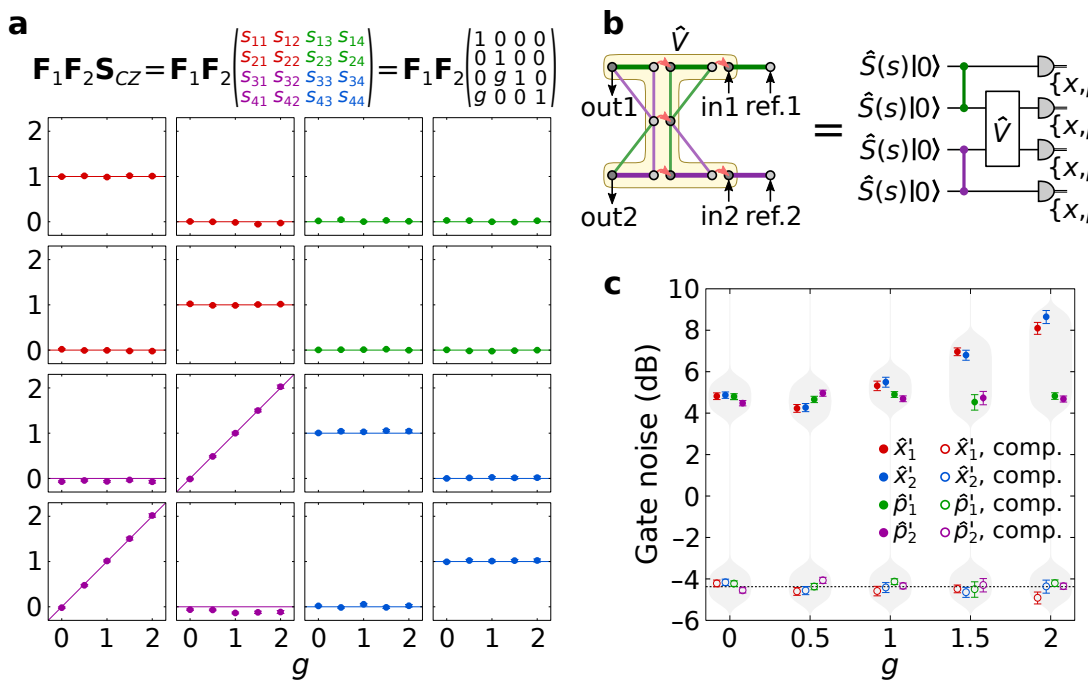

Figure 5.3: Two-mode gate. (a) Symplectic matrix corresponding to $\mathbf{G}$ in Eq. (5.1) for the implemented controlled-Z gate (modified by $\hat{F} \otimes \hat{F}$ ) as a function of the coupling coefficient $g$, measured by gate tomography (described in supplementary information section 5.5) with the corresponding circuit shown in (b). (c) Measured gate noise in the four output quadratures for each implemented controlled-Z gate (solid points), together with gate noise compensated for the effect of the gate noise matrix $\mathbf{N}$ in Eq. (5.1) (hollow points). The values for compensation are given in the supplementary information Fig. 5.10 for each value of $g$. The compensated gate noise can be compared with the initial squeezing variance for cluster state generation, measured to be $-4.4 \mathrm{~dB}$ (dashed line). Estimation of error bars are described in Fig. 5.2.

plotted in Fig. 5.2d as a function of the OPO pump power that controls the squeezing process. For vanishing squeezing (zero pump power) where the cluster state is simply a vacuum state, gate noise of $6 \mathrm{~dB}$ is measured, corresponding to the classical limit of our scheme. When increasing the OPO pump power, the gate noise reduces below this limit due to the increasing cluster state entanglement. The measured gate noise agrees well with that estimated from the experimental parameters of the setup described in section 3.6.2 [31]. Obviously, for fault-tolerant computation, much lower gate noise is required. Gate noise with potential improvements of the setup are estimated and plotted in Fig. 5.2d as well. It is clear that higher optical efficiencies and larger squeezing bandwidth significantly decrease the gate noise and can bring the system towards fault-tolerant computation. See supplementary information section 5.6 for a more comprehensive discussion on gate noise.

To complete the universal Gaussian gate set, we implemented a two-mode gate - a modified version of the controlled-Z gate, $\left(\hat{F} \otimes \hat{F}^{j}\right) \hat{C}_{Z}(g)=\left(\hat{F} \otimes \hat{F}^{j}\right) e^{i g \hat{x} \otimes \hat{x}}$. Here $w$ in $j=(-1)^{w}$ is the lower wire number of the two wires on which the gate is implemented. The required basis setting and resulting gate noise is derived in supplementary information section 5.4.2. To shorten the notation, in the following we denote $\left(\hat{F} \otimes \hat{F}^{j}\right)$ simply as $\hat{F} \hat{F}$. Note that the transformation $\hat{F} \hat{F}$ can be easily reversed in subsequent transformations to realize a pure $\hat{C}_{Z}(g)$ gate [32]. Together with the implemented single-mode gates, $\left\{\hat{F} \hat{F} \hat{C}_{Z}(g), \hat{R}(\theta), \hat{S}\left(e^{r}\right)\right\}$ constitutes a universal multimode Gaussian gate-set [118], while $\left\{\hat{F} \hat{F} \hat{C}_{Z}(1), \hat{R}(\pi / 2), \hat{F}^{j} \hat{P}(1)\right\}$ constitutes a multi-mode Clifford gate-set on GKP-encoded qubits [14]. For gate tomography of the implemented $\hat{F} \hat{F} \hat{C}_{Z}(g)$-gate, quadrature correlations of the output state and reference states entangled to the input states are measured (see supplementary information section 5.5.2). The resulting symplectic matrix, together with the corresponding gate tomography circuit, is shown in Figs. 5.3a and b, and the measured symplectic matrix elements are seen to agree well with the expected values. The gate noise, shown in Fig. 5.3c, is larger than for single-mode gates since two-mode gates are implemented in two computation steps and depend on $g$. By compensating for the effect of $\mathbf{N}$, we again retrieve the expected squeezing variance of $-4.4 \mathrm{~dB}$ with good agreement. 

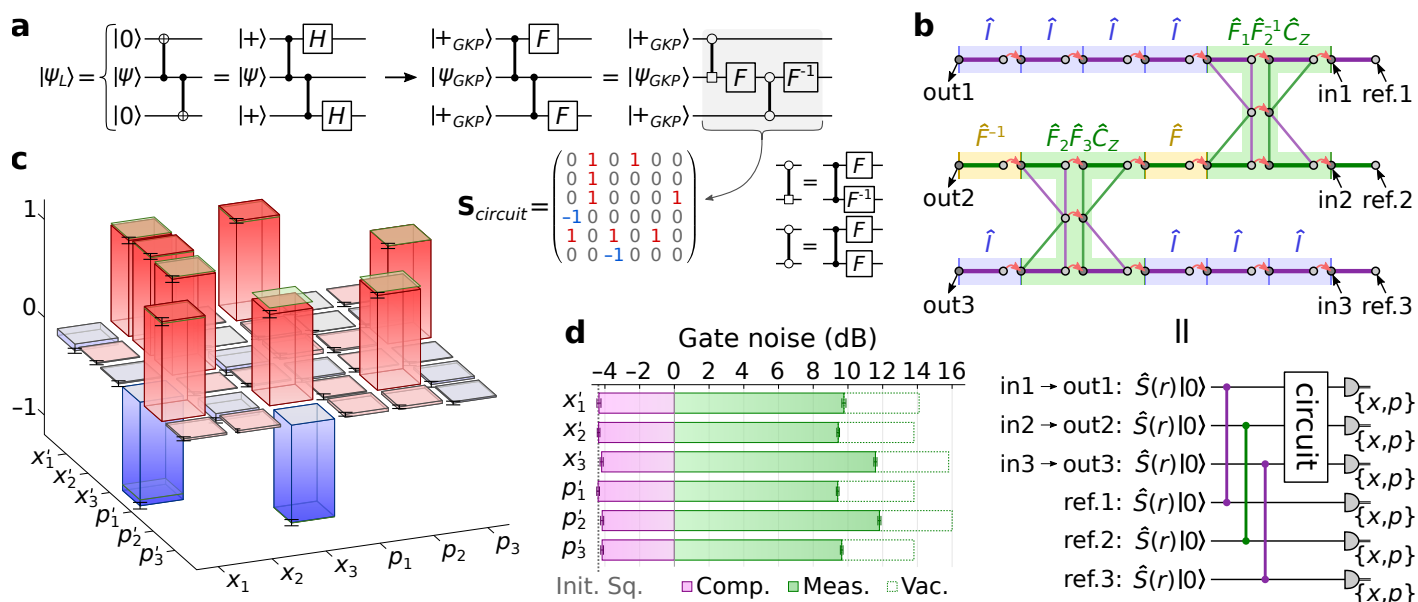

d Gate noise (dB)

II
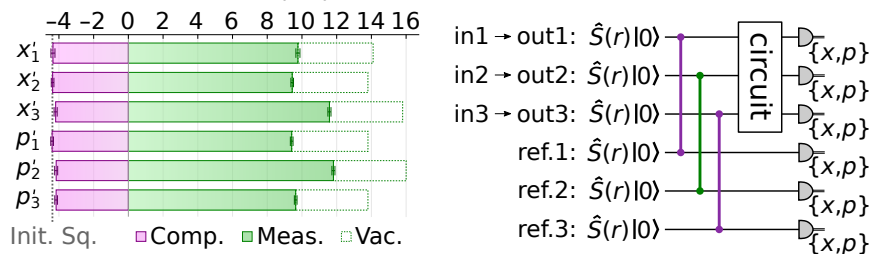

Figure 5.4: Quantum circuit. (a) Circuit encoding a logic qubit in the 3-qubit bit-flip error correction code, rewritten in terms of the CV $\hat{F} \hat{F} \hat{C}_{Z}(1)$ and $\hat{F}^{ \pm 1}=\hat{R}( \pm \pi / 2)$ gates to take GKPencoded qubits as input. (b) Implementation of the encoding circuit (marked by gray area in (a)) on three coupled cluster state wires, together with the corresponding circuit for circuit tomography described in supplementary information section 5.5.3. (c) Resulting circuit symplectic matrix estimated from quadrature correlations of input and reference modes in the circuit tomography. Here green pads show the expected values, listed for $\mathbf{S}_{\text {circuit }}$ in (a). (d) Measured gate noise (green bars), compared to expected gate noise for vacuum in place of the cluster state (dashed bars). Also, gate noise compensated for the combined circuit gate noise matrix $\mathbf{N}$ is shown (purple bars), with compensated values given in supplementary information Fig. 5.11. The compensated gate noise can be compared with the initial squeezing variance for cluster state generation of $-4.4 \mathrm{~dB}$ (dashed gray line). Estimation of error bars are described in Fig. 5.2.

\subsubsection{Quantum circuit}

To demonstrate the flexibility of combining gates into a quantum circuit, here we implement as an example a three-mode circuit, which for GKP qubits as inputs states encodes a logical qubit in the three-qubit bit-flip error correction code (see Fig. 5.4a). The implementation of the circuit on the cluster state is illustrated in Fig. 5.4b. It includes two $\hat{F} \hat{F} \hat{C}_{Z}(1)$ gates corresponding to qubit controlled-Z and Hadamard gates, two $\hat{F}^{ \pm 1}=\hat{R}( \pm \pi / 2)$ gates corresponding to qubit Hadamard gates, and eight identity gates which can be thought of as qubit memory and may be unnecessary depending on the surrounding circuit. The details of the implementation and associated gate noise are discussed in supplementary information section 5.4.3.

To characterize the performance of the implemented circuit, we perform circuit tomography similar to the strategy applied for gate transformations (see supplementary information section 5.5.3). The resulting gate symplectic matrix, shown in Fig. 5.4c, is seen to resemble the desired matrix. In Fig. 5.4d, the measured gate noise is shown and compared to the expected gate noise for a cluster state with no entanglement. It is clear that the entanglement of the cluster state leads to a reduction of the gate noise. To verify the measured gate noise values, we back-propagate the combined gate noise through the circuit by compensating for $\mathbf{N}$ (as presented in supplementary information Fig. 5.11) with the result shown in Fig. 5.4d, and the estimated values agree well with the initially measured degree of squeezing of $4.4 \mathrm{~dB}$. Note that the large total circuit noise stems from the accumulation of gate noise associated with multiple concatenated gates and the lack of error correction. To prevent gate noise accumulation as required for fault-tolerant computation, GKP quadrature error correction should be performed as often as possible, preferably in between each implemented gate [32]. 


\subsubsection{Outlook}

We have demonstrated the machinery for performing MBQC on our cluster state architecture, which relies on comparatively low-tech photonic technology at room temperature, and illustrated its computational flexibility by combining 12 gates into a simple quantum circuit. The single- and two-mode gates can be organized in any order on the six input modes of the cluster state, thereby allowing for the implementation of an arbitrary six-mode circuit transformation of, in principle, infinite depth. The demonstrated platform is currently restricted to a six-mode circuit, but due to its inherent deterministic nature, the platform can be efficiently up-scaled to allow for largescale computation. This can be attained by increasing the bandwidth of the optical squeezing process and complement it with broadband homodyne detectors. Bandwidths of several $\mathrm{GHz}$ are possible [35, 125], so the number of input modes can be increased to several thousands, bringing the platform well into NISQ (noisy intermediate-scale quantum technology) territory [102, 126]. Furthermore, with the platform's telecom compatibility, multiple processing units may straightforwardly be combined and scaled up without the need of complex quantum transduction. More generally, instead of the all-temporal encoding used here for constructing and scaling the optical cluster state, it is also possible to use spectral [20,96] and spatial [17] degrees of freedom.

To attain fault-tolerant universal quantum computing on our platform, the gate noise must be significantly decreased and the quantum information must be encoded as qubits, such as GKP qubits. Fault-tolerance is attained by increasing the amount of squeezing of the cluster state to lower the gate noise and by using GKP ancilla states for quantum error-correction to prevent the accumulation of noise. The squeezing threshold for fault-tolerance of similar MBQC schemes has been estimated to be in the range of $10-17 \mathrm{~dB}[38,43]$ which should be compared to the stateof-the-art of squeezing of $15 \mathrm{~dB}$ [115]. GKP states have been generated in vibrational modes of trapped ions [101] and in microwave cavity fields [100], but it remains a challenge to produce them in the optical spectrum [112, 127-130]. Once this challenge has been solved, all ingredients for fault-tolerant, universal, scalable quantum computing are available.

\subsection{Suppl. Inf.: Experimental setup}

The experimental setup in Fig. 5.1b is shown here in Fig. 5.5 with more details of the measurement device including control of the basis settings for different temporal modes. The cluster state generation scheme is similar to the scheme presented in chapter 3 [31], where a detailed description of the cluster state generation setup can be found in the corresponding methods section 3.3. The optical table of the experimental realization is presented in appendix A (page 147). To summarize: Two-mode squeezing in spatial mode $A$ and $B$ is generated by interfering two single mode squeezed states in a beam splitter denoted $\mathrm{BS}_{1}$. By delaying one mode of the two-mode squeezed states by $\tau$ and subsequently interfering the two modes $(A$ and $B)$ at beam-splitter $\mathrm{BS}_{2}$, a one-dimensional (1D) cluster state, namely a dual-rail wire [9, 19] with temporal mode duration of $\tau$, is formed. The $1 \mathrm{D}$ cluster state is coiled up by a long delay of $N \tau$ to form a cylinder with $N$ temporal modes in the circumference. Locally on the cylinder surface the coiled-up cluster state can be pictured as a 2-dimensional (2D) cluster state of parallel 1D cluster states. In chapter 3 the coiled-up 1D cluster state is then interfered with itself by beam-splitter $\mathrm{BS}_{3}$ to form a double bilayer square lattice [31, 32]. However, for cluster state computation, here we will consider the coiled-up cluster state just before $\mathrm{BS}_{3}$, while $\mathrm{BS}_{3}$ is part of a joint measurement device for implementing quantum gates by projective measurements. As such, in the experimental setup, the modes in computation are located just before $\mathrm{BS}_{3}$, and is marked as the logic level in Fig. 5.5. A computation scheme in this logic level is presented in chapter 4 [32], while here we use a slightly modified scheme as presented in section 5.4.

In chpater 3 [31], the generated coiled up $1 \mathrm{D}$ cluster state is in practice a $\mathcal{H}$-graph state [7]. Here, we rotate the phases of every mode in the logic level by $-\pi / 4$ to transform this $\mathcal{H}$-graph state into a cluster state with real edges. The $-\pi / 4$ phase-rotations on both spatial mode $A$ and $B$ commutes with $\mathrm{BS}_{3}$, and thus for simplicity, in the experimental realization we apply the 


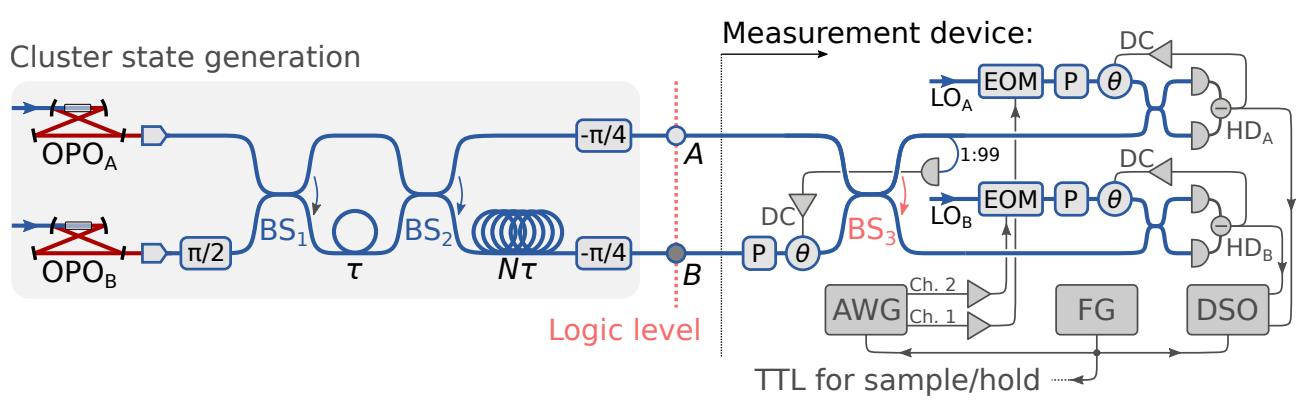

Figure 5.5: Schematics of the experimental setup showing the details of the measurement device for gate implementation by projective measurements, while details on the setup for cluster state generation can be found in the methods section 3.3 [31]. Red and blue represents free-space and single-mode fiber optics respectively. In the measurement device $\mathrm{P}$ indicates polarization control while $\theta$ indicates active phase control using a fiber stretcher [30]. The required basis setting sequence in the homodyne detectors (HD) to implement a desired gate is controlled using electrooptical modulators (EOM) in the local oscillators (LO). The EOM is driven using an amplified two-channel arbitrary waveform generator (AWG), and the measured quadrature time traces are acquired on a digital storage oscilloscope (DSO). The AWG, scope and sample/hold locking scheme is triggered from a function generator (FG).

$-\pi / 4$ phase-rotations directly onto the the local oscillators, $\mathrm{LO}_{A}$ and $\mathrm{LO}_{B}$, of the two homodyne detectors $\mathrm{HD}_{A}$ and $\mathrm{HD}_{B}$. Thus, in the following, the generated state is considered as a cluster state, and not a $\mathcal{H}$-graph state.

In the measurement device, the phase relation of the input modes of $\mathrm{BS}_{3}$ is actively locked by tapping off and detecting about $1 \%$ of the power in spatial mode $A$, and the DC measurement value is fed back via a PID to a fiber phase controller - a so-called DC-lock. The LO phases of the homodyne detectors are phase-locked in similar fashion using DC-locks, thereby enabling stable measurements of the $\hat{p}$ - and $\hat{x}$-quadrature of the spatial modes $A$ and $B$. Finally, using an electro-optical phase modulator (EOM) in each local oscillator, the homodyne detector bases can be individually and dynamically controlled in each spatial mode for different temporal modes. To prevent technical noise from the probe beams used for phase-locking of $\mathrm{BS}_{1-3}$ and the homodyne detectors, a sample/hold locking scheme is used, where a probe beam is chopped on and off as described in the methods section 3.3 [31]. All phase locks are activated when the probe is on, while when the probe is off, the phase-lock feedback is kept constant while acquiring data with basis settings controlled for each mode by the EOMs to implement a desired gate. Quadrature time traces from the homodyne detectors are acquired on a digital storage oscilloscope (DSO), and for each temporal mode $k$ the measurement outcome is extracted using the corresponding temporal mode function

$$
f_{k}(t)= \begin{cases}\mathcal{N}(t-k \tau) e^{-\kappa^{2} t^{2}} & ,|t-k \tau|<\tau / 2 \\ 0 & , \text { otherwise }\end{cases}
$$

where $\mathcal{N}$ is a normalization factor of units $\mathrm{s}^{-1}$. Here, $\kappa$ is optimized to be $2 \pi \times 2.0 \mathrm{MHz}$ to minimize the gate noise. In the data acquisition we measure 228 temporal modes, consecutively, corresponding to 19 turnarounds of the cylindrical cluster state for $N=12$. As shown in section 5.5 , this allows us to implemented and characterize multiple gates at once in parallel.

The experimental setup is operated at the telecom wavelength of $1550 \mathrm{~nm}$ to minimize propagation losses in optical fibers (blue lines in Fig. 5.5). For the short delay line a $50 \mathrm{~m}$ fiber is used leading to $\tau \approx 250 \mathrm{~ns}$ temporal mode duration, while for the long delay line $600 \mathrm{~m}$ is used leading to $N=12$. For phase locks, fiber stretchers of negligible optical losses are used as introduced in section 2.6.2 [30]. The EOMs for setting the homodyne detection bases are of model MPZ-LN-10 from iXblue with $10 \mathrm{GHz}$ bandwidth. To control the EOMs, a two channel arbitrary waveform generator (AWG) of model M4i.6631-x8 from Spectrum Instruments with 1.25 GS/s sampling rate 
and $400 \mathrm{MHz}$ bandwidth is used to generate the required waveforms to measure in a basis setting sequence implementing a desired gate. The waveform signals from the AWG driving the EOMs are amplified using THS3491 operational amplifiers (op-amps) from Texas Instrument with a $8000 \mathrm{~V} / \mu \mathrm{s}$ slew rate. To compensate for electrical responses in the AWG, op-amps and EOMs, before the experiment is carried out the AWG waveforms are optimized by inserting each EOM in a Mach-Zehnder interferometer, and feeding back the resulting applied phase shift from the EOMs to the AWG in order to update the waveform targeting a desired phase shift sequence. As a result, we are able to switch the homodyne detection basis from $-\pi / 2$ to $\pi / 2$ (both stable within $1 \%$ of the value) within $8 \mathrm{~ns}$, followed by a constant phase of the desired value after switching. More details on optimization of waveforms are presented in appendix C (page 153).

\subsection{Suppl. Inf.: Computation scheme}

In the experimental setup shown in Fig. 5.1b, and explained in detail in section 5.3, a dual-rail $1 \mathrm{D}$ cluster state $[9,19]$ is generated and coiled up into a cylinder with $N$ temporal modes in the circumference to form a cluster state with a local 2D topology. The 1D cluster state can be used for computation along the cluster state [10,25], but here, with the cluster state coiled up, the goal is instead to perform computation with information flowing along the cylinder, i.e. across the 1D cluster state. The scheme for doing so is explained in the following, and the required basis settings for implementing single-mode and two-mode gates are described in section 5.4.1 and 5.4.2, while in section 5.4.3 we combine single- and two-mode gates to implement a circuit.

In the language of graphical calculus of Gaussian states [7], the generated 1D cluster state has edge weights of $\pm t= \pm \tanh (2 r) / 2$ and self-loops of $i \operatorname{sech}(2 r)$ [9], where $r$ is the squeezing parameter of the initial momentum squeezed states. This is an approximate cluster state with the variance of the nullifiers,

$$
\begin{aligned}
& \hat{n}_{A, i}=\hat{p}_{A, i}-t\left(-\hat{x}_{A, i-1}-\hat{x}_{A, i+1}-\hat{x}_{B, i+N-1}+\hat{x}_{B, i+N+1}\right) \\
& \hat{n}_{B, i}=\hat{p}_{B, i}-t\left(\hat{x}_{A, i-N-1}-\hat{x}_{A, i-N+1}+\hat{x}_{B, i-1}+\hat{x}_{B, i+1}\right),
\end{aligned}
$$

vanishing in the limit of infinite squeezing:

$$
\operatorname{Var}\left\{\hat{n}_{A, i}\right\}=\operatorname{Var}\left\{\hat{n}_{B, i}\right\}=V_{0} \operatorname{sech}(2 r) \rightarrow 0 \quad, \quad \text { for } r \rightarrow \infty,
$$

where $V_{0}=1 / 2$ is the variance of vacuum for $\hbar=1$, and the subscript indicates the mode numbering in Fig. 5.6. Since the edge weight, $t$, depends on the squeezing parameter, $r$, the required basis setting for implementing a desired quantum gate will in general depend on $r$. Such scheme is described in detail in chapter 4 [32] for the cluster state considered here, namely the double bilayer square lattice (DBSL). However, in practice, it is inconvenient to have the basis settings depend on $r$, as the exact initial squeezing most often is unknown at the point the experiment is carried out and may vary slightly from time to time. Instead, here we redefine the generated state to have the same graph but with edge weights $\pm t= \pm 1 / 2$, i.e. we drop the squeezing dependent $\tanh (2 r)$ in the edge weights. The resulting $1 \mathrm{D}$ graph state is by the definition in Ref. [4] a cluster-type state $^{1}$ with the variance of the nullifiers in Eq. (5.4) equal

$$
\operatorname{Var}\left\{\hat{n}_{A, i}\right\}=\operatorname{Var}\left\{\hat{n}_{B, i}\right\}=2 V_{0} e^{-2 r} \rightarrow 0 \quad, \quad \text { for } r \rightarrow \infty,
$$

which as well vanish in the limit of infinite squeezing. Note that at high squeezing levels where $\operatorname{sech}(2 r) \approx 2 e^{-2 r}$, the nullifier variances for the approximate cluster state in Eq. (5.5) and the cluster-type state in Eq. (5.6) are equal, while for vanishing squeezing, (5.5) approaches the vacuum variance, $V_{0}$, while (5.6) approaches $2 V_{0}$. Thus, for finite squeezing, the redefined cluster-type state

\footnotetext{
${ }^{1}$ A cluster-type state, with graph vertices $a \in G$ and connected nodes $b \in N_{a}$ to $a$, is defined in [4] as a multi-mode Gaussian state where the variance of $\hat{p}_{a}-\sum_{b \in N_{a}} t_{b} \hat{x}_{b}$ (here the non-zero variable $t_{b}$ is added by us to generalize the cluster-type states to have variable edge weights) vanishes in the limit of infinite squeezing. Thus, cluster-type states are a more general group of states allowing cluster state approximations not covered by graphical calculus for Gaussian pure states [7].
} 


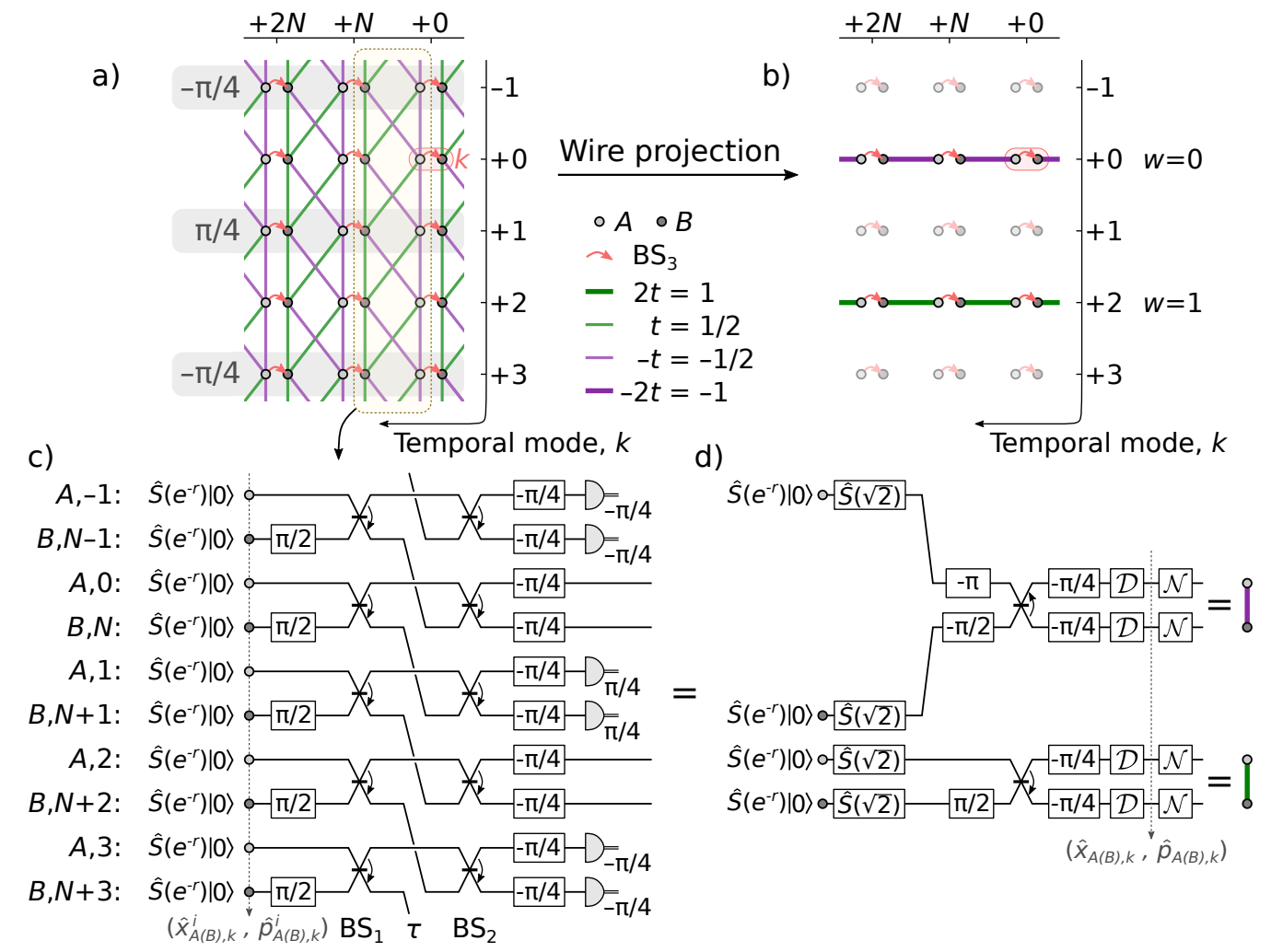

Figure 5.6: (a) Section of the coiled-up dual-rail 1D cluster state in the logic level of Fig. 5.1b, forming a local 2D cluster state of parallel vertical 1D cluster states. Here the bright and dark modes represent spatial mode $A$ and $B$, respectively, while temporal mode $k=0$ is marked as a red area. Modes in the gray shaded area are control modes, which are measured in basis $\hat{x}( \pm \pi / 4)$ to project the $2 \mathrm{D}$ cluster state into wires. The red arrow in each temporal mode indicates the beam-splitter of the measurement device, $\mathrm{BS}_{3}$, and can for the control modes be compensated for as shown in Eq. (5.10). (b) After measuring control modes, the cluster state is projected into wires with wire number $w=(k \bmod N) / 2$. (c) Shows the $1 \mathrm{D}$ cluster state generation and wire projection, while (d) shows the corresponding simplified circuit after measurements. Here $\mathcal{D}$ and $\mathcal{N}$ corresponds to displacements and noise as described in Eq. (5.7), (5.8) and (5.9).

with $t=1 / 2$ is more noisy than the more traditional approximate cluster state with $t=\tanh (2 r) / 2$. Fortunately, we find that the noise produced by gates implemented on the generated graph state considered as a cluster-type state is at the same level or, in some cases, even lower than if we use the graph state as an approximate cluster state. In the following, unless it may lead to confusion, we will use the term 'cluster state' for both cluster-type states and approximate cluster states.

The rules for graphical calculus of Ref. [7] applies to approximate cluster states and are therefore, to our knowledge, not necessarily valid for the more general cluster-type states. As a result, here we will derive the necessary graph transformation. A section of the coiled-up 1D cluster state is shown in Fig. 5.6a. To perform computation, we need to project the cluster state into wires along the cylinder, on which gates can be implemented [32]. To do so, control modes (in the grey shaded areas of Fig. 5.6a) are measured in alternating bases of $(-1)^{(k-1) / 2} \pi / 4$ where odd $k$ is the mode number of the temporal control modes in Fig. 5.6a, i.e. measuring $\hat{x}( \pm \pi / 4)=(\hat{x} \pm \hat{p}) / \sqrt{2}$ of control modes by homodyne detection. From standard graphical calculus of Ref. [7], we expect this measurement to form new edges of weight $\pm 2 t$ as shown in Fig. 5.6b. To see that this is indeed the case for the cluster-type state with $t=1 / 2$, we consider the $1 \mathrm{D}$ cluster state generation scheme in Fig. 5.6c: After measuring the control modes, the generation scheme can be simplified to separate the generation of two-mode entangled states as shown in Fig. 5.6d (to derive this, we 
have used the method of calculating quadrature transformations outlined in section 4.8 [32]). Here $\mathcal{D}$ symbolises a displacement in phase-space, $\hat{D}(\alpha)$, by $\operatorname{Re}\{\alpha\}$ and $\operatorname{Im}\{\alpha\}$ in $\hat{x}$ - and $\hat{p}$-quadrature, respectively, depending on the control mode measurement outcomes as

$$
\begin{aligned}
\hat{D}_{A, k}(\alpha) \quad, \quad \alpha=\frac{1}{2 \sqrt{2}}\left[(j-i) m_{A, k-1}\right. & +(-j-i) m_{A, k+1} \\
+ & \left.(j-i) m_{B, k+N-1}+(j+i) m_{B, k+N+1}\right], \\
\hat{D}_{B, k+N}(\beta) \quad, \quad \beta=\frac{1}{2 \sqrt{2}}\left[(-j+i) m_{A, k-1}\right. & +(-j-i) m_{A, k+1} \\
+ & \left.(-j+i) m_{B, k+N-1}+(j+i) m_{B, k+N+1}\right],
\end{aligned}
$$

where $j=(-1)^{w}$ with $w=(k \bmod N) / 2$ being the wire number, while $i$ is the imaginary unit, $i^{2}=$ -1 . Since the measurement outcomes, $m_{A(B), k}$, are known, this displacement can be compensated for by feeding the measurement results forward to displacement operations displacing the wire modes back. Or, we can simply keep track of the displacements and compensate for them in the final measurement outcomes since all operations implemented here are Gaussian operations $[2,5]$. $\mathcal{N}$ symbolises a quadrature symmetric noise operator, and originate from finite squeezing in the 1D cluster state generation. In the Heisenberg picture, this is represented by adding initial finite squeezed momentum quadratures, $\hat{p}_{A(B), k}^{i}$, to the the wire cluster state quadratures $\hat{x}_{A(B), k}$ and $\hat{p}_{A(B), k}$. Here $\left(\hat{x}_{A(B), k}^{i}, \hat{p}_{A(B), k}^{i}\right)$ and $\left(\hat{x}_{A(B), k}, \hat{p}_{A(B), k}\right)$ are marked on Fig. 5.6c,d. The resulting quadrature evolution of $\mathcal{N}$ on the wire mode quadratures is

$$
\left(\begin{array}{c}
\hat{x}_{A, k} \\
\hat{x}_{B, k+N} \\
\hat{p}_{A, k} \\
\hat{p}_{B, k+N}
\end{array}\right) \stackrel{\mathcal{N}}{\longrightarrow}\left(\begin{array}{c}
\hat{x}_{A, k} \\
\hat{x}_{B, k+N} \\
\hat{p}_{A, k} \\
\hat{p}_{B, k+N}
\end{array}\right)+\frac{1}{2 \sqrt{2}}\left(\begin{array}{cc}
-1 & -1 \\
-1 & 1 \\
1 & -1 \\
1 & 1
\end{array}\right)\left(\begin{array}{c}
\hat{p}_{A, k}^{i} \\
\hat{p}_{B, k+N}^{i}
\end{array}\right)
$$

for even wires $(w=(k \bmod N) / 2=$ even $)$, and

$$
\left(\begin{array}{c}
\hat{x}_{A, k} \\
\hat{x}_{B, k+N} \\
\hat{p}_{A, k} \\
\hat{p}_{B, k+N}
\end{array}\right) \stackrel{\mathcal{N}}{\longrightarrow}\left(\begin{array}{c}
\hat{x}_{A, k} \\
\hat{x}_{B, k+N} \\
\hat{p}_{A, k} \\
\hat{p}_{B, k+N}
\end{array}\right)+\frac{1}{2 \sqrt{2}}\left(\begin{array}{cc}
1 & -1 \\
-1 & -1 \\
-1 & -1 \\
1 & -1
\end{array}\right)\left(\begin{array}{c}
\hat{p}_{A, k-1}^{i} \\
\hat{p}_{B, k+N+1}^{i}
\end{array}\right)
$$

for odd wires $(w=(k \bmod N) / 2=$ odd $)$. Since the initial momentum squeezed quadratures, $\hat{p}_{A(B), k}^{i}$, squeezed by $e^{-r}$, has a Gaussian quadrature distribution with variance $\operatorname{Var}\left\{\hat{p}_{A(B), k}^{i}\right\}=$ $e^{-2 r} V_{0}$ and mean $\left\langle\hat{p}_{A(B), k}^{i}\right\rangle=0$, the $\mathcal{N}$-operation adds noise to the wire mode quadratures depending on the initial momentum squeezing, which vanish in the limit of infinite squeezing. From Eq. (5.8) and (5.9) the noise added by $\mathcal{N}$ is seen to be correlated in the quadratures between two connected wire modes. This is exactly in such a way, that $\mathcal{N}$ can be brought to the left side of the beam-splitter and phase shifts in Fig. 5.6d, in which case $\mathcal{N}$ adds uncorrelated noise in the two wire-modes before the beam-splitter. Fortunately, for the same reason, the quadrature noise added by $\mathcal{N}$ cancels out in the wire cluster state nullifiers and when implementing gates, as we will see later. Finally, note the $\sqrt{2}$ anti-squeezing on the initial momentum squeezed states in Fig. 5.6d. This anti-squeezing leads to a degradation of the "effective" initial momentum squeezed variance which becomes $2 e^{-2 r} V_{0}$ instead of $e^{-2 r} V_{0}$, and is the cost of projecting the cluster state into another cluster state useful for computation [32].

Similar to the 1D cluster state with edge weights $\pm t= \pm 1 / 2$, the projected two-mode entangled states in Fig. 5.6b,d are cluster-type states with edge weights $\pm 2 t= \pm 1$ : After compensating for the displacements $\mathcal{D}$, the nullifiers are

$$
\begin{aligned}
\hat{n}_{A, k} & =\hat{p}_{A, k}-(-1) \hat{x}_{B, k+N}=-\sqrt{2} \hat{p}_{A, k-1}^{i}-\sqrt{2} \hat{p}_{B, k+N+1}^{i} \\
\hat{n}_{B, k+N} & =\hat{p}_{B, k+N}-(-1) \hat{x}_{A, k}=\sqrt{2} \hat{p}_{A, k-1}^{i}-\sqrt{2} \hat{p}_{B, k+N+1}^{i}
\end{aligned}
$$


for even wires with $-2 t=-1$ edge weight, and

$$
\begin{aligned}
\hat{n}_{A, k} & =\hat{p}_{A, k}-\hat{x}_{B, k+N}=-\sqrt{2} \hat{p}_{A, k}^{i}+\sqrt{2} \hat{p}_{B, k+N}^{i} \\
\hat{n}_{B, k+N} & =\hat{p}_{B, k+N}-(-1) \hat{x}_{A, k}=\sqrt{2} \hat{p}_{A, k}^{i}+\sqrt{2} \hat{p}_{B, k+N}^{i}
\end{aligned}
$$

for odd wires with $2 t=1$ edge weight, such that the variances of all nullifiers become

$$
\operatorname{Var}\left\{\hat{n}_{A(B), k}\right\}=4 V_{0} e^{-2 r} \rightarrow 0 \text { for } r \rightarrow \infty,
$$

and thus vanish in the limit of infinite squeezing as required for cluster-type states. Note that the quadratures added by $\mathcal{N}$ in Eq. (5.8) and (5.9) are not present as they cancel out due to their (anti-)correlations between the connected wire modes in (even)odd wires.

During the projection of the wires in Fig. 5.6c,d we ignored the beam splitter of the measurement device, $\mathrm{BS}_{3}$, marked by red arrows in Fig. 5.6a,b (here we define a balanced beam-splitter operation as $\hat{B}=e^{-i \pi(\hat{x} \otimes \hat{p}-\hat{p} \otimes \hat{x}) / 4}$ similarly as in chaoter 3 and 4 [31,32], and its direction-dependency is marked below by an arrow pointing from the first to the second mode of the tensor products). This is possible when the two spatial modes $A$ and $B$ of the same temporal mode, $k$, are measured in the same basis, as is the case for the control modes, since

$$
\begin{aligned}
& -\hat{x}(\theta)=m_{A, k} \\
& -\hat{x}(\theta)=m_{B, k}=\left\{\begin{array}{l}
\hat{x}(\theta)=m_{-, k} \\
\hat{x}(\theta)=m_{+, k}
\end{array} \quad m_{ \pm, k}=\left(m_{A, k} \pm m_{B, k}\right) / \sqrt{2} .\right.
\end{aligned}
$$

Thus, the hypothetical measurement outcomes of the control modes before $\mathrm{BS}_{3}, m_{A(B), k}$, are extracted from the homodyne measurement outcomes of the measurement device after $\mathrm{BS}_{3}$ as $m_{A, k}=\left(m_{+, k}+m_{-, k}\right) / \sqrt{2}$ and $m_{B, k}=\left(m_{+, k}-m_{-, k}\right) / \sqrt{2}$. Note that, since we consider the computation from the logic-level point of view, the notation used here is opposite to that used in the language of macro-nodes in $[10,11,107]$. Here ' $A$ ' and ' $B$ ' refer to spatial mode $A$ and $B$ before $\mathrm{BS}_{3}$, while '-' and ' + ' refer to spatial mode $A$ and $B$ after $\mathrm{BS}_{3}$, respectively.

The wire modes in Fig. 5.6b, projected into two-mode cluster states, are now suitable for the implementation of gates. With $N=12$ temporal modes in the circumference of the cluster state cylinder generated in this work, the coiled-up $1 \mathrm{D}$ cluster state is projected into $N / 2=6$ wires, numbered as $w \in\{0,1,2,3,4,5\}$ with edge weight $-(-1)^{w}$. As a result, the generated cluster state may hold 6 modes in computation, while this can be scaled up simply by increasing $N$ corresponding to the ratio between the long and short delay lines in the experimental setup.

\subsubsection{Single mode gates}

Single-mode gates are implemented on each wire, and a single computation step is shown in Fig. 5.7. The input mode may be switched into the spatial mode $B$ at the logic level using an optical switch, or is, most often, the output mode of the previous computation step. With a joint measurement of the input mode and one mode of the two-mode wire cluster state, using the measurement device consisting of $\mathrm{BS}_{3}$ and two homodyne detectors, the input mode is teleported to the second mode of the wire cluster state. Depending on the basis setting the input mode undergoes a Gaussian operation, $\hat{U}$, while teleported:

$$
\left|\psi_{\text {out }}\right\rangle_{B, k+N}=\hat{U}\left|\psi_{\text {out }}\right\rangle_{B, k}
$$

Here, $\hat{U}$ consists partly of a desired gate, $\hat{G}$, a displacement by-product, and a noise operation due to finite squeezing. In the Heisenberg picture, $\hat{U}$ transforms the quadratures of the input mode $B, k$ to the output mode $B, k+N$ as

$$
\left(\begin{array}{c}
\hat{x}_{B, k+N}^{\prime} \\
\hat{p}_{B, k+N}^{\prime}
\end{array}\right)=\mathbf{G}\left(\begin{array}{c}
\hat{x}_{B, k} \\
\hat{p}_{B, k}
\end{array}\right)+\mathbf{N}\left(\begin{array}{c}
\hat{p}_{A, k}^{e i} \\
\hat{p}_{B, k+N}^{e i}
\end{array}\right)+\mathbf{D}\left(\begin{array}{c}
\tilde{m}_{-, k} \\
\tilde{m}_{+, k}
\end{array}\right)
$$




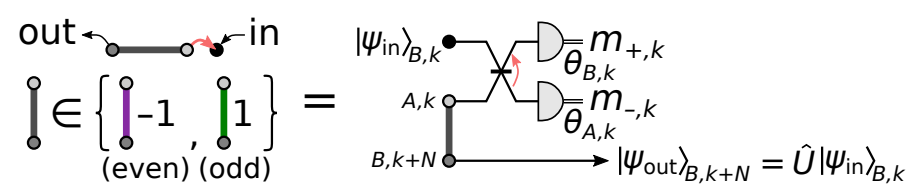

Figure 5.7: A single computation step from mode $B, k$ to $B, k+N$ on an even (purple with -1 edge weight) or odd (green with 1 edge weight) projected wire in Fig. 5.6, leading to the implemented operation with the quadrature transformation shown in Eq. (5.11). The black node represents the input mode, which may be switched into mode $B, k$ using an optical switch in the logic level at spatial mode $B$, or may, as most often, be the output of the previous computation step. The red arrow indicates the beam-splitter $\mathrm{BS}_{3}$ of the measurement device.

where the prime denotes the output mode quadratures after gate implementation. In the following we discuss each of the three terms on the right-hand-side. $\mathbf{G}$ is the symplectic matrix corresponding to the implemented gate and can be derived to be

$$
\hat{G}=(-1)^{w} \hat{R}\left(\frac{\theta_{+, k}}{2}\right) \hat{S}\left(\tan \frac{\theta_{-, k}}{2}\right) \hat{R}\left(\frac{\theta_{+, k}}{2}\right) \quad, \quad \theta_{ \pm, k}= \pm \theta_{A, k}+\theta_{B, k}
$$

where $\hat{R}(\theta)=e^{-i \theta\left(\hat{x}^{2}+\hat{p}^{2}\right) / 2}$ and $\hat{S}(s)=e^{i \ln (s)(\hat{x} \hat{p}+\hat{p} \hat{x}) / 2}$ are the rotation and squeezing operations, respectively, with the squeezing parameter $r=\ln (s)$ (leading to squeezing in the $\hat{x}$-quadrature for positive $r$ ). Note, here $\theta_{A, k}$ and $\theta_{B, k}$ are basis settings of the homodyne detectors in spatial mode $A$ and $B$ after $\mathrm{BS}_{3}$ and should not be confused with the hypothetical measurement outcomes $m_{A, k}$ and $m_{B, k}$ before $\mathrm{BS}_{3}$. The subscripts ' + ' and '-' of $\theta_{ \pm, k}$ simply notes the addition and difference of $\theta_{A, k}$ and $\theta_{B, k}$. The term $\mathbf{N}\left(\hat{p}_{A, k}^{e i}, \hat{p}_{B, k+N}^{e i}\right)^{T}$ represents the gate noise due to finite squeezing in the cluster state generation. Here $\hat{p}_{A, k}^{e i}$ and $\hat{p}_{B, k+N}^{e i}$ are the "effective" initial momentum quadratures for the two-mode wire cluster state including the $\sqrt{2}$ anti-squeezing contribution from the wire projection shown in Fig. 5.6d, and so, when including the wire projection, the second term in Eq. (5.11) is

$$
\mathbf{N}\left(\begin{array}{c}
\hat{p}_{A, k}^{e i} \\
\hat{p}_{B, k+N}^{e i}
\end{array}\right)= \begin{cases}\sqrt{2}\left(\begin{array}{cc}
-1 & -1 \\
1 & -1
\end{array}\right)\left(\begin{array}{c}
\hat{p}_{A, k-1}^{i} \\
\hat{p}_{B, k+N+1}^{i}
\end{array}\right) & , \text { for } w=\text { even } \\
\sqrt{2}\left(\begin{array}{cc}
-1 & 1 \\
1 & 1
\end{array}\right)\left(\begin{array}{c}
\hat{p}_{A, k}^{i} \\
\hat{p}_{B, k+N}^{i}
\end{array}\right) & , \text { for } w=\text { odd }\end{cases}
$$

Note, as mentioned previously, the noise added by $\mathcal{N}$ does not appear in the implementation of gates as the added noise is correlated between two connected wire-modes. As a result, with $\hat{p}_{A(B), k}^{i}$ being squeezed by $e^{-r}$, symmetric quadrature noise of variance $4 V_{0} e^{-2 r}$ is added as gate noise in each computation step, independent on the basis setting and wire number. Here, $\sum_{i} N_{q i}^{2}=4$, with $N_{q i}$ being the elements of the gate noise matrix $\mathbf{N}$ and $q=1,2$ for single-mode gates, are gate noise factors. Assuming identical squeezing in all cluster state modes, the gate noise variances of each quadrature can in general be written as the initial momentum squeezing variance scaled by the gate noise factors of each corresponding quadrature. Finally, $\mathbf{D}\left(\tilde{m}_{+, k}, \tilde{m}_{-, k}\right)^{T}$ is a displacement byproduct depending on the measurement outcomes. Here, the tilde indicates that the displacements in the wire projection $\mathcal{D}$ in Fig. 5.6 are included. Writing this out, the third term in Eq. (5.11) is

$$
\begin{array}{r}
\mathbf{D}\left(\begin{array}{c}
\tilde{m}_{-, k} \\
\tilde{m}_{+, k}
\end{array}\right)=\frac{j \sqrt{2}}{\sin \theta_{-, k}}\left(\begin{array}{cc}
-\cos \theta_{A, k} & \cos \theta_{B, k} \\
\sin \theta_{A, k} & -\sin \theta_{B, k}
\end{array}\right)\left(\begin{array}{l}
m_{+, k} \\
m_{-, k}
\end{array}\right) \\
+\frac{1}{\sqrt{2}}\left(\begin{array}{cccc}
-j & -j & -j & j \\
1 & -1 & 1 & 1
\end{array}\right)\left(\begin{array}{c}
m_{A, k-1} \\
m_{A, k+1} \\
m_{B, k+N-1} \\
m_{B, k+N+1}
\end{array}\right)
\end{array}
$$


where $j=(-1)^{w}$. Here, the first term is the displacement by-product from the gate implemented by teleportation, while the second term includes displacements from the wire projection. Note that $m_{ \pm, k}$ are the measurement outcomes in Fig. 5.7 after $\mathrm{BS}_{3}$, while $m_{A(B), k}$ are measurement outcomes of control modes before $\mathrm{BS}_{3}$ extracted using Eq. (5.10).

We implemented three single-mode gates, namely the rotation gate, $\hat{R}(\theta)=e^{-i \theta\left(\hat{x}^{2}+\hat{p}^{2}\right) / 2}$, the shearing gate $\hat{P}(\sigma)=e^{i \sigma \hat{x}^{2} / 2}$, and the squeezing gate $\hat{S}\left(e^{r}\right)=e^{i r(\hat{x} \hat{p}+\hat{p} \hat{x}) / 2}$, each with symplectic matrices

$$
\mathbf{R}_{\theta}=\left(\begin{array}{cc}
\cos \theta & \sin \theta \\
-\sin \theta & \cos \theta
\end{array}\right) \quad, \quad \mathbf{P}_{\sigma}=\left(\begin{array}{cc}
1 & 0 \\
\sigma & 1
\end{array}\right) \quad, \quad \mathbf{S}_{r}=\left(\begin{array}{cc}
e^{-r} & 0 \\
0 & e^{r}
\end{array}\right)
$$

transforming a quadrature vector $(\hat{x}, \hat{p})^{T} \cdot\left\{\hat{R}(\theta), \hat{S}\left(e^{r}\right)\right\}$ constitute a universal single-mode Gaussian gate set together with displacements in phase-space [6], while for GKP-encoded qubits on square grids in phase-space $\{\hat{R}(\pi / 2), \hat{P}(1)\}$, together with $\sqrt{\pi}$ displacements in phase-space, leads to a single-mode Clifford gate set in the encoded qubit subspace [14]. Note, phase-space displacements are ubiquitous in measurement-based quantum computation, but they are simply absorbed into the measurement results.

From Eq. (5.12), $\hat{R}(\theta)$ is implemented from mode $B, k$ to $B, k+N$ with basis setting

$$
\left(\begin{array}{l}
\theta_{A, k} \\
\theta_{B, k}
\end{array}\right)_{R}=\frac{1}{2}\left(\begin{array}{l}
\theta-(-1)^{w} \pi / 2 \\
\theta+(-1)^{w} \pi / 2
\end{array}\right),
$$

such that $\left(\theta_{+, k}, \theta_{-, k}\right)=\left(\theta,(-1)^{w} \pi / 2\right)$. Note that $(-1)^{w}$ in $\theta_{-, k}$ compensates for $(-1)^{w}$ in Eq. (5.12). From Eq. (5.12), $\hat{P}(\sigma)$ cannot be implemented in a single computation step. However, $\hat{F}^{j} \hat{P}(\sigma)=\hat{R}(j \pi / 2) \hat{P}(\sigma)$ can be implemented in a single computation step, in which $\hat{F}^{j}$ keeps a GKP-encoded qubit within the qubit subspace for $\sigma=1$, and may be compensated for in a following computation step if necessary. Here, with $j=(-1)^{w}, \hat{F}^{j}=\hat{F}$ for even wires and $\hat{F}^{j}=\hat{F}^{-1}=\hat{F}^{\dagger}$ for uneven wires. $\hat{F}^{j} \hat{P}(\sigma)$ is implemented from mode $B, k$ to $B, k+N$ with the basis setting

$$
\left(\begin{array}{c}
\theta_{A, k} \\
\theta_{B, k}
\end{array}\right)_{P}=\left(\begin{array}{c}
0 \\
\pi / 2-\arctan (\sigma / 2)
\end{array}\right),
$$

such that $\theta_{+, k}=\theta_{-, k}=\pi / 2-\arctan (\sigma / 2)$. Finally, $\hat{S}\left(e^{r}\right)$ is implemented from mode $B, k$ to $B, k+N$ with basis setting

$$
\left(\begin{array}{c}
\theta_{A, k} \\
\theta_{B, k}
\end{array}\right)_{S}=(-1)^{w} \arctan e^{r}\left(\begin{array}{c}
-1 \\
1
\end{array}\right),
$$

such that $\left(\theta_{+, k}, \theta_{-, k}\right)=\left(0,(-1)^{w} 2 \arctan e^{r}\right)$. For each of the implemented gates, the displacement by-product is compensated for in the measurement outcomes using Eq. (5.14), while with gate noise factors of 4 in each quadrature, we expect gate noise variance of $4 V_{0} e^{-2 r}$ (with $r$ here being the initial squeezing parameter in the cluster state generation) added to each quadrature, corresponding to $6 \mathrm{~dB}$ relative to the initial momentum squeezed quadrature variance.

\subsubsection{Two-mode gate}

If, instead of measuring the spatial modes $A$ and $B$ in the same basis for every temporal control mode, we measure them in different bases (such that Eq. (5.10) does not apply), two neighboring wires will be coupled [32]. This can then be used to implement two-mode gates as illustrated in Fig. 5.8.

In Fig. 5.8, the projected cluster state corresponds to the cluster-type state discussed for wire projection in Fig. 5.6. However, instead of measuring all control modes in the $\pm \pi / 4$-basis, one temporal control mode is only phase-rotated by $(-1)^{(k-1) / 2} \pi / 4$ but is left unmeasured. The result is a 10 -mode projected cluster state, which is then connected to two input modes, $B, k$ and $B, k+2$, via the joint measurement of the measurement device. Measuring all modes, except the output 


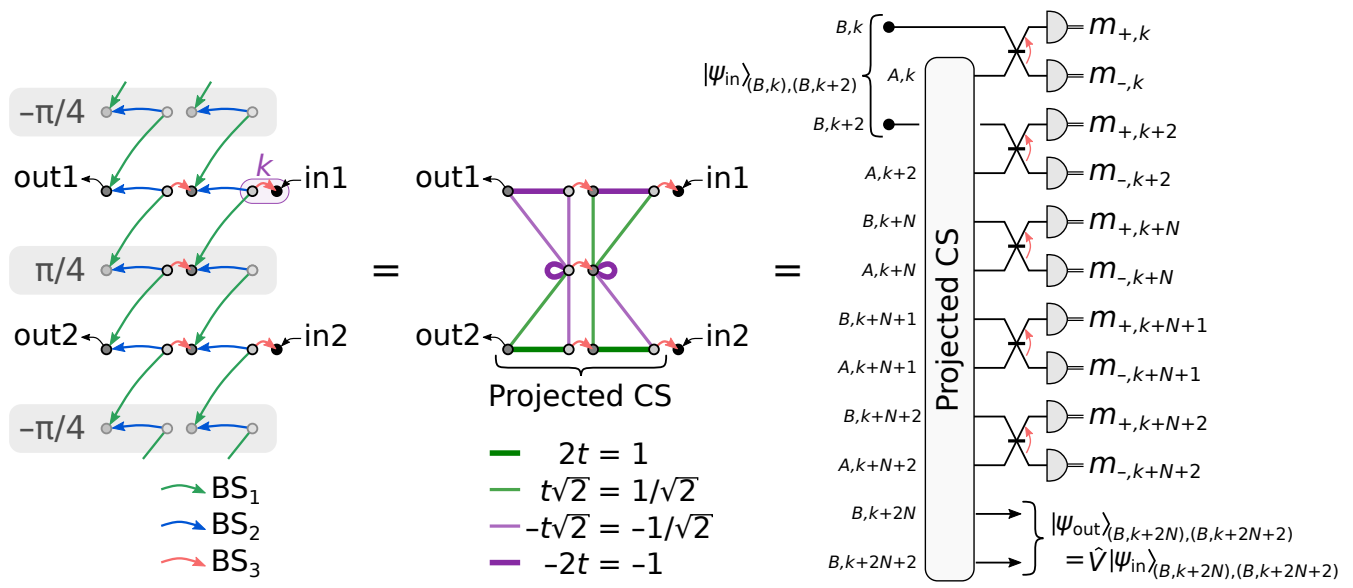

Figure 5.8: Scheme for implementing two-mode operations. Due to the many modes involved, the circuit for the cluster state generation and projection is shown graphically to the left with each beam splitter represented by an arrow. Here, control modes in the grey shaded areas are phase rotated by $\pm \pi / 4$, followed by measuring $\hat{x}$ of the faded modes. The resulting projected cluster-type state is shown in the center, consisting of two wires (here an even and odd wire on top and below) and a temporal control mode to couple the wires. By joint measurement of the input using the measurement device with $\mathrm{BS}_{3}$, as shown to the right, a two-mode operation, $\hat{V}$, with a desired two-mode gate is implemented depending on the basis setting.

modes $B, k+2 N$ and $B, k+2 N+2$, the states of the input modes are teleported to the output modes with a two-mode Gaussian operation, $\hat{V}$, applied depending on the basis settings,

$$
\left|\psi_{\text {out }}\right\rangle_{(B, k+2 N),(B, k+2 N+2)}=\hat{V}\left|\psi_{\text {in }}\right\rangle_{(B, k),(B, k+2)} \text {. }
$$

In general, $\hat{V}$ transforms the quadratures of the input to the output modes as

$$
\left(\begin{array}{c}
\hat{x}_{B, k+2 N}^{\prime} \\
\hat{x}_{B, k+2 N+2}^{\prime} \\
\hat{p}_{B, k+2 N}^{\prime} \\
\hat{p}_{B, k+2 N+2}^{\prime}
\end{array}\right)=\mathbf{G}_{2}\left(\begin{array}{c}
\hat{x}_{B, k} \\
\hat{x}_{B, k+2} \\
\hat{p}_{B, k} \\
\hat{p}_{B, k+2}
\end{array}\right)+\mathbf{N} \hat{\boldsymbol{p}}_{\boldsymbol{i}}+\mathbf{D} \boldsymbol{m},
$$

where, again, prime marks the output mode quadratures. Here $\mathbf{G}_{2}$ is the symplectic matrix corresponding to the implemented desired two-mode Gaussian gate, $\hat{G}_{2}$. $\mathbf{N} \hat{\boldsymbol{p}}_{\boldsymbol{i}}$ is the gate noise term with $\mathbf{N}$ being a gate noise matrix and $\hat{\boldsymbol{p}}_{\boldsymbol{i}}$ being a vector of initial momentum squeezed quadratures in the cluster state generation. Finally, $\mathbf{D} \boldsymbol{m}$ is the displacement by-product with $\mathbf{D}$ being a displacement matrix and $\boldsymbol{m}$ being a vector of measurement outcomes. Here, to shorten the discussion, instead of writing the quadrature transformation initially in terms of the projected cluster state quadratures and following measurement outcomes as for the single-mode operations in Eq. (5.11) (i.e. in terms of $\hat{p}_{A(B), k}$ and $\tilde{m}_{ \pm, k}$ ), in the following we will write the quadrature transformation directly from the initial momentum squeezed quadratures and measurement outcomes including the cluster state projection, similar to Eq. (5.13) and (5.14).

The two-mode controlled-Z gate, $\hat{C}_{Z}(g)=e^{i g \hat{x} \otimes \hat{x}}$ with $g$ being a coupling constant, constitutes, together with the single-mode gate set $\left\{\hat{R}(\theta), \hat{S}\left(e^{r}\right)\right\}$ in section 5.4.1, a universal multi-mode Gaussian gate set. Furthermore, for GKP-encoded qubits on square grids in phase-space, $\hat{C}_{Z}(1)$ leads, together with $\{\hat{F}=\hat{R}(\pi / 2), \hat{P}(1)\}$, to a multi-mode Clifford gate set in the encoded qubit subspace [14]. However, on the cluster state architecture considered here, $\hat{C}_{Z}(g)$ cannot be implemented directly. Instead, we implement $\left(\hat{F} \otimes \hat{F}^{j}\right) \hat{C}_{Z}(g)$, with short notation $\hat{F} \hat{F}^{j} \hat{C}_{Z}(g)$, where $j=(-1)^{w}$ with $w$ being the wire number of the input mode $B, k$ (again $\hat{F}^{+1}=\hat{F}$ and $\hat{F}^{-1}=\hat{F}^{\dagger}$ ). Here, $\hat{F}$ and $\hat{F}^{j}$ on the two output modes keep a GKP-encoded qubit within the encoded qubit subspace for 
$g=1$, and may be compensated in a subsequent computation step if necessary. The basis setting to implement $\hat{F} \hat{F}^{j} \hat{C}_{Z}(g)$ from modes $B, k$ and $B, k+2$ to modes $B, k+2 N$ and $B, k+2 N+2$ is

$$
\left(\begin{array}{c}
\theta_{A, k} \\
\theta_{B, k} \\
\theta_{A, k+2} \\
\theta_{B, k+2} \\
\theta_{A, k+N} \\
\theta_{B, k+N} \\
\theta_{A, k+N+1} \\
\theta_{B, k+N+1} \\
\theta_{A, k+N+2} \\
\theta_{B, k+N+2}
\end{array}\right)=\left(\begin{array}{c}
\pi / 4 \\
-\pi / 4 \\
(-1)^{w} \pi / 4 \\
-(-1)^{w} \pi / 4 \\
0 \\
(-1)^{w}[\pi / 2-\arctan (g / 2)] \\
(-1)^{w} \pi / 4 \\
(-1)^{w}[\pi / 4+2 \arctan (g / 2)] \\
0
\end{array}\right) .
$$

This basis setting includes the settings for the temporal control mode $k+N+1$ coupling the two wires $w$ and $w+1$ for the desired two-mode gates, while all other control modes surrounding the two wires are measured in the regular $\pm \pi / 4$ control mode basis as described at the beginning of section 5.4

The displacement matrix, D, and corresponding vector of measurement outcomes, $\boldsymbol{m}$, is shown in Fig. 5.9 for the different coupling constants $g$ implemented. For the measurement outcomes in $\boldsymbol{m}$ corresponding to the modes in Eq. (5.16), the direct homodyne measurement outcomes, $m_{ \pm, k}$, are used, while for control modes used to form the projected cluster state in Fig. 5.8, the measurement outcomes before $\mathrm{BS}_{3}, m_{A(B), k}$, are used, extracted using Eq. (5.10). In the characterization of the gate, the displacement is compensated for in the measurement outcomes of the output modes $B, k+2 N$ and $B, k+2 N+2$.

Finally, the gate noise matrix, $\mathbf{N}$, and corresponding vector of initial momentum squeezed quadratures, $\hat{\boldsymbol{p}}_{\boldsymbol{i}}$, is shown in Fig. 5.10. Assuming all initial momentum squeezed quadratures, $\hat{p}_{A(B), k}^{i}$, are equally squeezed by $e^{-r}$ such that the variances of the momentum quadrature distributions are $e^{-2 r} V_{0}$, gate noise of variance $e^{-2 r} V_{0} \sum_{i} N_{q i}^{2}$ is added in each output quadrature. Here, again, $\sum_{i} N_{q i}^{2}$ are gate noise factors scaling the initial momentum squeezing variance into gate noise variance with $q=1,2,3$, and 4 for the output quadratures $\hat{x}_{B, k+2 N}, \hat{x}_{B, k+2 N+2}, \hat{p}_{B, k+2 N}$ and $\hat{p}_{B, k+2 N+2}$, respectively. The resulting gate noise factors are shown in Fig. 5.10.

\subsubsection{Circuit}

Besides implementing individual gates of a multi-mode universal Gaussian gate set, the cluster state computation architecture in this work allows quantum gates to be combined universally, with single-mode gates on each wire by teleportation as described in section 5.4.1, and two-mode gates between neighboring wires coupled by control modes as described in section 5.4.2. To demonstrate this, we implement a quantum circuit consisting of 10 single-mode gates and 2 two-mode gates distributed on 3 modes as shown in Fig. 5.4b of the main text section 5.2. For GKP-encoded qubits encoded on square grids in phase-space, the implemented circuit corresponds to the encoding scheme of a logical qubit in the 3-qubit bit-flip error correction code as shown in Fig. 5.4a.

The circuit is implemented with the three input states on mode $(B, k),(B, k+2)$ and $(B, k+4)$, and output states on mode $(B, k+6 N),(B, k+6 N+2)$ and $(B, k+6 N+4)$. Since in this work the displacement by-product is compensated for in the measurement outcomes, the displacement of each gate will accumulate throughout the computation. By keeping track of each measurement outcome, and how they propagate through the subsequent gates towards the output modes, the displacements are compensated for in the measurements of the circuit's three output states. Here, using the expressions for displacements in single- and two-mode gates in Eq. (5.7) and in Fig. 5.9 for $g=1$, an accumulated displacement term, $\mathbf{D} \boldsymbol{m}$, on the circuit output modes is derived, and the resulting displacement matrix, D, and vector of measurement outcomes, $\boldsymbol{m}$, are shown in Fig. 5.11.

Each gate of the implemented circuit adds gate noise to the quadratures of the modes in computation proportional to the initial momentum squeezing in the cluster state generation. To 


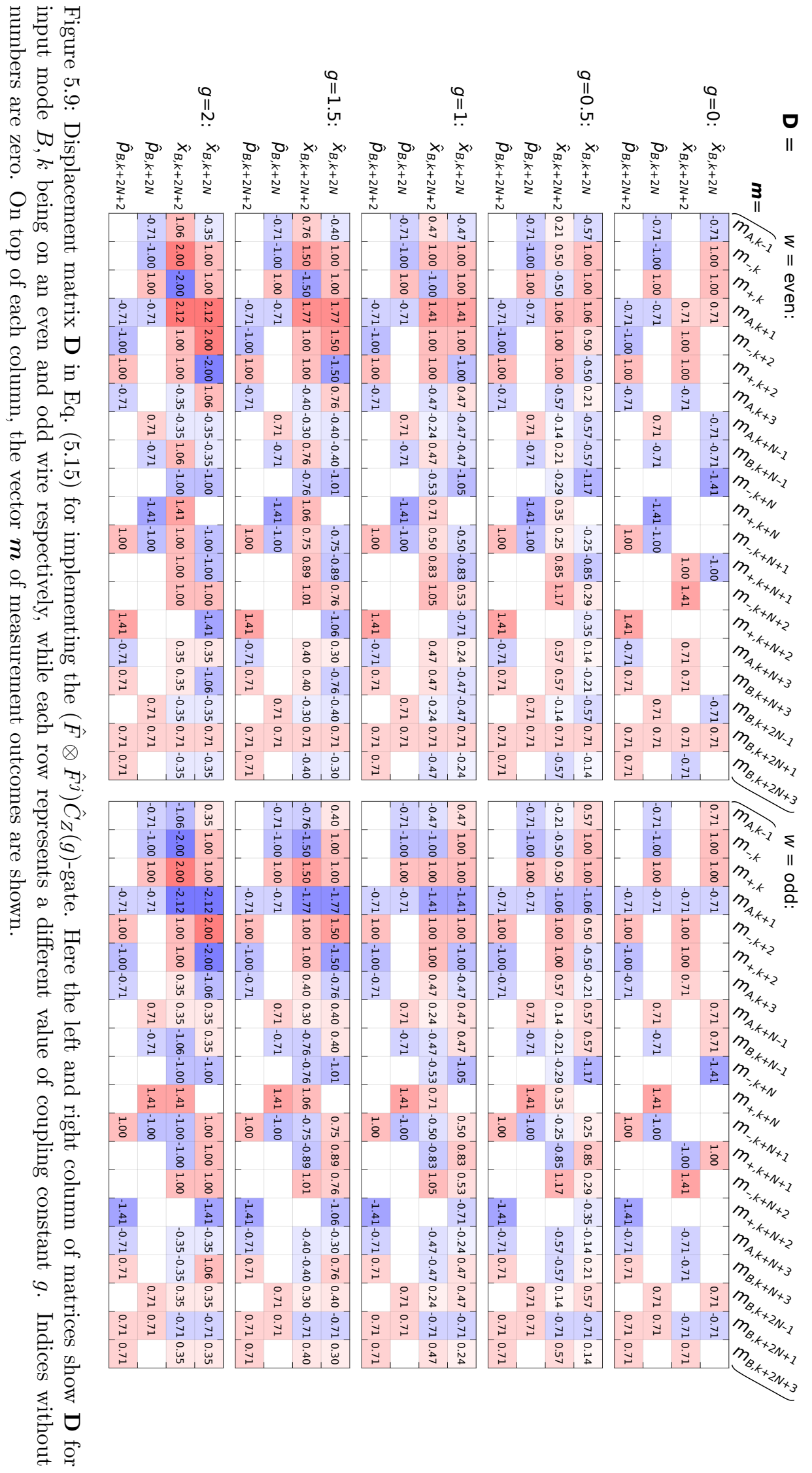




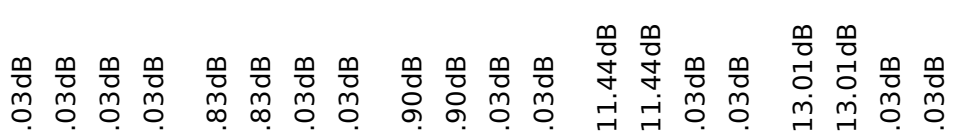
a ||

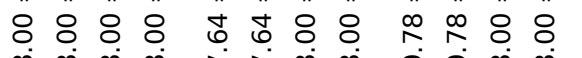
NF Nĩ̄

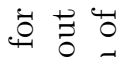
乙总青

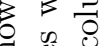
of : $\dot{\sigma} \dot{\sigma}$

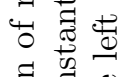

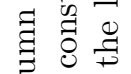

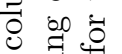

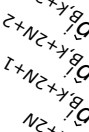

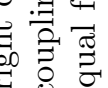

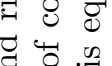

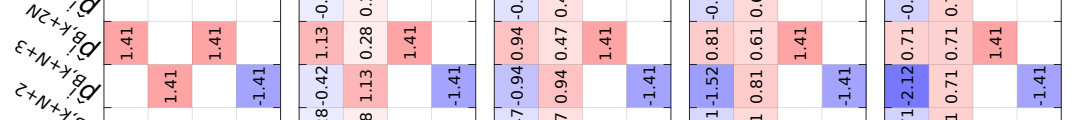

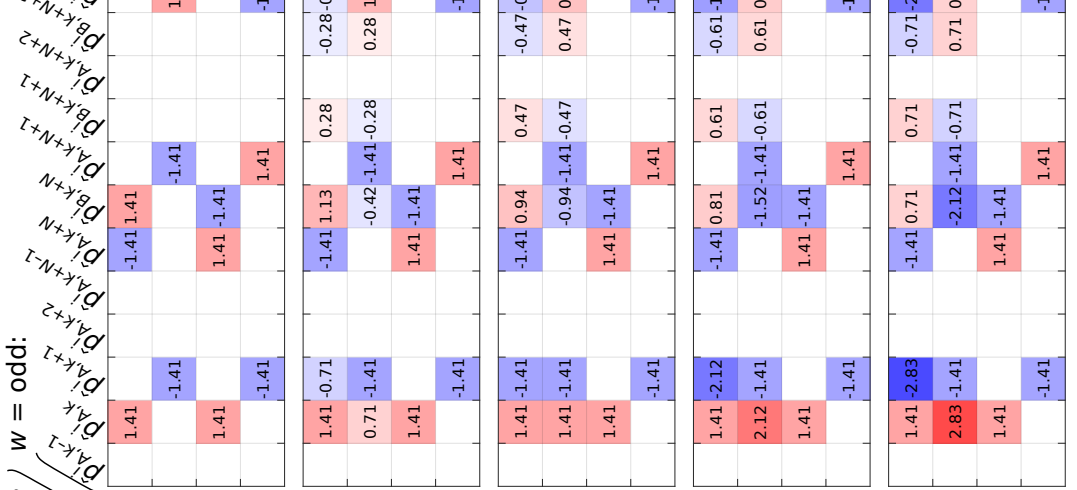

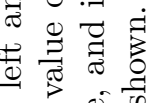

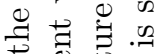

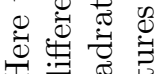
I :

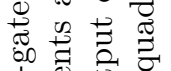

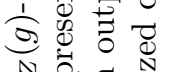
¿

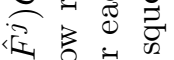
$\otimes$ 过

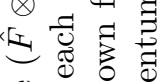

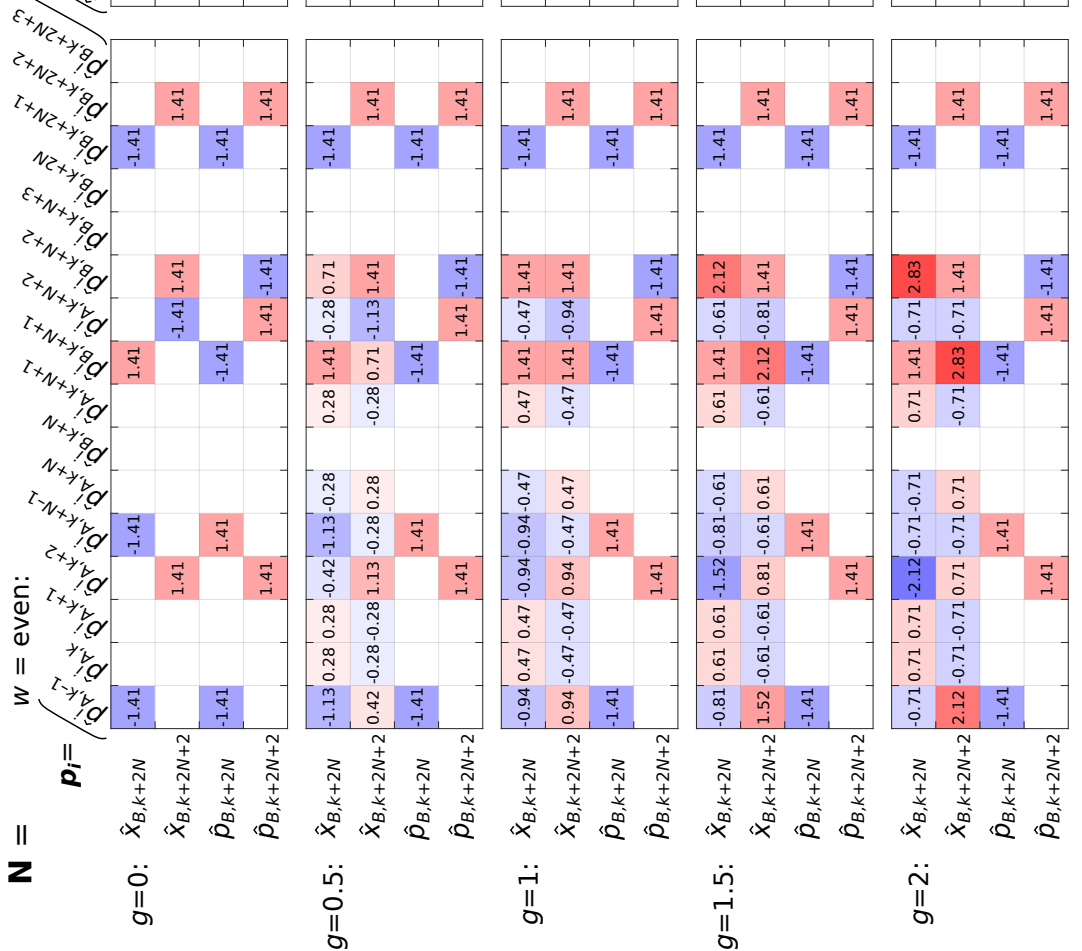

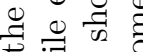
$\infty \frac{7}{3}$. 2

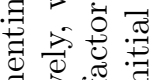
हृ .

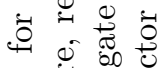
12.

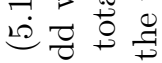

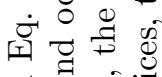
$\exists$ 政 乙

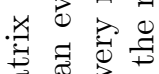
घี ซี .

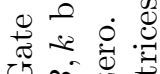
$\because 0$. 完卷 的

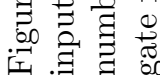



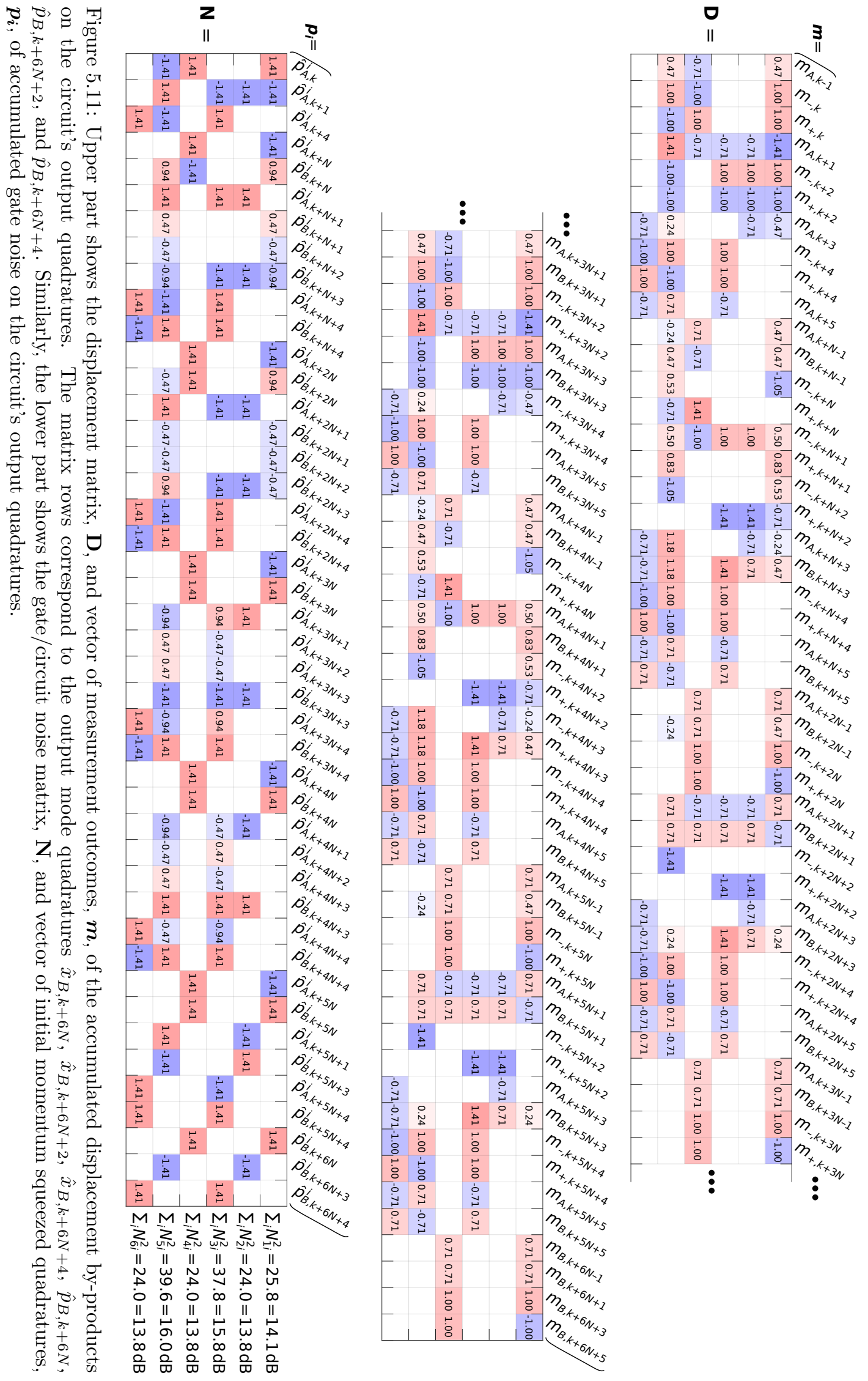
avoid gate noise accumulation, the noise must be removed after each gate implementation. This can be done by using GKP-encoded qubits for computation and applying GKP error correction of the computation mode quadratures [14] as often as possible, preferably after each implemented gate depending on the magnitude of the gate noise. In the work here, however, GKP-encoding and error correction is not implemented and as a result, the gate noise accumulates during the computation and thus adds to the circuit output modes. This noise is measured and compared to the theoretically expected accumulated gate noise which depends on the initial momentum squeezing. Using the expression for single- and two-mode gate noise in Eq. (5.13) and in Fig. 5.10 for $g=1$, and keeping track of the gate noise propagation through each gate of the implemented circuit, a combined circuit noise term of accumulated gate noise, $\mathbf{N} \hat{\boldsymbol{p}}_{\boldsymbol{i}}$, is derived with the noise matrix, $\mathbf{N}$, and the vector of initial momentum squeezed quadrature, $\hat{\boldsymbol{p}}_{\boldsymbol{i}}$, presented in Fig. 5.11. The resulting noise factors, $\sum_{j} N_{q j}^{2}$, of the output quadratures $\hat{x}_{B, k+6 N}, \hat{x}_{B, k+6 N+2}, \hat{x}_{B, k+6 N+4}$, $\hat{p}_{B, k+6 N}, \hat{p}_{B, k+6 N+2}$, and $\hat{p}_{B, k+6 N+4}$ for $q=1,2,3,4,5$, and 6 are also shown. Note that the large circuit noise is due to the lack of error correction. In fault-tolerant quantum computation the purpose of error correction is to prevent gate noise to accumulate into such large values.

\subsection{Suppl. Inf.: Gate tomography}

In section 5.4 we described how quantum gates and circuits are implemented by projective measurements on the generated cluster state presented in section 5.3. Here, we describe the method of gate and circuit characterization which is based on gate tomography. The procedure is based on probing the gates using entangled states as input and subsequently determine the quadrature correlations that remain after the gate operation. This method was also used in Ref. [25] but here we generalize it to multi-mode gates and circuits.

The implemented $n$-mode Gaussian gate/circuit, or operation, can be represented by a $2 n \times 2 n$ symplectic matrix $\mathbf{S}$ (referred to in section 5.4.1 and 5.4.2 as $\mathbf{G}$ and $\mathbf{G}_{2}$ ) plus gate noise. Here, we consider the case where the displacement by-products have been compensated for, either by feed-forward or, as in this work, in the measurement results. Thus, the goal is to estimate $\mathbf{S}$ and the variance of the gate noise in each quadrature. To do so, each of the $n$ input modes to the implemented operation is entangled to one of $n$ reference modes, and from measuring correlations of the reference modes and the operation's output modes we can extract $\mathbf{S}$, while the gate noise variance can be estimated from measured variances of the input and output modes together with the estimated $\mathbf{S}$. The corresponding circuit is presented in Fig. 5.12.

The entanglement probes in Fig. 5.12 are prepared by the wire projections shown in Fig. 5.6, and the resulting two-mode entangled states are formed as two-mode wire cluster states. One mode of each of the $n$ two-mode cluster state wires serves as an input mode to the implemented $n$-mode operation, while the other mode serves as the corresponding reference. The projected wires are two-mode squeezed states obtained by interfering two squeezed states, as shown in Fig. 5.6d, with the corresponding quadrature transformations

$$
\begin{aligned}
& \left(\begin{array}{l}
\hat{x}_{j}^{\text {ref. }} \\
\hat{p}_{j}^{\text {ref. }}
\end{array}\right)-\left(\begin{array}{l}
\hat{x}_{j}^{\text {ref. }} \\
\hat{p}_{j}^{\text {ref. }}
\end{array}\right)=\frac{1}{2}\left(\begin{array}{l}
\hat{x}_{j}^{\text {ref. }}-\hat{x}_{j}^{\text {in }}-\hat{p}_{j}^{\text {ref. }}-\hat{p}_{j}^{\text {in }} \\
\hat{x}_{j}^{\text {ref. }}+\hat{x}_{j}^{\text {in }}+\hat{p}_{j}^{\text {ref. }}-\hat{p}_{j}^{\text {in }}
\end{array}\right) \\
& \left(\begin{array}{c}
\hat{x}_{j}^{\text {in }} \\
\hat{p}_{j}^{\text {in }}
\end{array}\right)-\pi / 2 \\
& \left.\begin{array}{l}
\hat{x}_{j}^{\text {in }} \\
\hat{p}_{j}^{\text {in' }}
\end{array}\right)=\frac{1}{2}\left(\begin{array}{l}
\hat{x}_{j}^{\text {ref. }}+\hat{x}_{j}^{\text {in }}-\hat{p}_{j}^{\text {ref. }}+\hat{p}_{j}^{\text {in }} \\
\hat{x}_{j}^{\text {ref. }}-\hat{x}_{j}^{\text {in }}+\hat{p}_{j}^{\text {ref. }}+\hat{p}_{j}^{\text {in }}
\end{array}\right),
\end{aligned}
$$

for a $w=$ odd wire, with a similar transformation for an even wire. Here, the displacement, $\mathcal{D}$, is compensated for, while the $\sqrt{2}$ momentum anti-squeezing contribution and the effect of $\mathcal{N}$ (after moving $\mathcal{N}$ to the left of the beam-splitter and phase shifts in Fig. 5.6d where it adds uncorrelated noise in connected wire modes as discussed in section 5.4) are combined in an effective squeezing of $s>e^{-r}$ of the initial input and reference mode momentum quadratures such that

$$
\begin{aligned}
& \left\langle\left(\hat{x}_{j}^{\text {ref. }}\right)^{2}\right\rangle=\left\langle\left(\hat{x}_{j}^{\text {in }}\right)^{2}\right\rangle=a V_{0} / s^{2}, \\
& \left\langle\left(\hat{p}_{j}^{\text {ref. }}\right)^{2}\right\rangle=\left\langle\left(\hat{p}_{j}^{\text {in }}\right)^{2}\right\rangle=s^{2} V_{0},
\end{aligned}
$$




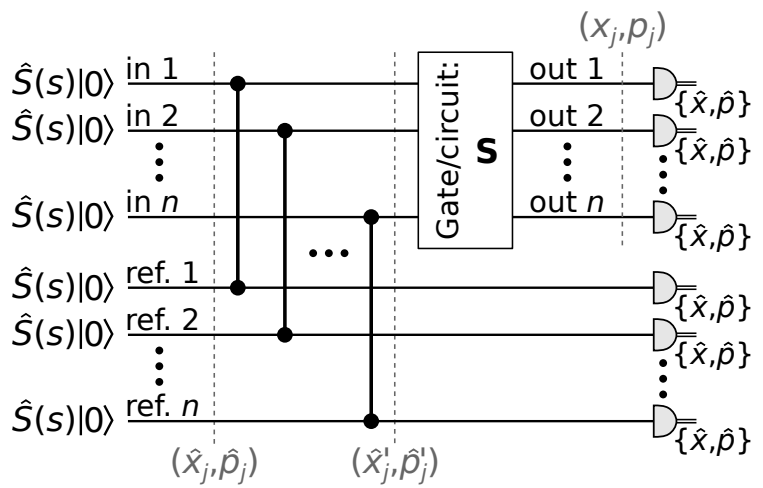

Figure 5.12: Corresponding circuit of the gate tomography. Here the controlled-Z gates applied to the input and reference modes correspond to the projected two-mode wire cluster states in Fig. 5.6, where $s$ is a combined squeezed coefficient including additional noise from the wire projection. By measuring the output and reference modes in $\hat{x}$ - and $\hat{p}$-quadrature, the implemented gate/circuit symplectic matrix $\mathbf{S}$ can be extracted using Eq. (5.19), while the gate noise can be estimated from the quadrature variances of the input and output modes. The primed and non-primed quadratures used in the text are marked with a dashed line.

$V_{0}=1 / 2$ is the variance of vacuum for $\hbar=1$, and $a=1$ for pure states while excess anti-squeezing is described by $a>1$. Note that here we assume the reference and input modes to be equally squeezed, while the exact values of $s$ and $a$ are unimportant for the purpose here. The resulting quadrature correlations between the input and reference modes are

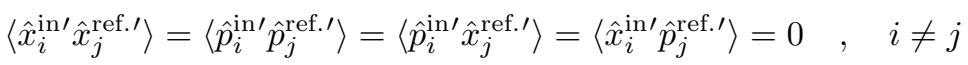

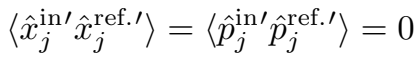

$$
\begin{aligned}
& \left\langle\hat{p}_{j}^{\text {in' }} \hat{x}_{j}^{\text {ref. } \prime}\right\rangle=\left\langle\hat{x}_{j}^{\text {in' }} \hat{p}_{j}^{\text {ref. } \prime}\right\rangle=\frac{1}{2}\left(\frac{a}{s^{2}}-s^{2}\right) V_{0} \equiv \varepsilon_{j}>0
\end{aligned}
$$

for odd wires with positive edge weight, while similar for even wires with opposite sign of $\varepsilon_{j}$. Here the first line shows quadrature correlations between input and reference modes of different two-mode wire states which are naturally zero.

After the gate/circuit operation the input mode quadratures are transformed by the symplectic matrix $\mathbf{S}$ with entries $s_{i, j}$ while gate noise is added to the output quadratures as

$$
\left(\begin{array}{c}
\hat{x}_{1}^{\text {out }} \\
\vdots \\
\hat{x}_{n}^{\text {out }} \\
\hat{p}_{1}^{\text {out }} \\
\vdots \\
\hat{p}_{n}^{\text {out }}
\end{array}\right)=\left(\begin{array}{cccccc}
s_{1,1} & \cdots & s_{1, n} & s_{1, n+1} & \cdots & s_{1,2 n} \\
\vdots & \ddots & \vdots & \vdots & \ddots & \vdots \\
s_{n, 1} & \cdots & s_{n, n} & s_{n, n+1} & \cdots & s_{n, 2 n} \\
s_{n+1,1} & \cdots & s_{n+1, n} & s_{n+1, n+1} & \cdots & s_{n+1,2 n} \\
\vdots & \ddots & \vdots & \vdots & \ddots & \vdots \\
s_{2 n, 1} & \cdots & s_{2 n, n} & s_{2 n, n+1} & \cdots & s_{2 n, 2 n}
\end{array}\right)\left(\begin{array}{c}
\hat{x}_{1}^{\text {in' }} \\
\vdots \\
\hat{x}_{n}^{\text {in' }} \\
\hat{p}_{1}^{\text {in' }} \\
\vdots \\
\hat{p}_{n}^{\text {in' }}
\end{array}\right)+\mathbf{N} \hat{\boldsymbol{p}}_{\boldsymbol{i}} .
$$

Here the gate noise vector of the initial momentum squeezed quadrature vector, $\hat{\boldsymbol{p}}_{\boldsymbol{i}}$, transformed by the gate noise matrix, $\mathbf{N}$, is described in section 5.4. Using Eq. (5.17) the quadrature correlations between the input and reference modes can then be used to extract $s_{i, j}$ since

$$
\begin{aligned}
\left\langle\hat{x}_{i}^{\text {out }} \hat{p}_{j}^{\text {ref.' }}\right\rangle & =\left\langle\left(s_{i, 1} \hat{x}_{1}^{\text {in' }}+\cdots+s_{i, n} \hat{x}_{n}^{\text {in' }}+s_{i, n+1} \hat{p}_{1}^{\text {in' }}+\cdots+s_{i, 2 n} \hat{p}_{n}^{\text {in' }}\right) \hat{p}_{j}^{\text {ref.' }}\right\rangle \\
& =s_{i, j}\left\langle\hat{x}_{j}^{\text {in' }} \hat{p}_{j}^{\text {ref.' }}\right\rangle+s_{i, n+j}\left\langle\hat{p}_{j}^{\text {in' }} \hat{p}_{j}^{\text {ref.' }}\right\rangle \\
& =s_{i, j} \varepsilon_{j}
\end{aligned}
$$




$$
\begin{aligned}
& \left\langle\hat{x}_{i}^{\text {out }} \hat{x}_{j}^{\text {ref. } \prime}\right\rangle=\left\langle\left(s_{i, 1} \hat{x}_{1}^{\text {in' }}+\cdots+s_{i, n} \hat{x}_{n}^{\text {in' }}+s_{i, n+1} \hat{p}_{1}^{\text {in' }}+\cdots+s_{i, 2 n} \hat{p}_{n}^{\text {in' }}\right) \hat{x}_{j}^{\text {ref.' }}\right\rangle \\
& =s_{i, j}\left\langle\hat{x}_{j}^{\text {in' }} \hat{x}_{j}^{\text {ref. } \prime}\right\rangle+s_{i, n+j}\left\langle\hat{p}_{j}^{\text {in' }} \hat{x}_{j}^{\text {ref. }}\right\rangle \\
& =s_{i, n+j} \varepsilon_{j} \\
& \left\langle\hat{p}_{i}^{\text {out }} \hat{p}_{j}^{\text {ref. } .}\right\rangle=\left\langle\left(s_{n+i, 1} \hat{x}_{1}^{\text {in }}+\cdots+s_{n+i, n} \hat{x}_{n}^{\text {in' }}+s_{n+i, n+1} \hat{p}_{1}^{\text {in }}+\cdots+s_{n+i, 2 n} \hat{p}_{n}^{\text {in }}\right) \hat{p}_{j}^{\text {ref. }}\right\rangle \\
& =s_{n+i, j}\left\langle\hat{x}_{j}^{\text {in' }} \hat{p}_{j}^{\text {ref.' }}\right\rangle+s_{n+i, n+j}\left\langle\hat{p}_{j}^{\text {in' }} \hat{p}_{j}^{\text {ref.' }}\right\rangle \\
& =s_{n+i, j} \varepsilon_{j} \\
& \left\langle\hat{p}_{i}^{\text {out }} \hat{x}_{j}^{\text {ref.' }}\right\rangle=\left\langle\left(s_{n+i, 1} \hat{x}_{1}^{\text {in' }}+\cdots+s_{n+i, n} \hat{x}_{n}^{\text {in' }}+s_{n+i, n+1} \hat{p}_{1}^{\text {in } \prime}+\cdots+s_{n+i, 2 n} \hat{p}_{n}^{\text {in' }}\right) \hat{x}_{j}^{\text {ref.' }}\right\rangle \\
& =s_{n+i, j}\left\langle\hat{x}_{j}^{\text {in' }} \hat{x}_{j}^{\text {ref. } \prime}\right\rangle+s_{n+i, n+j}\left\langle\hat{p}_{j}^{\text {in' }} \hat{x}_{j}^{\text {ref.' }}\right\rangle \\
& =s_{n+i, n+j} \varepsilon_{j}
\end{aligned}
$$

for $i, j \in\{1, \cdots, n\}$. Thus, finally, the symplectic matrix of the implemented operation can be estimated from quadrature correlations of output and reference modes as

$$
\mathbf{S}=\left(\begin{array}{ccc|ccc}
\vdots & & & \vdots & \\
\cdots & \left\langle\hat{x}_{i}^{\text {out }} \hat{p}_{j}^{\text {ref. }}\right\rangle / \varepsilon_{j} & \cdots & \cdots & \left\langle\hat{x}_{i}^{\text {out }} \hat{x}_{j}^{\text {ref. }}\right\rangle / \varepsilon_{j} & \cdots \\
\vdots & & & \vdots & \\
\hline & \vdots & & & \vdots & \\
\cdots & \left\langle\hat{p}_{i}^{\text {out }} \hat{p}_{j}^{\text {ref. }}\right\rangle / \varepsilon_{j} & \cdots & \cdots & \left\langle\hat{p}_{i}^{\text {out }} \hat{x}_{j}^{\text {ref. }}\right\rangle / \varepsilon_{j} & \cdots \\
\vdots & & & \vdots &
\end{array}\right)
$$

Here, $\varepsilon_{j}$ is estimated from the measurements of the quadrature correlations between input and reference modes on even and odd wire states individually side by side with the implemented operations. As such, within even and odd wires, we assume $\varepsilon_{j}$ to be identical for different $j$. This is a valid assumption, as the gate tomography of each implemented gate (including estimating $\varepsilon_{j}$ for even and odd wires) in this work is carried out within $19 N=228$ temporal modes, i.e. within a time period of $228 \times 247 \mathrm{~ns}=56.3 \mu \mathrm{s}$, while the setup stability allows for a stable setup without changes for much longer time periods.

Using the estimated symplectic matrix in Eq. (5.19), the variance of the gate noise in Eq. (5.18) can be estimated as the difference of the quadrature variance on the output modes and the expected quadrature variance from the input modes as

$$
\operatorname{Var}\left\{\mathbf{N} \hat{p}_{\boldsymbol{i}}\right\}=\operatorname{Var}\left\{\left(\begin{array}{c}
\hat{x}_{1}^{\text {out }} \\
\vdots \\
\hat{x}_{n}^{\text {out }} \\
\hat{p}_{1}^{\text {out }} \\
\vdots \\
\hat{p}_{n}^{\text {out }}
\end{array}\right)\right\}-\operatorname{Var}\left\{\mathbf{S}\left(\begin{array}{c}
\hat{x}_{1}^{\text {in }} \\
\vdots \\
\hat{x}_{n}^{\text {in }} \\
\hat{p}_{1}^{\text {in }} \\
\vdots \\
\hat{p}_{n}^{\text {in' }}
\end{array}\right)\right\} .
$$

Here the reference mode is traced out. Similar to $\varepsilon_{j}, \operatorname{Var}\left\{\hat{x}_{j}^{\text {in' }}\right\}$ and $\operatorname{Var}\left\{\hat{p}_{j}^{\text {in' }}\right\}$ are estimated by measurements on wire cluster state individually for even and odd wires, both before and after the gate tomography, but within $19 N=228$ temporal modes.

\subsubsection{Single-mode gates}

In Fig. 5.13 the basis setting layout on the generated cluster state for single-mode gate implementation and tomography is shown. Since $\mathbf{S}$ is a $2 \times 2$ matrix for single-mode gates, 4 different measurements of quadrature correlations of the output-reference mode are required to estimate $\mathbf{S}$. Thus, with a section of $19 N=228$ temporal modes, 8 different single-mode gates can be implemented and characterized at once in parallel on 4 wires. The remaining 2 of the 6 wires are used 


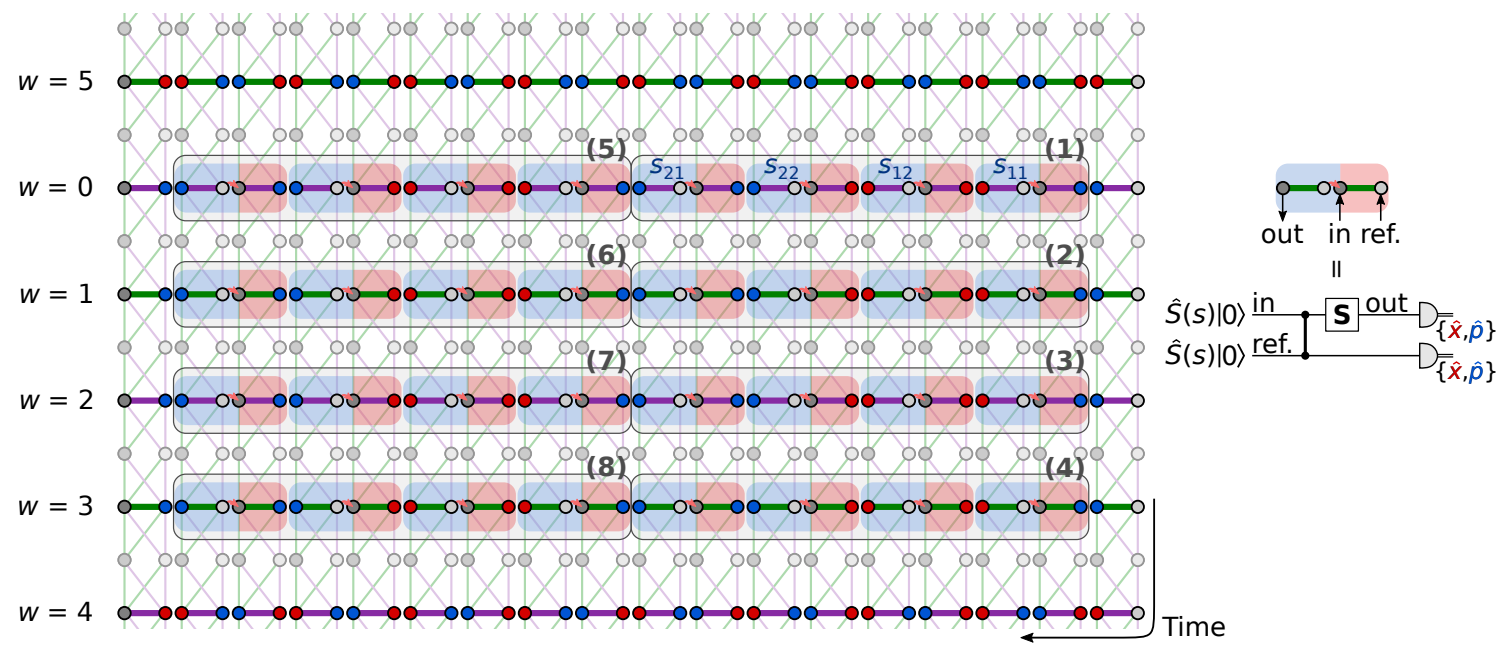

Figure 5.13: Basis setting layout with $19 N=228$ temporal modes for implementation and characterization by gate tomography of 8 different single-mode gates on wire $w=0-3$, each in the regions marked by (1-8). In each region the gate is implemented 4 times in order to measure $p x-$, $x x-, x p$ - and $p p$-correlations between the reference and output modes to estimate the indices $s_{1,1}$, $s_{1,2}, s_{2,2}$ and $s_{2,1}$, respectively, of the implemented gate symplectic matrix $\mathbf{S}$ by Eq. (5.19). Here, modes marked by red and blue are measured in $\hat{x}$ - and $\hat{p}$-basis, respectively, while faded modes are control modes measured in the $\pm \pi / 4$ basis to form wires. The faded edges to control modes are only shown to indicate the initial coiled up $1 \mathrm{D}$ cluster state before wire projection, and has now physical meaning here. Even though present in all temporal modes, the beam splitter $\mathrm{BS}_{3}$ is only shown between spatial modes measured in different basis in order to implement the desired gates. The measurement outcomes before $\mathrm{BS}_{3}$ on all other modes are extracted using Eq. (5.10). Wire $w=4$ and 5 are used to estimate the quadrature correlations between input and reference modes, $\varepsilon_{j}$, together with the quadrature variance of input modes in order to estimate the gate noise variance by Eq. (5.20). To the right of the cluster state basis layout, the corresponding single-mode gate tomography circuit of input, output, and reference modes is shown. 
to estimate the correlations between input and reference modes, $\varepsilon_{j}$, of the two-mode wire states in both odd and even wires, together with the input mode variance when tracing out the reference modes. To build up statistics, measurements with the layout in Fig. 5.13 are repeated 10000 times. In the following we will refer to such 10000 repeated measurements of the same layout as a set of measurements.

Each of the implemented single-mode gates, $\hat{R}(\theta), \hat{P}(\sigma)$ and $\hat{S}\left(e^{-r}\right)$, are implemented in different sets of measurements with 7 different values of $\theta, \sigma$ and $r$. To fill out one set, which can implement and characterize 8 different gates, the value $\theta, \sigma, r=0$ are repeated twice in each set. From wire $w=4$ in each set the input-reference quadrature correlations are estimated to be $\varepsilon_{j}=-2.14 \pm 0.03,-2.15 \pm 0.03$ and $-2.14 \pm 0.03$ for the sets with $\hat{R}(\theta), \hat{P}(\sigma)$ and $\hat{S}\left(e^{-r}\right)$, respectively, and are used for gate tomography on $w=$ even wires with negative edge weight. Similarly, from wire $w=5, \varepsilon_{j}=2.11 \pm 0.04,2.12 \pm 0.03$ and $2.11 \pm 0.02$ are estimated and used for gate tomography on $w=$ odd wires with positive edge weight. The uncertainties on $\varepsilon_{j}$ are estimated as the standard deviation of the 16 measured quadrature correlations in each wire $w=4$ and 5 for each measurement set. The resulting symplectic matrix for each implemented single-mode gates, extracted using Eq. (5.19), are shown in Fig. 5.2a as a function of $\theta, \sigma$ and $r$.

Also, using wires $w=4$ and 5 , the quadrature variances of the input modes when tracing out the reference mode (i.e. ignoring the reference mode measurement outcome) are estimated to be $\left(\operatorname{Var}\left\{x_{j}^{\text {in' }}\right\}, \operatorname{Var}\left\{p_{j}^{\text {in' }}\right\}\right)=(2.28 \pm 0.04,2.67 \pm 0.05),(2.33 \pm 0.03,2.64 \pm 0.03)$ and $(2.31 \pm$ $0.02,2.66 \pm 0.02)$ on wire $w=4$ for measurement sets with $\hat{R}(\theta), \hat{P}(\sigma)$ and $\hat{S}\left(e^{-r}\right)$ respectively, and similarly $\left(\operatorname{Var}\left\{x_{j}^{\text {in }}\right\}, \operatorname{Var}\left\{p_{j}^{\text {in }}\right\}\right)=(2.30 \pm 0.03,2.78 \pm 0.04),(2.34 \pm 0.03,2.75 \pm 0.03)$ and $(2.31 \pm 0.03,2.75 \pm 0.03)$, respectively, on wire $w=5$. The uncertainties in the variances of the $\hat{x}$ and $\hat{p}$ quadratures in each measurement set are estimated as standard deviation of the 8 measurements of input modes in each quadrature on wires $w=4$ and 5 . The resulting gate noise variances, extracted using Eq. (5.20) with the variance of the output modes measured in each gate tomography, are shown in Fig. $5.2 \mathrm{c}$. Also, the gate noise variance compensated by the theoretical gate noise factor of $6 \mathrm{~dB}$ is shown, in which case it agrees with the initial momentum squeezing of $4.4 \mathrm{~dB}$ below the vacuum variance as discussed at the end of section 5.4.1 (a more general discussion of gate noise is presented in section 5.6).

To show how the gate noise behaves as a function of squeezing, in Fig. $5.2 \mathrm{~d}$ of the main text section 5.2 the gate noise of $\hat{R}(\theta)$, averaged over $\hat{x}$ and $\hat{p}$ and over the 7 different implemented values of $\theta$, is shown as a function of pump power relative to the OPO thresholds. The measured gate noise is seen to agree well with the gate noise expected for the estimated properties of the experimental setup and is discussed further in section 5.6.

\subsubsection{Two-mode gate}

When performing gate tomography on the two-mode controlled-Z gate, $\hat{C}_{Z}(g)$, the $4 \times 4=16$ entries $s_{i, j}$ of the corresponding symplectic matrix $\mathbf{S}$ can be estimated by executing the $\hat{C}_{Z}(g)$ gate four times, since 4 different input-reference mode quadrature correlations can be estimated simultaneously using the two reference and output modes of each implementation. The basis setting layout of 228 temporal modes is shown in Fig. 5.14, in which $\hat{C}_{Z}(g)$ can be implemented with three different values of $g$. In total, $\hat{C}_{Z}(g)$ is implemented with 5 different values of $g$ distributed on two sets of measurements with $g=0$ repeated twice to fill out the sets.

Similar to the gate tomography of single-mode gates in section 5.5.1, the input-reference quadrature correlations, $\varepsilon_{j}$, averaged on the two measurement sets, are estimated from wires $w=4$ and 5 to be $\varepsilon_{j}=-2.15 \pm 0.04$ and $2.13 \pm 0.03$, respectively, with uncertainties estimated as the standard deviation of 16 measured quadrature correlations in each wire. From the gate tomography, the resulting symplectic matrix estimated using Eq. (5.19) is shown in main text section Fig. 5.3a as a function of $g$.

From wires $w=4$ and 5 the input mode variances, averaged over the two measurement sets, are estimated to be $\left(\operatorname{Var}\left\{x_{j}^{\text {in' }}\right\}, \operatorname{Var}\left\{p_{j}^{\text {in }}\right\}\right)=(2.36 \pm 0.06,2.72 \pm 0.04)$ and $(2.34 \pm 0.03,2.65 \pm 0.04)$ on even and odd wires respectively, with uncertainties estimated as the standard deviation of the 


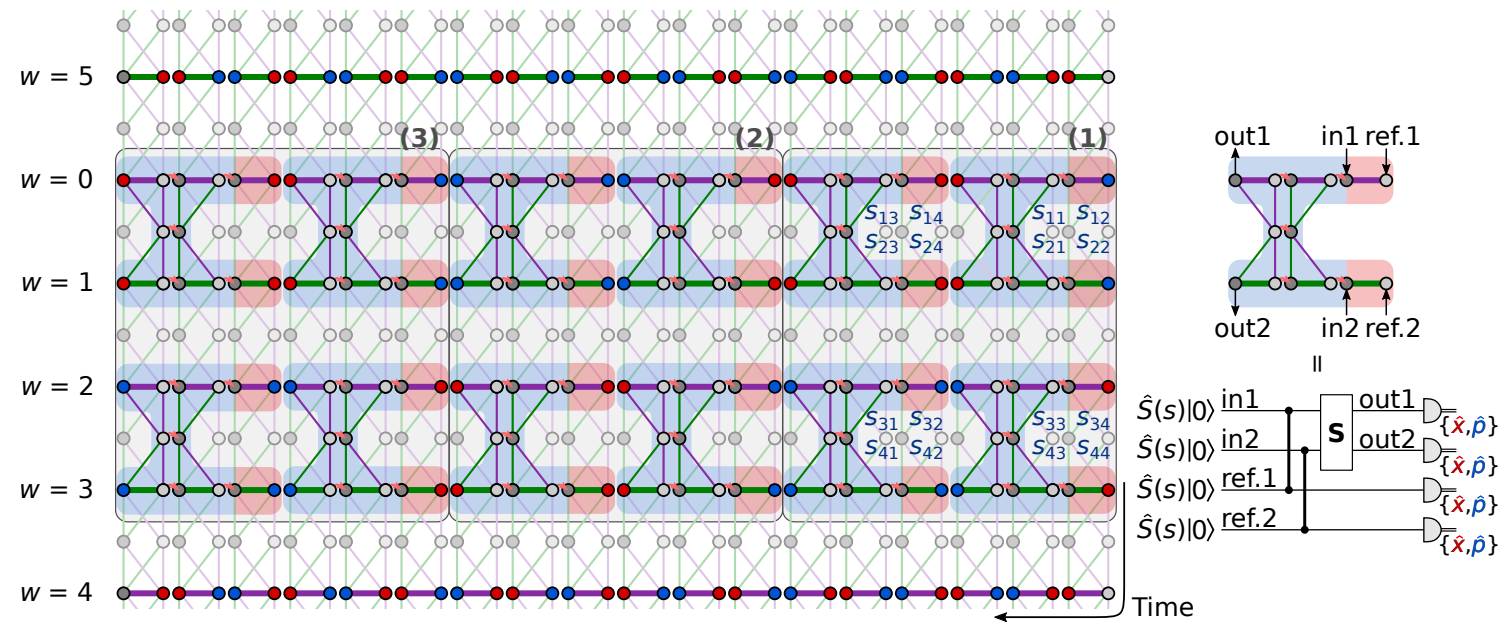

Figure 5.14: Basis setting layout for implementation and characterization by gate tomography of the two-mode $\hat{C}_{Z}(g)$-gate. The notation is the same as in Fig. 5.13 for single-mode gates. The same $\hat{C}_{Z}(g)$-gate is implemented 4 times for gate tomography. As a result, 3 different $\hat{C}_{Z}(g)$-gates can be implemented and characterized within one measurement set of $19 \mathrm{~N}=228$ temporal modes in the regions marked (1-3). $\hat{C}_{Z}(g)$ is implemented and characterized for 5 different values of $g$ in two sets of measurements. To the right, the corresponding circuit of the two-mode gate tomography circuit of input, output, and reference modes is shown.

8 measured input modes per $\hat{x}$ - and $\hat{p}$-quadrature of each wire. Using this, and the estimated $\mathbf{S}$, the gate noise variance is estimated using Eq. (5.20), and shown in Fig. 5.3c as a function of $g$. Here, the gate noise variance is also compensated for by the theoretical gate noise factor presented in Fig. 5.10, in which case the gate noise agrees well with the initial momentum squeezed variance of $4.4 \mathrm{~dB}$ below the vacuum variance.

\subsubsection{Circuit}

The quantum circuit, described in section 5.4.3, is implemented on wires $w=1,2$ and 3 as shown in the basis setting layout in Fig. 5.15. Similar to the $\hat{C}_{Z}(g)$-gate in section 5.5 .2 , all $6 \times 6=36$ entries of the corresponding symplectic matrix $\mathbf{S}$ can be extracted from implementing the circuit 4 times, as $3 \times 3=9$ indices can be estimated from the three reference and output modes of one implementation. Two measurement sets are required for full gate tomography of the circuit implemented on wires $w=1,2$ and 3: One as in Fig. 5.15 where the first 6 columns of $\mathbf{S}$ are estimated, and a similar set where the $\hat{x}$ - and $\hat{p}$-basis settings are swapped on the reference and output modes in order to estimate the last 6 columns of $\mathbf{S}$.

The first and last two time steps on all wires, $w=0-5$ (though only wire $w=1,2$ and 3 are shown in Fig. 5.15), are used to estimate the input-reference mode quadrature correlations, $\varepsilon_{j}$, and are estimated to be $\varepsilon_{j}=-2.14 \pm 0.03$ and $2.12 \pm 0.06$ on even and odd wires, respectively, averaged over the two measurement sets. Uncertainties are estimated as the standard deviation of 12 measured quadrature correlations each for even and odd wires. The resulting estimated symplectic matrix using Eq. (5.19) is shown in the main text Fig. 5.4c.

Similar to $\varepsilon_{j}$, the quadrature variances of input modes are estimated from the first and last two time steps in each wire when tracing out reference modes by ignoring the reference mode measurement outcomes. The input mode quadrature variances are estimated to be $\left(\operatorname{Var}\left\{x_{j}^{\text {in' }}\right\}, \operatorname{Var}\left\{p_{j}^{\text {in }}\right\}\right)=$ $(2.34 \pm 0.05,2.72 \pm 0.03)$ and $(2.34 \pm 0.04,2.68 \pm 0.08)$ on even and odd wires respectively. Uncertainties are estimated as the standard deviation from 6 measured input modes per $\hat{x}$ - and $\hat{p}$-quadrature each in even and odd wires. Using this, and the estimated $\mathbf{S}$, the gate noise variance is estimated using Eq. (5.20), and shown in Fig. 5.4d. The gate noise is also shown when compensating for the 


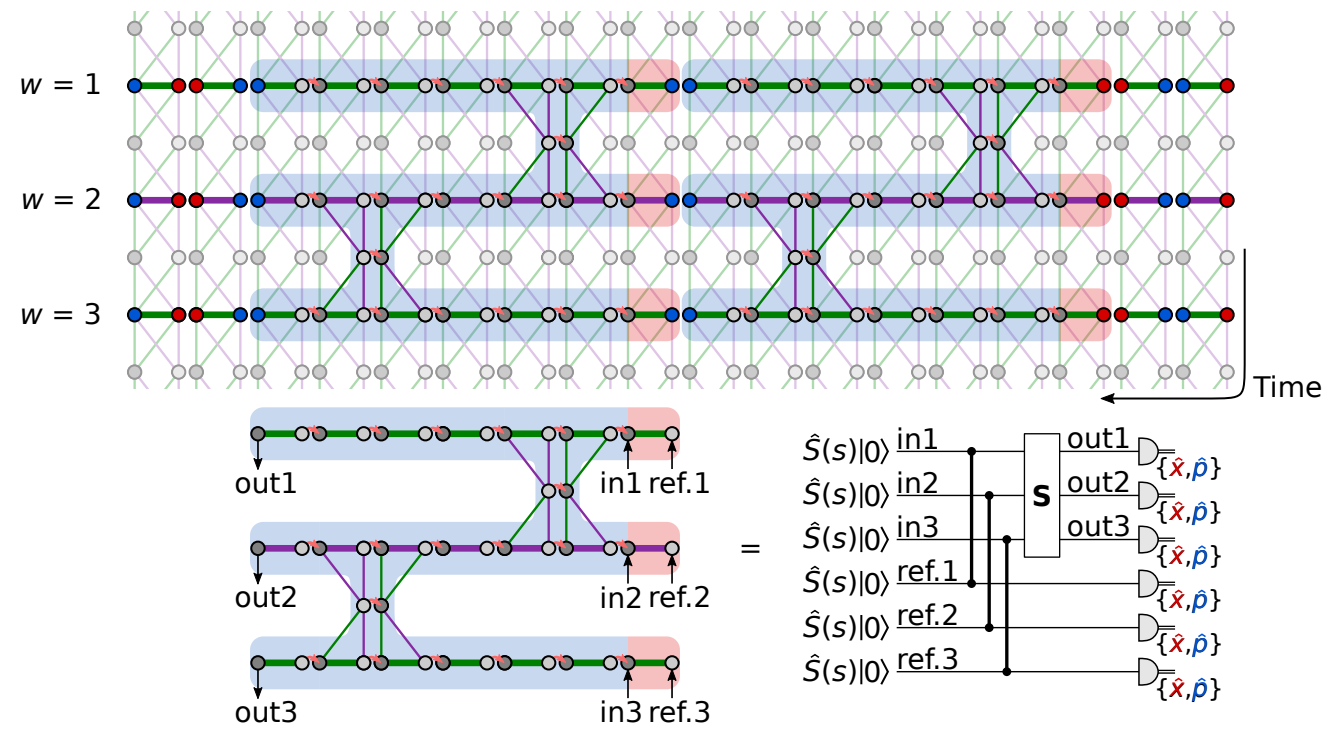

Figure 5.15: Basis setting layout for implementation and characterization of the circuit described in section 5.4.3, here implemented on wire $w=1,2$ and 3, while wire $w=0,4$ and 5 are not shown here. The notation is the same as in Fig. 5.13 for single-mode gates. For full tomography the circuit is implemented 4 times, distributed on two sets of measurements. Here, the first set is shown from which the first 6 columns of the symplectic matrix, $\mathbf{S}$, is estimated. In the second set for estimating the last 6 columns of $\mathbf{S}$, the $\hat{x}$ - and $\hat{p}$-basis settings on all output and reference modes are swapped. Below the basis setting layout, the corresponding circuit of the multi-mode circuit tomography with input, output, and reference modes is shown.

theoretical gate noise factors presented in Fig. 5.11, and the resulting gate noise agrees well with the initial momentum squeezed variance of $4.4 \mathrm{~dB}$ below the vacuum variance.

\subsection{Suppl. Inf.: Gate noise}

In this section, we discuss the performance with respect to measured gate noise in the cluster state computation scheme of this work. Ideal cluster states have nullifiers of zero variance. However, such ideal cluster states, generated from infinite squeezed states, are non-physical. Instead, as discussed in section 5.4, we generate approximate cluster states, or cluster-type states, from finitely squeezed states with nullifier variances that vanish in the limit of infinite squeezing but are otherwise nonzero. When implementing gates on the cluster state by projective measurement, the inherent noise in the cluster state from finite squeezing leads to gate noise proportional to the initial squeezing in the cluster state generation as discussed in section 5.4.1 and 5.4.2- the gate noise variance in each quadrature is the product of the initial squeezing variance and the corresponding gate noise factor.

To prevent gate noise from accumulating on the states in computation, error correction is required. This can be achieved by encoding the computational states as qubits using GottesmanKitaev-Preskill (GKP) encoding, where qubits are encoded in the bosonic mode quadratures [14]. The gate noise, which appears as noise in the quadratures, can then be corrected with GKP quadrature error correction with a success probability depending on the amount of squeezing. In the event of an error in the quadrature error correction, a bit-flip and/or phase-flip error is induced on the encoded qubit, which can then be corrected with a concatenated qubit error correction code. A detailed review of GKP encoding and error correction can be found in [14, 112, 113], while in $[3,32]$ GKP error correction and resulting qubit error probability is reviewed in terms of gate noise. To achieve fault-tolerant quantum computation, the probability of inducing a qubit error 
on the GKP-encoded qubits should be lower than the error probability threshold allowed by the concatenated qubit error correction code. Since this qubit error probability depends on the gate noise and thereby the initial squeezing, the amount of required squeezing for fault-tolerance depends on the error probability that the concatenated qubit error correction code can tolerate. Different squeezing levels have been estimated in the settings of cluster state computation, ranging from at highest $20 \mathrm{~dB}$ below the vacuum variance [3], to $17 \mathrm{~dB}$ in [38] where excess anti-squeezing is shown to not affect the squeezing threshold, and further to $10 \mathrm{~dB}$ in [43] using topological qubit error correction codes (though this requires a 3-dimensional cluster state instead of the 2-dimensional cluster state used in this work).

In the other limit of no squeezing, the generated cluster state is simply a vacuum state. In this case, a desired gate can still be implemented, as the quadrature transformation is mainly realized by the feedforward of the displacement by-product, and as such, this is equivalent to classical teleportation [124]. In this limit, considering single-mode gates, the gate noise is 4 units of vacuum noise, $4 V_{0}$, as discussed in section 5.4.1, i.e. $6 \mathrm{~dB}$ above the vacuum variance. Though single-mode gates can be implemented with less gate noise in classical teleportation, in the cluster state computation scheme in this work, it is not possible to lower the gate noise below $4 V_{0}=$ $6 \mathrm{~dB}$ without squeezing in the cluster state generation leading to quantum correlations between the cluster state modes. With $e^{-r}$ momentum quadrature squeezing, the single-mode gate noise becomes $4 e^{-2 r} V_{0}$. In chapter 3 [31] the cluster state modes are shown to be inseparable when the cluster state is generated with more than $3 \mathrm{~dB}$ of squeezing, while in the work here the cluster state is generated with $4.4 \mathrm{~dB}$ of squeezing, which leads to expected single-mode gate noise of $-4.4 \mathrm{~dB}+6 \mathrm{~dB}=1.6 \mathrm{~dB}=1.4 V_{0}$. This expected gate noise agrees with the measured gate noise shown in the main text Fig. $5.2 \mathrm{c}$, where the gate noise compensated for by $6 \mathrm{~dB}$ (hollow points) is compared to the $4.4 \mathrm{~dB}$ initial squeezing (dashed line). The same analysis is shown for the twomode $\hat{C}_{Z}(g)$-gate and accumulated gate noise of the implemented circuit in the main text Fig. $5.3 \mathrm{c}$ and $5.4 \mathrm{~d}$.

In conclusion, even though the gate noise measured in this work is larger than what can be achieved with different architectures, it is less than what can be achieved with the computation scheme used here without the presence of a cluster state [32]. In the cluster state computation scheme here, the function of the cluster state can be thought of as to decrease the gate noise depending on the cluster state quality in terms of initial squeezing. In the main text Fig. 5.2d, the measured single-mode gate noise is shown as a function of pump power. At zero pump power where the cluster state is simply vacuum, we measured $6 \mathrm{~dB}$ gate noise as expected, while increasing the pump power, generating a cluster state, decreases the gate noise. At some point the gate noise saturates due to optical losses and phase fluctuations, limiting the cluster state quality achievable in the given setup. This is not a fundamental limit but merely a technical challenge. For fault-tolerant computation, optical losses and phase control need to be optimized to improve the cluster state quality, while the computation scheme will remain the same as demonstrated here. Even though some cluster state architectures, like the quad-rail lattice $[9,107]$, can offer an improvement in the gate noise by $3 \mathrm{~dB}$ [32], to achieve fault-tolerant computation it is not enough to simply measure gate noise less than the vacuum variance, $V_{0}=0 \mathrm{~dB}$; optical losses and phase control need to be optimized for any architecture. As such, although the gate noise is a good figure of merit, a gate noise below $V_{0}$ is not the target - it is gate noise below some fault-tolerant squeezing threshold which is the target. Below we estimate how the gate noise would scale in more optimal settings of the computation scheme in this work.

\subsubsection{Gate noise in optimal settings}

To estimate the gate noise, it is sufficient to estimate the initial quadrature squeezing in the cluster state generation and multiply with the gate noise factors $\sum_{i} N_{q i}^{2}$ with $N_{q i}$ being entries of the gate noise matrix $\mathbf{N}$ as discussed in section 5.4. For single-mode gates, the gate noise factor is $\sum_{i} N_{1 i}^{2}=\sum_{i} N_{2 i}^{2}=4$ in both $\hat{x}$ - and $\hat{p}$-quadrature, corresponding to adding $6 \mathrm{~dB}$ to the initial squeezing, while the gate noise factor for the $\hat{C}_{Z}(g)$-gate is given in Fig. 5.10. Thus, we need to first estimate the initial momentum squeezing in the cluster state temporal modes. 
The temporal modes are defined by the temporal mode function, $f_{k}(t)$, in Eq. (5.3). For the initial momentum squeezed states in the cluster state generation $\langle\hat{x}\rangle=\langle\hat{p}\rangle=0$, and the quadrature variance of a temporal mode $k$ becomes

$$
\begin{aligned}
\operatorname{Var}\left\{\hat{q}_{k}\right\}=\left\langle\hat{q}^{2}\right\rangle & =\left\langle\int f_{k}(t) \hat{q}(t) \mathrm{d} t \int f_{k}\left(t^{\prime}\right) \hat{q}\left(t^{\prime}\right) \mathrm{d} t^{\prime}\right\rangle \\
& =\iint f_{k}(t) f_{k}\left(t^{\prime}\right)\left\langle\hat{q}(t) \hat{q}\left(t^{\prime}\right)\right\rangle \mathrm{d} t \mathrm{~d} t^{\prime} \quad, \hat{q}=\hat{x}, \hat{p}
\end{aligned}
$$

where $\left\langle\hat{x}(t) \hat{x}\left(t^{\prime}\right)\right\rangle$ and $\left\langle\hat{p}(t) \hat{p}\left(t^{\prime}\right)\right\rangle$ are the quadrature auto-covariance functions. For momentum squeezed states generated as output from an optical parametric oscillator (OPO) pumped below threshold, the quadrature auto-covariance functions are

$$
\begin{aligned}
\left\langle\hat{x}(t) \hat{x}\left(t^{\prime}\right)\right\rangle & =\frac{1}{2} \delta\left(t-t^{\prime}\right)+\frac{\eta \gamma \varepsilon}{\gamma-\varepsilon} e^{-(\gamma-\varepsilon)\left|t-t^{\prime}\right|}, \\
\left\langle\hat{p}(t) \hat{p}\left(t^{\prime}\right)\right\rangle & =\frac{1}{2} \delta\left(t-t^{\prime}\right)-\frac{\eta \gamma \varepsilon}{\gamma+\varepsilon} e^{-(\gamma+\varepsilon)\left|t-t^{\prime}\right|}
\end{aligned}
$$

where $\eta, \gamma$, and $\varepsilon$ are the overall efficiency, the OPO decay rate (or bandwidth), and pump rate, respectively [79]. In the experimental setup $\gamma=2 \pi \times 7.7 \mathrm{MHz}$ on average for the two OPO squeezing sources [31].

Inserting Eq. (5.3) and (5.22) into Eq. (5.21), and using that $\varepsilon=\gamma \sqrt{P / P_{\text {thr. }}}$ where $P / P_{\text {thr. }}$ is the pump power relative to the OPO threshold, we estimate the quadrature squeezing and antisqueezing as a function of pump power for different efficiencies. Adding $6 \mathrm{~dB}$ to the noise variance, we estimate the resulting single-mode gate noise, which is presented in the main text Fig. 5.2d for some optimal settings with high efficiencies of $\eta \geq 90 \%$. Here, the gate noise is presented with two different OPO bandwidths, namely $\gamma=2 \pi \times 7.7 \mathrm{MHz}$ as in the experimental setup, and $\gamma=2 \pi \times 100 \mathrm{MHz}$ for more optimal settings. Predicted gate noise for the current experimental efficiency of $\eta=77.7 \%$ (an average of $78.9 \%$ and $76.4 \%$ of two fitted efficiencies in section 3.6.2 [31]), together with an estimated phase fluctuation of $\sigma=4^{\circ}$, is shown as well and is seen to agree well with the experimentally measured gate noise. The same analysis is possible for the two-mode $\hat{C}_{Z}(g)$-gate using the gate noise factors in Fig. 5.10. In general, the gate noise is seen to decrease with increasing efficiencies and pump power. Furthermore, the gate noise is also seen to decrease with increasing squeezing bandwidth. This is explained by a better coverage of the temporal mode frequency response by the wider squeezing bandwidth of $100 \mathrm{MHz}$.

In conclusion, for high enough efficiency and phase control where the gate noise decrease persistently with increasing pump power instead of saturating, the gate noise in the cluster state computation scheme here can be brought down and eventually allow fault-tolerant computation, depending on the squeezing threshold set by the concatenated qubit error correction code. Besides this, the squeezing source bandwidth should be broad enough to cover the temporal modes in the frequency domain well. This latter condition is especially important to keep in mind when scaling up the number of encoded modes by reducing the short delay line, $\tau$, shortening the temporal modes in time domain and thereby broadening them in frequency domain. 


\section{Chapter 6}

\section{A fault-tolerant continuous-variable measurement-based quantum computation architecture}

In this chapter, the paper "A fault-tolerant continuous-variable measurement-based quantum computation architecture" of Ref. [34] is presented. This paper is authored by Mikkel V. Larsen, Christopher Chamberland, Kyungjoo Noh, Jonas S. Neergaard-Nielsen, and Ulrik L. Andersen, and is submitted for publication.

Throughout chapter 3,4 , and 5 [31-33] the focus has been on cluster state generation and implementation of gates by projective measurements. Due to finite squeezing in the cluster state generation, the implemented gates will always be noisy, and quantum error correction is required for fault-tolerant computation. In this final theoretical work, we study efficient implementation of error correction, and we propose a complete and fault-tolerant computation scheme.

While this work utilizes the experience of chapter 2,3 , and $5[30,31,33]$ to propose an experimentally feasible computation scheme, this work has largely come forward through the theoretical study in chapter 4 [32]. Note, in this work, instead of $\hat{x}$ as in chapter $2-5, \hat{q}$ represents the electric field amplitude (or position) quadrature operator-I apologize for any inconvenience this change of notation brings the reader.

From www.arXiv.org as pre-print arXiv:2101.03014 (2021).

\subsection{Abstract}

Continuous variable measurement-based quantum computation on cluster states has in recent years shown great potential for scalable, universal, and fault-tolerant quantum computation when combined with the Gottesman-Kitaev-Preskill (GKP) code and quantum error correction. However, no complete fault-tolerant architecture exists that includes everything from cluster state generation with finite squeezing to gate implementations with realistic noise and error correction. In this work, we propose a simple architecture for the preparation of a cluster state in three dimensions in which gates by gate teleportation can be efficiently implemented. To accommodate scalability, we propose architectures that allow for both spatial and temporal multiplexing, with the temporal encoded version requiring as little as two squeezed light sources. Due to its three-dimensional structure, the architecture supports topological qubit error correction, while GKP error correction is efficiently realized within the architecture by teleportation. To validate fault-tolerance, the architecture is simulated using surface-GKP codes, including noise from GKP-states as well as gate 
noise caused by finite squeezing in the cluster state. We find a fault-tolerant squeezing threshold of $13.2 \mathrm{~dB}$ with room for further improvement.

\subsection{Introduction}

In measurement-based quantum computation (MBQC), gates are implemented by projective measurements on a multi-mode entangled cluster state, circumventing the complex coherent unitary dynamics required in conventional gate-based quantum computation [1]. As such, the cluster state is a critical resource for MBQC, and its number of modes and structural design defines the size of a potential measurement-induced algorithm. A particularly promising platform for scaling and controlling the structure of a cluster state is the optical continuous variable $(\mathrm{CV})$ platform $[2,5]$, where large cluster states can be deterministically generated and controlled, and efficiently measured by homodyne detection. This has been proven by the realizations of large-scale CV cluster states in both one dimension [19, 20] and two dimensions [31, 37] (where Ref. [31] is the content of chapter 3). Moreover, the versatility of the CV optical platform has been further corroborated by the recent demonstrations of single- and multi-mode gates using high-efficiency projective measurements on one-dimensional [25] and two-dimensional cluster states [33] (where Ref. [33] is the content of chapter 5 ).

MBQC based on CV is however inherently noisy due to the impossibility of generating maximally entangled CV cluster states: The generation of maximal CV entanglement requires squeezed states of infinite squeezing and thereby infinite energy, which is not feasible. Therefore, inevitably, Gaussian noise will be added to the quantum information during computation. To combat this additive noise, information is encoded as special qubits in CV bosonic modes of infinite dimension [13-15]. These CV qubits introduce a certain structure that makes them correctable against noise using tailored syndrome measurements. However, this comes with the cost of inducing bit-flip and phase-flip errors on the encoded qubits which must then be corrected by some qubit quantum error correction scheme. Implementing qubit error correction efficiently in MBQC puts stringent requirements on the underlying cluster state. As an example, the local connectivity in the cluster states support only coupling between nearest-neighbor modes, so topological error correction is a natural choice for qubit error correction [131]. This, in turn, requires a three-dimensional (3D) cluster state for MBQC [132-134].

Different proposals on 3D cluster state generation and topological MBQC exist. Fukui et al. [43] suggested a scheme for fault-tolerant MBQC based on topological error correction, but their scheme assumes the availability of a highly complex 3D cluster state of encoded qubits. Wu et al. [44] proposed an optical setup for the generation of a 3D cluster state using time and frequency multiplexing. However, in their proposal gates are implemented by gate teleportation through four-mode square cluster states leading to increased gate noise. In another work of Fukui et al. [45], an all-temporally encoded 3D cluster state is proposed, but this scheme is experimentally highly challenging as it requires the construction of 12 squeezing sources and real-time feed-forward operations. Moreover, no schemes for qubit encoding and qubit error correction was put forward. The most complete work on CV MBQC to date is carried out by Bourassa et al. in Ref. [46] in which a computation architecture for the generation of a 3D cluster state combined with topological MBQC is proposed. However, the suggested architecture is based on spatial encoding, rendering the number of spatial resources very large (as this number scales linearly with the computation size). Moreover, their scheme relies on a very large number of experimentally challenging on-line swap and sum gates which they assume to be ideal.

In our work, we propose a simple, scalable, and complete architecture for topological MBQC and validate the fault-tolerance of the computation scheme. It is based on gate teleportation on parallel one-dimensional (1D) cluster states, or wires, arranged in a 3D lattice and coupled by variable beam-splitters for two-mode gates. As such, the setup is a variation of the well-demonstrated 1D cluster state generation $[19,25,31]$ with added variable beam-splitters. Combined with GottesmanKitaev-Preskill (GKP) encoded qubits [14], the scheme allows for universal computation, while fault-tolerance is achievable by encoding logical qubits in the topological surface code [135-137]. 
Furthermore, the scheme, being based on gate teleportation, is compatible with a recently proposed GKP correction protocol that dispenses with demanding coupling to ancillary GKP-qubits [42]. We validate the fault-tolerance of the full scheme by a thorough simulation that includes both noise in the GKP-qubits and gate noise caused by finite squeezing in the cluster state. As a result, when combining the topological surface code with GKP error correction in the surface-GKP code [121], we find a squeezing threshold of $17.3 \mathrm{~dB}$. We continue to propose a variation of the surfaceGKP code - the surface-4-GKP code with four GKP corrections during the surface code syndrome measurements - by which we upgrade the squeezing threshold to $13.2 \mathrm{~dB}$ while leaving room for further improvements. If gate noise is ignored, the threshold drops to $10.6 \mathrm{~dB}$, which is comparable to that found in $[43,46]$.

The paper is organized as follows. In section 6.3 we present the computation scheme and describe the implementation of the required gates. In section 6.4 we focus on GKP error correction within the computation scheme, and in section 6.5 we implement the surface code for qubit error correction and validate the fault-tolerance properties by performing simulations. In section 6.6 we discuss the results and conclude the paper.

\subsection{Computation scheme}

Our computation scheme supports temporal as well as spatial encoding of the cluster state nodes. The architectural design of the setups for temporal and spatial encoding are shown in Fig. 6.1a and Fig. 6.2, respectively. Despite the significant architectural differences of the two designs, the computation schemes for temporal and spatial encoding are identical. Therefore, while focusing on the temporally encoded scheme in this paper, all the presented methods, results, and conclusions also hold true for the spatially encoded scheme - even a combination of the spatial and temporal encoding architectures might lead to a similar computation scheme with identical conclusions.

The temporally encoded scheme consists of three parts: the preparation of resource states, the injection of input states at the computational level, and the measurements, enabled by a temporally delocalized measurement device (TDMD). Note, the term 'computational level' refers to the location in the setup at which information is encoded and computation takes place. In some of our previous works (chapter 4 and 5) this computational level is referred to as a 'logic level' [32, 33]. However, in this chapter we reserve the term 'logic' for qubit error correction in section 6.5. As ancillary input for the resource preparation, we switch between squeezed vacuum states, $|0\rangle_{\mathrm{sq}}$, when implementing gates by projective measurements, and GKP qunaught states [42], $|\varnothing\rangle_{\mathrm{GKP}}$, when performing GKP error correction. In section 6.4, GKP error correction with ancillary $|\varnothing\rangle_{\mathrm{GKP}}$-states is described, while throughout this section we focus on gate implementation with ancillary $|0\rangle_{\mathrm{sq}}$-states.

At the resource preparation stage, the spatial modes $A$ and $B$ are initially occupied by squeezed vacuum states, $|0\rangle_{\text {sq }}$, which are squeezed along the orthogonal quadratures $(\hat{q}-\hat{p}) / \sqrt{2}$ and $(\hat{q}+$ $\hat{p}) / \sqrt{2}$, respectively. Here, $\hat{q}$ and $\hat{p}$ are the electric field amplitude and phase (or position and momentum) quadratures for which we use the $\hbar=1$ convention, corresponding to a vacuum variance of $1 / 2$. Each pair of squeezed states is then interfered on a balanced beam-splitter, leading to two-mode entanglement with $\hat{q} \hat{p}$-correlations. This is an approximate cluster state equivalent to a conventional two-mode squeezed state (with $\hat{q} \hat{q}-$ and $\hat{p} \hat{p}$-correlations) that is phaserotated by $\pi / 4$ in both modes $[4,7]$. As a unitary operator for the balanced beam-splitter, we use $\hat{B}=e^{-i \pi\left(\hat{q}_{i} \otimes \hat{p}_{j}-\hat{p}_{i} \otimes \hat{q}_{j}\right) / 4}$ with corresponding symplectic matrix

$$
\mathbf{B}=\frac{1}{\sqrt{2}}\left(\begin{array}{cccc}
1 & -1 & 0 & 0 \\
1 & 1 & 0 & 0 \\
0 & 0 & 1 & -1 \\
0 & 0 & 1 & 1
\end{array}\right)
$$

acting on $\left(\hat{q}_{i}, \hat{q}_{j}, \hat{p}_{i}, \hat{p}_{j}\right)^{T}$ quadrature vectors, and represented graphically with an arrow pointing from mode $i$ to $j$. Note, in this work we prepare two-mode cluster states, however, we could 


\section{(a)}

(b)

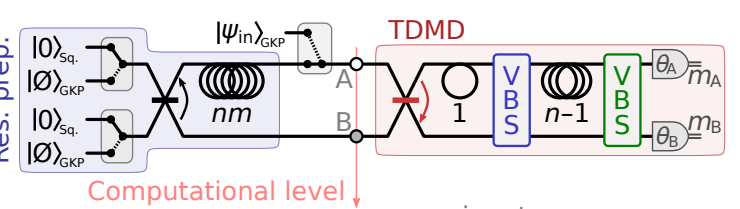

(b)

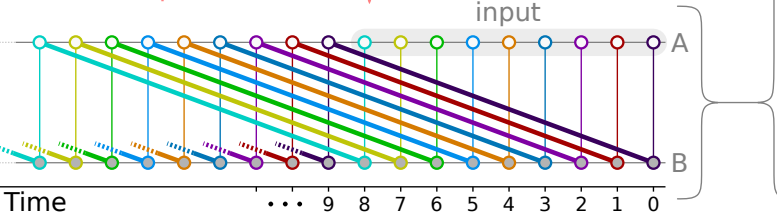

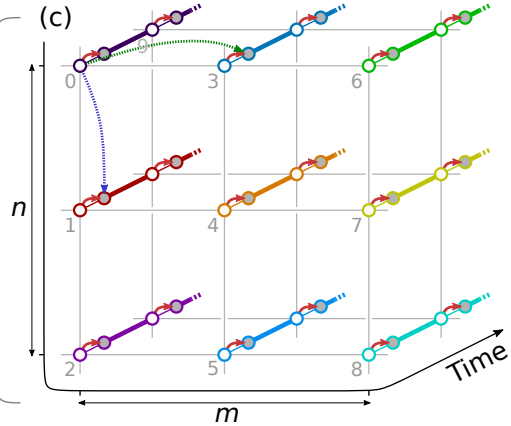

Figure 6.1: (a) Temporally encoded computational setup of our scheme. The scheme consists of three parts: The resource preparation (res. prep.); the computational level where the computation takes place; and the temporally delocalized measurement device (TDMD) for gate implementation by projective measurements. This scheme utilizes temporal multiplexing of two spatial modes, $A$ and $B$, marked in the computational level. (b) Wires of two-mode entanglement at the computational level shown in the time domain, here for the simple case of $n m=9$. Bold lines represent two-mode entanglement, while thin lines indicate the temporal overlap of $A$ and $B$. The wires begin with $n m$ input states in temporal modes 0 to 8 , switched in using an optical switch in $A$ of the computational level. The colors of the wires have no physical meaning and are merely used to indicate different wires. (c) Wires rearranged into a 3D time lattice where the input is encoded onto the $n \times m$ end surface while gates are implemented by teleportation along the wires in the third dimension. Here, the red arrows represent the first beam-splitter of the TDMD, while the dotted blue and green arrows represent the variable beam-splitters of the TDMD. The first 10 temporal modes of (b) from 0 to 9 are labeled in (c).

as well have considered preparation of conventional two-mode squeezed states (with $\hat{q} \hat{q}-$ and $\hat{p} \hat{p}$ correlations) which are equivalent to cluster states under phase-rotation that may be absorbed into the measurement bases.

After the interference at the beam splitter, the modes of $A$ are delayed by $n m$ temporal modes, leading to synchronization of the modes $A$ and $B$ of two-mode entangled states that initially are separated by $n m$ temporal modes. ${ }^{1}$ The result is $n m$ decoupled wires of two-mode entangled states, illustrated in the time domain in Fig. $6.1 \mathrm{~b}$ for $n m=9$. Here, each color indicates different wires with the bold lines indicating two-mode entanglement, while the thin lines indicate temporal overlap of $A$ and $B$. The $n m$ wires constitute the computational level in which computation is performed. Using an optical switch, $n m$ input modes to the computation can be switched into the computational level at $A$-optical switching has been demonstrated in a continuous variable quantum setting in $[30,114]$. In Fig. $6.1 \mathrm{~b}$ nine input modes have been switched into $A$ in temporal modes 0 to 8 .

The $n m$ wires can be arranged in a 2D grid such that they form a 3D square lattice, as shown in Fig. 6.1c. The third dimension is in principle arbitrarily deep. As such, information is encoded on a surface while computation proceeds along the third dimension by teleportation using the TDMD. The TDMD consists of a balanced beam-splitter, two variable beam-splitters (VBS), two delays of 1 and $n-1$ temporal modes, and two homodyne detectors (HD) measuring $A$ and $B$ in bases $\hat{q}(\theta)=\hat{q} \cos \theta+\hat{p} \sin \theta$. The arrangement is illustrated in Fig. 6.1a. Each VBS can vary between two settings: when implementing single-mode gates, the VBSs are left 'open' such that the modes $A$ and $B$ do not interfere, corresponding to $\hat{I}_{A} \otimes \hat{I}_{B}$; when implementing two-mode gates, one of the two VBSs are 'enabled' to be functioning as a balanced beam-splitter with the symplectic matrix in Eq. (6.1) interfering $A$ and $B$. Such a variable beam-splitter may be implemented in various ways, for instance as a Mach-Zehnder interferometer with a controllable phase in one arm, or by

\footnotetext{
${ }^{1}$ We assume the temporal mode duration and spacing to be equal. In practice, for a pulsed scheme, the temporal mode duration corresponds to the pulse width, while the $n m$-delay corresponds to a delay of $n m$ times the temporal pulse spacing.
} 


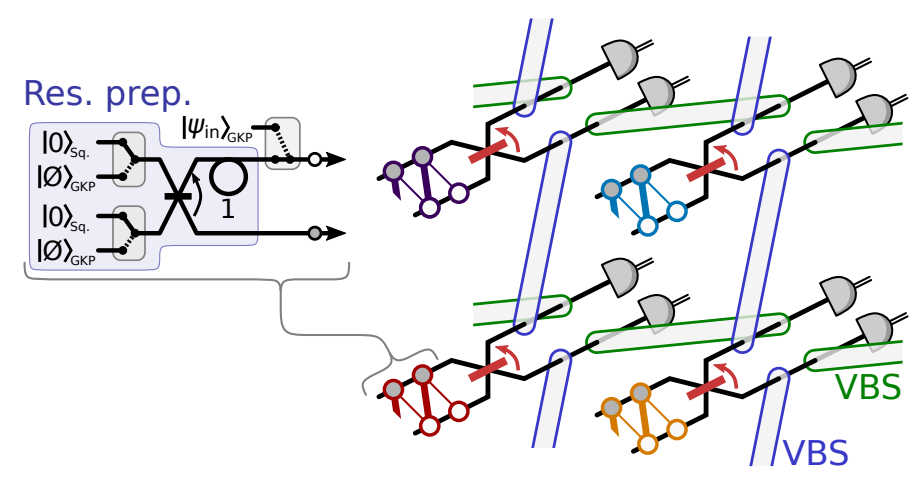

Figure 6.2: Spatially encoded version of the computation scheme illustrated in Fig. 6.1. Here, the wires originate from individual resource preparations and are coupled through a grid of variable beam-splitters (VBS). The resulting computation scheme is exactly the same as in Fig. 6.1, but with the 3D lattice of Fig. $6.1 \mathrm{c}$ being encoded in (space $)^{2} \times$ time instead of (time $)^{3}$. Spatial encoding has the advantage of the computation time not being affected when scaling up the lattice. However, this comes at the cost of requiring spatially scalable resources. As such, spatial encoding may be possible on platforms with integrated photonics, whereas temporal encoding may be advantageous in free-space and fiber optical platforms.

polarization control combined with polarization-dependent beam-splitters [114, 138, 139].

When the VBSs are left open, the TDMD simply implements a two-mode joint Bell measurement which enacts a single-mode gate teleportation through the two-mode entangled resource state $[10,25]$. The state in computation is teleported from temporal mode $k$ in $A,(A, k)$, to mode $(A, k+n m)$. In this process, depending on the HD basis settings, $\theta_{A, k}$ and $\theta_{B, k}$, the gate operation

$$
\hat{R}\left(\theta_{+}\right) \hat{S}\left(\tan \theta_{-}\right) \hat{R}\left(\theta_{+}\right)
$$

is implemented on the teleported state, where $\theta_{ \pm}=\left( \pm \theta_{A, k}+\theta_{B, k}\right) / 2 .^{2}$ Here $\hat{R}(\theta)=e^{-i \theta\left(\hat{q}^{2}+\hat{p}^{2}\right) / 2}$ and $\hat{S}(s)=e^{i \ln (s)(\hat{q} \hat{p}+\hat{p} \hat{q}) / 2}$ are the rotation and squeezing operators. Note, $k$ labels the temporal modes at the computational level, while at the HDs, modes in $A$ are delayed by $n$ temporal modes relative to modes in $B$. All single-mode Gaussian gates can be implemented with two iterations of Eq. (6.2) [6].

Enabling one of the two VBSs, two-mode gates can be implemented between nearest neighbours in the $3 \mathrm{D}$ time lattice. Two-mode gates between $(A, k)$ and $(A, k+1)$ are implemented by enabling the first VBS, while enabling the second VBS allows two-mode gates between $(A, k)$ and $(A, k+n)$. In the 3D time lattice of Fig. 6.1c, the VBSs are represented by dotted arrows. To encode the surface code described in section 6.5, we implement two different symmetric two-mode gates: $\hat{C}_{Z}(g)=e^{i g \hat{q} \otimes \hat{q}}$ and $\hat{C}_{X}(g)=e^{-i g \hat{p} \otimes \hat{p}}$. They are controlled-phase gates that displace one mode in $\hat{p}$ (or $\hat{q}$ ) by an amount $g \hat{q}$ (or $g \hat{p}$ ) controlled by the other mode. We note that $\hat{C}_{X}(g)$ does not correspond to a controlled-not gate. $\hat{C}_{Z}(g)$, or $\hat{C}_{X}(g)$, constitutes together with Eq. (6.2) a universal Gaussian gate set. In practice, $\hat{C}_{Z}(g)$ and $\hat{C}_{X}(g)$ cannot be implemented in a single computation step without some Fourier by-products of $\pi / 2$ phase-rotations, $\hat{F}=\hat{R}(\pi / 2)$. To implement the surface code in section 6.5 with a minimum number of computation steps, we make use of 4 variations of $\hat{C}_{Z}(g)$ and $\hat{C}_{X}(g)$ with different by-products, each listed in table 6.1 with their required basis settings for implementation. These are with by-products of $\hat{F} \otimes \hat{F}^{\dagger}$ or $\hat{F}^{\dagger} \otimes \hat{F}$ when implemented on modes $(A, k+n m) \otimes(A, k+n m+j)$ where $j=1$ or $n$ depending on which VBS is enabled. When implementing the surface code, the gates are arranged such that the Fourier by-products cancel.

\footnotetext{
${ }^{2}$ Comparing with Ref. [10], a squeezing operator, dependent on the squeezing of the ancillary $|0\rangle_{\text {sq }}{ }^{-s t a t e s, ~ i s ~}$ missing in Eq. (6.2). This is because in this work the two-mode entangled states prepared in the resource preparation are considered cluster-type states with edge weight 1 [4], similar to in chapter 5 [33], instead of approximate cluster states in the language of Ref. [7].
} 


\begin{tabular}{lr}
\hline \hline Two-mode gate & Basis setting, $\left(\theta_{A, k}, \theta_{B, k}, \theta_{A, k+j}, \theta_{B, k+j}\right)$ \\
\hline$\left(\hat{F}^{\dagger} \otimes \hat{F}\right) \hat{C}_{Z}(g)$ & $\left(-\arctan \frac{2}{g}, 0,0, \arctan \frac{2}{g}\right)$ \\
$\hat{C}_{Z}(g)\left(\hat{F} \otimes \hat{F}^{\dagger}\right)$ & $\left(-\frac{\pi}{2}+\arctan \frac{2}{g}, \frac{\pi}{2},-\frac{\pi}{2}, \frac{\pi}{2}-\arctan \frac{2}{g}\right)$ \\
$\left(\hat{F} \otimes \hat{F}^{\dagger}\right) \hat{C}_{X}(g)$ & $\left(-\frac{\pi}{2}+\arctan \frac{2}{g}, \frac{\pi}{2},-\frac{\pi}{2}, \frac{\pi}{2}-\arctan \frac{2}{g}\right)$ \\
$\hat{C}_{X}(g)\left(\hat{F}^{\dagger} \otimes \hat{F}\right)$ & $\left(-\arctan \frac{2}{g}, 0,0, \arctan \frac{2}{g}\right)$ \\
\hline \hline
\end{tabular}

Table 6.1: Two-mode gates with input and output in modes $(A, k) \otimes(A, k+j)$ and $(A, k+n m) \otimes$ $(A, k+n m+j)$, respectively, and their required basis settings. Here, $j=1$ when enabling the first VBS (marked by blue in Fig. 6.1), and $j=n$ when enabling the second VBS (marked by green in Fig. 6.1). The order of the tensor products are arranged with earlier temporal modes first. Note, as apparent from the basis settings, $\left(\hat{F}^{\dagger} \otimes \hat{F}\right) \hat{C}_{Z}(g)=\hat{C}_{X}(g)\left(\hat{F}^{\dagger} \otimes \hat{F}\right)$ and $\hat{C}_{Z}(g)\left(\hat{F} \otimes \hat{F}^{\dagger}\right)=$ $\hat{C}_{X}(g)\left(\hat{F}^{\dagger} \otimes \hat{F}\right)$. However, when implementing the gates in the surface code in section 6.5 , it is useful to consider them individually, as we will have the Fourier by-products to cancel.

Finally, as the resource squeezed states $|0\rangle_{\mathrm{sq}}$ are finitely squeezed, all gate implementations will inevitably produce excess noise which accumulates on the computational modes throughout the computation. Due to the Gaussian nature of the quadrature distribution of $|0\rangle_{\text {sq }}$, this gate noise leads to a Gaussian convolution of the quadratures of all computational modes [10, 32, 33]. Assuming the variance of the squeezed quadrature of $|0\rangle_{\text {sq }}$ to be $\sigma^{2}=e^{-2 r} / 2$ (where $r$ is the squeezing parameter), the uncorrelated gate noise is

$$
\sigma_{\text {gate }}^{2}=2 \sigma^{2}=e^{-2 r}
$$

which will be added symmetrically in each quadrature of the computational modes. In addition to gate noise, an implemented gate also results in a displacement of the computational modes depending on the projective measurement outcomes. Since the measurement outcomes are known, this displacement can be compensated for by another cancelling displacement operation. However, in practice, these ubiquitous displacement operations need not be executed directly onto the output modes; they can simply be accounted for in post-processing of the measurement outcomes. Therefore, in this work, we will ignore these displacements, while for practical implementation, one has to keep these in mind when analysing the measurement outcomes.

For a derivation of implemented gates considered in this section, together with their resulting gate noise and displacements, see appendix section 6.7 .

\subsection{GKP quadrature correction}

As mentioned above, noise will be added to the computation modes at each gate implementation due to the finite amount of squeezing of the resource states. To correct for this noise and thus prevent noise accumulation, we consider a quadrature noise correction scheme that relies on bosonic qubit encoding in the infinite-dimensional Hilbert space. This noise correction scheme, however, comes with the cost of introducing qubit errors which must be subsequently corrected by a qubit error correction scheme. The first correction layer, the quadrature correction scheme, will be discussed in this section while the second correction layer, the qubit error correction, will be the subject of section 6.5.

Several schemes for encoding qubits into bosonic harmonic oscillators of infinite Hilbert space dimension exist, including cat-codes [13], binomial codes [15], and the Gottesman-Kitaev-Preskill (GKP) code [14]. Since the gate noise of our computation scheme is additive quadrature noise, GKP-encoding where a qubit is encoded in the mode quadratures as Dirac combs is most suitable. The GKP code is also suitable for correcting excitation loss errors since excitation loss can be converted via quantum-limited amplification into additive quadrature noise [109, 140-142]. Furthermore, as the gate noise (with variance given in Eq. (6.3)) is added symmetrically in phase 
space, we consider GKP qubits encoded on square grids in phase space with a $2 \sqrt{\pi} \times 2 \sqrt{\pi}$ unit cell. For such encoded qubits, a universal Clifford gate set is realized by the Gaussian gates $\left\{\hat{R}(\pi / 2), \hat{P}(1), \hat{C}_{Z}(1)\right\}$ together with $\sqrt{\pi}$ displacements in phase space. For a comprehensive review of the GKP code, see $[14,112,113]$.

Information encoded in GKP qubit states, $\left|\psi_{\text {in }}\right\rangle_{\mathrm{GKP}}$, is launched into the computation scheme at the computational level as shown in Fig. 6.1a. These states are not ideal as they are subjected to the finite energy constraints (similar to the squeezed states). This means that the uncertainties of the individual spikes of the quadrature comb of the GKP state are not zero but have a finite value. Mathematically, the delta functions of the Dirac comb in the GKP state quadrature wave function are replaced by finitely squeezed Gaussian functions, each with a variance of $\sigma_{\mathrm{GKP}}^{2}$, and an overall envelope that satisfies the Fourier relations between the orthogonal $\hat{q}$ and $\hat{p}$ quadratures with equally squeezed spikes. In the following, we ignore the overall envelope, which is valid for sufficiently high squeezing, while we assume that the squeezing of the GKP spikes is the same as that of the $|0\rangle_{\mathrm{sq}}$ states in the resource preparation,

$$
\sigma_{\mathrm{GKP}}^{2}=\sigma^{2}=e^{-2 r} / 2 .
$$

The Gaussian noise accompanying gate implementation results in the variance of the GKP spikes increasing by $\sigma_{\text {gate }}^{2}$ in both quadratures for every single gate. To prevent this, GKP quadrature correction is performed, preferably after every gate. Traditionally, this is done by coupling each quadrature to ancillary GKP states, which are then measured, and the result is fed forward to displacements of the computational qubit (or compensated for in following measurement outcomes) [14]. This approach, however, requires active two-mode gates which are experimentally hard to realize online and noisy to implement by projective measurements. Instead, we use the new approach by Walshe et al. [42] where GKP quadrature correction is realized by qubit teleportation using ancillary GKP qunaught states and is directly compatible with our computation scheme. The GKP qunaught state, $|\varnothing\rangle_{\mathrm{GKP}}$, is the 1-level version of the generalized GKP qudit state with a $\sqrt{2 \pi}$ spacing between the spikes in the quadrature wave functions $[42,143]$. As such, $|\varnothing\rangle_{\text {GKP }}$ holds no information, but interfering two $|\varnothing\rangle_{\mathrm{GKP}}$ states on a beam-splitter results in a two-mode GKP-qubit Bell state. This state can then be used for GKP-qubit teleportation with support only on the GKP grid in phase-space so that a noisy GKP qubit is projected into a purified GKP qubit by the teleportation.

The implementation of the GKP quadrature correction in [42] is shown in Fig. 6.3. In the resource preparation, we switch from $|0\rangle_{\mathrm{sq}}$ to $|\varnothing\rangle_{\mathrm{GKP}}$ states. After interference on the first beamsplitter, a GKP Bell state is prepared at the computational level instead of a two-mode CV cluster state. For teleportation of a noisy GKP qubit through the GKP Bell state, a Bell-measurement of the noisy GKP qubit and one mode of the Bell state should be carried out by the TDMD. This is done by leaving the two VBSs open and measuring in the $\hat{q}$ and $\hat{p}$ basis in spatial modes $A$ and $B$, respectively. The corresponding graph in a small section of the 3D time lattice is shown in Fig. 6.3 together with the corresponding circuit. The resulting Kraus operator,

$$
\hat{K}\left(m_{A}, m_{B}\right)=\mathcal{N} \hat{\bar{\Pi}}_{\mathrm{GKP}} \hat{X}\left(-m_{A} \sqrt{2}\right) \hat{Z}\left(-m_{B} \sqrt{2}\right),
$$

projects the noisy input state into a purified GKP qubit state. Here, $\hat{X}\left(-m_{A} \sqrt{2}\right)=e^{i m_{A} \sqrt{2} \hat{p}}$ and $\hat{Z}\left(-m_{B} \sqrt{2}\right)=e^{-i m_{B} \sqrt{2} \hat{q}}$ are displacements in the $\hat{q}$ and $\hat{p}$ quadratures, respectively, depending on the measurement outcomes $m_{A}$ and $m_{B}, \mathcal{N}$ is a normalization factor, also depending on the measurement outcomes, and

$$
\hat{\bar{\Pi}}_{\mathrm{GKP}}=\left|\overline{0}_{\mathrm{GKP}}\right\rangle\left\langle\overline{0}_{\mathrm{GKP}}|+| \overline{1}_{\mathrm{GKP}}\right\rangle\left\langle\overline{1}_{\mathrm{GKP}}\right|
$$

is a noisy GKP projector. $\left|\bar{j}_{\mathrm{GKP}}\right\rangle$ (where $j=0,1$ ) are approximate GKP Pauli-Z eigenstates with spike variance identical to that of the initial $|\varnothing\rangle_{\mathrm{GKP}}$ states. For simplicity, we assume the spikes in the $|\varnothing\rangle_{\mathrm{GKP}}$ states - and hence also the newly projected GKP qubit - to have variance $\sigma_{\text {GKP }}^{2}$, just as we did for $\left|\psi_{\text {in }}\right\rangle_{\mathrm{GKP}}$. 


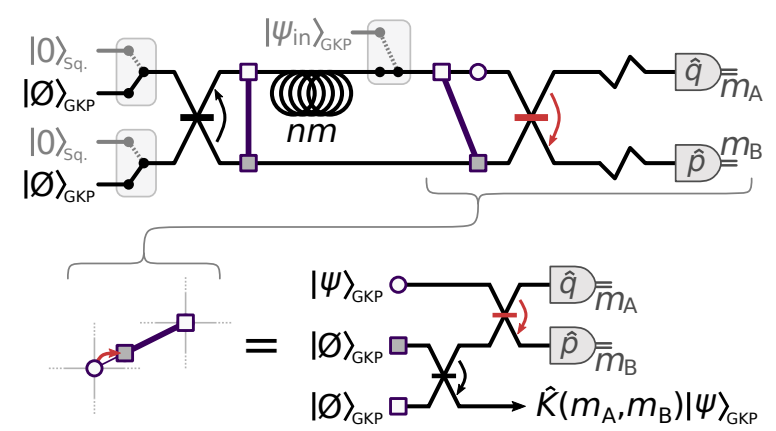

Figure 6.3: Implementation of GKP quadrature correction by qubit teleportation. Qunaught states, $|\varnothing\rangle_{\mathrm{GKP}}$, are injected at the resource preparation state, thereby preparing a two-mode GKPqubit Bell state shown as two connected rectangles before the $\mathrm{nm}$-delay. In the computational level after the $n m$-delay, one part of the Bell state overlaps in time with the GKP-qubit state to be corrected, $|\psi\rangle_{\mathrm{GKP}}$, shown as a circle. Setting the TDMD to perform a Bell measurement (the two VBSs are open and left out in the figure) $|\psi\rangle_{\mathrm{GKP}}$ is teleported through the Bell state and projected into a purified GKP qubit state by the Kraus operator in Eq. (6.5). Below is the corresponding graph as it will appear in the 3D time lattice of Fig. 6.1c as well as the corresponding circuit diagram.

The output values, $m_{A}$ and $m_{B}$, are integer multiples of $\sqrt{\pi / 2}$ plus some noise associated with the finite squeezing of the GKP qubit and qunaught states. As such, the $\hat{X}\left(-m_{A} \sqrt{2}\right)$ and $\hat{Z}\left(-m_{B} \sqrt{2}\right)$ displacements in Eq. (6.5) mainly corresponds to Pauli-X and Pauli-Z operations on the encoded qubit, and is a natural result of the teleportation similar to regular qubit teleportation. These displacements may be compensated for by unitarily displacing the teleported state back in $\hat{q}(\hat{p})$ by $m_{A(B)} \sqrt{2}$ rounded to the nearest integer of $\sqrt{\pi}$, or simply by shifting the final measurement outcomes. However, due to the inevitable noise in $m_{A}$ and $m_{B}$, occasionally $m_{A(B)} \sqrt{2}$ will be rounded to the wrong integer of $\sqrt{\pi}$ which then results in a faulty displacement operation. This induces a qubit error. The probability for this error to occur is [121]

$$
p_{\sigma}(z)=\frac{\sum_{n \in \mathbb{Z}} \exp \left[-(z-(2 n+1) \sqrt{\pi})^{2} /\left(2 \sigma^{2}\right)\right]}{\sum_{n \in \mathbb{Z}} \exp \left[-(z-n \sqrt{\pi})^{2} /\left(2 \sigma^{2}\right)\right]}
$$

where the residual analogue information, $z=\mathcal{R}\left(m_{A(B)} \sqrt{2}\right)$, when rounding is given by

$$
\mathcal{R}\left(m_{A(B)} \sqrt{2}\right)=m_{A(B)} \sqrt{2}-\sqrt{\pi}\left\lfloor\frac{m_{A(B)} \sqrt{2}}{\sqrt{\pi}}+\frac{1}{2}\right\rfloor,
$$

In Eq. (6.6), $\sigma^{2}=\sigma_{\text {in }}^{2}+\sigma_{\text {GKP }}^{2}$ is the variance of $z$ with $\sigma_{\text {in }}^{2}$ being the spike variance of the GKP qubit before teleportation. For example, if the GKP qubit to be corrected has gone through one gate, then $\sigma_{\text {in }}^{2}=\sigma_{\text {GKP }}^{2}+\sigma_{\text {gate }}^{2}$ where $\sigma_{\text {GKP }}^{2}$ was the GKP qubit spike variance before the gate and $\sigma_{\text {gate }}^{2}$ is the gate noise variance in Eq. (6.3). Using the analogue information from the GKP quadrature correction to improve the concatenated qubit error correction was proposed in [144], and here, similar to in [121], we use this information through the probability in Eq. (6.6) to improve the second layer of error correction, the surface code, which is the subject of the next section.

\subsection{Surface code}

In section 6.4, we showed how to project the continuous variable noise from the finite squeezing in section 6.3 into qubit Pauli errors by GKP quadrature correction. However, in order to perform fault-tolerant quantum computation, such Pauli errors must be corrected using an outer quantum error correcting code. Given the nearest neighbour interactions of the computation scheme in 
section 6.3, topological error correction is a natural choice to use as an outer code. With information encoded on a surface of the computation scheme's 3D time lattice, and gates implemented in the third dimension, we consider the surface code [135-137]. Specifically, to compute logical $X$ or $Z$ error rates, we implement the simulation methods of [121] applied to the rotated surface code $[145,146]$. Such simulation methods are adapted to the computation scheme as described in the appendix section 6.8. We note that the rotated surface code may not be the most resource-efficient code for our computation scheme since it is rotated $45^{\circ}$ with respect to the $3 \mathrm{D}$ time lattice, and thereby, computation modes located in the corner of the 3D time lattice may not be utilizedthe rotated surface code was chosen for straightforwardly reusing the simulation method of [121]. Below, in section 6.5.1, we first describe the implementation of the surface code, and then consider it combined with GKP quadrature correction. In section 6.5.2, we then present simulation results of logical error rates and provide a squeezing threshold.

\subsubsection{Implementation of the rotated surface code}

A logical qubit is shown in Fig. 6.4a for a distance $d=5$ rotated surface code. Information is encoded in $d^{2}$ data qubits (white and gray circles). The stabilizers of the code are measured using $\left(d^{2}-1\right) / 2$ ancilla qubits prepared in $|+\rangle_{\mathrm{GKP}}$ (green circles) and $\left(d^{2}-1\right) / 2$ ancilla qubits prepared in $|0\rangle_{\mathrm{GKP}}$ (red circles). In what follows, we refer to green and red ancillas as measure- $Z$ and measure- $X$ ancillas.

One round of $Z$ and $X$-type stabilizer measurements is shown in Fig. 6.4b,c. Each stabilizer measurement consists of four two-qubit gates and is thus implemented in four time steps along the third dimension of the 3D time lattice in which the surface code is implemented as shown in Fig. 6.4d. Using the optical input-switch in spatial mode $A$ of the setup described in section 6.3, the ancilla qubits, initialized beforehand in the $|0\rangle_{\mathrm{GKP}}$ and $|+\rangle_{\mathrm{GKP}} \propto|0\rangle_{\mathrm{GKP}}+|1\rangle_{\mathrm{GKP}}$ states, are switched into the computational level in the temporal modes corresponding to ancillary modes of the surface code. The measure- $Z$ and $-X$ ancillas are then coupled to neighbouring data qubits using $\hat{C}_{Z}(1)=e^{i \hat{q} \otimes \hat{q}}$ and $\hat{C}_{X}( \pm 1)=e^{\mp i \hat{p} \otimes \hat{p}}$ gates, before being measured in the $\hat{p}$ and $\hat{q}$ basis, respectively. To measure such ancillas using the TDMD, the VBSs are left open while the same basis is chosen in spatial modes $A$ and $B$ in which case the measurements commute with the beamsplitter of the TDMD. Note that the state initialization and measurement basis for the ancillas are opposite of what is traditionally used in the surface code since they are coupled to data qubits via $\hat{C}_{Z}$ and $\hat{C}_{X}$ gates instead of sum-gates, $\hat{C}_{\mathrm{NOT}}=e^{-i \hat{q} \otimes \hat{p}}$. The reason for not using sum-gates is that such gates cannot be implemented in the MBQC scheme considered in this work in a single set of projective measurements. As such, using sum-gates would lead to larger gate error rates compared to the error rates of the $\hat{C}_{Z}$ and $\hat{C}_{X}$ gates.

While the measure- $Z$ ancillas are coupled to data qubits with a constant coupling rate through $\hat{C}_{Z}(1)$, the measure- $X$ ancillas are coupled to data qubits with $\hat{C}_{X}(1)$ in step 1 and 4 and $\hat{C}_{X}(-1)$ in step 2 and 3 . This is to prevent the propagation of finite squeezing noise among measure-qubits [121] (though this does not matter in the case of GKP quadrature correction during the stabilizer measurements as discussed later). Furthermore, since the $\hat{C}_{Z}(1)$ and $\hat{C}_{X}( \pm 1)$ gates cannot be implemented in a single computation step without Fourier by-products as described in section 6.3, the surface code is implemented with the two-mode gates listed in table 6.1, and so, for the different two-mode gates in Fig. 6.4 we use

$$
\begin{aligned}
& \mathfrak{l}=\left\{\begin{array}{ll}
\left(\hat{F}^{\dagger} \otimes \hat{F}\right) \hat{C}_{Z}(1) & , \text { step } 1 \& 3 \\
\hat{C}_{Z}(1)\left(\hat{F} \otimes \hat{F}^{\dagger}\right) & , \text { step } 2 \& 4
\end{array},\right. \\
& \mathcal{I}=\left\{\begin{array}{ll}
\left(\hat{F} \otimes \hat{F}^{\dagger}\right) \hat{C}_{X}(1) & , \text { step } 1 \\
\hat{C}_{X}(1)\left(\hat{F}^{\dagger} \otimes \hat{F}\right) & , \text { step } 4
\end{array},\right. \\
& \stackrel{O}{o}=\left\{\begin{array}{ll}
\left(\hat{F} \otimes \hat{F}^{\dagger}\right) \hat{C}_{X}(-1), & \text { step } 3 \\
\hat{C}_{X}(-1)\left(\hat{F}^{\dagger} \otimes \hat{F}\right), & \text { step } 2
\end{array},\right.
\end{aligned}
$$

where the first term in the tensor products is the earlier temporal mode in the computational level. 


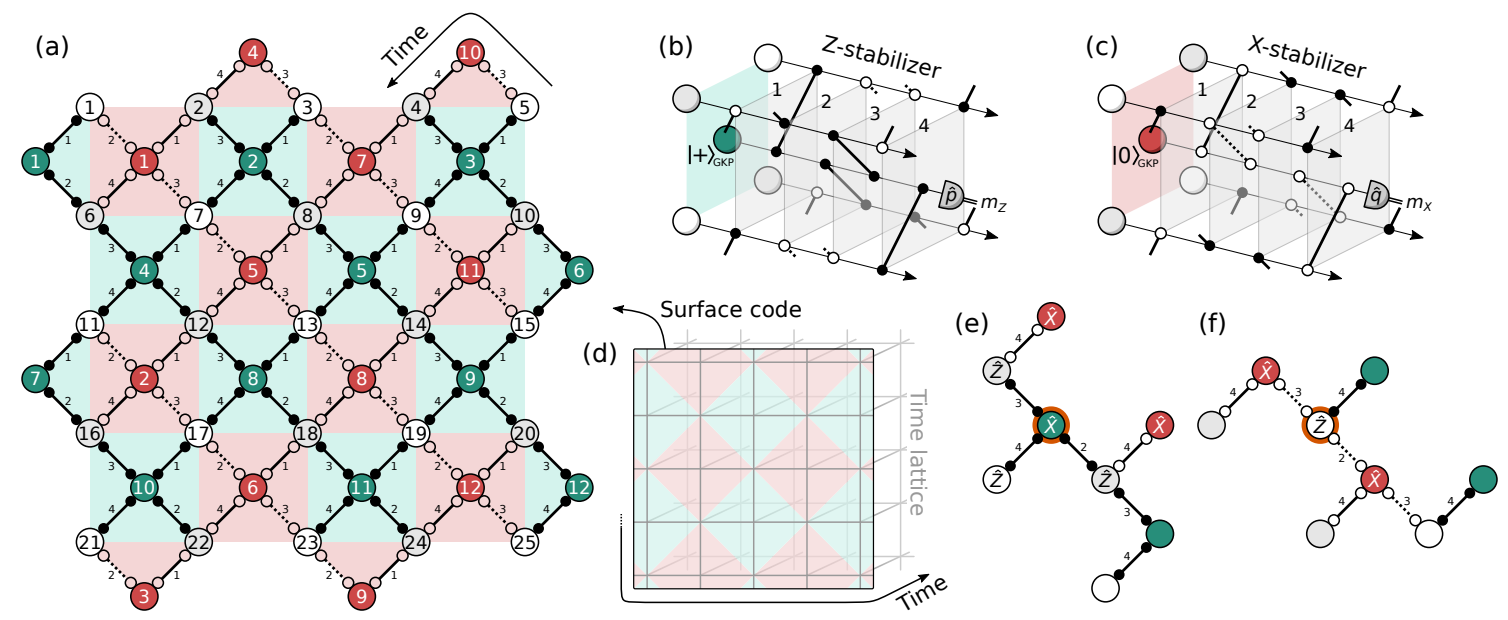

Figure 6.4: a Illustration of a logical qubit for the $d=5$ rotated surface code. White and gray circles represent odd and even data qubits, respectively. Green and red circles represent $Z$ - and $X$-measure qubits, respectively. The two-mode gate operations are listed in Eq. (6.8) and the labels 1 to 4 indicate the time steps in which those gates are implemented. (b,c) Illustration of one round of syndrome measurements including the initialization of the encoded GKP ancilla qubits, 4 time steps for the coupling between the data and ancilla qubits by 4 measurement-induced twomode gates, and a measurement using the TDMD. For the surface-GKP code, GKP corrections are performed on data qubits at the beginning of a surface code syndrome measurement cycle. For the surface-4-GKP code, GKP quadrature corrections are performed on all data and ancilla qubits after each gate. (d) Orientation of the surface code in the 3D time lattice of the computation scheme in Fig. 6.1. Here, the surface code is encoded in two dimensions of the time lattice with vertices corresponding to encoded GKP qubits, while gates are encoded in each step along the third dimension of the time lattice. (e,f) Example of qubit errors induced by GKP quadrature correction in the surface-4-GKP code. In (e), an $\hat{X}$ Pauli error occurs on a measure- $Z$ ancilla after the first two-mode gate of a surface code syndrome measurement round. The error then propagates to neighbouring data and measure- $X$ ancillas via the subsequent two-mode gates used to measure the surface code stabilizers. In (f), a $\hat{Z}$ Pauli error occurs on a data qubit after the first two-mode gate and propagates to two measure- $X$ ancillas. 
In this way, the Fourier by-products of step $1(3)$ and 2(4) cancel as $\hat{F} \hat{F}^{\dagger}=\hat{F}^{\dagger} \hat{F}=\hat{I}$ on measure$Z$ and odd data qubits, and becomes $\hat{F} \hat{F}=\hat{F}^{\dagger} \hat{F}^{\dagger}=-\hat{I}$ on measure- $X$ and even data qubits. Hence such terms have no influence on the encoded information and do not propagate errors. For CV noise, $-\hat{I}$ on even data qubits cancels with $-\hat{I}$ on measure- $X$ qubits when phase-space displacements propagate in-between measure qubits.

We proceed to combine the surface code with GKP quadrature correction, the so-called surfaceGKP code. Commonly, in the surface-GKP code, each round of syndrome measurements consists of correction of the GKP data qubits followed by measurements of the surface code stabilizers. In this way, qubit errors induced in the GKP quadrature correction is corrected by the surface code $[43,121,147]$. However, in the usual surface-GKP code, gate noise accumulates during all four gates of the stabilizer measurements in Fig. 6.4b,c. We propose to modify the scheme to perform GKP quadrature correction of each mode after every implemented gate. In other words, for each $Z$ - and $X$-stabilizer measurement, GKP quadrature correction is performed four times, and we refer to this as the surface-4-GKP code. Unfortunately, when doing so, qubit errors are induced during the surface code stabilizer measurements with a large impact on the fault-tolerant error threshold [137]. Two examples of induced qubits errors, and how they propagate during the stabilizer measurements, are shown in Fig. 6.4e,f. Here, a qubit $\hat{X}$ error on a measure- $Z$ qubit, induced in the GKP quadrature correction after the first gate of the stabilizer measurement, propagates to three data qubits as $\hat{Z}$ errors through the $\hat{C}_{Z}$ gates (while an initial $\hat{Z}$ error will not propagate through $\hat{C}_{Z}$ ). From there it further propagates to two measure- $X$ qubits through $\hat{C}_{X}$ gates. Similarly, a $\hat{Z}$ error on a data qubit after the first two-mode gate propagates as $\hat{X}$ errors to measure- $X$ qubits through $\hat{C}_{X}$. These errors lead to faulty syndrome measurements, and may therefore lead to wrong error recovery inducing logic errors, but even then, we will find a significant improvement of the surface-4-GKP code over the surface-GKP code. Note, in the case here with GKP quadrature correction after every gate, having -1 coupling rate in $\hat{C}_{X}(-1)$ of step 2 and 3 is unnecessary, as all $\mathrm{CV}$ noise is immediately corrected. However, since a -1 coupling rate requires no extra resources and is solely controlled by the basis settings in table 6.1, we keep it like this to compare with the surface-GKP code.

Finally, the surface code $Z$ - and $X$-stabilizer measurement outcomes from $d$ rounds of syndrome measurements are recorded in 3D $Z$ - and $X$-spacetime graphs. Here, 'space' traditionally refers to the plane of qubits in the surface code, but in our implementation this 'space'-plane is encoded in time on a surface of the 3D time lattice. Minimum-weight perfect matching (MWPM) $[148,149]$ on these graphs is then used as the decoding algorithm to determine data qubit errors and the resulting error recovery. In practice, the error recovery is simply handled by using and updating a Pauli frame [111, 150-152], similar to how feed-forward can be handled in MBQC by compensating for by-products in the following measurement outcomes $[2,5]$. Using the residual analogue information from the GKP quadrature correction to infer the probability of inducing a qubit error, the edges of the spacetime graphs are weighted for the MWPM algorithm to find error paths of highest probability. With four GKP quadrature corrections of each mode in one round of syndrome measurements, multiple error probabilities are combined in each edge weight as

$$
p_{\text {tot }}=\frac{1}{2}\left(1-\prod_{i}\left[1-2 p_{i}\right]\right),
$$

where $p_{i}$ is the probability given by Eq. (6.6) for one GKP quadrature correction taking values between 0 (no error) and 1/2 (minimal error information). With propagating qubit errors, it is not straightforward how to combine error probabilities of different GKP qubits, but here we have done as follows: Errors on a measure qubit, both induced by GKP quadrature correction and propagated from other modes, are combined on the corresponding vertical edge representing a measurement error. Errors on a data qubit, both induced by GKP quadrature correction and propagated from other modes, are combined on the corresponding horizontal edge of the following round of syndrome measurements where the data qubit errors will be detected, except for $\hat{Z}(\hat{X})$ errors on odd(even) data qubits induced in the first step, which are instead included in horizontal edges of the current round of syndrome measurements as they will be correctly detected there. For 
(a)

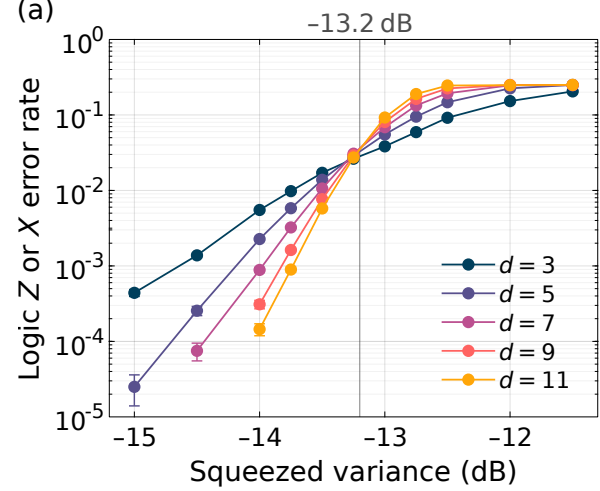

(b)

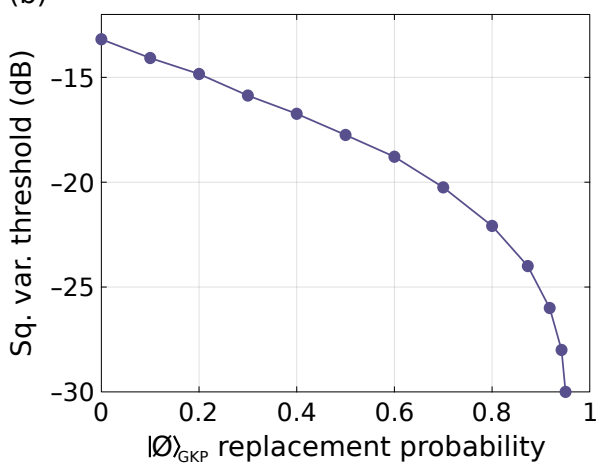

Figure 6.5: (a) Simulated logic $\hat{Z}$ and $\hat{X}$ error probability of the surface-4-GKP code as a function of the (identical) squeezing of the $|0\rangle_{\mathrm{sq}}$-states used for gate implementation, the GKP qubits encoding the surface code and the $|\varnothing\rangle_{\mathrm{GKP}}$-states used for quadrature correction. The logic error probability is shown for different code distances $d$, and the fault-tolerant threshold where the logic error rate decreases with increasing code distance is seen to be at $13.2 \mathrm{~dB}$ of squeezing. Error bars of standard deviations are estimated by bootstrapping. (b) Squeezing threshold of the surface-4GKP code as a function of the probability with which a $|\varnothing\rangle_{\mathrm{GKP}}$-state is replaced with a $|0\rangle_{\mathrm{sq}}$-state in the resource preparation. Here, the threshold is estimated as the crossing point of the $d=7$ and $d=9$ logic error rates. For zero replacement probability, the threshold is that of (a).

the probabilities to correctly add up in the MWPM, the edge weights in the spacetime graphs are taken to be $\log _{2}\left(p_{\text {tot }}\right)$.

\subsubsection{Simulation results}

To establish a fault-tolerant error threshold, we numerically simulate the complete scheme. The GKP-encoded data and measure qubits and the qunaught states, $|\varnothing\rangle_{\mathrm{GKP}}$, are all initialized with $\sigma_{\text {GKP }}^{2}=\sigma^{2}=e^{-2 r} / 2$ variance of the wave functions' GKP spikes as described in section 6.4. The ancillary squeezed vacuum states for gate implementation, $|0\rangle_{\mathrm{sq}}$, are equally squeezed by $\sigma^{2}=e^{-2 r} / 2$ leading to quadrature-symmetric gate noise of variance $\sigma_{\text {gate }}^{2}=2 \sigma^{2}=e^{-2 r}$ as described in section 6.3. Using the Monte Carlo method, logical qubit error rates are simulated as a function of squeezing using up to 100000 simulation samples with a stopping condition at the occurrence of 500 combined logic $\hat{Z}$ and $\hat{X}$ qubit error events. The resulting logical $\hat{Z}$ or $\hat{X}$ error rate (they are equal) is shown in Fig. 6.5a for different code distances $d$ as a function of squeezing level, while the logical $\hat{Y}$ error rate is smaller. The decibel scale is defined relative to the vacuum variance, $10 \log _{10}\left[\sigma^{2} /(1 / 2)\right]$. The resulting squeezing threshold from where the logic error rate decrease with increasing code distance is found to be $13.2 \mathrm{~dB}$ of squeezing.

For comparison, in the appendix section 6.8 we also simulate the error rates of other scenarios with the simulation results shown in Fig. 6.6. For the surface-GKP code with a single GKP quadrature correction before the surface code stabilizer measurements, the squeezing threshold increases to $17.3 \mathrm{~dB}$. This is significantly higher than the $13.2 \mathrm{~dB}$ squeezing threshold of the surface-4-GKP code due to accumulation of gate noise during the stabilizer measurements. To compare with other MBQC schemes with topological error correction where gate noise is typically not taken into account, and so only includes finite squeezing noise from GKP states, we simulate the surface-4-GKP code with $\sigma_{\text {gate }}^{2}=0$. The resulting threshold is $10.6 \mathrm{~dB}$ of squeezing which agrees well with the $10 \mathrm{~dB}$ reported in [43] and the $10.5 \mathrm{~dB}$ reported in [46]. Finally, to see the impact of using the residual analogue information of the GKP quadrature correction in the weighting of the spacetime graphs for MWPM decoding, we simulate the surface-4-GKP code with fixed weighting based on variances of each mode at each point in the code similar to [121]. As expected, the result is a slightly larger squeezing threshold of $14.1 \mathrm{~dB}$. 
While the GKP code has been experimentally realized in trapped-ion and circuit QED systems $[100,101,153]$, GKP state generation in optical platforms is yet to be demonstrated, although there are many recent proposals [112, 128-130, 154-156]. At first, GKP state generation is most likely going to be probabilistic. In [46] it is proposed to combine multiple GKP state generators with optical switches, and then switch between generators with a successful preparation of a GKP state. In this way, the success probability of the GKP state generation, $p_{\varnothing}$, can in principle be brought arbitrarily close to 1 . Since the surface-4-GKP code requires a large supply of $|\varnothing\rangle_{\text {GKP }}$ states, we consider as our final analysis the multi-GKP state generation scheme of [46] for $|\varnothing\rangle_{\text {GKP }}$ resource state preparation. If all the generators fail to prepare a $|\varnothing\rangle_{\mathrm{GKP}}$ state in a given temporal mode for GKP correction, a deterministically generated squeezed vacuum state, $|0\rangle_{\mathrm{sq}}$, is used instead. In this case, if $|\varnothing\rangle_{\mathrm{GKP}}$ is replaced by $|0\rangle_{\mathrm{sq}}$ in spatial mode $A(B)$, only the $\hat{q}(\hat{p})$ quadrature is corrected in the GKP quadrature correction, while the other quadrature accumulates gate noise of variance $\sigma_{\text {gate }}^{2}$ during the correction [42]. The resulting fault-tolerance squeezing threshold is shown in Fig. $6.5 \mathrm{~b}$ as a function of the probability $1-p_{\varnothing}$ of replacing $|\varnothing\rangle_{\mathrm{GKP}}$ states by $|0\rangle_{\mathrm{sq}}$ states. Here, for each point, the threshold is estimated as the crossing point of the logic error rates for surface code distances $d=7$ and $d=9$. For zero replacement probability, $1-p_{\varnothing}=0$, where $|\varnothing\rangle_{\mathrm{GKP}}$ states are always successfully generated, the squeezing threshold is the same as in Fig. 6.5a. For increasing replacement probability, the squeezing threshold level increases as expected, while it seems to converge to infinite squeezing around $1-p_{\varnothing} \approx 0.95$. Note, here we still assume successful encoding of the surface code. I.e., the data and measure qubits switched into the setup as $\left|\psi_{\text {in }}\right\rangle_{\text {GKP }}$ in Fig. 6.1a are successfully prepared as GKP qubit states. With probabilistic optical GKP state generation, this may be possible using state storage of a probabilistically prepared GKP state until it is switched into the computation scheme [157-160].

\subsection{Discussion and conclusion}

In this work, we have proposed a simple but complete architecture for optical CV MBQC that includes quadrature noise correction and qubit error correction using topological codes. The setup consists of simple optical devices such as beam-splitters, delays, optical switches, and variable beam-splitters, where the latter two can be decomposed into beam-splitters and optical phase shifters. The scheme allows for both spatial and temporal encoding, with the temporally encoded version requiring just two squeezing sources. A universal Gaussian gate set is directly implementable, while universal qubit computation is made possible by feeding the setup with GKP states, thereby supplying the required non-Gaussianity [39-41]. As the computation scheme is based on gate teleportation on wires of two-mode entangled states, the setup naturally supports the new GKP quadrature correction scheme in Ref. [42], circumventing the need for on-line active two-mode gates coupling to ancillary GKP states. Finally, by arranging the GKP qubits in a 2D plane of the cluster state that allows for nearest-neighbor interactions, topological codes can be realized. By encoding a variation of the surface-GKP code - the surface-4-GKP code - we show fault-tolerant computation to be possible above a certain squeezing threshold by simulating a logic qubit memory, or an identity gate, of the surface-4-GKP code. In the surface code, Clifford gates can be implemented by braiding [137] or lattice surgery [161] implemented by regulating the surface code syndrome measurements, while non-Clifford gates may be realized using magic states distilled from GKP qubits prepared in a magic state [39, 40] and injected into the surface code as input states [137, 162].

The fault-tolerant squeezing threshold is found to be $13.2 \mathrm{~dB}$. The estimation of this number takes into account the finite squeezing values of GKP states as well as the gate noise stemming from the finite squeezing values of the generated cluster state on which gates are implemented by projective measurements. However, this squeezing threshold leaves room for improvements: The decoding algorithm from Ref. [121] used here is optimized to detect qubit errors occurring in between the surface code stabilizer measurements. For the surface-4-GKP code, due to the repeated GKP stabilizer measurements in one surface-code cycle, Pauli errors arising from gate failures are induced during the stabilizer measurements which can result in errors that are correlated 
on both data and ancilla qubits and can reduce the effective code distance of the surface code. However, the full effective code distance of the surface code can be retained by adding space-time correlated edges to the spacetime matching graphs of the surface code decoder, which may also improve the squeezing threshold [163-166]. Note, that such modifications are solely implemented at the software level of the error correction decoder and thus requires no modifications to the setup. Another improvement may be found in the gate implementation: Due to the similarity of the GKP quadrature correction and gate implementation, it might be possible to combine the two transformations in one step, that is, implementing a gate while correcting the quadratures. Although the quadrature correction is only considered on single wires [42], it might be possible to generalize it to the two-wire case by which two-mode gates could be implemented during GKP quadrature correction, thereby eliminating gate noise coursed by finite squeezing. If this is possible while maintaining the GKP quadrature correction quality of Ref. [42], the resulting squeezing threshold reduces to $10.6 \mathrm{~dB}$ as shown in the appendix section 6.8.

The squeezing thresholds in this work are derived by assuming a particular noise model in which all resource states are finitely squeezed while all optical propagation and detection losses are set to zero. In practice, however, losses cannot be neglected. Let us denote the transmission of the setup by $\eta$. For Gaussian states, $0<\eta<1$ leads to the formation of mixed states with reduced effective squeezing. This can be reformulated as an ideal, loss-less setup $(\eta=1)$ with mixed squeezed vacuum states as input having a lower effective squeezing, and some excess antisqueezing that does not affect the measurement-based computation [38]. As a result, for $\eta<1$ the $13.2 \mathrm{~dB}$ squeezing threshold corresponds to the effectively measured squeezing. Now, for the GKP states, besides a Gaussian convolution in the quadratures, $\eta<1$ leads to a "shrinking" of a GKP state in phase-space. To see this, consider the Heisenberg picture with $\eta$ modeled as a beam-splitter of $\eta$ transmission. In this case, an amount $1-\eta$ of vacuum is mixed into the state, adding noise to the quadratures, while a share $1-\eta$ of the state is lost, "shrinking" the state in the quadratures by $\sqrt{\eta}$. The quadrature shrinking is more detrimental to GKP spikes far from the phase-space origin, which are naturally delimited in GKP states of finite squeezing due to the overall envelope in the quadrature wave function. For GKP-states with $13.2 \mathrm{~dB}$ of squeezing, we assume this effect to be negligible on the qubit error probabilities for reasonably high efficiencieswe estimate $\eta \gtrsim 0.95$ to be doable on optical platforms. We also note that the shrinking effect can be counteracted by linear amplification which on the other hand will further reduce the amount of squeezing [109, 140-142], effectively resetting $\eta$ to unity at the cost of lowering the effective squeezing of the GKP state. Again, the estimated threshold of $13.2 \mathrm{~dB}$ refers to the required squeezing after such actions have been implemented. Another detrimental effect that has not been directly accounted for is interferometric phase fluctuations. Similar to optical loss, phase fluctuations lead to mixed squeezed states of reduced squeezing and excess anti-squeezing as well as mixed GKP states with an impact that increases with the quadrature value.

Finally, we comment on the scalability of the computation scheme. For the temporal encoding in Fig. 6.1, the number of modes in which GKP qubits can be encoded for computation, i.e. the size of the encoding plane in the 3D time lattice, depends on the $n m$-delay in the resource preparation. Increasing the delay length increases the number of encoding modes. However, doing so also increases the optical propagation loss, which puts a limit on the useful delay length. Thus, to continue scaling up, $\mathrm{nm}$ must be increased by shortening the temporal modes, in turn increasing the demands on the squeezing and detection bandwidth. In [35], squeezed light with a bandwidth of $2.5 \mathrm{THz}$ was demonstrated, limited by the phase-matching condition of the non-linear down conversion process, while in [36], detection of squeezing up to $3 \mathrm{THz}$ sideband frequency was demonstrated. Assuming proper squeezing, experimental control, and detection in a $2.5 \mathrm{THz}$ bandwidth defining temporal modes of $\sim 1 / 2.5 \mathrm{THz}$ duration, and assuming a propagation efficiency above 0.95 ( $0.23 \mathrm{~dB}$ attenuation) in a low-loss optical fiber with low optical attenuation of $0.15 \mathrm{~dB} / \mathrm{km}$, up to $n m \approx 10^{7}$ computation modes may be realized in the temporally encoded computation scheme. For the spatial architecture in Fig. 6.2, scalability is similar to other schemes based on spatial encoding. It relies on the availability of resources, and is suitable for integrated photonics [167]. Finally, temporal and spatial encoding may be combined: Consider multiple tem- 
porally encoded computational devices, each as in Fig. 6.1. Using the optical switch at the setup computational level, computation modes can be switched in and out between different devices. Since the setup is optical, the devices are simply connected by optical fibers between the switches of each device without the need of quantum transducers. Furthermore, with the switch being mode selective, each mode of an encoded logical qubit in the surface code can be transferred without the need of decoding and re-encoding the logical quantum state, while measurement of the surface code stabilizers after transfer may be used for error-correcting the transfer line. This is not only suitable for combining temporal and spatial encoding for up-scaling, but is also useful in a quantum internet scheme $[168,169]$, and is made possible by the optical architecture combined with temporal multiplexing on the transfer lines.

\subsection{Appendix: Gates by projective measurements}

To derive the gates implemented by projective measurements in section 6.3 , consider one computation step on two parallel wires,

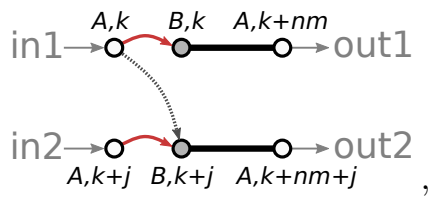

where the red arrows represent the first beam-splitter in the TDMD, and the gray arrow represents the blue or green VBS in the TDMD for $j=1$ or $j=n$, respectively. Here, a two-mode input state (separable or not) is encoded in modes $(A, k),(A, k+j)$, while the two-mode entangled states are prepared at the resource preparation stage, and can be written as

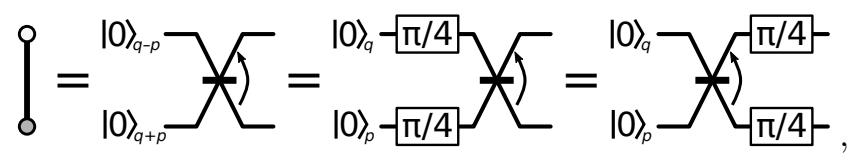

where $|0\rangle_{q \pm p}$ is a squeezed vacuum state, squeezed along the $(\hat{q} \pm \hat{p}) / \sqrt{2}$ quadrature, and similarly, $|0\rangle_{q}$ and $|0\rangle_{p}$ are squeezed along the $\hat{q}$ and $\hat{p}$, respectively. As such, the two-mode entangled states correspond to two-mode squeezed states rotated in phase-space by $\pi / 4$, turning them into approximate cluster states with tanh $2 r$ edge weight and $i \operatorname{sech} 2 r$ self-loops, where $r$ is the squeezing parameter of the initial squeezed vacuum states [7, 44]. Alternatively, we can consider the twomode entangled states more generally as cluster-type states [4], here with edge weight 1 , for which the implemented gate is independent on the squeezing, $r$, which then only affects the gate noise [33]. The two situations are equivalent: One can change from the former to the latter by normalizing the edge weight [10]. Here, we will consider cluster-type states, since implementing a desired gate in practice (without considering the resulting gate noise) then requires no prior knowledge of the squeezing level.

For single-mode gates the VBSs of the TDMD are left open, and the dashed arrow in Eq. (6.9) represents $\hat{I}_{A, k} \otimes \hat{I}_{B, k+j}$. In this case, we can ignore the second wire, and focus on a joint projective measurement of the input mode $(A, k)$ and one mode of the two-mode entangled state, $(B, k)$, resulting in gate teleportation to the output mode $(A, k+n m)$ - exactly the same derivation can be made on the second wire of mode $(A, k+j),(B, k+j)$, and $(B, k+n m+j)$. The corresponding circuit is

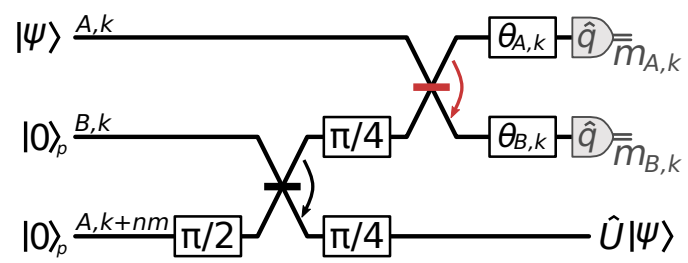


where $|0\rangle_{q}$ in $(A, k+n m)$ is replaced by $\hat{R}(\pi / 2)|0\rangle_{p}$ only to follow the traditional convention of cluster states with initial squeezing in $\hat{p}$ quadratures. Using the method in the appendix section 4.8 [32] with $\left(\theta_{A, k}, \theta_{B, k}\right)$ being the basis setting determining the implemented gate, the corresponding quadrature transformation in the Heisenberg picture can be derived to be

$$
\left(\begin{array}{c}
\hat{q}_{A, k+n m}^{\prime} \\
\hat{p}_{A, k+n m}^{\prime}
\end{array}\right)=\mathbf{G}\left(\begin{array}{c}
\hat{q}_{A, k} \\
\hat{p}_{A, k}
\end{array}\right)+\mathbf{N}\left(\begin{array}{c}
\hat{p}_{B, k} \\
\hat{p}_{A, k+n m}
\end{array}\right)+\mathbf{D}\left(\begin{array}{c}
m_{A, k} \\
m_{B, k}
\end{array}\right) .
$$

Here, $\mathbf{G}$ is the symplectic matrix corresponding to the desired single-mode gate operation in Eq. (6.2),

$$
\mathbf{N}=\left(\begin{array}{cc}
1 & 1 \\
1 & -1
\end{array}\right)
$$

is a gate noise matrix, and

$$
\mathbf{D}=\frac{\sqrt{2}}{\sin \left(2 \theta_{-}\right)}\left(\begin{array}{cc}
-\cos \theta_{B, k} & -\cos \theta_{A, k} \\
\sin \theta_{B, k} & \sin \theta_{A, k}
\end{array}\right)
$$

is a displacement matrix. Since $\left(\theta_{A, k}, \theta_{B, k}\right), m_{A, k}$ and $m_{B, k}$ are known, $\mathbf{D}\left(m_{A, k}, m_{B, k}\right)^{T}$ can be compensated for by displacing the teleported state back by $-\mathbf{D}\left(m_{A, k}, m_{B, k}\right)^{T}$, or simply by taking this displacement into account in the following measurement outcomes. With finite squeezing in the ancillary modes such that $\operatorname{Var}\left\{\hat{p}_{B, k}\right\}=\operatorname{Var}\left\{\hat{p}_{A, k+n m}\right\}=\sigma^{2}=e^{-2 r} / 2$, the noise term $\mathbf{N}\left(\hat{p}_{B, k}, \hat{p}_{A, k+n m}\right)^{T}$ leads to quadrature-symmetric gate noise in $\hat{q}_{A, k+n m}^{\prime}$ and $\hat{p}_{A, k+n m}^{\prime}$ of

$$
\sigma_{\text {gate }}^{2}=\operatorname{Var}\left\{\hat{p}_{B, k}\right\}+\operatorname{Var}\left\{\hat{p}_{A, k+n m}\right\}=e^{-2 r} .
$$

In the Wigner function picture, this gate noise corresponds to convolutions in both quadratures by a Gaussian function of variance $\sigma_{\text {gate }}^{2}$, each followed by the application of a corresponding Gaussian envelope due to the Fourier relation between $\hat{q}$ and $\hat{p}[10,32]$.

When implementing two-mode gates by enabling the first or second VBS of the TDMD, the corresponding circuit is

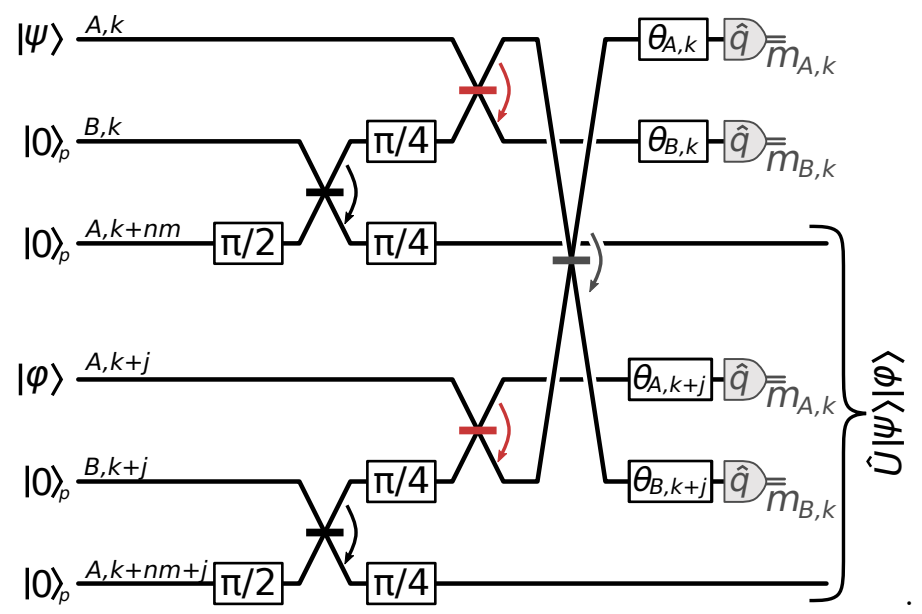

We do not derive a general expression for the implemented gate as a function of the basis setting $\left(\theta_{A, k}, \theta_{B, k}, \theta_{A, k+j}, \theta_{B, k+j}\right)$. Instead, we use the method introduced in chapter 4 [32]: A cost function is defined based on the implemented gate, a desired target gate, and the gate noise, which is then used in a global search to find the basis setting that implements a desired gate with the minimum gate noise. The resulting basis settings for the gates required to implement the surface code are shown in Table 6.1. We note that those settings only implement two different gates since $\left(\hat{F}^{\dagger} \otimes \hat{F}\right) \hat{C}_{Z}(g)=\hat{C}_{X}(g)\left(\hat{F}^{\dagger} \otimes \hat{F}\right)$ and $\hat{C}_{Z}(g)\left(\hat{F} \otimes \hat{F}^{\dagger}\right)=\hat{C}_{X}(g)\left(\hat{F}^{\dagger} \otimes \hat{F}\right)$. The reason for considering them as four different gates is to make the implementation of the surface code more intuitive. The 
basis settings are not unique: other settings exist that implement the same gates with equal gate noise.

The quadrature transformation when applying the basis settings for two-mode gates is

$$
\begin{aligned}
\left(\begin{array}{c}
\hat{q}_{A, k+n m}^{\prime} \\
\hat{q}_{A, k+n m+j}^{\prime} \\
\hat{p}_{A, k+n m}^{\prime} \\
\hat{p}_{A, k+n m+j}^{\prime}
\end{array}\right) & =\mathbf{G}\left(\begin{array}{c}
\hat{q}_{A, k} \\
\hat{q}_{A, k+j} \\
\hat{p}_{A, k} \\
\hat{p}_{A, k+j}
\end{array}\right) \\
+\mathbf{N} & \left(\begin{array}{c}
\hat{p}_{B, k} \\
\hat{p}_{A, k+n m} \\
\hat{p}_{B, k+j} \\
\hat{p}_{A, k+n m+j}
\end{array}\right)+\mathbf{D}\left(\begin{array}{c}
m_{A, k} \\
m_{B, k} \\
m_{A, k+j} \\
m_{B, k+j}
\end{array}\right) .
\end{aligned}
$$

Again, $\mathbf{G}$ is the symplectic matrix corresponding the implemented two-mode gate.

$\mathbf{D}\left(m_{A, k}, m_{B, k}, m_{A, k+j}, m_{B, k+j}\right)^{T}$ is a displacement in phase-space with

$$
\mathbf{D}=\left(\begin{array}{cccc}
-\sqrt{5} / 2 & 0 & 1 / \sqrt{2} & \sqrt{5} / 2 \\
-\sqrt{5} / 2 & -1 / \sqrt{2} & 0 & -\sqrt{5} / 2 \\
0 & -\sqrt{2} & 0 & 0 \\
0 & 0 & \sqrt{2} & 0
\end{array}\right)
$$

for $\left(\hat{F}^{\dagger} \otimes \hat{F}\right) \hat{C}_{Z}(g)$ and $\hat{C}_{X}(g)\left(\hat{F}^{\dagger} \otimes \hat{F}\right)$, and

$$
\mathbf{D}=\left(\begin{array}{cccc}
0 & -\sqrt{2} & 0 & 0 \\
0 & 0 & -\sqrt{2} & 0 \\
\sqrt{5} / 2 & 0 & -1 / \sqrt{2} & \sqrt{5} / 2 \\
\sqrt{5} / 2 & -1 / \sqrt{2} & 0 & -\sqrt{5} / 2
\end{array}\right)
$$

for $\hat{C}_{Z}(g)\left(\hat{F} \otimes \hat{F}^{\dagger}\right)$ and $\hat{C}_{X}(g)\left(\hat{F}^{\dagger} \otimes \hat{F}\right)$, both of which can be compensated for, just like for singlemode gates. $\mathbf{N}\left(\hat{p}_{B, k}, \hat{p}_{A, k+n m}, \hat{p}_{B, k+j}, \hat{p}_{A, k+n m+j}\right)^{T}$ represents gate noise where

$$
\mathbf{N}=\left(\begin{array}{cccc}
1 & 1 & 0 & 0 \\
0 & 0 & 1 & 1 \\
1 & -1 & 0 & 0 \\
0 & 0 & 1 & -1
\end{array}\right)
$$

leads to quadrature-symmetric gate noise of variance

$$
\begin{aligned}
\sigma_{\text {gate }}^{2} & =\operatorname{Var}\left\{\hat{p}_{B, k}\right\}+\operatorname{Var}\left\{\hat{p}_{A, k+n m}\right\} \\
& =\operatorname{Var}\left\{\hat{p}_{B, k+j}\right\}+\operatorname{Var}\left\{\hat{p}_{A, k+n m+j}\right\} \\
& =e^{-2 r}
\end{aligned}
$$

which conveniently equals the gate noise variance of single-mode gates.

\subsection{Appendix: Simulation}

To simulate the logic qubit error rate of the surface code, we adopted and modified the simulation in Ref. [121] to the computation scheme of this work. The simulation method is well-described in appendix B of Ref. [121] and is summarized here with focus on the modifications. In the simulation, quadrature noise is simulated as stochastic normally-distributed variables for each quadrature of each mode $i, \xi_{q}^{i}$ and $\xi_{p}^{i}$. For GKP-states, $\xi_{q}^{i}$ and $\xi_{p}^{i}$ are initialized with random samples from $\mathcal{N}\left(0, \sigma_{\mathrm{GKP}}\right)$, where $\mathcal{N}(0, \sigma)$ is a normal distribution of zero mean and $\sigma^{2}$ variance. After each gate, independent random samples from $\mathcal{N}\left(0, \sigma_{\text {gate }}\right)$ are added to $\xi_{q}^{i}$ and $\xi_{p}^{i}$ as gate noise. As for 
homodyne measurements, $\xi_{q}^{i}$ or $\xi_{p}^{i}$ is read out, and the logic value is determined from the closest integer multiple of $\sqrt{\pi}$. Note that, unlike in Ref. [121], we do not consider measure noise or idle noise. In optical platforms, homodyne measurements are carried out with near-unity efficiency (any loss is assumed to just degrade the squeezing as discussed in section 6.6). Furthermore, in MBQC no modes are idle since modes not performing any tasks still have to teleport through the computation step and thereby acquire gate noise instead of idle noise.

For the two-mode gates in the surface code, the simulation here differs from Ref. [121] by using $\hat{C}_{Z}(1)$ and $\hat{C}_{X}( \pm 1)$ gates instead of sum-gates. For a two-mode gate between modes $i$ and $j$, the quadrature noise variables are updated as

$$
\begin{aligned}
& \xi_{q}^{i} \leftarrow \xi_{q}^{i} \quad+\operatorname{randG}\left(\sigma_{\text {gate }}^{2}\right) \\
& \hat{C}_{Z}(1): \begin{array}{ll}
\xi_{q}^{i} \leftarrow \xi_{p}^{i}+\xi_{q}^{j} & +\operatorname{randG}\left(\sigma_{\text {gate }}^{2}\right) \\
\xi^{j} \leftarrow \xi^{j} & +\operatorname{randG}\left(\sigma^{2}\right)
\end{array} \\
& \xi_{q}^{j} \leftarrow \xi_{q}^{j} \quad+\operatorname{randG}\left(\sigma_{\text {gate }}^{2}\right) \\
& \xi_{q}^{j} \leftarrow \xi_{p}^{j}+\xi_{q}^{i}+\operatorname{randG}\left(\sigma_{\text {gate }}^{2}\right) \\
& \hat{C}_{X}( \pm 1): \begin{array}{ll}
\xi_{q}^{i} \leftarrow \xi_{q}^{i} \pm \xi_{p}^{j} & +\operatorname{randG}\left(\sigma_{\text {gate }}^{2}\right) \\
\xi_{q}^{i} \leftarrow \xi_{p}^{i} & +\operatorname{randG}\left(\sigma_{\text {gate }}^{2}\right) \\
\xi_{q}^{j} \leftarrow \xi_{q}^{j} \pm \xi_{p}^{i} & +\operatorname{randG}\left(\sigma_{\text {gate }}^{2}\right) \\
\xi_{q}^{j} \leftarrow \xi_{p}^{j} & +\operatorname{randG}\left(\sigma_{\text {gate }}^{2}\right)
\end{array}
\end{aligned}
$$

where randG $\left(\sigma^{2}\right)$ returns a random value from $\mathcal{N}(0, \sigma)$.

For GKP quadrature correction, instead of coupling to ancillary GKP qubits through sum gates as in Ref. [121], the mode to be corrected is teleported through a two-mode GKP qubit Bell state as described in section 6.4. The Bell state is prepared by interfering two GKP qunaught states, denoted $\varnothing 1$ and $\varnothing 2$,

$$
\begin{aligned}
& \xi_{q}^{\varnothing 1}=\operatorname{randG}\left(\sigma_{\mathrm{GKP}}^{2}\right) \quad \xi_{q}^{\varnothing 2}=\operatorname{randG}\left(\sigma_{\mathrm{GKP}}^{2}\right) \\
& \xi_{p}^{\varnothing 1}=\operatorname{randG}\left(\sigma_{\mathrm{GKP}}^{2}\right) \quad, \quad \xi_{p}^{\varnothing 2}=\operatorname{randG}\left(\sigma_{\mathrm{GKP}}^{2}\right)
\end{aligned}
$$

on a beam-splitter,

$$
\begin{aligned}
& \xi_{q}^{\varnothing 1} \leftarrow\left(\xi_{q}^{\varnothing 1}-\xi_{q}^{\varnothing 2}\right) / \sqrt{2} \\
& \xi_{p}^{\varnothing 1} \leftarrow\left(\xi_{p}^{\varnothing 1}-\xi_{p}^{\varnothing 2}\right) / \sqrt{2} \\
& \xi_{q}^{\varnothing 2} \leftarrow\left(\xi_{q}^{\varnothing 1}+\xi_{q}^{\varnothing 2}\right) / \sqrt{2} \\
& \xi_{p}^{\varnothing 2} \leftarrow\left(\xi_{p}^{\varnothing 1}+\xi_{p}^{\varnothing 2}\right) / \sqrt{2} .
\end{aligned}
$$

To teleport, the mode to be corrected, $i$, and $\varnothing 1$ are interfered on a beam-splitter and measured in $\hat{q}$ and $\hat{p}$, respectively, with outcomes

$$
m_{A}=\left(\xi_{q}^{i}-\xi_{q}^{\varnothing 1}\right) / \sqrt{2} \quad, \quad m_{B}=\left(\xi_{p}^{i}+\xi_{p}^{\varnothing 1}\right) / \sqrt{2} .
$$

Finally, to compensate for the Pauli by-products of the qubit teleportation (displacements by $\sqrt{\pi}$ ), $m_{A} \sqrt{2}$ and $m_{B} \sqrt{2}$ are rounded to the nearest integer multiple of $\sqrt{\pi}$,

$$
\mathcal{P}\left(m_{A(B)} \sqrt{2}\right)=\sqrt{\pi}\left\lfloor\frac{m_{A(B)} \sqrt{2}}{\sqrt{\pi}}+\frac{1}{2}\right\rfloor,
$$

which is then used to displace the teleportation output mode, $\varnothing 2$, back,

$$
\begin{aligned}
& \xi_{q}^{\varnothing 2} \leftarrow \xi_{q}^{\varnothing 2}+\mathcal{P}\left(m_{A} \sqrt{2}\right) \\
& \xi_{p}^{\varnothing 2} \leftarrow \xi_{p}^{\varnothing 2}+\mathcal{P}\left(m_{B} \sqrt{2}\right) .
\end{aligned}
$$

For the sake of simulation, we pass the corrected output mode to the input mode, $\xi_{q}^{i} \leftarrow \xi_{q}^{\varnothing 2}$ and $\xi_{p}^{i} \leftarrow \xi_{p}^{\varnothing 2}$, such that mode $i$ can be reused in the following simulation. The probability of having induced a qubit error by rounding to a wrong integer of $\sqrt{\pi}$ in Eq. (6.10) due to input 


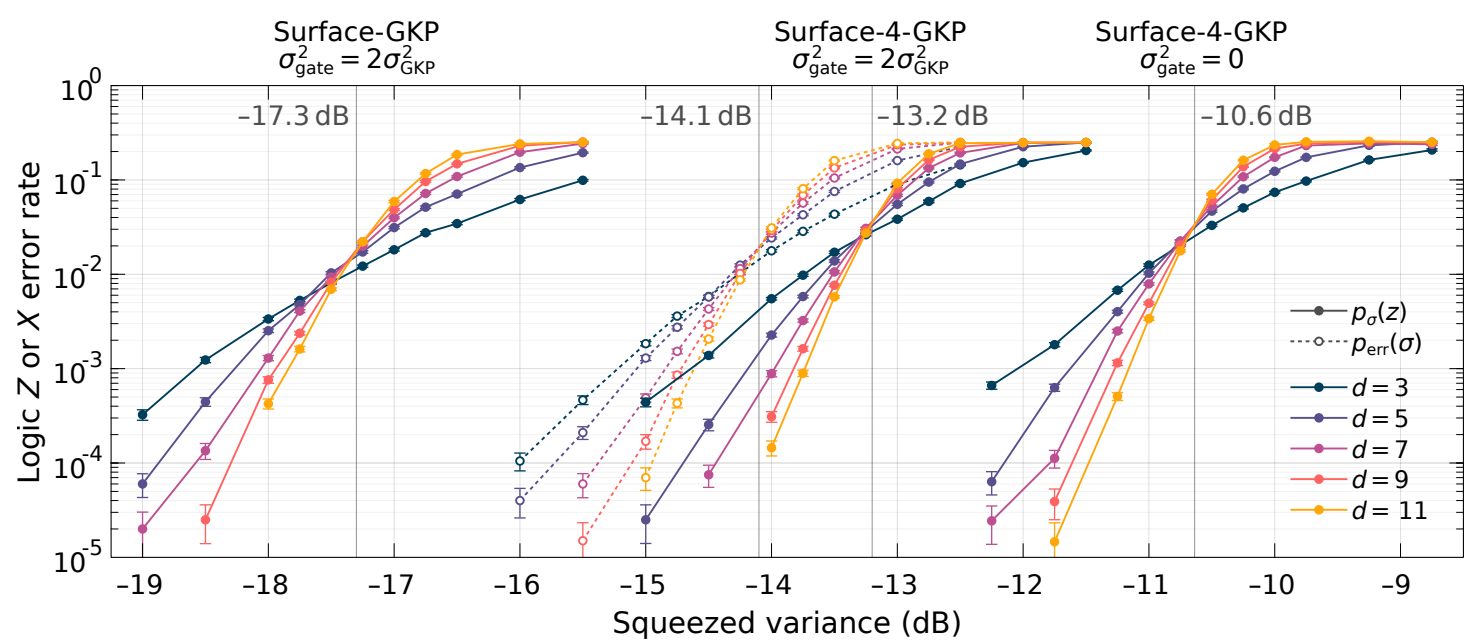

Figure 6.6: Simulation results of all four simulated cases. Here, the case of the surface-4-GKP code using $p_{\sigma}(z)$ is the results shown in the main text Fig. 6.5. Error bars of standard deviation are estimated by bootstrapping.

noise in $\xi_{q}^{i}$ and $\xi_{p}^{i}$, together with initialization noise of $\xi_{q}^{\varnothing 1}, \xi_{p}^{\varnothing 1}, \xi_{q}^{\varnothing 2}$, and $\xi_{p}^{\varnothing 2}$, is inferred using the residual analogue information, $\mathcal{R}\left(m_{A(B)} \sqrt{2}\right)=m_{A(B)} \sqrt{2}-\mathcal{P}\left(m_{A(B)} \sqrt{2}\right)$, in Eq. (6.7) through the probability in Eq. (6.6). Finally, it is used for weighting the spacetime graphs of stabilizer measurement outcomes for the MWPM decoding. Here, $\sigma_{\text {in }}^{2}$ in $\sigma^{2}=\sigma_{\text {in }}^{2}+\sigma_{\text {GKP }}^{2}$ of Eq. (6.6) is the quadrature variance of the input mode, and is carefully kept track of in the simulation based on previous gates and corrections.

In one simulation, $d+1$ rounds of surface code stabilizer measurements are carried out. Data GKP qubits are initialized in round 1 with $\sigma_{\text {GKP }}^{2}$ variance. To stabilize the data qubits, measure GKP qubits and qunaught states are initialized in round 1 to $d$ with $\sigma_{\text {GKP }}^{2}$ variance, followed by noisy gates and measurements to build up the spacetime graphs. In the last round, $d+1$, measure GKP qubits and qunaught states are initialized with zero variance to carry out ideal syndrome measurements for determining logic qubit errors induced in round 1 to $d$. To build up statistics, for each squeezing level and code distance, $d$, this process is repeated 100000 times, or until a total of $500 \operatorname{logic} \hat{X}$ and $\hat{Z}$ errors are detected.

We have simulated four different cases, shown in Fig. 6.6. In three cases, GKP states (qubits and qunaught states) and squeezed vacuum states are initialized with equal variance, $\sigma_{\mathrm{GKP}}^{2}=$ $\sigma^{2}=e^{-2 r} / 2$, which from Eq. (6.3) and (6.4) leads to $\sigma_{\text {gate }}^{2}=2 \sigma_{\mathrm{GKP}}^{2}$. In this way, the surface-GKP code with GKP quadrature correction before the surface code stabilizer measurements, and the surface-4-GKP code with four GKP quadrature corrections during the stabilizer measurements, was simulated. To see the impact of using the analogue information from the GKP correction in the weighting of the spacetime graphs, the surface-4-GKP code was simulated using

$$
p_{\mathrm{err}}(\sigma)=\sum_{n \in \mathbb{Z}} \frac{1}{\sqrt{2 \pi \sigma^{2}}} \int_{\left(2 n+\frac{1}{2}\right) \sqrt{\pi}}^{\left(2 n+\frac{3}{2}\right) \sqrt{\pi}} d \xi e^{-\xi^{2} /\left(2 \sigma^{2}\right)}
$$

instead of Eq. (6.6) [121]. By integrating the wave function marginal distribution in the odd GKP bins, $p_{\text {err }}(\sigma)$ infers the qubit error probability only based on variances without taking the projective measurement outcome into account. Finally, to compare with other MBQC schemes supporting topological error correction, but only taking noise from GKP-states into account, the surface-4-GKP code is simulated using $\sigma_{\text {gate }}^{2}=0$. 


\section{Chapter 7}

\section{Conclusion}

In this thesis, work further towards the realization of continuous variable (CV) measurementbased quantum computation (MBQC) in Ref. [30-34] was presented. This includes experimental work on cluster state generation, gate implementation, and temporal multiplexing, as well as theoretical work on noise performance analysis, error correctable computation schemes, and faulttolerant computation. In chapter 3, the generation of a two-dimensional (2D) cluster state was realized, while a computation scheme on this cluster state was presented in chapter 4 . In chapter 5 , the implementation of a universal Gaussian gate set on the generated cluster state was then presented. Both of these experimental demonstrations operates at the telecom wavelength, and take advantage of fiber optics utilizing the developed techniques in chapter 2 . The generated cluster state and implemented gates are noisy due to finite squeezed and experimental imperfections as optical inefficiencies and phase fluctuations. In chapter 4, a noise analysis was carried out of the demonstrated computation scheme together with other proposed schemes [9, 11, 37]. From this analysis, it was concluded that certain architectures are preferable for most efficiently utilizing the available squeezing, and the requirements for error correction was discussed. Based on this, in chapter 6 a new computation scheme was presented that allows error correction, and the scheme was shown to be fault-tolerant for a certain amount of squeezing. Below, the work of each chapter 2-6 are summarized and concluded individually.

In chapter 2 [30], an optical switch was used for temporally multiplexing a single squeezing source to prepare two-mode entangled states in two spatial modes. Although this is not directly relevant to the following experimental work of this thesis, experimental techniques were developed including optical delay, efficient in-fiber phase control, and fiber-based homodyne detection, each of which has been applied in chapter 3 and 5. Furthermore, in fully functional MBQC architectures, optical switching plays an important role in switching different quantum states in and out of a computation, routing between different computations, and connecting to different detectors $[5,12$, 46, 60, 170].

In chapter 3 [31], a 2D cluster state generation scheme was proposed and demonstrated. This work was done in parallel with a similar work by Asavanant et al. demonstrating a 2D cluster state [37]. A cluster state of two dimensions is the minimum number of dimensions required for universal computation: One dimension for encoding a multi-mode input state in multiple cluster state nodes, and another dimension for implementing gates by projective measurements. As such, this work opens for the implementation of a programmable multi-mode quantum circuit. In the validation of the generated cluster state using the van Loock-Furusawa separability criteria [103], linear combinations of nullifiers are demonstrated to successfully bring down the required squeezing threshold to $3 \mathrm{~dB}$ for validating genuine inseparability of the cluster state. This improvement is to be compared with a squeezing threshold of $6 \mathrm{~dB}$ when following previous applications of the van Loock-Furusawa separability criteria [19, 37] without considering linear combinations of nullifiers.

In chapter 4 [32], a computation scheme was proposed for the cluster state demonstrated in chapter 3, and noise analysis was carried out on this scheme as well as on other computation schemes on $2 \mathrm{D}$ cluster states, namely the cluster state demonstrated by Asavanent et al. [37], the 
bilayer-square lattice (BSL) in Ref. [11], and the quad-rail lattice (QRL) in Ref. [9]. All of the considered schemes allow for universal computation when combined with the Gottesman-KitaevPreskill (GKP) encoded qubits [14]. The three cluster states of chapter 3, by Asavanant et al., and the BSL, are all shown to have similar noise performance. This is despite the fact that the cluster state generation scheme in chapter 3 is experimentally simpler comprising only two squeezing sources and three interfering beam-splitters. The QRL cluster state is shown to be optimal due to a simpler entanglement structure in the logical level (or in between the corresponding macronodes in the language of Ref. $[9,11])$.

In chapter 5 [33], following the computation scheme proposed in chapter 4, a multi-mode Gaussian gate set was implemented on the generated $2 \mathrm{D}$ cluster state by projective measurements. Besides implementing a multi-mode gate set, in chapter 5 different gates are combined in a simple quantum circuit to demonstrate the programmability of the scheme. The implemented gates and circuit are characterized by multi-mode gate tomography. This was done by transmitting single modes of two-mode entangled states through the gates/circuit and measuring the quadrature correlations of the two-mode entangled states after applying the gates/circuit. Due to finite squeezing in the cluster state generation, the implemented gates are noisy. To characterize the gate performance, gate noise, comprising Gaussian noise added in the quadratures, is derived from the gate tomography. Although the resulting gate noise is large, the gate noise is shown to agree well with the available squeezing, and it was discussed how the gate noise can be made arbitrarily small by improving optical efficiencies, phase fluctuations, and squeezing bandwidth.

Finally, in chapter 6 [34], a computation architecture was proposed, supporting error correction to combat noise due to finite squeezing. Based on the analysis in chapter 4 , the proposed scheme is designed with a simple cluster state structure that matches the noise performance of the QRL. With GKP states as inputs, the scheme supports the GKP quadrature correction scheme recently proposed in Ref. [42], preventing active on-line coupling to ancillary GKP qubits. To correct GKP qubit errors, gates are implemented on a three-dimensional cluster state supporting topological qubit error correction. By combining GKP quadrature correction with the topological surface code, the scheme is simulated to be fault-tolerant given $13.2 \mathrm{~dB}$ of squeezing in both the cluster state generation and the supplied GKP states, with room left for further improvements.

\subsection{Outlook}

In recent years, multiple simplifications have appeared to ease the realization of universal and fault-tolerant MBQC in the CV regime. These simplifications include cluster state generation by linear optics and off-line squeezing [7], improved squeezing thresholds using the residual analog information from GKP quadrature correction [43, 144], GKP magic state distillation, and universal computation with Gaussian gates rendering non-Gaussian gates unnecessary [39, 40], etc. However, for universal fault-tolerant MBQC in the CV regime, important challenges are still to be tackled. Most obvious is the preparation and preservation of high quality squeezing to minimize CV noise, as well as the encoding of a qubit (or another discrete level system) into the infinite-dimensional Hilbert space of bosonic modes to allow correction of CV noise. For the latter, GKP-states has shown advantages [109, 112, 113, 140]. While GKP-states have been prepared in ion-traps [101] and in the micro-wave regime [100], generation of GKP-states on an optical platform is still an experimental challenge although proposals exist [112, 128-130, 154-156]. For high-quality squeezing, as of today, squeezing of $15 \mathrm{~dB}$ has been demonstrated [115], while maintaining similar squeezing quality in a cluster state generation and computation setup is a matter of experimental improvements. Below, few other, possibly less challenging, problems that deserve attention are discussed.

Throughout the work presented in this thesis, temporal encoding is considered by time multiplexing of the spatial resources. This has the advantage of keeping the number of required spatial resources at a manageable level. In temporal encoded computation schemes, scalability is achieved by adjusting the delay lengths. However, due to propagation losses, an upper bound on the maximum delay length exist, and to keep scaling up, the temporal mode duration is reduced as discussed in section 6.6. Doing so requires increasing the squeezing bandwidth, which is an active 
research topic with a $2.5 \mathrm{THz}$ squeezing bandwidth recently demonstrated in Ref. [35], limited only by phase-matching in the parametric down-conversion process. However, experimental control of increasing bandwidth is required as well. The short temporal modes need to be detectable, and towards achieving this, there have been recent demonstrations on the detection of squeezed light in the tera-hertz regime using an optical parametric amplifier [36]. Additionally, phase control of local oscillators, as well as other active components, is required to operate at an even larger bandwidth to change settings in between temporal modes. In the proof-of-principle work in chapter 5, basis settings are changed within $8 \mathrm{~ns}$ between temporal modes, while in Ref. [25], Asavanant et al. managed to change basis settings within $2 \mathrm{~ns}$. However, to properly utilize tera-hertz squeezing bandwidths, the ability to change computation settings within pico-seconds is to be developed.

An alternative to all-temporal encoding is the frequency and spatial encoding. In spatial encoding, information is encoded in space while gates are typically implemented in time as proposed in [46]. Such realization scales similar to other spatially encoded platforms including superconducting qubits and ion-traps, each based on coherent dynamics instead of gates implemented by projective measurements. In frequency encoded platforms, information is encoded in frequency modes, while gates can be implemented in the frequency or time domain $[8,11,44,171,172]$. Similar to all-temporal encoded schemes, scalability in frequency encoding is as well limited by the squeezing bandwidth. 2D cluster states and implementation of programmable gates are still to be demonstrated in spatial and frequency encoded schemes.

For optical switching of quantum states of light in and out of an optical computation device, an efficient and fast optical switch is to be developed. In chapter 2, optical switching was demonstrated with a switching time of $\sim 50 \mathrm{~ns}$ and $17 \%$ optical transmission loss. To achieve fault-tolerant computation $17 \%$ loss is unacceptable, and fast optical switches with high transmission efficiency are required. In Ref. [114] optical switching of $<7 \%$ loss and $10 \mathrm{~ns}$ switching time was realized with polarization control and a polarizing beam-splitter (PBS), and similar performance can be expected using phase control in an interferometer. In such settings, not just on/off-switching is realized, but a variable beam-splitter is realized by the continuous polarization or phase control. Thus, on the experimental side of the work presented in this thesis, a straightforward next step is to implement such variable beam-splitter in the cluster state generation and gate implementation setup of chapter 3 and 5 , both for switching input states into the computation, but also to work towards architectures with active on-line optical control as in the proposed schemes of chapter 6 and Ref. [46].

Another requirement to experimental control is the ability to feed measurement results forward in the computation and use them to implement gates conditioned on the measurement results. We may consider two kinds of feedforward: feedforward to implement conditioned displacement gates, and feedforward to implement other conditioned operations. For circuits implemented with all-Gaussian gates (remember, non-Clifford gates can be implemented using GKP magic states and Gaussian gates [39-41]), conditioned displacement can simply be accounted for in the final homodyne measurement outcomes, since displacements continue to be displacements when propoagated through Gaussian gates (although with different displacement coefficients). As a result, active feedforward is not required for conditioned displacement gates. This includes conditioned displacements in GKP quadrature correction, compensation of by-product displacements when implementing gates by projective measurements, and implementing Pauli operations on encoded GKP qubits. For feedforward to implement other kinds of conditioned operations, active feedforward is required when the conditioned operations cannot simply be taken into account in the measurement results. An important example hereof is the case of non-Clifford gates implemented using magic states. For instance, the qubit $\pi / 8$-phase-gate implemented by gate teleportation using a GKP qubit Hadamard eigenstate requires a conditioned shear-gate [14]. As a result, in this case, active feedforward is required to adjust the basis settings of some following projective measurements to implement the desired shear-gate based on previous measurement outcomes. The gates implemented by projective measurements in chapter 5 are without active feedforward, while for universal computation active feedforward becomes necessary, either if implementing non-Gaussian gates [5], or if implementing non-Clifford GKP-qubit gates using magic states. Thus, future work 
can be the implementation of active feedforward on the setup presented in chapter 5 and requires fast processing of the measurement outcomes and feedforward to dynamic phase control of the local oscillators for homodyne detectors. While feedforward is applied in the field of quantum sensing using fast processing with a field-programmable gate array (FPGA) [173-175], active feedforward is still to be demonstrated in the settings of CV MBQC.

Finally, besides experimental improvements, it is important to keep developing the theoretical side to simplify the experimental requirements. Examples hereof are the work by Baragiola et al. by wich non-Gaussian gates has become unnecessary for universal computation on GKP encoded qubits [39], and the work by Walshe et al. by witch active on-line coupling to ancilla GKP qubits through sum-gates can be avoided in GKP quadrature correction [42]. A next step in the theoretical work of this thesis would be to improve the squeezing threshold for fault-tolerant computation in the architecture presented in chapter 6. As discussed in section 6.6, few improvements may be considered. This includes optimizing the decoding applied in the topological surface error correction code, while gate noise may be reduced if it is possible to combine two-mode gates and GKP quadrature correction in a single computation step as similar to single-mode gates in Ref. [42]. 


\section{Appendix A}

\section{Optical table}

The optical table with the experimental setup of $2 \mathrm{D}$ cluster state generation and gate implementation in chapter 3 and 5 is shown in Fig. A.2. Feedback and control electronics for the setup are shown in Fig. A.1.

For demodulation and PI-controllers, the setup includes 9 RedPitayas (STEMlab 125-14) (RP) FPGA boards as shown on Fig. A.1. Here the RPs of RP rack 1 are synchronized by using the crystal oscillator of RP3 for RP5 and RP13 as well. This is in order to use the modulation of RP3, driving the free-space EOM, to demodulate in RP5 and RP13 for the OPO cavity Pound-DreverHall lock. Similar, in RP rack 2 the RPs are synchronized by using crystal oscillator of RP9 for RP4, RP6, and RP11 as well. In this way, the modulation from the parametric gain lock (Probe-1 pzt. and Probe-2 pzt.) can be used for demodulation in a AC phase locks in RP4, RP6, and RP11 as well. This is used for the homodyne detector as described in appendix B.

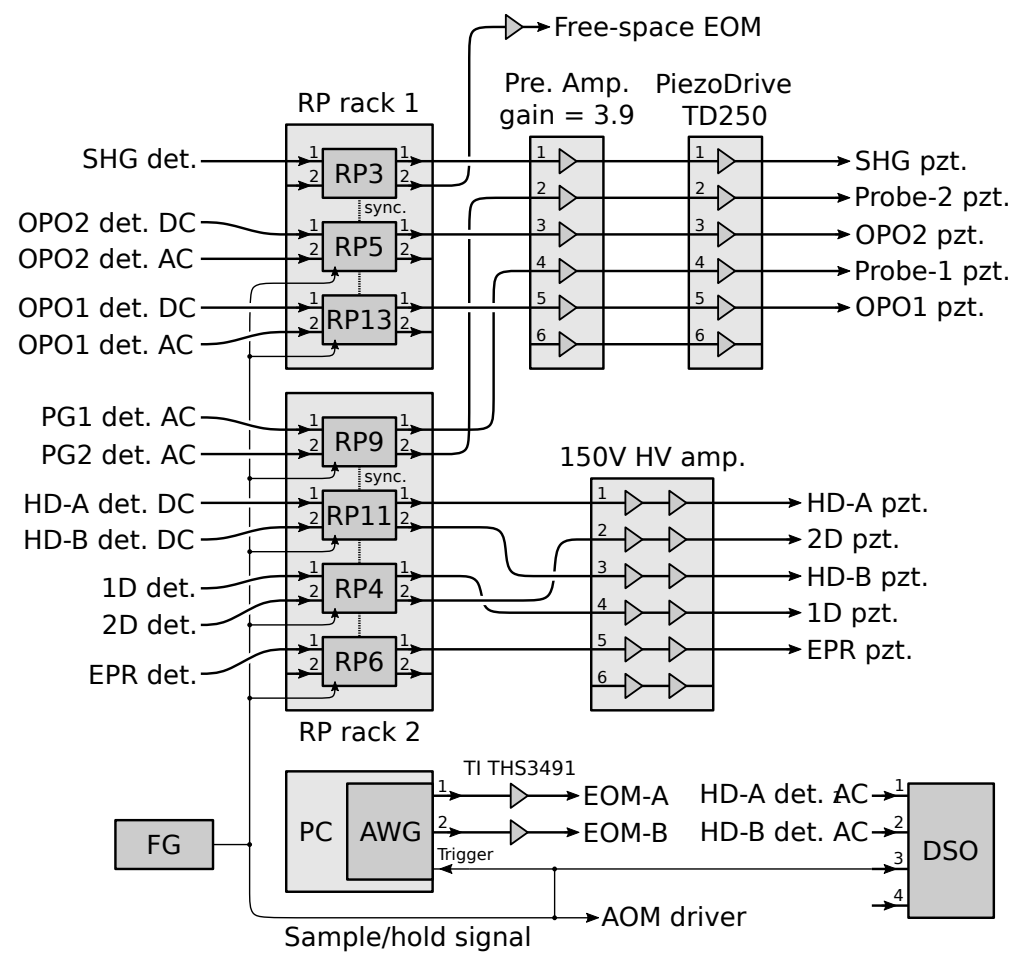

Figure A.1: Feedback and control electronics of the experimental setup in Fig. A.2. Abbreviations: RedPitaya (RP); function generator (FG); computer (PC); arbitrary waveform generator (AWG); digital storage digital storage oscilloscope (DSO). Inputs and outputs are shown in Fig. A.2. 


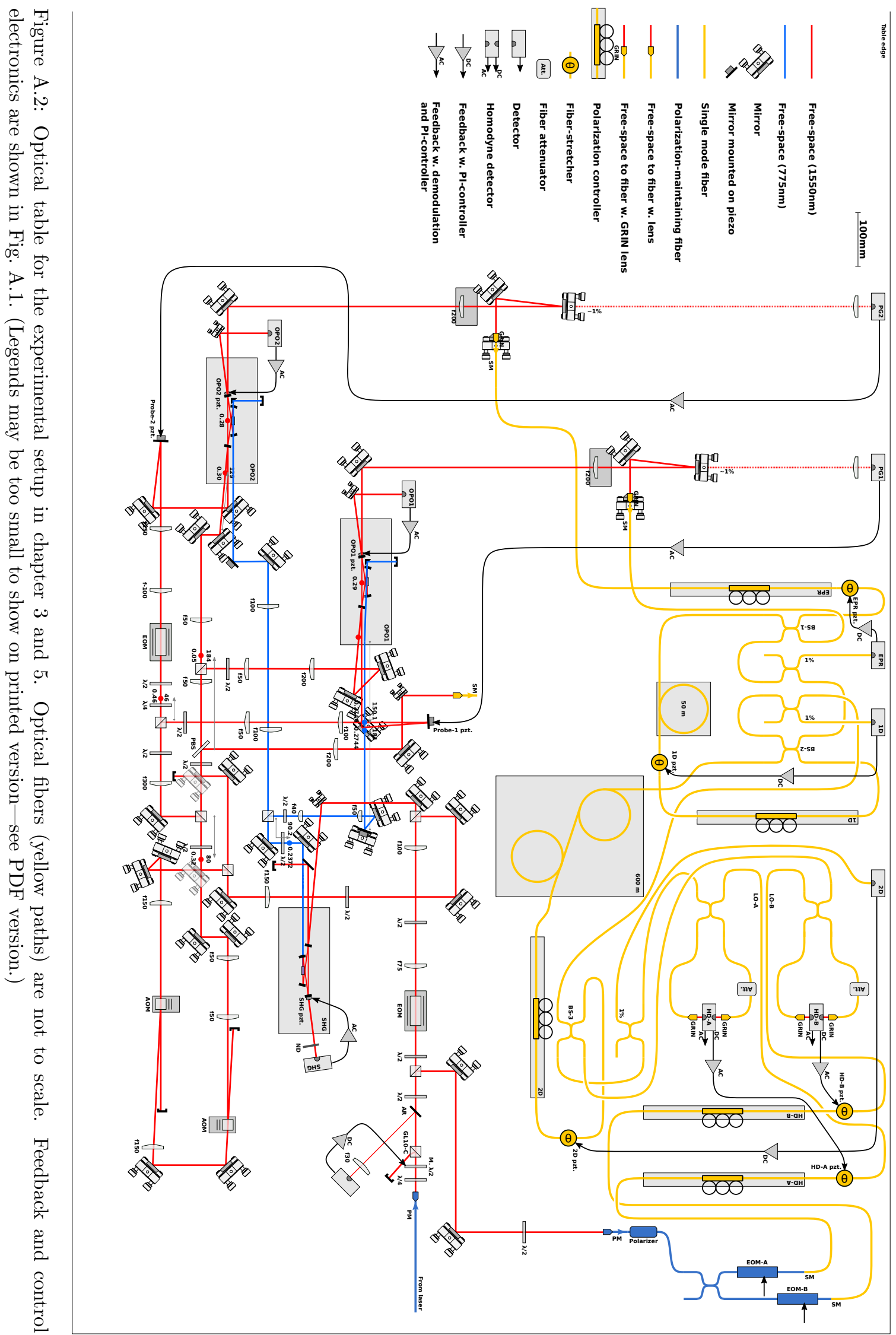




\section{Appendix B}

\section{Phase locking in 2D cluster state generation}

In chapter 3 , a $2 \mathrm{D}$ cluster state is generated by interfering two spatial modes three times with optical delays in between. For each point of interference, the relative phase on the balanced beamsplitters are controlled using phase locking. In this appendix the required configuration of each phase lock is derived.

Part of the experimental setup for 2D cluster state generation is shown in Fig. B.1. The input to the setup is squeezed vacuum states, squeezed along the $\hat{x}$-quadrature, but for phase locking, a coherent seed beam is injected into each of the two inputs during the locking period of a sample/hold locking scheme as described in section 3.3. From the parametric gain lock of the two squeezing sources, the seed beams are modulated with frequencies $f_{A}$ and $f_{B}$ in spatial modes $A$ and $B$. This modulation is shown in Fig. B.1 as arrows on the seed beams in phase-space. In general, frequencies less than $100 \mathrm{kHz}$ is used for modulation (in chapter $3 f_{A}=90 \mathrm{kHz}$ and $f_{B}=55 \mathrm{kHz}$ was used). For such modulation frequencies, in the following we can ignore the effect of the two optical delays of $50 \mathrm{~m}$ and $600 \mathrm{~m}$, corresponding to $250 \mathrm{~ns}$ and $3 \mu \mathrm{s}$, on the modulated coherent seed beams.

To start with, we review the effect of a $\pi / 2$ phase rotation and the balanced beam-splitter in phase-space. We use the same definitions as in chapter 3-6 (in chapter 2 slightly different definitions was used). The $\pi / 2$ phase rotation transforms the quadratures as

$$
\left(\begin{array}{l}
\hat{x} \\
\hat{p}
\end{array}\right) \stackrel{\leftrightarrow}{\longrightarrow} \cdot\left(\begin{array}{l}
\hat{x}^{\prime} \\
\hat{p}^{\prime}
\end{array}\right)=\left(\begin{array}{cc}
0 & 1 \\
-1 & 0
\end{array}\right)\left(\begin{array}{l}
\hat{x} \\
\hat{p}
\end{array}\right)=\left(\begin{array}{c}
\hat{p} \\
-\hat{x}
\end{array}\right) .
$$

Here the quadrature transformation is shown in phase-space as well, which will become useful later.

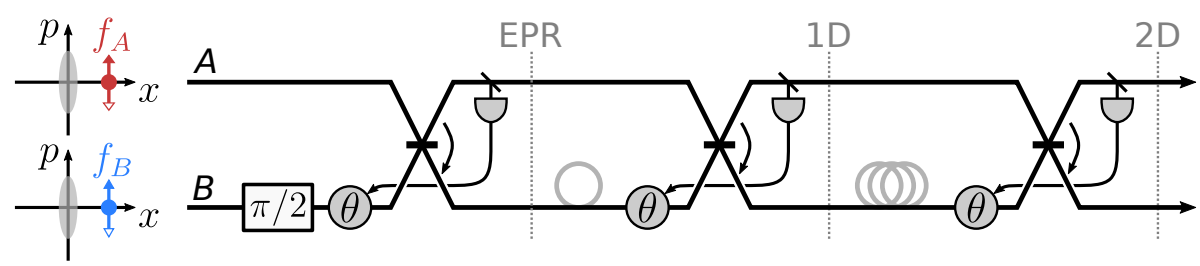

Figure B.1: Schematics of the $\pi / 2$ phase rotation and three beam-splitters in the $2 \mathrm{D}$ cluster state generation. In the experimental implementation, the beam-splitters are implemented with 50:50 fiber-couplers. For more details on the setup, see section 3.3. For the used modulation frequencies, $f_{A}$ and $f_{B}$, the $50 \mathrm{~m}$ and $600 \mathrm{~m}$ delay can be ignored in this appendix. 
The balanced beam-splitter transforms the quadratures as

$$
\left(\begin{array}{l}
\hat{x}_{1} \\
\hat{x}_{2} \\
\hat{p}_{1} \\
\hat{p}_{2}
\end{array}\right) \longrightarrow \longrightarrow\left(\begin{array}{l}
\hat{x}_{1}^{\prime} \\
\hat{x}_{2}^{\prime} \\
\hat{p}_{1}^{\prime} \\
\hat{p}_{2}^{\prime}
\end{array}\right)=\frac{1}{\sqrt{2}}\left(\begin{array}{cccc}
1 & -1 & 0 & 0 \\
1 & 1 & 0 & 0 \\
0 & 0 & 1 & -1 \\
0 & 0 & 1 & 1
\end{array}\right)\left(\begin{array}{l}
\hat{x}_{1} \\
\hat{x}_{2} \\
\hat{p}_{1} \\
\hat{p}_{2}
\end{array}\right)=\left(\begin{array}{l}
\hat{x}_{1}-\hat{x}_{2} \\
\hat{x}_{1}+\hat{x}_{2} \\
\hat{p}_{1}-\hat{p}_{2} \\
\hat{p}_{1}+\hat{p}_{2}
\end{array}\right) .
$$

We start with the first phase-lock used to prepare two-mode squeezed states (or EPR-states):

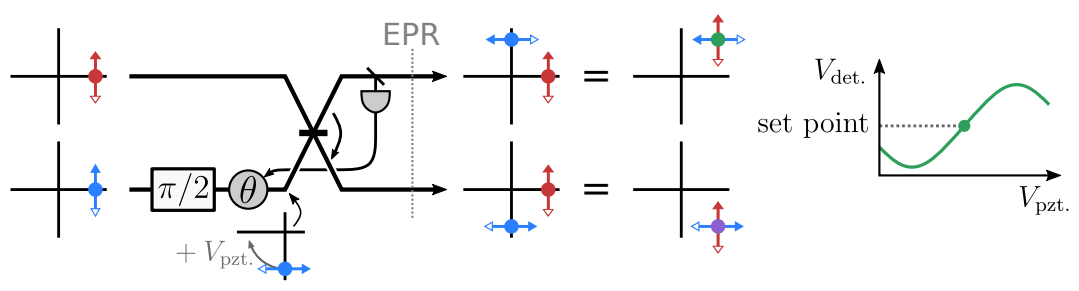

From the squeezing sources, the seed beams are located along the amplitude quadratures, $\hat{x}$, and modulated in the phase quadratures, $\hat{p}$, as shown above (this is done by locking the parametric gain in the squeezing sources to de-amplification of the seed beams). After the $\pi / 2$ phase rotation using Eq. (B.1), the seed in spatial mode $B$ is along the $-\hat{p}$-quadrature. Using Eq. (B.2), after the beam-splitter the two seed beams are interfered as shown above to form seed beams in spatial mode $A$ and $B$ along $\hat{x}+\hat{p}$ and $\hat{x}-\hat{p}$, respectively. Using a tapping mirror, the interference is detected in spatial mode $A$ and fed back to a phase-controller, $\theta$, in spatial mode $B$ before the beam-splitter. The phase-controller consist of a fiber-stretcher driven by a piezoelectric actuator. Here we will define, that when increasing the voltage to the piezoelectric actuator, $V_{\text {pzt. }}$, it will cause a delay leading to the seed beam rotating clockwise in phase-space. In this case the detected signal, $V_{\text {det., }}$ will increase with increasing $V_{\text {pzt. }}$ around the set point as shown above to the right. To stabilize the phase, $V_{\text {det }}$ is fed back through a PI-controller as

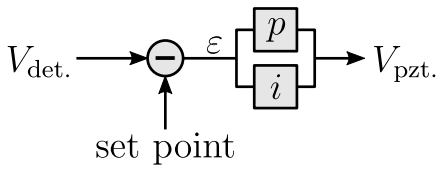

If $V_{\text {det. }}$ is above the set point, then the error signal, $\varepsilon=V_{\text {det. }}-$ [set point], is positive and $V_{\text {pzt. }}$. should be decreased. This is done using negative proportional and integration gain in the PIcontroller, $p, i<0$. To summarize, the first phase lock for EPR-state generation is locked using a DC lock with a set point set to the middle of an interference fringe, and using negative $p$ - and $i$-gain to lock on the positive slope.

The phase lock for generating a $1 \mathrm{D}$ cluster state (or $\mathcal{H}$-graph state) is similar to the first phase lock, but without $\pi / 2$ phase rotation:

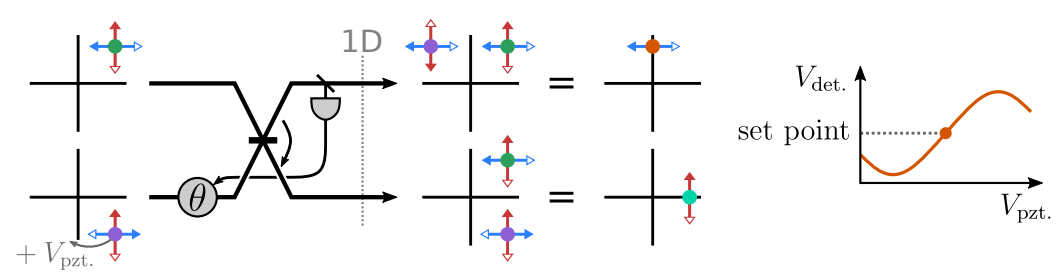

Using Eq. (B.2), after the beam-splitter the seed beams are interfered to be along $\hat{p}$ and $\hat{x}$ in spatial mode $A$ and $B$, respectively. Following the same arguments as for the first phase lock, when $V_{\text {pzt. }}$ is increased, the detected tapped off signal, $V_{\text {det. }}$, increases. To stabilize the phase, a PI-controller is used and, similar to the first phase-lock, the set point is the middle of an interference fringe while negative $p$ - and $i$-gains should be used to lock on the positive slope. Note how the mixed 
modulation frequencies cancels after the phase lock for 1D cluster state generation. This is expected since two beam-splitter operations of Eq. (B.1) becomes

$$
\frac{1}{\sqrt{2}}\left(\begin{array}{cccc}
1 & -1 & 0 & 0 \\
1 & 1 & 0 & 0 \\
0 & 0 & 1 & -1 \\
0 & 0 & 1 & 1
\end{array}\right) \frac{1}{\sqrt{2}}\left(\begin{array}{cccc}
1 & -1 & 0 & 0 \\
1 & 1 & 0 & 0 \\
0 & 0 & 1 & -1 \\
0 & 0 & 1 & 1
\end{array}\right)=\left(\begin{array}{cccc}
0 & -1 & 0 & 0 \\
1 & 0 & 0 & 0 \\
0 & 0 & 0 & -1 \\
0 & 0 & 1 & 0
\end{array}\right),
$$

and the input quadratures are no longer mixed.

Finally, we consider the last phase lock for generating the coiled-up 2D cluster state (or $\mathcal{H}$-graph state):

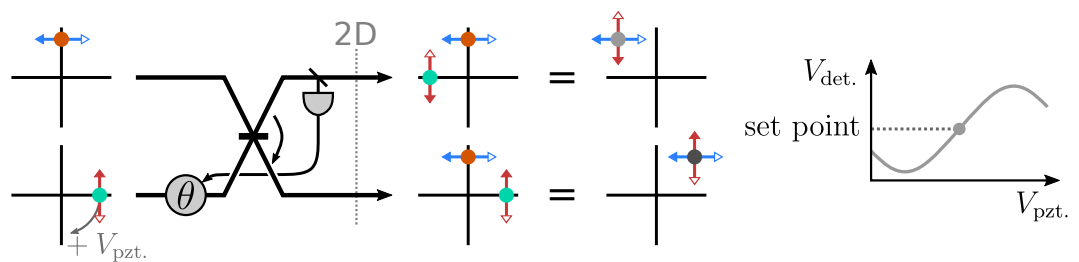

After applying Eq. (B.2) the seed beams are interfered to be along $-\hat{x}+\hat{p}$ and $\hat{x}+\hat{p}$ in spatial mode $A$ and $B$, respectively. Again, increasing $V_{\text {pzt. }}$ leads to an increased detected interference signal, $V_{\text {det. }}$. Thus, again, the phase is locked to the middle of an interference fringe with negative $p$ - and $i$-gain.

Note how the modulation is mixed after the last phase lock. Both seed beams in spatial mode $A$ and $B$ are modulated in $\hat{x}$-quadrature by $f_{B}$ and in $\hat{p}$-quadrature by $f_{A}$. This is convenient for AC-locking in the the following homodyne detection: To measure the $\hat{x}(\hat{p})$ quadratures of spatial modes $A$ and $B$, the detected signals in the two homodyne detectors are demodulated by $f_{A}\left(f_{B}\right)$. The resulting demodulated signals are fed back through a PI-controllers to phase controls in the two local oscillator paths. To determine the sign of the $p$ - and $i$-gains, consider first homodyne detection in spatial mode $B$ : The seed beam is along the $\hat{x}+\hat{p}$ quadratures, so when measuring the $\hat{x}$ - or $\hat{p}$-quadrature, if $p$ and $i$ are set correctly, we expect to see a positive offset in the detected signal before demodulation. If a negative offset is observed, the signs of $p$ and $i$ are swapped for the local oscillator phase lock in spatial mode $B$. For spatial mode $A$ the seed beam is along the $-\hat{x}+\hat{p}$ quadrature. As a result, if measuring the $\hat{x}$-quadrature, we expect to see a negative offset of the detected signal before demodulation, while, if measuring the $\hat{p}$-quadrature, we expect to see a positive offset. If not, the signs of $p$ and $i$ are swapped for the local oscillator phase lock in spatial mode $A$. 


\section{Appendix C}

\section{Waveform optimization for gate implentation}

In chapter 5, using two homodyne detectors (HD), Gaussian gates are implemented by projective measurements on the $2 \mathrm{D}$ cluster state generated in chapter 3 . This requires control of basis settings in the two HDs to measure each temporal mode in different bases depending on the desired gate to be implemented. For each HD this is done by controlling the phase of the local oscillator using an electro-optic modulator (EOM). The two EOMs are driven from a two-channel arbitrary waveform generator (AWG), and to implement a given bases sequence for a desired gate, we need to derive the required waveforms on the two channels of the AWG, which is the topic of this appendix.

The two waveforms for the HDs are derived experimentally using the setup shown in Fig. C.1, and the two waveforms are derived individually (one at a time). First, using a balanced fibercoupler, light is divided in two local oscillators $(\mathrm{LO})$ for the two $\mathrm{HDs}, \mathrm{LO}_{\mathrm{A}}$ and $\mathrm{LO}_{\mathrm{B}}$. Each $\mathrm{LO}$ passes through an EOM, polarization control $(\mathrm{P})$, and phase control for phase-locking $(\theta)$. When implementing gates, the LOs continue to each their HD, but to derive the optimal waveform for a desired bases sequence, the two LOs are interfered in a second balanced fiber-coupler to form a Mach-Zehnder-interferometer (MZI). One port of the MZI outputs is detected and used to phaselock the MZI using a DC lock. The other port is detected using a high-speed detector (Thorlabs DET08CFC with $5 \mathrm{GHz}$ bandwidth), and monitored on a digital storage oscilloscope (DSO) with a $2.5 \mathrm{GHz}$ sampling rate. When triggered from a function generator (FG), the AWG (Spectrum Instruments M4i.6631-x8 with $1.25 \mathrm{GS} / \mathrm{s}$ sampling rate and $400 \mathrm{MHz}$ bandwidth) drives an EOM

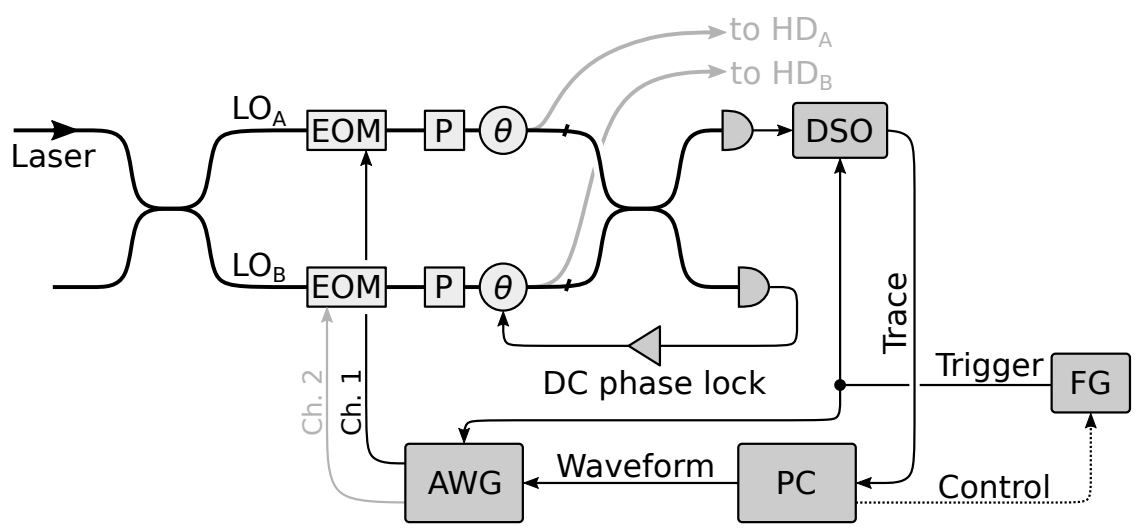

Figure C.1: Setup used for optimizing the waveforms used in the AWG to drive the EOMs to control the basis settings for gate implementation. Abbreviation and explanation are given in text. Note, one waveform is optimized at a time. 


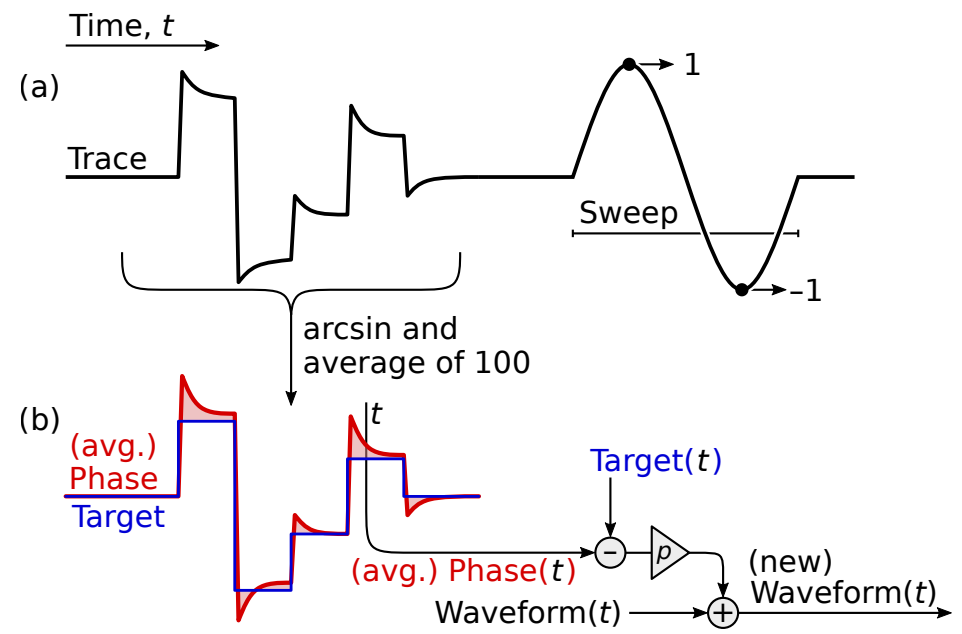

Figure C.2: Illustration of the data processing in the PC of Fig. C.1. (a) shows one acquired trace including an appended sweep used for normalizing the trace. The waveforms used are at maximum $100 \mu \mathrm{s}$ long including the appended sweep. After normalization using the sweep-part of the trace, the corresponding phase is calculated by arcsin and any offset is removed. This is repeated by acquiring 100 traces with the same waveform in order to build up statistics of the measured phase. In (b) the resulting average phase is compared to a target, and the waveform is updated depending on the difference.

(iXblue MPZ-LN-10 with $10 \mathrm{GHz}$ bandwidth) based on a given waveform, and the DSO acquires a trace. The waveforms used are at maximum $100 \mu \mathrm{s}$ long and applied with a $100 \mathrm{~Hz}$ repetition rate, and so it is not necessary to hold the DC phase lock during this process since the feedback is low-pass filtered by few kilo-hertz and does not see the fast change in phase caused by the EOM.

The acquired trace from the DSO is processed in a computer (PC) to update the waveform used by the AWG. This processing is illustrated in Fig. C.2. Every waveform has a "sweep" appended to it that cause a sine-wave in the trace, which is first used for normalizing the trace. This is done by fitting a sine-curve to the sweep, and the trace is normalized using the resulting fitting parameters. Following the normalization, the corresponding phase is derived by

$$
\text { Phase }=\arcsin (\text { Trace }) \text {. }
$$

A possible offset from the DC phase locking (not shown in Fig. C.2) is removed by subtracting an average over time of the phase before the bases sequence (where the phase is flat and should be zero if no offset is present). The process of driving an EOM, acquiring a trace, normalizing the trace, calculating the corresponding phase, and removing any offset, is repeated 100 times with the same waveform to build up statistics of the measured phase. The average phase is then compared to a target phase, and at each time $t$ the difference is calculated, scaled by a gain factor $p$ and added to the waveform in order to compensate for the difference:

$$
\text { Waveform }(t) \leftarrow \text { Waveform }(t)+p\left[\text { Phase }_{\text {avg. }}(t)-\operatorname{Target}(t)\right] \quad, \quad \text { for all } t \text {. }
$$

With this newly updated waveform the whole process is repeated, and the measured phase goes towards the target for each iteration. The absolute value of the gain factor $p$ depends on the setup (optical power, electrical gain, etc.), and should be adjusted before optimizing a waveform. The sign of $p$ depends on which waveform is optimized: The same phase shift in each of the two EOMs leads to opposite change in the acquired traces. The sign of $p$ should be chosen such that $p\left[\right.$ Phase $_{\text {avg. }}(t)$ - Target $\left.(t)\right]$ compensates the waveform for the difference between the phase and the target, while the absolute value should be chosen small enough to not overcompensate in each iteration. 
There is a limit to how close an implemented phase sequence can be made to a target sequence due mainly to limited bandwidths of the AWG and operational amplifiers between the AWG and EOMs (Texas Instruments THS3491). To improve on this, the target should be made with soft edges (transitions between bases), and in this work edges following error-functions was used with a 8 ns transition time. With optimal gain factor, $p$, the change in the measured phase is observed to become negligible after approximately 10 iterations.

Another limitation is the phase range to which the waveform can be optimized. If we are to optimize the waveform to a phase close to $\pi / 2$, we reach the \pm 1 limit in the trace (after normalizing using the sweep of the trace), and the estimated phase is uncertain. Worse is it above $\pi / 2$ since the trace is periodic with the phase. Note though, $\pi$ can always be added or subtracted to a basis with absolute value larger than $\pi / 2$ to move it into the range $[-\pi / 2 ; \pi / 2]$ (such $\pm \pi$ added to the basis setting is compensated in the measurement outcomes by changing the sign of the measurement result of the corresponding mode when implementing gates). However, sometimes it is preferable to keep one basis setting just outside this range to prevent large phase-shifts to a neighbouring basis setting when adding/subtracting $\pi$. The waveform optimization is observed to work best in a smaller range of around $[-\pi / 4 ; \pi / 4]$ where the trace is close to linearly dependent on the phase. Different parts of the waveform is therefore optimized separately: By adding an offset in the MZI DC phase lock, the trace is moved up or down corresponding to adding a phase $\phi$ to the optimal phase optimization range, $[-\pi / 4 ; \pi / 4]+\phi$. In this way, we optimize different parts of the waveform when it falls within the optimization phase range $[-\pi / 4 ; \pi / 4]+\phi$ for different $\phi$ set in the MZI phase lock, while phases outside $[-\pi / 4 ; \pi / 4]+\phi$ are ignored.

With the waveform optimization method presented in this appendix, the resulting optimized waveform takes electronic response into account and avoids capacitive effects, overshooting the phase after each edge (unless too short transition times are used), as well as ringing and other common electronic effects. An example of an optimized and non-optimized waveform is shown in Fig. C.3 for a simple square phase shift. For the non-optimized waveform some capacitive effect is seen in the resulting phase when changing the phase between 0 and $\pi / 4$. This capacitive effect is compensated for in the optimized waveform with some overshooting in the waveform, and the resulting phase is seen to follow the target well. Note, here a transition time less than $8 \mathrm{~ns}$ is possible due to the short phase shift of $\pi / 4$, while for gate implementation in general larger phase shifts are required and $8 \mathrm{~ns}$ transition time is used. Finally, in Fig. C.4 phase of a simple basis setting sequence of $\{0, \pi / 4,0,-\pi / 4,0, \pi / 8,0\}$ is shown using a non-optimized waveform of square functions, and an optimized waveform. Again, the optimized waveform is seen to both compensate for some capacitive effect as well as some ringing effect, and the resulting phase is seen to follow the target well. 


\section{(a) Non-optimized waveform}
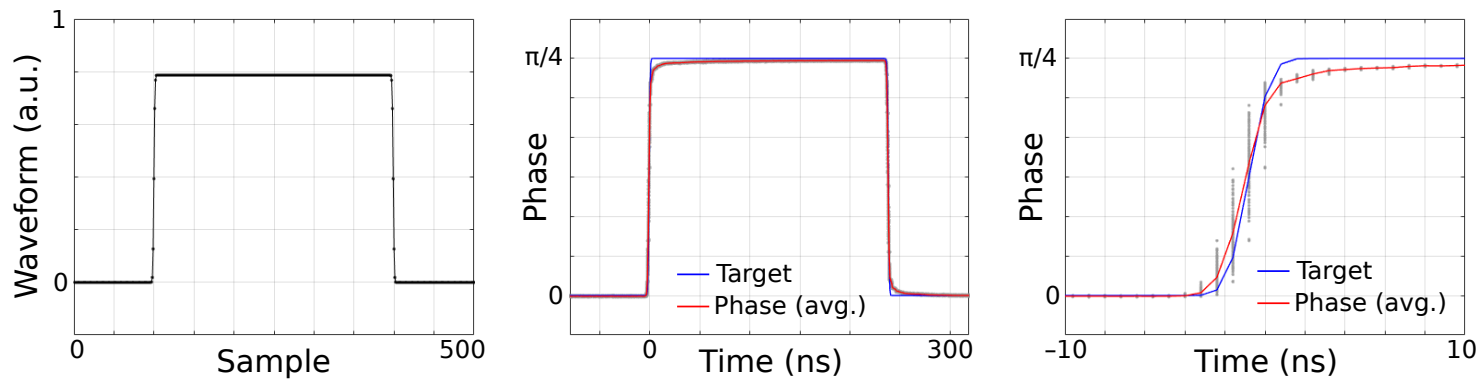

(b) Optimized waveform
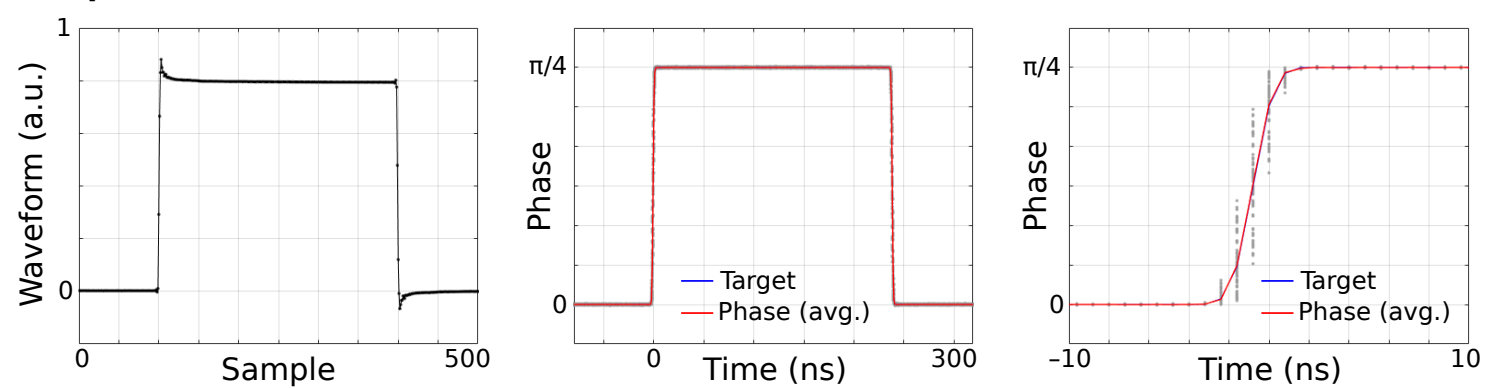

Figure C.3: (a) Phase shift using a non-optimized waveform. To the left a square waveform with $1.25 \mathrm{GS} / \mathrm{s}$ is shown. In the middle the resulting phase is shown with a zoom-in around the rising edge to the right. The phase is an average from 100 traces, and the corresponding 100 phases are shown with grey points. (b) Similar to (a) but with an optimized waveform. The optimized waveform is seen to have some overshooting after each edge to compensate for the capacitive effect seen in (a). The resulting phase follows the target well (note that the target and phase overlap, and the target is barely visible below the phase).
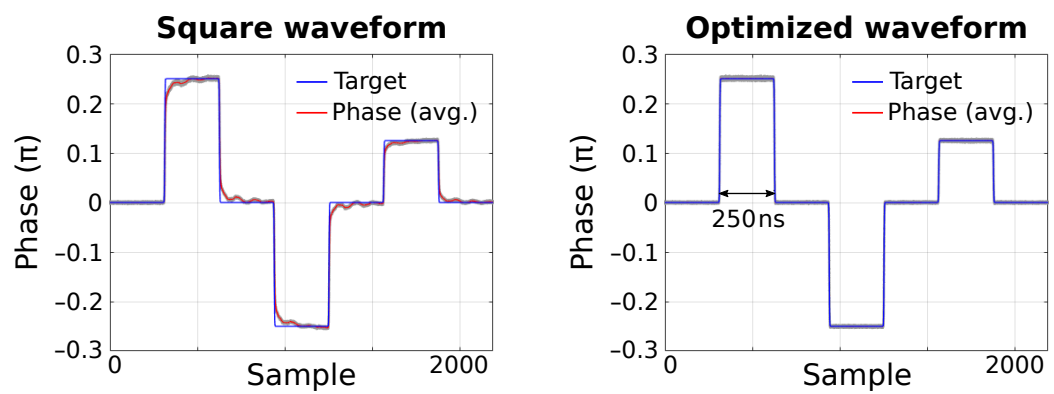

Figure C.4: Phase shift sequence of $\{0, \pi / 4,0,-\pi / 4,0, \pi / 8,0\}$. (left) shows the resulting phase using a non-optimized wave form of square functions. Both a capacitive and ringing effect is seen in the resulting phase. To the right the resulting phase is shown when using the optimized waveform. The phase is shown to follow the target well (note that the target and phase overlap, and the measured phase is barely visible below the target). 


\section{Bibliography}

[1] R. Raussendorf and H. J. Briegel, "A one-way quantum computer," Phys. Rev. Lett. 86, 5188 (2001).

[2] N. C. Menicucci, P. van Loock, M. Gu, C. Weedbrook, T. C. Ralph, and M. A. Nielsen, "Universal quantum computation with continuous-variable cluster states," Phys. Rev. Lett. 97, 110501 (2006).

[3] N. C. Menicucci, "Fault-tolerant measurement-based quantum computing with continuousvariable cluster states," Phys. Rev. Lett. 112, 120504 (2014).

[4] P. van Loock, C. Weedbrook, and M. Gu, "Building Gaussian cluster states by linear optics," Phys. Rev. A 76, 032321 (2007).

[5] M. Gu, C. Weedbrook, N. C. Menicucci, T. C. Ralph, and P. van Loock, "Quantum computing with continuous-variable clusters," Phys. Rev. A 79, 062318 (2009).

[6] R. Ukai, J.-i. Yoshikawa, N. Iwata, P. van Loock, and A. Furusawa, "Universal linear Bogoliubov transformations through one-way quantum computation," Phys. Rev. A 81, 032315 (2010).

[7] N. C. Menicucci, S. T. Flammia, and P. van Loock, "Graphical calculus of Gaussian pure states," Phys. Rev. A 83, 042335 (2011).

[8] N. C. Menicucci, S. T. Flammia, and O. Pfister, "One-way quantum computing in the optical frequency comb," Phys. Rev. Lett. 101, 130501 (2008).

[9] N. C. Menicucci, "Temporal-mode continuous-variable cluster states using linear optics," Phys. Rev. A 83, 062314 (2011).

[10] R. N. Alexander, S. C. Armstrong, R. Ukai, and N. C. Menicucci, "Noise analysis of singlemode Gaussian operations using continuous-variable cluster states," Phys. Rev. A 90, 062324 (2014).

[11] R. N. Alexander, P. Wang, N. Sridhar, M. Chen, O. Pfister, and N. C. Menicucci, "One-way quantum computing with arbitrarily large time-frequency continuous-variable cluster states from a single optical parametric oscillator," Phys. Rev. A 94, 032327 (2016).

[12] R. N. Alexander, S. Yokoyama, A. Furusawa, and N. C. Menicucci, "Universal quantum computation with temporal-mode bilayer square lattices," Phys. Rev. A 97, 032302 (2018).

[13] P. T. Cochrane, G. J. Milburn, and W. J. Munro, "Macroscopically distinct quantumsuperposition states as a bosonic code for amplitude damping," Phys. Rev. A 59, 2631 (1999).

[14] D. Gottesman, A. Kitaev, and J. Preskill, "Encoding a qubit in an oscillator," Phys. Rev. A 64, 012310 (2001). 
[15] M. H. Michael, M. Silveri, R. T. Brierley, V. V. Albert, J. Salmilehto, L. Jiang, and S. M. Girvin, "New class of quantum error-correcting codes for a bosonic mode," Phys. Rev. X 6, 031006 (2016).

[16] M. Pysher, Y. Miwa, R. Shahrokhshahi, R. Bloomer, and O. Pfister, "Parallel generation of quadripartite cluster entanglement in the optical frequency comb," Phys. Rev. Lett. 030505, 107 (2011).

[17] S. Armstrong, J. Morizur, J. Janousek, B. Hage, N. Treps, P. K. Lam, and H. Bachor, "Programmable multimode quantum networks," Nat. Commun. 3, 1026 (2012).

[18] J. Roslund, R. Medeiros de Araújo, S. Jiang, C. Fabre, and N. Treps, "Wavelengthmultiplexed quantum networks with ultrafast frequency combs," Nat. Photonics 8, 109 (2014).

[19] S. Yokoyama, R. Ukai, S. C. Armstrong, C. Sornphiphatphong, T. Kaji, S. Suzuki, J. Yoshikawa, H. Yonezawa, N. C. Menicucci, and A. Furusawa, "Ultra-large-scale continuousvariable cluster states multiplexed in the time domain," Nat. Photonics 7, 982 (2013).

[20] M. Chen, N. C. Menicucci, and O. Pfister, "Experimental realization of multipartite entanglement of 60 modes of a quantum optical frequency comb," Phys. Rev. Lett. 112, 120505 (2014).

[21] J.-i. Yoshikawa, S. Yokoyama, T. Kaji, C. Sornphiphatphong, Y. Shiozawa, K. Makino, and A. Furusawa, "Invited article: Generation of one-million-mode continuous-variable cluster state by unlimited time-domain multiplexing," APL Photonics 1, 060801 (2016).

[22] R. Ukai, N. Iwata, Y. Shimokawa, S. C. Armstrong, A. Politi, J.-i. Yoshikawa, P. van Loock, and A. Furusawa, "Demonstration of unconditional one-way quantum computations for continuous variables," Phys. Rev. Lett. 106, 240504 (2011).

[23] R. Ukai, S. Yokoyama, J.-i. Yoshikawa, P. van Loock, and A. Furusawa, "Demonstration of a controlled-phase gate for continuous-variable one-way quantum computation," Phys. Rev. Lett. 107, 250501 (2011).

[24] X. Su, S. Hao, X. Deng, L. Ma, M. Wang, X. Jia, C. Xie, and K. Peng, "Gate sequence for continuous variable one-way quantum computation," Nat. Commun. 4, 2828 (2013).

[25] W. Asavanant, B. Charoensombutamon, S. Yokoyama, T. Ebihara, T. Nakamura, R. N. Alexander, M. Endo, J.-i. Yoshikawa, N. C. Menicucci, H. Yonezawa, and A. Furusawa, "Onehundred step measurement-based quantum computation multiplexed in the time domain with $25 \mathrm{MHz}$ clock frequency," arXiv:2006.11537 (2020).

[26] C. Reimer, S. Sciara, P. Roztocki, M. Islam, L. R. Cortés, Y. Zhang, B. Fischer, S. Loranger, R. Kashyap, A. Cino, S. T. Chu, B. E. Little, D. J. Moss, L. Caspani, W. J. Munro, J. Azaña, M. Kues, and R. Morandotti, "High-dimensional one-way quantum processing implemented on d-level cluster states," Nat. Phys. 15, 148-154 (2019).

[27] W. Gao, X. Yao, J. Cai, H. Lu, P. Xu, T. Yang, C. Lu, Y. Chen, Z. Chen, and J. Pan, "Experimental measurement-based quantum computing beyond the cluster-state model," Nat. Photonics 5, 117-123 (2011).

[28] P. Walther, K. J. Resch, T. Rudolph, E. Schenck, H. Weinfurter, V. Vedral, M. Aspelmeyer, and A Zeilinger, "Experimental one-way quantum computing," Nature 434, 169 (2005).

[29] B. P. Lanyon, P. Jurcevic, M. Zwerger, C. Hempel, E. A. Martinez, W. Dür, H. J. Briegel, R. Blatt, and C. F. Roos, "Measurement-based quantum computation with trapped ions," Phys. Rev. Lett. 111, 210501 (2013). 
[30] M. V. Larsen, X. Guo, C. R. Breum, J. S. Neergaard-Nielsen, and U. L. Andersen, "Fiber coupled EPR-state generation using a single temporally multiplexed squeezed light source," npj Quantum Information 5, 46 (2019).

[31] M. V. Larsen, X. Guo, C. R. Breum, J. S. Neergaard-Nielsen, and U. L. Andersen, "Deterministic generation of a two-dimensional cluster state," Science 366, 369 (2019).

[32] M. V. Larsen, J. S. Neergaard-Nielsen, and U. L. Andersen, "Architecture and noise analysis of continuous-variable quantum gates using two-dimensional cluster states," Phys. Rev. A 102, $042608(2020)$.

[33] M. V. Larsen, X. Guo, C. R. Breum, J. S. Neergaard-Nielsen, and U. L. Andersen, "Deterministic multi-mode gates on a scalable photonic quantum computing platform," arXiv:2010.14422 (2020).

[34] M. V. Larsen, C. Chamberland, K. Noh, J. S. Neergaard-Nielsen, and U. L. Andersen, "A fault-tolerant continuous-variable measurement-based quantum computation architecture," arXiv:2101.03014 (2021).

[35] T. Kashiwazaki, N. Takanashi, T. Yamashima, T. Kazama, K. Enbutsu, R. Kasahara, T. Umeki, and A. Furusawa, "Continuous-wave 6-dB-squeezed light with 2.5-THz-bandwidth from single-mode PPLN waveguide," APL Photonics 5, 036104 (2020).

[36] N. Takanashi, A. Inoue, T. Kashiwazaki, T. Kazama, K. Enbutsu, R. Kasahara, T. Umeki, and A. Furusawa, "All-optical phase-sensitive detection for ultra-fast quantum computation," Opt. Express 28, 34916 (2020).

[37] W. Asavanant, Y. Shiozawa, S. Yokoyama, B. Charoensombutamon, H. Emura, R. N. Alexander, S. Takeda, J. Yoshikawa, N. C. Menicucci, H. Yonezawa, and A. Furusawa, "Generation of time-domain-multiplexed two-dimensional cluster state," Science 366, 373 (2019).

[38] B. W. Walshe, L. J. Mensen, B. Q. Baragiola, and N. C. Menicucci, "Robust fault tolerance for continuous-variable cluster states with excess antisqueezing," Phys. Rev. A 100, 010301(R) (2019).

[39] B. Q. Baragiola, G. Pantaleoni, R. N. Alexander, A. Karanjai, and N. C. Menicucci, "AllGaussian universality and fault tolerance with the Gottesman-Kitaev-Preskill code," Phys. Rev. Lett. 123, 200502 (2019).

[40] H. Yamasaki, T. Matsuura, and M. Koashi, "Cost-reduced all-Gaussian universality with the Gottesman-Kitaev-Preskill code: Resource-theoretic approach to cost analysis," Phys. Rev. Research 2, 023270 (2020).

[41] J. Hastrup, M. V. Larsen, J. S. Neergaard-Nielsen, N. C. Menicucci, and U. L. Andersen, "Cubic phase gates are not suitable for non-Clifford operations on GKP states," arXiv:2009.05309 (2020).

[42] B. W. Walshe, B. Q. Baragiola, R. N. Alexander, and N. C. Menicucci, "Continuous-variable gate teleportation and bosonic-code error correction," Phys. Rev. A 102, 062411 (2020).

[43] K. Fukui, A. Tomita, A. Okamoto, and K. Fujii, "High-threshold fault-tolerant quantum computation with analog quantum error correction," Phys. Rev. X 8, 021054 (2018).

[44] B. H. Wu, R. N. Alexander, S. Liu, and Z. Zhang, "Quantum computing with multidimensional continuous-variable cluster states in a scalable photonic platform," Phys. Rev. Research 2, 023138 (2020).

[45] K. Fukui, W. Asavanant, and A. Furusawa, "Temporal-mode continuous-variable threedimensional cluster state for topologically protected measurement-based quantum computation," Phys. Rev. A 102, 032614 (2020). 
[46] J. E. Bourassa, R. N. Alexander, M. Vasmer, A. Patil, I. Tzitrin, T. Matsuura, D. Su, B. Q. Baragiola, S. Guha, G. Dauphinais, K. K. Sabapathy, N. C. Menicucci, and I. Dhand, "Blueprint for a scalable photonic fault-tolerant quantum computer," arXiv:2010.02905 (2020).

[47] M. A. Nielsen and I. L. Chuang, "Quantum Computation and Quantum Information", Cambridge University Press, England (2000).

[48] C. H. Bennett and D. P. DiVincenzo, "Quantum information and computation," Nature 404, $247(2000)$.

[49] A. M. Dalzell, A. W. Harrow, D. E. Koh, and R. L. La Placa, "How many qubits are needed for quantum computational supremacy?" Quantum 4, 264 (2020).

[50] D. Gottesman and I. L. Chuang, "Quantum teleportation as a universal computational primitive," Nature 402, 390 (1999).

[51] R. Raussendorf, D. E. Browne, and H. J. Briegel, "Measurement-based quantum computation with cluster states," Phys. Rev. A 68, 022312 (2003).

[52] O. Glöckl, S. Lorenz, C. Marquardt, J. Heersink, M. Brownnutt, C. Silberhorn, Q. Pan, P. van Loock, N. Korolkova, and G. Leuchs, "Experiment towards continuous-variable entanglement swapping: Highly correlated four-partite quantum state," Phys. Rev. A 68, 012319 (2003).

[53] X. Su, A. Tan, X. Jia, J. Zhang, C. Xie, and K. Peng, "Experimental preparation of quadripartite cluster and Greenberger-Horne-Zeilinger entangled states for continuous variables," Phys. Rev. Lett. 98, 070502 (2007).

[54] R. Dong, J. Heersink, J. Yoshikawa, O. Glöckl, U. L. Andersen, and G. Leuchs, "An efficient source of continuous variable polarization entanglement," New J. Phys. 9, 410 (2007).

[55] M. Yukawa, R. Ukai, P. van Loock, and A. Furusawa, "Experimental generation of four-mode continuous-variable cluster states," Phys. Rev. A 78, 012301 (2008).

[56] T. Aoki, G. Takahashi, T. Kajiya, J. Yoshikawa, S. L. Braunstein, P. van Loock, and A. Furusawa, "Quantum error correction beyond qubits," Nat. Phys. 5, 541 (2009).

[57] U. L. Andersen, T. Gehring, C. Marquardt, and G. Leuchs, "30 years of squeezed light generation," Physica Scripta 91, 053001 (2016).

[58] R. N. Alexander, N. C. Gabay, P. P. Rohde, and N. C. Menicucci, "Measurement-based linear optics," Phys. Rev. Lett. 118, 110503 (2017).

[59] K. R. Motes, A. Gilchrist, J. P. Dowling, and P. P. Rohde, "Scalable boson sampling with time-bin encoding using a loop-based architecture," Phys. Rev. Lett. 113, 120501 (2014).

[60] S. Takeda and A. Furusawa, "Universal quantum computing with measurement-induced continuous-variable gate sequence in a loop-based architecture," Phys. Rev. Lett. 119, 120504 (2017).

[61] M. D. Reid, P. D. Drummond, W. P. Bowen, E. G. Cavalcanti, P. K. Lam, H. A. Bachor, U. L. Andersen, and G. Leuchs, "Colloquium: The Einstein-Podolsky-Rosen paradox: From concepts to applications," Rev. Mod. Phys. 81, 1727 (2009).

[62] A. Furusawa, J. L. Sørensen, S. L. Braunstein, C. A. Fuchs, H. J. Kimble, and E. S. Polzik, "Unconditional quantum teleportation," Science 282, 706 (1998).

[63] L. S. Madsen, V. C. Usenko, M. Lassen, R. Filip, and U. L. Andersen, "Continuous variable quantum key distribution with modulated entangled states," Nat. Commun. 3, 1083 (2012). 
[64] Z. Y. Ou, S. F. Pereira, H. J. Kimble, and K. C. Peng, "Realization of the Einstein-PodolskyRosen paradox for continuous variable," Phys. Rev. Lett. 68, 3663 (1992).

[65] P. J. Bardroff and S. Stenholm, "Two-mode laser with excess noise," Phys. Rev. A 62, 023814 (2000)

[66] C. Schori, J. L. Sørensen, and E. S. Polzik, "Narrow-band frequency tunable light source of continuous quadrature entanglement," Phys. Rev. A 66, 033802 (2002).

[67] W. P. Bowen, N. Treps, R. Schnabel, and P. K. Lam, "Experimental demonstration of continuous variable polarization entanglement," Phys. Rev. Lett. 89, 253601 (2002).

[68] K. Hayasaka, Y. Zhang, and K. Kasai, "Generation of twin beams from an optical parametric oscillator pumped by a frequency-doubled diode laser," Optics Lett. 29, 1665 (2004).

[69] J. Laurat, T. Coudreau, G. Keller, N. Treps, and C. Fabre, "Effects of mode coupling on the generation of quadrature Einstein-Podolsky-Rosen entanglement in a type-II optical parametric oscillator below threshold," Phys. Rev. A 71, 022313 (2005).

[70] J. Wenger, A. Ourjoumtsev, R. Tualle-Brouri, and P. Grangier, "Time-resolved homodyne characterization of individual quadrature-entangled pulses," Eur. Phys. J. D 32, 391 (2005).

[71] A. S. Villar, M. Martinelli, C. Fabre, and P. Nussenzveig, "Direct production of tripartite pump-signal-idler entanglement in the above-threshold optical parametric oscillator," Phys. Rev. Lett. 97, 140504 (2006).

[72] C. Silberhorn, P. K. Lam, O. Weiß, F. König, N. Korolkova, and G. Leuchs, "Generation of continuous variable Einstein-Podolsky-Rosen entanglement via the Kerr nonlinearity in an optical fiber," Phys. Rev. Lett. 86, 4267 (2001).

[73] W. P. Bowen, R. Schnabel, P. K. Lam, and T. C. Ralph, "Experimental investigation of criteria for continuous variable entanglement," Phys. Rev. Lett. 90, 043601 (2003).

[74] N. Takei, H. Yonezawa, T. Aoki, and A. Furusawa, "High-fidelity teleportation beyond the no-cloning limit and entanglement swapping for continuous variables," Phys. Rev. Lett. 94, 220502 (2005).

[75] L. Duan, G. Giedke, J. I. Cirac, and P. Zoller, "Inseparability criterion for continuous variable systems," Phys. Rev. Lett. 84, 2722 (2000).

[76] C. Weedbrook, S. Pirandola, R. García-Patrón, N. J. Cerf, T. C. Ralph, J. H. Shapiro, and S. Lloyd, "Gaussian quantum information," Rev. Mod. Phys. 84, 621 (2012).

[77] M. D. Reid, "Demonstration of the Einstein-Podolsky-Rosen paradox using nondegenerate parametric amplification," Phys. Rev. A 40, 913 (1989).

[78] T. Aoki, G. Takahashi, and A. Furusawa, "Squeezing at 946nm with periodically poled KTiOPO4," Opt. Express 14, 6930 (2006).

[79] M. J. Collett and C. W. Gardiner, "Squeezing of intracavity and traveling-wave light fields produced in parametric amplification," Phys. Rev. A 30, 1386 (1984).

[80] F. Kaneda and P. G. Kwiat, "High-efficiency single-photon generation via large-scale active time multiplexing," arXiv:1803.04803 (2018).

[81] C. Weedbrook A. Furusawa S. Pirandola, J. Eisert and S. Braunstein, "Advances in quantum teleportation," Nature Photonics 9, 641 (2015).

[82] X. Guo, C. R. Breum, J. Borregaard, S. Izumi, M. V. Larsen, T. Gehring, M. Christandl, J. S. Neergaard-Nielsen, and U. L. Andersen, "Distributed quantum sensing in a continuous variable entangled network," Nat. Phys. 16, 281 (2020). 
[83] L. Neuhaus, R. Metzdorff, S. Chua, T. Jacqmin, T. Briant, A. Heidmann, P.-F. Cohadon, and S. Deléglise, "PyRPL (Python Red Pitaya Lockbox) - An open-source software package for FPGA-controlled quantum optics experiments," CLEO/Europe-EQEC IEEE, www.pyrpl.org (2017).

[84] H. Mei, B. Li, H. Huang, and R. Rao, "Piezoelectric optical fiber stretcher for application in an atmospheric optical turbulence sensor," Appl. Opt. 46, 4371 (2007).

[85] N. Takei, N. Lee, D. Moriyama, J. S. Neergaard-Nielsen, and A. Furusawa, "Time-gated Einstein-Podolsky-Rosen correlation," Phys. Rev. A 74, 060101 (2006).

[86] L. Mandel and E. Wolf, Optical Coherence and Quantum Optics, Cambridge University Press (1995).

[87] R. Simon, "Peres-Horodecki separability criterion for continuous variable systems," Phys. Rev. Lett. 84, 2726 (2000).

[88] M. V. Larsen, X. Guo, C. R. Breum, J. S. Neergaard-Nielsen, and U. L. Andersen, "Data and analysis code for 'Deterministic generation of a two-dimensional cluster state'," figshare.com: http://doi.org/10.11583/DTU.8647211 (2020).

[89] T. D. Ladd, F. Jelezko, R. Laflamme, Y. Nakamura, C. Monroe, and J. L. O'Brien, "Quantum computers," Nature 464, 45 (2010).

[90] Y. Wang, Y. Li, Z. Yin, and B. Zeng, "16-qubit IBM universal quantum computer can be fully entangled," npj Quantum Information 4, 46 (2018).

[91] O. Mandel, M. Greiner, A. Widera, T. Rom, T. W. Hänsch, and Immanuel Bloch, "Controlled collisions for multi-particle entanglement of optically trapped atoms," Nature 425, 937 (2003).

[92] Y. Tokunaga, S. Kuwashiro, T. Yamamoto, M. Koashi, and N. Imoto, "Generation of highfidelity four-photon cluster state and quantum-domain demonstration of one-way quantum computing," Phys. Rev. Lett. 100, 210501 (2008).

[93] I. Schwartz, D. Cogan, E. R. Schmidgall, Y. Don, L. Gantz, O. Kenneth, N. H. Lindner, and D. Gershoni, "Deterministic generation of a cluster state of entangled photons," Science 112, 434 (2016).

[94] S. E. Economou, N. Lindner, and T. Rudolph, "Optically generated 2-dimensional photonic cluster state from coupled quantum dots," Phys. Rev. Lett. 105, 093601 (2010).

[95] P. Wang, M. Chen, N. C. Menicucci, and O. Pfister, "Weaving quantum optical frequency combs into continuous-variable hypercubic cluster states," Phys. Rev. A 90, 032325 (2014).

[96] Y. Cai, J. Roslund, G. Ferrini, F. Arzani, X. Xu, C. Fabre, and N. Treps, "Multimode entanglement in reconfigurable graph states using optical frequency combs," Nat. Commun. 8, 15645 (2017).

[97] S. D. Bartlett, B. C. Sanders, S. L. Braunstein, and K. Nemoto, "Efficient classical simulation of continuous variable quantum information processes," Phys. Rev. Lett. 88, 097904 (2002).

[98] G. S. Thekkadath, D. S. Phillips, J. F. F. Bulmer, W. R. Clements, A. Eckstein, B. A. Bell, J. Lugani, T. A. W. Wolterink, A. Lita, S. W. Nam, T. Gerrits, C. G. Wade, and I. A. Walmsley, "Tuning between photon-number and quadrature measurements with weak-field homodyne detection," Phys. Rev. A 101, 031801(R) (2020).

[99] B. Hacker, S. Welte, S. Daiss, A. Shaukat, S. Ritter, L. Li, and G. Rempe, "Deterministic creation of entangled atom-light Schrödinger-cat states," Nat. Photonics 13, 110 (2019). 
[100] P. Campagne-Ibarcq, A. Eickbusch, S. Touzard, E. Zalys-Geller, N. E. Frattini, V. V. Sivak, P. Reinhold, S. Puri, S. Shankar, R. J. Schoelkopf, L. Frunzio, M. Mirrahimi, and M. H. Devoret, "Quantum error correction of a qubit encoded in grid states of an oscillator," Nature 584, 368 (2020).

[101] C. Flühmann, T. L. Nguyen, M. Marinelli, V. Negnevitsky, K. Mehta, and J. P. Home, "Encoding a qubit in a trapped-ion mechanical oscillator," Nature 566, 513 (2019).

[102] D. Su, K. K. Sabapathy, C. R. Myers, H. Qi, C. Weedbrook, and K. Brádler, "Implementing quantum algorithms on temporal photonic cluster states," Phys. Rev. A 98, 032316 (2018).

[103] P. van Loock and A. Furusawa, "Detecting genuine multipartite continuous-variable entanglement," Phys. Rev. A 67, 052315 (2003).

[104] Y. Miwa, J.-i. Yoshikawa, P. van Loock, and A. Furusawa, "Demonstration of a universal one-way quantum quadratic phase gate," Phys. Rev. A 80, 050303(R) (2009).

[105] Y. Wang, X. Su, H. Shen, A. Tan, C. Xie, and K. Peng, "Toward demonstrating controlled-X operation based on continuous variable four-partite cluster state and quantum teleporters," Phys. Rev. A 81, 022311 (2010).

[106] S. T. Flammia, N. C. Menicucci, and O. Pfister, "The optical frequency comb as a one-way quantum computer," J. Phys. B: At. Mol. Opt. Phys. 42, 114009 (2009).

[107] R. N. Alexander and N. C. Menicucci, "Flexible quantum circuits using scalable continuousvariable cluster states," Phys. Rev. A 93, 062326 (2016).

[108] A. P. Lund, T. C. Ralph, and H. L. Haselgrove, "Fault-tolerant linear optical quantum computing with small-amplitude coherent states," Phys. Rev. Lett. 100, 030503 (2008).

[109] V. V. Albert, K. Noh, K. Duivenvoorden, D. J. Young, R. T. Brierley, P. Reinhold, C. Vuillot, L. Li, C. Shen, S. M. Girvin, B. M. Terhal, and L. Jiang, "Performance and structure of single-mode bosonic codes," Phys. Rev. A 97, 032346 (2018).

[110] A. M. Steane, "Overhead and noise threshold of fault-tolerant quantum error correction," Phys. Rev. A 68, 042322 (2003).

[111] E. Knill, "Quantum computing with realistically noisy devices," Nature 434, 39 (2005).

[112] I. Tzitrin, J. E. Bourassa, N. C. Menicucci, and K. K. Sabapathy, "Progress towards practical qubit computation using approximate Gottesman-Kitaev-Preskill codes," Phys. Rev. A 101, 032315 (2020).

[113] B. M. Terhal, J. Conrad, and C. Vuillot, "Towards scalable bosonic quantum error correction," Quantum Science and Technology 5, 043001 (2020).

[114] S. Takeda, K. Takase, and A. Furusawa, "On-demand photonic entanglement synthesizer," Science Advances 5, eaaw4530 (2019).

[115] H. Vahlbruch, M. Mehmet, K. Danzmann, and R. Schnabel, "Detection of 15 dB squeezed states of light and their application for the absolute calibration of photoelectric quantum efficiency," Phys. Rev. Lett. 117, 110801 (2016).

[116] S. L. Braunstein, "Squeezing as an irreducible resource," Phys. Rev. A 71, 055801 (2005).

[117] S. D. Bartlett and B. C. Sanders, "Efficient classical simulation of optical quantum information circuits," Phys. Rev. Lett. 89, 207903 (2002).

[118] S. Lloyd and S. L. Braunstein, "Quantum computation over continuous variables," Phys. Rev. Lett. 82, 1784 (1999). 
[119] U. L. Andersen, J. S. Neergaard-Nielsen, P. van Loock, and A. Furusawa, "Hybrid discreteand continuous-variable quantum information," Nat. Phys. 11, 713 (2015).

[120] Y. Ra, A. Dufour, M. Walschaers, C. Jacquard, T. Michel, C. Fabre, and N. Treps, "NonGaussian quantum states of a multimode light field," Nat. Phys. 16, 144 (2020).

[121] K. Noh and C. Chamberland, "Fault-tolerant bosonic quantum error correction with the surface-Gottesman-Kitaev-Preskil code," Phys. Rev. A 101, 012316 (2020).

[122] L. Egan, D. M. Debroy, C. Noel, A. Risinger, D. Zhu, D. Biswas, M. Newman, M. Li, K. R. Brown, M. Cetina, and C. Monroe, "Fault-tolerant operation of a quantum error-correction code," arXiv:2009.11482 (2020).

[123] F. Arute, K. Arya, R. Babbush, D. Bacon, J. C. Bardin, R. Barends, R. Biswas, S. Boixo, F. G. S. L. Brandao, D. A. Buell, B. Burkett, Y. Chen, Z. Chen, B. Chiaro, R. Collins, W. Courtney, A. Dunsworth, E. Farhi, B. Foxen, A. Fowler, C. Gidney, M. Giustina, R. Graff, K. Guerin, S. Habegger, M. P. Harrigan, M. J. Hartmann, A. Ho, M. Hoffmann, T. Huang, T. S. Humble, S. V. Isakov, E. Jeffrey, Z. Jiang, D. Kafri, K. Kechedzhi, J. Kelly, P. V. Klimov, S. Knysh, A. Korotkov, F. Kostritsa, D. Landhuis, M. Lindmark, E. Lucero, D. Lyakh, Sa. Mandrà, J. R. McClean, M. McEwen, A. Megrant, X. Mi, K. Michielsen, M. Mohseni, J. Mutus, O. Naaman, M. Neeley, C. Neill, M. Y. Niu, E. Ostby, A. Petukhov, J. C. Platt, C. Quintana, E. G. Rieffel, P. Roushan, N. C. Rubin, D. Sank, K. J. Satzinger, V. Smelyanskiy, K. J. Sung, M. D. Trevithick, A. Vainsencher, B. Villalonga, T. White, Z. J. Yao, P. Yeh, A. Zalcman, H. Neven, and J. M. Martinis, "Quantum supremacy using a programmable superconducting processor," Nature 574, 505 (2019).

[124] S. L. Braunstein and H. J. Kimble, "Teleportation of continuous quantum variables," Phys. Rev. Lett. 80, 869 (1990).

[125] J. F. Tasker, J. Frazer, G. Ferranti, E. J. Allen, L. F. Brunel, S. Tanzilli, V. D'Auria, and J. C. F. Matthews, "Silicon photonics interfaced with integrated electronics for $9 \mathrm{GHz}$ measurement of squeezed light," Nat. Photonics 15, 11 (2021).

[126] J. Preskill, "Quantum computing in the NISQ era and beyond," Quantum 2, 79 (2018).

[127] S. Pirandola, S. Mancini, D. Vitali, and P. Tombesi, "Generating continuous variable quantum codewords in the near-field atomic lithography," J. Phys. B 39, 997 (2006).

[128] K. R. Motes, B. Q. Baragiola, A. Gilchrist, and N. C. Menicucci, "Encoding qubits into oscillators with atomic ensembles and squeezed light," Phys. Rev. A 95, 053819 (2017).

[129] D. J. Weigand and B. M. Terhal, "Generating grid states from Schrödinger-cat states without postselection," Phys. Rev. A 97, 022341 (2018).

[130] M. Eaton, R. Nehra, and O. Pfister, "Non-Gaussian and Gottesman-Kitaev-Preskill state preparation by photon catalysis," New J. Phys. 21, 113034 (2019).

[131] A. Y. Kitaev, "Fault-tolerant quantum computation by anyons," Ann. Phys. (Amsterdam) 303, 2 (2003).

[132] R. Raussendorf, J. Harrington, and G. Goyal, "A fault-tolerant one-way quantum computer," Ann. Phys. (Amsterdam) 321, 2242 (2006).

[133] R. Raussendorf, J. Harrington, and G. Goyal, "Topological fault-tolerance in cluster state quantum computation," New J. Phys. 9, 199 (2007).

[134] R. Raussendorf and J. Harrington, "Fault-tolerant quantum computation with high threshold in two dimensions," Phys. Rev. Lett. 98, 190504 (2007). 
[135] S. B. Bravyi and A. Y Kitaev, "Quantum codes on a lattice with boundary," arXiv:9811052 (1998).

[136] E. Dennis, A. Kitaev, A. Landahl, and J. Preskill, "Topological quantum memory," J. Math. Phys. 43, 4452 (2002).

[137] A. G. Fowler, M. Mariantoni, J. M. Martinis, and A. N. Cleland, "Surface codes: Towards practical large-scale quantum computation," Phys. Rev. A 86, 032324 (2012).

[138] D. Bonneau, M. Lobino, P. Jiang, C. M. Natarajan, M. G. Tanner, R. H. Hadfield, S. N. Dorenbos, V. Zwiller, M. G. Thompson, and J. L. O'Brien, "Fast path and polarization manipulation of telecom wavelength single photons in lithium niobate waveguide devices," Phys. Rev. Lett. 108, 053601 (2012).

[139] Y. He, X. Ding, Z.-E. Su, H.-L. Huang, J. Qin, C. Wang, S. Unsleber, C. Chen, H. Wang, Y.M. He, X.-L. Wang, W.-J. Zhang, S.-J. Chen, C. Schneider, M. Kamp, L.-X. You, Z. Wang, S. Höfling, C.-Y. Lu, , and J.-W. Pan, "Time-bin-encoded boson sampling with a singlephoton device," Phys. Rev. Lett. 118, 190501 (2017).

[140] K. Noh, V. V. Albert, and L. Jiang, "Quantum capacity bounds of Gaussian thermal loss channels and achievable rates with Gottesman-Kitaev-Preskill codes," IEEE Transactions on Information Theory 65, 2563 (2019).

[141] J. S. Ivan, K. K. Sabapathy, and R. Simon, "Operator-sum representation for bosonic Gaussian channels," Phys. Rev. A 84, 042311 (2011).

[142] R. García-Patrón, C. Navarrete-Benlloch, S. Lloyd, J. H. Shapiro, and N. J. Cerf, "Majorization theory approach to the Gaussian channel minimum entropy conjecture," Phys. Rev. Lett. 108, 110505 (2012).

[143] K. Duivenvoorden, B. M. Terhal, and D. Weigand, "Single-mode displacement sensor," Phys. Rev. A 95, 012305 (2017).

[144] K. Fukui, A. Tomita, and A. Okamoto, "Analog quantum error correction with encoding a qubit into an oscillator," Phys. Rev. Lett. 119, 180507 (2017).

[145] H. Bombin and M. A. Martin-Delgado, "Optimal resources for topological two-dimensional stabilizer codes: Comparative study," Phys. Rev. A 76, 012305 (2007).

[146] Y. Tomita and K. M. Svore, "Low-distance surface codes under realistic quantum noise," Phys. Rev. A 90, 062320 (2014).

[147] C. Vuillot, H. Asasi, Y. Wang, L. P. Pryadko, and B. M. Terhal, "Quantum error correction with the toric Gottesman-Kitaev-Preskill code," Phys. Rev. A 99, 032344 (2019).

[148] J. Edmonds, "Paths, trees, and flowers," Can. J. Math. 17, 449 (1965).

[149] J. Edmonds, "Maximum matching and a polyhedron with 0,1 vertices," J. Res. Natl. Bur. Stand., Sect. B 69 B, 125 (1965).

[150] D. P. DiVincenzo and P. Aliferis, "Effective fault-tolerant quantum computation with slow measurements," Phys. Rev. Lett. 98, 020501 (2007).

[151] B. M. Terhal, "Quantum error correction for quantum memories," Rev. Mod. Phys. 87, 307 (2015).

[152] C. Chamberland, P. Iyer, and D. Poulin, "Fault-tolerant quantum computing in the Pauli or Clifford frame with slow error diagnostics," Quantum 2, 43 (2018). 
[153] B. de Neeve, T. L. Nguyen, T. Behrle, and J. Home, "Error correction of a logical grid state qubit by dissipative pumping," arXiv:2010.09681 (2020).

[154] H. M. Vasconcelos, L. Sanz, and S. Glancy, "All-optical generation of states for 'Encoding a qubit in an oscillator'," Opt. Lett. 34, 3261 (2010).

[155] Y. Shi, C. Chamberland, and A. Cross, "Fault-tolerant preparation of approximate GKP states," New J. Phys. 21, 093007 (2019).

[156] D. Su, C. R. Myers, and K. K. Sabapathy, "Conversion of Gaussian states to non-Gaussian states using photon-number-resolving detectors," Phys. Rev. A 100, 052301 (2019).

[157] A. I. Lvovsky, B. C. Sanders, and W. Tittel, "Optical quantum memory," Nat. Photonics 3, 706 (2009).

[158] T. Zhong, J. M. Kindem, J. G. Bartholomew, J. Rochman, I. Craiciu, E. Miyazono, M. Bettinelli, E. Cavalli, V. Verma, S. W. Nam, F. Marsili, M. D. Shaw, A. D. Beyer, and A. Faraon, "Nanophotonic rare-earth quantum memory with optically controlled retrieval," Science $\mathbf{3 5 7}$, 1392 (2017).

[159] M. Bouillard, G. Boucher, J. F. Ortas, B. Pointard, and R. Tualle-Brouri, "Quantum storage of single-photon and two-photon Fock states with an all-optical quantum memory," Phys. Rev. Lett. 122, 210501 (2019).

[160] Y. Hashimoto, T. Toyama, J.-i. Yoshikawa, K. Makino, F. Okamoto, R. Sakakibara, S. Takeda, P. van Loock, and A. Furusawa, "All-optical storage of phase-sensitive quantum states of light," Phys. Rev. Lett. 123, 113603 (2019).

[161] C. Horsman, A. G. Fowler, S. Devitt, and R. van Meter, "Surface code quantum computing by lattice surgery," New J. Phys. 14, 123011 (2012).

[162] D. Litinski, "Magic state distillation: Not as costly as you think," Qauntum 3, 205 (2019).

[163] D. S. Wang, A. G. Fowler, and L. C. L. Hollenberg, "Surface code quantum computing with error rates over 1\%," Phys. Rev. A 83, 020302(R) (2011).

[164] C. Chamberland, G. Zhu, T. J. Yoder, J. B. Hertzberg, and A. W. Cross, "Topological and subsystem codes on low-degree graphs with flag qubits," Phys. Rev. X 10, 011022 (2020).

[165] C. Chamberland, A. Kubica, T. J. Yoder, and G. Zhu, "Triangular color codes on trivalent graphs with flag qubits," New Journal of Physics 22, 023019 (2020).

[166] C. Chamberland, K. Noh, P. Arrangoiz-Arriola, E. T. Campbell, C. T. Hann, J. Iverson, H. Putterman, T. C. Bohdanowicz, S. T. Flammia, A. Keller, G. Refael, J. Preskill, L. Jiang, A. H. Safavi-Naeini, O. Painter, and F. G. S. L. Brandão, "Building a fault-tolerant quantum computer using concatenated cat codes," arXiv:2012.04108 (2020).

[167] J. Wang, F. Sciarrino, A. Laing, and M. G. Thompson, "Integrated photonic quantum technologies," Nat. Photonics 14, 273 (2020).

[168] H. J. Kimble, "The quantum internet," Nature 453, 1023 (2008).

[169] S. Wehner, D. Elkouss, and R. Hanson, "Quantum internet: A vision for the road ahead," Science 362, eaam9288 (2018).

[170] S. Takeda and A. Furusawa, "Toward large-scale fault-tolerant universal photonic quantum computing," APL Photonics 4, 060902 (2019).

[171] X. Zhu, C.-H. Chang, C. González-Arciniegas, A. Pe'er, J. Higgins, and O. Pfister, "Hypercubic cluster states in the phase modulated quantum optical frequency comb," arXiv:1912.11215 (2019). 
[172] O. Pfister, "Continuous-variable quantum computing in the quantum optical frequency comb," J. Phys. B: At. Mol. Opt. Phys. 53, 012001 (2019).

[173] F. E. Becerra, J. Fan, G. Baumgartner, J. Goldhar, J. T. Kosloski, and A. Migdall, "Experimental demonstration of a receiver beating the standard quantum limit for multiple nonorthogonal state discrimination," Nat. Photonics 7, 147 (2013).

[174] F. E. Becerra, J. Fan, and A. Migdall, "Photon number resolution enables quantum receiver for realistic coherent optical communications," Nat. Photonics 9, 48 (2015).

[175] S. Izumi, J. S. Neergaard-Nielsen, and U. L. Andersen, "Tomography of a feedback measurement with photon detection," Phys. Rev. Lett. 124, 070502 (2020). 